\title{
ॠUSGS
}

science for a changing world

In cooperation with the Bureau of Land Management

\section{Potential Demographic and Genetic Effects of a Sterilant Applied to Wild Horse Mares}

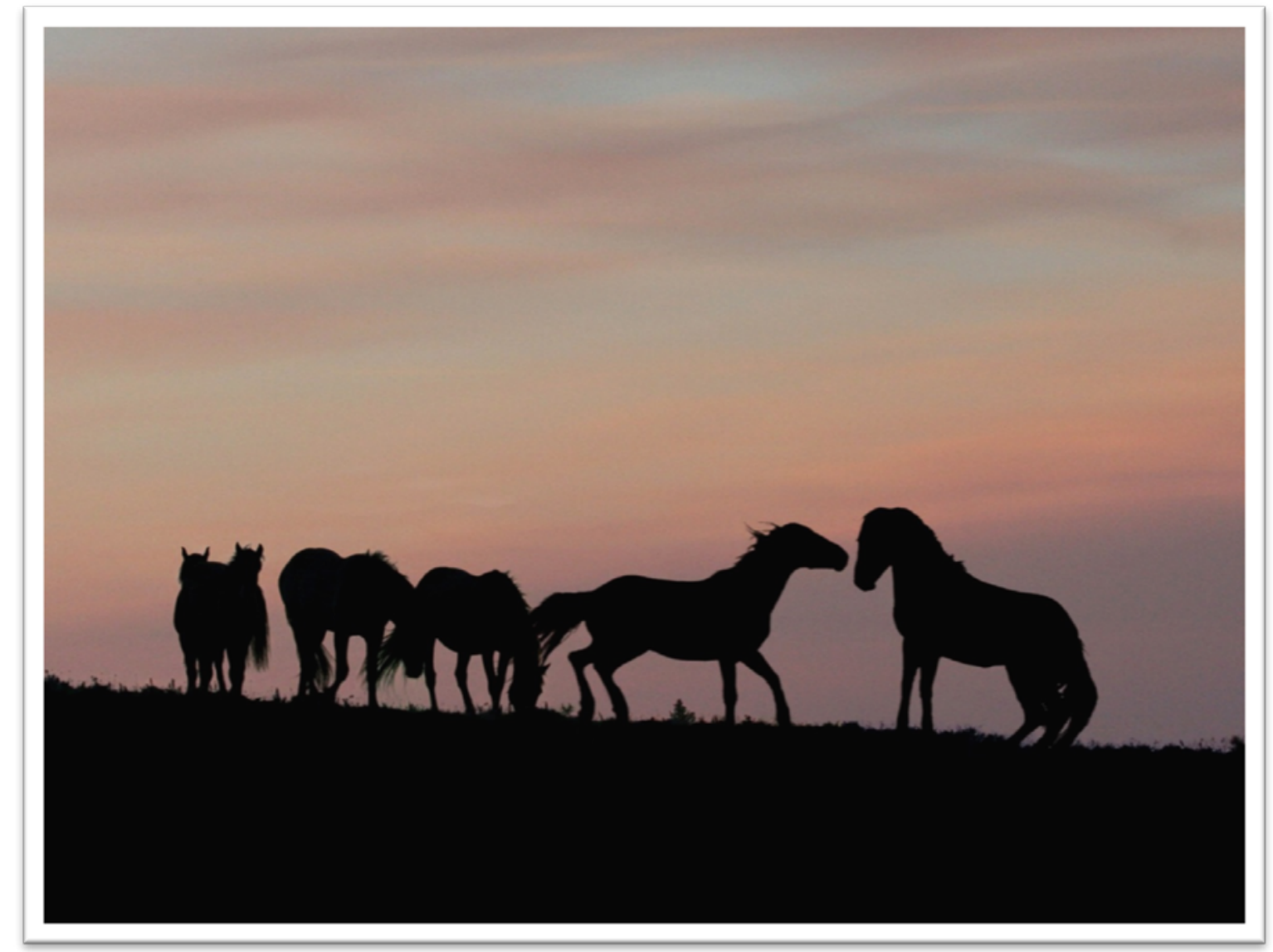

Open-File Report 2015-1045

U.S. Department of the Interior

U.S. Geological Survey 
Photo credit: Jason Ransom, USGS Fort Collins Science Center 


\section{Potential Demographic and Genetic Effects of a Sterilant Applied to Wild Horse Mares}

By James E. Roelle and Sara J. Oyler-McCance

In cooperation with the Bureau of Land Management

Open-File Report 2015-1045

U.S. Department of the Interior

U.S. Geological Survey 


\section{U.S. Department of the Interior \\ SALLY JEWELL, Secretary}

\section{U.S. Geological Survey \\ Suzette M. Kimball, Acting Director}

U.S. Geological Survey, Reston, Virginia: 2015

For more information on the USGS-the Federal source for science about the Earth,

its natural and living resources, natural hazards, and the environment-visit

http://www.usgs.gov or call 1-888-ASK-USGS

For an overview of USGS information products, including maps, imagery, and publications, visit http://www.usgs.gov/pubprod

To order this and other USGS information products, visit http://store.usgs.gov

Any use of trade, firm, or product names is for descriptive purposes only and does not imply endorsement by the U.S. Government.

Although this information product, for the most part, is in the public domain, it also may contain copyrighted materials as noted in the text. Permission to reproduce copyrighted items must be secured from the copyright owner.

Suggested citation:

Roelle, J.E., and Oyler-McCance, S.J., 2015, Potential demographic and genetic effects of a sterilant applied to wild horse mares: U.S. Geological Survey Open-File Report 2015-1045, 153 p., http://dx.doi.org/10.3133/ofr20151045.

ISSN 2331-1258 (online) 


\section{Contents}

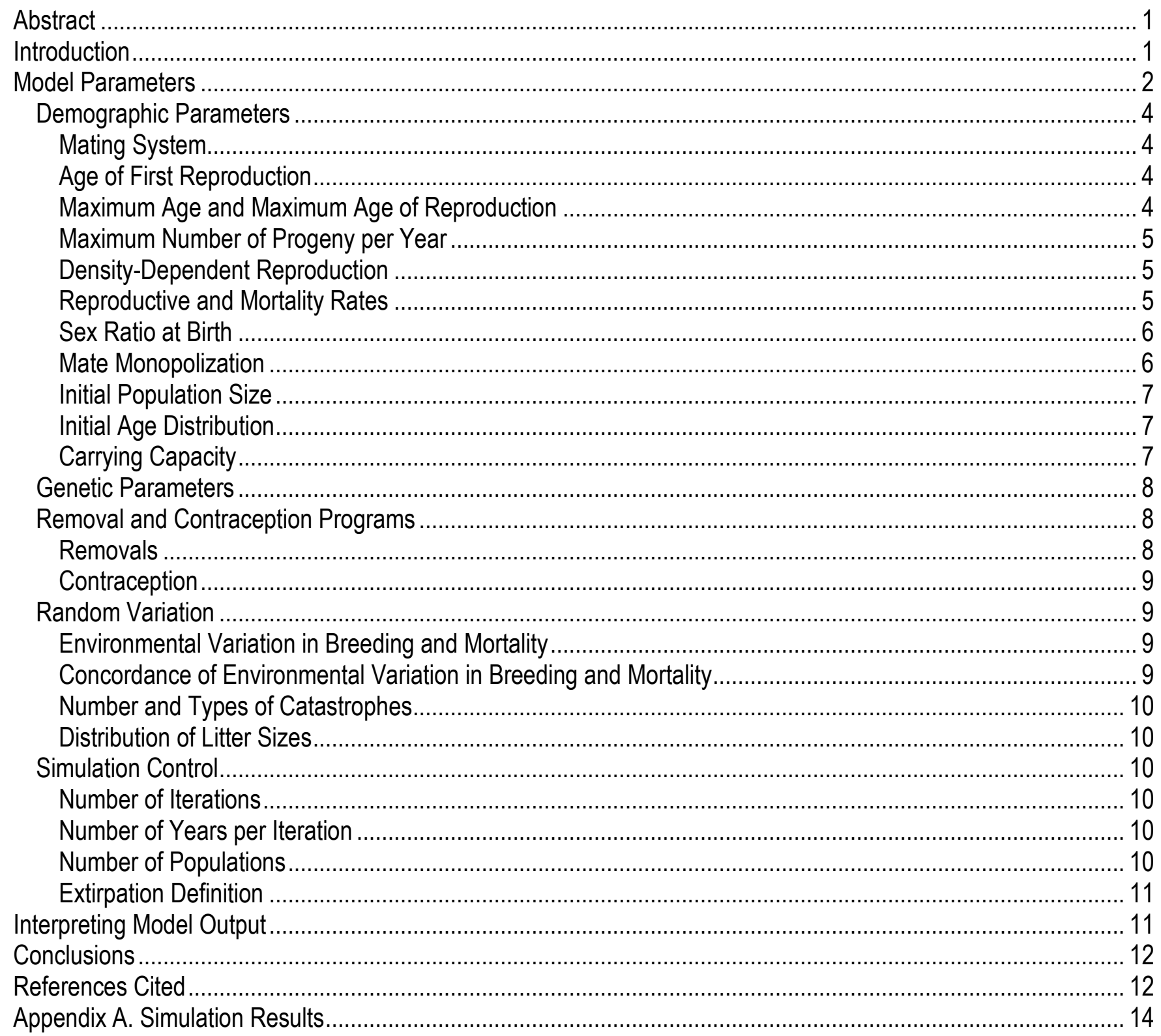

\section{Tables}

1. Parameter values and options used in simulations of the effects of a mare sterilant applied to wild horses.

2. Annual survival and reproductive rates used to achieve various annual population growth rates.

3. Translation of the annual rates shown in table 1 into VORTEX parameter values for an annual population growth rate of 20 percent $(\lambda=1.20)$ 


\title{
Abbreviations Used in This Report
}

\author{
$\geq \quad$ Greater than or equal to \\ $<\quad$ Less than \\ $\lambda \quad$ lambda; annual population growth rate multiplier for a herd \\ BLM Bureau of Land Management \\ $\mathrm{K} \quad$ carrying capacity \\ $\mathrm{N}$ population size \\ U.S. United States
}




\title{
Potential Demographic and Genetic Effects of a Sterilant Applied to Wild Horse Mares
}

\author{
By James E. Roelle and Sara J. Oyler-McCance
}

\begin{abstract}
Wild horse populations on western ranges can increase rapidly, resulting in the need for the Bureau of Land Management (BLM) to remove animals in order to protect the habitat that horses share with numerous other species. As an alternative to removals, BLM has sought to develop a long-term, perhaps even permanent, contraceptive to aid in reducing population growth rates. With long-term (perhaps even permanent) efficacy of contraception, however, comes increased concern about the genetic health of populations and about the potential for local extirpation. We used simulation modeling to examine the potential demographic and genetic consequences of applying a mare sterilant to wild horse populations. Using the VORTEX software package, we modeled the potential effects of a sterilant on 70 simulated populations having different initial sizes ( 7 values), growth rates (5 values), and genetic diversity ( 2 values). For each population, we varied the treatment rate of mares from 0 to 100 percent in increments of 10 percent. For each combination of these treatment levels, we ran 100 stochastic simulations, and we present the results in the form of tables and graphs showing mean population size after 20 years, mean number of removals after 20 years, mean probability of extirpation after 50 years, and mean heterozygosity after 50 years. By choosing one or two combinations of initial population size, population growth rate, and genetic diversity that best represent a herd of interest, a manager can assess the likely effects of a contraceptive program by examining the output tables and graphs representing the selected conditions.
\end{abstract}

\section{Introduction}

As the primary agency responsible for management of wild horses (Equus caballus) on public lands in the United States (U.S.), the Bureau of Land Management (BLM) has a need for a long-lasting, non-lethal method to control population growth rates. Given the protection afforded by The Wild FreeRoaming Horses and Burros Act of 1971 (P.L. 92-195 as amended) and general lack of natural predators, wild horse populations commonly increase at rates of 15-25 percent per year (Eberhardt and others, 1982; Garrott and others, 1991a) and quickly exceed the carrying capacity of their ranges. In the past, BLM has managed excess horses through a gather, removal, and adoption program, with unadopted horses held in short- and long-term holding facilities until the end of their natural lives. The sustainability of this strategy has recently been called into question, however (U.S. Government Accountability Office, 2008; Garrott and Oli, 2013; National Research Council, 2013). Therefore, BLM has increased efforts to develop a contraceptive that will reduce population growth rates in wild horse herds and thus reduce the number of excess animals that must be removed and cared for. 
The contraceptive SpayVac has recently demonstrated the potential for long-lasting efficacy (at least 4 years) in wild horses (Killian and others, 2008). Maximizing the duration of contraceptive effectiveness is especially important in managing wild horses, which in most cases must be captured in order to successfully administer the drug. With extended efficacy (or perhaps even permanent sterility), however, comes increased concern for both the genetic health of a horse herd and the possibility of extirpation. Simulation modeling can be a useful tool in understanding the likely consequences of alternative management actions. We used the VORTEX software package (Lacy and others, 2014; Lacy and Pollak, 2014) to simulate the effects of treating various proportions of mares with a permanent sterilant. Response variables included population size, number of animals removed, average heterozygosity, and probability of local extirpation. (The VORTEX manual (Lacy and others, 2014) uses the term "extinction" rather than "extirpation." We believe that extirpation is more appropriate in this case, as we are simulating local populations, not the entire species.) We chose the VORTEX software because it can be used to simulate both demographic and genetic effects, and because it has been useful in simulating management of the horse population on Assateague Island (Ballou and others, 2008).

VORTEX is an individual-based model in which events happen or fail to happen on the basis of probabilities for each modeled individual. For example, if 100 females are of breeding age, each of which can produce at most one offspring, and fecundity is set to 80 percent, there is no guarantee that exactly 80 offspring will be born. Rather, for each female of breeding age, a random number chosen from a uniform distribution between 0 and 1 is compared to the probability of producing an offspring (in this example, 0.8). If the random number is less than 0.8 , then the female is tagged in the model to give birth but otherwise does not. Thus, no two simulations are identical even when they use the same demographic parameters, and multiple iterations must be run in order to examine the possible range of outcomes. In general, we present results in the form of mean values for the multiple simulations; however, it is important to remember that extreme values can also be of interest. In addition, it is important to note that these simulations represent the results of management prescriptions applied consistently through time, without adaptation based on monitoring of results. In the real world, there would be many opportunities to adjust management strategies based on previous results.

\section{Model Parameters}

Parameter values and VORTEX options used in our simulations are summarized in table 1 and described in some detail in the sections that follow. Our knowledge of the biology and demography of horse populations and the efficacy of SpayVac and other forms of contraception is incomplete; what is applicable to one herd may not be applicable to all herds. In light of that fact, in cases where specific parameter values were in question, we tried to select the value or option that would have the largest effect on one or more of the response variables. For example, we don't know that SpayVac is a sterilant, but it may be. Furthermore, BLM is considering the use of other techniques (for instance, ovariectomy) that definitely will be permanent. So, for modeling purposes, we assume a permanent sterilant; once a mare is treated and the treatment works, she is infertile for life. This assumption is likely to minimize the number of animals tagged for removal during a simulation, while at the same time maximizing any decline in heterozygosity. We were similarly conservative in our choice of other parameter values and options, always choosing those that were likely to have the most detrimental effect on population demography and genetics. 


\section{Table 1. Parameter values and options used in simulations of the effects of a mare sterilant applied to wild}

horses.

$\left[\mathrm{H}_{0}\right.$, observed heterozygosity; $\geq$, equal to or greater than; BLM, Bureau of Land Management $]$

\begin{tabular}{|c|c|}
\hline Parameter or option & Value selected for simulations \\
\hline Mating system & Long-term polygyny \\
\hline Age of first reproduction & Females $=3$ years, males $=5$ years \\
\hline Maximum age (life span) & 25 years \\
\hline Maximum age of reproduction & 20 years \\
\hline Basic time cycle & 1 year \\
\hline Maximum litters per year & 1 \\
\hline Maximum progeny per litter & 1 \\
\hline Density-dependent reproduction & Not used \\
\hline Reproductive rates & See tables 2 and 3 \\
\hline Mortality or survival rates & See tables 2 and 3 \\
\hline Sex ratio at birth & 50 percent males \\
\hline Mate monopolization & Mean of 3.0 mates per successful sire \\
\hline Initial population size & $100,250,500,750,1,000,1,250$, or 1,500 \\
\hline Initial age distribution & Stable age distribution calculated from age-specific schedules in table 2 and 3 \\
\hline Carrying capacity & $\begin{array}{l}\text { Arbitrarily set at } 5 \times \text { the initial population size, but the contraception and removal programs prevent } \\
\text { carrying capacity from having any effect }\end{array}$ \\
\hline Initial heterozygosity & $\begin{array}{l}\text { Actual allele frequencies at } 13 \text { microsatellite loci from the Lost Creek herd in Wyoming (high diversity, } \\
\qquad \mathrm{H}_{\mathrm{o}}=0.78 \text { ) or the Cibola-Trigo herd in Arizona (low diversity, } \mathrm{H}_{\mathrm{o}}=0.49 \text { ) }\end{array}$ \\
\hline Removals & $\begin{array}{l}\text { When the simulated population reaches } \geq 150 \text { percent of the initial population size, try to remove enough } \\
\text { animals to get back to the initial population size. BLM's age-class removal criteria are used, but are } \\
\text { constrained such that no more than } 90 \text { percent of any age and sex class are removed. }\end{array}$ \\
\hline Efficacy of treatment & Treatment is efficacious in 90 percent of all newly treated mares \\
\hline Duration of efficacy & If treatment is efficacious, female remains contracepted for life \\
\hline Proportion of females treated & 0 percent- 100 percent, in 10 percent increments \\
\hline Standard deviation due to environmental variability & $\mathrm{S}_{\mathrm{EV}}=0.12$ for fecundity; $\mathrm{S}_{\mathrm{EV}}=0.06$ for mortality \\
\hline Concordance & Yes. Breeding and mortality are affected simultaneously. \\
\hline Probability of catastrophe & 10 percent annually \\
\hline Impact of catastrophe & Reduce reproduction to 50 percent of normal; reduce survival to 75 percent of normal \\
\hline Distribution of litter sizes & Not used; litter size in horses is virtually always 1 \\
\hline Number of iterations per simulation & 100 \\
\hline Number of years per iteration & 20 years for population size and removals; 50 years for heterozygosity and probability of extirpation \\
\hline Number of populations in each simulation & 1 \\
\hline Definition of extirpation & The population is considered to be extirpated when it no longer has individuals of both sexes. \\
\hline
\end{tabular}




\title{
Demographic Parameters
}

\author{
Mating System
}

VORTEX models breeding systems as monogamous or polygamous and short-term or longterm. With monogamous breeding, a male is needed for every breeding female; males may therefore become a limiting factor restricting breeding. In polygamous models, only one male is needed for all females to have an opportunity to breed. However, another parameter (see the section Mate Monopolization) can specify that only a subset of males has an opportunity to breed. For example, a polygynous system can be created in which successful males control harems of, say, five females on average, while the remaining males are excluded from breeding.

If a short-term option is chosen, then VORTEX assumes that mates are randomly reshuffled each year and that all available individuals have an equal probability of breeding. If a long-term option is chosen, then once harem relationships between stallions and mares are formed, those relationships remain intact across years of the simulation until a member dies or disperses to a different population. Demographically, it does not matter whether long-term relationships or rearrangement of harems each year is chosen. Genetically, there may be a small effect on the rate of loss of genetic diversity from the population, with greater loss of diversity expected in populations with higher harem fidelity.

We realize that wild horses do not necessarily mate for life (mares do move between harems) but, of the two choices available in VORTEX, long-term polygyny comes closer to representing the mating system of wild horses. We therefore used long-term polygyny as the mating system in all of our simulations. This is a conservative assumption, in the sense that it represents the greatest opportunity for loss of genetic diversity. Any additional interchange that does occur in the real world will tend to ameliorate effects and result in higher genetic diversity than predicted by our models.

\section{Age of First Reproduction}

VORTEX describes reproduction in terms of the age when the first offspring are born, not the age at onset of sexual maturity or the age at first conception. The program also assumes that breeding (and, for that matter, all other events) takes place at discrete intervals, usually years. Thus, age at first reproduction is entered as an integer value. The value used should represent something like the median age of first reproduction, not the earliest age ever observed, because the earliest observed age may not be typical of the population. We therefore used 3 years as the age of first reproduction in females and 5 years as the age of first reproduction in males (Garrott and Taylor, 1990; Garrott and others, 1991b; Roelle and others, 2010).

\section{Maximum Age and Maximum Age of Reproduction}

If the species being modeled does not reproduce throughout its entire adult life, the age when the species ceases to reproduce must be specified. For example, humans typically reach reproductive senescence before they die. If the typical life expectancy is 70 years but reproduction ceases at 50 years, then 50 years would be the maximum age of reproduction. VORTEX requires that the maximum age be the same for both sexes, but allows the maximum age of reproduction to vary by sex. Establishing the maximum age of reproduction is difficult because old animals are hard to age (unless they were marked or identified in the year of birth) and because sample sizes of old animals are typically small. Garrott and others (1991b) present information showing that some mares can reproduce at greater than 20 years 
of age, whereas Roelle and others (2010) found no evidence of reproduction by females older than 16 years. Roelle and others (2010) also found that very few harems are held by stallions older than age 20. On the basis of available evidence, we used 20 years as the maximum age of reproduction and 25 years as the maximum age.

\section{Maximum Number of Progeny per Year}

VORTEX provides a great deal of flexibility in specifying the maximum number of progeny that can be born to a given female in a year (or other time interval), thus allowing species that produce multiple clutches or litters to be modeled. However, most of this flexibility is not needed for modeling a horse population. Given that horses produce only a single litter per year (the average gestation period is 335-342 days; Card and Hillman, 1993) and that twins are virtually unknown, we used 1 for the maximum number of litters per year, 1 for the maximum number of progeny per litter, and a year (365 days) as the basic time cycle.

\section{Density-Dependent Reproduction}

VORTEX allows the reproductive rate of the population being modeled to change with changing population size. For example, reproduction could be low when the population is small owing to a difficulty in finding mates (Allee effect) or, conversely, reproduction could drop off when the population is large (more specifically, at high density) owing to limited resources or territories, intraspecific competition, crowding, or stress.

Density-dependent reproduction, also known as compensatory reproduction, has been reported for wild horses (Kirkpatrick and Turner, 1991; Jenkins, 2000; Roelle and others, 2010), but quantifying the magnitude of the effect is difficult, and the information available is insufficient to allow generalization or calculation of some average function. In addition, many of our simulated populations differed only about 50 percent (that is, between $\mathrm{N}$ and $1.5 \times \mathrm{N}$ ) because of the way we implemented the removal function (see section Removal and Contraception Programs). In our estimation, reproductive rates are not likely to change substantially owing to density in that relatively small range. We therefore did not use density-dependent reproduction in our simulations.

\section{Reproductive and Mortality Rates}

VORTEX models reproductive rate as the proportion of adult females giving birth each year (or other time interval), where an adult female is defined by the age of first reproduction (see previous discussion). Similarly, mortality rates are modeled as the proportion of animals dying in each age and sex class. From age 0 to the age of first reproduction, each age and sex class can have a different mortality rate; after the age of first reproduction, all animals are considered adults, and the mortality rate can vary only by sex.

While these parameters are fundamental to modeling population dynamics, values are well known for perhaps only a handful of wild horse populations. In our estimation, however, wild horse managers are more likely to have at least some idea of the annual population growth rate multiplier for a herd $(\lambda$, lambda), which is just a different way of expressing the growth rate itself. We therefore modeled a set of scenarios in which $\lambda=1.05,1.10,1.15,1.20$, or 1.25 (that is, annual growth rates of 5 percent, 10 percent, 15 percent, 20 percent, or 25 percent in the absence of contraception). To produce these growth rates, we used reproductive and survival rates (table 2) derived largely from Garrott 
(1991a). We arrived at the values for $\lambda=1.25$ by experimenting with parameters in the model. Table 3 provides an example of how we translated these parameters into VORTEX.

Table 2. Annual survival and reproductive rates used to simulate various annual population growth rates.

\begin{tabular}{cccc}
\hline $\begin{array}{c}\text { Population } \\
\text { growth rate } \\
\text { (percent) }\end{array}$ & $\begin{array}{c}\text { Juvenile } \\
\text { survival rate } \\
\text { (percent) }\end{array}$ & $\begin{array}{c}\text { Adult } \\
\text { survival rate } \\
\text { (percent) }\end{array}$ & $\begin{array}{c}\text { Mares foaling } \\
\text { (percent) }\end{array}$ \\
\hline 5 & 80 & 91 & 50 \\
10 & 80 & 92 & 65 \\
15 & 80 & 97 & 65 \\
20 & 80 & 99 & 80 \\
25 & 90 & 99 & 92 \\
\hline
\end{tabular}

Table 3. Translation of the annual rates shown in table 1 into VORTEX parameter values for an annual population growth rate of 20 percent $(\lambda=1.20)$.

$[\lambda$, annual population growth rate multiplier for a herd; $<$, less than; $\geq$, equal to or greater than $]$

\begin{tabular}{cccccc}
\hline \multicolumn{2}{c}{ Reproduction } & \multicolumn{2}{c}{ Female Mortality } & \multicolumn{2}{c}{ Male Mortality } \\
\hline $\begin{array}{c}\text { Mare age } \\
\text { (years) }\end{array}$ & $\begin{array}{c}\text { Mares foaling } \\
\text { (percent) }\end{array}$ & $\begin{array}{c}\text { Age } \\
\text { (years) }\end{array}$ & $\begin{array}{c}\text { Rate } \\
\text { (percent) }\end{array}$ & $\begin{array}{c}\text { Age } \\
\text { (years) }\end{array}$ & $\begin{array}{c}\text { Rate } \\
\text { (percent) }\end{array}$ \\
\hline 1 & 0 & $<1$ & 20 & $<1$ & 20 \\
2 & 0 & 1 to $<2$ & 1 & 1 to $<2$ & 2 to $<3$ \\
$\geq 3$ & $\geq 2$ & 1 & 3 to $<4$ & 1 \\
& & & & 4 to $<5$ & 1 \\
& & & & $\geq 5$ & 1 \\
\hline
\end{tabular}

We believe that, in the absence of contraception, growth rates of most wild horse herds will fall within the range shown in table 2 . Rather than having to specify reproductive and mortality rates, a manager will need only to choose one or perhaps two values for the annual population growth rate to use the output from the model.

\section{Sex Ratio at Birth}

While foal sex ratio can differ from 50:50 in small herds in a given year (Roelle and others, 2010), over time the ratio tends to be fairly even in most herds. For example, Garrott (1991b) found that for a 12-year period 65 of 74 (88 percent) herds sampled in Nevada, Oregon, and Wyoming had a foal sex ratio that did not differ from 50:50. We therefore used 50 percent males in the foal crop in our simulations.

\section{Mate Monopolization}

As noted in the previous section, we ran our simulations using a polygynous breeding system. In order to do so, VORTEX requires that one of the following three parameters be specified: percentage of males in the breeding pool, percentage of males successfully siring offspring, or mean number of mates per successful sire. The remaining two parameter values are calculated from the one that is supplied based on the assumptions that some males are excluded from the breeding pool and that breeding 
success among males in the pool of available breeders is described by a Poisson distribution. Only the first parameter (percentage of males in the breeding pool) is used by VORTEX.

Of the three ways in which mate monopolization can be described in VORTEX, mean number of mates per successful sire is the one most commonly discussed in the literature on feral horses, but comparisons among studies are hampered by inconsistencies in defining what constitutes a potential mate (that is, the age classes of mares). Roelle and others (2010) defined potential mates as all mares 2 years of age and older, and found a mean harem size of 2.2 mares (range 1.9 to 2.7) during 12 years. Using the same age criteria, Kaseda and Khalil (1996) found average harem sizes of 1.8 to 5.3 mares in many years.

Potential effects of using different values for the mean number of mates per successful sire are not intuitive; to a first approximation, however, one might expect that varying this parameter would affect genetics more than demography. We explored sensitivity of model results to mean number of mates per successful sire in a series of simulations with initial population size $=500$, proportion of mares treated $=0.5$, lambda $=1.05$ (in the absence of contraception and removals), and initial heterozygosity $=0.77$. We varied mean number of mates per successful sire systematically between 1.5 and 5.0. The fairly low growth rate and fairly high proportion of mares treated consistently kept the simulated population after 50 years at 200-250 animals; however, final heterozygosity ranged only from 0.74 to 0.75 . Given the apparent lack of sensitivity of the model, we decided to set the value of mean number of mates per successful sire to 3.0 for all simulations.

\section{Initial Population Size}

Horse herds managed by BLM vary considerably in size, and the size of the herd is potentially very important relative to demographic and genetic results of contraception. We approached this problem by simulating herds with initial populations of $100,250,500,750,1,000,1,250$, and 1,500 animals. These initial populations should encompass the appropriate management level (AML; population goal) for many herds. We recommend a more detailed, site-specific analysis before a sterilant is applied to any herd with fewer than 100 animals. In addition, we implicitly assume that a herd is at its appropriate size before the results of these simulations can be used to evaluate a fertility control program. For example, the results of the simulations having an initial population and appropriate management level of 500 animals cannot be used if the population in fact contains, say, 1,000 animals.

\section{Initial Age Distribution}

VORTEX allows two options for specifying the initial age distribution used in a simulation. A specific age distribution (the number of individuals in each sex and age class) can be entered, or VORTEX can calculate the stable age distribution (proportion of animals) that would result from the age-specific schedules of reproduction and mortality described previously. We used the latter option, and the proportions were applied to the initial population size to determine the number of animals initially in each sex and age class.

\section{Carrying Capacity}

Carrying capacity $(\mathrm{K})$ describes a theoretical upper limit to the size of a population. In VORTEX, if the actual population, $\mathrm{N}$, exceeds $\mathrm{K}$ at the end of a particular year, then additional mortality is applied equally across all age and sex classes to reduce the population such that $\mathrm{N}=\mathrm{K}$. While carrying capacity is an important theoretical construct, values for $\mathrm{K}$ are difficult to estimate. We 
somewhat arbitrarily set the value for $\mathrm{K}$ at 5 times the initial population size. With the contraception and removal programs specified as described in subsequent sections, simulated populations never reach carrying capacity.

\section{Genetic Parameters}

VORTEX allows inbreeding depression to be incorporated into the population model as a reduction in first-year survival among inbred individuals. Although some diploid species that have been studied show depressed fitness when inbred (O'Brien and Evermann, 1988; Quattro and Vrijenhoek, 1989), the rate at which inbreeding affects survival and reproduction is not well understood for most species. Therefore, we elected not to use this function in the wild horse model. A better approach for investigating the potential genetic consequences of contraception and gathers (round-ups) is to track the degree of genetic diversity, which can be loosely linked to fitness and an ability to adapt to environmental change.

VORTEX tracks changes in genetic diversity using heterozygosity (the frequency of two different alleles at a given gene) as a metric. Heterozygosity ranges from 0 (all individuals are homozygous and the same at all genes) to 1 (all individuals are heterozygous with different alleles at all genes). It is generally believed that higher levels of heterozygosity are associated with more genetic variation and lower probability of inbreeding depression.

VORTEX can track heterozygosity in two ways. Because VORTEX includes a detailed simulation of genetic change in the populations, one way to measure changes in heterozygosity is to start with maximum heterozygosity (1) by assigning each founder individual two unique alleles at a number of loci (genes). Each offspring is randomly assigned one of the two alleles from each parent at each locus. This method tracks relative loss of heterozygosity through time. Perhaps a more realistic way to examine loss of heterozygosity is to mimic real populations by starting with individuals that have a known level of heterozygosity. VORTEX allows this option by allowing real allele frequencies to be used as a starting point for model simulations. Thus, if we know the level of heterozygosity for a population, we can enter real allele frequencies such that the starting point of the simulations will have exactly that level of heterozygosity. We used the latter of these two approaches and ran the simulations for two different initial levels of heterozygosity (high and low) as reported for two real-world herds (Gus Cothran, Texas A\&M University, unpublished reports prepared for BLM). Actual allele frequencies at 13 microsatellite loci were taken from the Lost Creek herd in Wyoming (high genetic diversity, $\mathrm{H}_{\mathrm{o}}=0.78$; report prepared in 2010) and the Cibola-Trigo herd in Arizona (low genetic diversity, $\mathrm{H}_{\mathrm{o}}=0.49$; report prepared in 2008). Absent site-specific information, we typically expect larger herds to have higher genetic diversity.

\section{Removal and Contraception Programs}

\section{Removals}

Given the limitations of available contraceptive methods, and facing population sizes that greatly exceed the desired number of animals in many herd management areas, BLM currently views contraception as a means of reducing the number of animals that must be removed, and perhaps increasing the interval between gathers, rather than as a replacement for gathers. We therefore simulated a gather-and-removal program that is as similar as possible to the program that BLM uses in the field. We modeled a gather-and-removal event in any year in which the simulated population was 150 percent or more of the initial population, and we attempted to remove sufficient animals to return the population 
to its initial size. We removed animals from age classes according to BLM's removal criteria, which specify the following order: ages 4 and under, followed by ages 11-19, followed by ages $5-10$. We constrained the number of animals removed, however, such that no more than 90 percent of any group was taken (because it would rarely be possible or desirable to remove every animal from a given age class). Our response variable is the total number of animals removed in 20 years averaged for 100 iterations.

\section{Contraception}

We implemented a simulated contraception program using capabilities in VORTEX called "population state" variables and "individual state" variables. Briefly, we used these variables to define the proportion of previously untreated females that are treated in each application and the probability that the treatment works if it is given (efficacy). In most cases, animals in BLM herds need to be gathered in order to apply a fertility control agent; we therefore treated mares following every gather and removal. Killian and others (2008) found that 17 percent of mares treated with SpayVac were pregnant in years 2 through 4 of their study, which implies efficacy of 83 percent. The most aggressive assumption would be that the treatment is 100 percent efficacious. However, it is probably also fair to assume that some mares will not respond to an immunocontraceptive such as SpayVac. We therefore assumed efficacy of 90 percent for all simulations. We also assumed that once a mare is successfully contracepted, she remains contracepted for life without further treatment (we assumed permanent sterility). We varied the proportion of mares treated from 0 to 100 percent in increments of 10 percent to illustrate the range of possible contraceptive effects. For example, when the proportion of mares treated is set at 20 percent, each mare not successfully treated in the past has a 20 percent chance of being caught and treated, and there is a 90 percent chance that the treatment will be effective. Note that high treatment rates (90-100 percent, for example) would likely be very difficult to achieve in the real world because some animals are difficult or impossible to gather.

\section{Random Variation}

\section{Environmental Variation in Breeding and Mortality}

In addition to the variability inherent in an individual-based model, VORTEX allows specification of variability (standard deviation) in the proportion of females breeding and in the proportion of both sexes dying due to factors external to the population (such as weather, predation, parasite loads). Estimates of variability in these parameters are generally not available for wild horse herds, but sufficient information was available to calculate standard deviations in fecundity and mortality for the Pryor Mountain herd (Roelle and others, 2010).

The standard deviation due to environmental variability, $s_{e v}$, for either fecundity or mortality,

can be expressed as $s_{e v}=\sqrt{s_{t o t}^{2}-s_{d s}^{2}}$, where $s_{t o t}^{2}$ is the total variance in the parameter for all years and $s_{d s}^{2}$ is the sampling (binomial) variance for all individual rates. From the Pryor Mountain data set (Roelle and others, 2010), we calculated $s_{e v}=0.12$ for fecundity and $s_{e v}=0.06$ for mortality. We combined ages and sexes for both of these calculations and used the resulting values in all simulations.

\section{Concordance of Environmental Variation in Breeding and Mortality}

Factors such as those already described (weather, predation, parasite loads) can affect reproduction and survival independently or simultaneously. Simultaneously means that good years for 
reproduction are also good years for survival. We know of no information that can be brought to bear on this subject in regard to wild horse demography, but it seems logical that demographic effects would be most pronounced if these parameters vary simultaneously. We therefore opted for concordance in environmental variation.

\section{Number and Types of Catastrophes}

The VORTEX software allows specification of catastrophes (floods, forest fires, epidemic diseases) through particular effects on reproduction and survival. Perhaps the most common such event on western ranges is drought, and although droughts occur fairly frequently, we know of no specific information relating drought to reproduction and survival in wild horses. Nevertheless, we felt that it was important to include some form of catastrophe in our simulations. We therefore specified that drought would have a 10 percent probability in any year and that it would reduce reproduction to 50 percent of the normal level and survival to 75 percent of the normal level.

\section{Distribution of Litter Sizes}

VORTEX allows for one additional form of random variation if females can produce litters of different sizes. Because wild horses almost never produce more than one offspring, we did not use this parameter.

\section{Simulation Control}

\section{Number of Iterations}

As noted in the previous section, VORTEX uses a random number generator to simulate random events in an organism's life cycle. Therefore, no two runs or iterations of the model are identical even with a single set of input data. To obtain a more complete picture of demographic behavior, multiple iterations are required. We explored changes in the standard deviation of both mean population size and heterozygosity after 50 years while varying the number of iterations from 25 to 1,000 . Because of the complexity of the removal and contraception functions, no consistent pattern emerged in the standard deviations of these parameters. More particularly, increasing the number of iterations to more than 100 did not consistently lead to lower variance about the mean. We therefore chose 100 iterations for all of our simulations.

\section{Number of Years per Iteration}

Initial simulations with some basic data indicated that significant changes in heterozygosity are rare in less than 50 years. On the other hand, significant demographic changes can occur in as little as 20 years. We therefore evaluated mean population size and removals at the end of 20 years of simulation, but we used 50 years to evaluate heterozygosity and probability of extirpation.

\section{Number of Populations}

VORTEX can be used to model a single, isolated population or a complex metapopulation composed of as many as 50 populations with interchange of animals between them. Although it is clear that some animals move between certain populations of wild horses, we know of no information on 
rates of such movement. We therefore modeled only a single population without introduction of animals from outside, which is the worst case from the perspective of genetic diversity.

\section{Extirpation Definition}

VORTEX offers several methods for defining extirpation of a population. We used the definition that says a population is extirpated when it no longer contains individuals of both sexes.

\section{Interpreting Model Output}

Appendix A contains output from 70 sets of simulations representing two levels of genetic diversity (low or high), seven initial population sizes $(100,250,500,750,1,000,1,250$, or 1,500 animals), and five population growth multipliers $(1.05,1.10,1.15,1.20$, or 1.25). Each set of simulations is represented by a figure, and each figure contains five graphs and a table of supporting data. The order of the figures varies first by annual growth rate, then by population size, and then by genetic diversity. Thus, figure 1 has low genetic diversity, an initial population size of 100, and a growth multiplier of 1.05, and is followed by figure 2 with low genetic diversity, an initial population size of 100, and a growth multiplier of 1.10, and so on. In each figure, the first four (black and white) graphs and the table represent the same set of simulations, and the graphs and table have identical values. Due to a nuance in the way VORTEX operates, however, the final (color) graph represents a different set of simulations having the same parameter values but differing slightly due to random variation as already discussed. Thus, the value for average population size at 20 years on the color graph will not in general be identical to that in the table, but the two values will usually be very similar.

The black and white graphs present mean values (that is, averaged for 100 iterations) of four response variables on the vertical axes: mean population size after 20 years, mean number of removals after 20 years, mean probability of extirpation after 50 years, and mean heterozygosity in the herd after 50 years. In all cases, the horizontal axis is the percentage of previously untreated mares that are treated following each gather, varying from 0 percent to 100 percent. The colored graph represents the mean trajectory of the population size for a subset of the treatment levels ( 0 percent, 20 percent, 40 percent, and so on) depicted as lines of different color. It is very important to understand that there is some information that cannot be conveyed by mean values. For example, there are many cases where the table and black and white graphs show that animals were removed, but the colored graph lines never plot higher than $1.5 \times$ the initial population. The appropriate interpretation is that on average the population stayed below the threshold $(1.5 \times$ the initial population), but in some individual iterations the population grew sufficiently to trigger a removal.

As an example application of model results, suppose a manager wants to know what proportion of mares can be treated with a sterilant in a herd having low genetic diversity, an initial population size of about 750, and a population growth multiplier of 1.15 . This set of conditions is represented by figure 18 in appendix A. The first thing to notice about figure 18 is that no treatment level has any real effect on either mean probability of extirpation after 50 years or mean heterozygosity after 50 years. The graph of mean population size after 20 years, however, shows that treatment levels greater than 50 percent of the mares result in populations somewhat less than 750 . We also see that if 50 percent of the mares are treated, the mean number of removals after 20 years is around 900, or about 500 fewer than in the scenario where no mares are treated. The number of removals can be further reduced by treating a higher proportion of mares, but only if average population levels below 750 are acceptable. The average trajectory of the population size through time can be examined by looking at the last (color) graph in the 
figure 18, which also shows that treatment rates more than about 50 percent will result in populations that are commonly below the starting point of 750 animals.

Finally, uncertainties about level of genetic diversity, population size, or growth rate can be addressed by examining figures representing nearby values in the parameter space. Thus, in the example in the preceding paragraph, a manager might also wish to look at the figures representing 500 and 1,000 animals in the population and growth multipliers of 1.10 and 1.20 to gain some understanding of how sensitive model results are to these assumptions.

\section{Conclusions}

We believe that simulation results presented herein represent a useful starting point for evaluating a management program involving a contraceptive technique that is permanent (or nearly so). In particular, the results show that only in the most extreme circumstances (such as low initial genetic diversity, low population growth rate, high proportion of mares treated, no change in management for 50 years) would there likely be any noticeable effect on genetic diversity or a significant probability of extirpation of a herd. Monitoring and adaptive management would reduce the probability of unacceptable results even further. For example, a simple exchange program in which an animal or two were moved between herds periodically would help to maintain genetic diversity, and the contraceptive program could always be modified or discontinued in the event that the reduction of population was greater than anticipated. In short, we see nothing in our results that should steer wild horse managers away from permanent contraceptive techniques, as long as results are monitored and adjustments are made if necessary.

\section{References Cited}

Ballou, J.D., Traylor-Holzer, K., Turner, A., Malo, A.F., Powell, D., Maldonado, J., and Eggert, L., 2008, Simulation model for contraceptive management of the Assateague Island feral horse population using individual based data: Wildlife Research, v. 35, p. 502-512.

Card, C.E., and Hillman, R.B., 1993, Parturition, in McKinnon, A.O., and Voss, J.L., eds., Equine reproduction: Philadelphia, Pa., Lea and Febiger, p. 567-573.

Eberhardt, L.L., Majorowicz, A.K., and Wilcox, J.A., 1982, Apparent rates of increase for two feral horse herds: Journal of Wildlife Management, v. 46, p. 367-374.

Garrott, R.A., 1991a, Feral horse fertility control—Potential and limitations: Wildlife Society Bulletin, v. 19, p. 52-58.

Garrott, R.A., 1991b, Sex ratios and differential survival of feral horses: Journal of Animal Ecology, v. 60, p. 929-937.

Garrott, R.A., Eagle, T.C., and Plotka, E.D., 1991b, Age-specific reproduction in feral horses: Canadian Journal of Zoology, v. 69, p. 738-743.

Garrott, R.A., and Oli, M.K., 2013, A critical crossroad for BLM's wild horse program: Science, v. 341, p. 847-848.

Garrott, R.A., Siniff, D.B., and Eberhardt, L.L., 1991a, Growth rates of feral horse populations: Journal of Wildlife Management, v. 55, p. 641-648.

Garrott, R.A., and Taylor, L., 1990, Dynamics of a feral horse population in Montana: Journal of Wildlife Management, v. 54, p. 603-612.

Jenkins, S.H., 2000, Density dependence in population dynamics of feral horses: Bureau of Land Management, National Science and Technology Center, Resource Notes 26, 2 p. 
Kaseda, Y., and Khalil, A.M., 1996, Harem size and reproductive success of stallions in Misaki feral horses: Applied Animal Behaviour Science, v. 47, p. 163-173.

Killian, Gary, Thain, D., Diehl, N.K., Rhyan, J., and Miller, L., 2008, Four-year contraception rates of mares treated with single-injection porcine zona pellucida and $\mathrm{GnRH}$ vaccines and intrauterine devices: Wildlife Research, v. 35, p. 531-539.

Kirkpatrick, J.F., and Turner, J.W., Jr., 1991, Compensatory reproduction in feral horses: Journal of Wildlife Management, v. 55, p. 649-652.

Lacy, R.C., Miller, P.S., and Traylor-Holzer, K., 2014, VORTEX-A stochastic simulation of the extinction process, Version 10.0.0.4 User's Manual: Apple Valley, Minn., IUCN SSC Conservation Breeding Specialist Group and Chicago Zoological Society, $175 \mathrm{p}$.

Lacy, R.C., and Pollak, J.P., 2014, Vortex-A stochastic simulation of the extinction process, Version 10.0: Brookfield, Ill., Chicago Zoological Society. (software)

National Research Council, 2013, Using science to improve the BLM wild horse and burro program-A way forward: Washington, D.C., National Acadamies Press, 383 p.

O’Brien, S.J., and Evermann, J.F., 1988, Interactive influence of infectious disease on genetic diversity of natural populations: Trends in Ecology and Evolution, v 3, p. 254-259.

Quattro, J.M., and Vrijenhoek, R.C., 1989, Fitness differences among remnant populations of the endangered Sonoran topminnow: Science, v. 245, p. 976-978.

Roelle, J.E., Singer, F.J., Zeigenfuss, L.C., Ransom, J.I., Coates-Markle, L., and Schoenecker, K.A., 2010, Demography of the Pryor Mountain wild horses, 1993-2007: U.S. Geological Survey Scientific Investigations Report 2010-5125, $31 \mathrm{p}$.

U.S. Government Accountability Office, 2008, Effective long-term options needed to manage unadoptable wild horses: Washington, D.C., GAO-09-77, 81 p. 


\section{Appendix A. Simulation Results}

Mean population size after 20 years

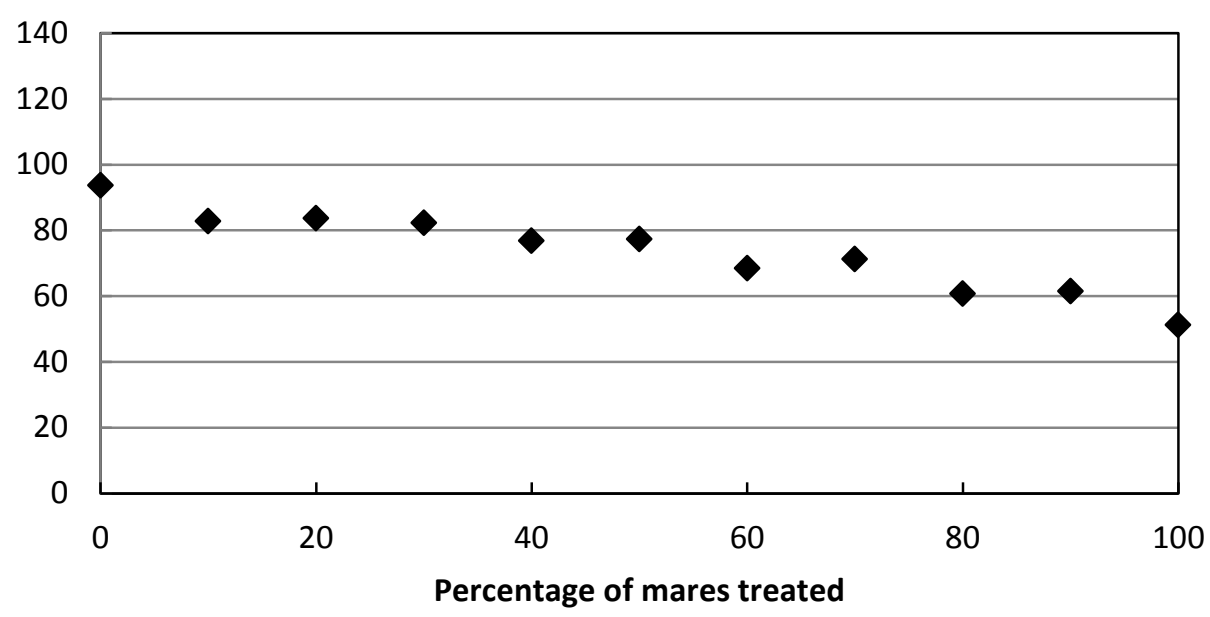

Mean probability of extirpation after 50 years

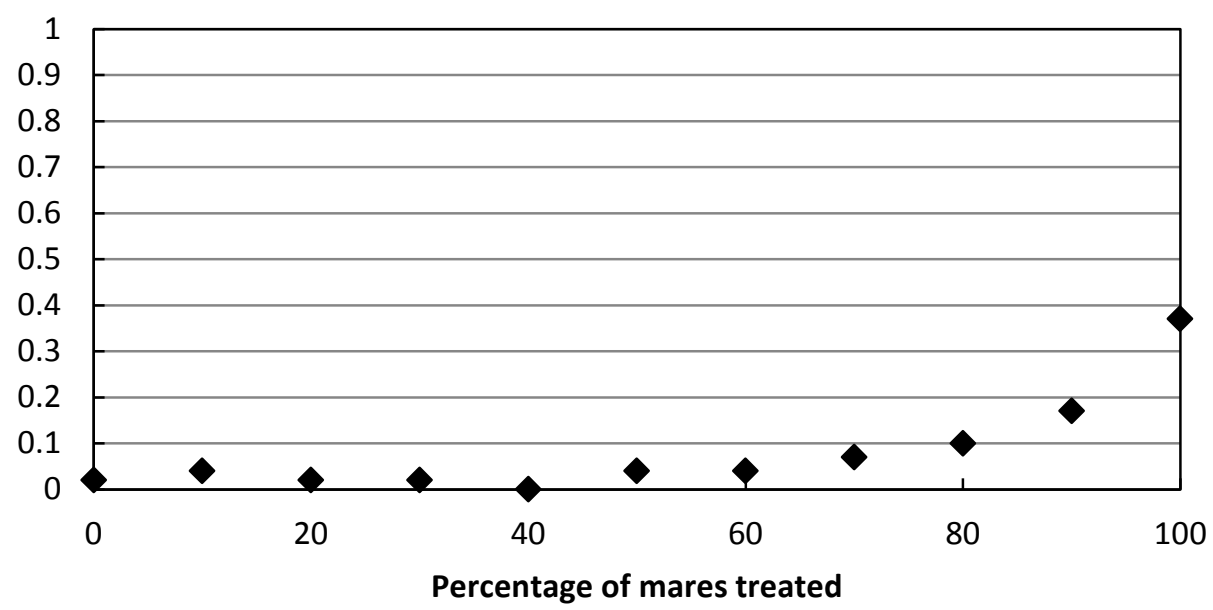

Figure 1. Initial population size $=100$, lambda $=1.05$, genetic diversity $=$ low .
Mean number of removals after 20 years

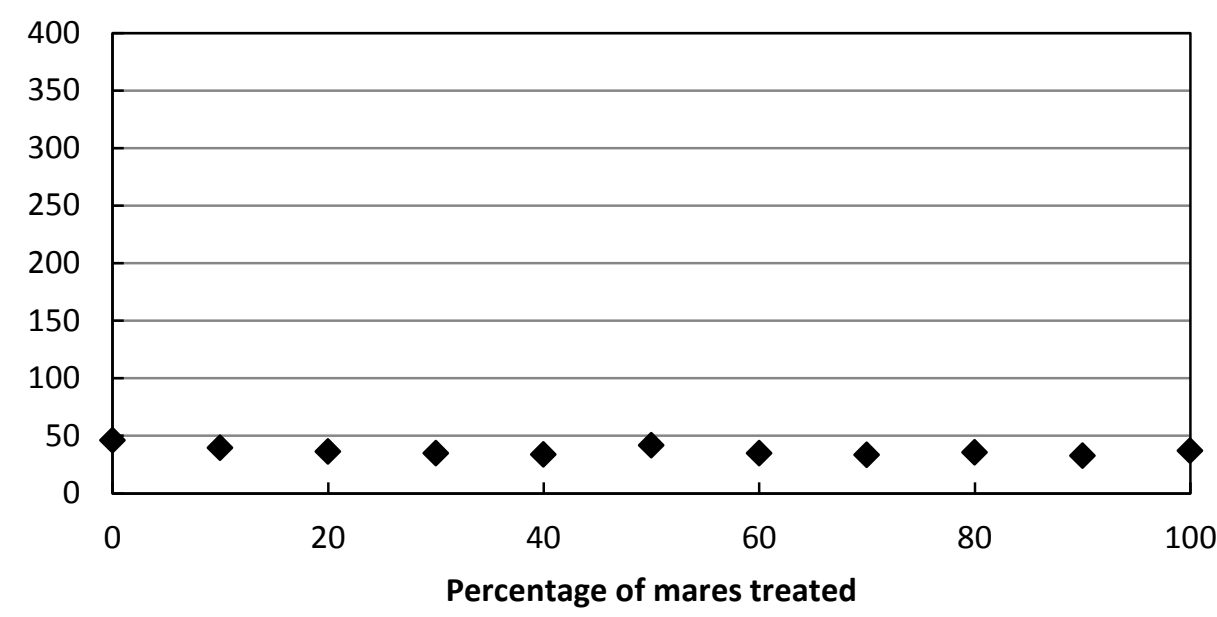

Mean heterozygosity after $\mathbf{5 0}$ years

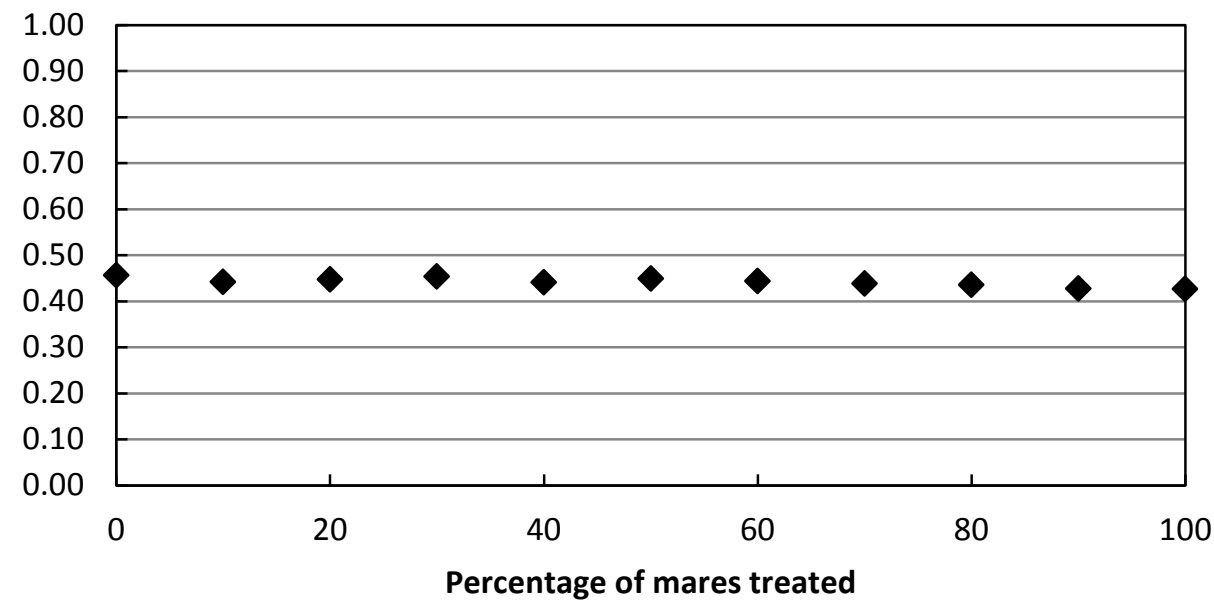




\begin{tabular}{|c|c|c|c|c|c|c|c|c|c|c|c|}
\hline Percentage of mares treated & 0 & 10 & 20 & 30 & 40 & 50 & 60 & 70 & 80 & 90 & 100 \\
\hline Mean population size after 20 years & 93.71 & 82.73 & 83.68 & 82.28 & 76.81 & 77.32 & 68.49 & 71.19 & 60.66 & 61.53 & 51.22 \\
\hline Mean number of removals after 20 years & 45.83 & 39.52 & 36.34 & 34.74 & 33.65 & 41.69 & 34.88 & 33.26 & 35.4 & 32.7 & 36.94 \\
\hline Mean probability of extirpation after 50 years & 0.02 & 0.04 & 0.02 & 0.02 & 0 & 0.04 & 0.04 & 0.07 & 0.1 & 0.17 & 0.37 \\
\hline Mean heterozygosity after 50 years & 0.46 & 0.44 & 0.45 & 0.45 & 0.44 & 0.45 & 0.44 & 0.44 & 0.44 & 0.43 & 0.43 \\
\hline
\end{tabular}

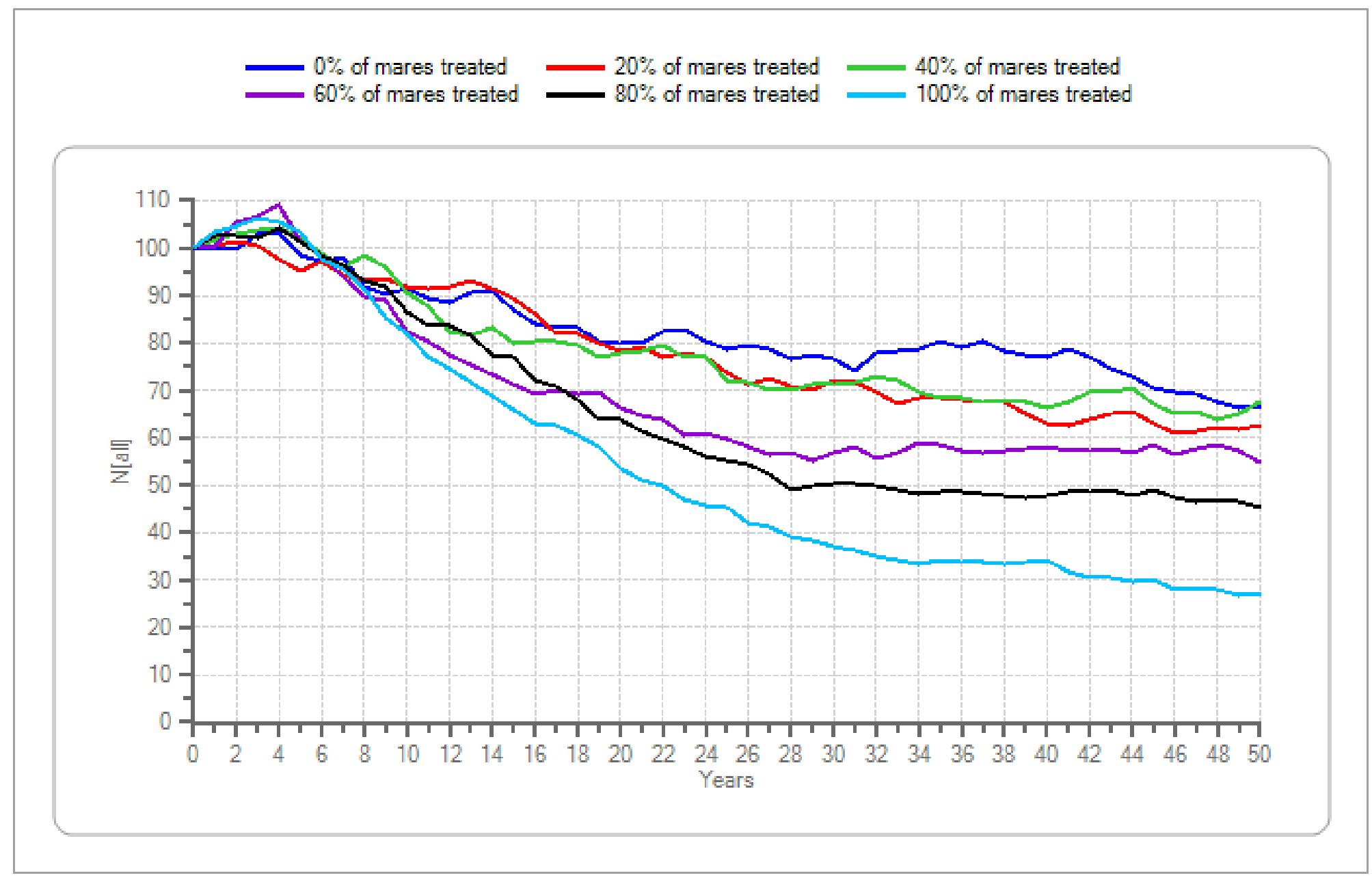

Figure 1. Initial population size $=100$, lambda $=1.05$, genetic diversity $=$ low - Continued 
Mean population size after 20 years

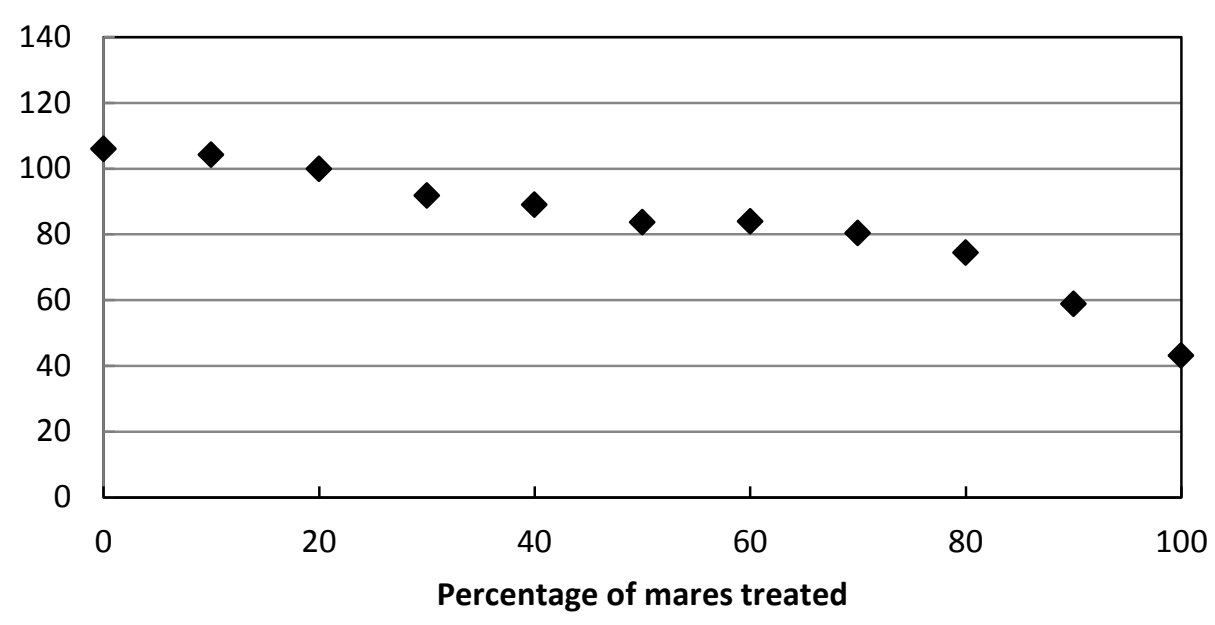

Mean probability of extirpation after $\mathbf{5 0}$ years

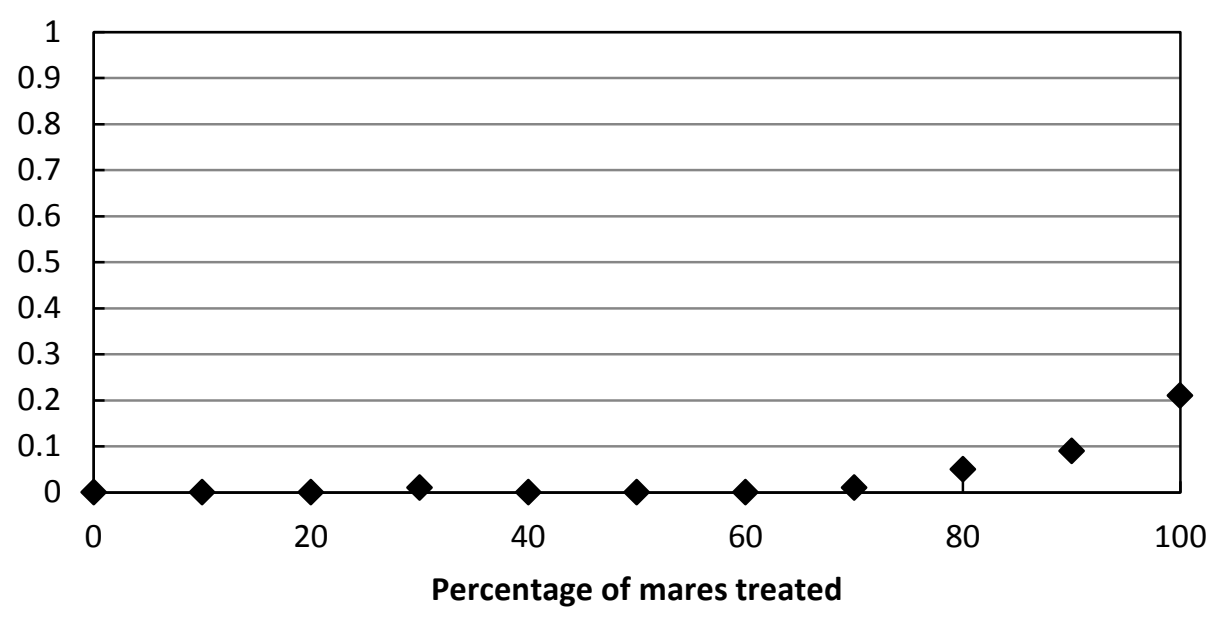

Figure 2. Initial population size $=100$, lambda $=1.10$, genetic diversity $=$ low .
Mean number of removals after 20 years

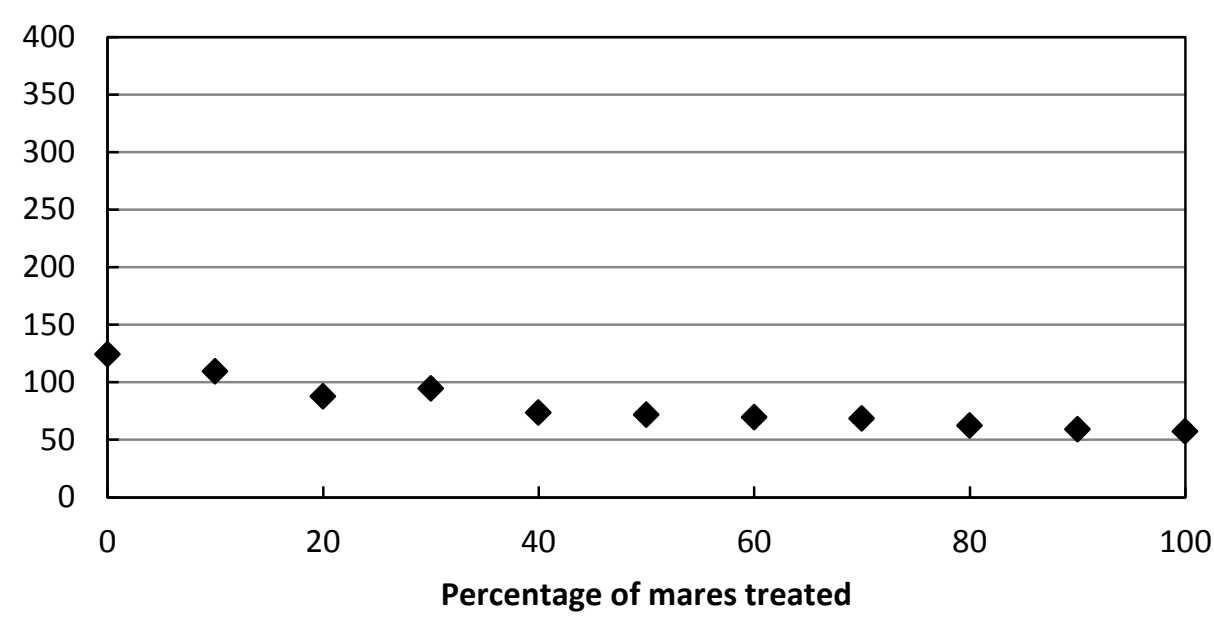

Mean heterozygosity after $\mathbf{5 0}$ years

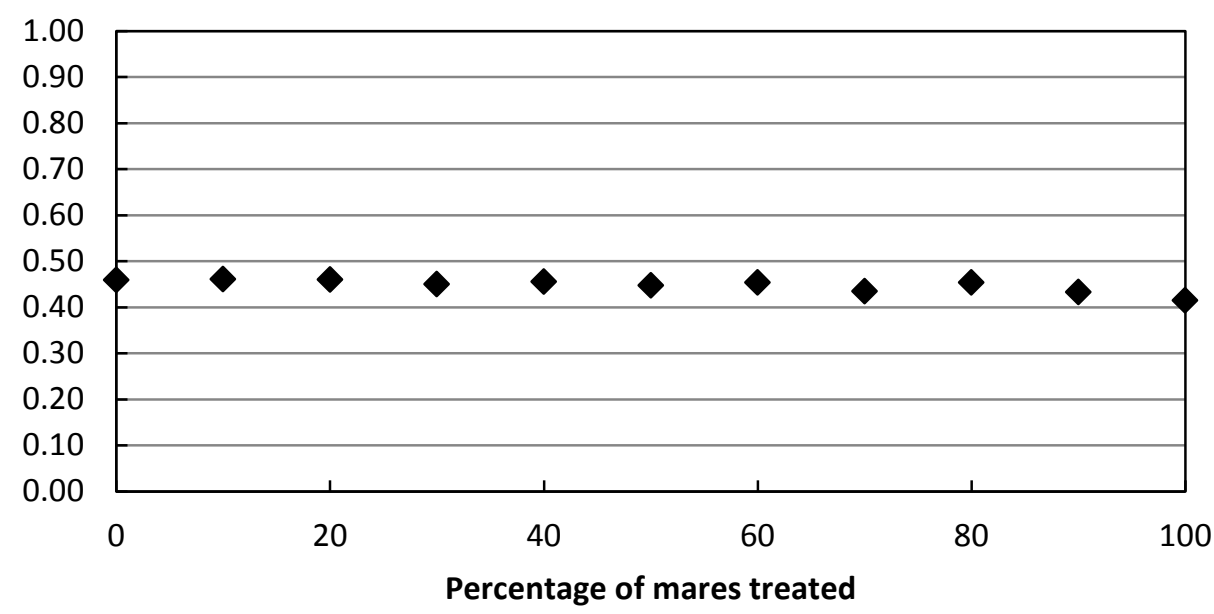




\begin{tabular}{|c|c|c|c|c|c|c|c|c|c|c|c|}
\hline Percentage of mares treated & 0 & 10 & 20 & 30 & 40 & 50 & 60 & 70 & 80 & 90 & 100 \\
\hline Mean population size after 20 years & 105.96 & 104.13 & 99.93 & 91.74 & 89 & 83.71 & 83.91 & 80.41 & 74.37 & 58.79 & 43.07 \\
\hline Mean number of removals after 20 years & 124.34 & 109.22 & 87.59 & 94.47 & 73.54 & 71.53 & 69.56 & 68.28 & 62.33 & 58.98 & 57.08 \\
\hline Mean probability of extirpation after 50 years & 0 & 0 & 0 & 0.01 & 0 & 0 & 0 & 0.01 & 0.05 & 0.09 & 0.21 \\
\hline Mean heterozygosity after 50 years & 0.46 & 0.46 & 0.46 & 0.45 & 0.46 & 0.45 & 0.45 & 0.43 & 0.45 & 0.43 & 0.41 \\
\hline
\end{tabular}

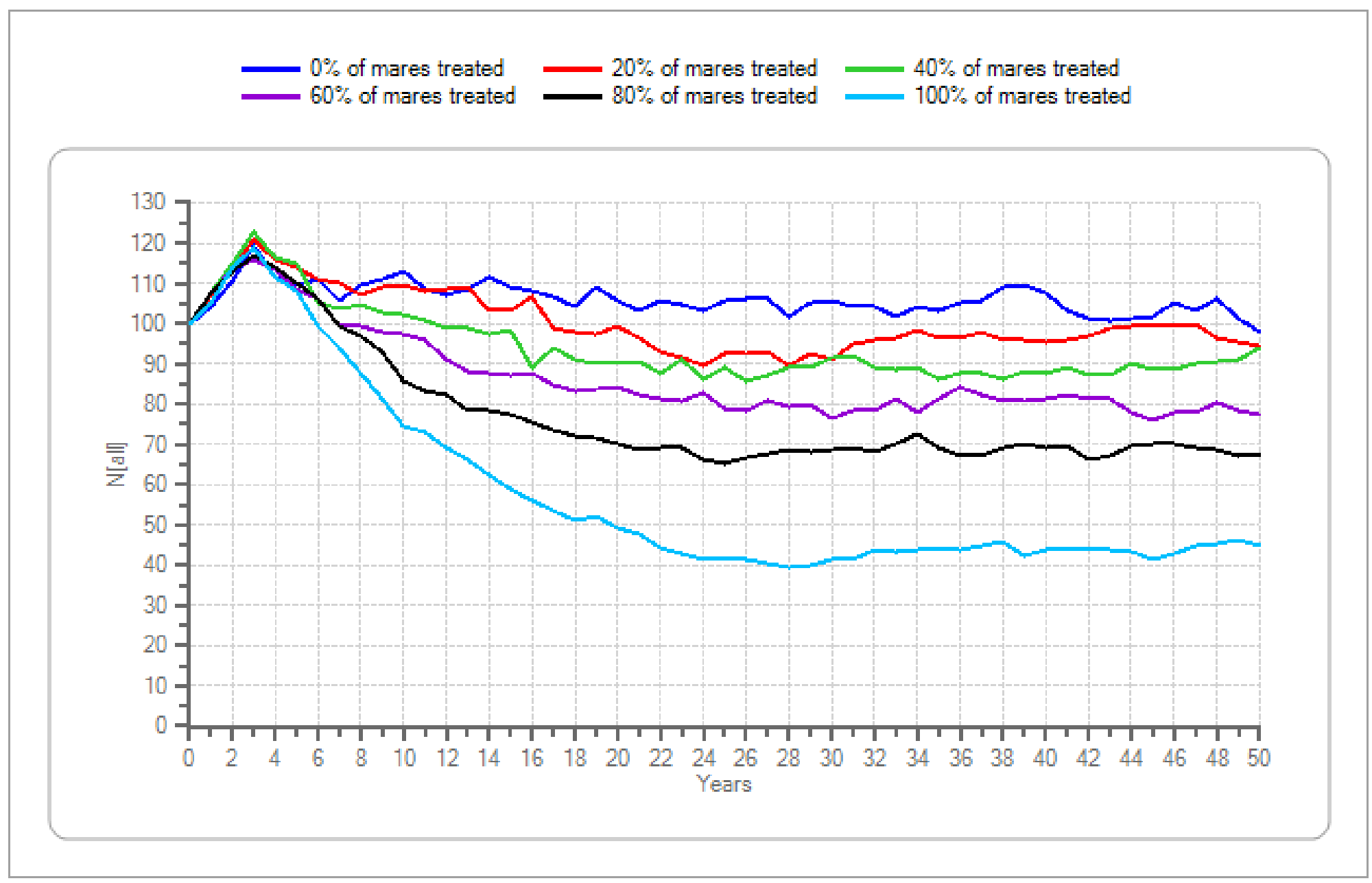

Figure 2. Initial population size $=100$, lambda $=1.10$, genetic diversity $=$ low -Continued 
Mean population size after 20 years

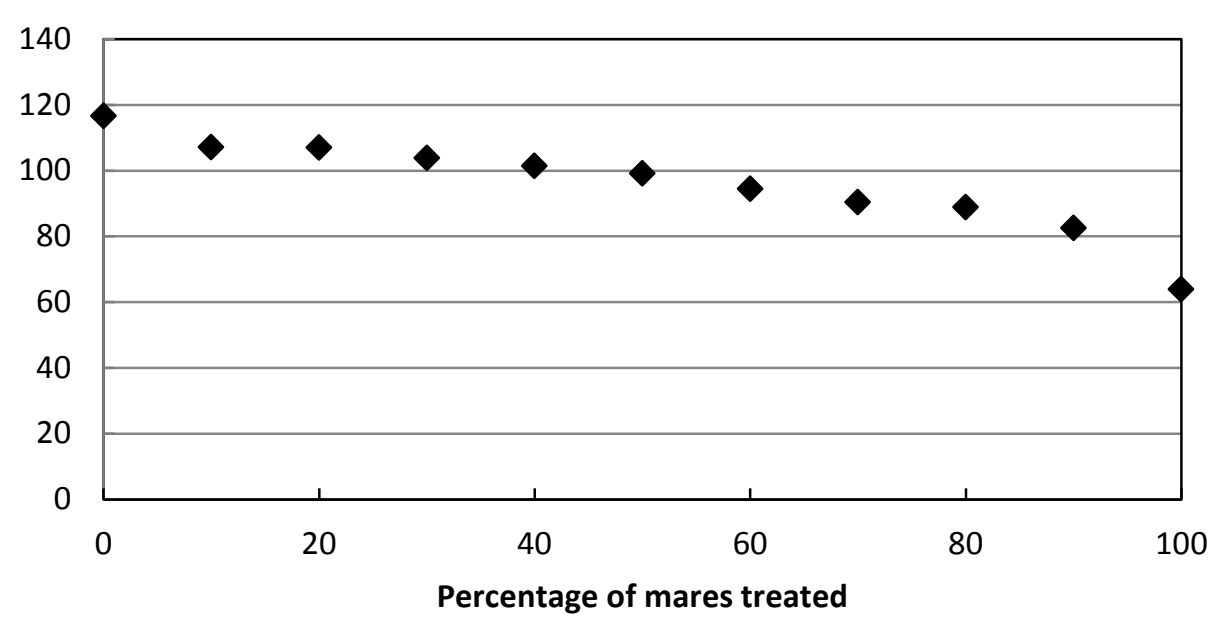

Mean probability of extirpation after $\mathbf{5 0}$ years

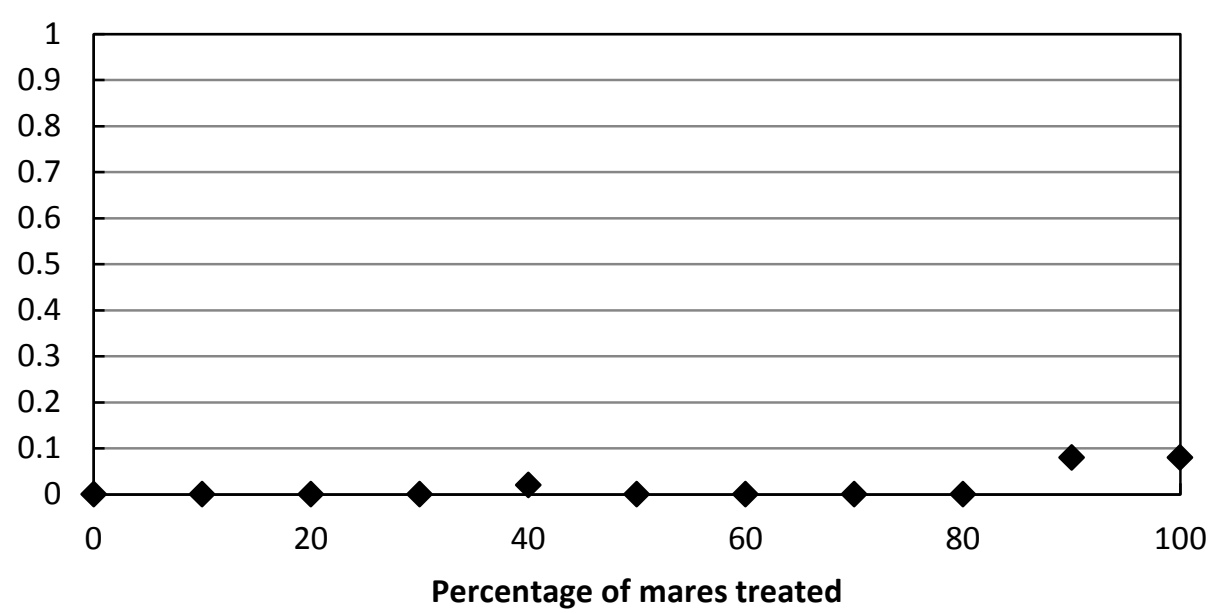

Figure 3. Initial population size $=100$, lambda $=1.15$, genetic diversity $=$ low .
Mean number of removals after 20 years

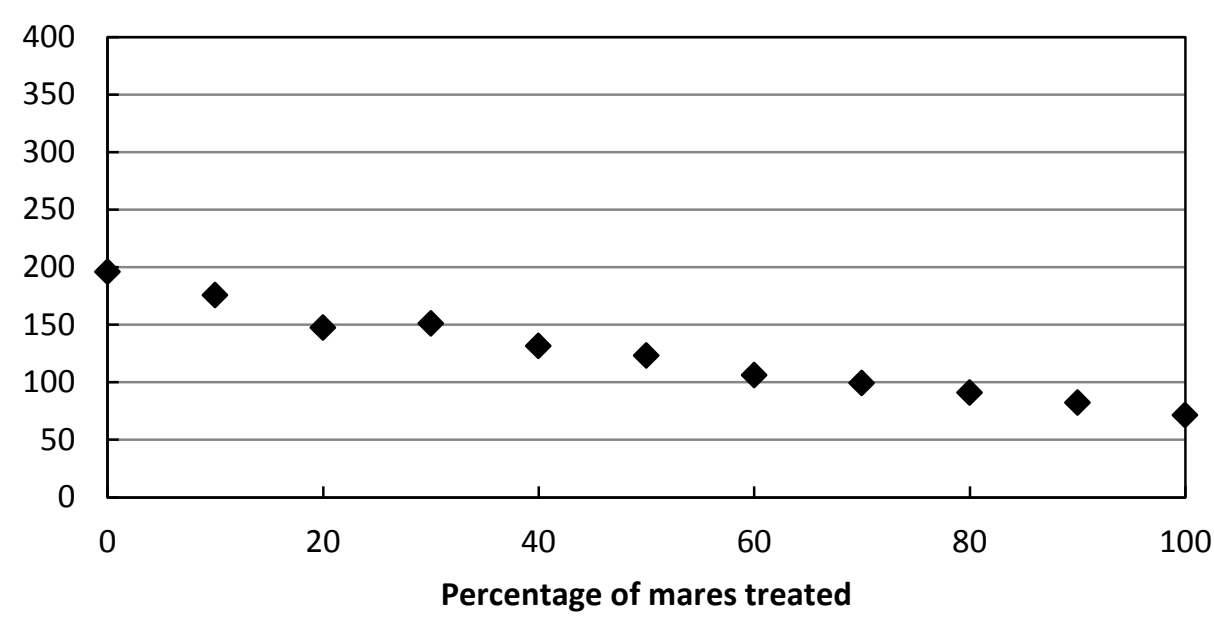

Mean heterozygosity after $\mathbf{5 0}$ years

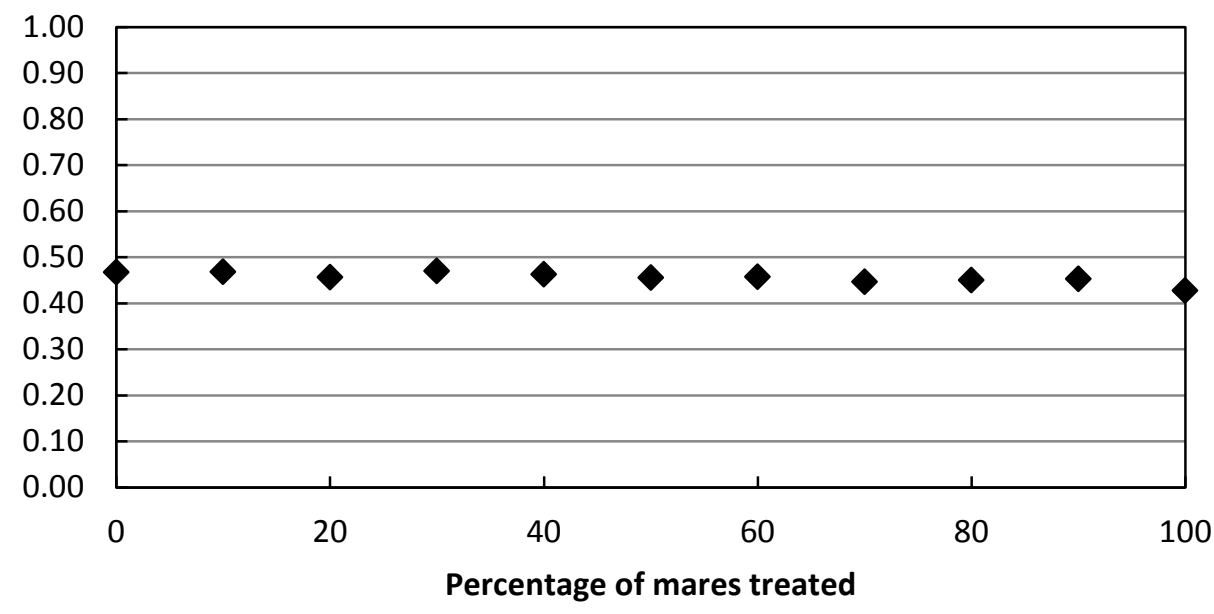




\begin{tabular}{|c|c|c|c|c|c|c|c|c|c|c|c|}
\hline Percentage of mares treated & 0 & 10 & 20 & 30 & 40 & 50 & 60 & 70 & 80 & 90 & 100 \\
\hline Mean population size after 20 years & 116.67 & 107.04 & 106.93 & 103.8 & 101.36 & 99.18 & 94.47 & 90.36 & 88.82 & 82.5 & 63.9 \\
\hline Mean number of removals after 20 years & 196.1 & 175.66 & 147.22 & 150.85 & 131.53 & 123.08 & 106.05 & 99.21 & 90.8 & 82.28 & 71.23 \\
\hline Mean probability of extirpation after 50 years & 0 & 0 & 0 & 0 & 0.02 & 0 & 0 & 0 & 0 & 0.08 & 0.08 \\
\hline Mean heterozygosity after 50 years & 0.47 & 0.47 & 0.46 & 0.47 & 0.46 & 0.46 & 0.46 & 0.45 & 0.45 & 0.45 & 0.43 \\
\hline
\end{tabular}

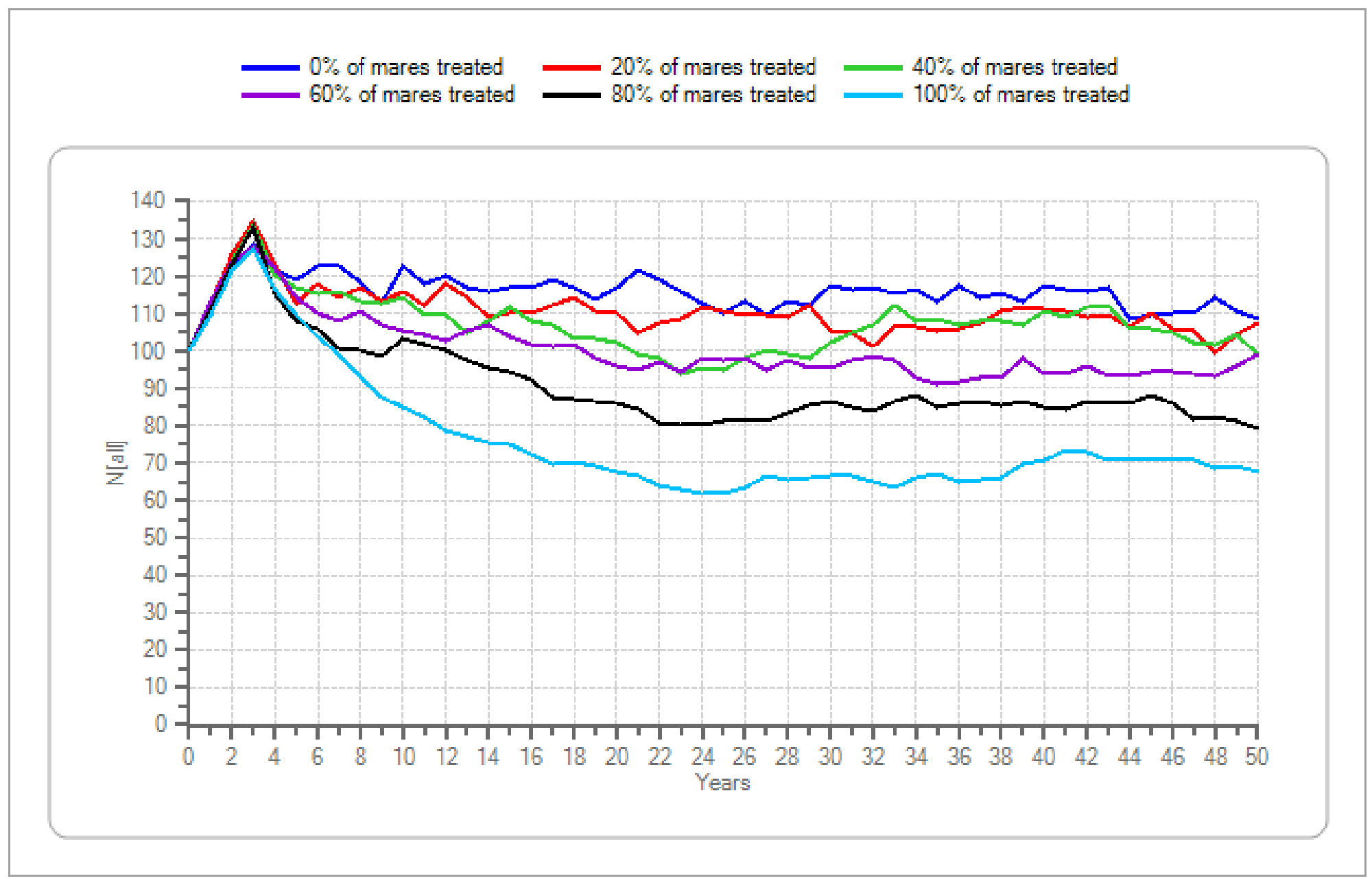

Figure 3. Initial population size $=100$, lambda $=1.15$, genetic diversity $=$ low -Continued 
Mean population size after 20 years

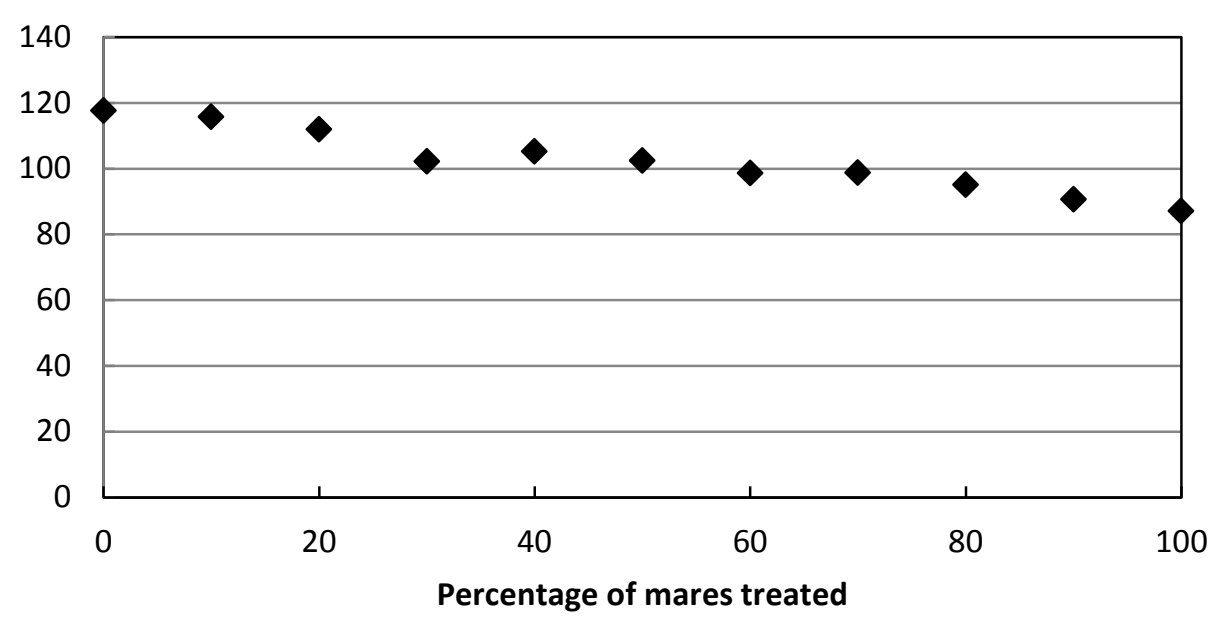

Mean probability of extirpation after $\mathbf{5 0}$ years

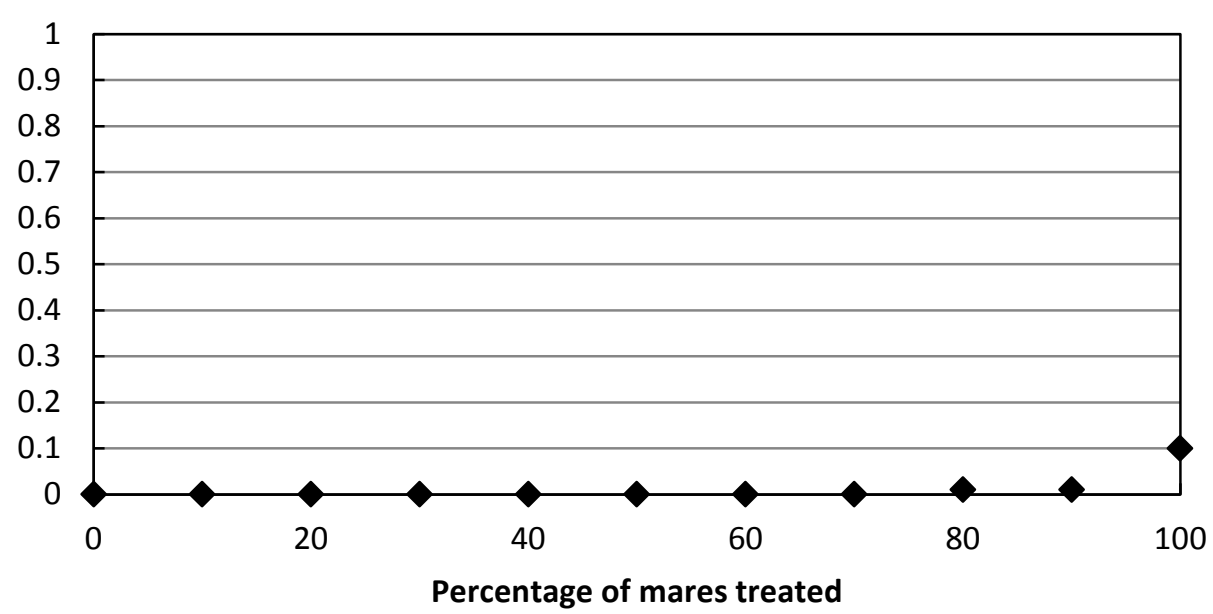

Figure 4. Initial population size $=100$, lambda $=1.20$, genetic diversity $=$ low .
Mean number of removals after 20 years

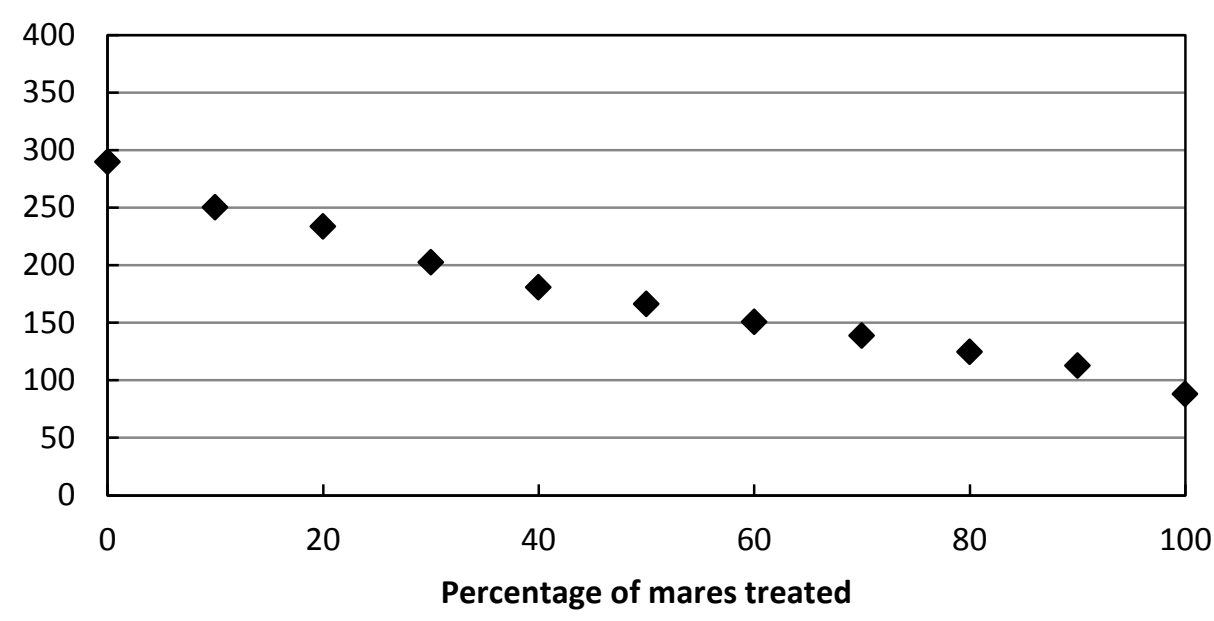

Mean heterozygosity after $\mathbf{5 0}$ years

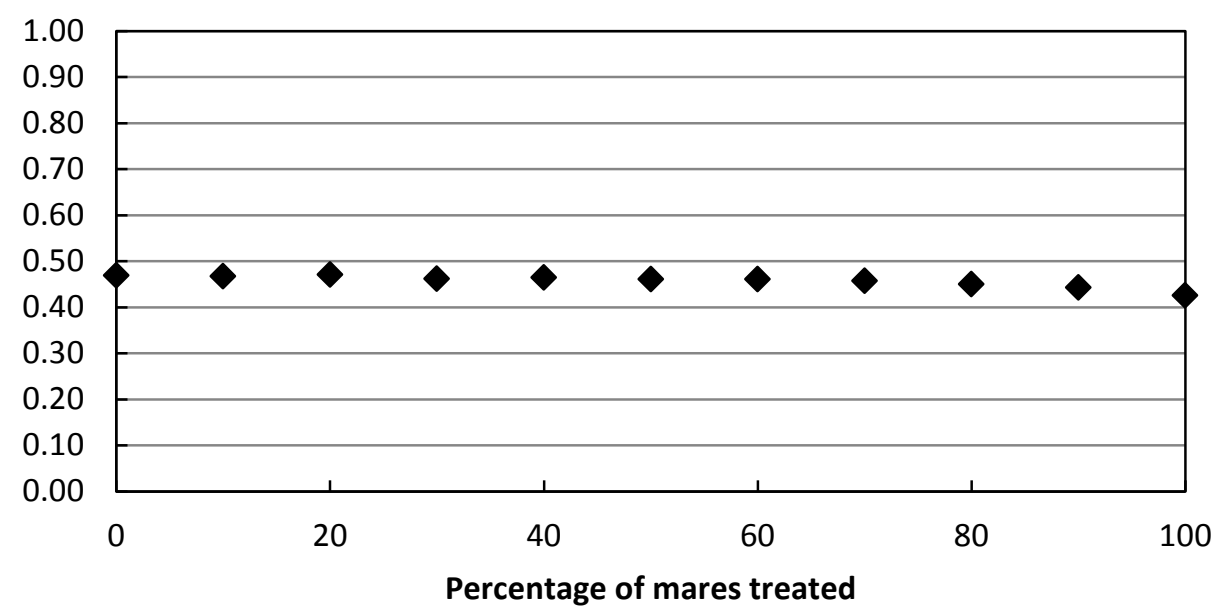




\begin{tabular}{|c|c|c|c|c|c|c|c|c|c|c|c|}
\hline Percentage of mares treated & 0 & 10 & 20 & 30 & 40 & 50 & 60 & 70 & 80 & 90 & 100 \\
\hline Mean population size after 20 years & 117.66 & 115.72 & 111.98 & 102.21 & 105.14 & 102.37 & 98.63 & 98.73 & 95.01 & 90.61 & 87.04 \\
\hline Mean number of removals after 20 years & 289.73 & 250.34 & 233.51 & 202.32 & 180.67 & 166.39 & 150.74 & 138.75 & 124.57 & 112.71 & 88.13 \\
\hline Mean probability of extirpation after 50 years & 0 & 0 & 0 & 0 & 0 & 0 & 0 & 0 & 0.01 & 0.01 & 0.1 \\
\hline Mean heterozygosity after 50 years & 0.47 & 0.47 & 0.47 & 0.46 & 0.46 & 0.46 & 0.46 & 0.46 & 0.45 & 0.44 & 0.43 \\
\hline
\end{tabular}

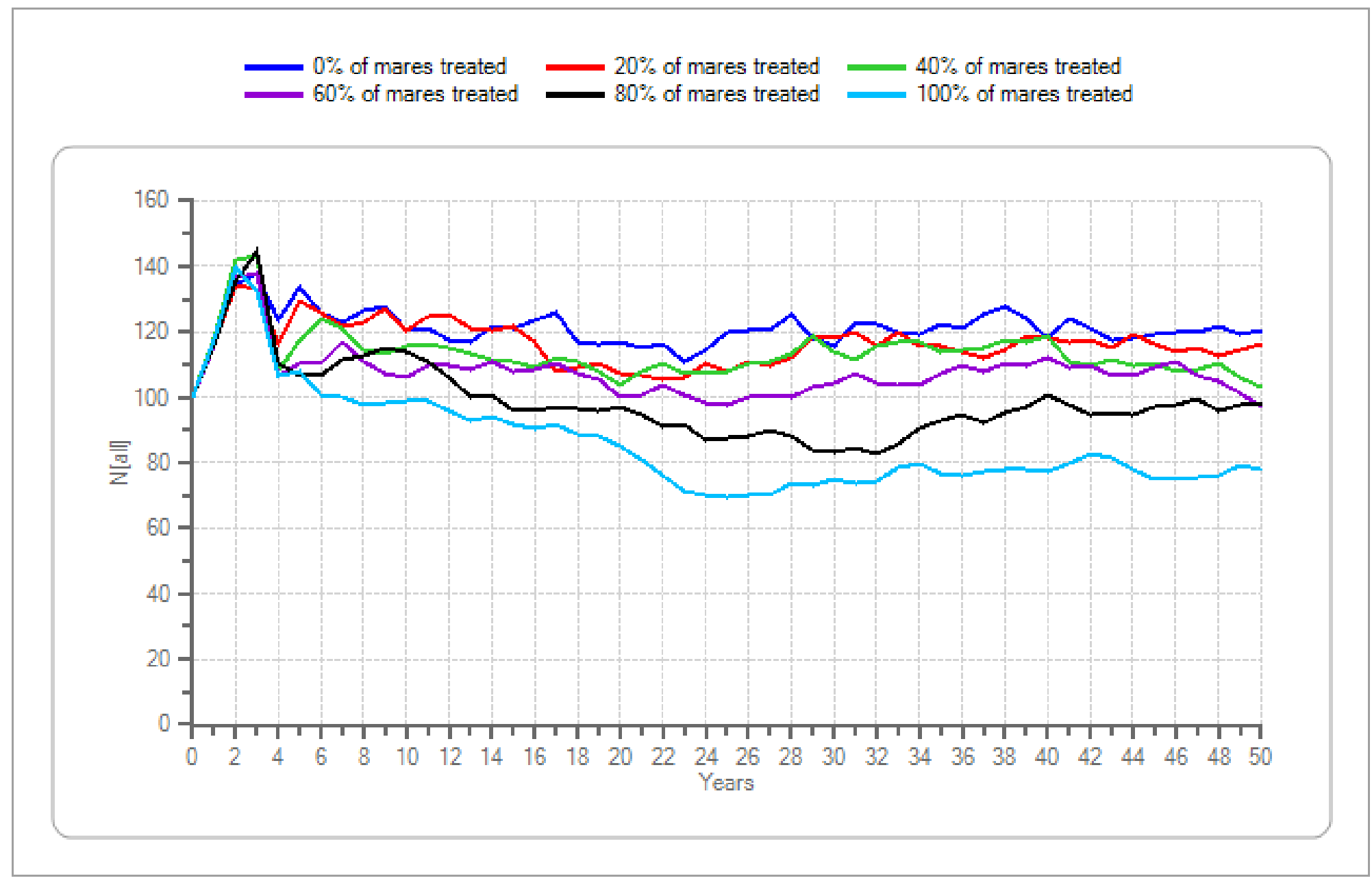

Figure 4. Initial population size $=100$, lambda $=1.20$, genetic diversity $=$ low - Continued 
Mean population size after 20 years

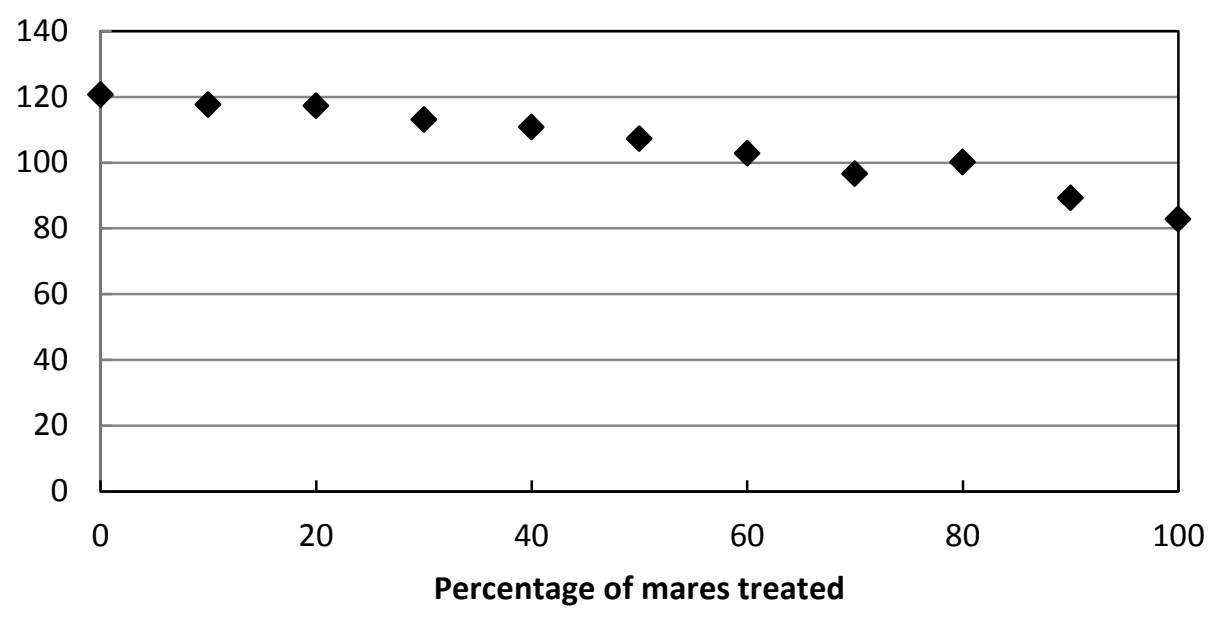

Mean probability of extirpation after $\mathbf{5 0}$ years

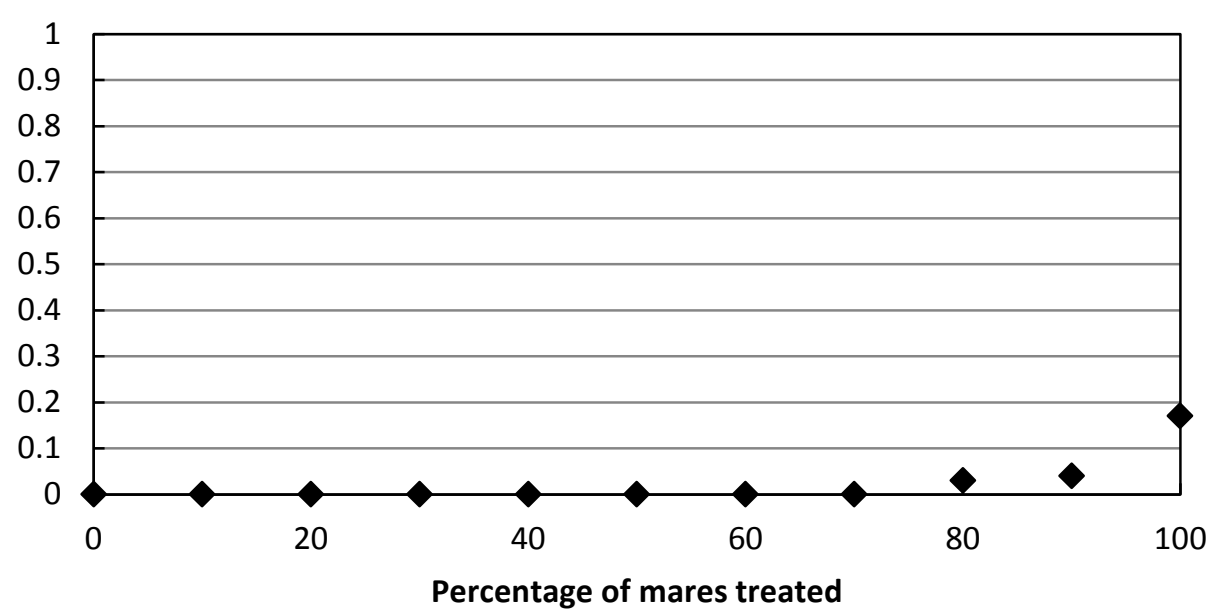

Figure 5. Initial population size $=100$, lambda $=1.25$, genetic diversity $=$ low .
Mean number of removals after 20 years

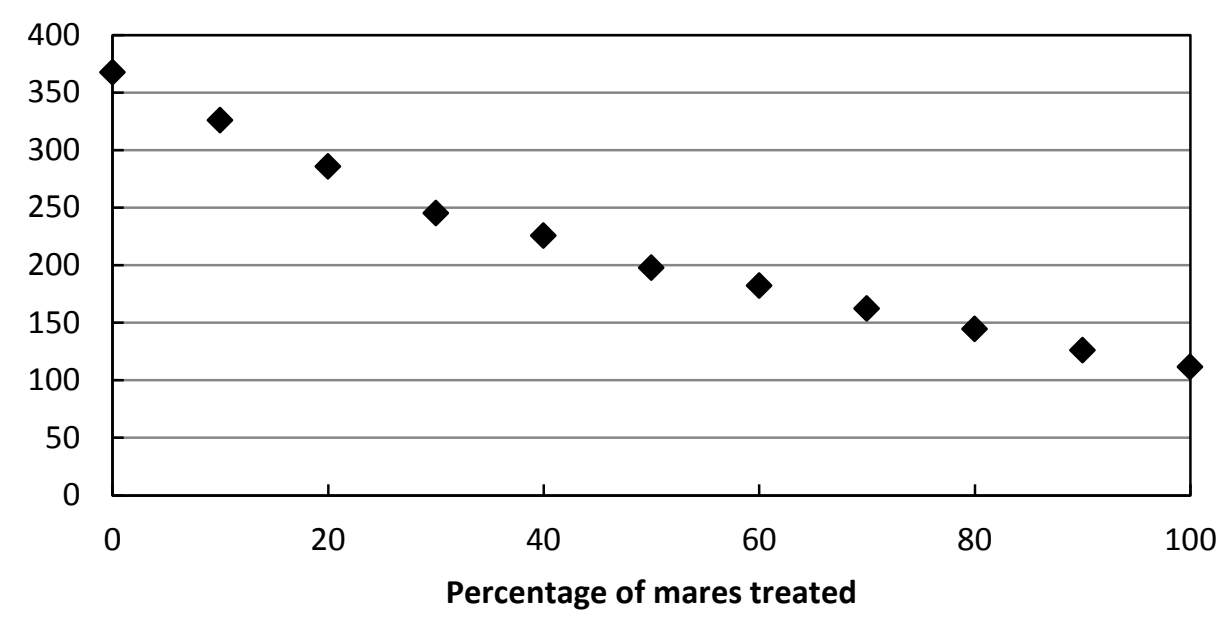

Mean heterozygosity after $\mathbf{5 0}$ years

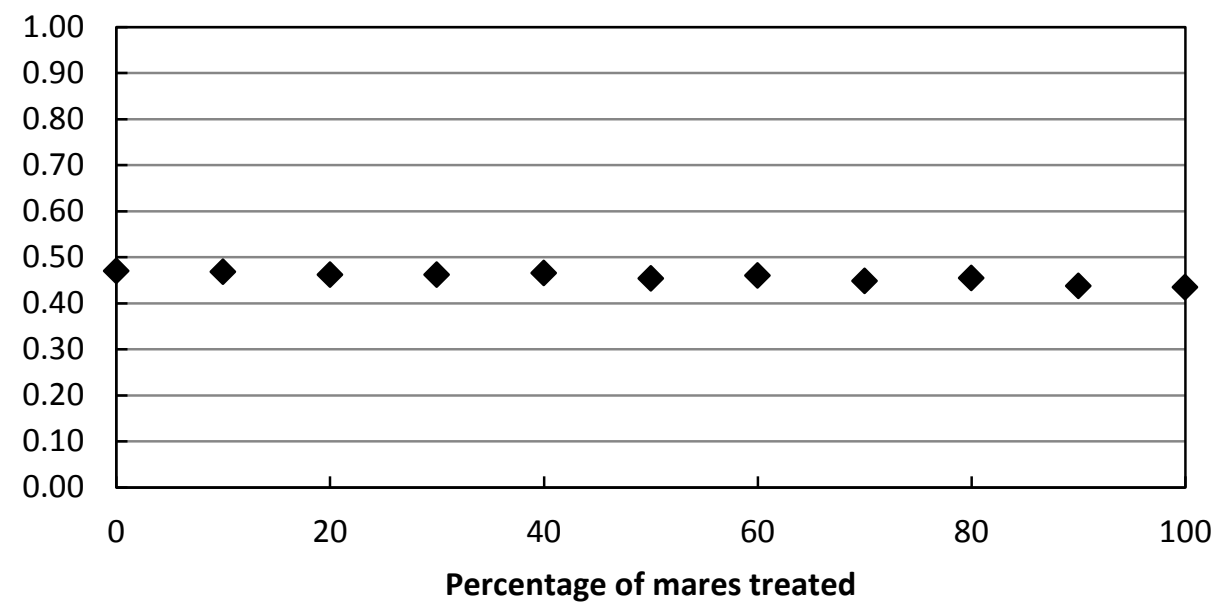




\begin{tabular}{lccccccccccc}
\hline \multicolumn{1}{c}{ Percentage of mares treated } & $\mathbf{0}$ & $\mathbf{1 0}$ & $\mathbf{2 0}$ & $\mathbf{3 0}$ & $\mathbf{4 0}$ & $\mathbf{5 0}$ & $\mathbf{6 0}$ & $\mathbf{7 0}$ & $\mathbf{8 0}$ & $\mathbf{9 0}$ & $\mathbf{1 0 0}$ \\
\hline Mean population size after 20 years & 120.71 & 117.59 & 117.22 & 113.11 & 110.75 & 107.17 & 102.75 & 96.64 & 100.1 & 89.18 & 82.72 \\
Mean number of removals after 20 years & 367.4 & 325.91 & 285.86 & 245.19 & 225.62 & 197.56 & 182.24 & 162.3 & 144.4 & 126.13 & 111.72 \\
Mean probability of extirpation after 50 years & 0 & 0 & 0 & 0 & 0 & 0 & 0 & 0 & 0.03 & 0.04 & 0.17 \\
Mean heterozygosity after 50 years & 0.47 & 0.47 & 0.46 & 0.46 & 0.47 & 0.45 & 0.46 & 0.45 & 0.45 & 0.44 & 0.43 \\
\hline
\end{tabular}

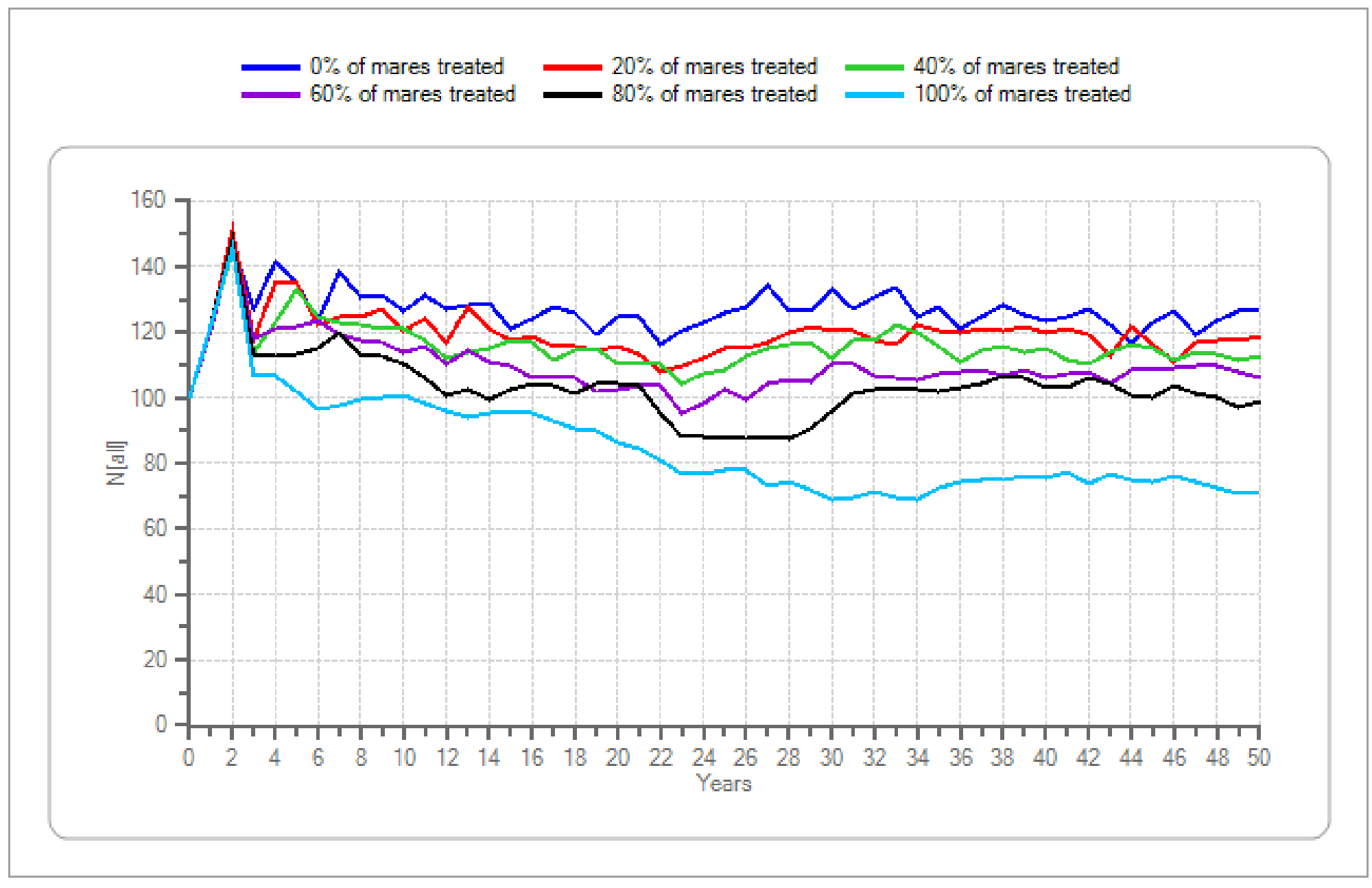

Figure 5. Initial population size $=100$, lambda $=1.25$, genetic diversity $=$ low - Continued 
Mean population size after 20 years

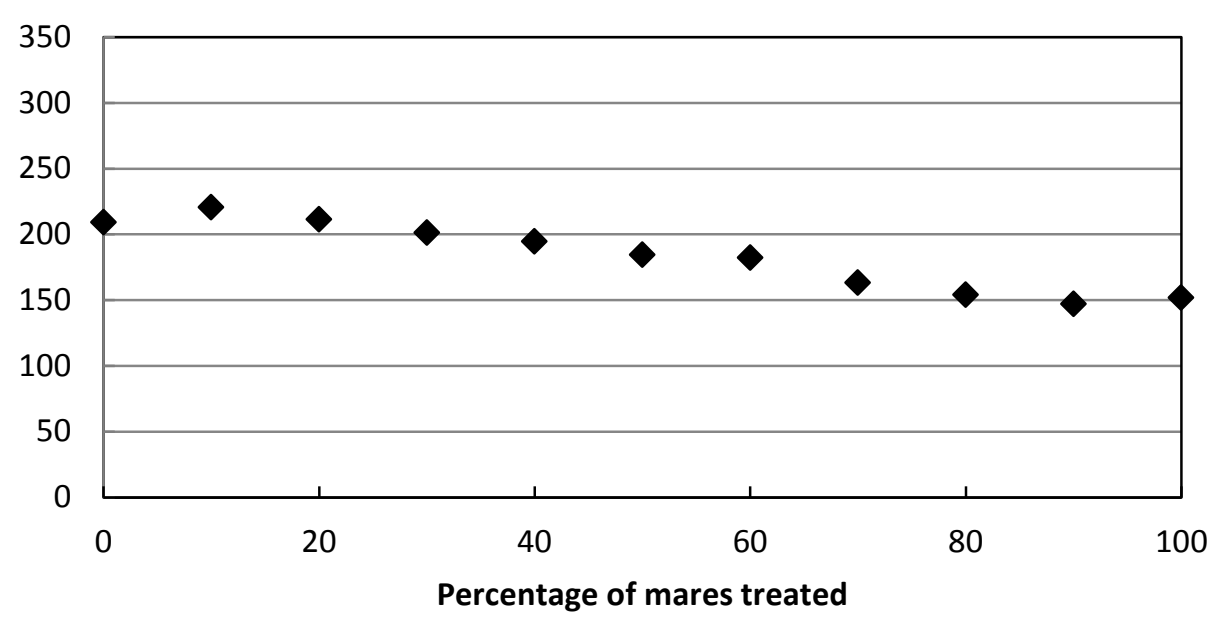

Mean probability of extirpation after $\mathbf{5 0}$ years

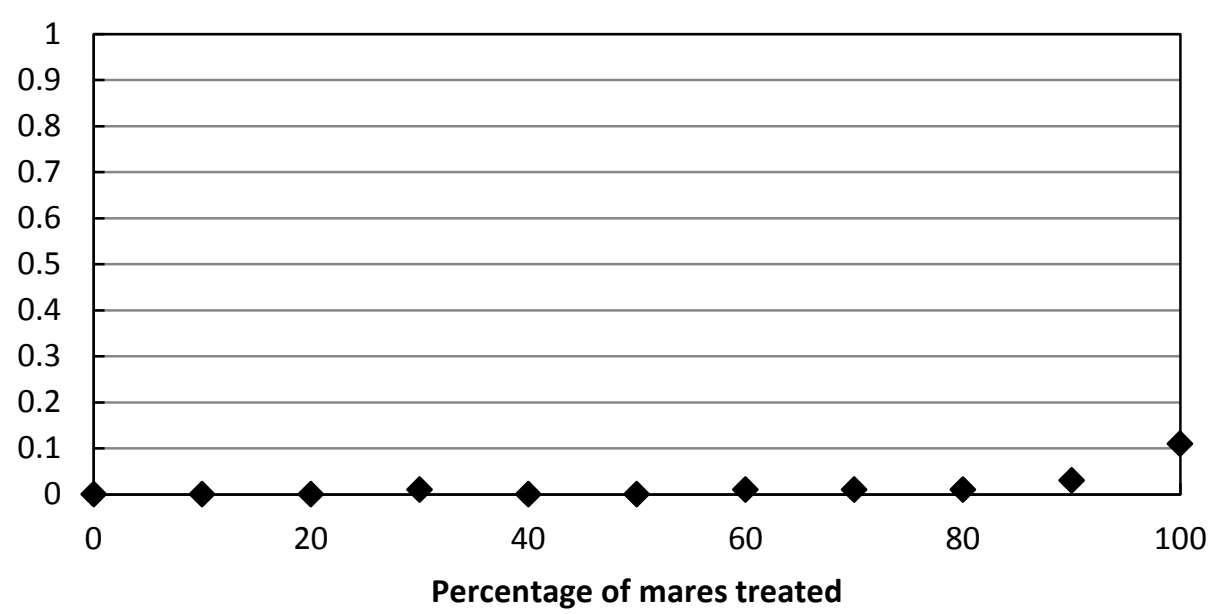

Figure 6. Initial population size $=250$, lambda $=1.05$, genetic diversity $=$ low .
Mean number of removals after 20 years

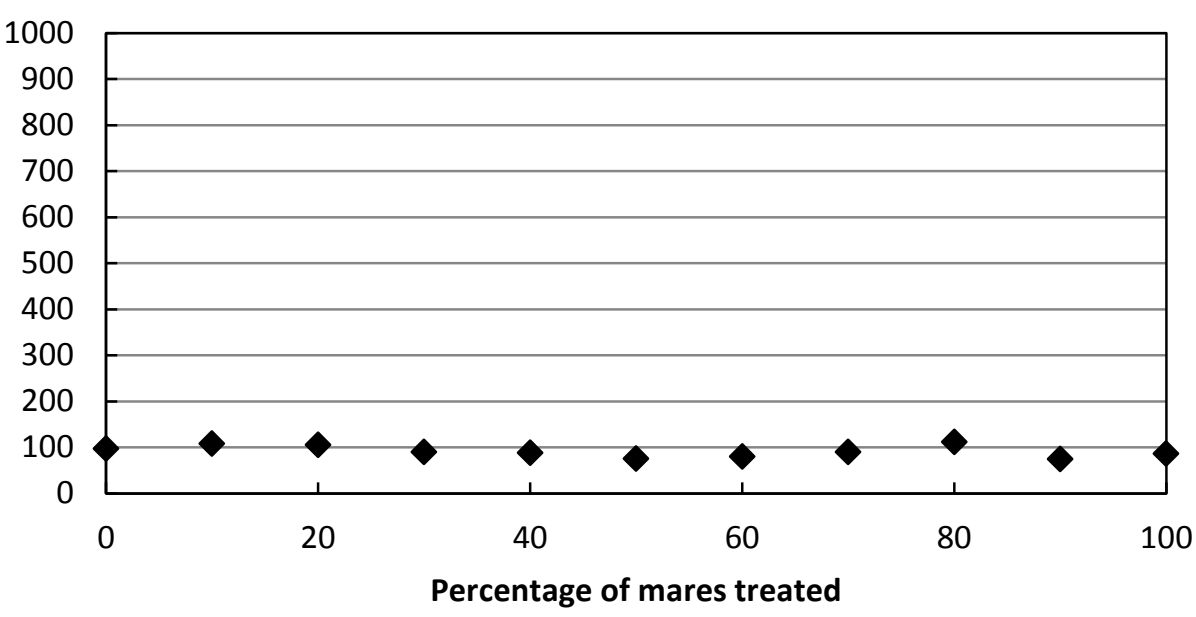

Mean heterozygosity after $\mathbf{5 0}$ years

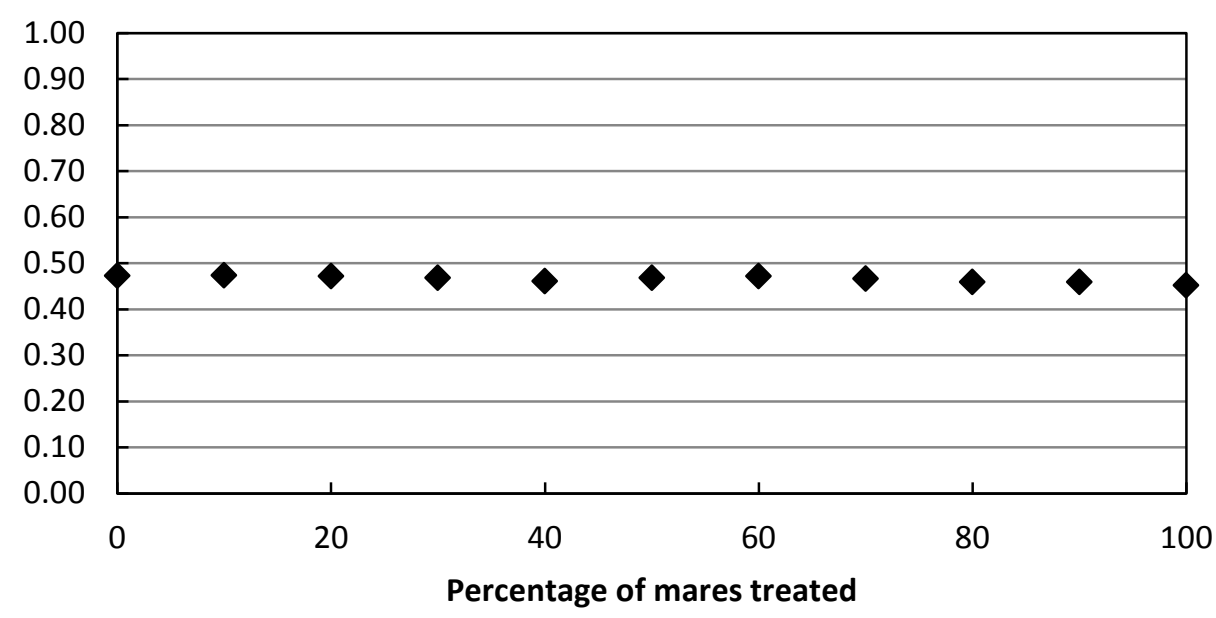




\begin{tabular}{lccccccccccc}
\hline \multicolumn{1}{c}{ Percentage of mares treated } & $\mathbf{0}$ & $\mathbf{1 0}$ & $\mathbf{2 0}$ & $\mathbf{3 0}$ & $\mathbf{4 0}$ & $\mathbf{5 0}$ & $\mathbf{6 0}$ & $\mathbf{7 0}$ & $\mathbf{8 0}$ & $\mathbf{9 0}$ & $\mathbf{1 0 0}$ \\
\hline Mean population size after 20 years & 209.16 & 220.46 & 211.25 & 201.33 & 194.71 & 184.37 & 182.2 & 163.33 & 153.91 & 147.12 & 151.88 \\
Mean number of removals after 20 years & 96.65 & 107.54 & 105.05 & 89.47 & 87.49 & 75.07 & 79.56 & 89.29 & 111.56 & 74 & 85.79 \\
Mean probability of extirpation after 50 years & 0 & 0 & 0 & 0.01 & 0 & 0 & 0.01 & 0.01 & 0.01 & 0.03 & 0.11 \\
Mean heterozygosity after 50 years & 0.47 & 0.47 & 0.47 & 0.47 & 0.46 & 0.47 & 0.47 & 0.47 & 0.46 & 0.46 & 0.45 \\
\hline
\end{tabular}

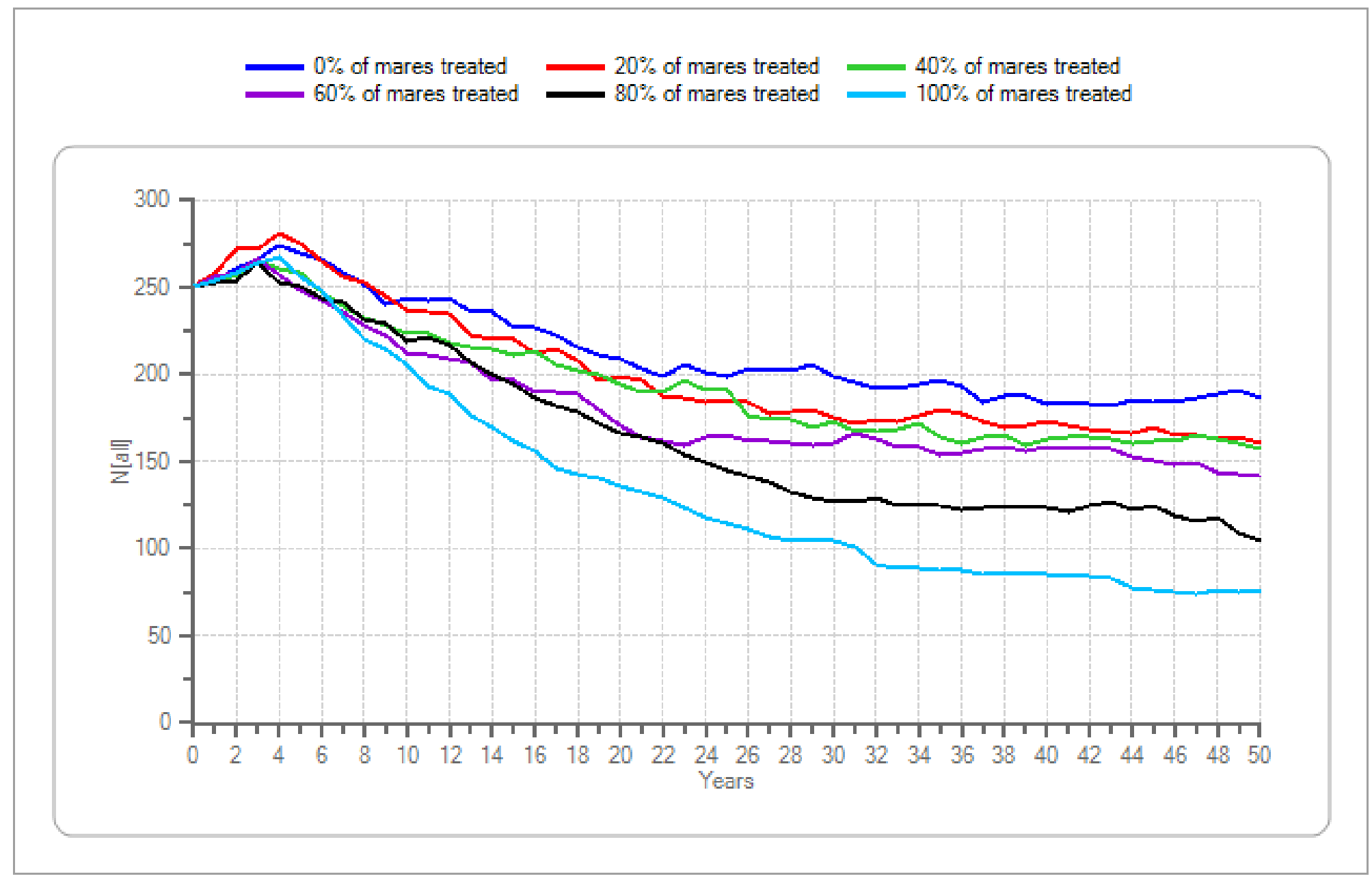

Figure 6. Initial population size $=250$, lambda $=1.05$, genetic diversity $=$ low - Continued 
Mean population size after 20 years

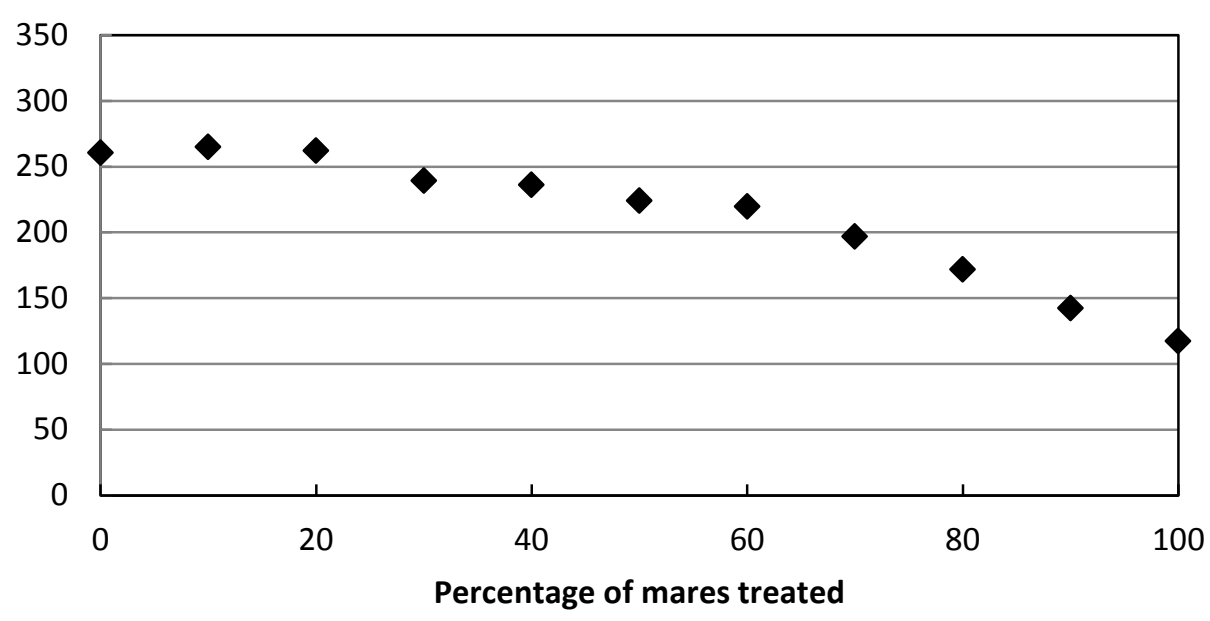

Mean probability of extirpation after $\mathbf{5 0}$ years

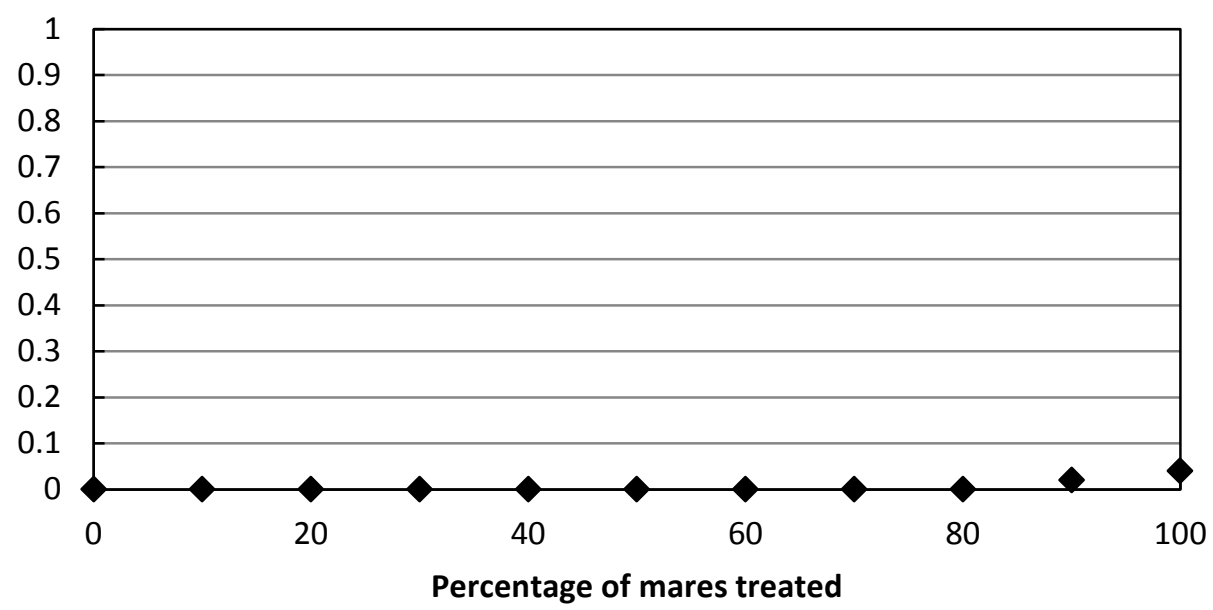

Figure 7. Initial population size $=250$, lambda $=1.10$, genetic diversity $=$ low .
Mean number of removals after 20 years

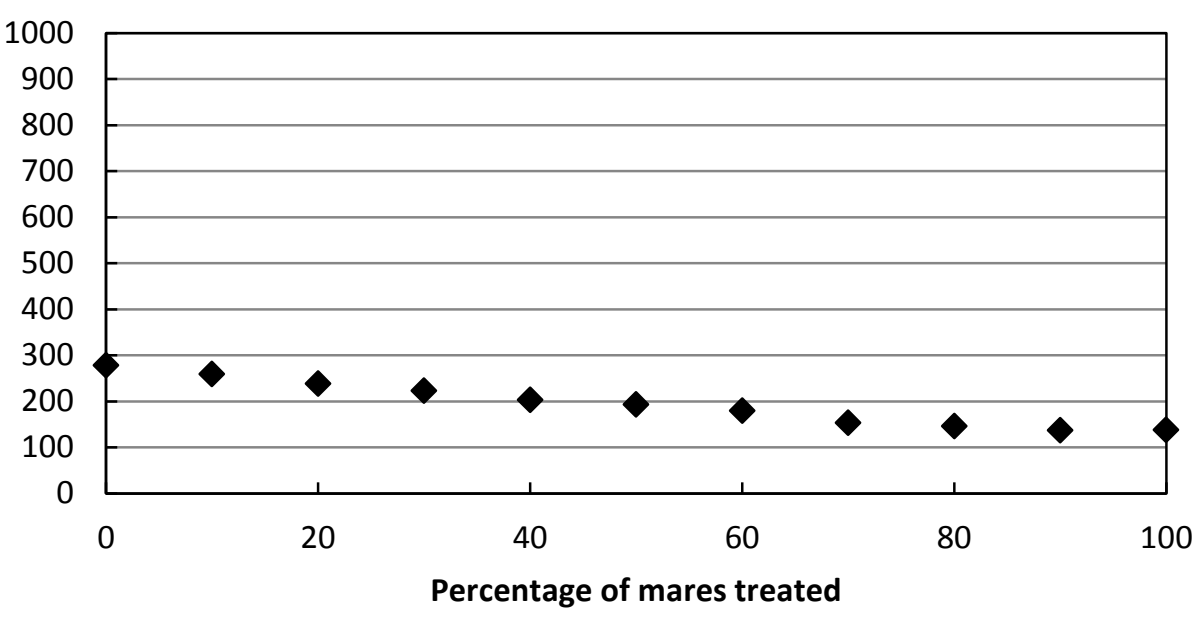

Mean heterozygosity after $\mathbf{5 0}$ years

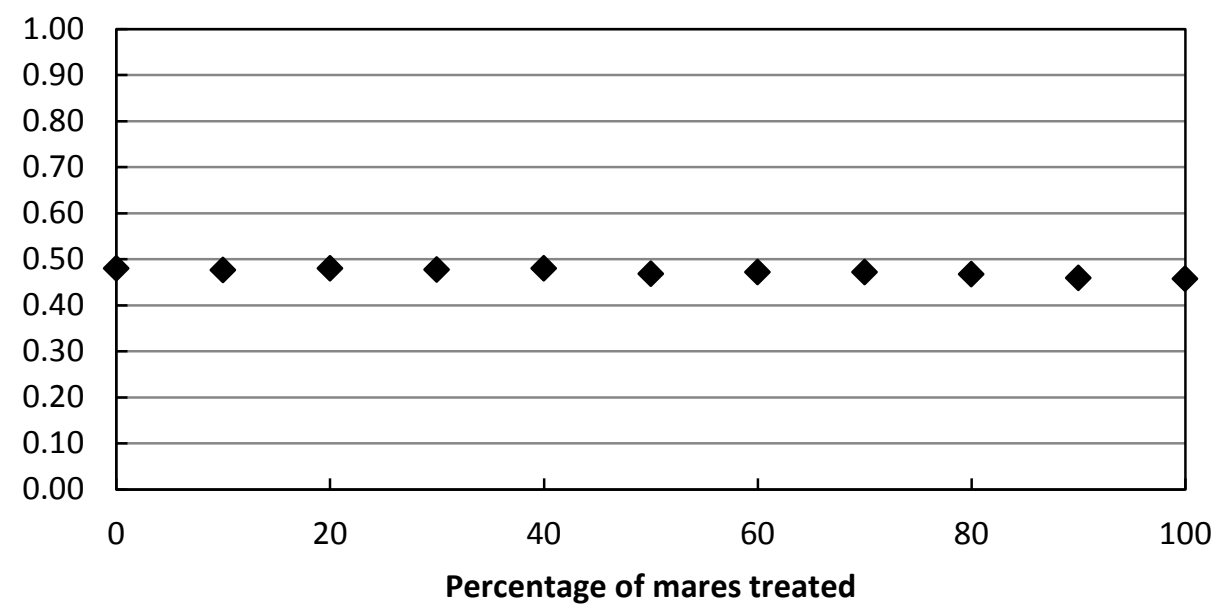




\begin{tabular}{lccccccccccc}
\hline \multicolumn{1}{c}{ Percentage of mares treated } & $\mathbf{0}$ & $\mathbf{1 0}$ & $\mathbf{2 0}$ & $\mathbf{3 0}$ & $\mathbf{4 0}$ & $\mathbf{5 0}$ & $\mathbf{6 0}$ & $\mathbf{7 0}$ & $\mathbf{8 0}$ & $\mathbf{9 0}$ & $\mathbf{1 0 0}$ \\
\hline Mean population size after 20 years & 260.38 & 264.77 & 262.05 & 239.17 & 236 & 224.01 & 219.74 & 196.91 & 171.78 & 142.36 & 117.4 \\
Mean number of removals after 20 years & 278.3 & 259.21 & 237.83 & 223.07 & 202.93 & 192.93 & 179.33 & 153.23 & 145.94 & 137.16 & 137.79 \\
Mean probability of extirpation after 50 years & 0 & 0 & 0 & 0 & 0 & 0 & 0 & 0 & 0 & 0.02 & 0.04 \\
Mean heterozygosity after 50 years & 0.48 & 0.48 & 0.48 & 0.48 & 0.48 & 0.47 & 0.47 & 0.47 & 0.47 & 0.46 & 0.46 \\
\hline
\end{tabular}

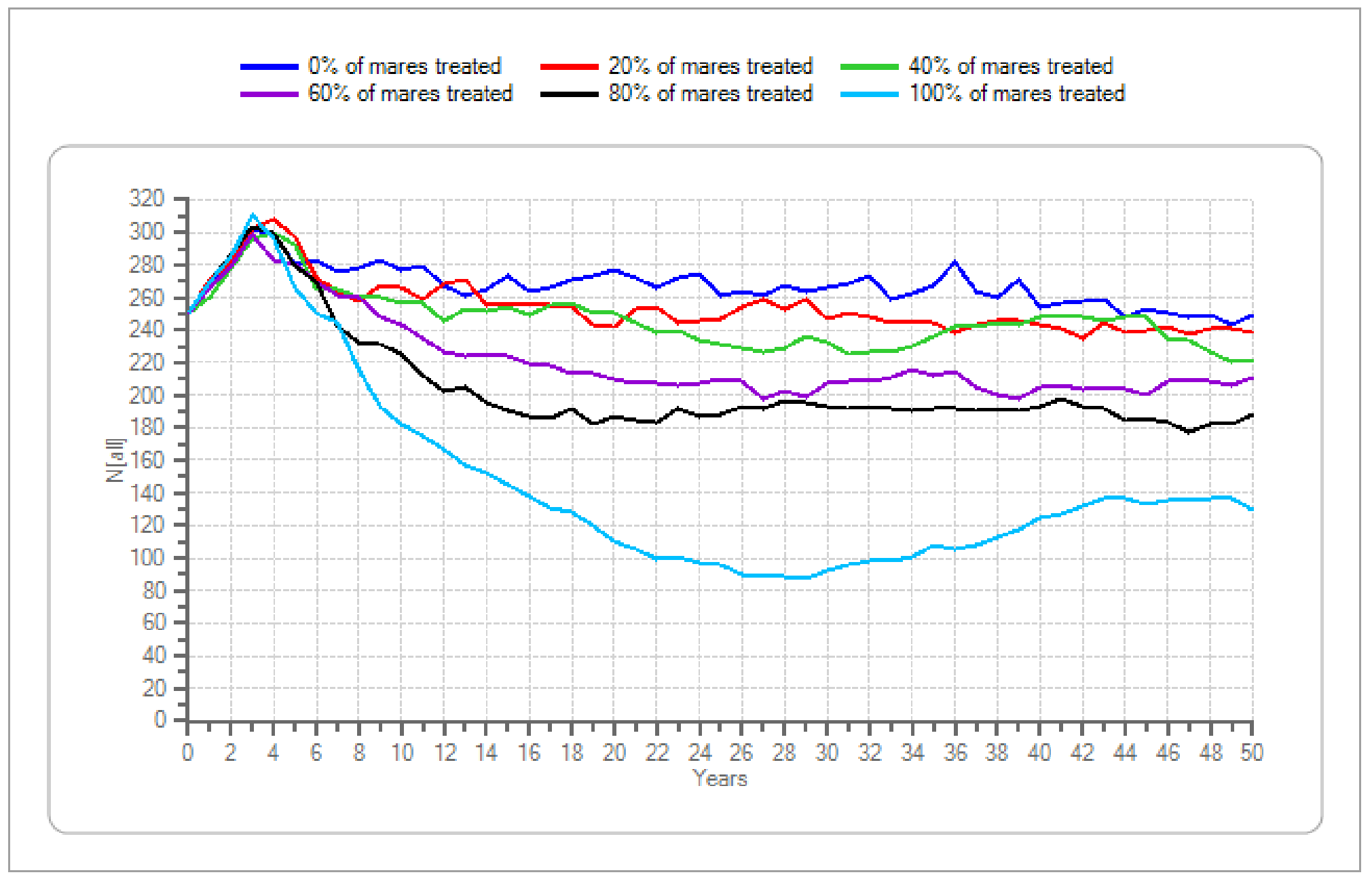

Figure 7. Initial population size $=250$, lambda $=1.10$, genetic diversity $=$ low - Continued 
Mean population size after 20 years

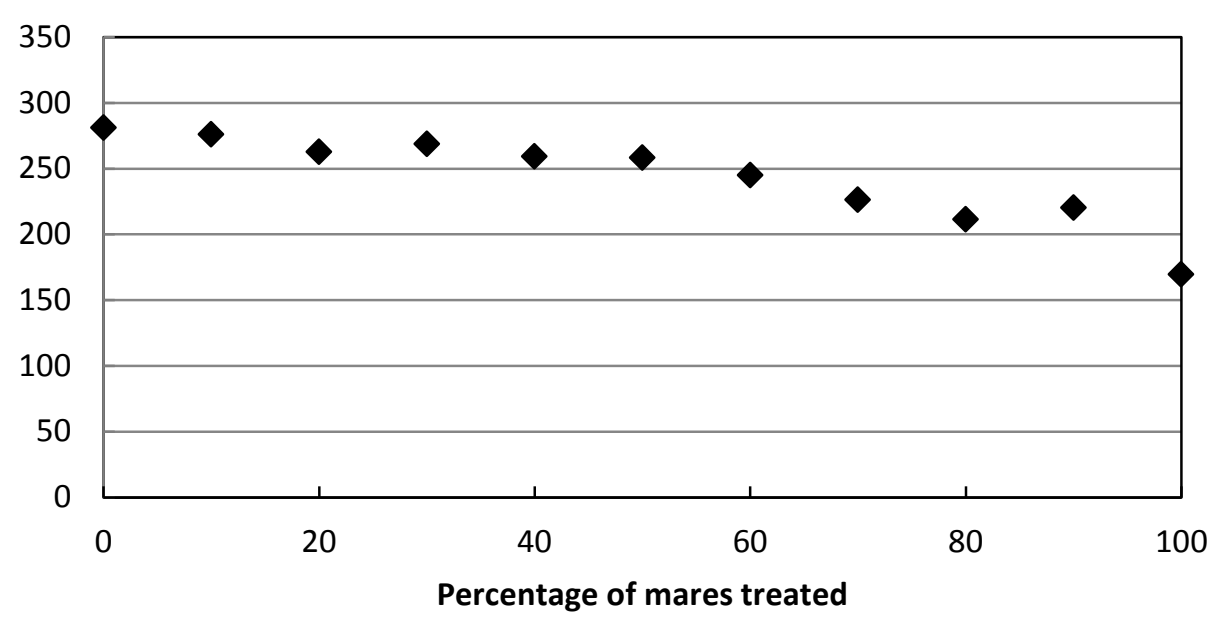

Mean probability of extirpation after $\mathbf{5 0}$ years

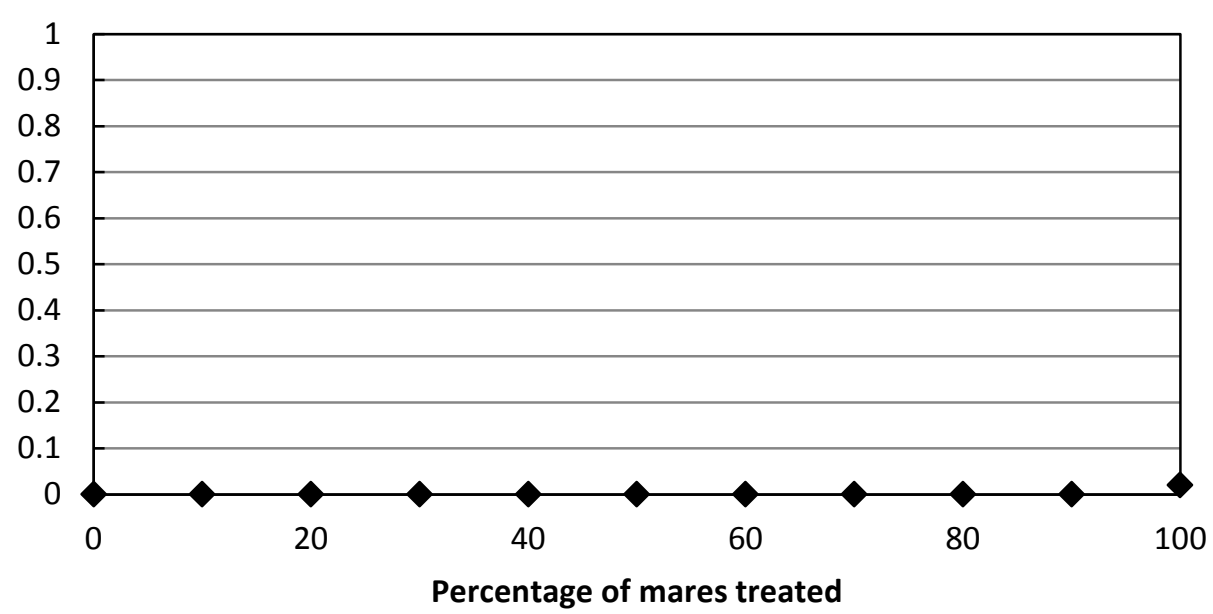

Figure 8. Initial population size $=250$, lambda $=1.15$, genetic diversity $=$ low .

\section{Mean number of removals after 20 years}

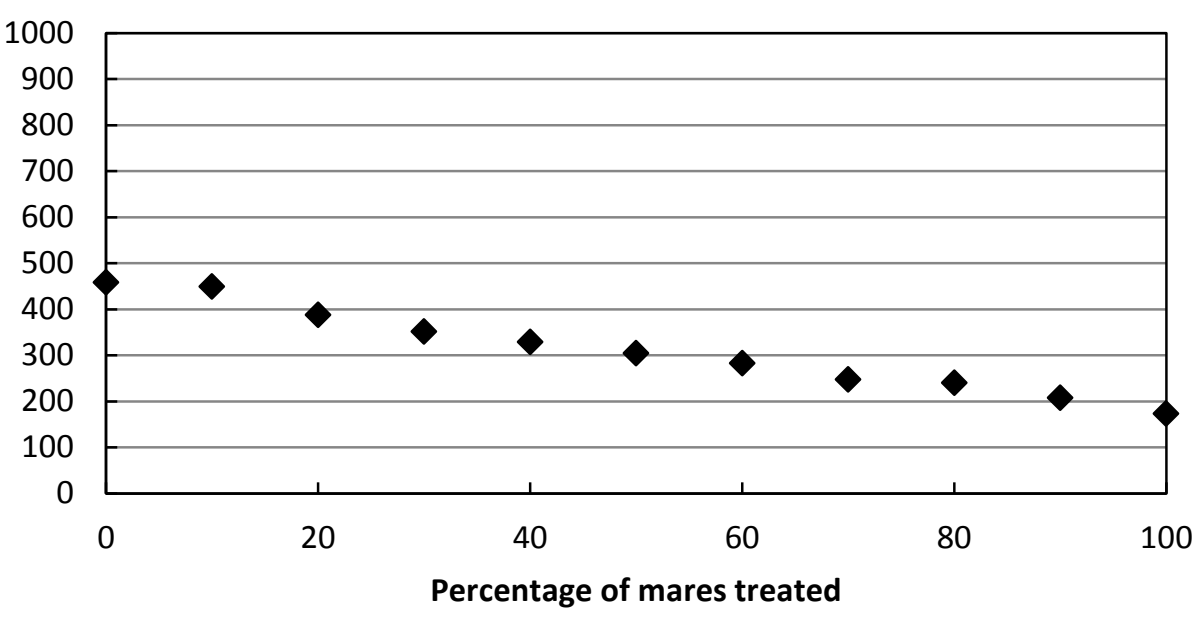

Mean heterozygosity after $\mathbf{5 0}$ years

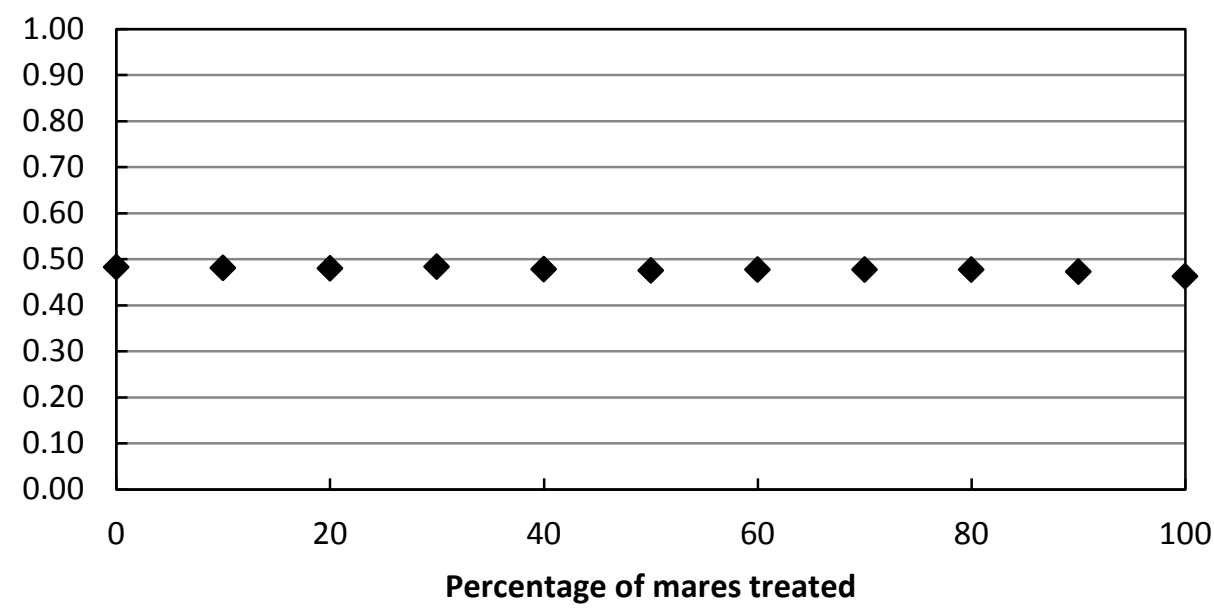




\begin{tabular}{lccccccccccc}
\hline \multicolumn{1}{c}{ Percentage of mares treated } & $\mathbf{0}$ & $\mathbf{1 0}$ & $\mathbf{2 0}$ & $\mathbf{3 0}$ & $\mathbf{4 0}$ & $\mathbf{5 0}$ & $\mathbf{6 0}$ & $\mathbf{7 0}$ & $\mathbf{8 0}$ & $\mathbf{9 0}$ & $\mathbf{1 0 0}$ \\
\hline Mean population size after 20 years & 281.13 & 275.97 & 262.64 & 268.65 & 259.12 & 258.31 & 244.81 & 226.37 & 211.37 & 220.14 & 169.63 \\
Mean number of removals after 20 years & 458.22 & 448.92 & 387.51 & 351.03 & 328.21 & 304.61 & 282.41 & 247.07 & 239.97 & 207.47 & 173.26 \\
Mean probability of extirpation after 50 years & 0 & 0 & 0 & 0 & 0 & 0 & 0 & 0 & 0 & 0 & 0.02 \\
Mean heterozygosity after 50 years & 0.48 & 0.48 & 0.48 & 0.48 & 0.48 & 0.48 & 0.48 & 0.48 & 0.48 & 0.47 & 0.46 \\
\hline
\end{tabular}

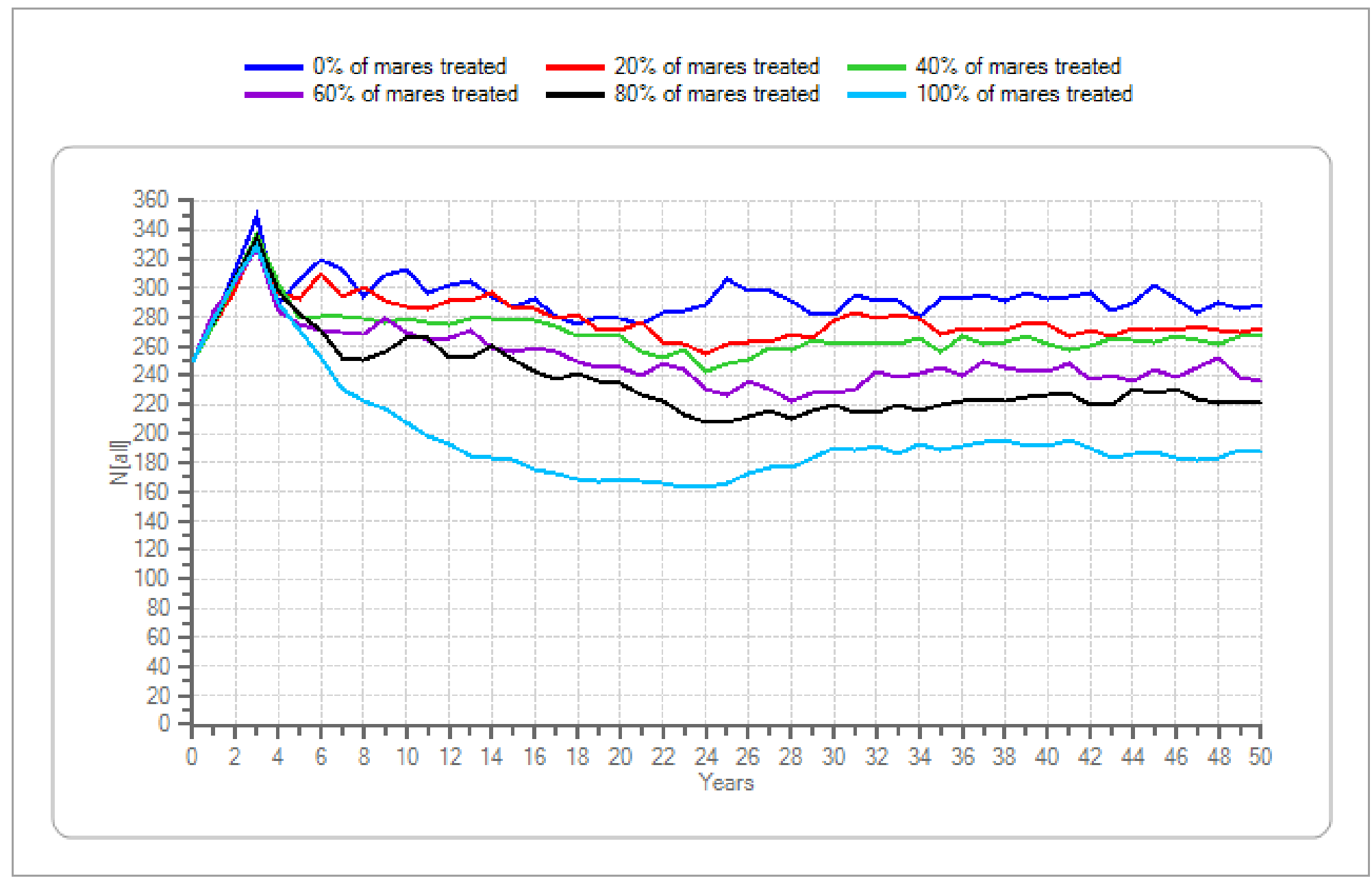

Figure 8. Initial population size $=250$, lambda $=1.15$, genetic diversity $=$ low - Continued 
Mean population size after 20 years

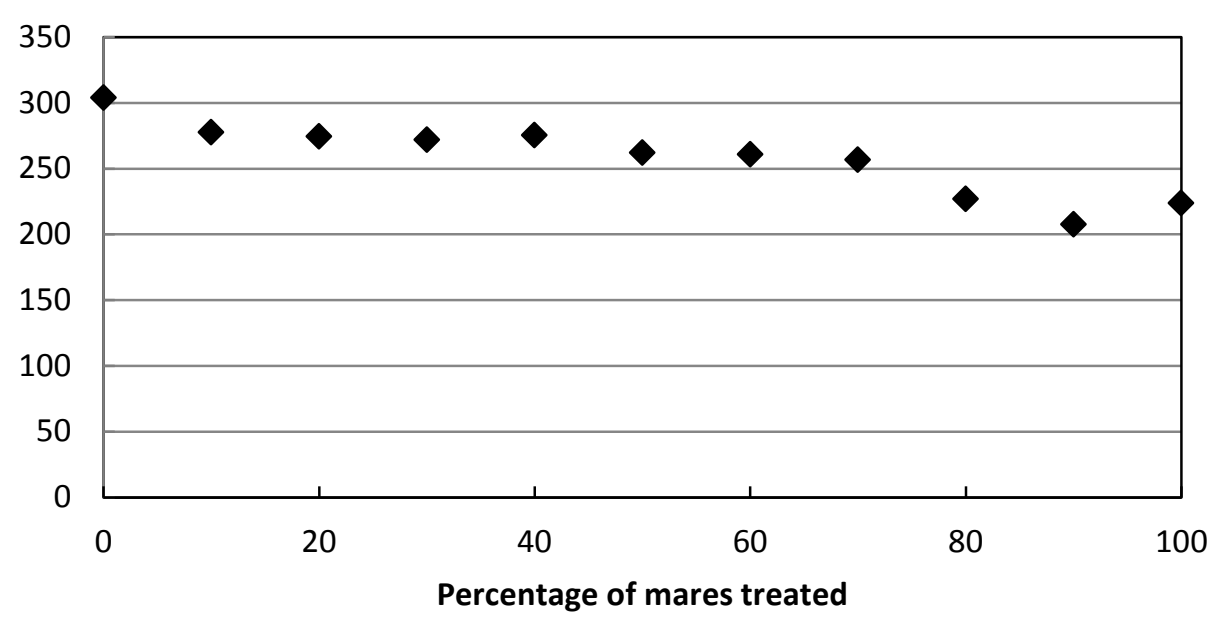

Mean probability of extirpation after $\mathbf{5 0}$ years

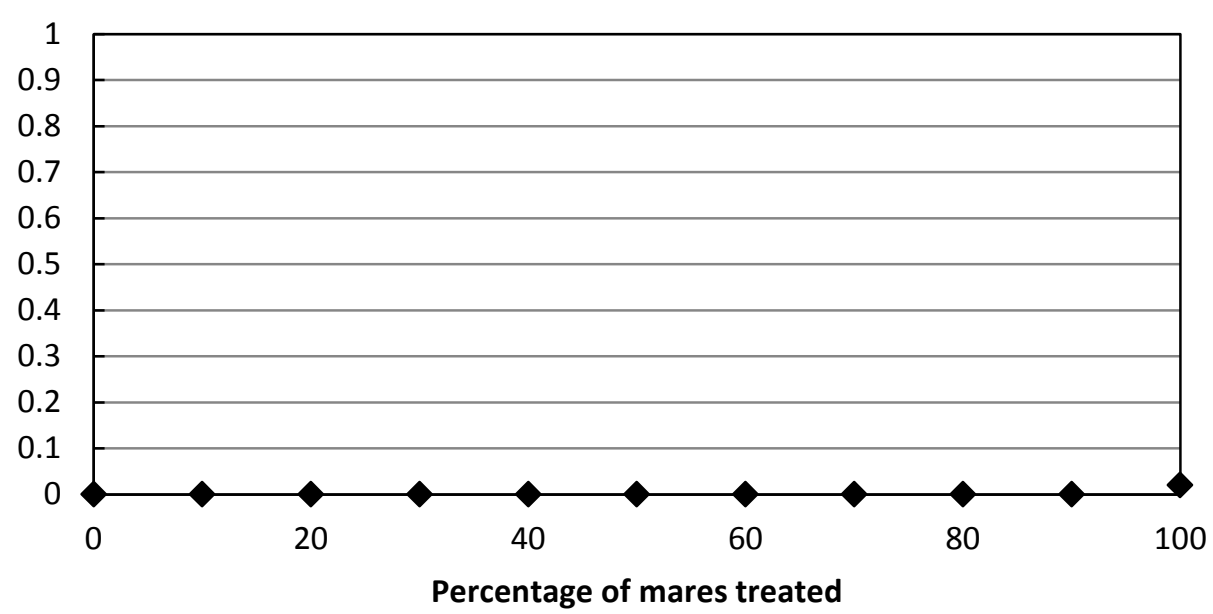

Figure 9. Initial population size $=250$, lambda $=1.20$, genetic diversity $=$ low .
Mean number of removals after 20 years

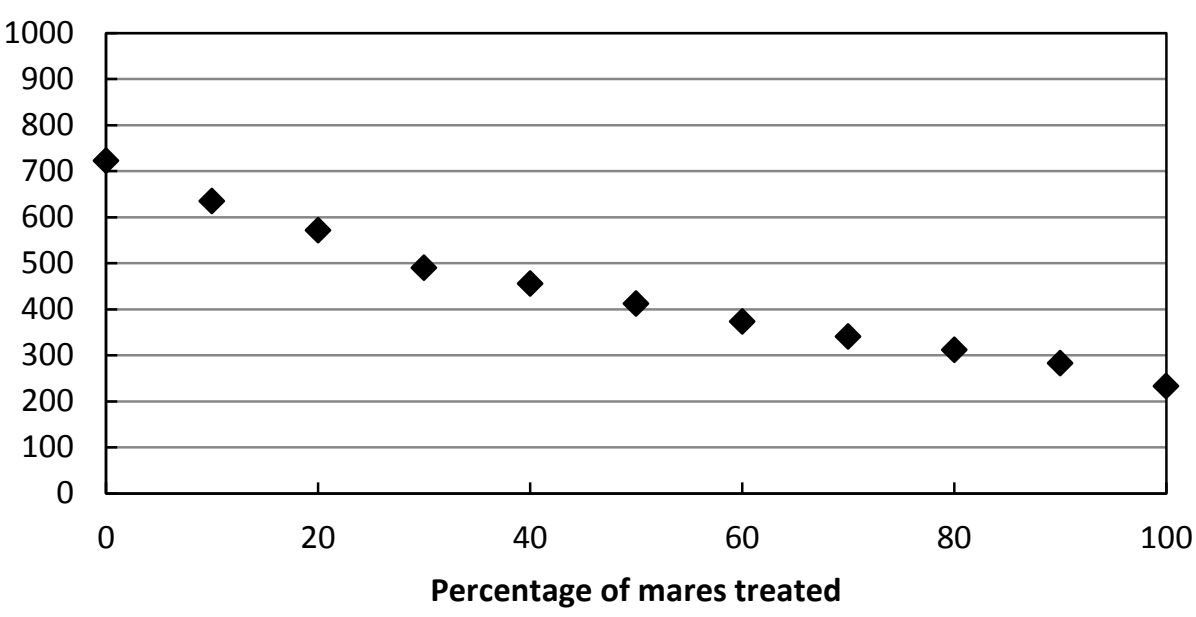

Mean heterozygosity after $\mathbf{5 0}$ years

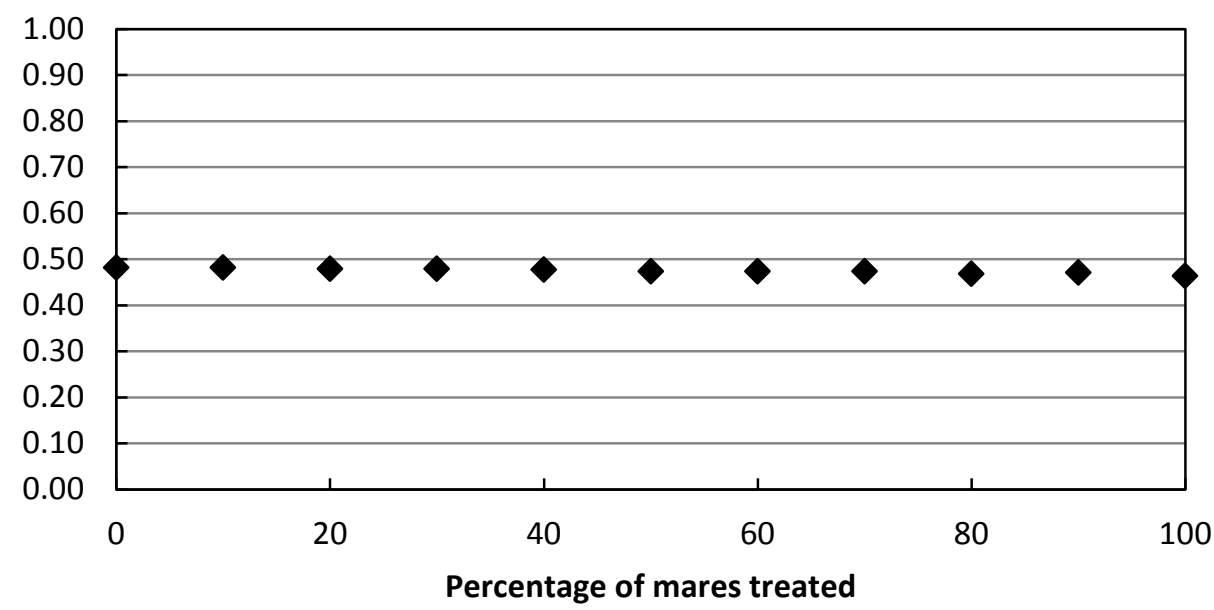




\begin{tabular}{lccccccccccc}
\hline \multicolumn{1}{c}{ Percentage of mares treated } & $\mathbf{0}$ & $\mathbf{1 0}$ & $\mathbf{2 0}$ & $\mathbf{3 0}$ & $\mathbf{4 0}$ & $\mathbf{5 0}$ & $\mathbf{6 0}$ & $\mathbf{7 0}$ & $\mathbf{8 0}$ & $\mathbf{9 0}$ & $\mathbf{1 0 0}$ \\
\hline Mean population size after 20 years & 303.72 & 277.47 & 274.27 & 271.98 & 275.36 & 262.1 & 260.81 & 256.62 & 226.89 & 207.59 & 223.76 \\
Mean number of removals after 20 years & 722.53 & 634.9 & 570.99 & 490.14 & 455.85 & 412 & 373.4 & 340.01 & 311.73 & 282.63 & 232.31 \\
Mean probability of extirpation after 50 years & 0 & 0 & 0 & 0 & 0 & 0 & 0 & 0 & 0 & 0 & 0.02 \\
Mean heterozygosity after 50 years & 0.48 & 0.48 & 0.48 & 0.48 & 0.48 & 0.47 & 0.47 & 0.47 & 0.47 & 0.47 & 0.46 \\
\hline
\end{tabular}

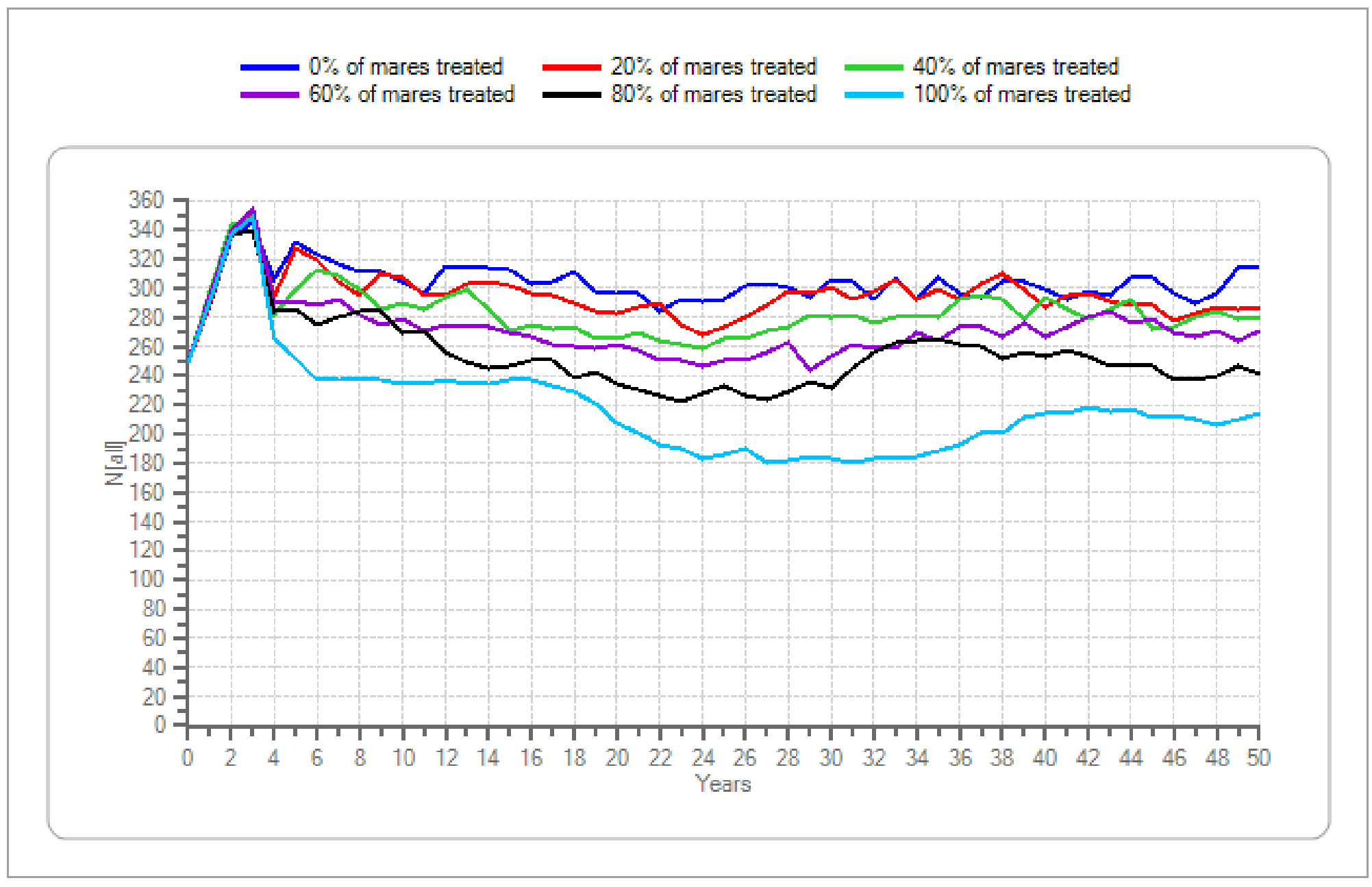

Figure 9. Initial population size $=250$, lambda $=1.20$, genetic diversity $=$ low - Continued 
Mean population size after 20 years

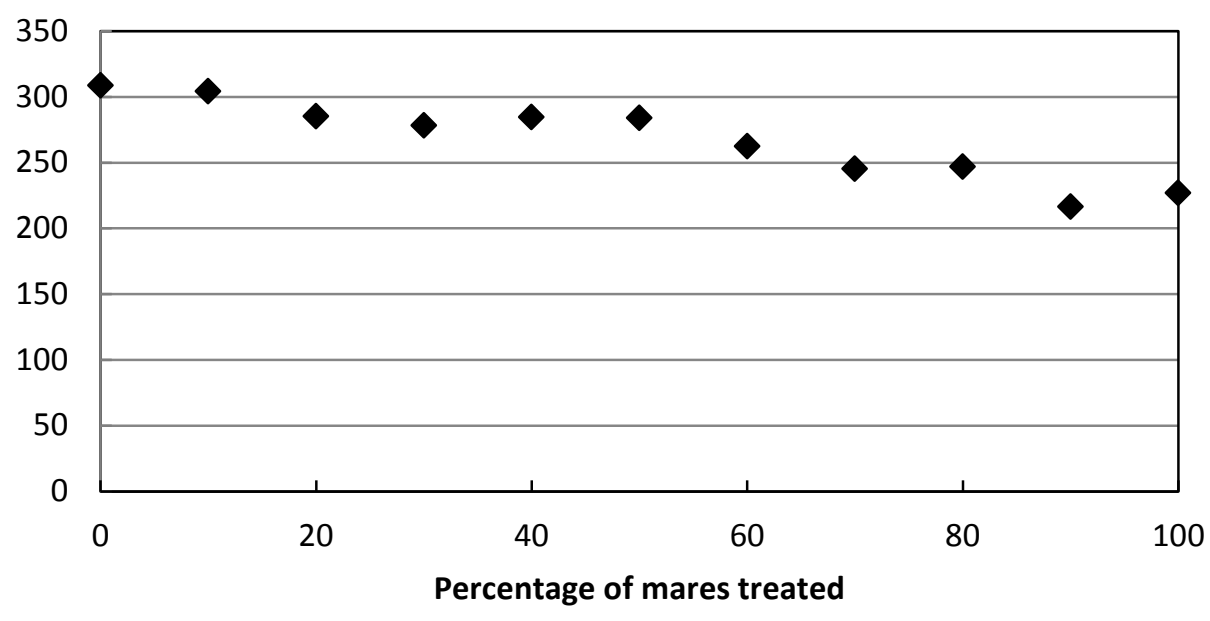

Mean probability of extirpation after $\mathbf{5 0}$ years

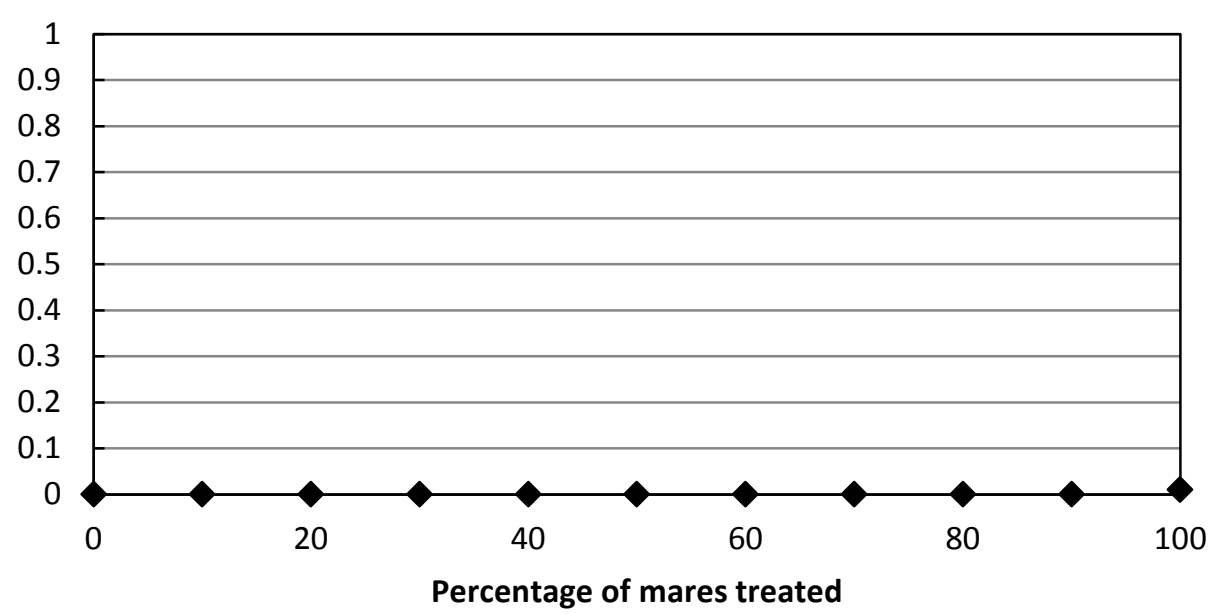

Figure 10. Initial population size $=250$, lambda $=1.25$, genetic diversity $=$ low .
Mean number of removals after 20 years

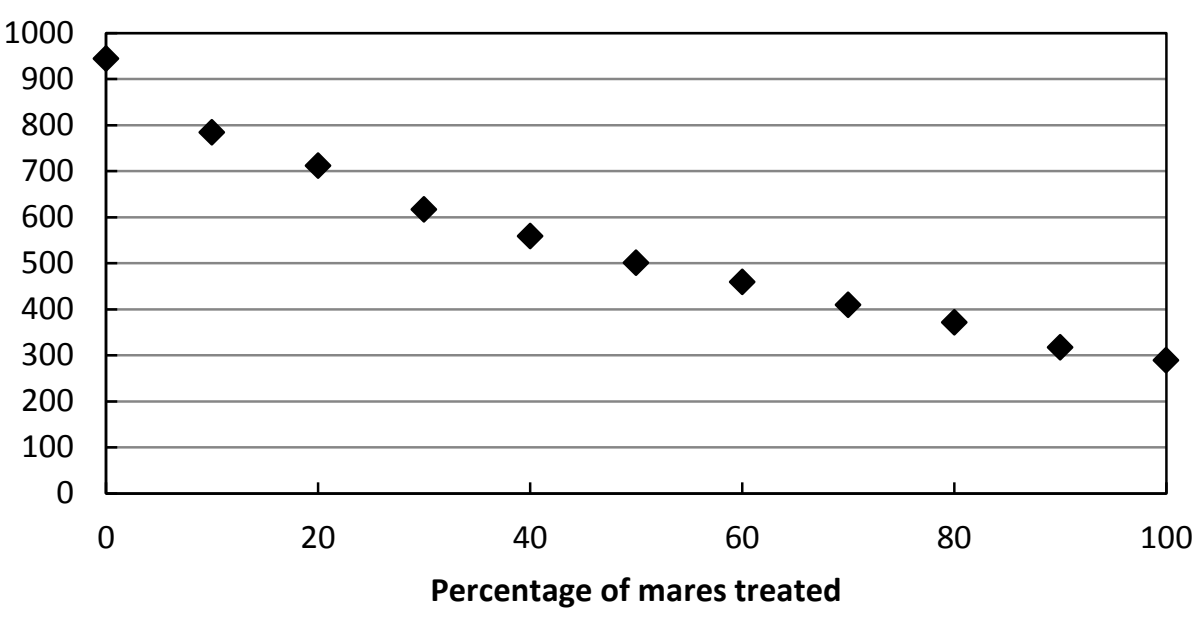

Mean heterozygosity after $\mathbf{5 0}$ years

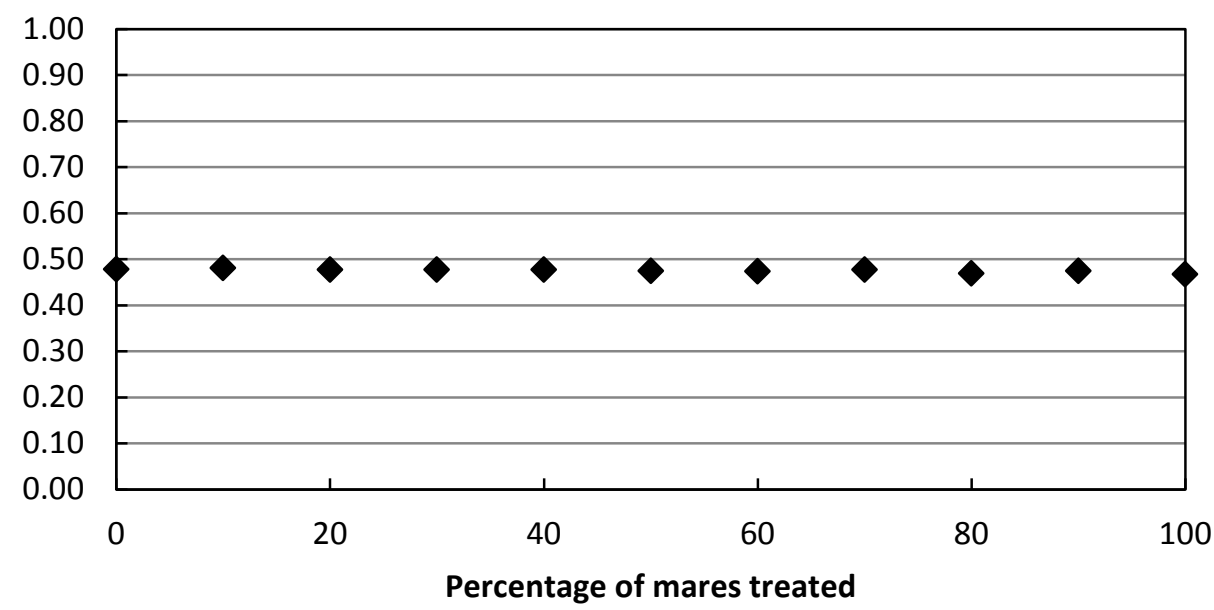




\begin{tabular}{lccccccccccc}
\hline \multicolumn{1}{c}{ Percentage of mares treated } & $\mathbf{0}$ & $\mathbf{1 0}$ & $\mathbf{2 0}$ & $\mathbf{3 0}$ & $\mathbf{4 0}$ & $\mathbf{5 0}$ & $\mathbf{6 0}$ & $\mathbf{7 0}$ & $\mathbf{8 0}$ & $\mathbf{9 0}$ & $\mathbf{1 0 0}$ \\
\hline Mean population size after 20 years & 308.52 & 304.15 & 285.11 & 278.16 & 284.69 & 283.85 & 262.37 & 245.28 & 246.89 & 216.51 & 226.76 \\
Mean number of removals after 20 years & 944.59 & 783.74 & 711.97 & 616.4 & 558.71 & 501.11 & 459.28 & 409.28 & 370.9 & 316.51 & 288.81 \\
Mean probability of extirpation after 50 years & 0 & 0 & 0 & 0 & 0 & 0 & 0 & 0 & 0 & 0 & 0.01 \\
Mean heterozygosity after 50 years & 0.48 & 0.48 & 0.48 & 0.48 & 0.48 & 0.47 & 0.47 & 0.48 & 0.47 & 0.47 & 0.47 \\
\hline
\end{tabular}

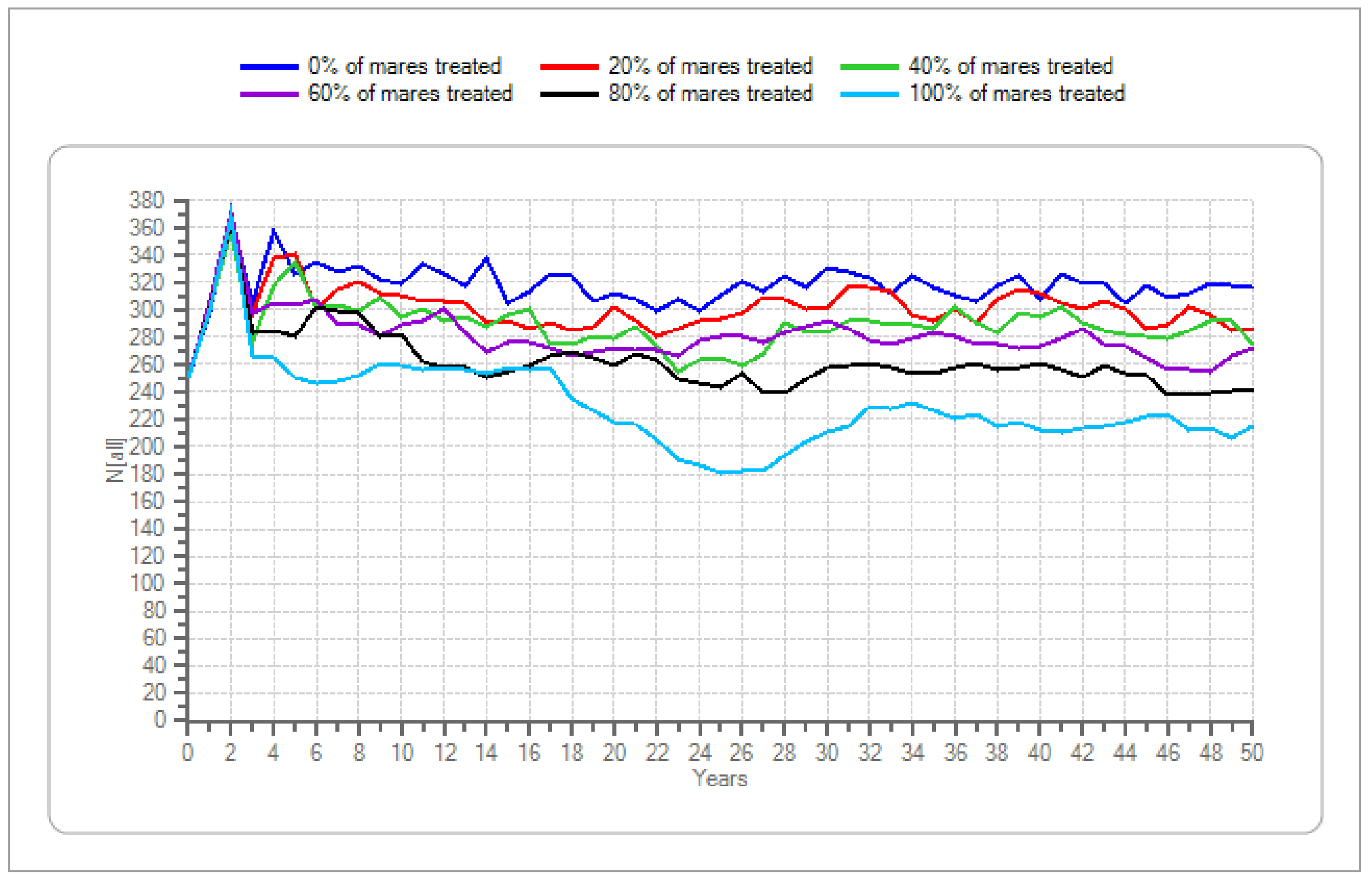

Figure 10. Initial population size $=250$, lambda $=1.25$, genetic diversity $=$ low - Continued 
Mean population size after 20 years

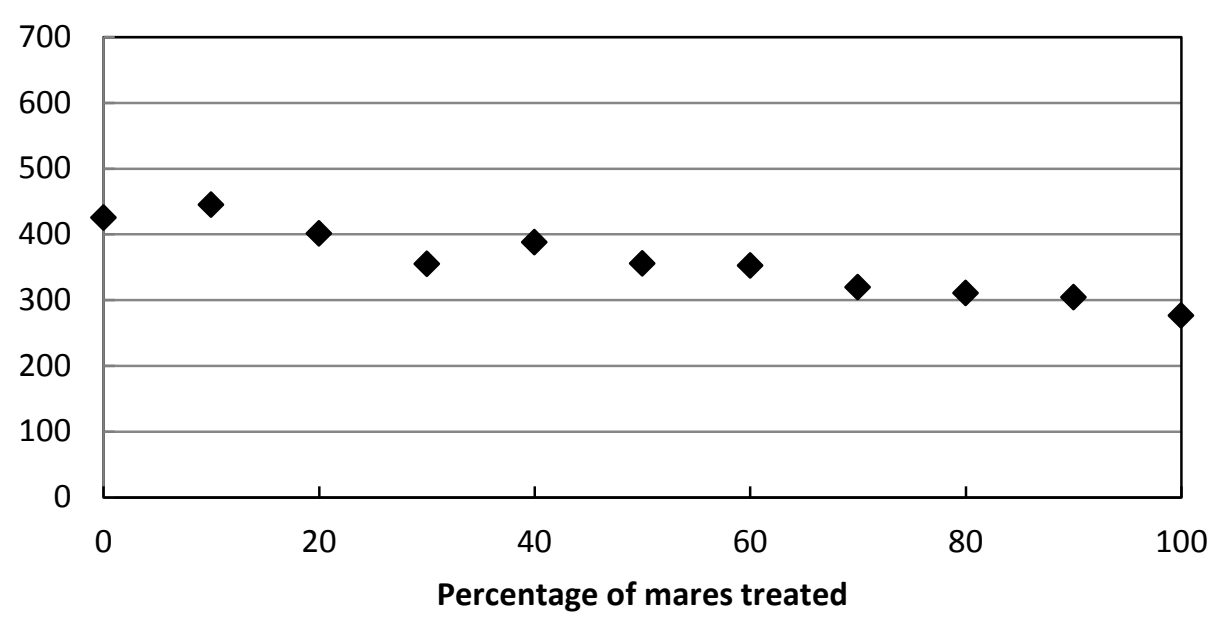

Mean probability of extirpation after $\mathbf{5 0}$ years

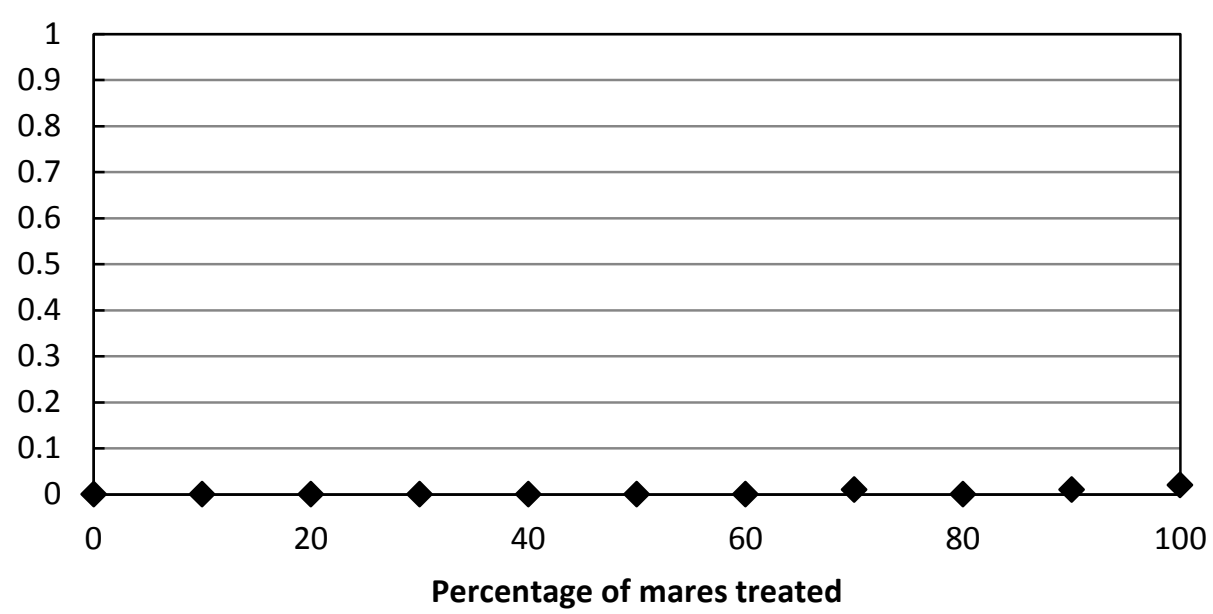

Figure 11. Initial population size $=500$, lambda $=1.05$, genetic diversity $=$ low .
Mean number of removals after 20 years

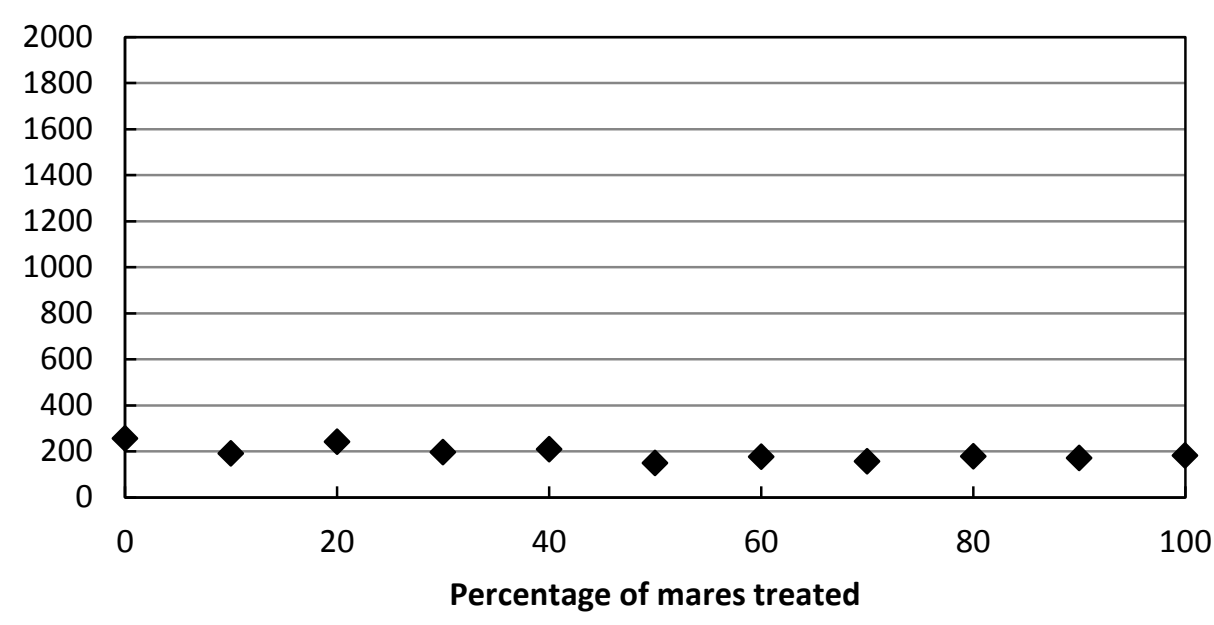

Mean heterozygosity after $\mathbf{5 0}$ years

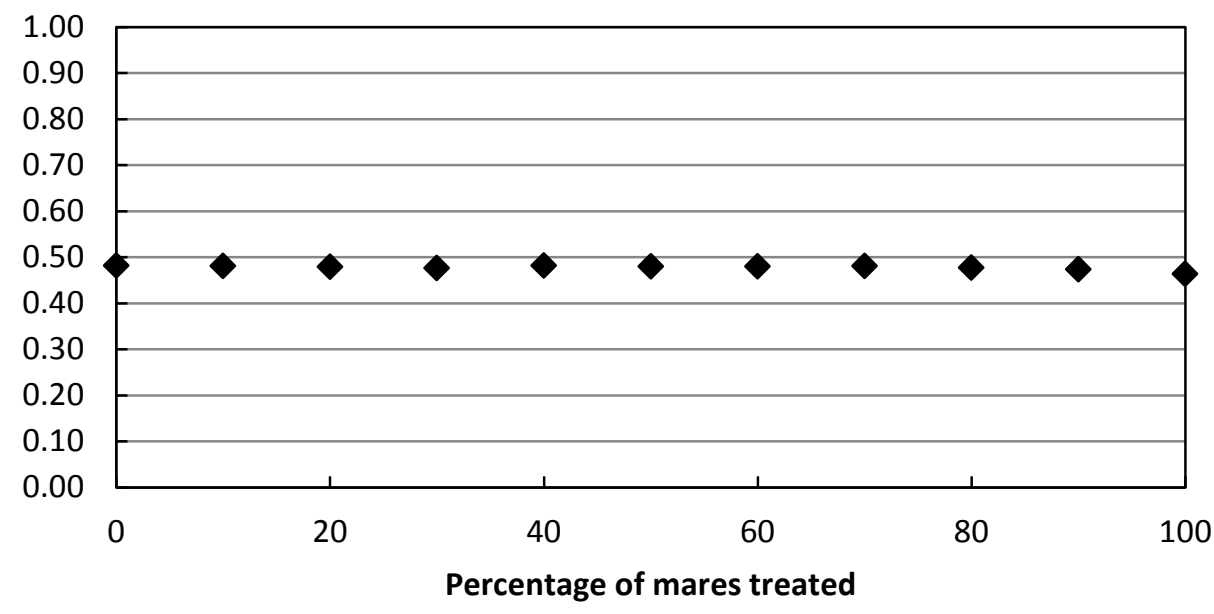




\begin{tabular}{lccccccccccc}
\hline \multicolumn{1}{c}{ Percentage of mares treated } & $\mathbf{0}$ & $\mathbf{1 0}$ & $\mathbf{2 0}$ & $\mathbf{3 0}$ & $\mathbf{4 0}$ & $\mathbf{5 0}$ & $\mathbf{6 0}$ & $\mathbf{7 0}$ & $\mathbf{8 0}$ & $\mathbf{9 0}$ & $\mathbf{1 0 0}$ \\
\hline Mean population size after 20 years & 425.35 & 444.78 & 401.43 & 354.61 & 387.64 & 355.61 & 352.23 & 319.32 & 310.27 & 304.15 & 276.61 \\
Mean number of removals after 20 years & 255.36 & 190.95 & 241.46 & 196.02 & 208.87 & 147.97 & 176.38 & 156.08 & 177.73 & 169.95 & 181.87 \\
Mean probability of extirpation after 50 years & 0 & 0 & 0 & 0 & 0 & 0 & 0 & 0.01 & 0 & 0.01 & 0.02 \\
Mean heterozygosity after 50 years & 0.48 & 0.48 & 0.48 & 0.48 & 0.48 & 0.48 & 0.48 & 0.48 & 0.48 & 0.47 & 0.46 \\
\hline
\end{tabular}

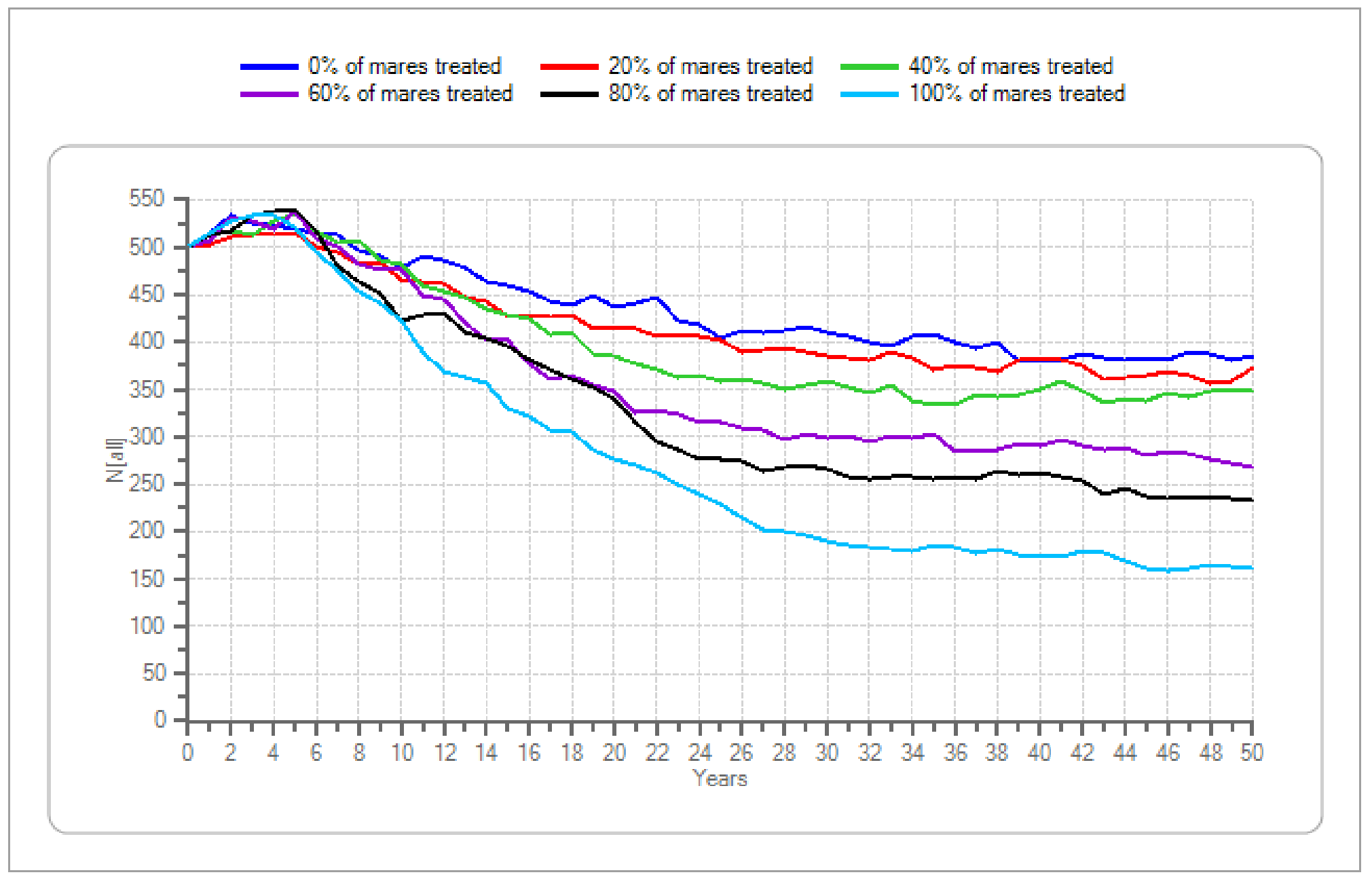

Figure 11. Initial population size $=500$, lambda $=1.05$, genetic diversity $=$ low - Continued 
Mean population size after 20 years

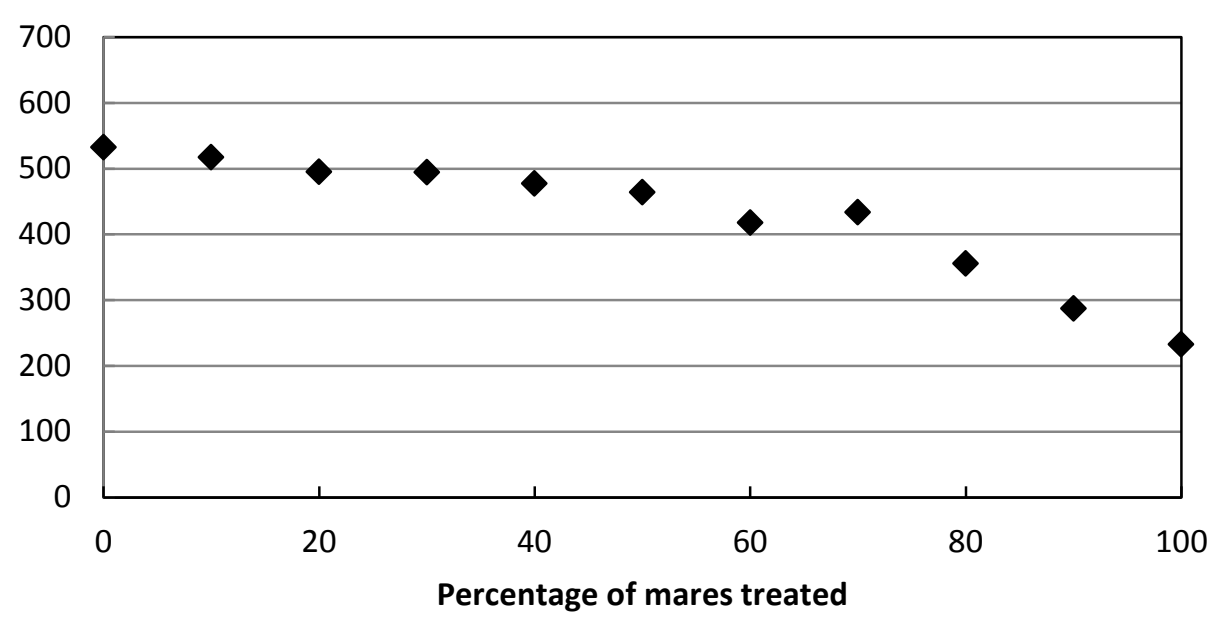

Mean probability of extirpation after $\mathbf{5 0}$ years

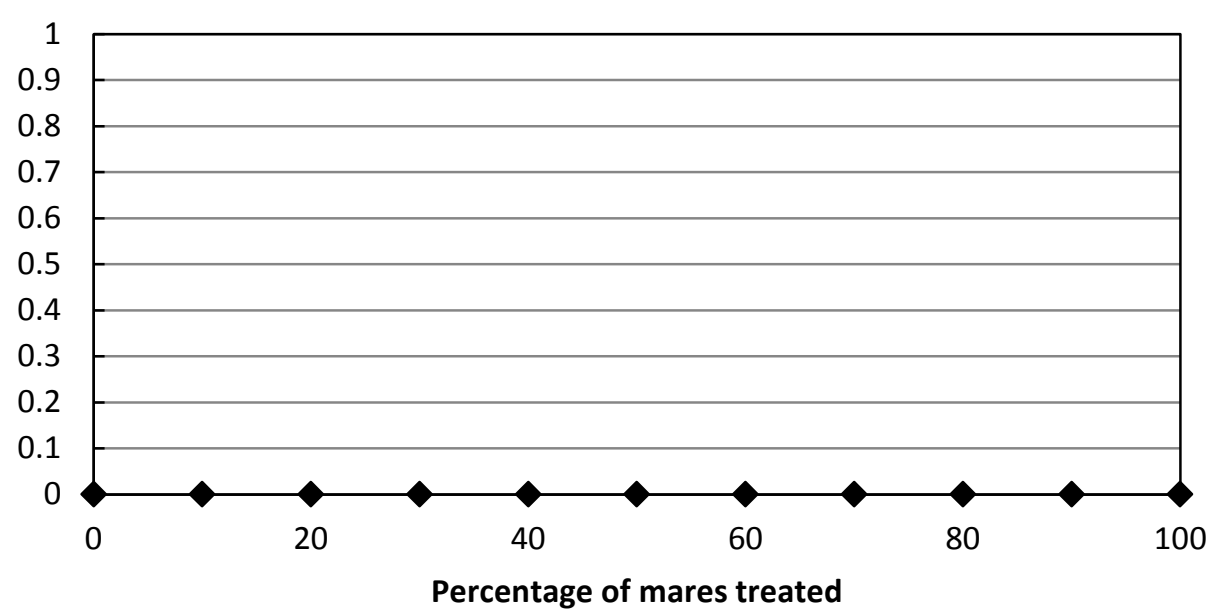

Figure 12. Initial population size $=500$, lambda $=1.10$, genetic diversity $=$ low .
Mean number of removals after 20 years

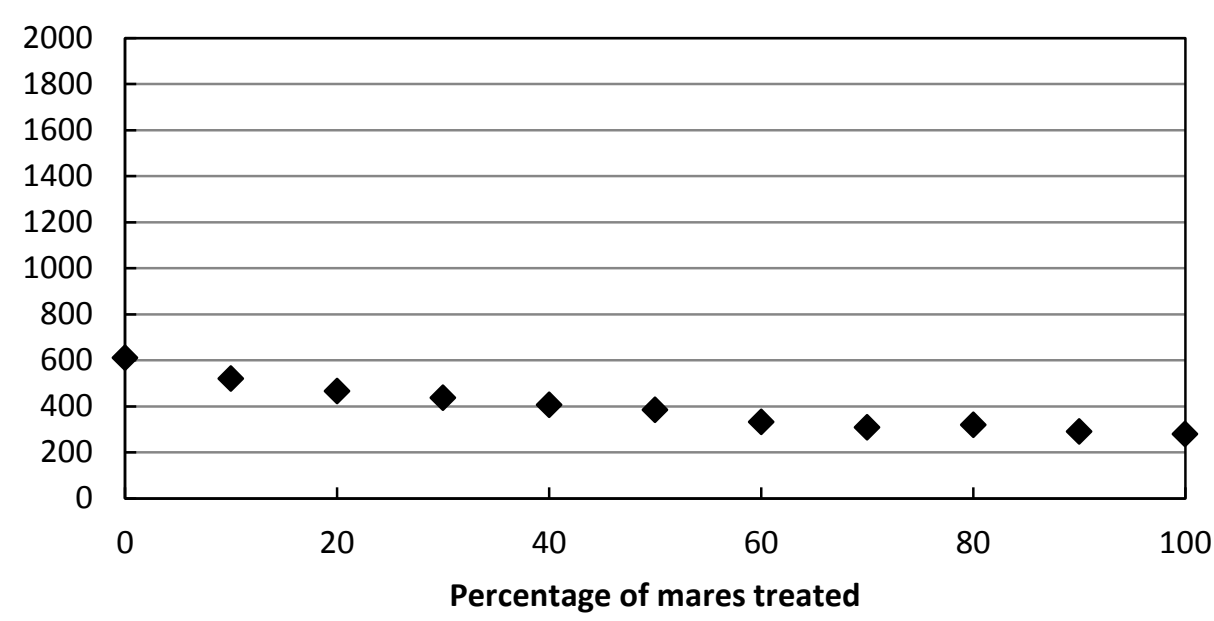

Mean heterozygosity after $\mathbf{5 0}$ years

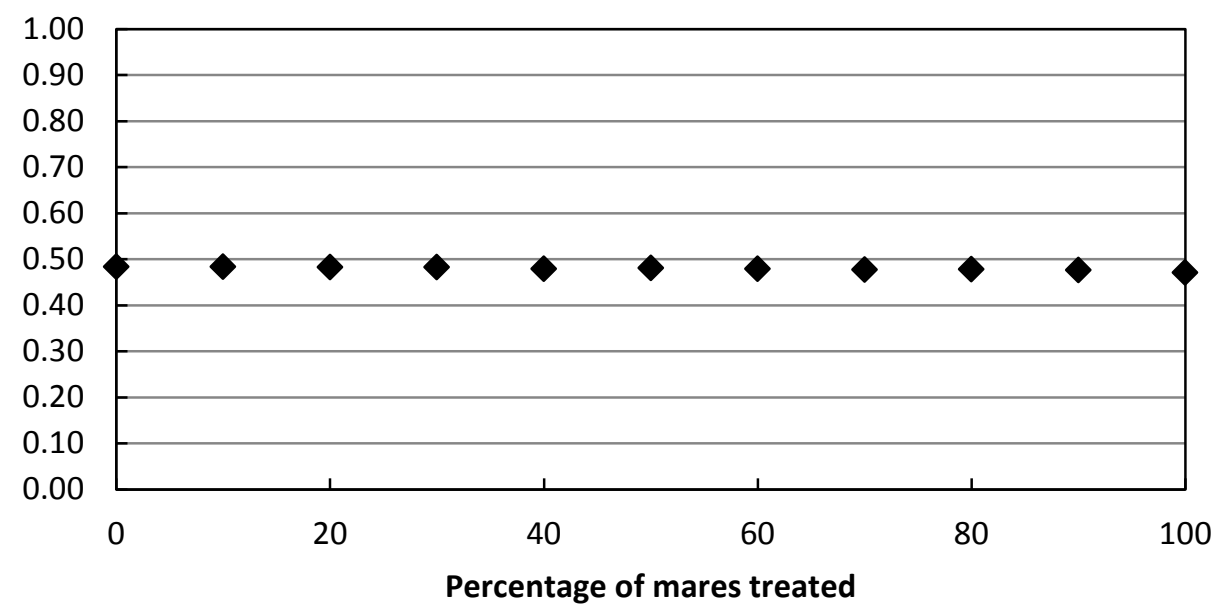




\begin{tabular}{lccccccccccc}
\hline \multicolumn{1}{c}{ Percentage of mares treated } & $\mathbf{0}$ & $\mathbf{1 0}$ & $\mathbf{2 0}$ & $\mathbf{3 0}$ & $\mathbf{4 0}$ & $\mathbf{5 0}$ & $\mathbf{6 0}$ & $\mathbf{7 0}$ & $\mathbf{8 0}$ & $\mathbf{9 0}$ & $\mathbf{1 0 0}$ \\
\hline Mean population size after 20 years & 532.14 & 517.32 & 494.85 & 494.04 & 476.94 & 463.89 & 417.36 & 433.64 & 355.55 & 286.94 & 232.49 \\
Mean number of removals after 20 years & 610.21 & 520.05 & 465.8 & 436.08 & 406.01 & 384.76 & 332.11 & 307.18 & 318.48 & 289.02 & 279.16 \\
Mean probability of extirpation after 50 years & 0 & 0 & 0 & 0 & 0 & 0 & 0 & 0 & 0 & 0 & 0 \\
Mean heterozygosity after 50 years & 0.48 & 0.48 & 0.48 & 0.48 & 0.48 & 0.48 & 0.48 & 0.48 & 0.48 & 0.48 & 0.47 \\
\hline
\end{tabular}

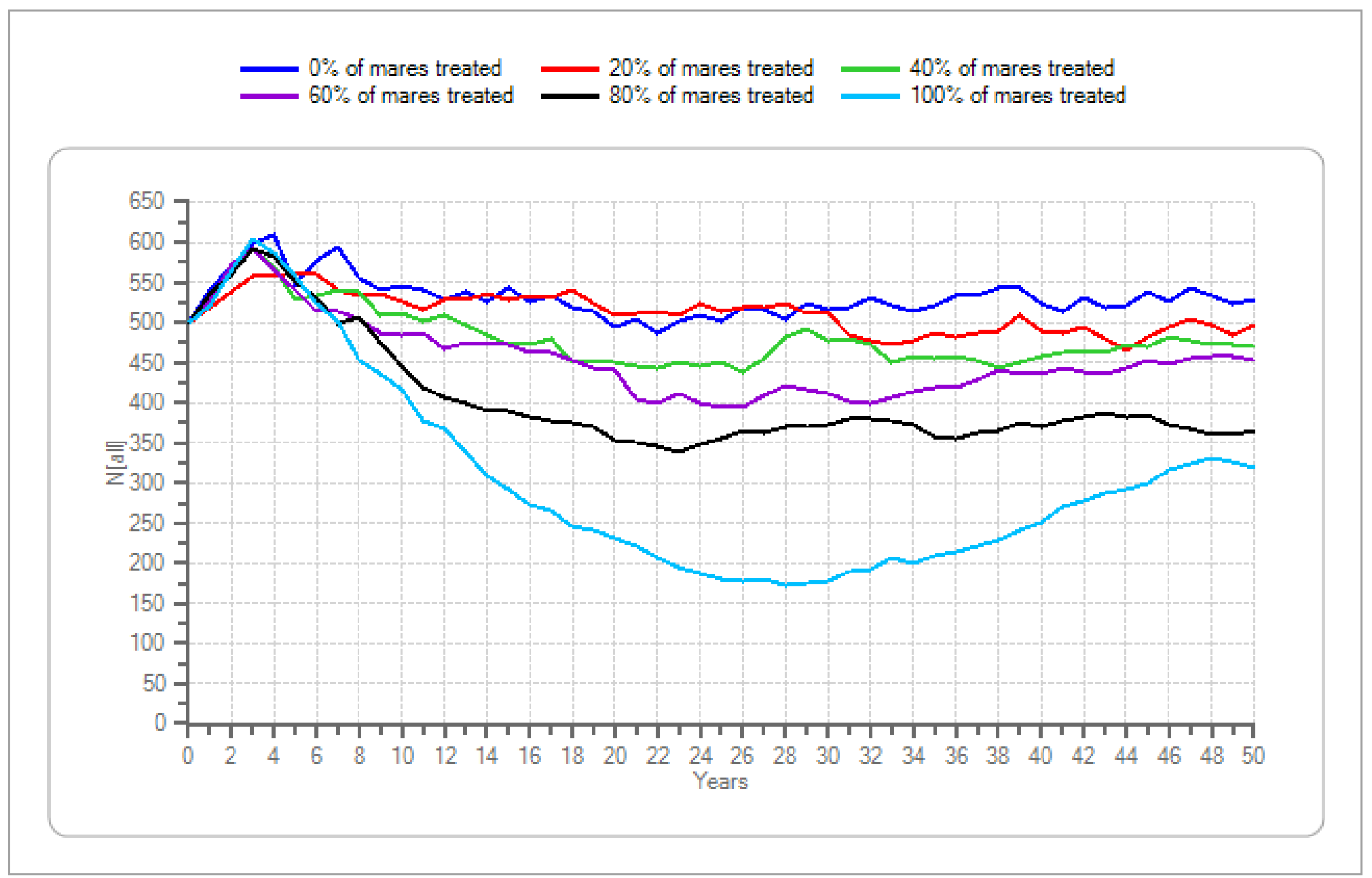

Figure 12. Initial population size $=500$, lambda $=1.10$, genetic diversity $=$ low - Continued 
Mean population size after 20 years

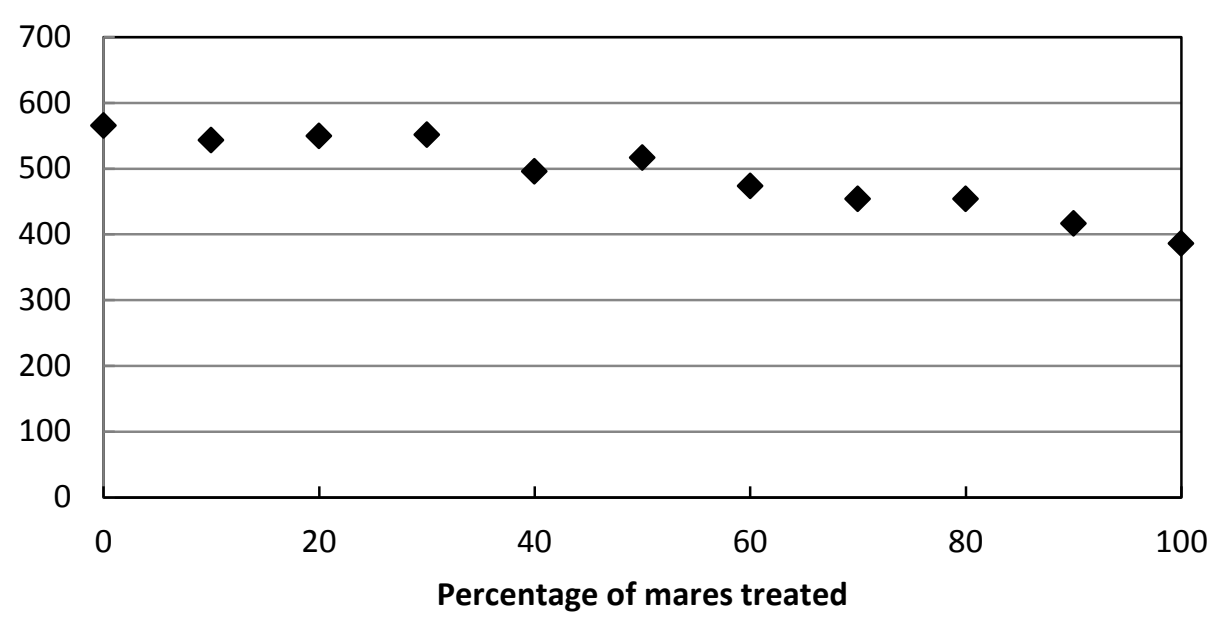

Mean probability of extirpation after $\mathbf{5 0}$ years

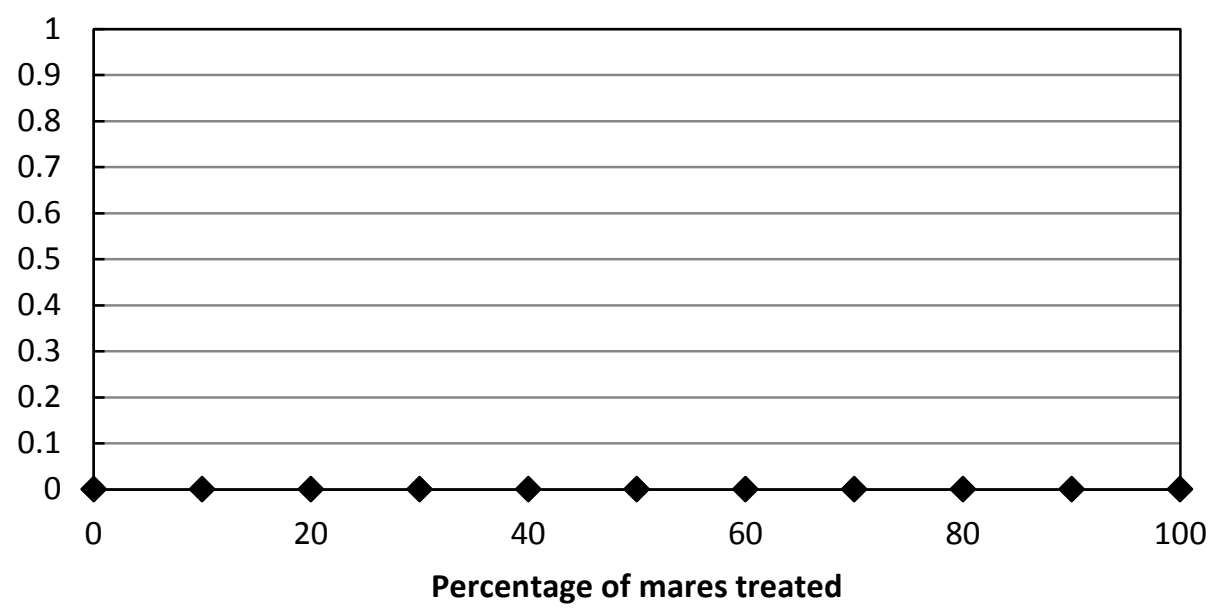

Figure 13. Initial population size $=500$, lambda $=1.15$, genetic diversity $=$ low .
Mean number of removals after 20 years

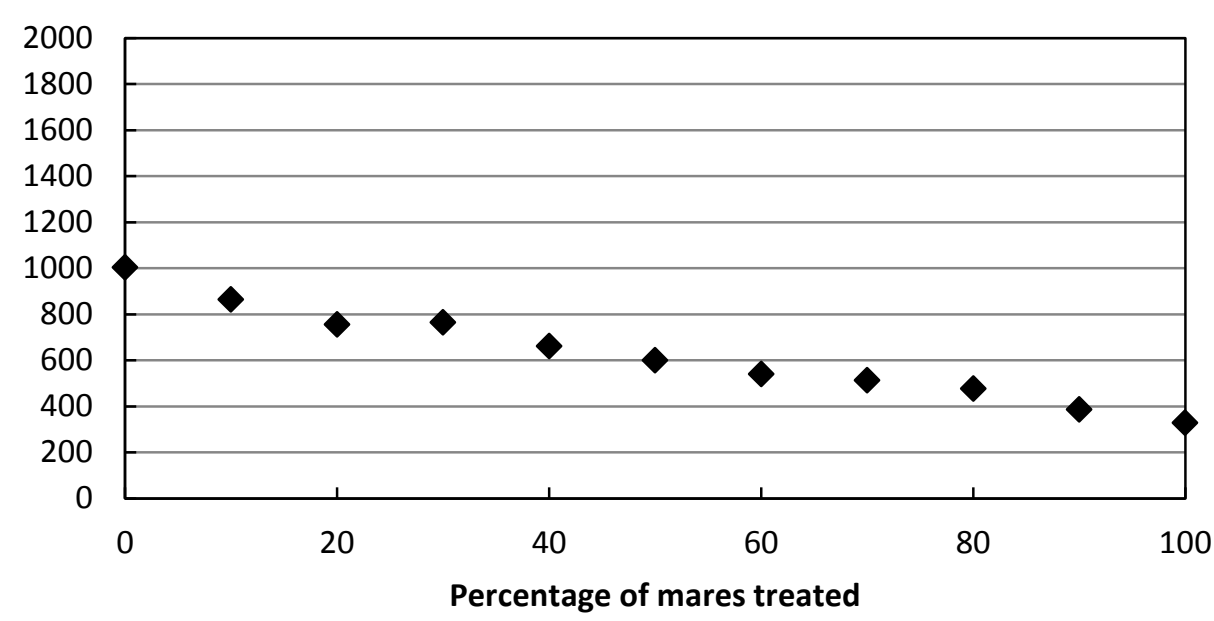

Mean heterozygosity after $\mathbf{5 0}$ years

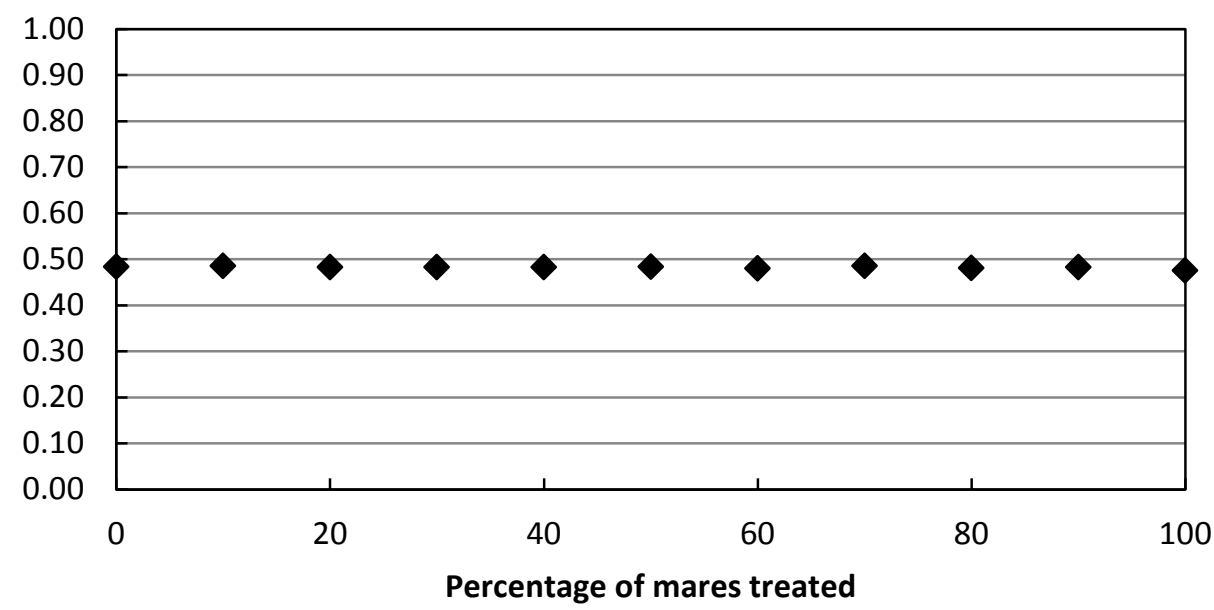




\begin{tabular}{lccccccccccc}
\hline \multicolumn{1}{c}{ Percentage of mares treated } & $\mathbf{0}$ & $\mathbf{1 0}$ & $\mathbf{2 0}$ & $\mathbf{3 0}$ & $\mathbf{4 0}$ & $\mathbf{5 0}$ & $\mathbf{6 0}$ & $\mathbf{7 0}$ & $\mathbf{8 0}$ & $\mathbf{9 0}$ & $\mathbf{1 0 0}$ \\
\hline Mean population size after 20 years & 565.34 & 543.12 & 549.14 & 551.57 & 495.37 & 516.42 & 473.26 & 453.73 & 453.48 & 416.08 & 385.94 \\
Mean number of removals after 20 years & 1002.92 & 863.83 & 755.3 & 764.15 & 660.85 & 598.87 & 539.63 & 512.76 & 475.42 & 384.89 & 328.2 \\
Mean probability of extirpation after 50 years & 0 & 0 & 0 & 0 & 0 & 0 & 0 & 0 & 0 & 0 & 0 \\
Mean heterozygosity after 50 years & 0.48 & 0.49 & 0.48 & 0.48 & 0.48 & 0.48 & 0.48 & 0.49 & 0.48 & 0.48 & 0.48 \\
\hline
\end{tabular}

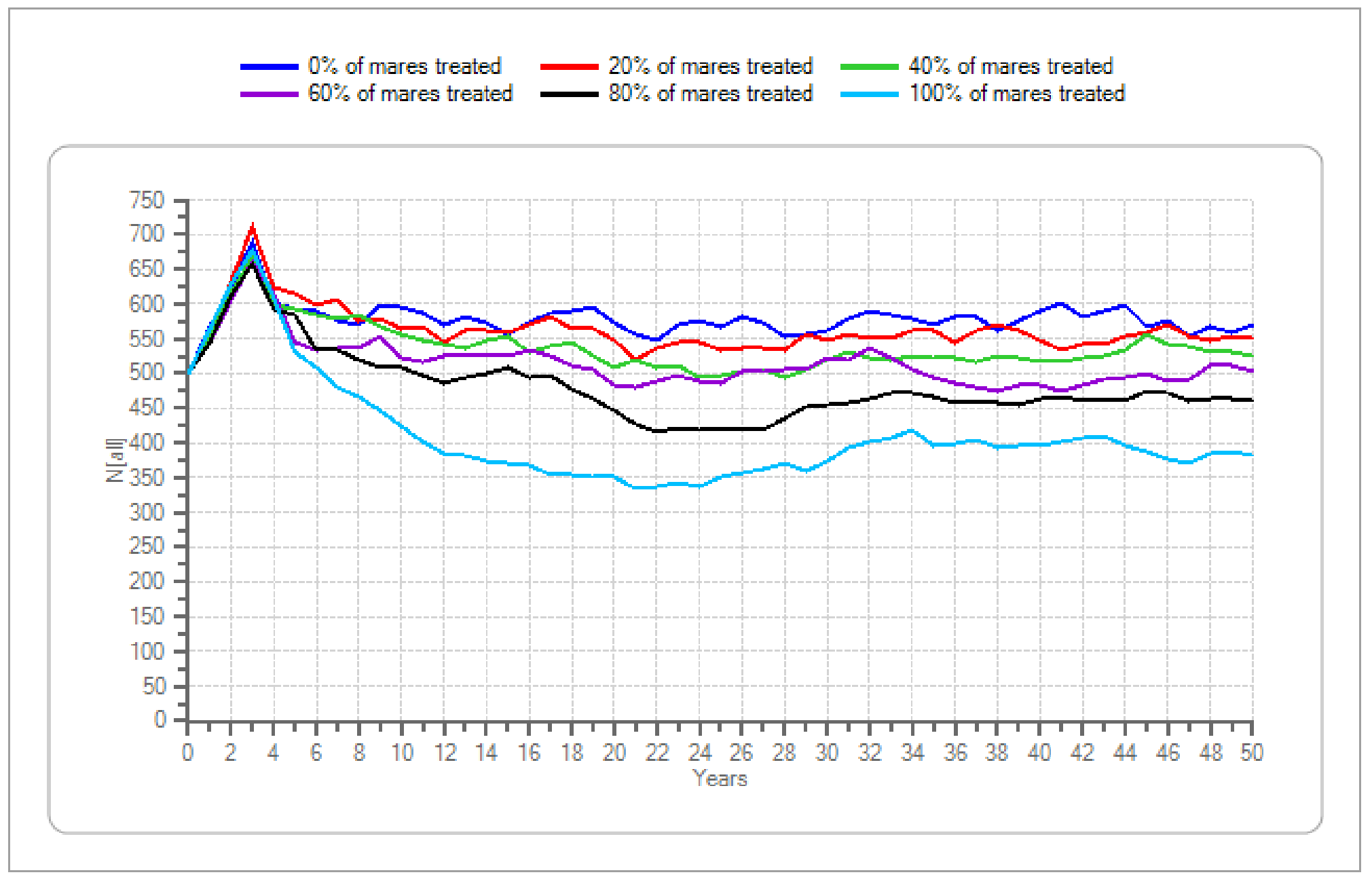

Figure 13. Initial population size $=500$, lambda $=1.15$, genetic diversity $=$ low - Continued 
Mean population size after 20 years

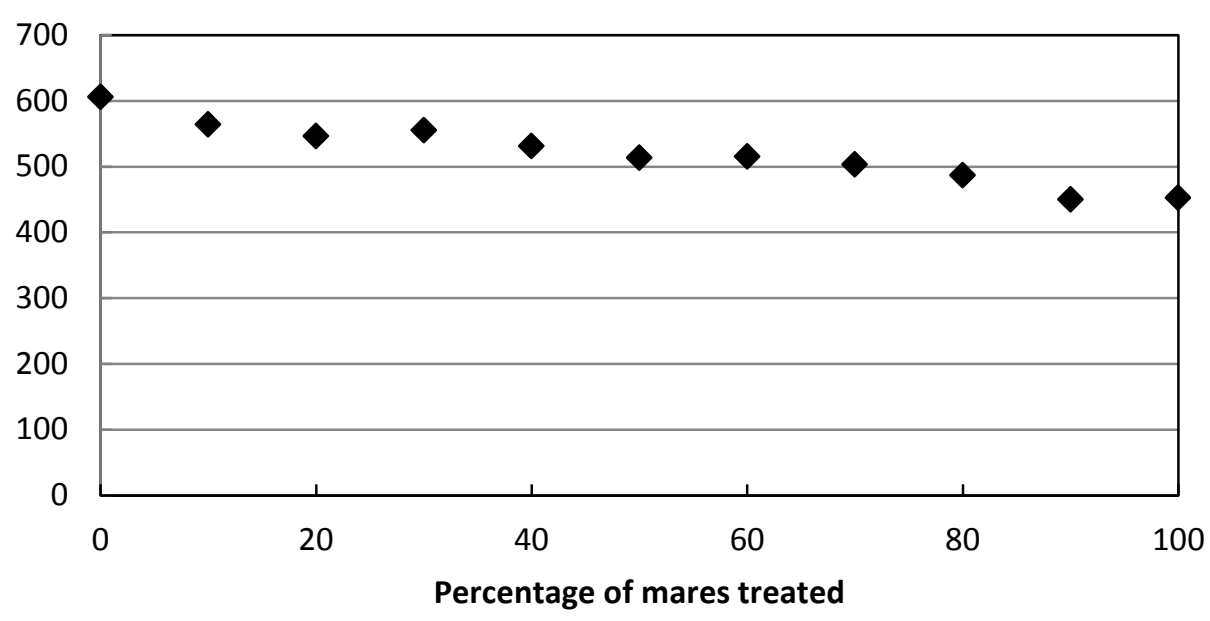

Mean probability of extirpation after $\mathbf{5 0}$ years

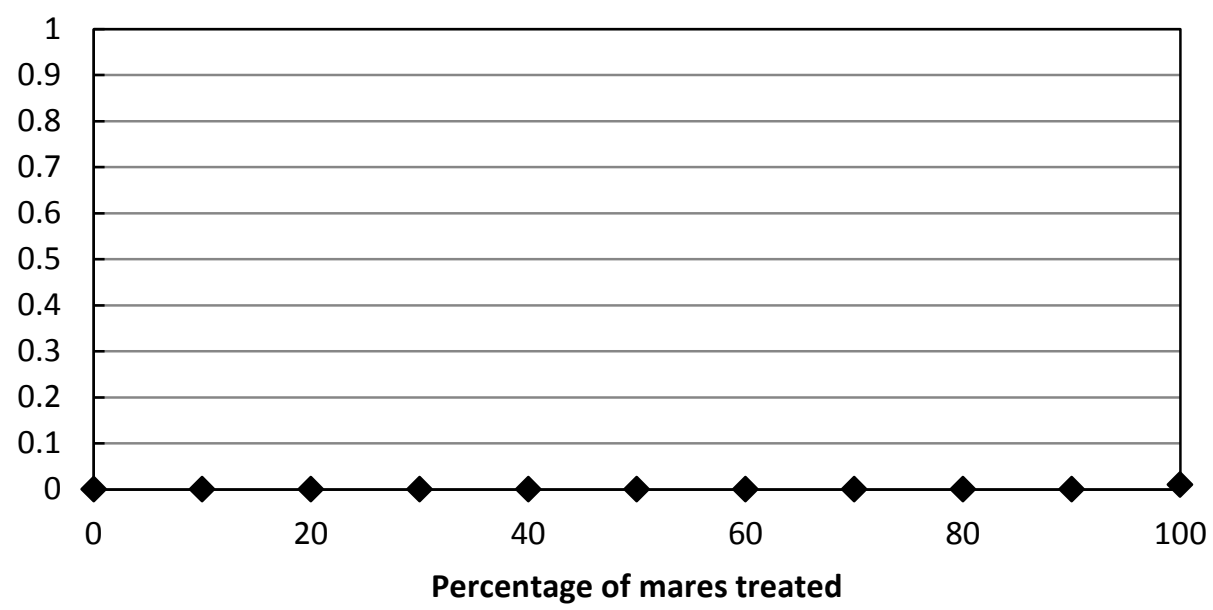

Figure 14. Initial population size $=500$, lambda $=1.20$, genetic diversity $=$ low .
Mean number of removals after 20 years

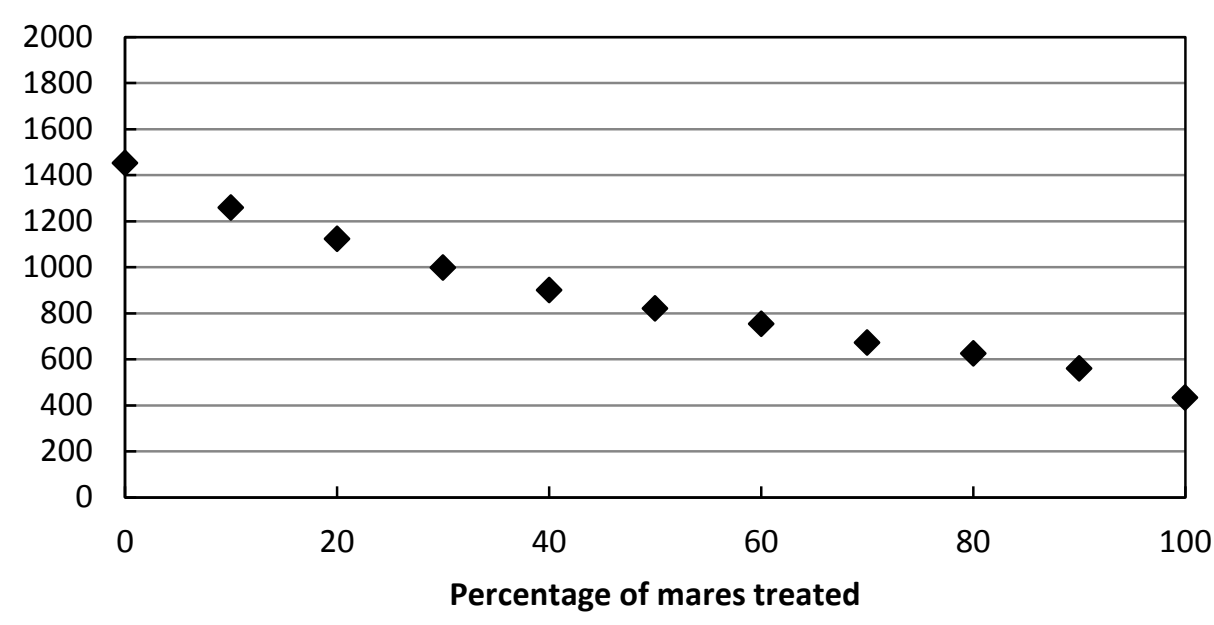

Mean heterozygosity after 50 years

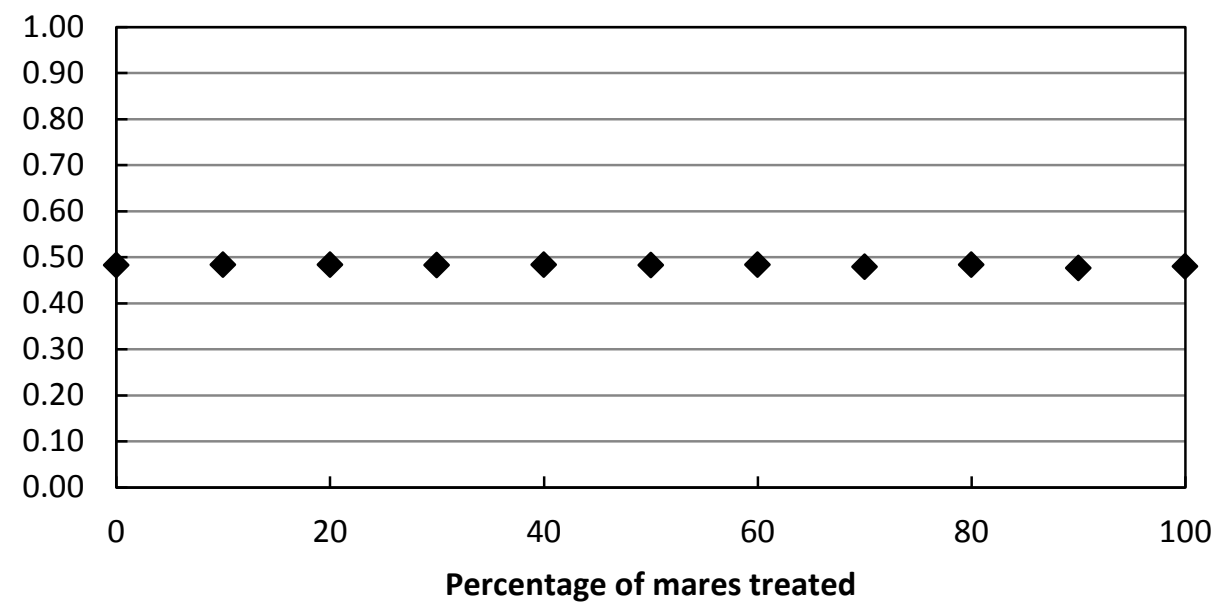




\begin{tabular}{lccccccccccc}
\hline \multicolumn{1}{c}{ Percentage of mares treated } & $\mathbf{0}$ & $\mathbf{1 0}$ & $\mathbf{2 0}$ & $\mathbf{3 0}$ & $\mathbf{4 0}$ & $\mathbf{5 0}$ & $\mathbf{6 0}$ & $\mathbf{7 0}$ & $\mathbf{8 0}$ & $\mathbf{9 0}$ & $\mathbf{1 0 0}$ \\
\hline Mean population size after 20 years & 606.16 & 564.12 & 546.4 & 555.28 & 531.1 & 513.41 & 515.42 & 502.94 & 486.58 & 450.15 & 452.21 \\
Mean number of removals after 20 years & 1451.75 & 1258.73 & 1122.91 & 997.36 & 900.23 & 820.29 & 752.55 & 671.84 & 624.39 & 559.54 & 432.37 \\
Mean probability of extirpation after 50 years & 0 & 0 & 0 & 0 & 0 & 0 & 0 & 0 & 0 & 0 & 0.01 \\
Mean heterozygosity after 50 years & 0.48 & 0.48 & 0.48 & 0.48 & 0.48 & 0.48 & 0.48 & 0.48 & 0.48 & 0.48 & 0.48 \\
\hline
\end{tabular}

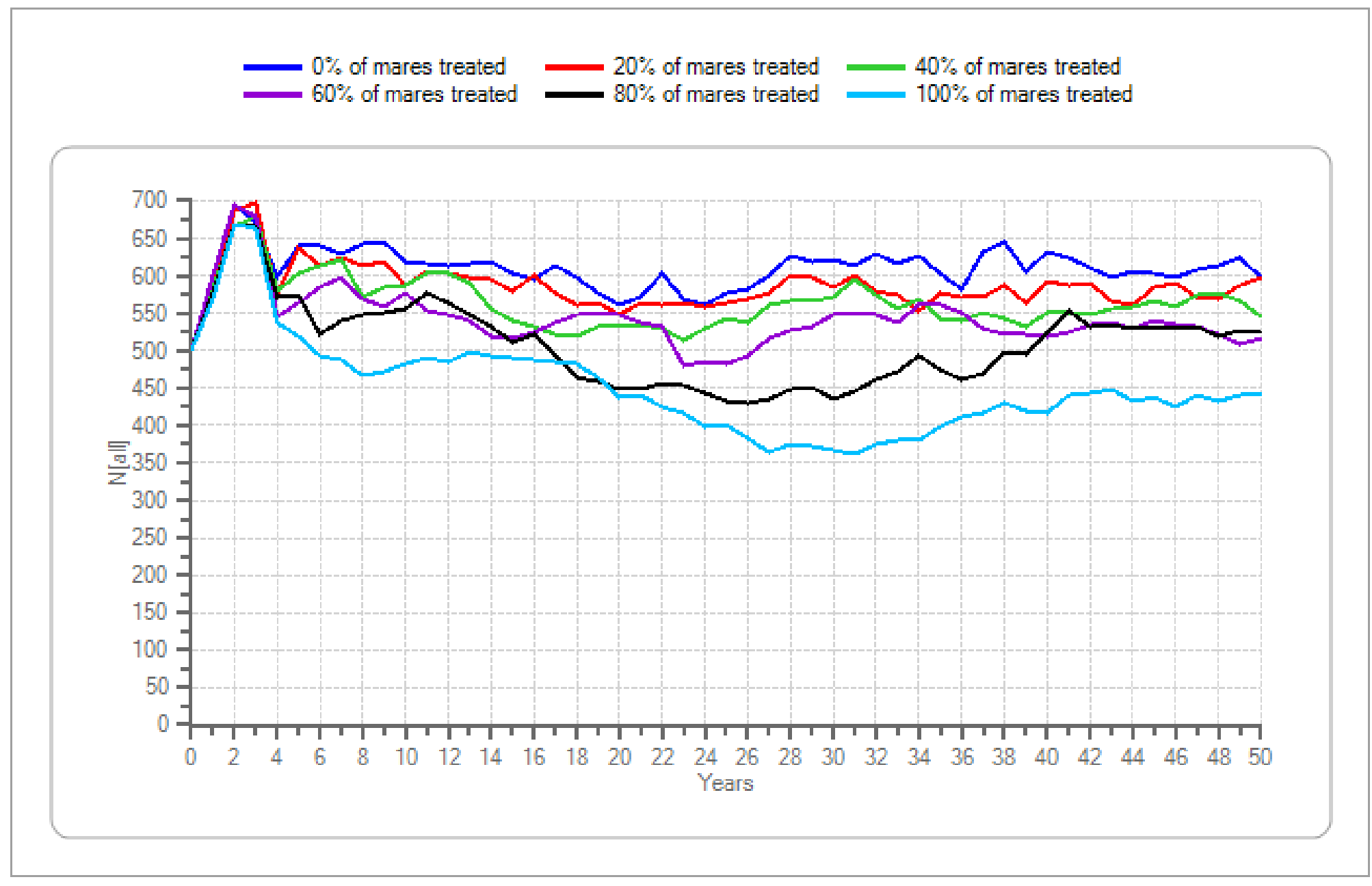

Figure 14. Initial population size $=500$, lambda $=1.20$, genetic diversity $=$ low - Continued 
Mean population size after 20 years

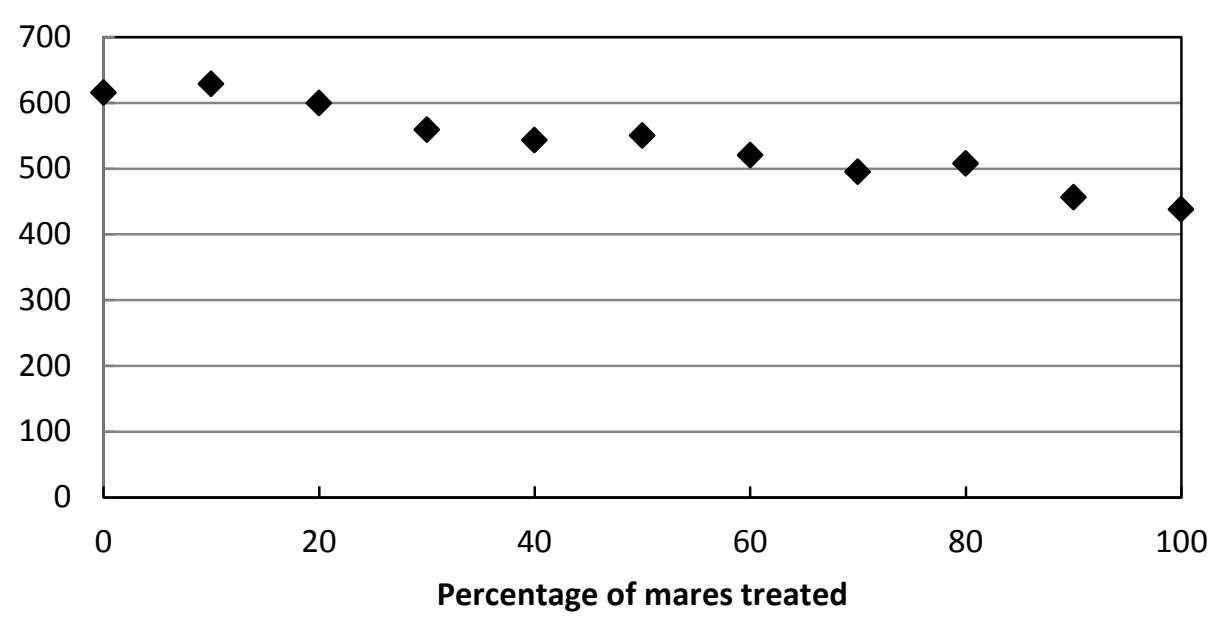

Mean probability of extirpation after $\mathbf{5 0}$ years

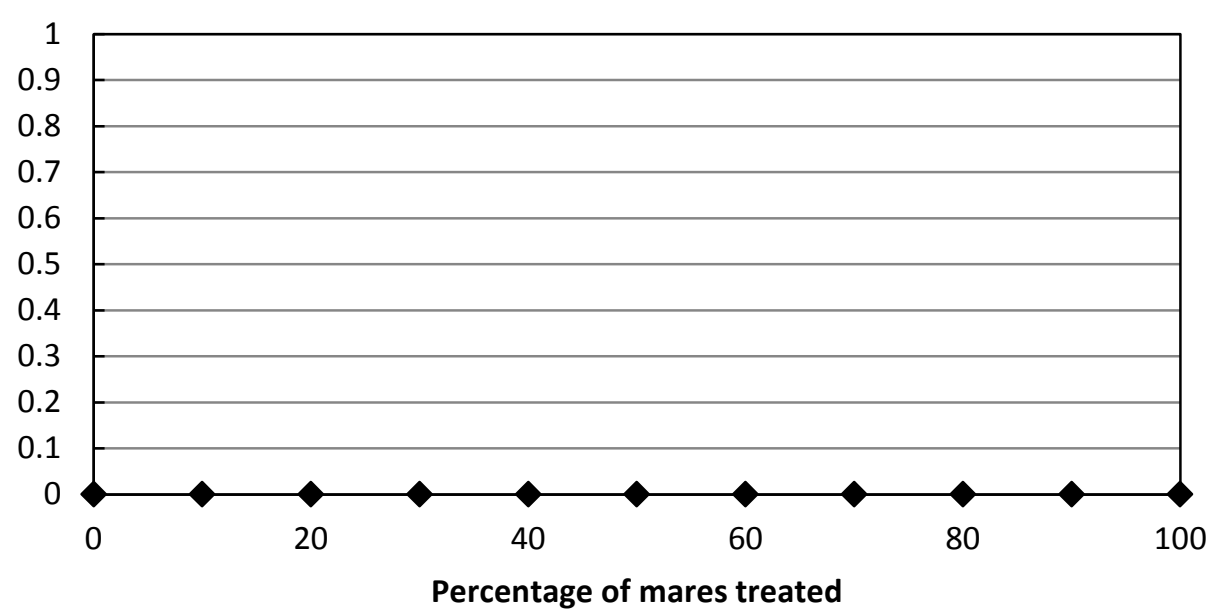

Figure 15. Initial population size $=500$, lambda $=1.25$, genetic diversity $=$ low .
Mean number of removals after 20 years

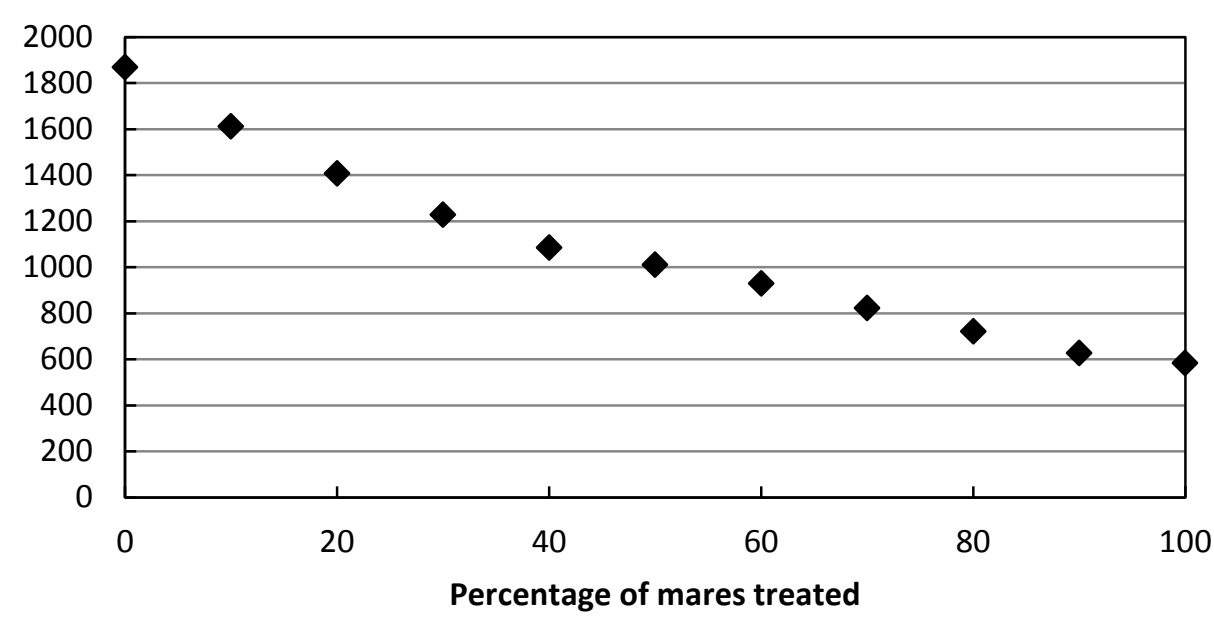

Mean heterozygosity after $\mathbf{5 0}$ years

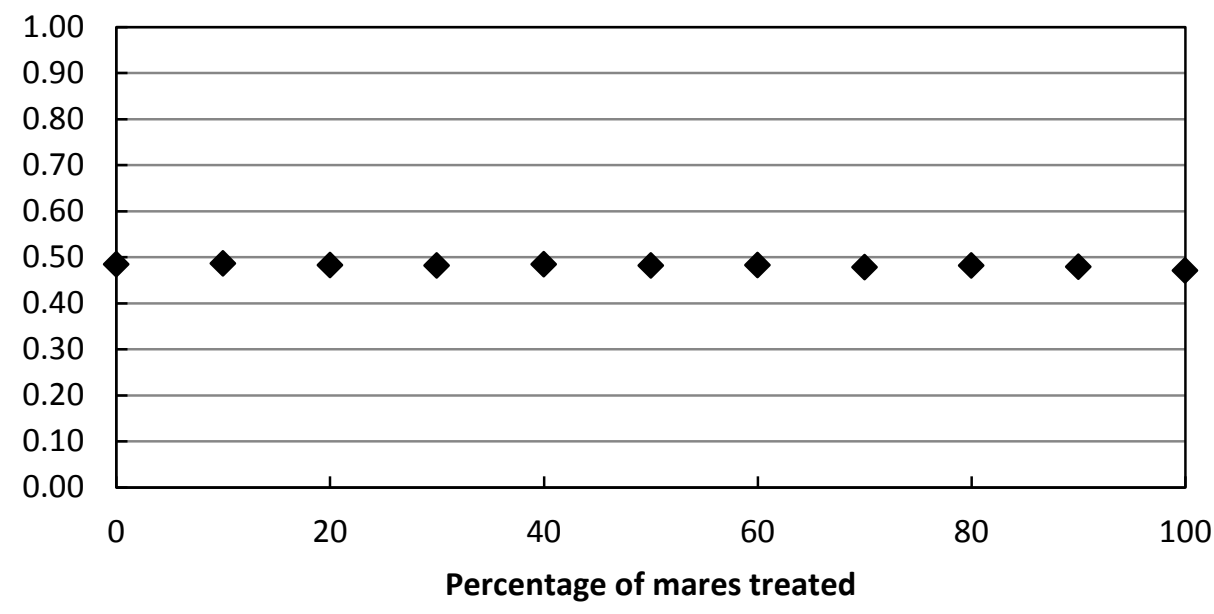




\begin{tabular}{lccccccccccc}
\hline \multicolumn{1}{c}{ Percentage of mares treated } & $\mathbf{0}$ & $\mathbf{1 0}$ & $\mathbf{2 0}$ & $\mathbf{3 0}$ & $\mathbf{4 0}$ & $\mathbf{5 0}$ & $\mathbf{6 0}$ & $\mathbf{7 0}$ & $\mathbf{8 0}$ & $\mathbf{9 0}$ & $\mathbf{1 0 0}$ \\
\hline Mean population size after 20 years & 615.29 & 628.52 & 599.27 & 559.22 & 543.09 & 550.12 & 520.04 & 495.03 & 507.73 & 456.42 & 437.97 \\
Mean number of removals after 20 years & 1868.95 & 1612.35 & 1406.33 & 1227.35 & 1083.85 & 1009.6 & 928.44 & 822.54 & 721.17 & 626.66 & 583.8 \\
Mean probability of extirpation after 50 years & 0 & 0 & 0 & 0 & 0 & 0 & 0 & 0 & 0 & 0 & 0 \\
Mean heterozygosity after 50 years & 0.48 & 0.49 & 0.48 & 0.48 & 0.48 & 0.48 & 0.48 & 0.48 & 0.48 & 0.48 & 0.47 \\
\hline
\end{tabular}

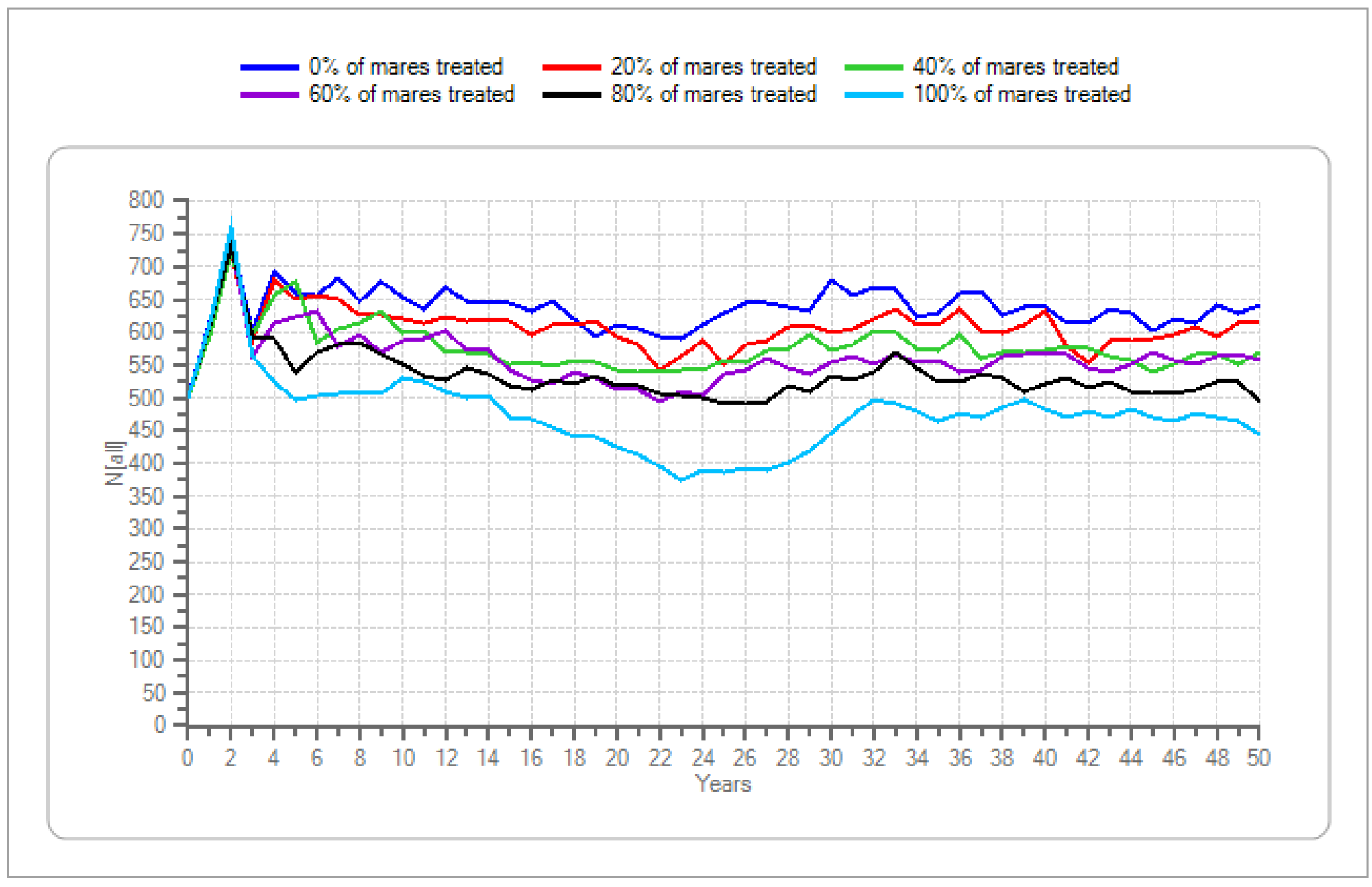

Figure 15. Initial population size $=500$, lambda $=1.25$, genetic diversity $=$ low - Continued 
Mean population size after 20 years

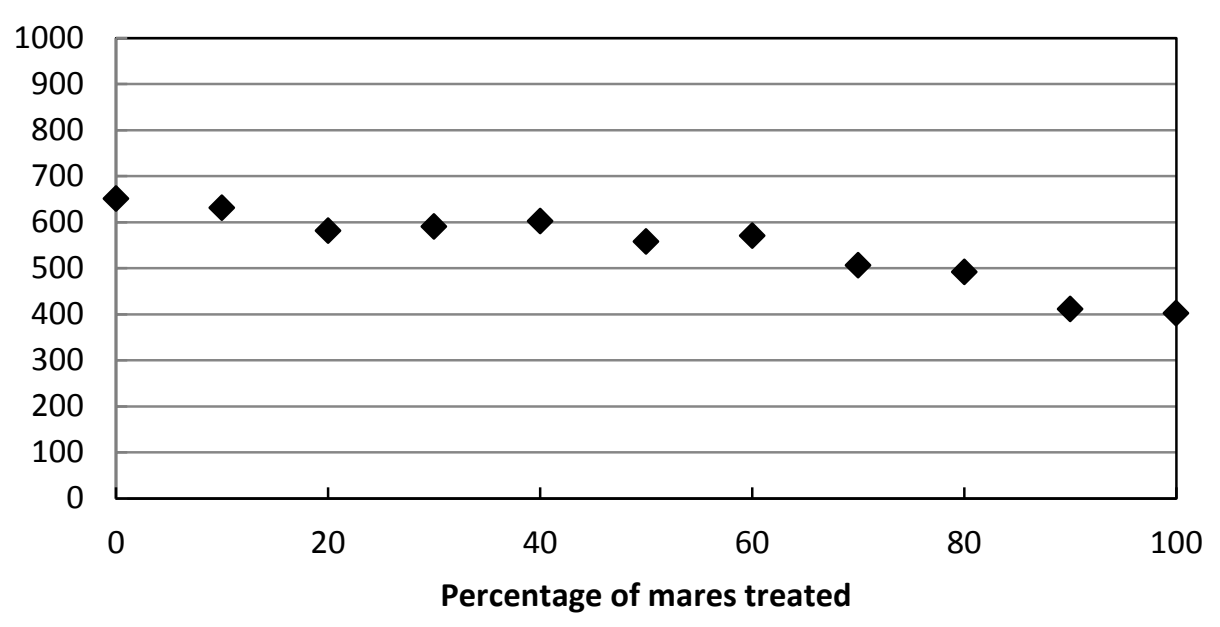

Mean probability of extirpation after $\mathbf{5 0}$ years

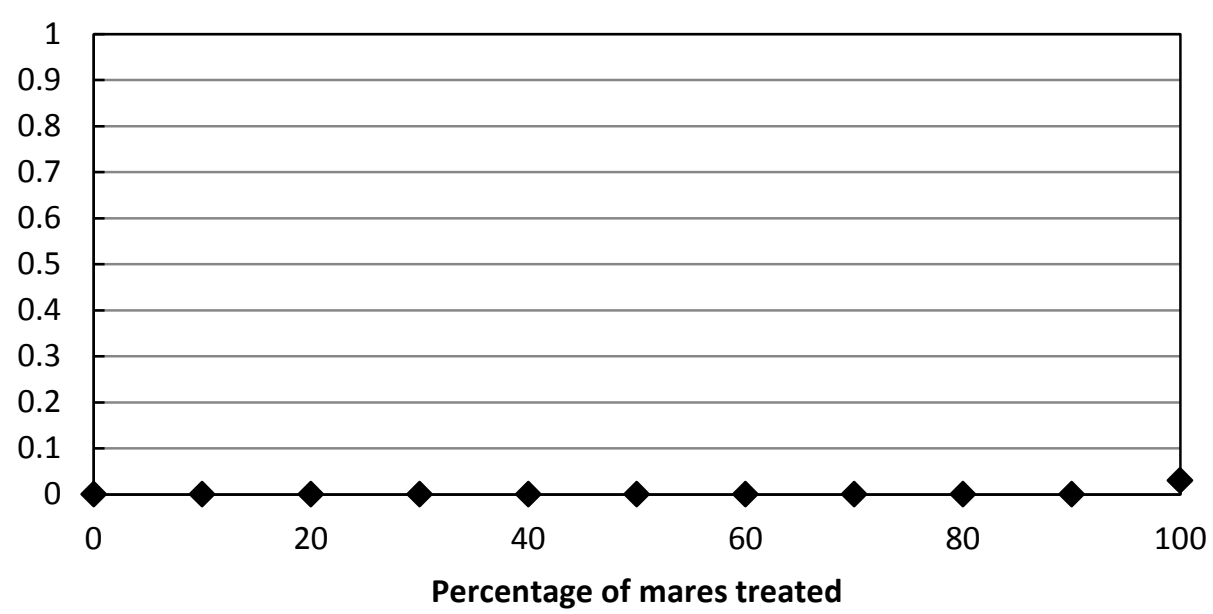

Figure 16. Initial population size $=750$, lambda $=1.05$, genetic diversity $=$ low .
Mean number of removals after 20 years

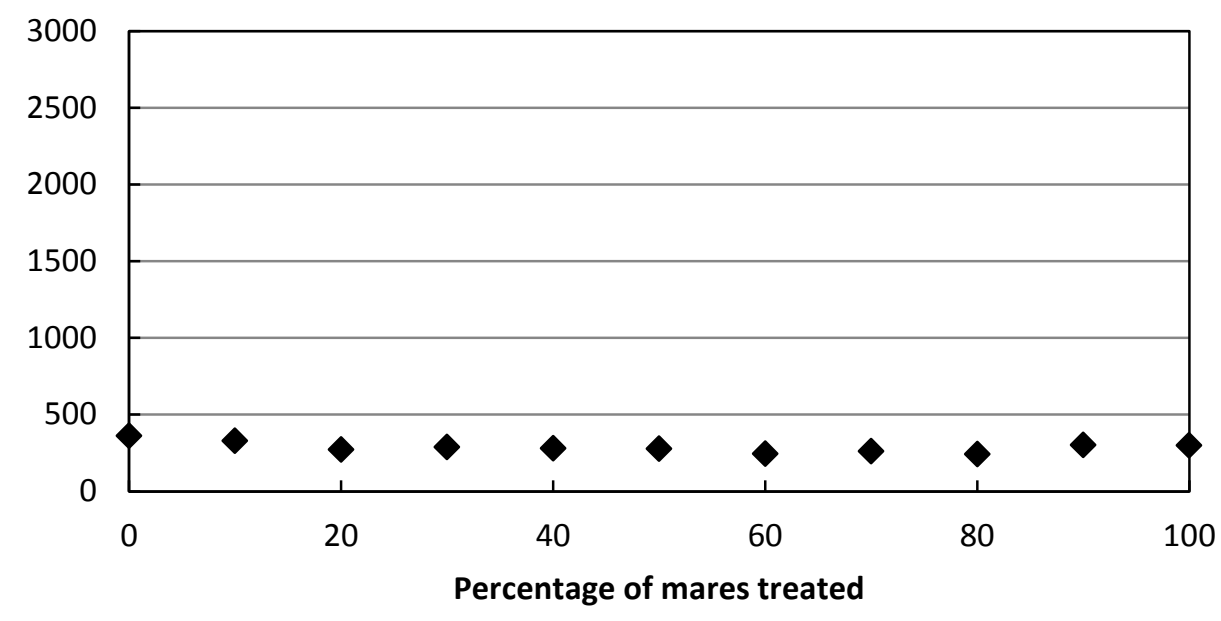

Mean heterozygosity after $\mathbf{5 0}$ years

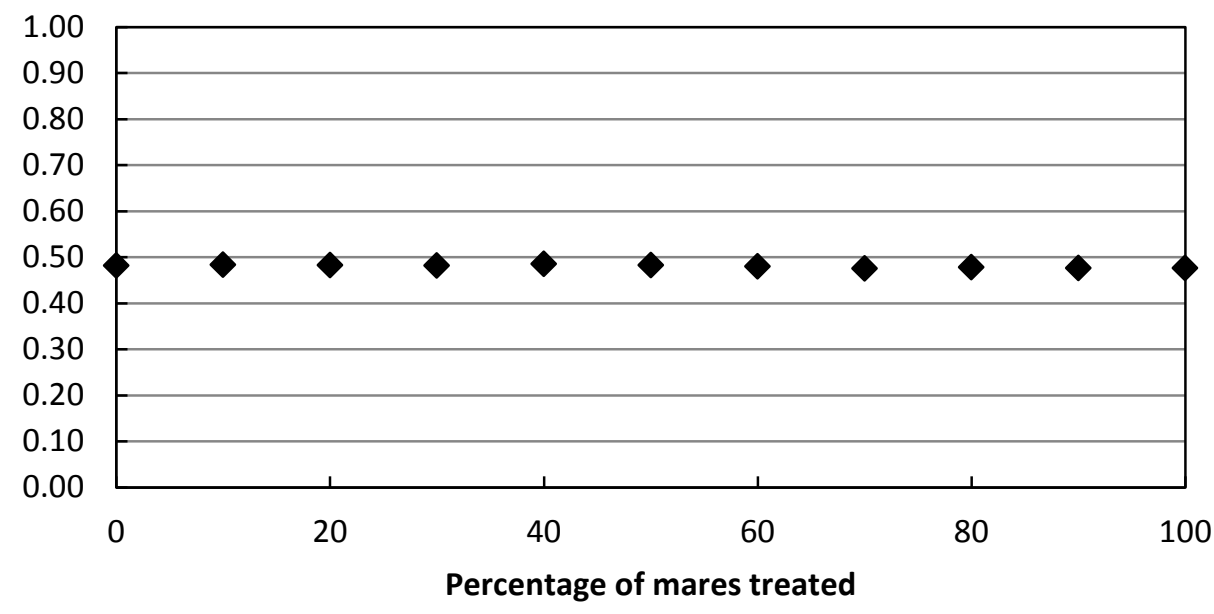




\begin{tabular}{lccccccccccc}
\hline \multicolumn{1}{c}{ Percentage of mares treated } & $\mathbf{0}$ & $\mathbf{1 0}$ & $\mathbf{2 0}$ & $\mathbf{3 0}$ & $\mathbf{4 0}$ & $\mathbf{5 0}$ & $\mathbf{6 0}$ & $\mathbf{7 0}$ & $\mathbf{8 0}$ & $\mathbf{9 0}$ & $\mathbf{1 0 0}$ \\
\hline Mean population size after 20 years & 650.88 & 631.43 & 581.13 & 590.12 & 602.15 & 557.46 & 570.81 & 506.51 & 491.17 & 411.2 & 401.89 \\
Mean number of removals after 20 years & 361.95 & 328.24 & 272.67 & 288.49 & 279.12 & 276.66 & 243.67 & 261.64 & 241.29 & 302.35 & 297.91 \\
Mean probability of extirpation after 50 years & 0 & 0 & 0 & 0 & 0 & 0 & 0 & 0 & 0 & 0 & 0.03 \\
Mean heterozygosity after 50 years & 0.48 & 0.48 & 0.48 & 0.48 & 0.49 & 0.48 & 0.48 & 0.48 & 0.48 & 0.48 & 0.48 \\
\hline
\end{tabular}

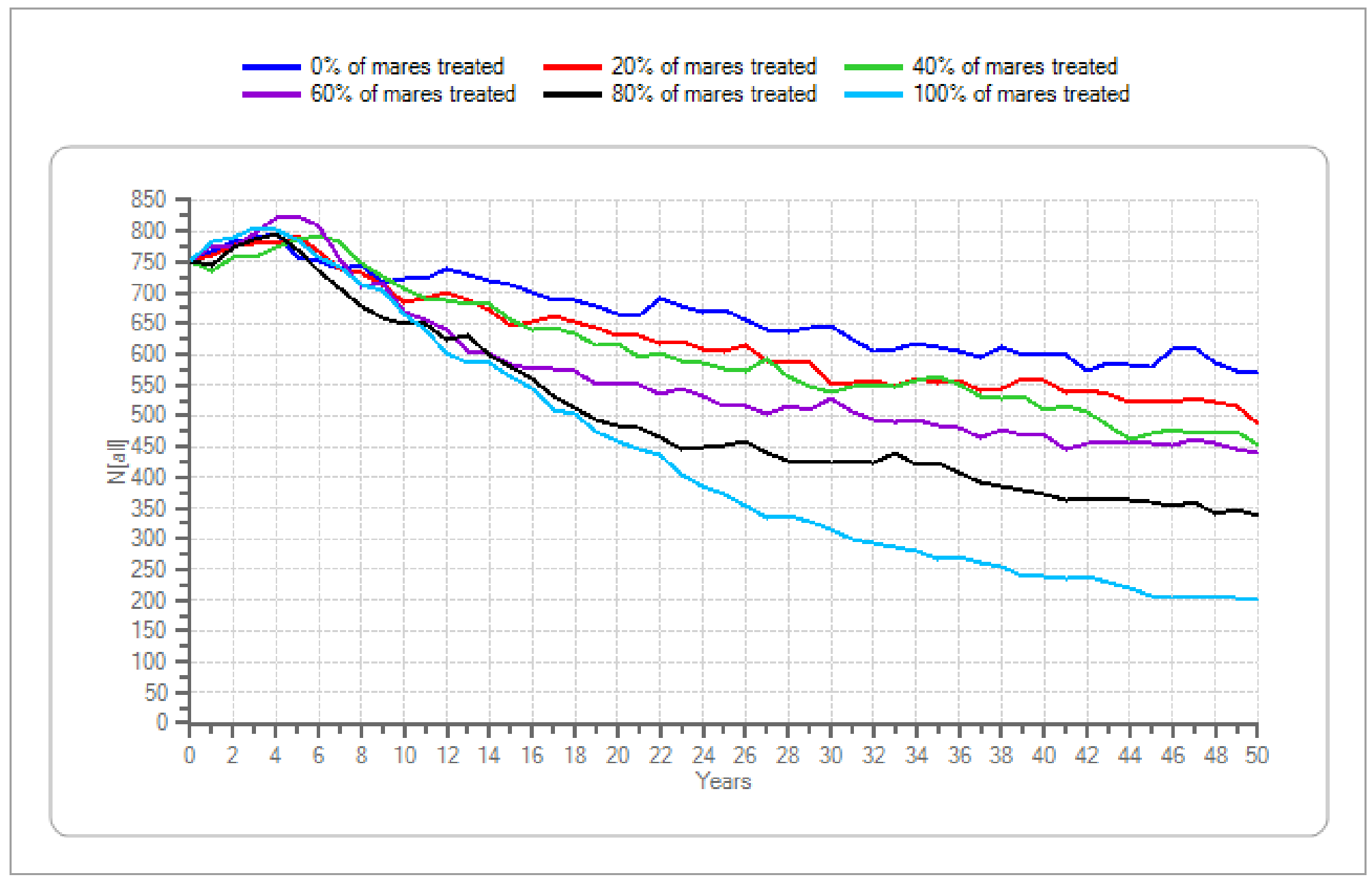

Figure 16. Initial population size $=750$, lambda $=1.05$, genetic diversity $=$ low - Continued 
Mean population size after 20 years

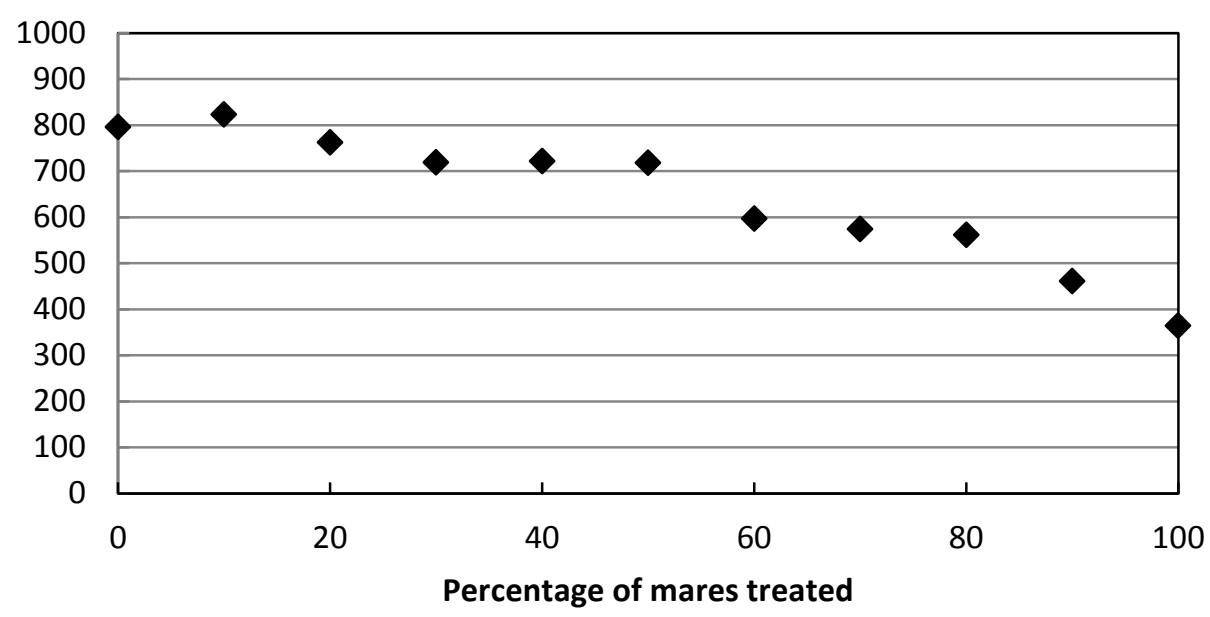

Mean probability of extirpation after $\mathbf{5 0}$ years

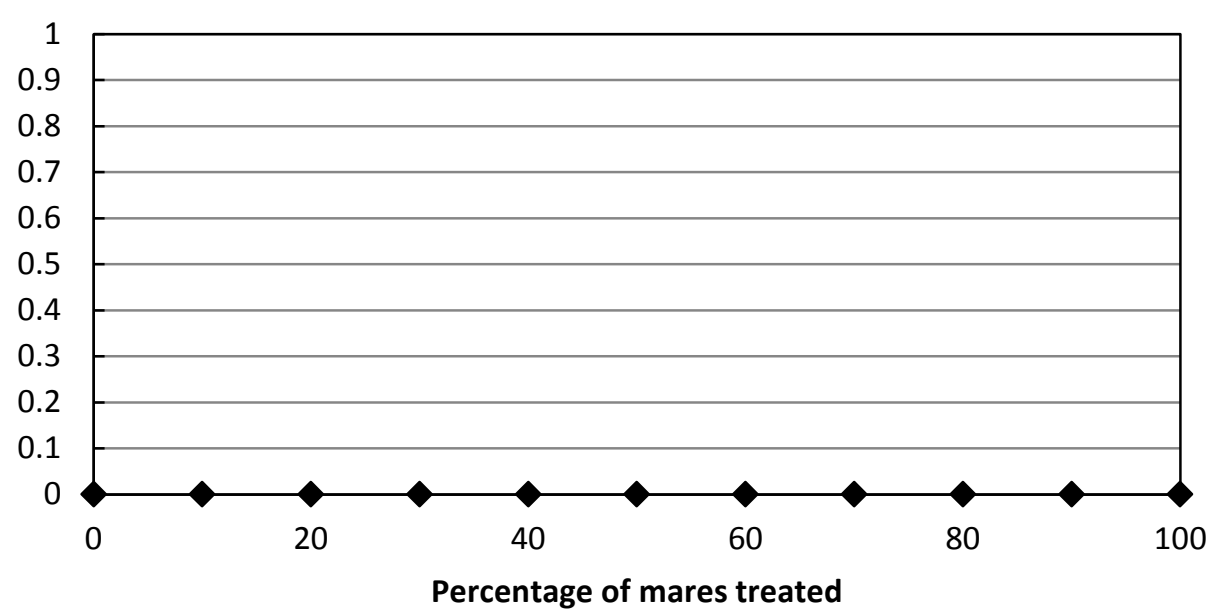

Figure 17. Initial population size $=750$, lambda $=1.10$, genetic diversity $=$ low .
Mean number of removals after 20 years

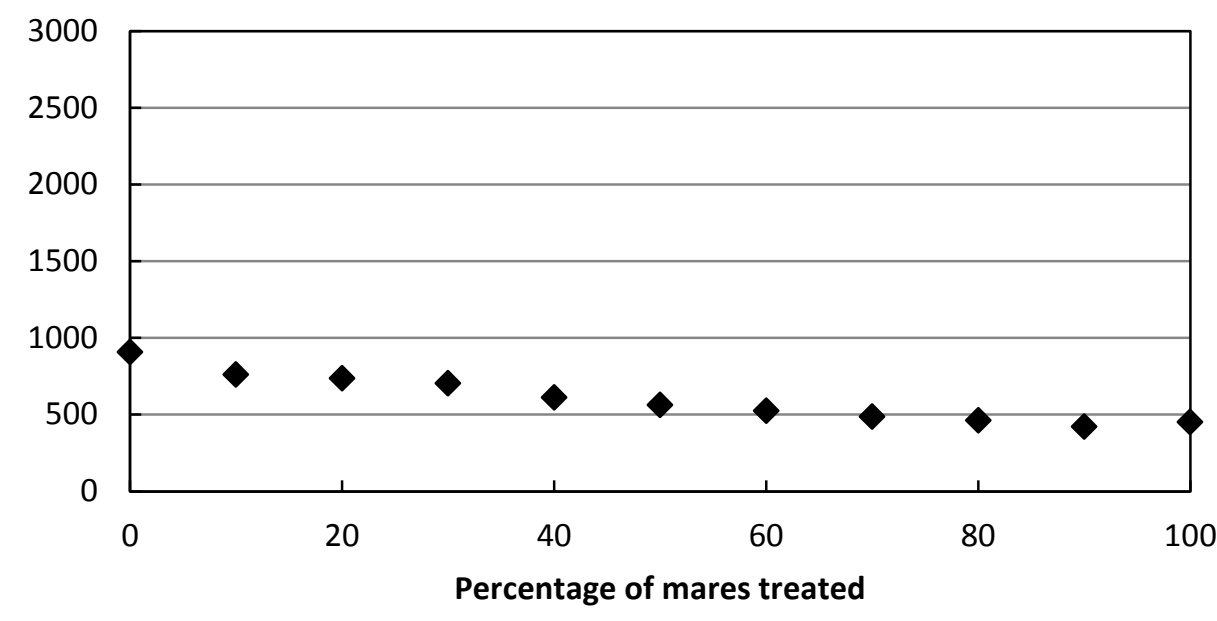

Mean heterozygosity after $\mathbf{5 0}$ years

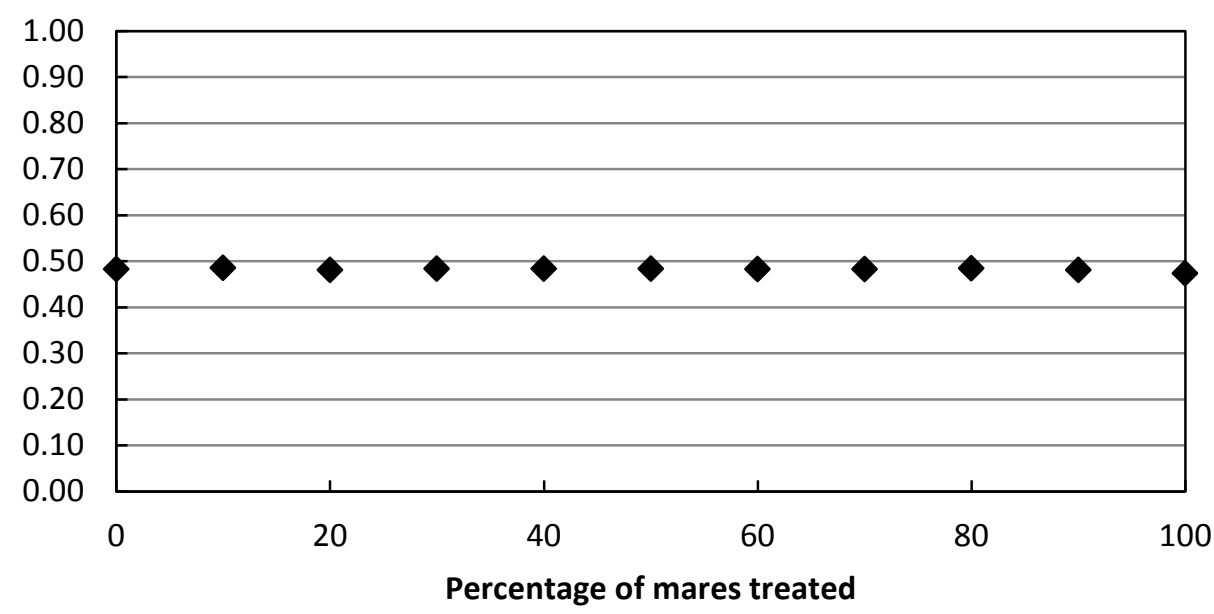




\begin{tabular}{lccccccccccc}
\hline \multicolumn{1}{c}{ Percentage of mares treated } & $\mathbf{0}$ & $\mathbf{1 0}$ & $\mathbf{2 0}$ & $\mathbf{3 0}$ & $\mathbf{4 0}$ & $\mathbf{5 0}$ & $\mathbf{6 0}$ & $\mathbf{7 0}$ & $\mathbf{8 0}$ & $\mathbf{9 0}$ & $\mathbf{1 0 0}$ \\
\hline Mean population size after 20 years & 795.56 & 823.24 & 762.24 & 718.7 & 721.89 & 717.77 & 597.06 & 573.89 & 561.16 & 460.9 & 363.9 \\
Mean number of removals after 20 years & 908.52 & 760.43 & 736.27 & 703.39 & 611.56 & 562.49 & 523.38 & 486.72 & 462.27 & 420.49 & 450.7 \\
Mean probability of extirpation after 50 years & 0 & 0 & 0 & 0 & 0 & 0 & 0 & 0 & 0 & 0 & 0 \\
Mean heterozygosity after 50 years & 0.48 & 0.49 & 0.48 & 0.48 & 0.48 & 0.48 & 0.48 & 0.48 & 0.48 & 0.48 & 0.47 \\
\hline
\end{tabular}

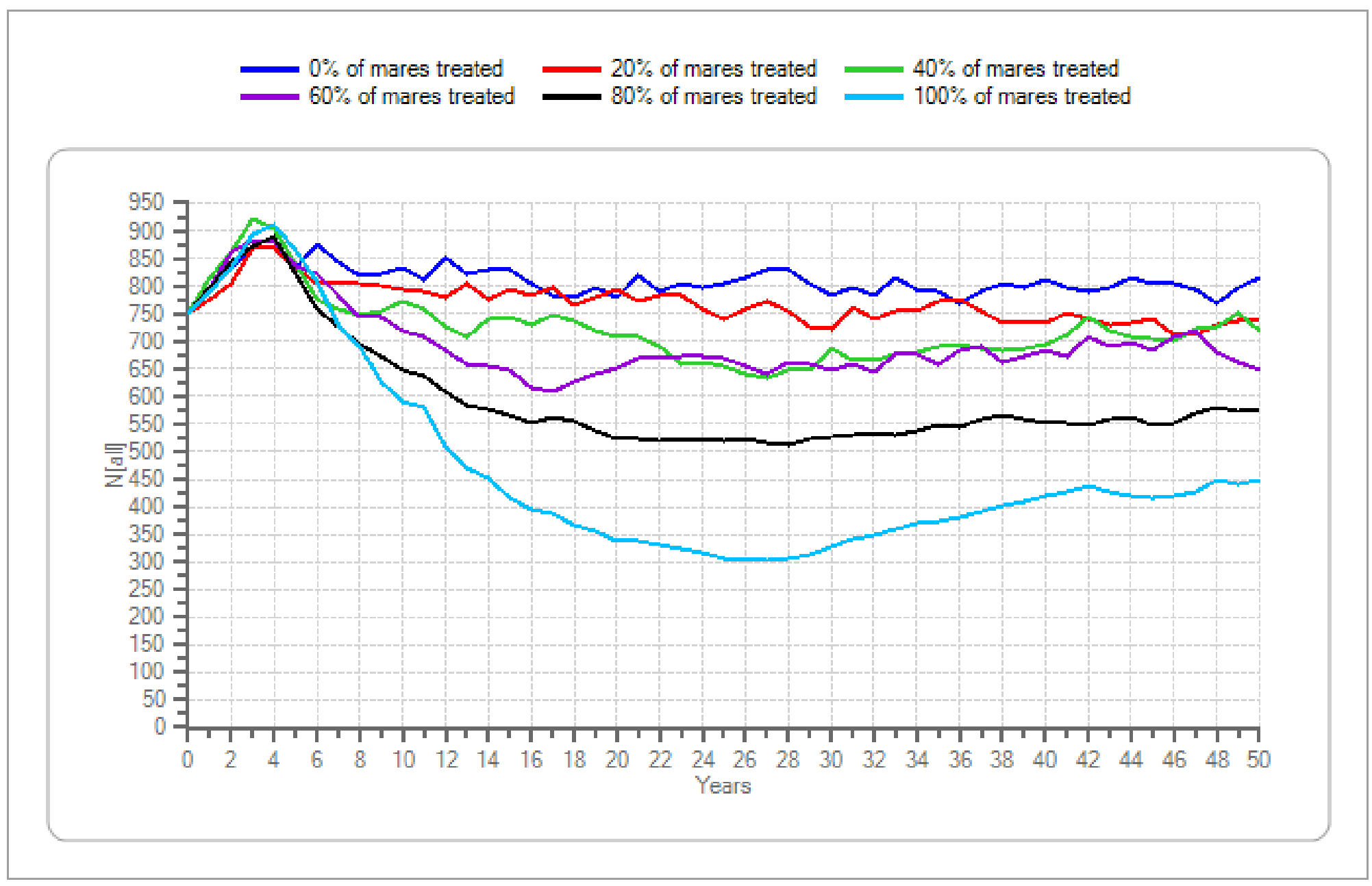

Figure 17. Initial population size $=750$, lambda $=1.10$, genetic diversity $=$ low - Continued 
Mean population size after 20 years

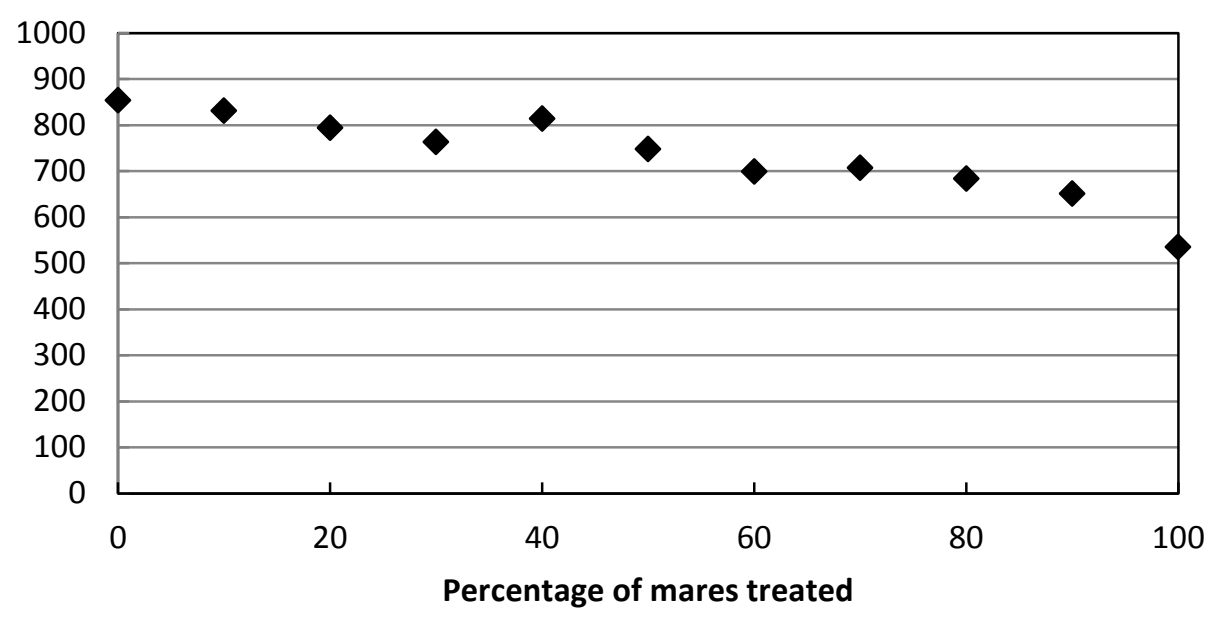

Mean probability of extirpation after $\mathbf{5 0}$ years

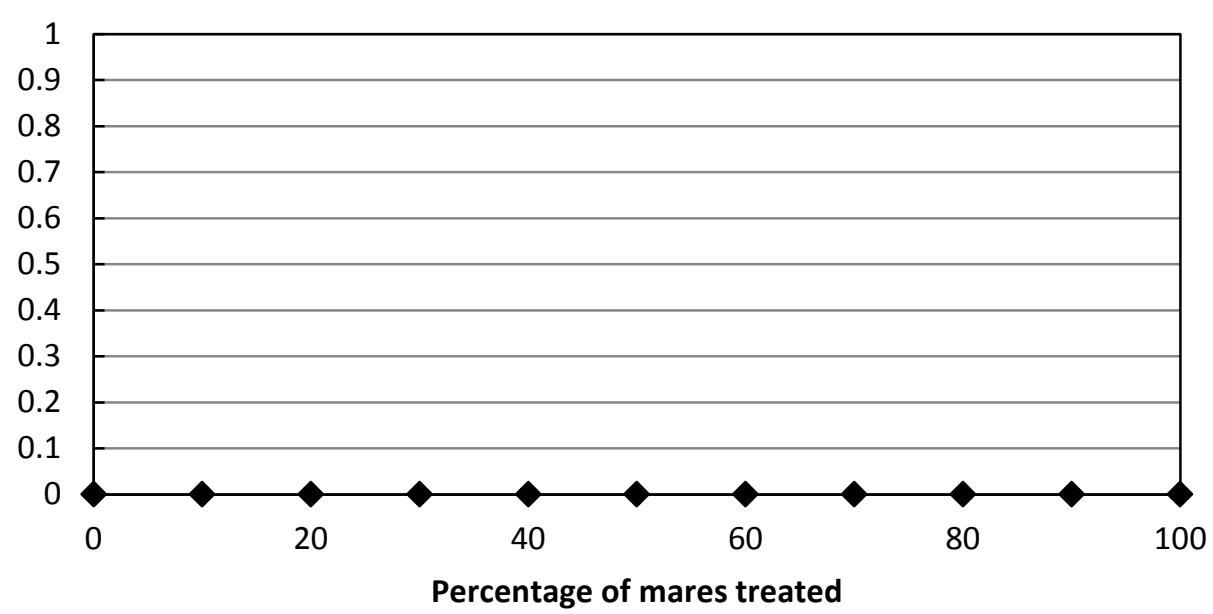

Figure 18. Initial population size $=750$, lambda $=1.15$, genetic diversity $=$ low .
Mean number of removals after 20 years

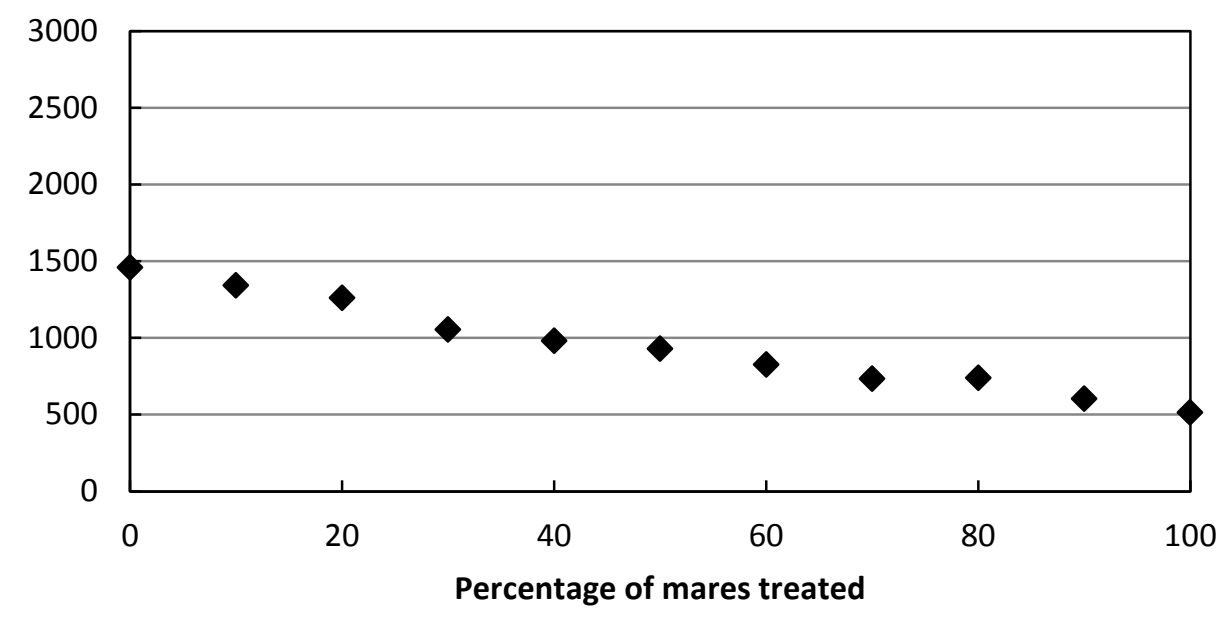

Mean heterozygosity after $\mathbf{5 0}$ years

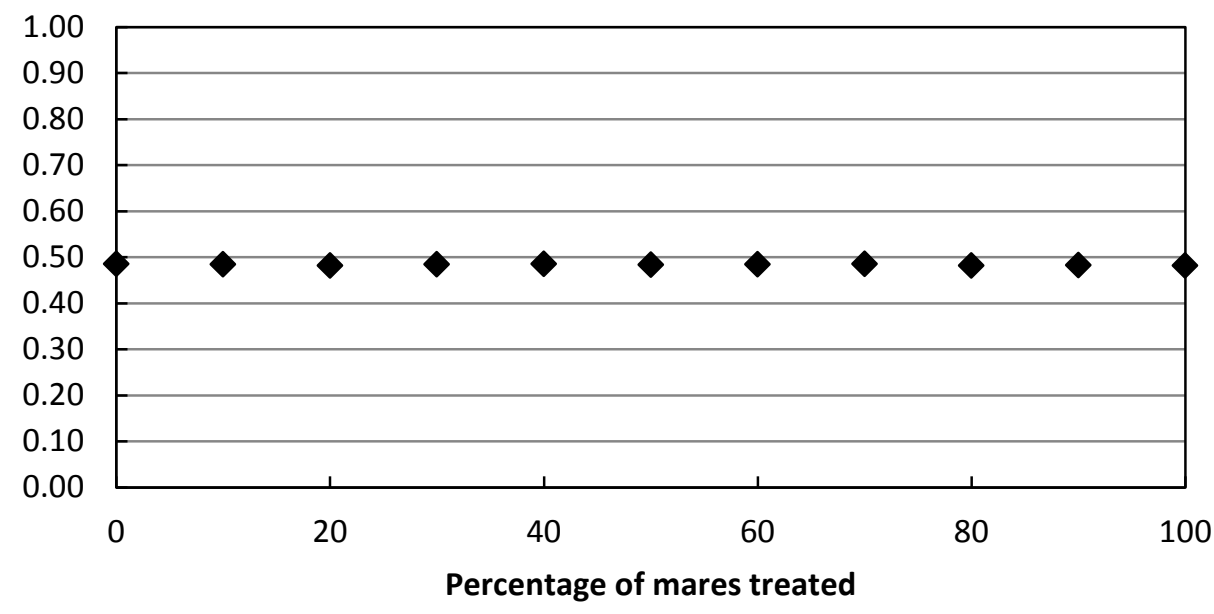




\begin{tabular}{lccccccccccc}
\hline \multicolumn{1}{c}{ Percentage of mares treated } & $\mathbf{0}$ & $\mathbf{1 0}$ & $\mathbf{2 0}$ & $\mathbf{3 0}$ & $\mathbf{4 0}$ & $\mathbf{5 0}$ & $\mathbf{6 0}$ & $\mathbf{7 0}$ & $\mathbf{8 0}$ & $\mathbf{9 0}$ & $\mathbf{1 0 0}$ \\
\hline Mean population size after 20 years & 853.53 & 831.08 & 794.36 & 763.4 & 813.88 & 747.49 & 698.91 & 707.4 & 683.74 & 650.95 & 535.33 \\
Mean number of removals after 20 years & 1458.62 & 1341.8 & 1260.79 & 1054.92 & 979.41 & 928.62 & 825.18 & 733.54 & 738.13 & 602.52 & 512.15 \\
Mean probability of extirpation after 50 years & 0 & 0 & 0 & 0 & 0 & 0 & 0 & 0 & 0 & 0 & 0 \\
Mean heterozygosity after 50 years & 0.49 & 0.48 & 0.48 & 0.48 & 0.49 & 0.48 & 0.48 & 0.49 & 0.48 & 0.48 & 0.48 \\
\hline
\end{tabular}

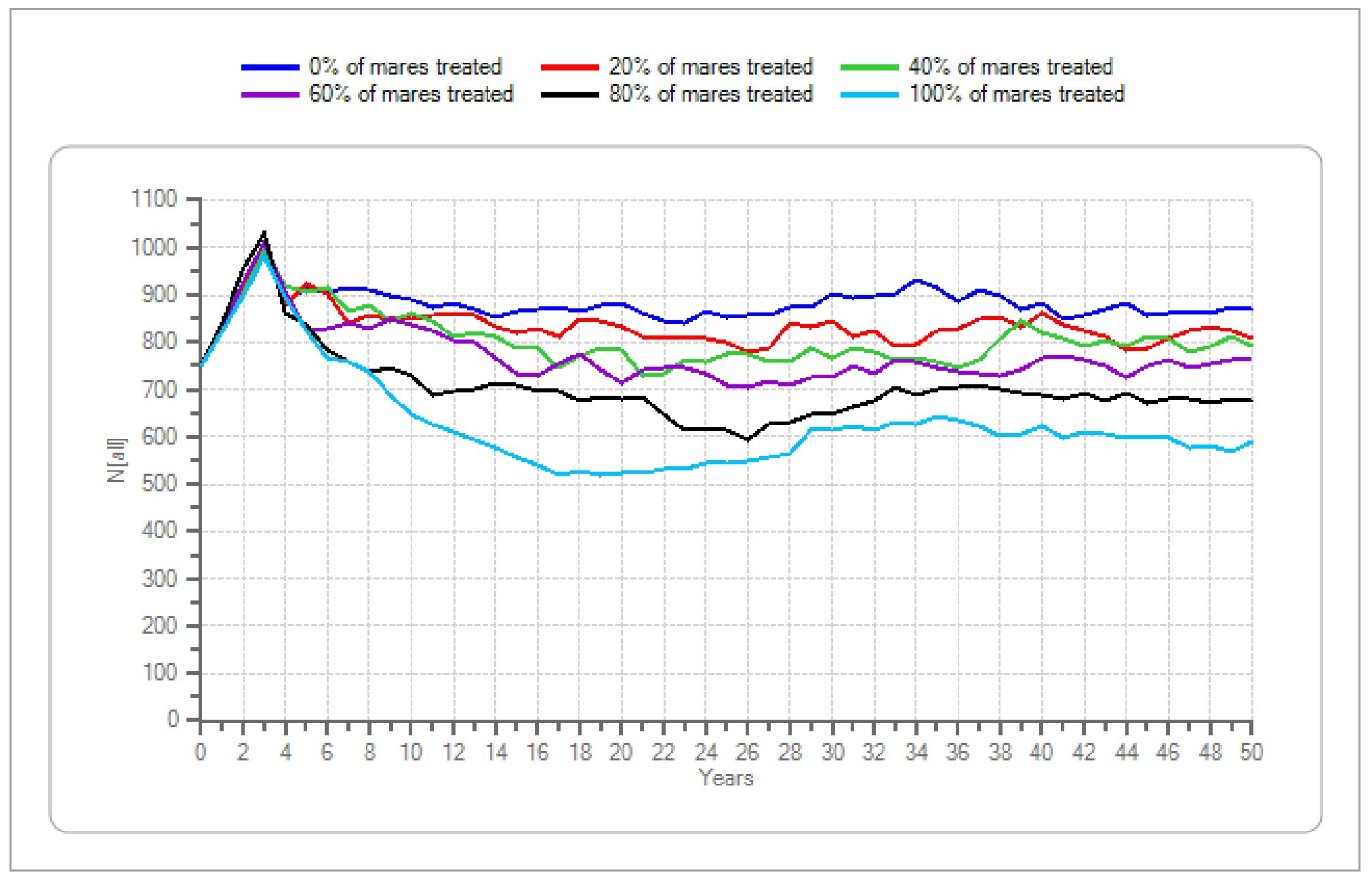

Figure 18. Initial population size $=750$, lambda $=1.15$, genetic diversity $=$ low - Continued 
Mean population size after 20 years

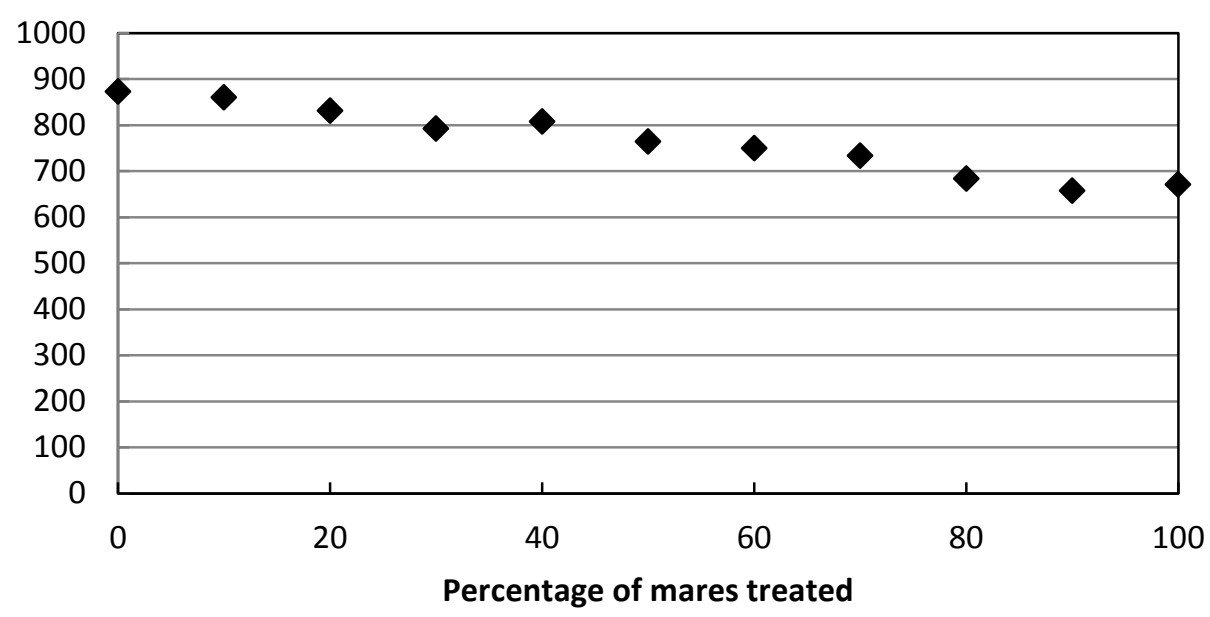

Mean probability of extirpation after $\mathbf{5 0}$ years

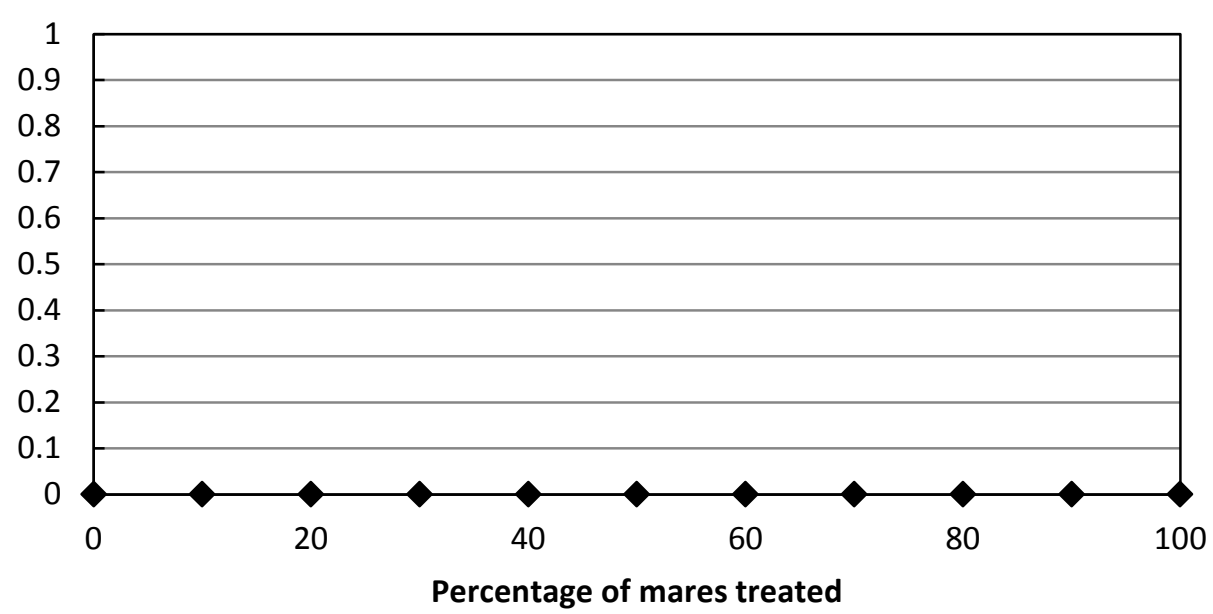

Figure 19. Initial population size $=750$, lambda $=1.20$, genetic diversity $=$ low .
Mean number of removals after 20 years

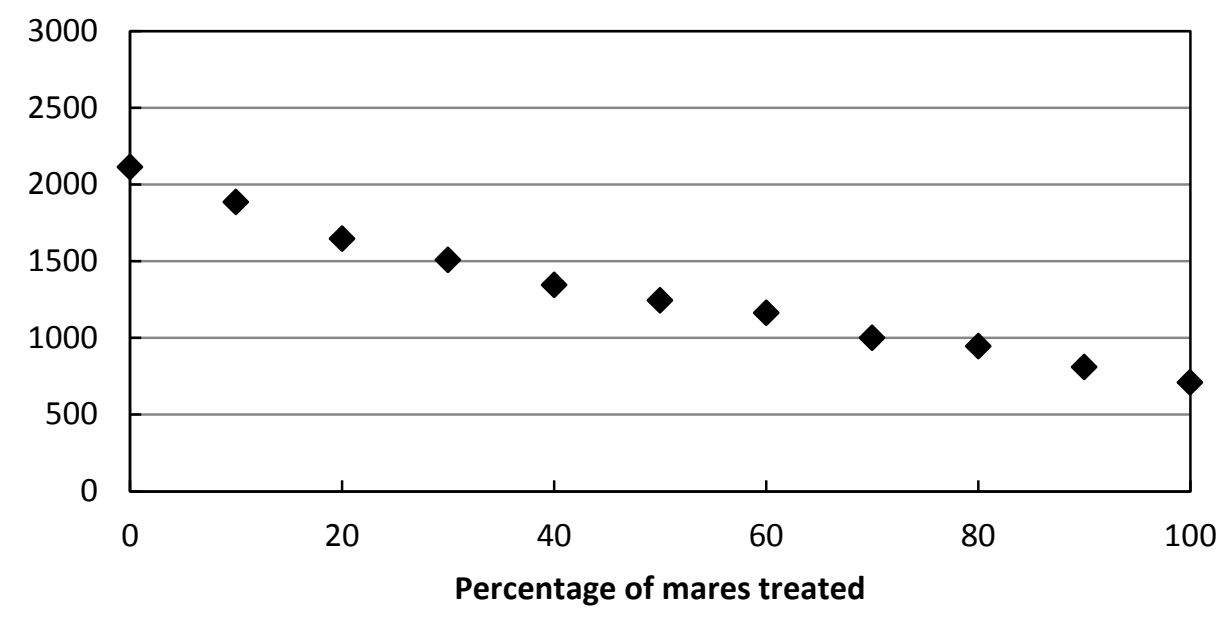

Mean heterozygosity after $\mathbf{5 0}$ years

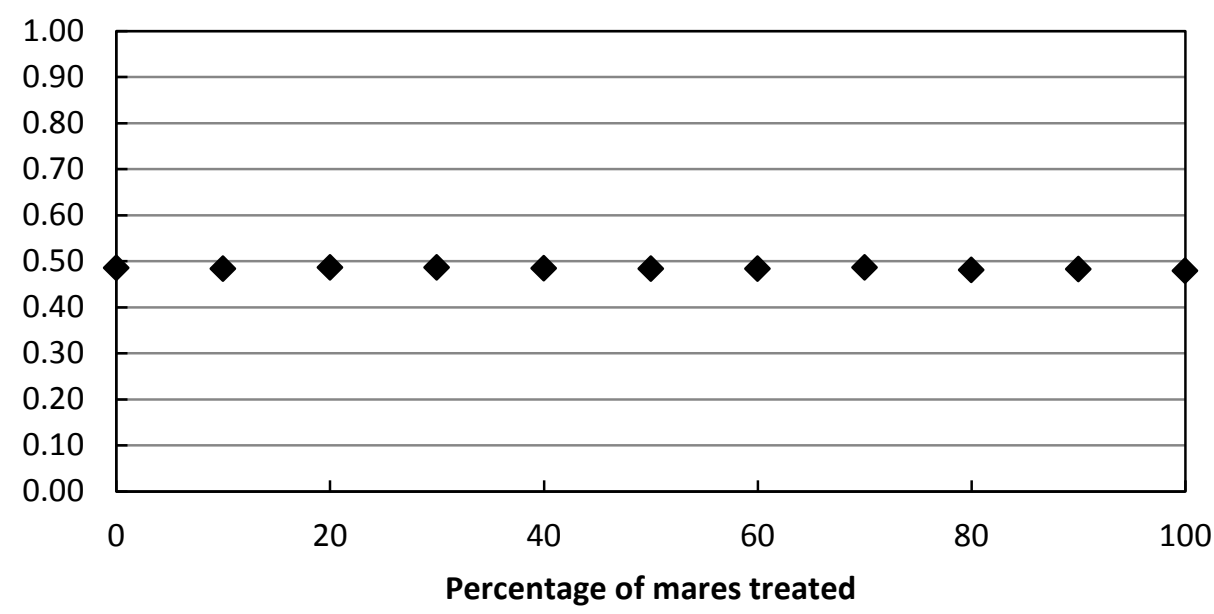




\begin{tabular}{lccccccccccc}
\hline \multicolumn{1}{c}{ Percentage of mares treated } & $\mathbf{0}$ & $\mathbf{1 0}$ & $\mathbf{2 0}$ & $\mathbf{3 0}$ & $\mathbf{4 0}$ & $\mathbf{5 0}$ & $\mathbf{6 0}$ & $\mathbf{7 0}$ & $\mathbf{8 0}$ & $\mathbf{9 0}$ & $\mathbf{1 0 0}$ \\
\hline Mean population size after 20 years & 872.42 & 860.25 & 830.74 & 792.41 & 807.75 & 763.95 & 749.48 & 733.6 & 683.58 & 657.07 & 670.54 \\
Mean number of removals after 20 years & 2112.42 & 1885.73 & 1646.6 & 1507.2 & 1345.72 & 1244.22 & 1162.02 & 999.18 & 944.68 & 809.02 & 709.93 \\
Mean probability of extirpation after 50 years & 0 & 0 & 0 & 0 & 0 & 0 & 0 & 0 & 0 & 0 & 0 \\
Mean heterozygosity after 50 years & 0.49 & 0.48 & 0.49 & 0.49 & 0.48 & 0.48 & 0.48 & 0.49 & 0.48 & 0.48 & 0.48 \\
\hline
\end{tabular}

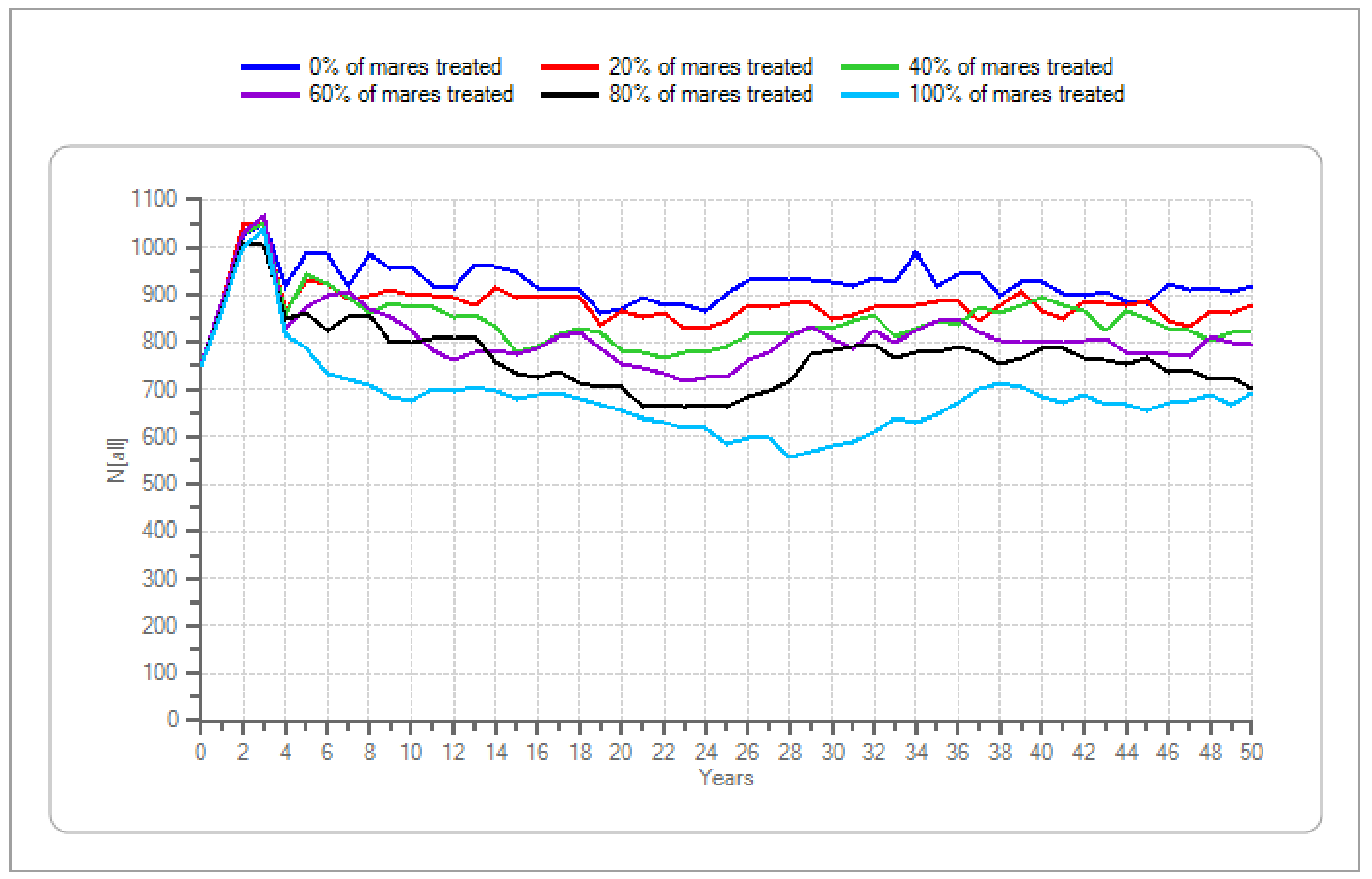

Figure 19. Initial population size $=750$, lambda $=1.20$, genetic diversity $=$ low - Continued 
Mean population size after 20 years

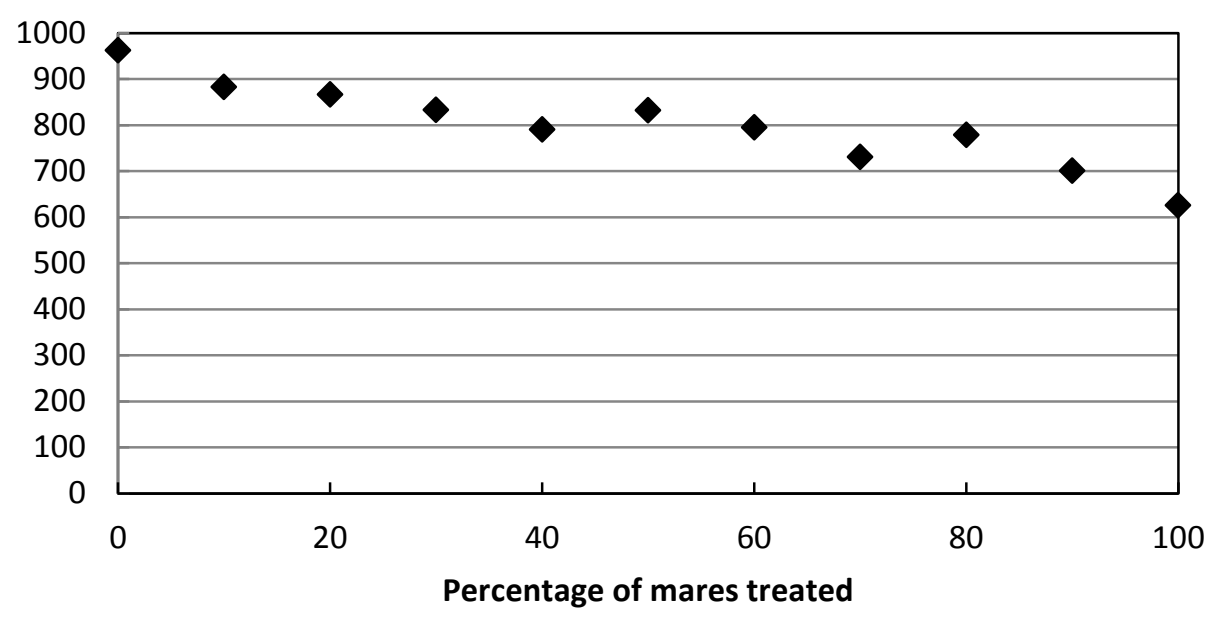

Mean probability of extirpation after $\mathbf{5 0}$ years

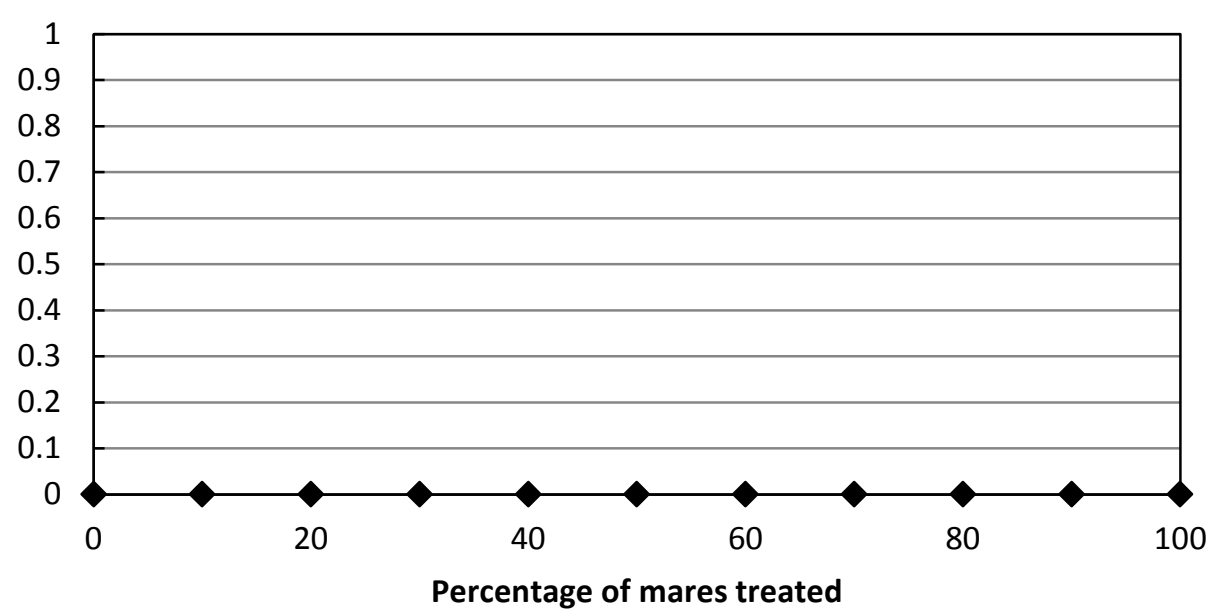

Figure 20. Initial population size $=750$, lambda $=1.25$, genetic diversity $=$ low .
Mean number of removals after 20 years

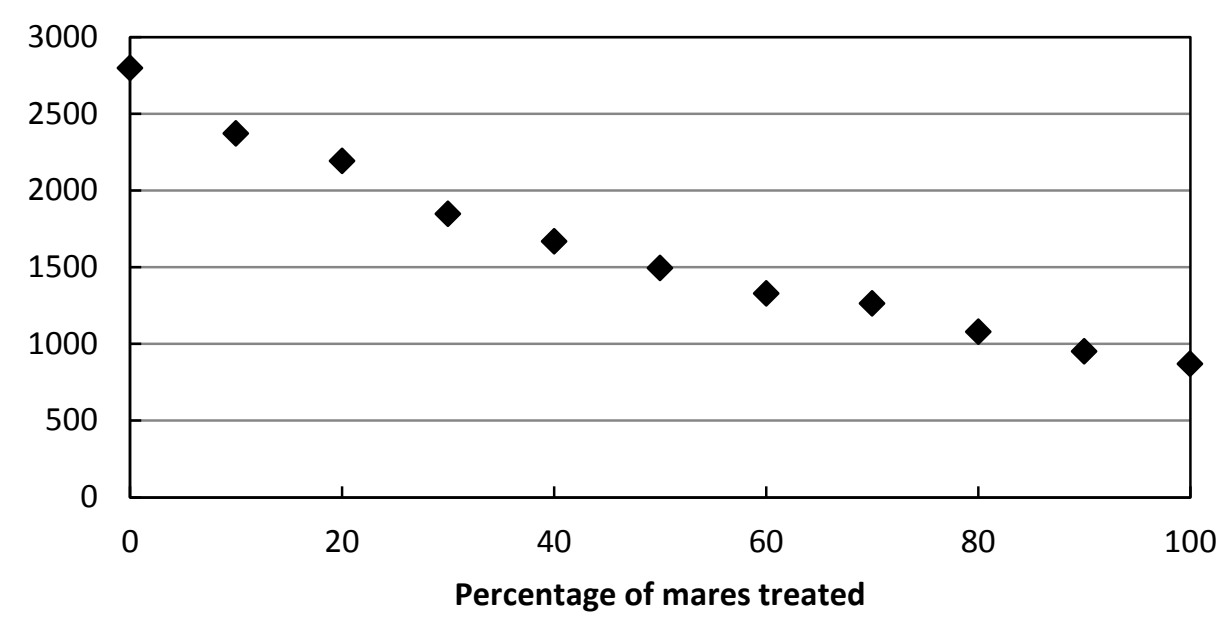

Mean heterozygosity after $\mathbf{5 0}$ years

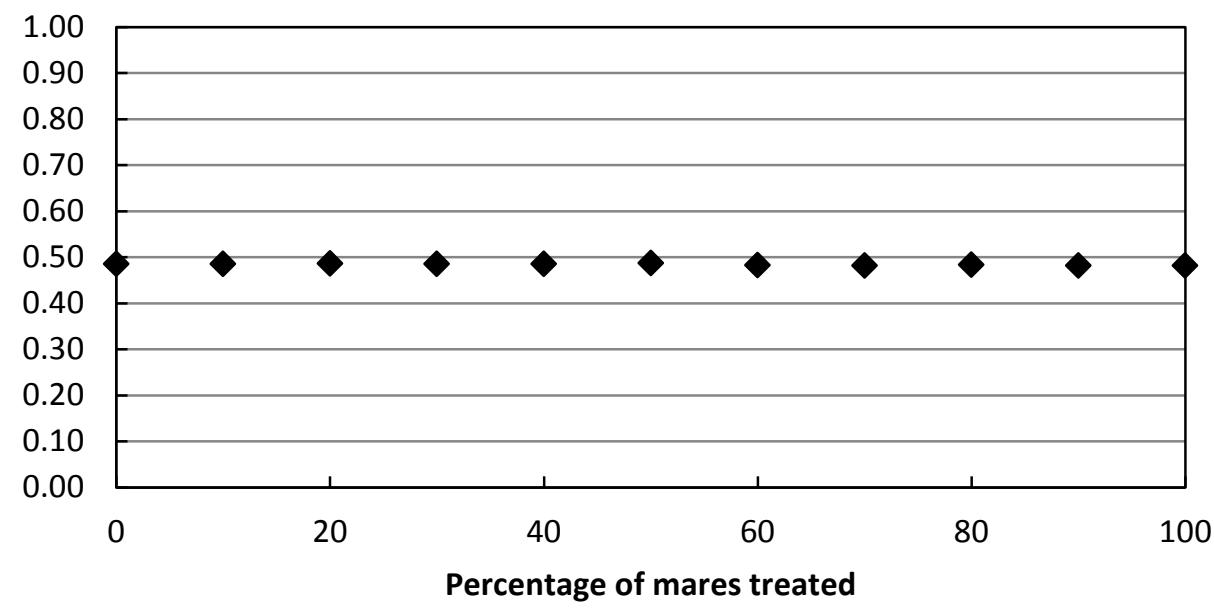




\begin{tabular}{lcccccccccccc}
\hline \multicolumn{1}{c}{ Percentage of mares treated } & $\mathbf{0}$ & $\mathbf{1 0}$ & $\mathbf{2 0}$ & $\mathbf{3 0}$ & $\mathbf{4 0}$ & $\mathbf{5 0}$ & $\mathbf{6 0}$ & $\mathbf{7 0}$ & $\mathbf{8 0}$ & $\mathbf{9 0}$ & $\mathbf{1 0 0}$ \\
\hline Mean population size after 20 years & 962.21 & 882.4 & 866.81 & 832.94 & 790.31 & 832.37 & 794.81 & 730.52 & 778.33 & 701.12 & 625.35 \\
Mean number of removals after 20 years & 2796.8 & 2371.7 & 2191.6 & 1846.0 & 1667.5 & 1492.6 & 1328.4 & 1264.1 & 1078.9 & 951.3 & 870.4 \\
Mean probability of extirpation after 50 years & 0 & 0 & 0 & 0 & 0 & 0 & 0 & 0 & 0 & 0 & 0 \\
Mean heterozygosity after 50 years & 0.49 & 0.49 & 0.49 & 0.49 & 0.49 & 0.49 & 0.48 & 0.48 & 0.48 & 0.48 & 0.48 \\
\hline
\end{tabular}

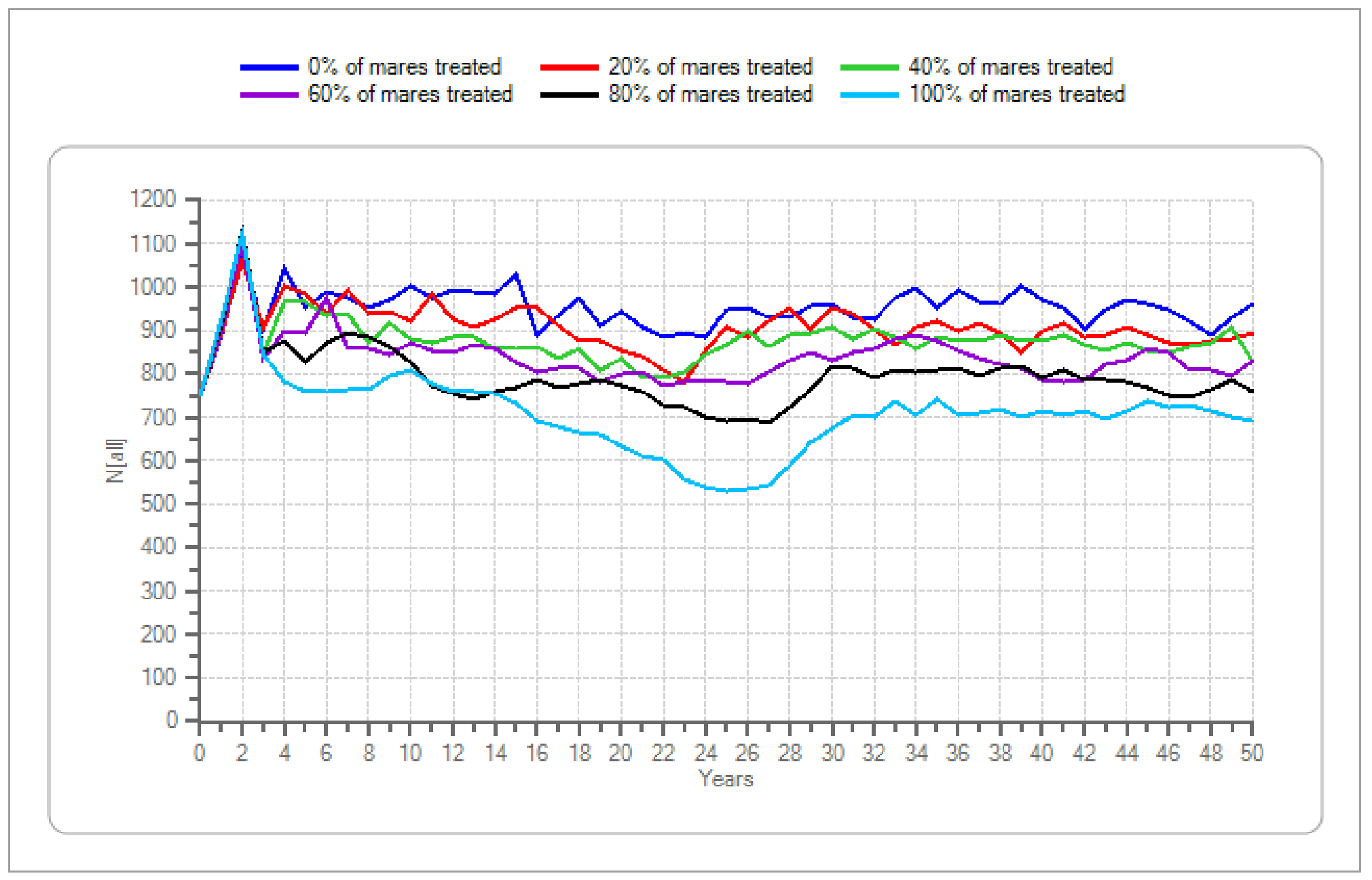

Figure 20. Initial population size $=750$, lambda $=1.25$, genetic diversity $=$ low - Continued 
Mean population size after 20 years

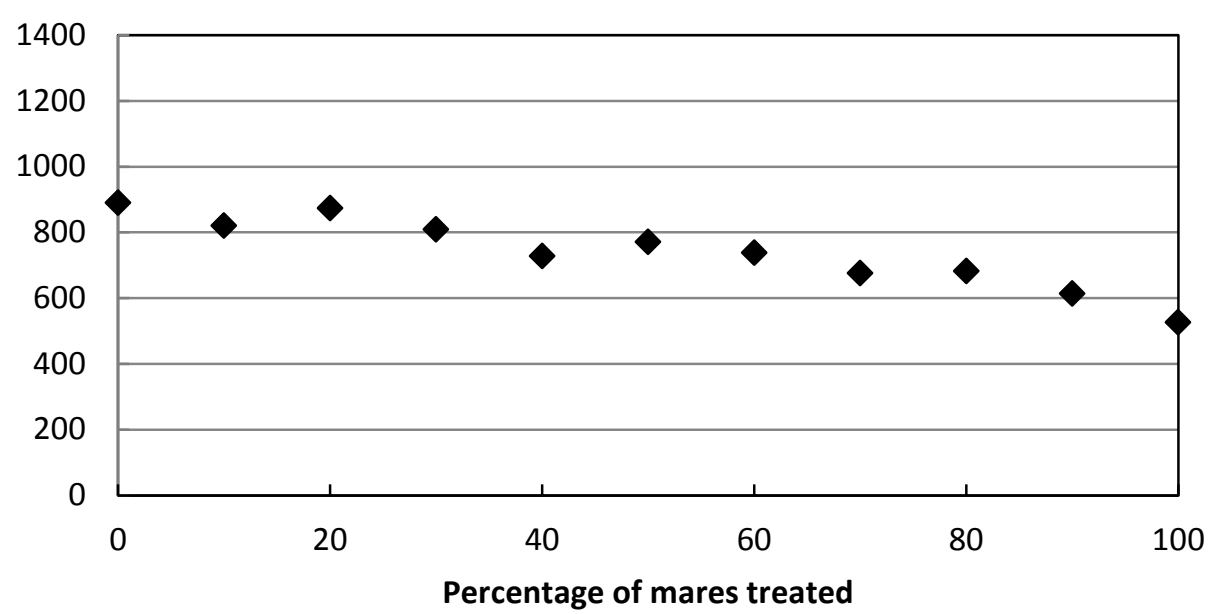

Mean probability of extirpation after 50 years

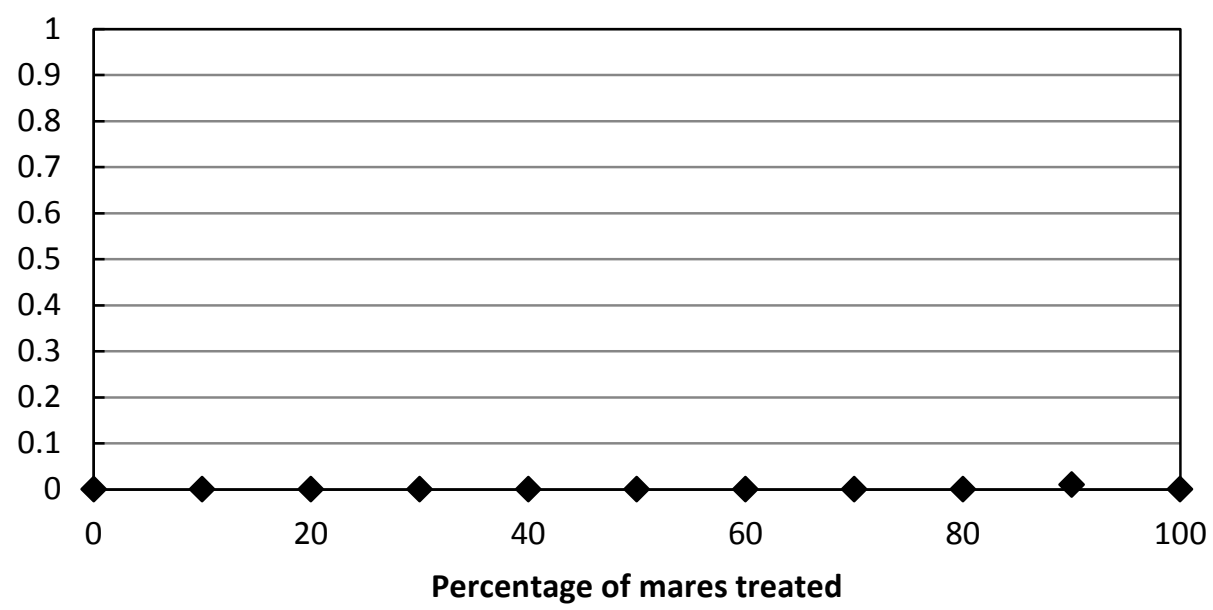

Figure 21. Initial population size $=1000$, lambda $=1.05$, genetic diversity $=$ low .
Mean number of removals after 20 years

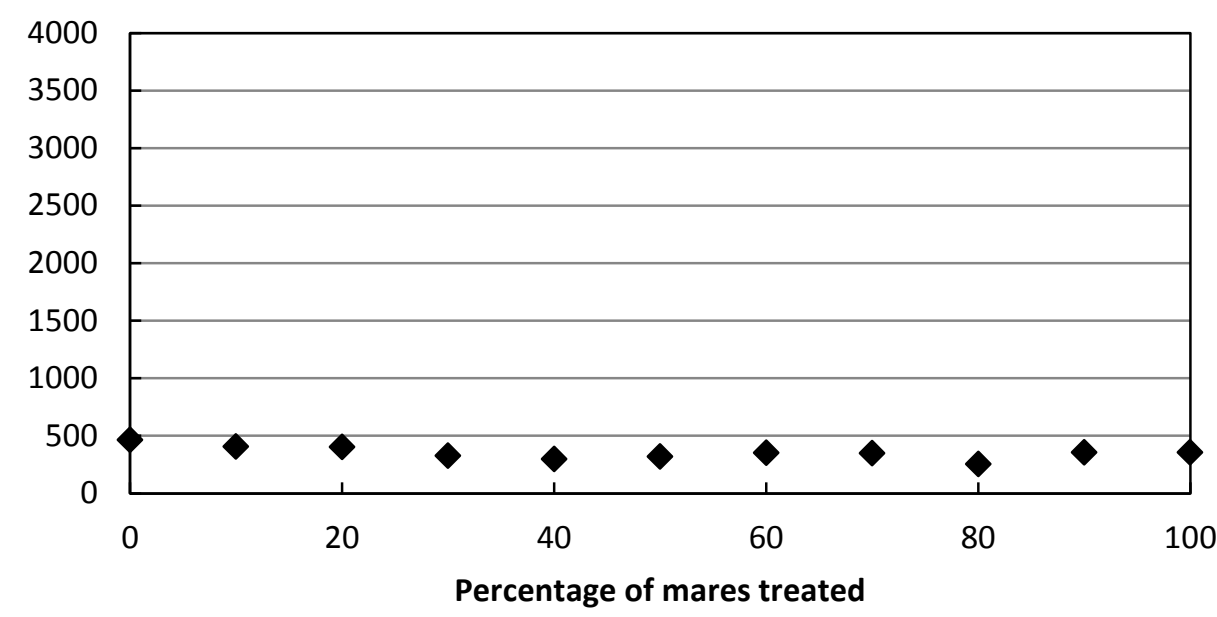

Mean heterozygosity after $\mathbf{5 0}$ years

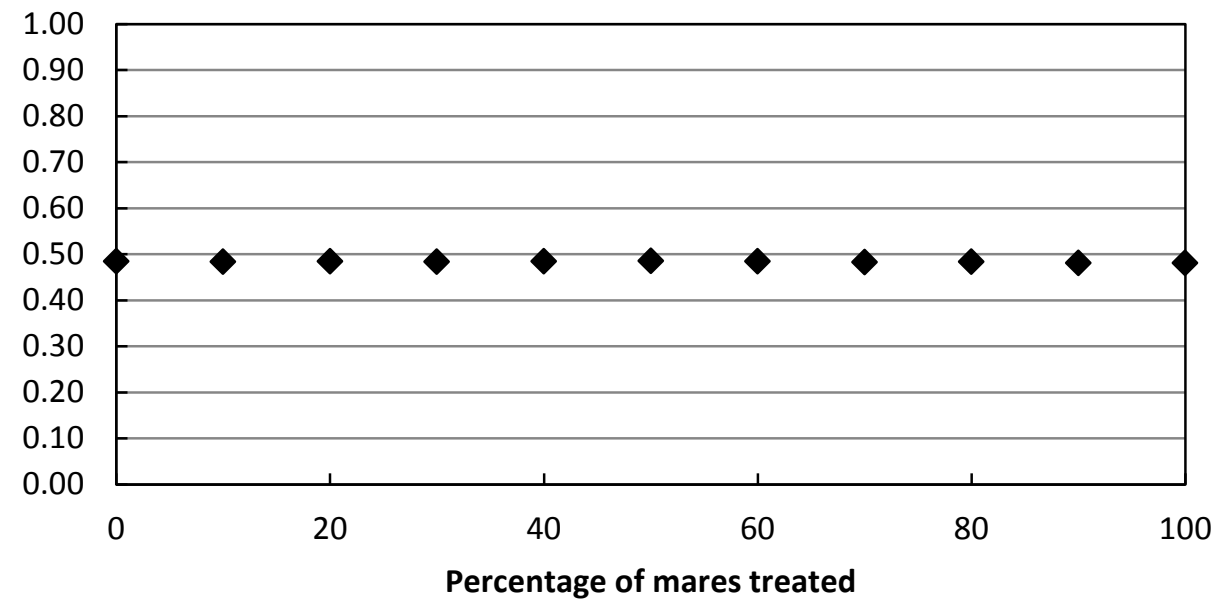




\begin{tabular}{lcccccccccccc}
\hline \multicolumn{1}{c}{ Percentage of mares treated } & $\mathbf{0}$ & $\mathbf{1 0}$ & $\mathbf{2 0}$ & $\mathbf{3 0}$ & $\mathbf{4 0}$ & $\mathbf{5 0}$ & $\mathbf{6 0}$ & $\mathbf{7 0}$ & $\mathbf{8 0}$ & $\mathbf{9 0}$ & $\mathbf{1 0 0}$ \\
\hline Mean population size after 20 years & 889.31 & 819.79 & 873.34 & 808.05 & 727.98 & 770.69 & 737.69 & 675.8 & 681.41 & 613.88 & 525.62 \\
Mean number of removals after 20 years & 462.82 & 404.15 & 400.39 & 327.35 & 297.35 & 318.94 & 349.73 & 348.62 & 252.02 & 354.25 & 356.61 \\
Mean probability of extirpation after 50 years & 0 & 0 & 0 & 0 & 0 & 0 & 0 & 0 & 0 & 0.01 & 0 \\
Mean heterozygosity after 50 years & 0.48 & 0.48 & 0.48 & 0.48 & 0.48 & 0.49 & 0.48 & 0.48 & 0.48 & 0.48 & 0.48 \\
\hline
\end{tabular}

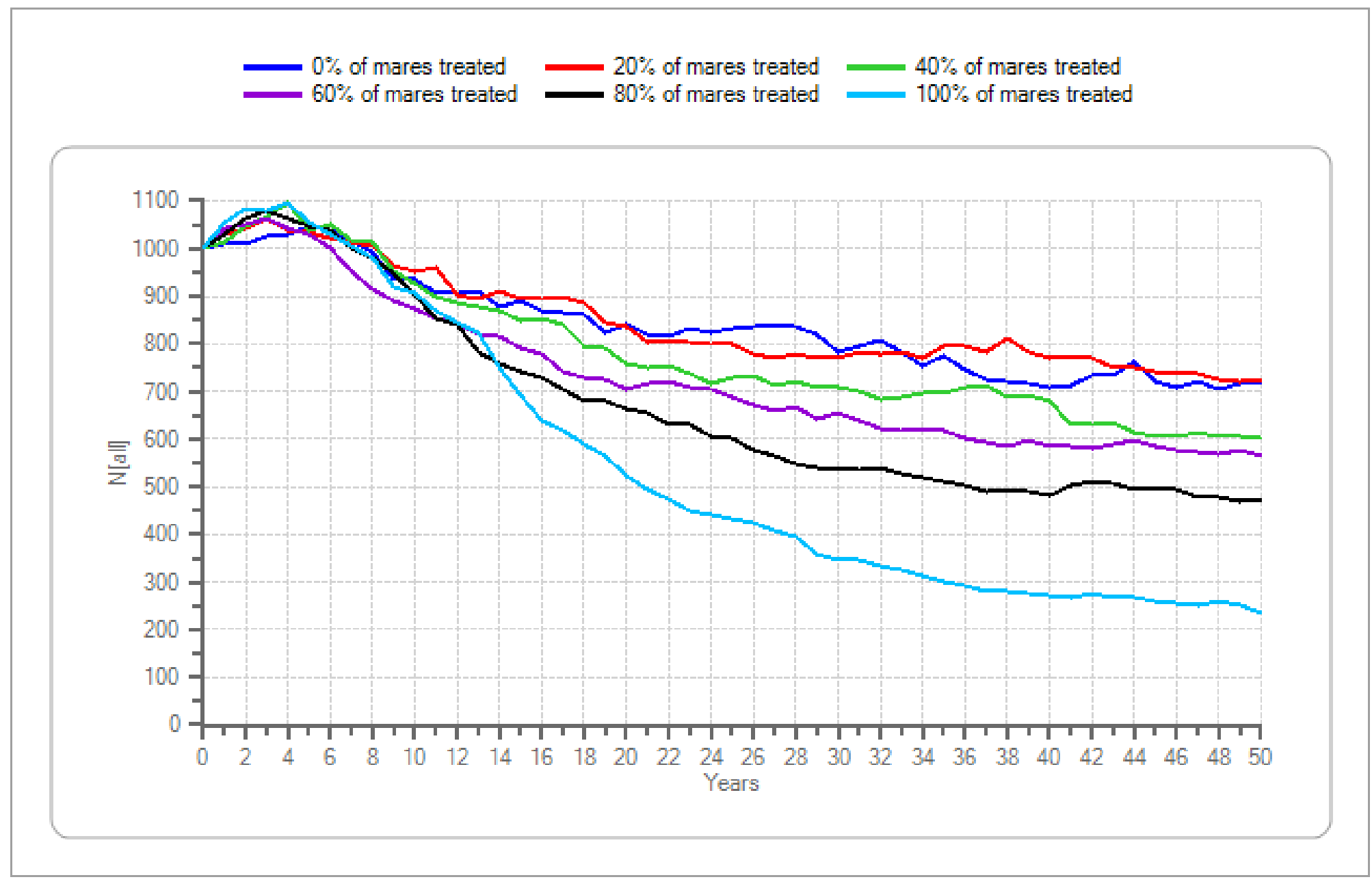

Figure 21. Initial population size $=1000$, lambda $=1.05$, genetic diversity $=$ low - Continued 
Mean population size after 20 years

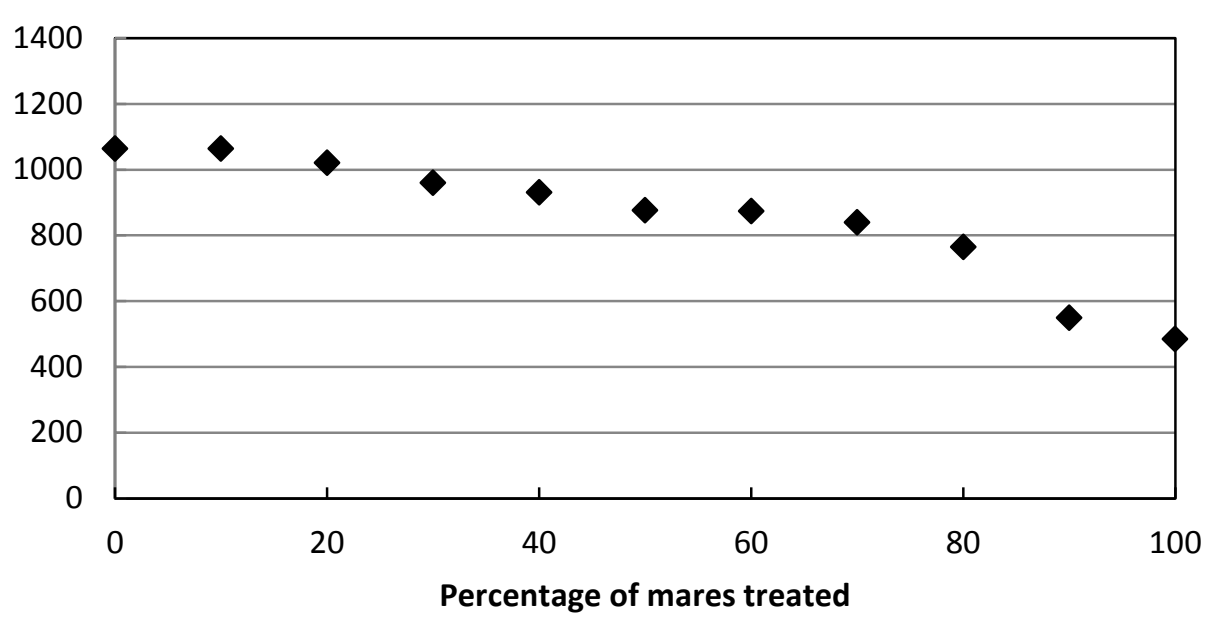

Mean probability of extirpation after $\mathbf{5 0}$ years

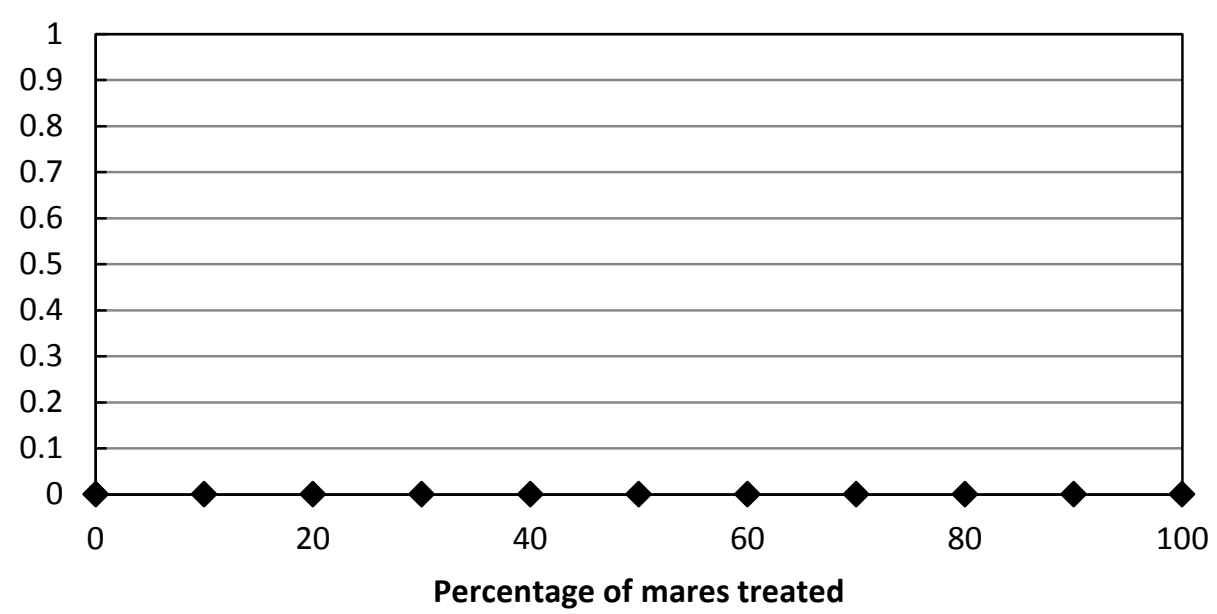

Figure 22. Initial population size $=1000$, lambda $=1.10$, genetic diversity $=$ low .
Mean number of removals after 20 years

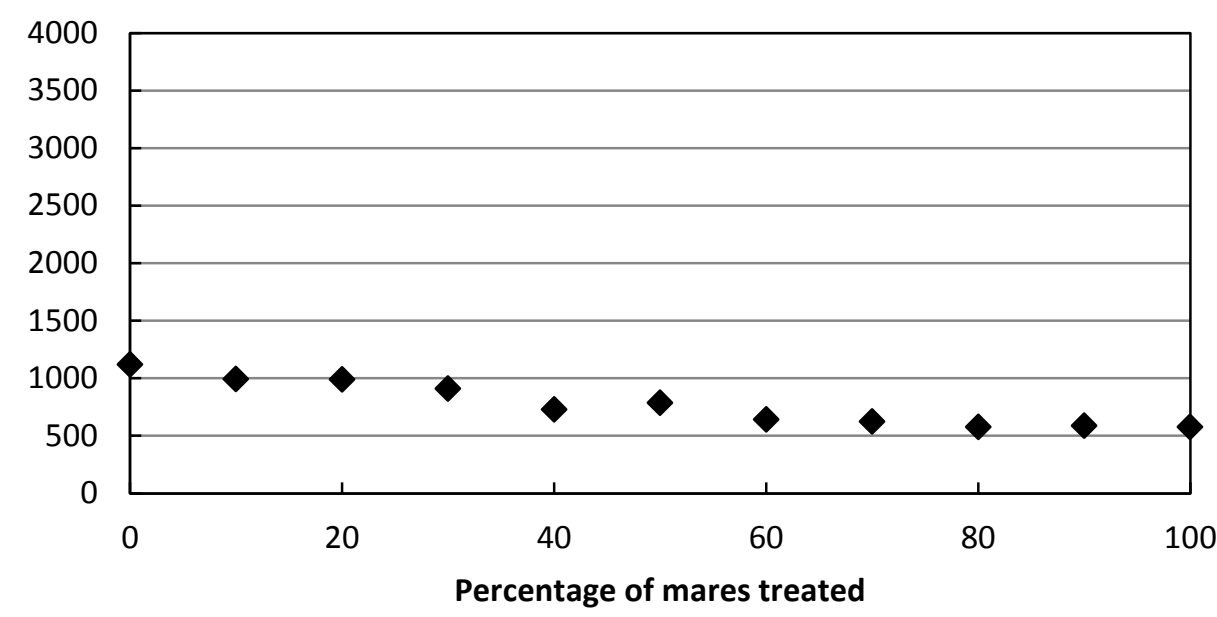

Mean heterozygosity after $\mathbf{5 0}$ years

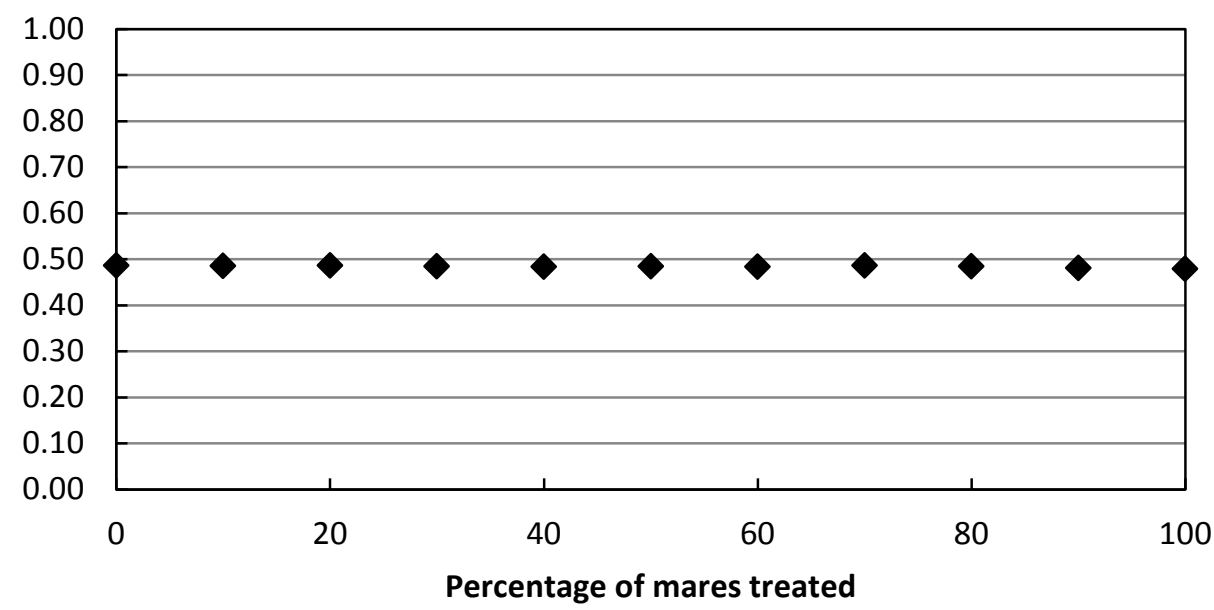




\begin{tabular}{lcccccccccccc}
\hline \multicolumn{1}{c}{ Percentage of mares treated } & $\mathbf{0}$ & $\mathbf{1 0}$ & $\mathbf{2 0}$ & $\mathbf{3 0}$ & $\mathbf{4 0}$ & $\mathbf{5 0}$ & $\mathbf{6 0}$ & $\mathbf{7 0}$ & $\mathbf{8 0}$ & $\mathbf{9 0}$ & $\mathbf{1 0 0}$ \\
\hline Mean population size after 20 years & 1063.82 & 1064.02 & 1019.82 & 959.43 & 930.43 & 875.89 & 872.81 & 839.08 & 764.81 & 549.04 & 483.72 \\
Mean number of removals after 20 years & 1118.7 & 991.5 & 988.6 & 908.4 & 728.6 & 785.9 & 642.2 & 621.2 & 577.2 & 585.4 & 577.7 \\
Mean probability of extirpation after 50 years & 0 & 0 & 0 & 0 & 0 & 0 & 0 & 0 & 0 & 0 & 0 \\
Mean heterozygosity after 50 years & 0.49 & 0.49 & 0.49 & 0.48 & 0.48 & 0.48 & 0.48 & 0.49 & 0.48 & 0.48 & 0.48 \\
\hline
\end{tabular}

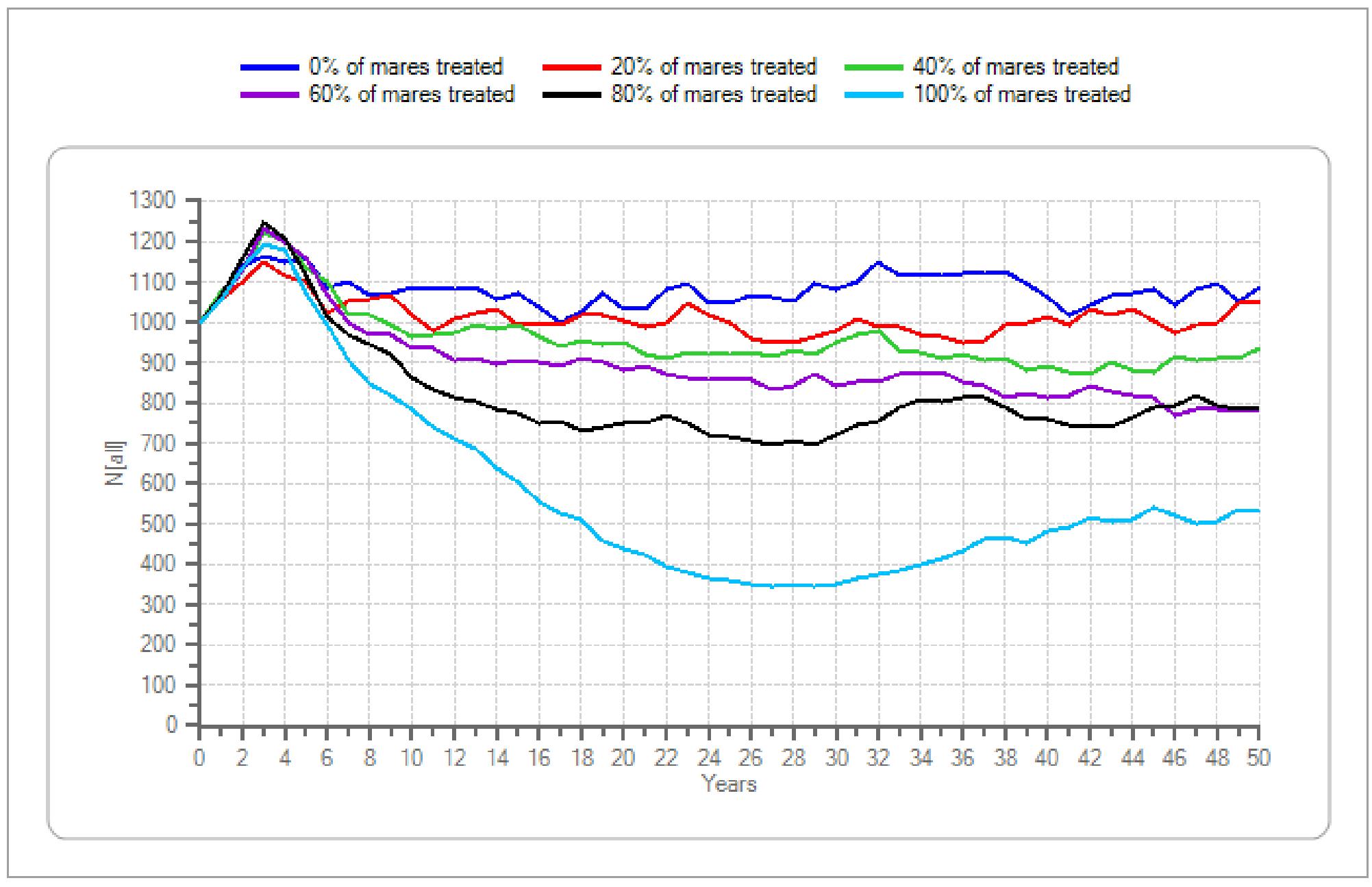

Figure 22. Initial population size $=1000$, lambda $=1.10$, genetic diversity $=$ low - Continued 
Mean population size after 20 years

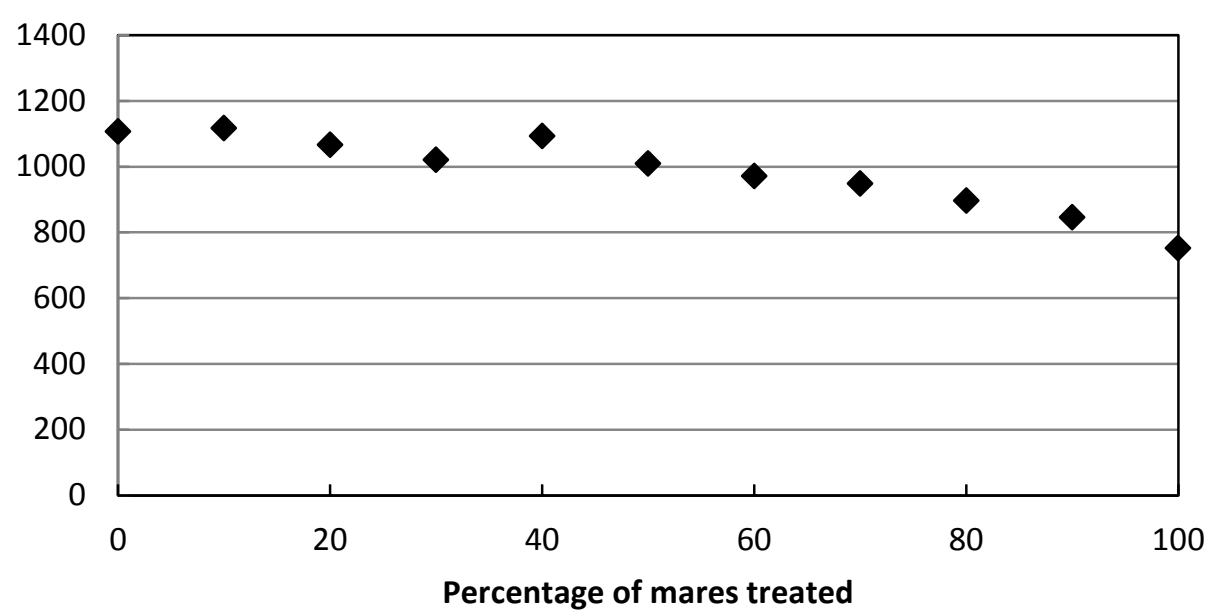

Mean probability of extirpation after 50 years

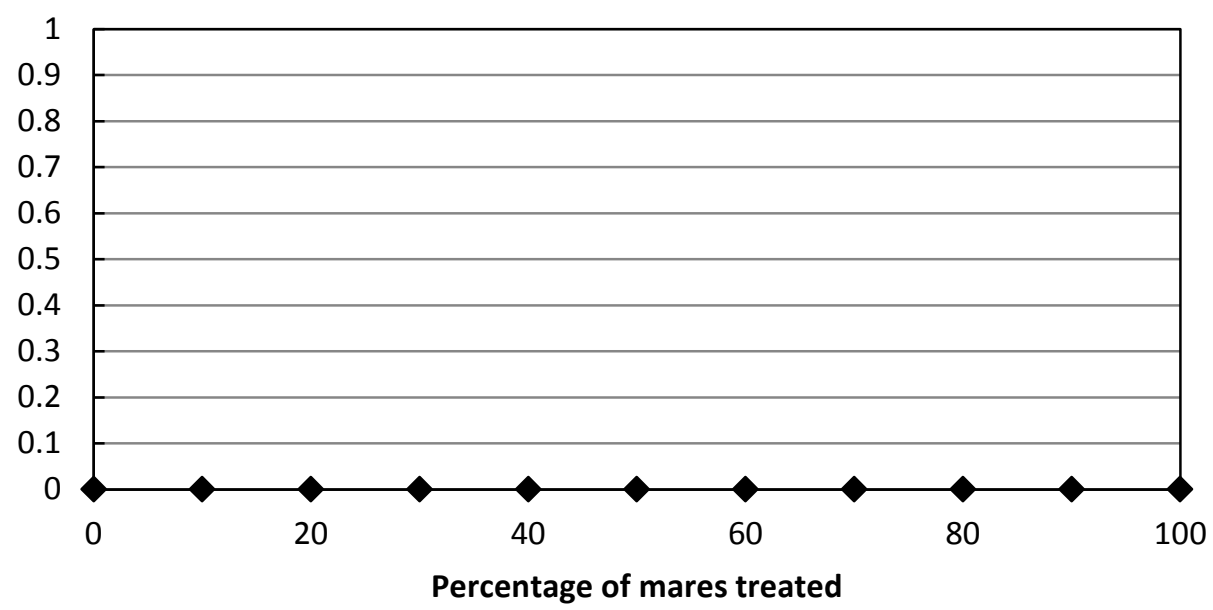

Figure 23. Initial population size $=1000$, lambda $=1.15$, genetic diversity $=$ low .
Mean number of removals after 20 years

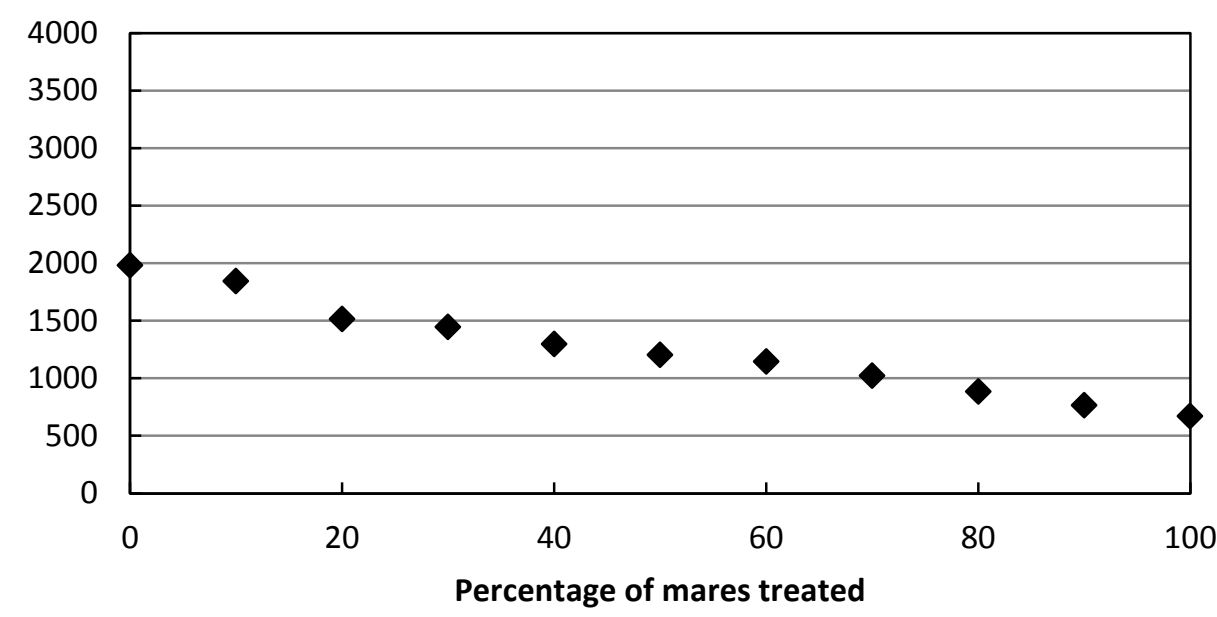

Mean heterozygosity after $\mathbf{5 0}$ years

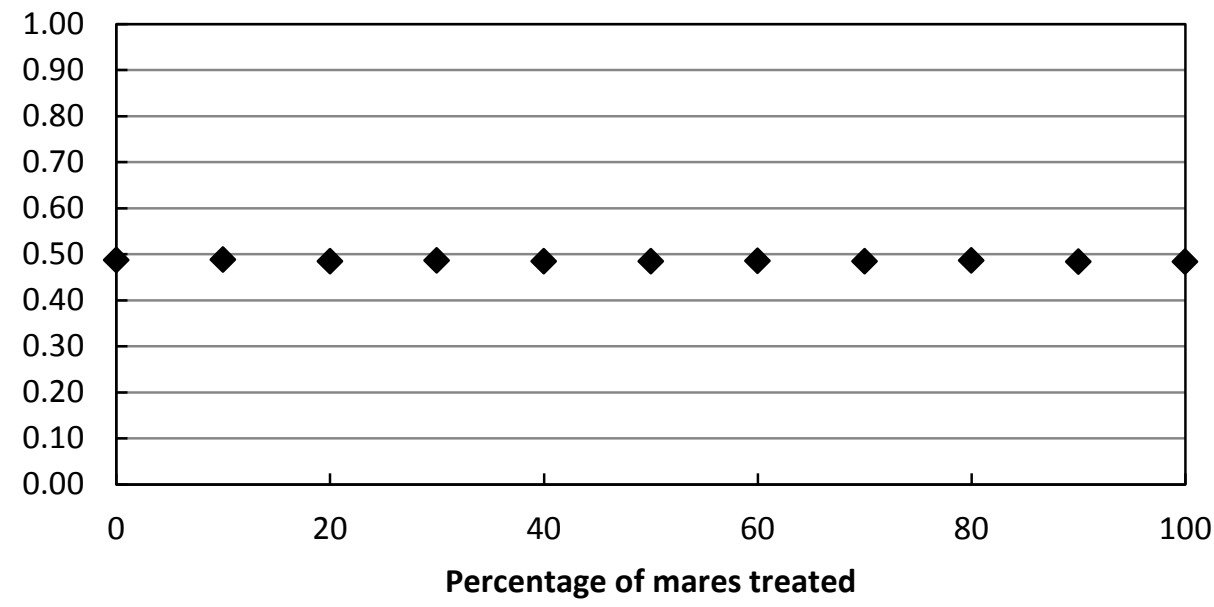




\begin{tabular}{lcccccccccccc}
\hline \multicolumn{1}{c}{ Percentage of mares treated } & $\mathbf{0}$ & $\mathbf{1 0}$ & $\mathbf{2 0}$ & $\mathbf{3 0}$ & $\mathbf{4 0}$ & $\mathbf{5 0}$ & $\mathbf{6 0}$ & $\mathbf{7 0}$ & $\mathbf{8 0}$ & $\mathbf{9 0}$ & $\mathbf{1 0 0}$ \\
\hline Mean population size after 20 years & 1106.82 & 1117.06 & 1065.72 & 1020.7 & 1093.2 & 1008.49 & 970.31 & 948.69 & 895.72 & 845.6 & 752.22 \\
Mean number of removals after 20 years & 1979.8 & 1841.8 & 1512.9 & 1445.2 & 1296.8 & 1201.1 & 1143.0 & 1020.5 & 883.9 & 764.1 & 669.3 \\
Mean probability of extirpation after 50 years & 0 & 0 & 0 & 0 & 0 & 0 & 0 & 0 & 0 & 0 & 0 & 0 \\
Mean heterozygosity after 50 years & 0.49 & 0.49 & 0.48 & 0.49 & 0.48 & 0.48 & 0.49 & 0.48 & 0.49 & 0.48 & 0.48 \\
\hline
\end{tabular}

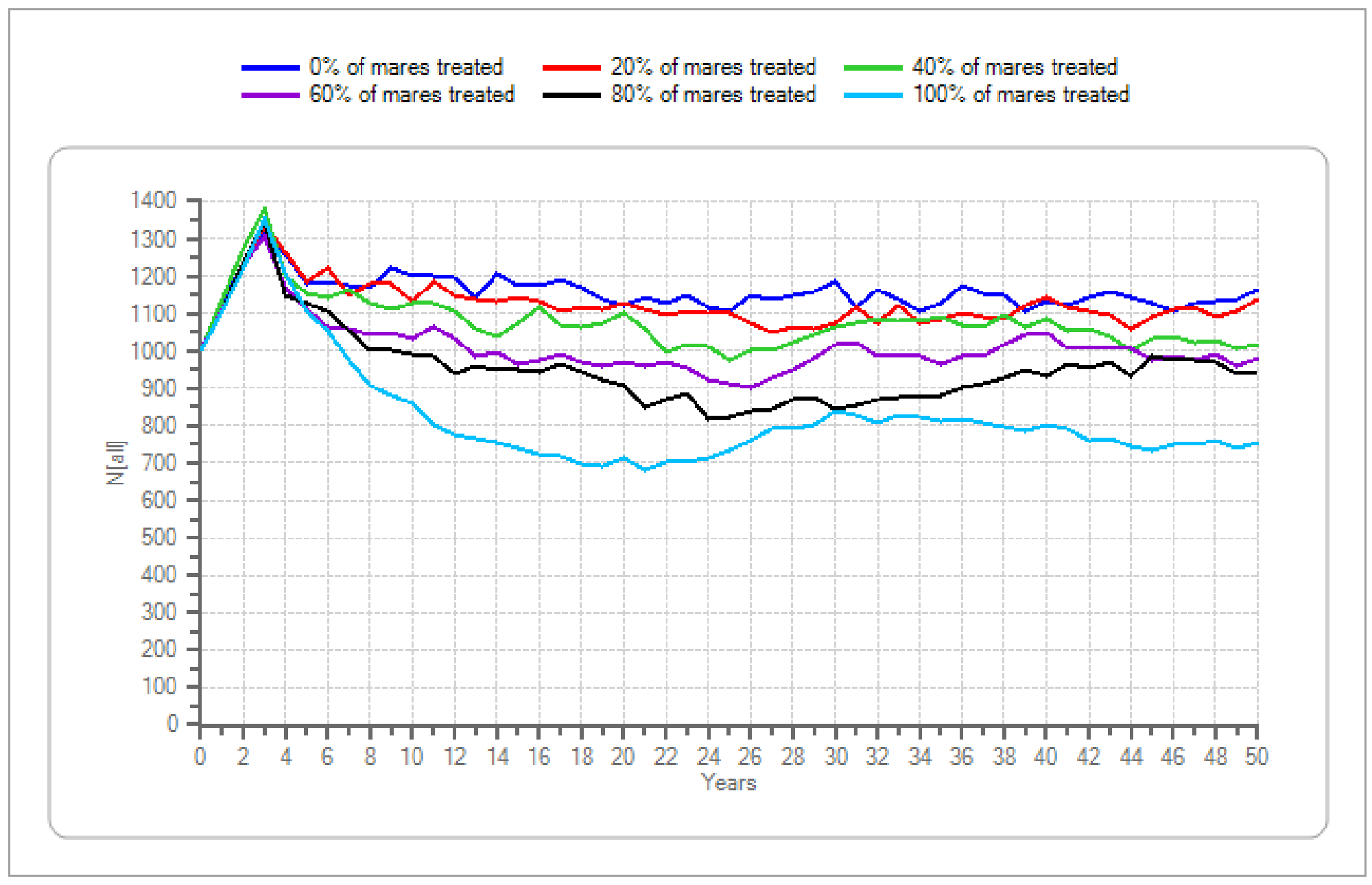

Figure 23. Initial population size $=1000$, lambda $=1.15$, genetic diversity $=$ low - Continued 
Mean population size after 20 years

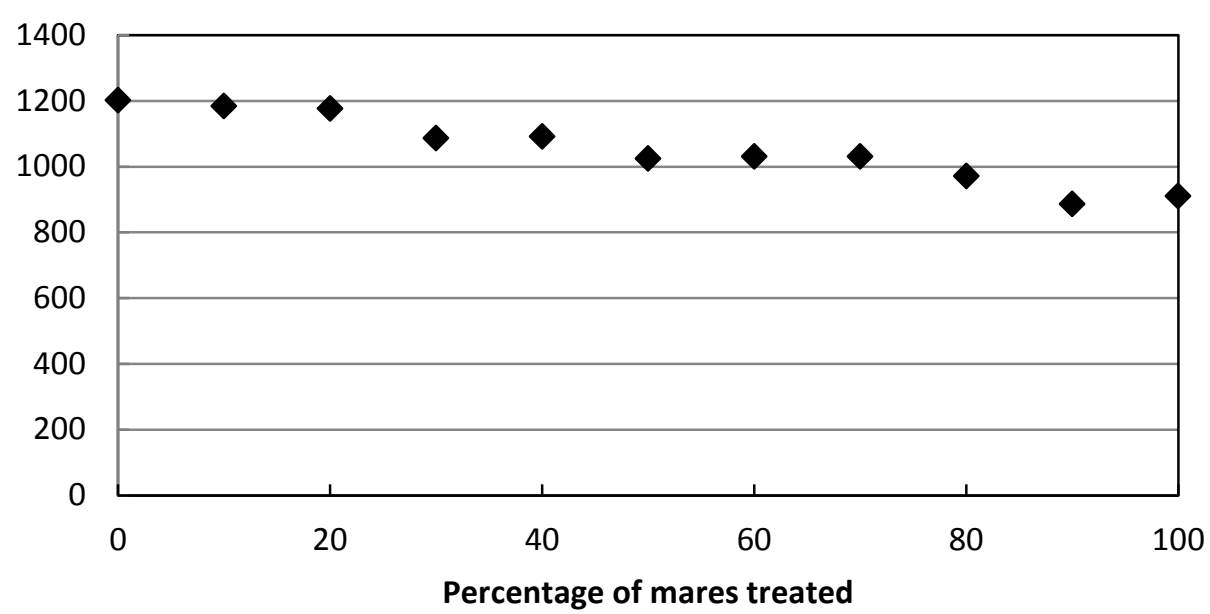

Mean probability of extirpation after 50 years

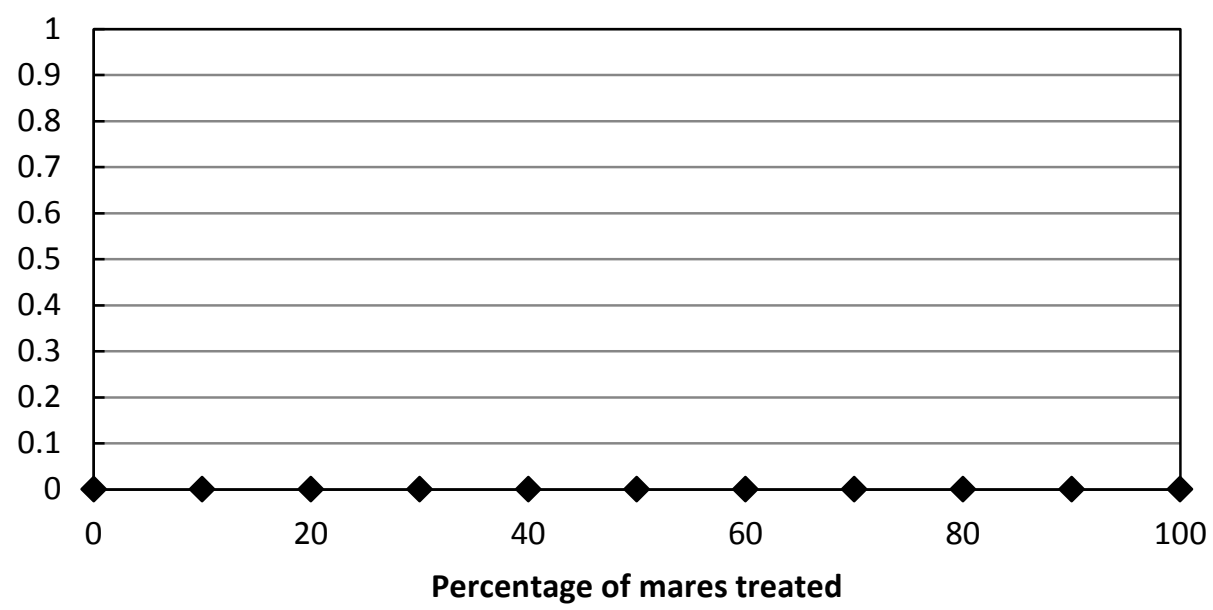

Figure 24. Initial population size $=1000$, lambda $=1.20$, genetic diversity $=$ low .
Mean number of removals after 20 years

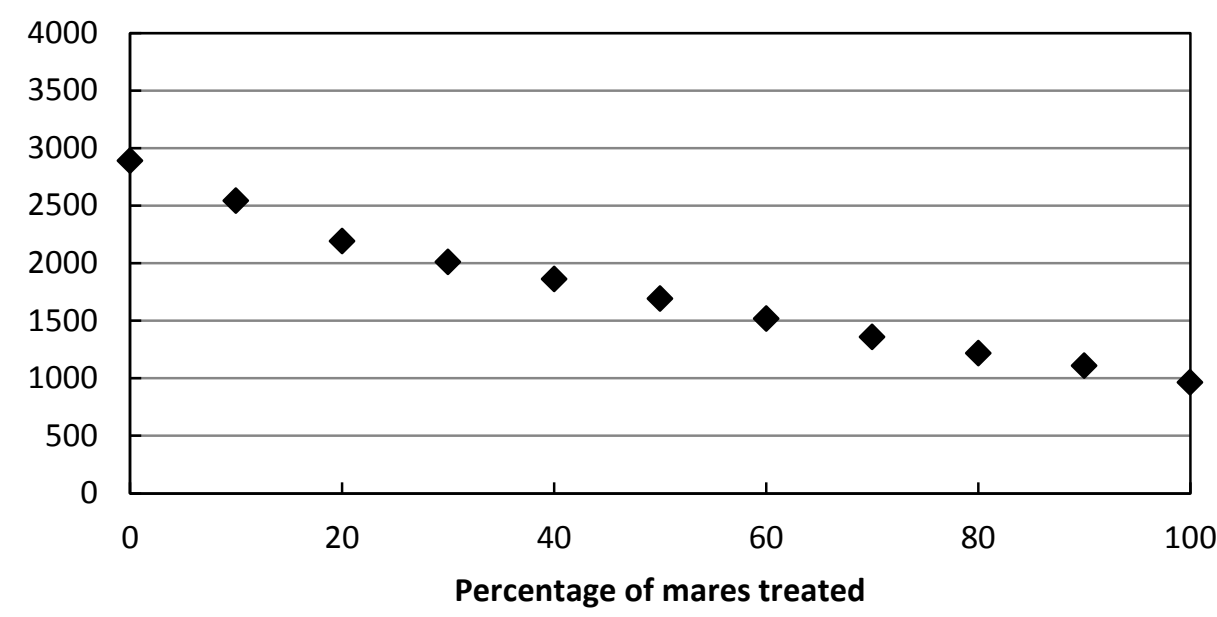

Mean heterozygosity after $\mathbf{5 0}$ years

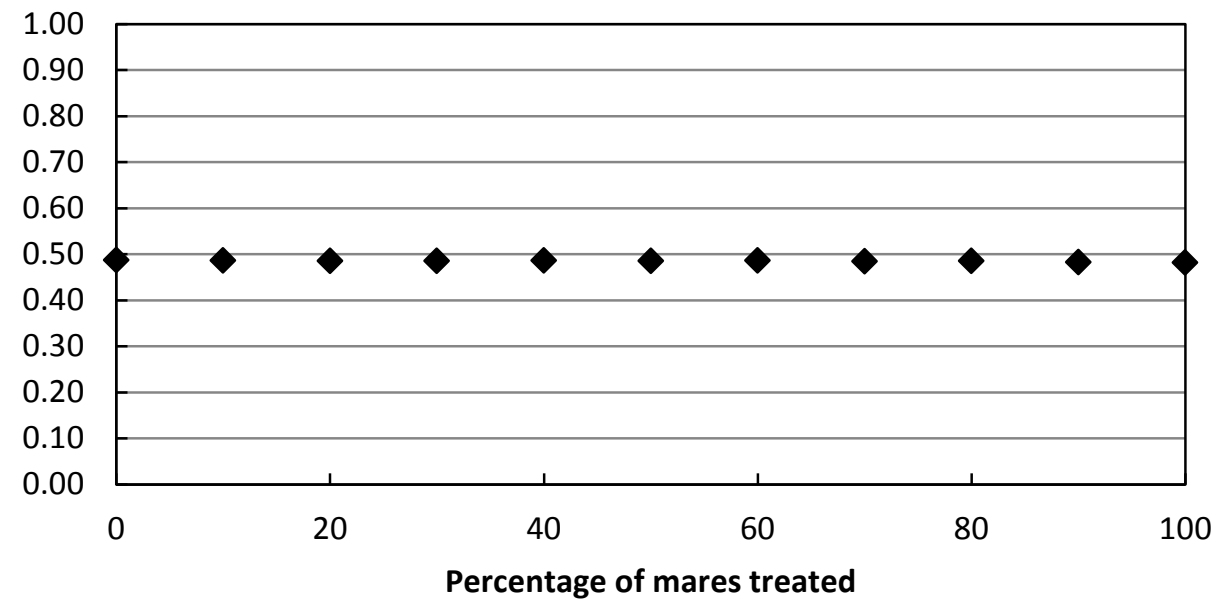




\begin{tabular}{lccccccccccccc}
\hline \multicolumn{1}{c}{ Percentage of mares treated } & $\mathbf{0}$ & $\mathbf{1 0}$ & $\mathbf{2 0}$ & $\mathbf{3 0}$ & $\mathbf{4 0}$ & $\mathbf{5 0}$ & $\mathbf{6 0}$ & $\mathbf{7 0}$ & $\mathbf{8 0}$ & $\mathbf{9 0}$ & $\mathbf{1 0 0}$ \\
\hline Mean population size after 20 years & 1201.08 & 1184.02 & 1176.54 & 1085.77 & 1090.8 & 1024.31 & 1030.98 & 1029.85 & 970.74 & 885.67 & 910.27 \\
Mean number of removals after 20 years & 2890.6 & 2543.6 & 2191.6 & 2009.4 & 1861.7 & 1689.8 & 1515.9 & 1357.8 & 1215.4 & 1107.5 & 961.8 \\
Mean probability of extirpation after 50 years & 0 & 0 & 0 & 0 & 0 & 0 & 0 & 0 & 0 & 0 & 0 \\
Mean heterozygosity after 50 years & 0.49 & 0.49 & 0.49 & 0.49 & 0.49 & 0.49 & 0.49 & 0.48 & 0.48 & 0.48 & 0.48 \\
\hline
\end{tabular}

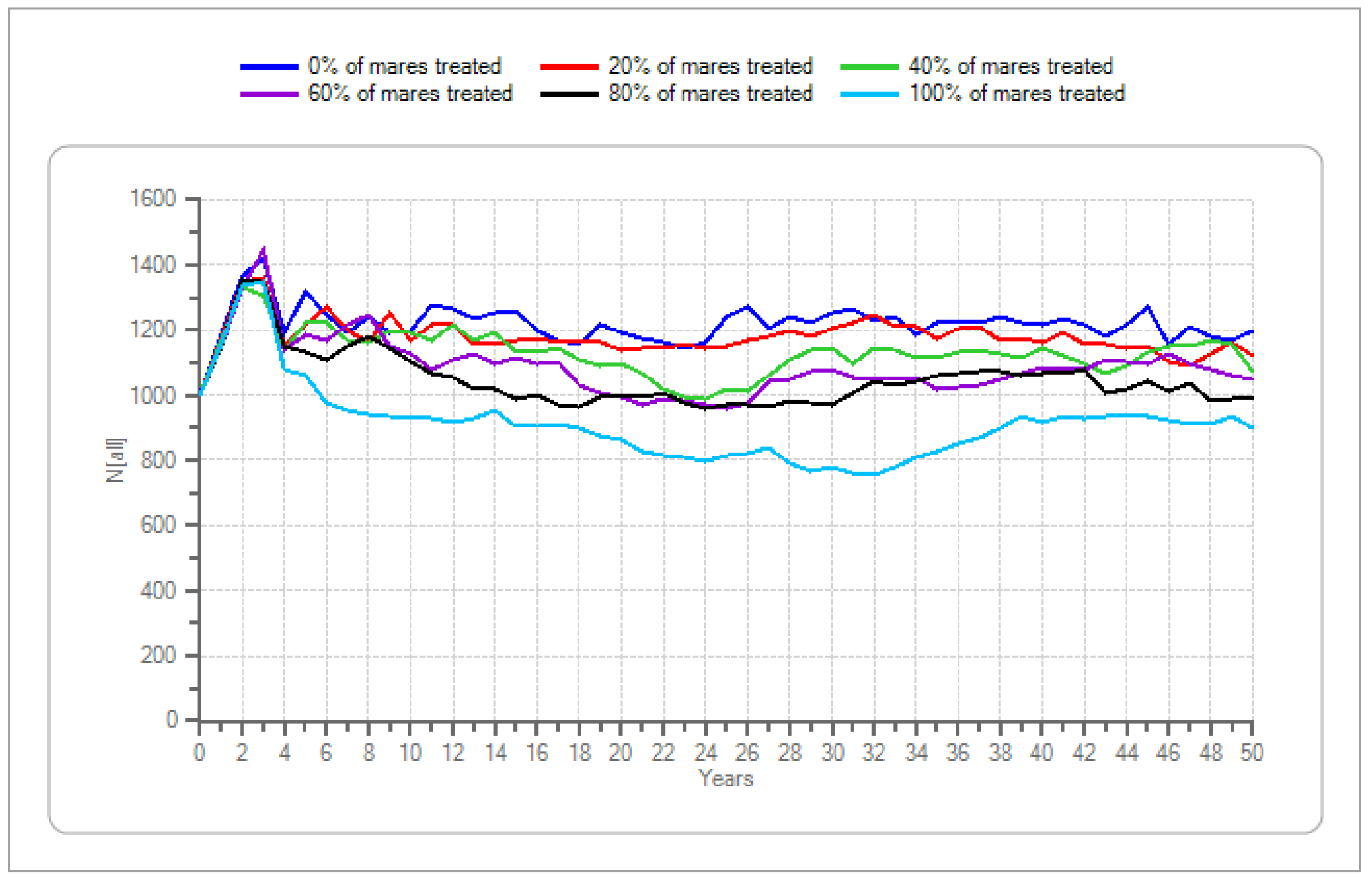

Figure 24. Initial population size $=1000$, lambda $=1.20$, genetic diversity $=$ low - Continued 
Mean population size after 20 years

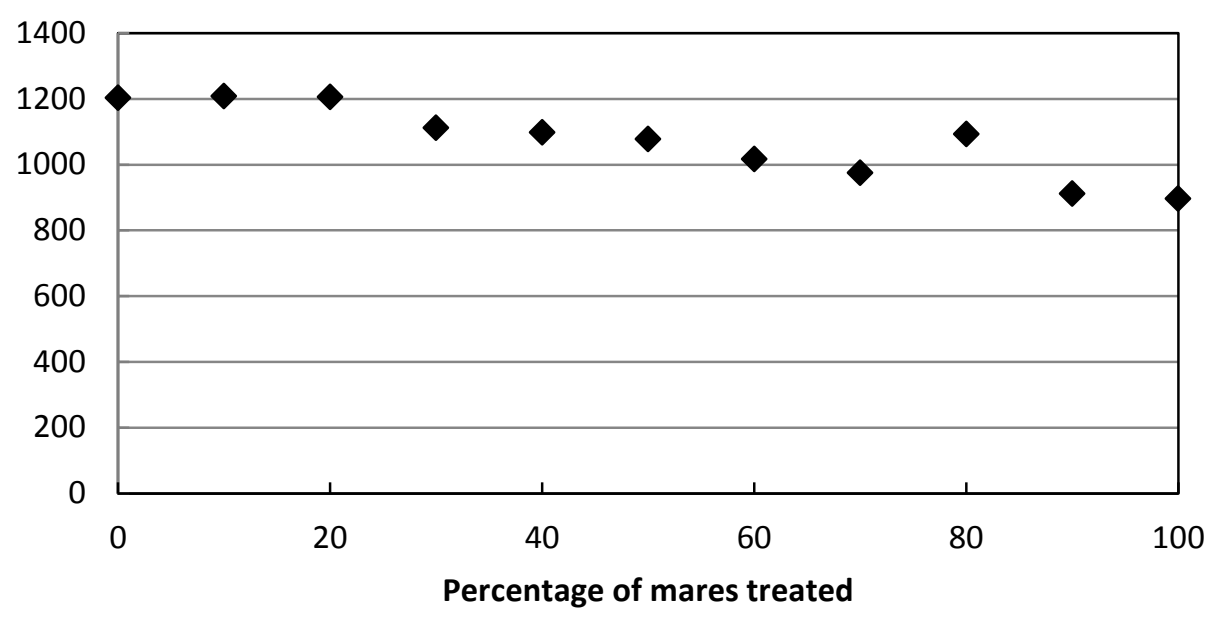

Mean probability of extirpation after 50 years

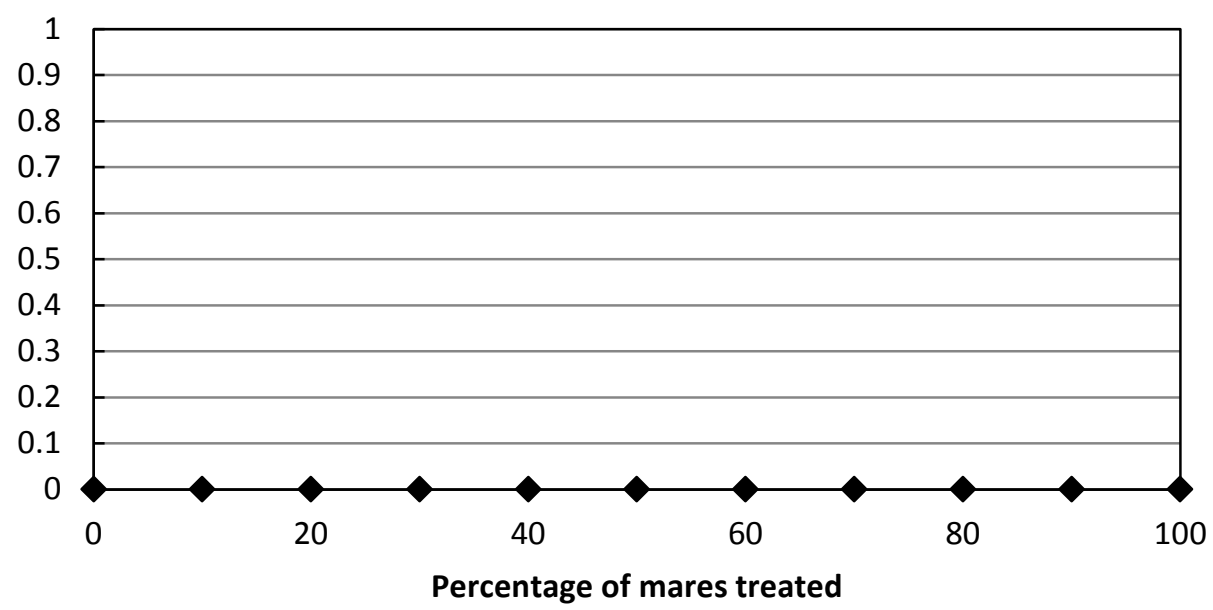

Figure 25. Initial population size $=1000$, lambda $=1.25$, genetic diversity $=$ low .
Mean number of removals after 20 years

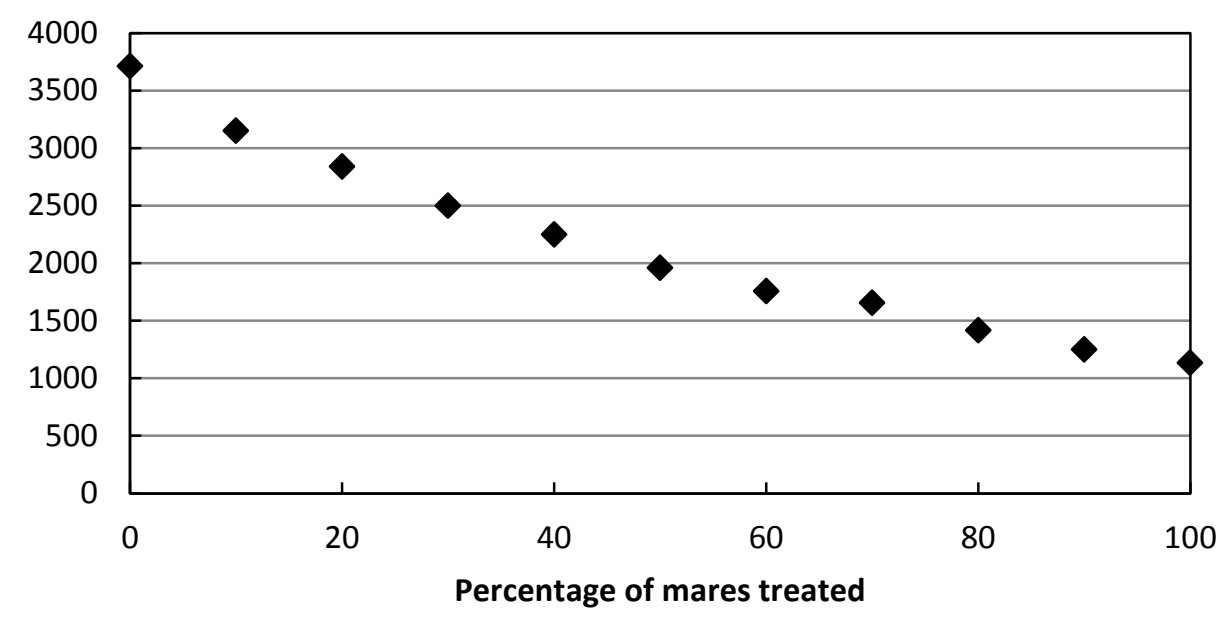

Mean heterozygosity after $\mathbf{5 0}$ years

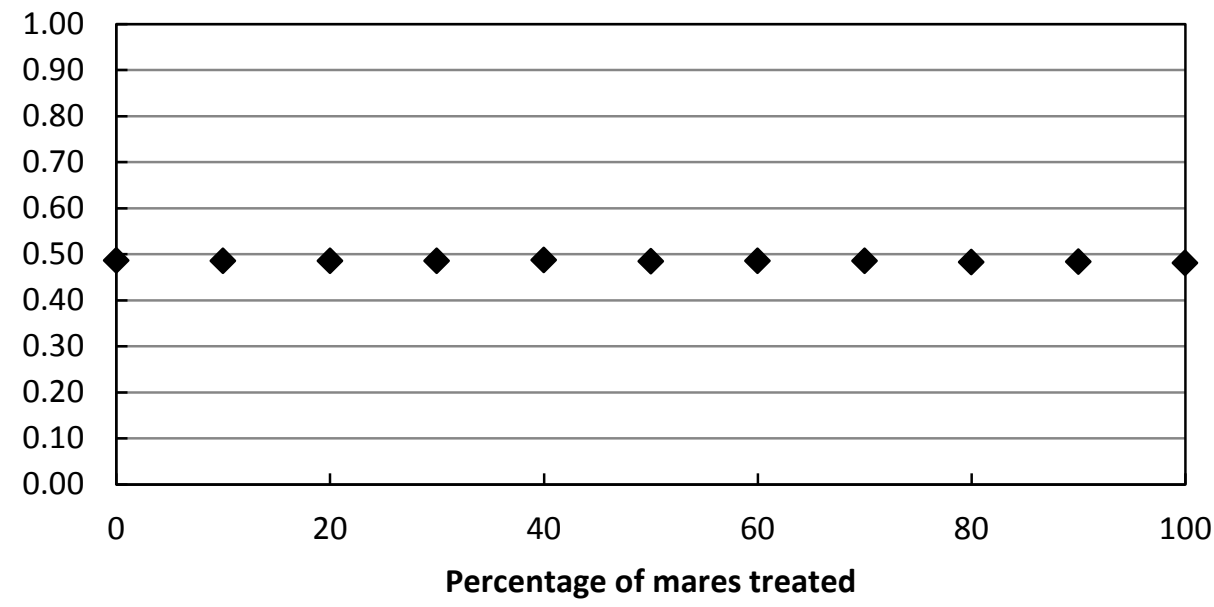




\begin{tabular}{lcccccccccccc}
\hline \multicolumn{1}{c}{ Percentage of mares treated } & $\mathbf{0}$ & $\mathbf{1 0}$ & $\mathbf{2 0}$ & $\mathbf{3 0}$ & $\mathbf{4 0}$ & $\mathbf{5 0}$ & $\mathbf{6 0}$ & $\mathbf{7 0}$ & $\mathbf{8 0}$ & $\mathbf{9 0}$ & $\mathbf{1 0 0}$ \\
\hline Mean population size after 20 years & 1202.9 & 1207.36 & 1205.51 & 1111.97 & 1097.25 & 1076.74 & 1016.51 & 975.02 & 1092.2 & 911.9 & 896.56 \\
Mean number of removals after 20 years & 3713.4 & 3151.0 & 2838.8 & 2498.7 & 2250.0 & 1960.2 & 1756.7 & 1654.6 & 1417.1 & 1247.7 & 1131.8 \\
Mean probability of extirpation after 50 years & 0 & 0 & 0 & 0 & 0 & 0 & 0 & 0 & 0 & 0 & 0 & 0 \\
Mean heterozygosity after 50 years & 0.49 & 0.49 & 0.49 & 0.49 & 0.49 & 0.48 & 0.49 & 0.49 & 0.48 & 0.48 & 0.48 \\
\hline
\end{tabular}

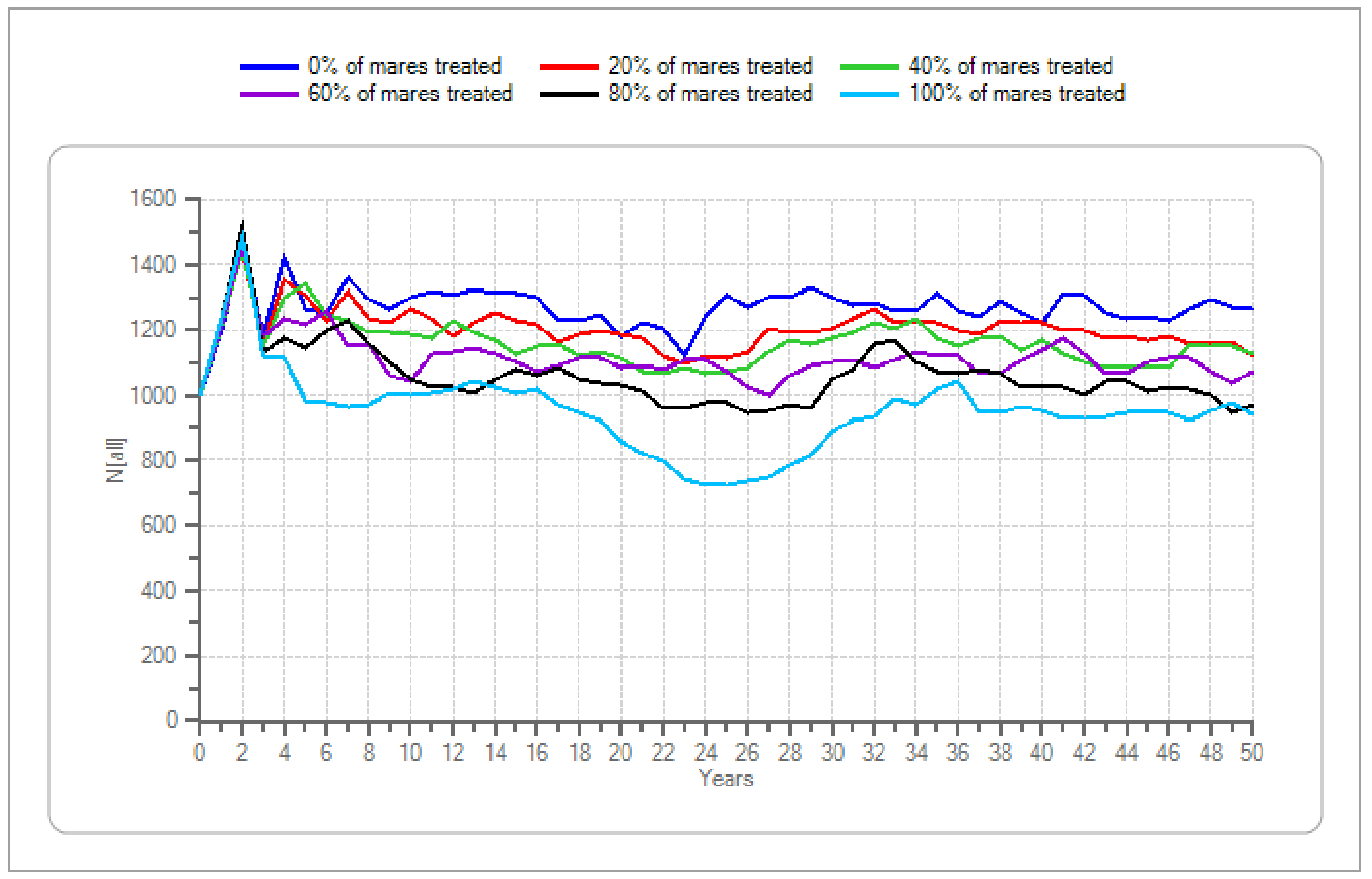

Figure 25. Initial population size $=1000$, lambda $=1.25$, genetic diversity $=$ low - Continued 
Mean population size after 20 years

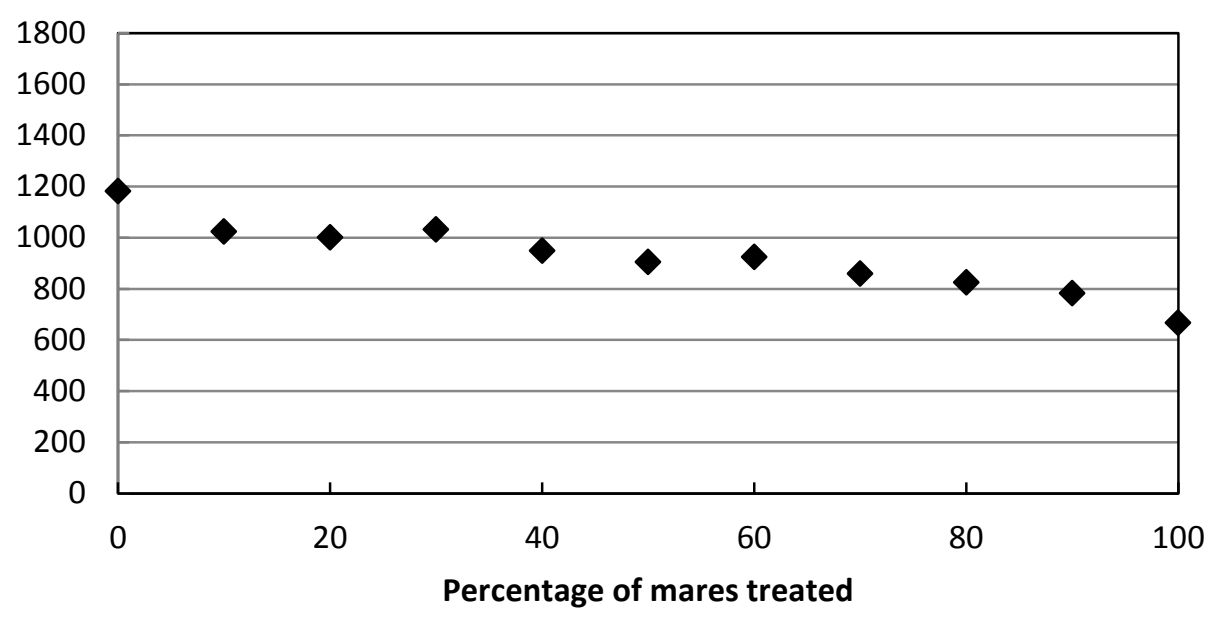

Mean probability of extirpation after $\mathbf{5 0}$ years

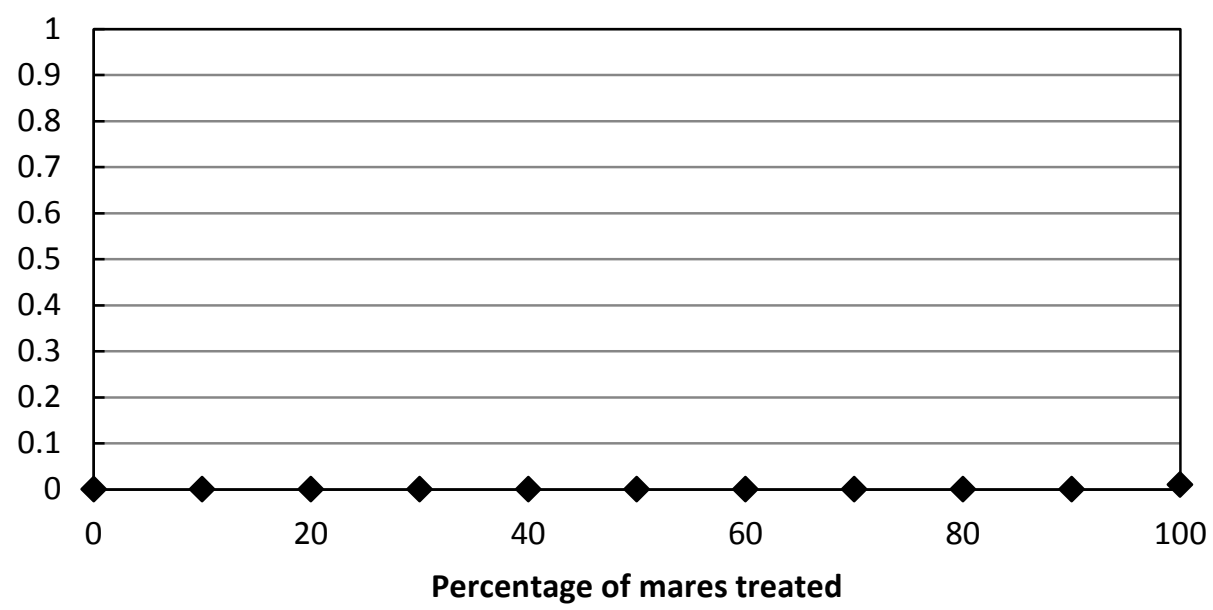

Figure 26. Initial population size $=1250$, lambda $=1.05$, genetic diversity $=$ low .
Mean number of removals after 20 years

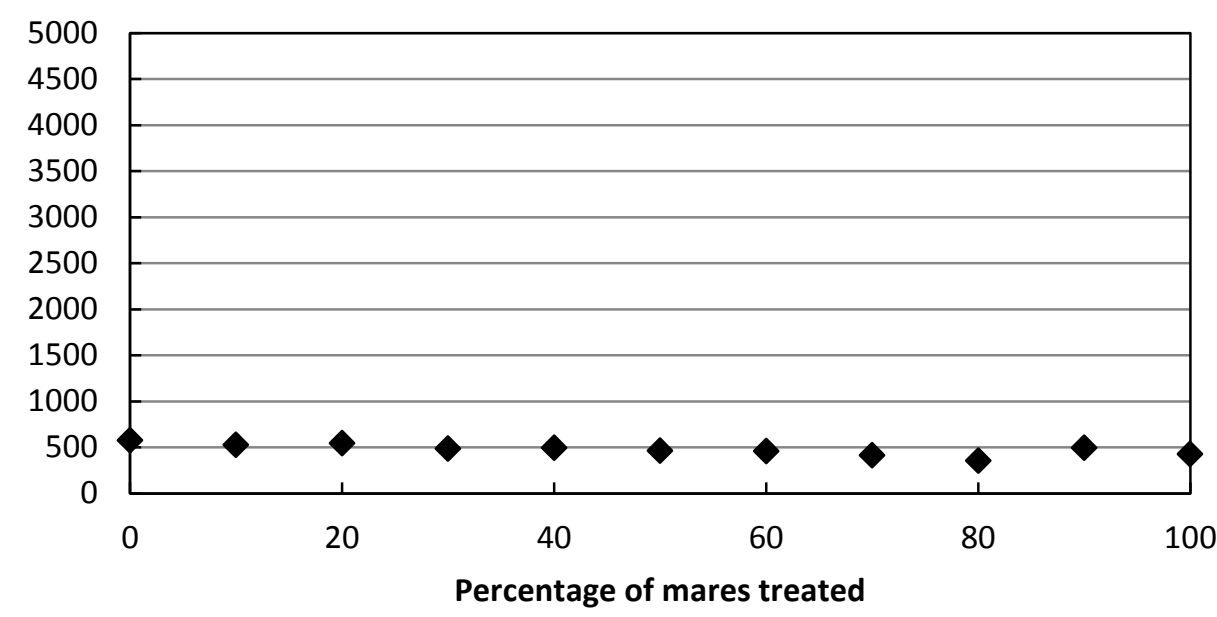

Mean heterozygosity after $\mathbf{5 0}$ years

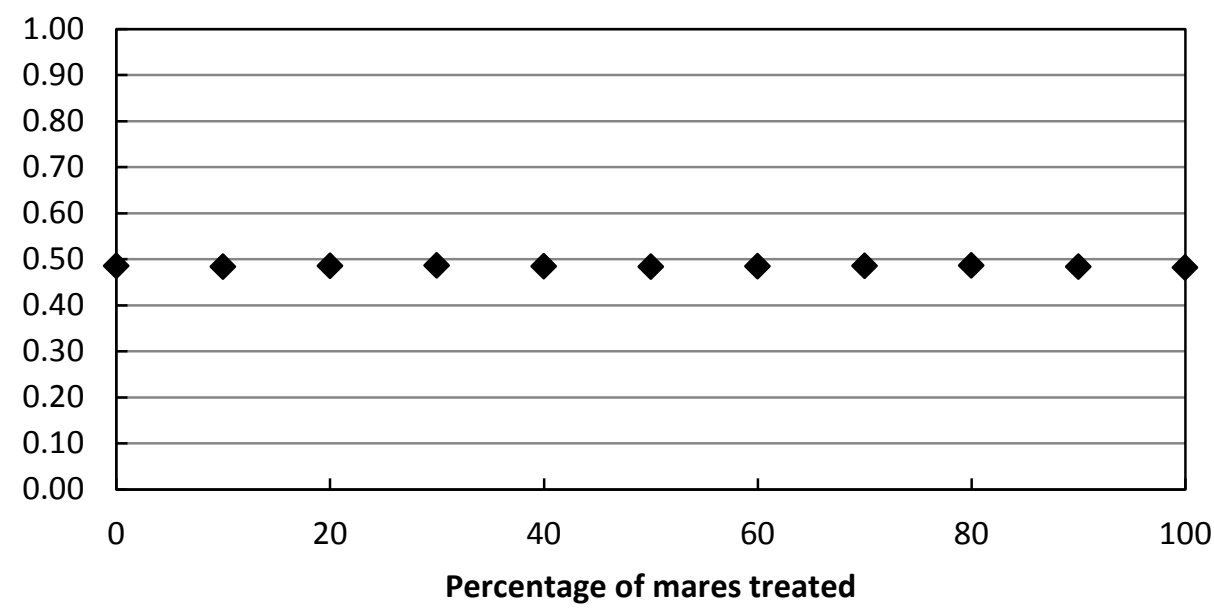




\begin{tabular}{lcccccccccccc}
\hline \multicolumn{1}{c}{ Percentage of mares treated } & $\mathbf{0}$ & $\mathbf{1 0}$ & $\mathbf{2 0}$ & $\mathbf{3 0}$ & $\mathbf{4 0}$ & $\mathbf{5 0}$ & $\mathbf{6 0}$ & $\mathbf{7 0}$ & $\mathbf{8 0}$ & $\mathbf{9 0}$ & $\mathbf{1 0 0}$ \\
\hline Mean population size after 20 years & 1181.85 & 1024.21 & 1000.58 & 1032.15 & 948.63 & 904.73 & 923.82 & 859.2 & 824.58 & 782.46 & 666.31 \\
Mean number of removals after 20 years & 574.25 & 523.43 & 544.18 & 483.72 & 493.96 & 463.54 & 459.15 & 412.01 & 352.83 & 491.73 & 425.11 \\
Mean probability of extirpation after 50 years & 0 & 0 & 0 & 0 & 0 & 0 & 0 & 0 & 0 & 0 & 0.01 \\
Mean heterozygosity after 50 years & 0.49 & 0.48 & 0.49 & 0.49 & 0.48 & 0.48 & 0.48 & 0.49 & 0.49 & 0.48 & 0.48 \\
\hline
\end{tabular}

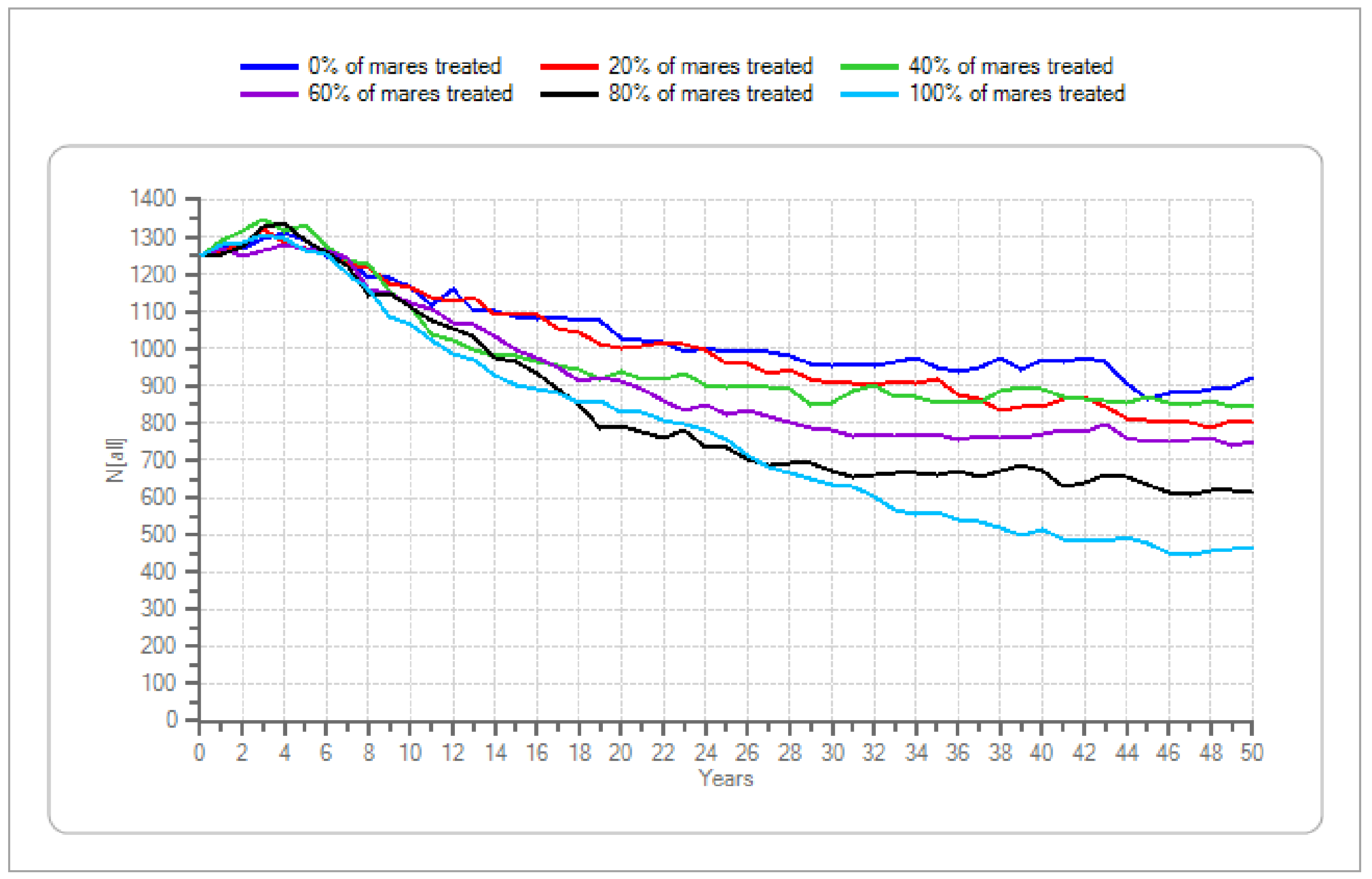

Figure 26. Initial population size $=1250$, lambda $=1.05$, genetic diversity $=$ low - Continued 
Mean population size after 20 years

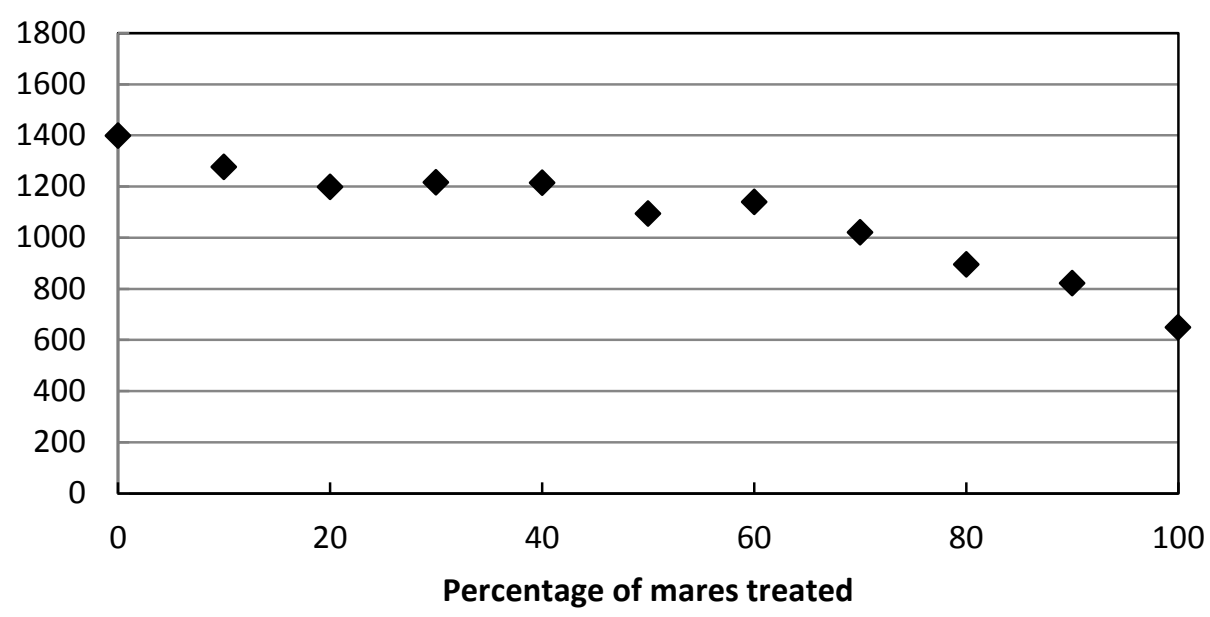

Mean probability of extirpation after $\mathbf{5 0}$ years

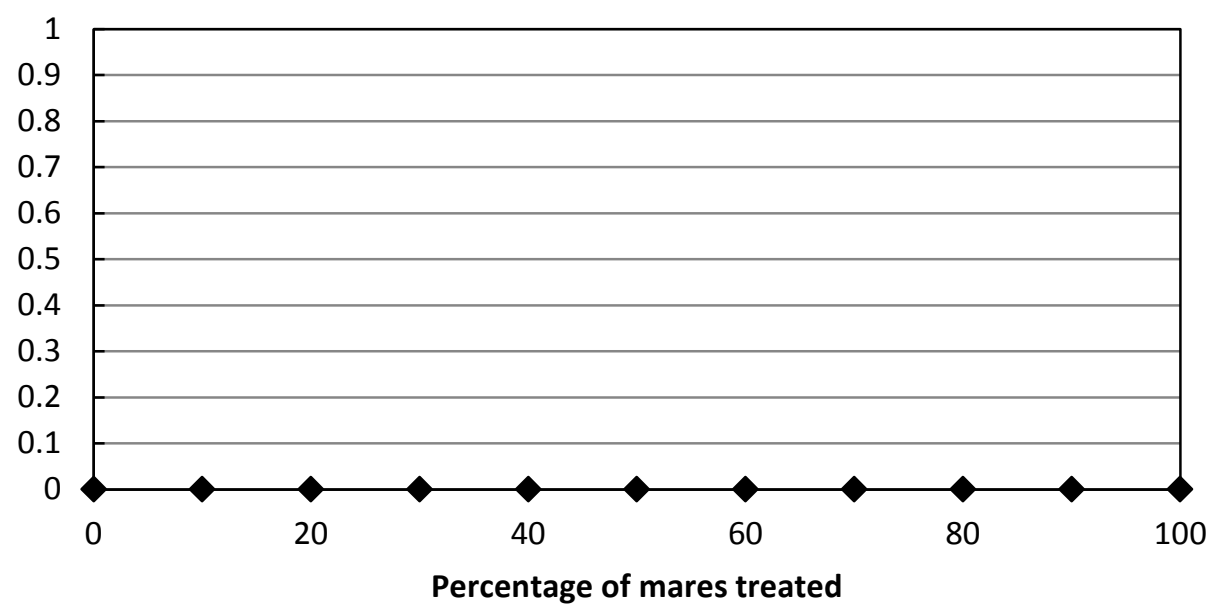

Figure 27. Initial population size $=1250$, lambda $=1.10$, genetic diversity $=$ low .
Mean number of removals after 20 years

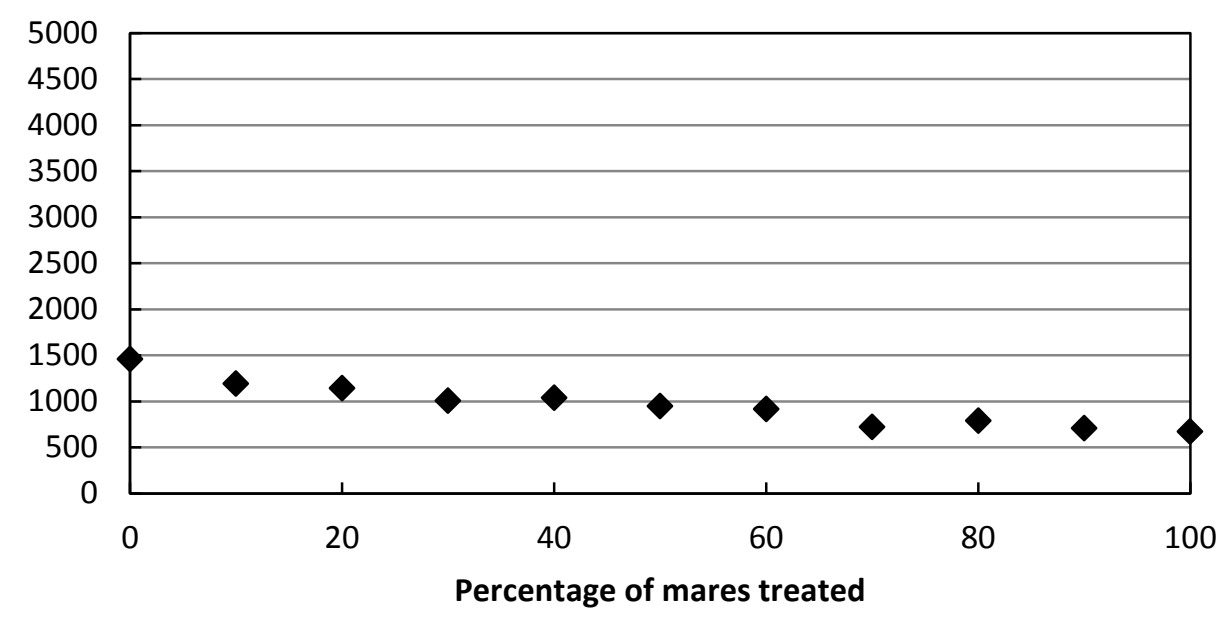

Mean heterozygosity after $\mathbf{5 0}$ years

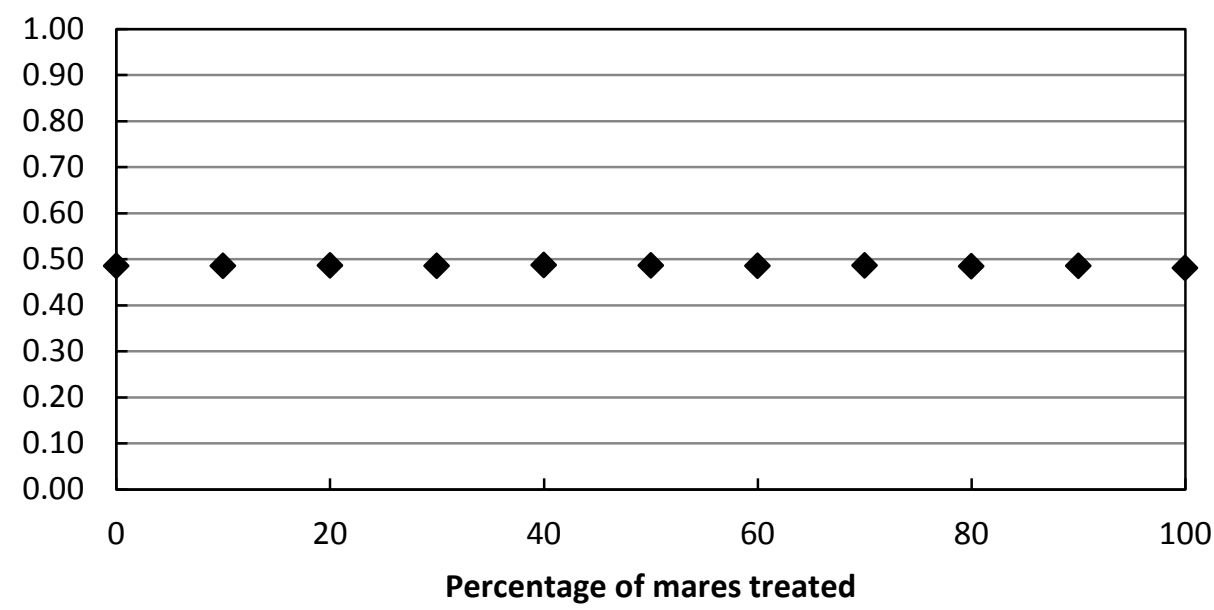




\begin{tabular}{lcccccccccccc}
\hline \multicolumn{1}{c}{ Percentage of mares treated } & $\mathbf{0}$ & $\mathbf{1 0}$ & $\mathbf{2 0}$ & $\mathbf{3 0}$ & $\mathbf{4 0}$ & $\mathbf{5 0}$ & $\mathbf{6 0}$ & $\mathbf{7 0}$ & $\mathbf{8 0}$ & $\mathbf{9 0}$ & $\mathbf{1 0 0}$ \\
\hline Mean population size after 20 years & 1398.94 & 1275.26 & 1198.43 & 1215.27 & 1214.22 & 1094.1 & 1138.43 & 1019.54 & 893.99 & 821.65 & 649.09 \\
Mean number of removals after 20 years & 1458.6 & 1191.7 & 1140.2 & 1004.3 & 1038.6 & 948.0 & 915.8 & 720.1 & 788.2 & 708.3 & 672.1 \\
Mean probability of extirpation after 50 years & 0 & 0 & 0 & 0 & 0 & 0 & 0 & 0 & 0 & 0 & 0 \\
Mean heterozygosity after 50 years & 0.49 & 0.48 & 0.49 & 0.49 & 0.49 & 0.49 & 0.49 & 0.49 & 0.48 & 0.49 & 0.48 \\
\hline
\end{tabular}

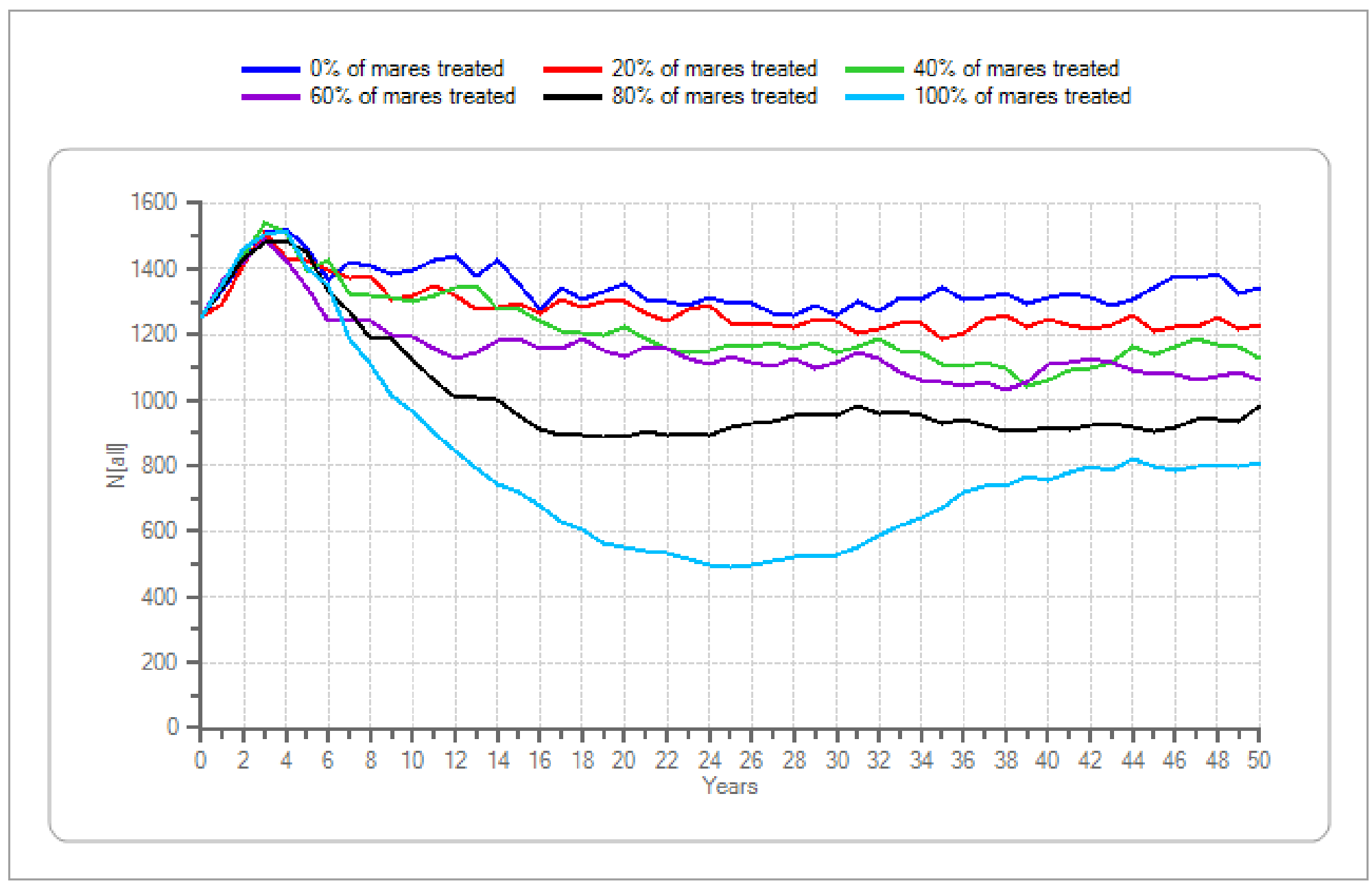

Figure 27. Initial population size $=1250$, lambda $=1.10$, genetic diversity $=$ low - Continued 
Mean population size after 20 years

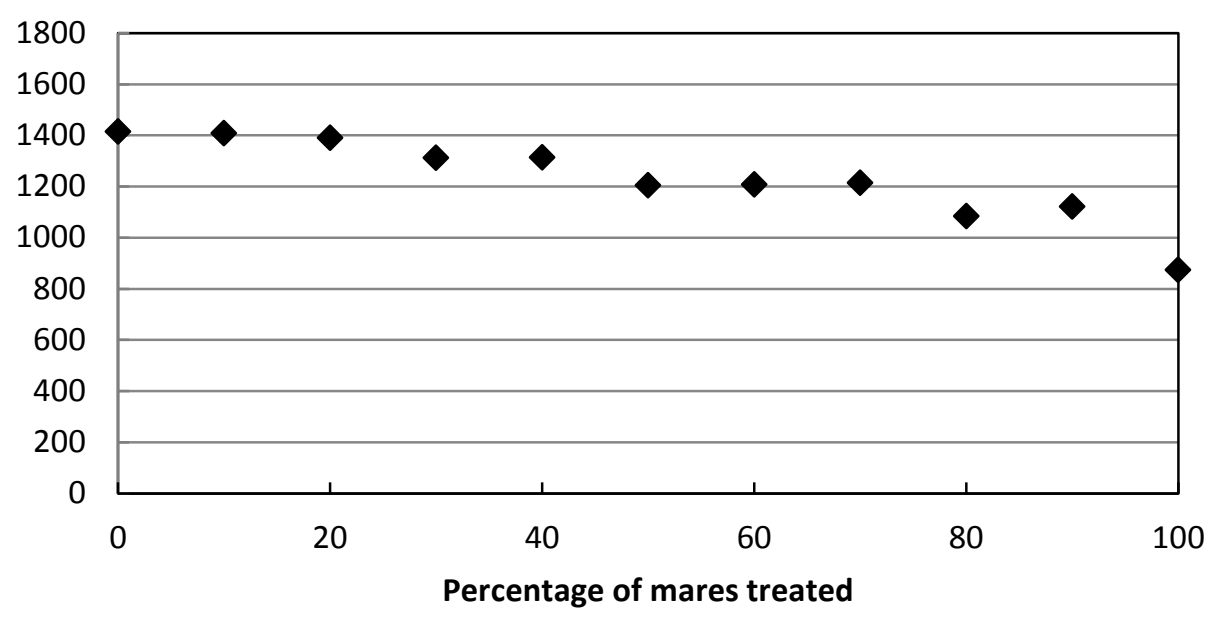

Mean probability of extirpation after $\mathbf{5 0}$ years

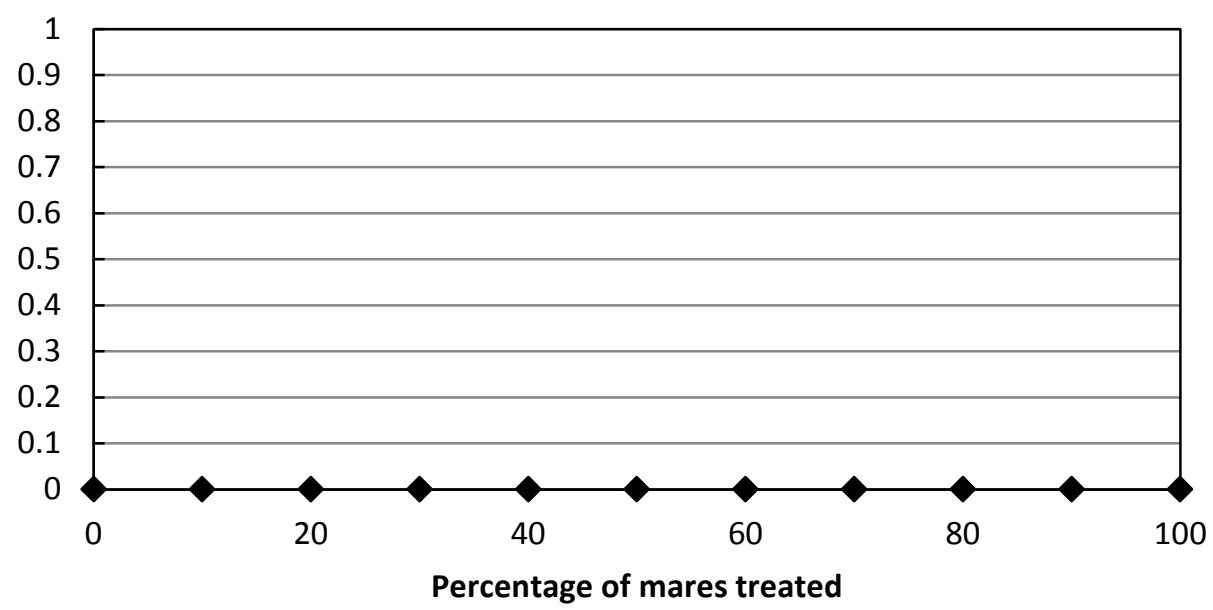

Figure 28. Initial population size $=1250$, lambda $=1.15$, genetic diversity $=$ low .
Mean number of removals after 20 years

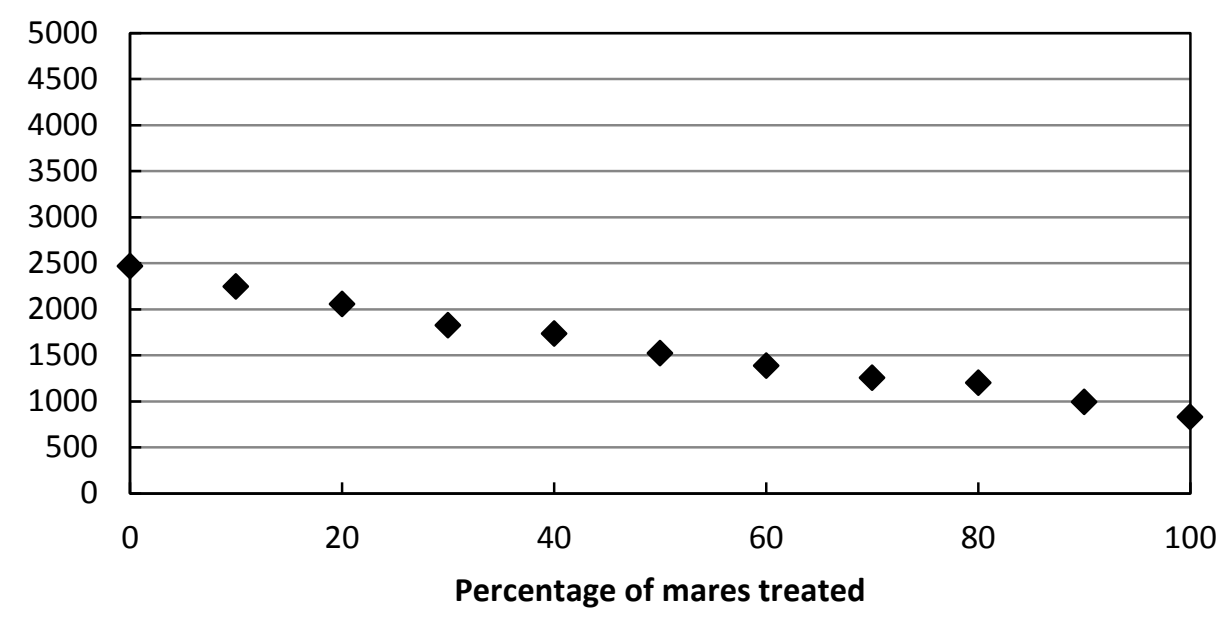

Mean heterozygosity after $\mathbf{5 0}$ years

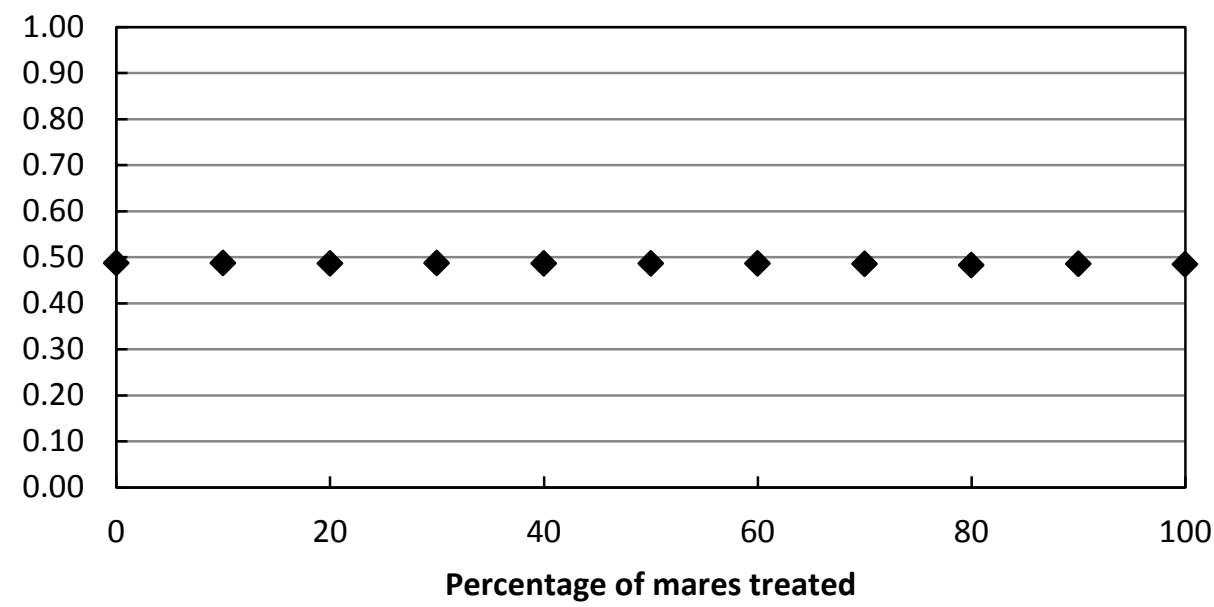




\begin{tabular}{lccccccccccccc}
\hline \multicolumn{1}{c}{ Percentage of mares treated } & $\mathbf{0}$ & $\mathbf{1 0}$ & $\mathbf{2 0}$ & $\mathbf{3 0}$ & $\mathbf{4 0}$ & $\mathbf{5 0}$ & $\mathbf{6 0}$ & $\mathbf{7 0}$ & $\mathbf{8 0}$ & $\mathbf{9 0}$ & $\mathbf{1 0 0}$ \\
\hline Mean population size after 20 years & 1413.8 & 1407.5 & 1390.5 & 1311.6 & 1313.5 & 1204.9 & 1207.4 & 1213.3 & 1084.0 & 1120.9 & 874.2 \\
Mean number of removals after 20 years & 2465.8 & 2243.6 & 2057.3 & 1822.6 & 1733.3 & 1522.9 & 1385.8 & 1252.5 & 1198.7 & 990.3 & 829.2 \\
Mean probability of extirpation after 50 years & 0 & 0 & 0 & 0 & 0 & 0 & 0 & 0 & 0 & 0 & 0 \\
Mean heterozygosity after 50 years & 0.49 & 0.49 & 0.49 & 0.49 & 0.49 & 0.49 & 0.49 & 0.49 & 0.48 & 0.49 & 0.48 \\
\hline
\end{tabular}

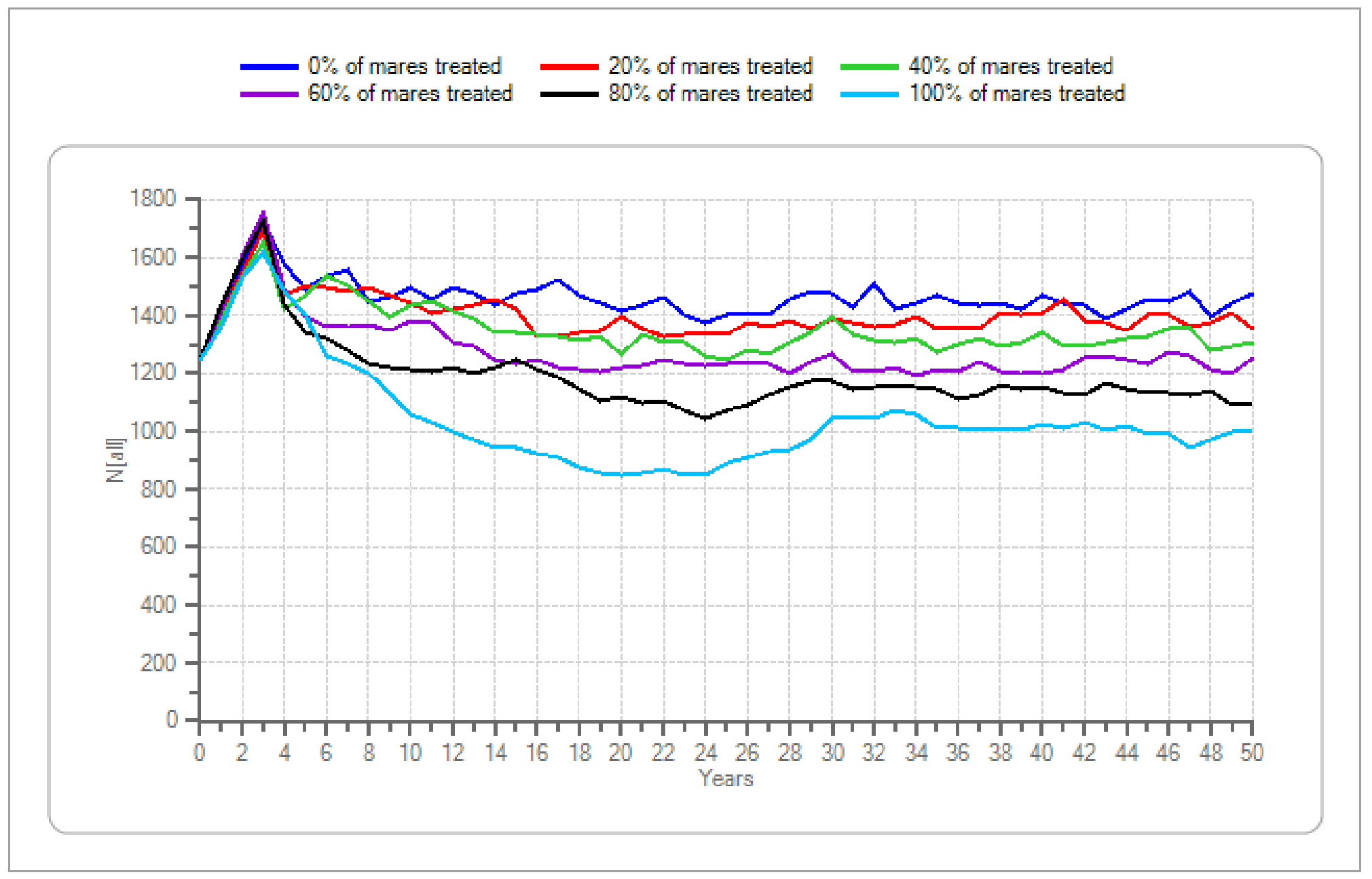

Figure 28. Initial population size $=1250$, lambda $=1.15$, genetic diversity $=$ low - Continued 
Mean population size after 20 years

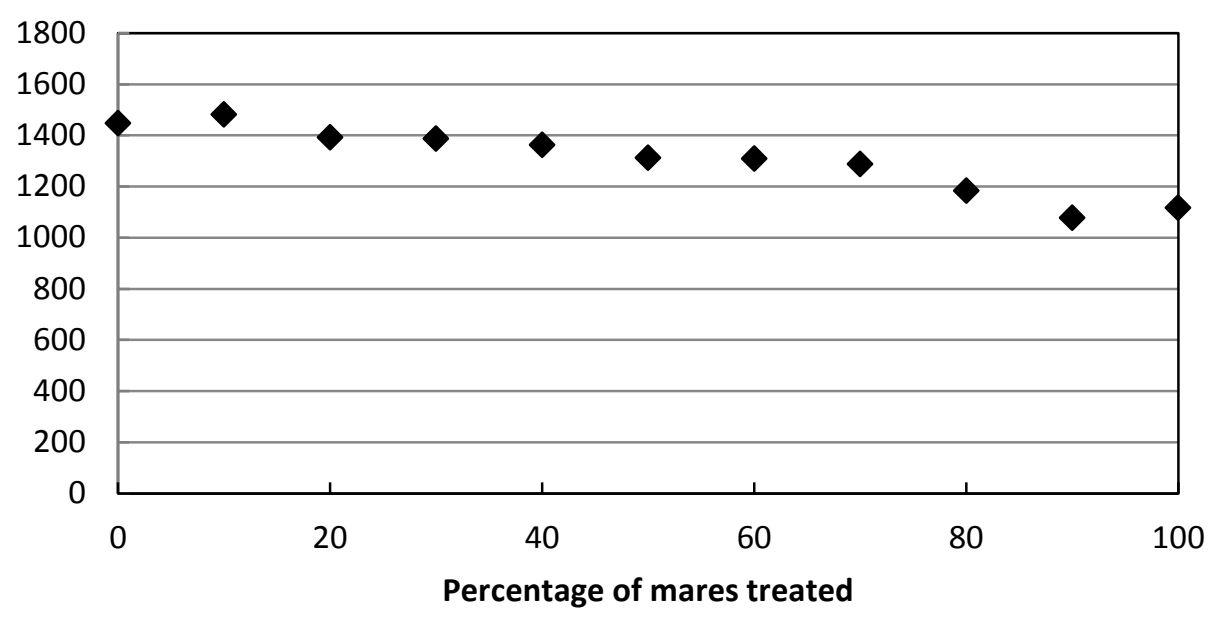

Mean probability of extirpation after $\mathbf{5 0}$ years

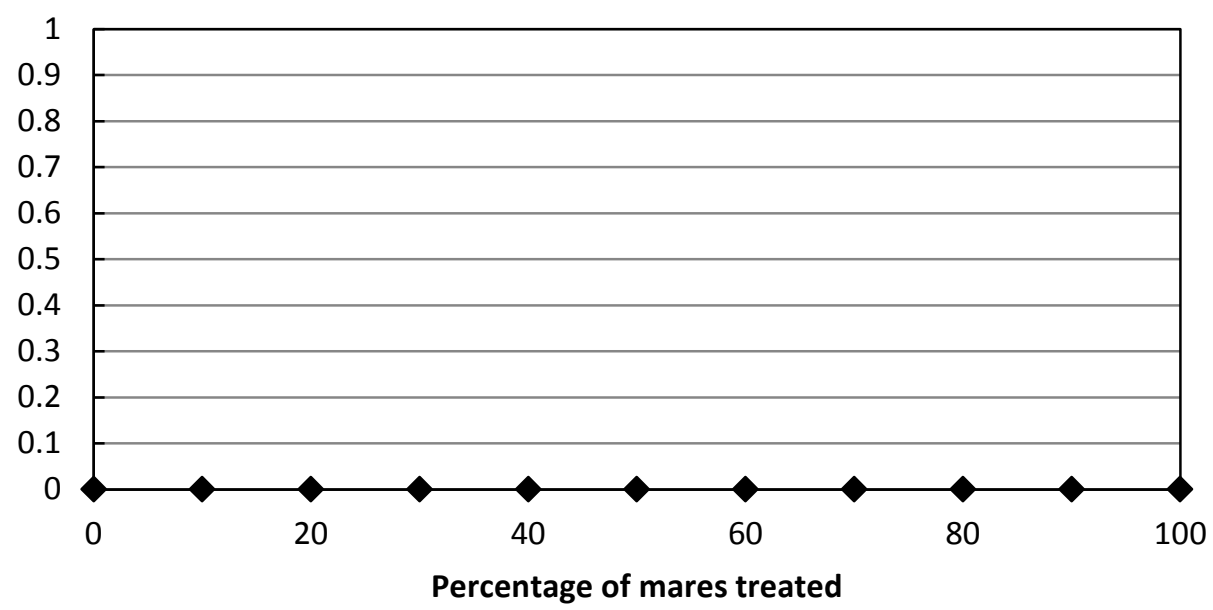

Figure 29. Initial population size $=1250$, lambda $=1.20$, genetic diversity $=$ low .
Mean number of removals after 20 years

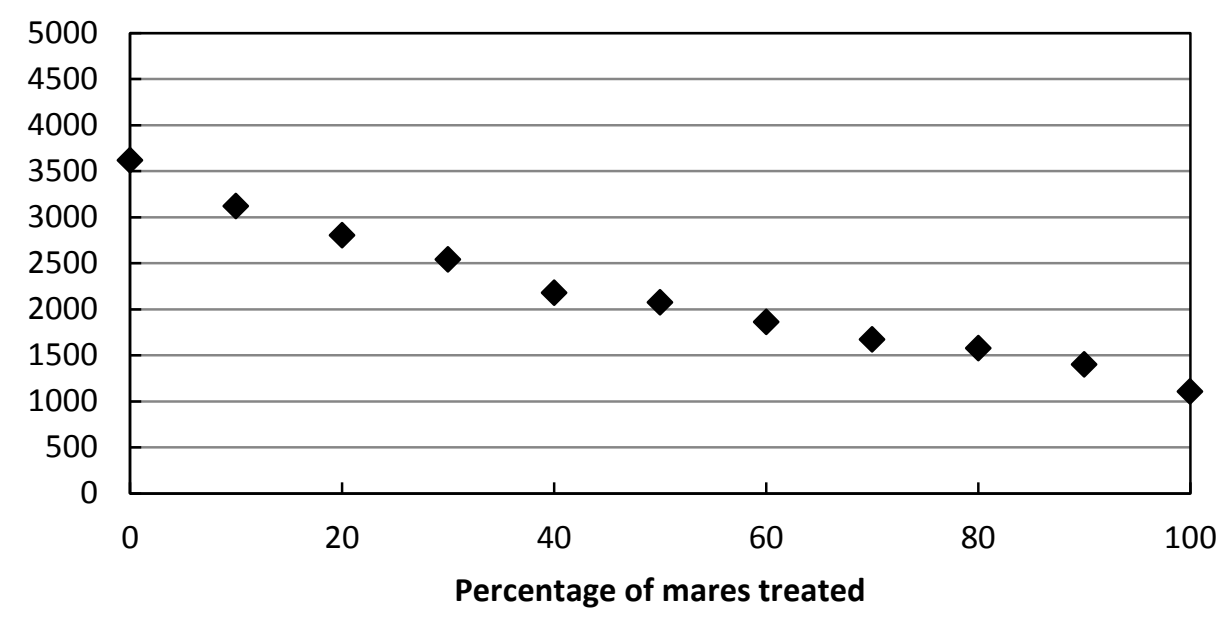

Mean heterozygosity after $\mathbf{5 0}$ years

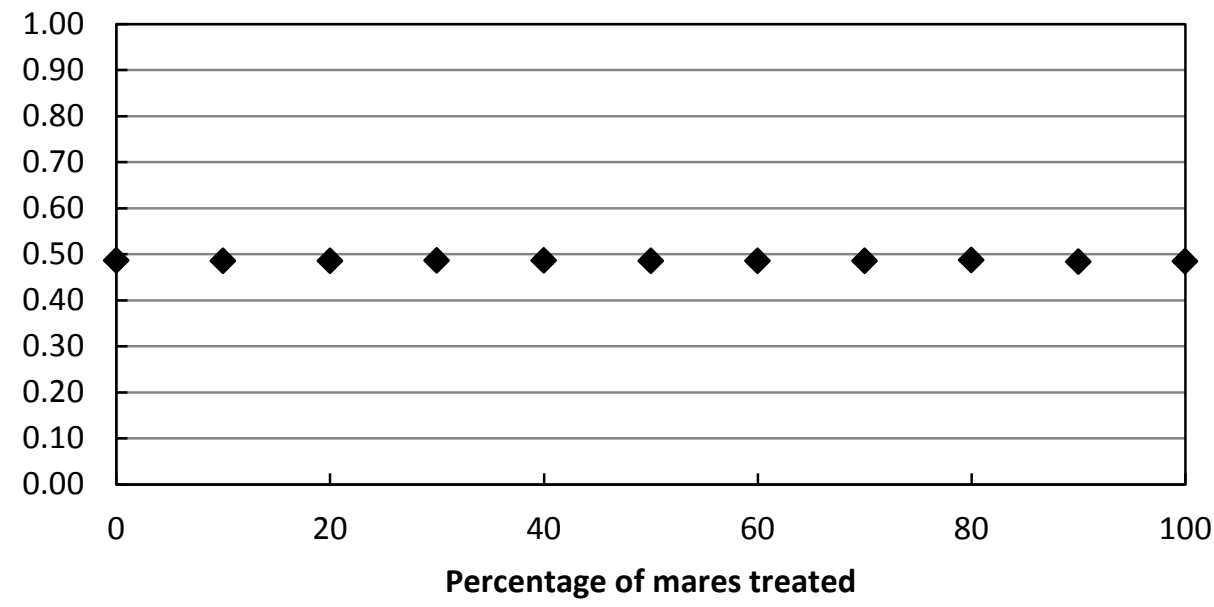




\begin{tabular}{lccccccccccccc}
\hline \multicolumn{1}{c}{ Percentage of mares treated } & $\mathbf{0}$ & $\mathbf{1 0}$ & $\mathbf{2 0}$ & $\mathbf{3 0}$ & $\mathbf{4 0}$ & $\mathbf{5 0}$ & $\mathbf{6 0}$ & $\mathbf{7 0}$ & $\mathbf{8 0}$ & $\mathbf{9 0}$ & $\mathbf{1 0 0}$ \\
\hline Mean population size after 20 years & 1447.5 & 1481.5 & 1392.3 & 1386.1 & 1362.7 & 1312.3 & 1309.2 & 1287.2 & 1182.8 & 1077.5 & 1116.7 \\
Mean number of removals after 20 years & 3615.2 & 3121.2 & 2802.8 & 2538.6 & 2179.6 & 2071.5 & 1859.4 & 1671.5 & 1576.1 & 1398.1 & 1103.3 \\
Mean probability of extirpation after 50 years & 0 & 0 & 0 & 0 & 0 & 0 & 0 & 0 & 0 & 0 & 0 & 0 & 0 \\
Mean heterozygosity after 50 years & 0.49 & 0.49 & 0.49 & 0.49 & 0.49 & 0.49 & 0.49 & 0.48 & 0.49 & 0.48 & 0.48 \\
\hline
\end{tabular}

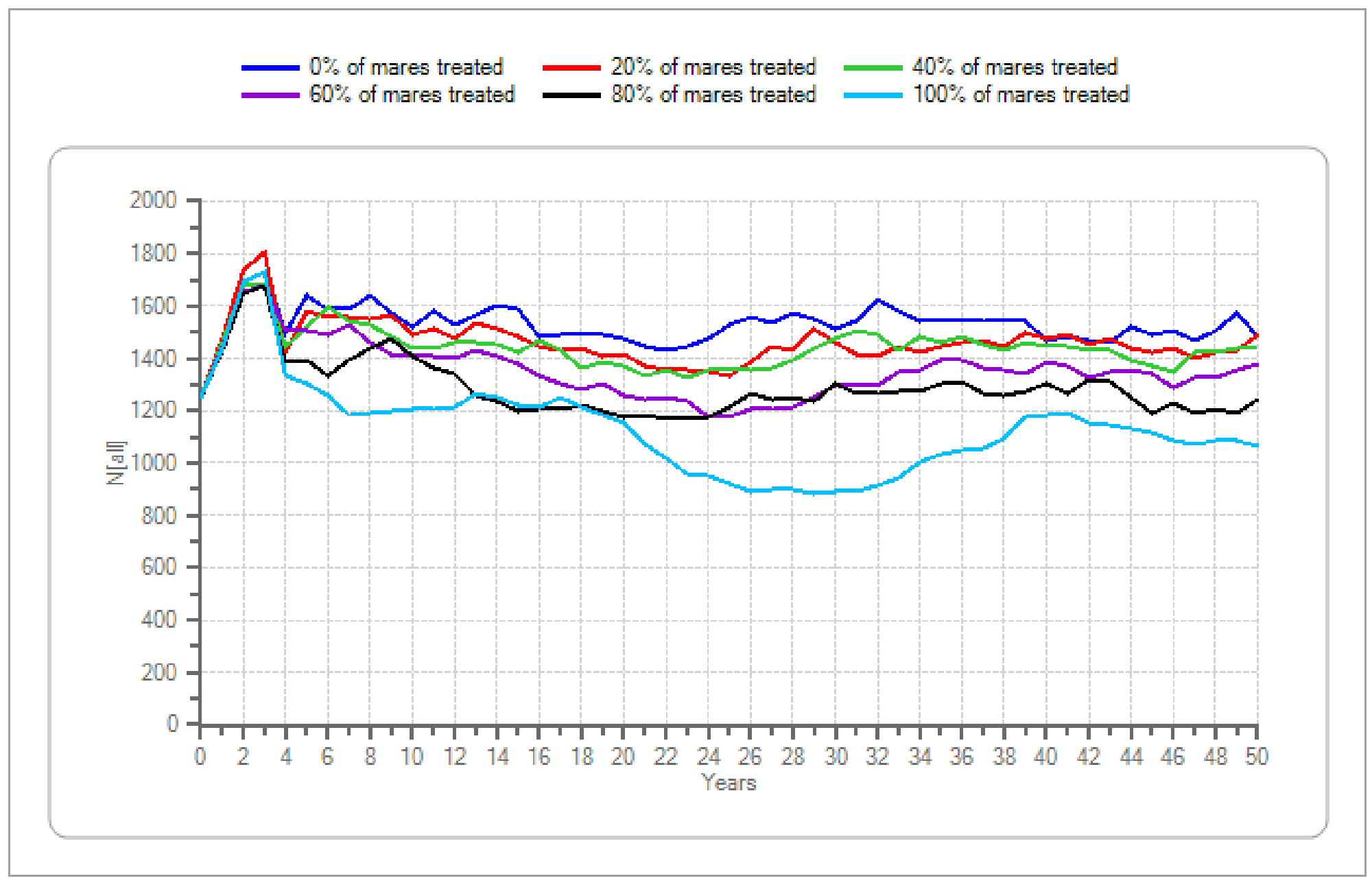

Figure 29. Initial population size $=1250$, lambda $=1.20$, genetic diversity $=$ low - Continued 
Mean population size after 20 years

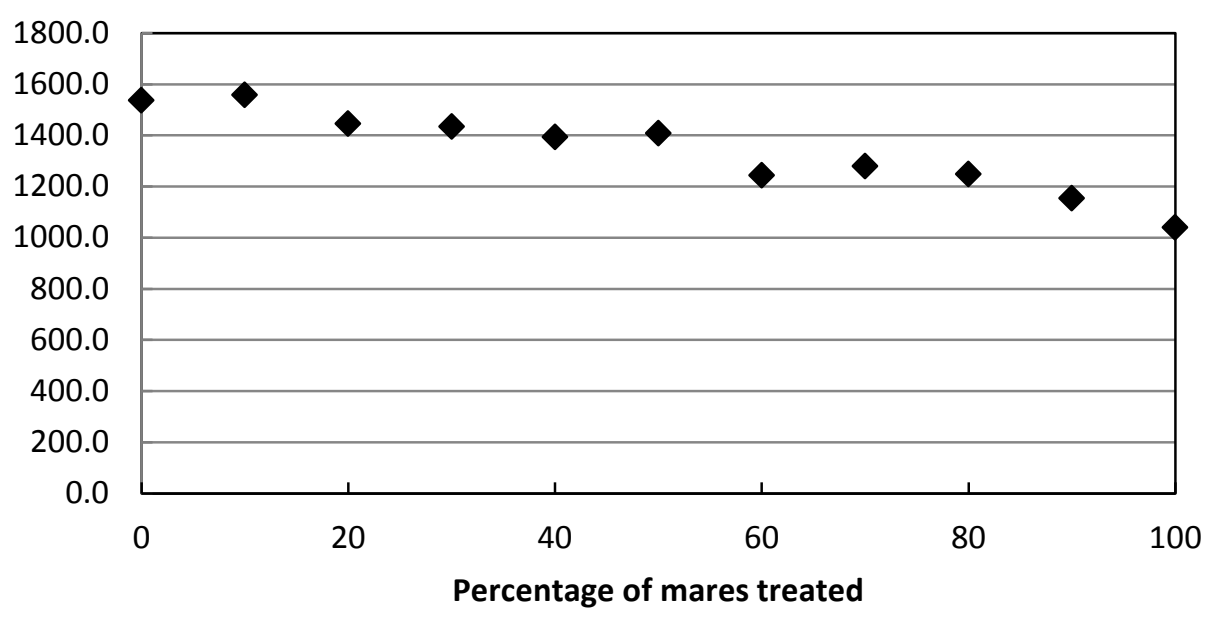

Mean probability of extirpation after $\mathbf{5 0}$ years

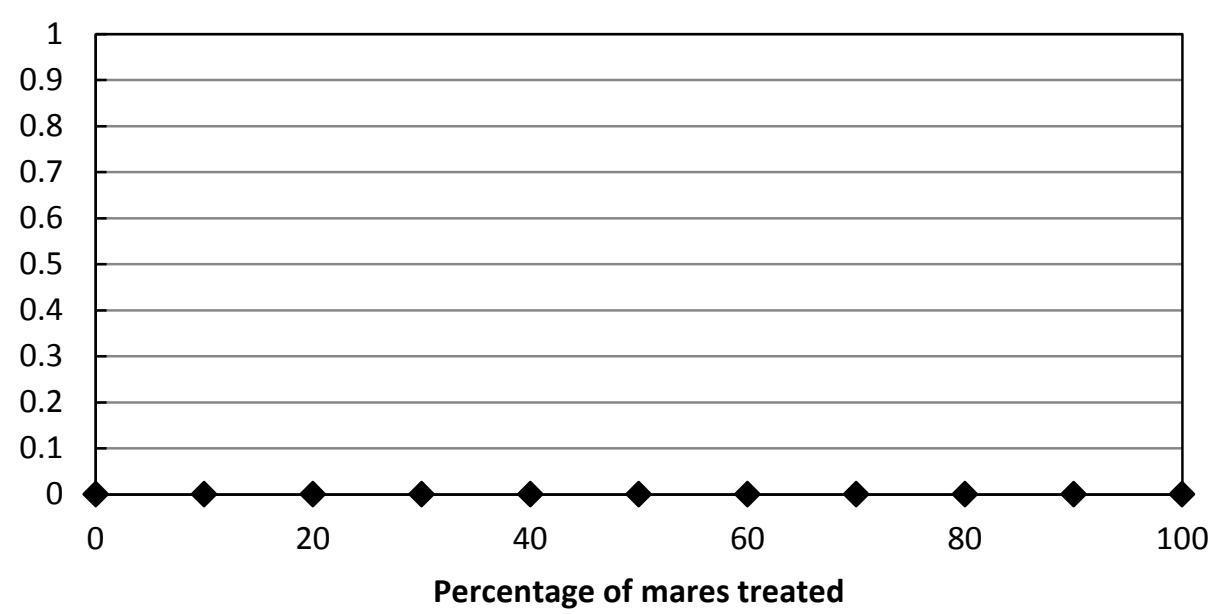

Figure 30. Initial population size $=1250$, lambda $=1.25$, genetic diversity $=$ low .
Mean number of removals after 20 years

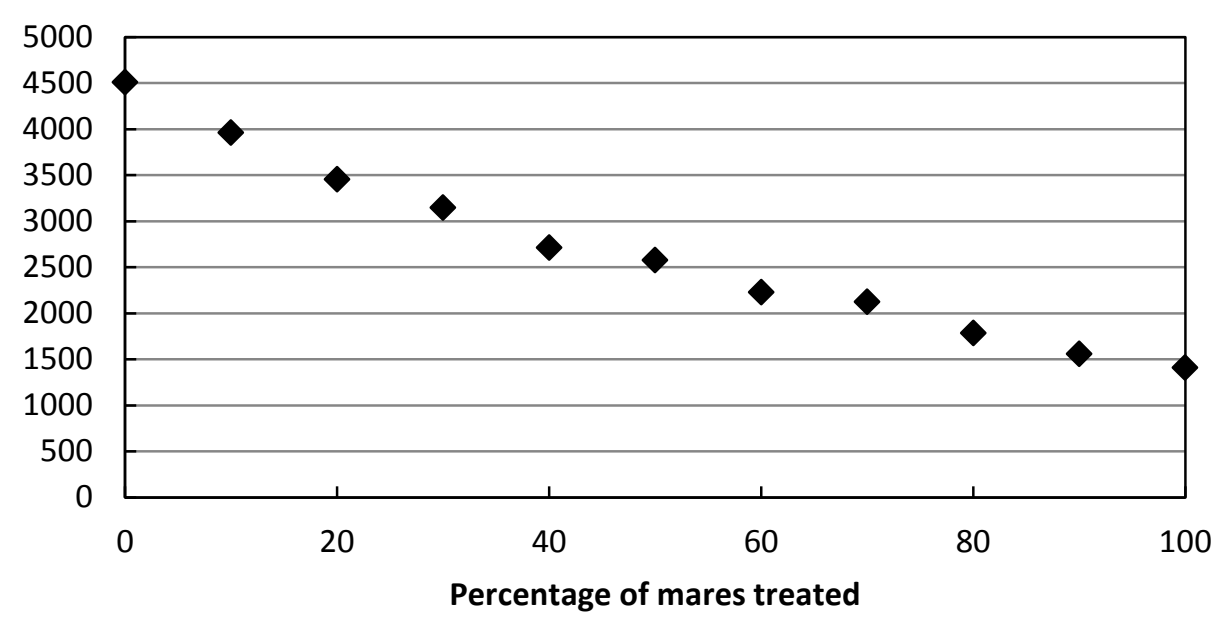

Mean heterozygosity after 50 years

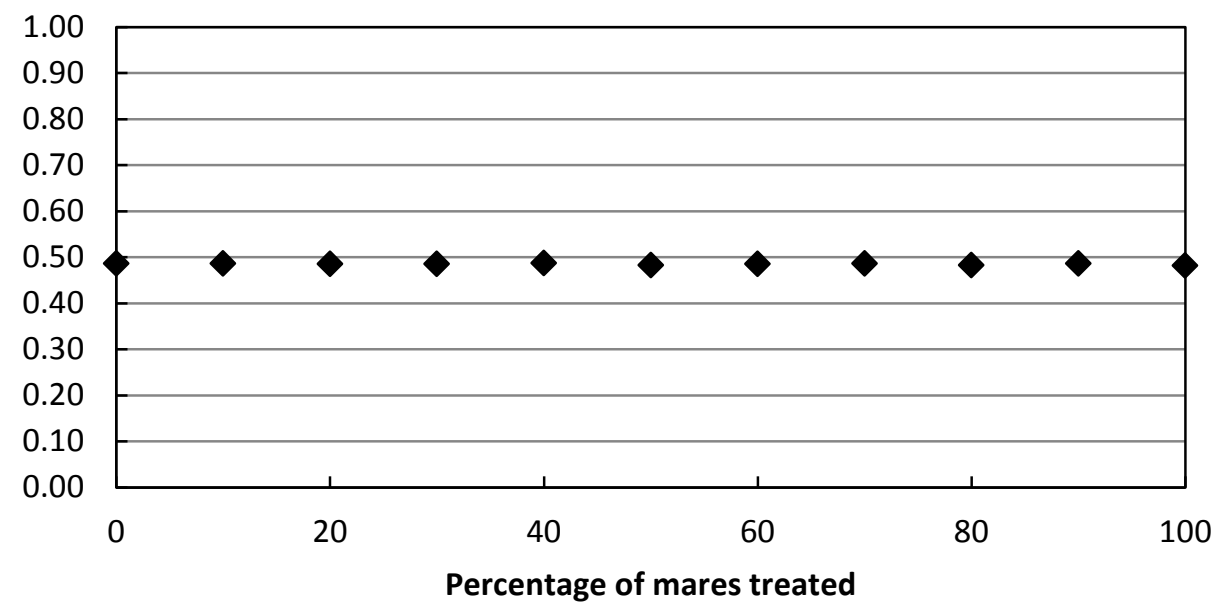




\begin{tabular}{lccccccccccccc}
\hline \multicolumn{1}{c}{ Percentage of mares treated } & $\mathbf{0}$ & $\mathbf{1 0}$ & $\mathbf{2 0}$ & $\mathbf{3 0}$ & $\mathbf{4 0}$ & $\mathbf{5 0}$ & $\mathbf{6 0}$ & $\mathbf{7 0}$ & $\mathbf{8 0}$ & $\mathbf{9 0}$ & $\mathbf{1 0 0}$ \\
\hline Mean population size after 20 years & 1536.3 & 1557.9 & 1445.1 & 1433.6 & 1394.1 & 1407.8 & 1243.9 & 1279.1 & 1248.4 & 1154.4 & 1039.9 \\
Mean number of removals after 20 years & 4509.1 & 3960.3 & 3453.9 & 3146.1 & 2711.4 & 2577.9 & 2226.4 & 2122.1 & 1782.3 & 1559.0 & 1409.3 \\
Mean probability of extirpation after 50 years & 0 & 0 & 0 & 0 & 0 & 0 & 0 & 0 & 0 & 0 & 0 & 0 & 0 \\
Mean heterozygosity after 50 years & 0.49 & 0.49 & 0.49 & 0.48 & 0.49 & 0.48 & 0.49 & 0.49 & 0.48 & 0.49 & 0.48 \\
\hline
\end{tabular}

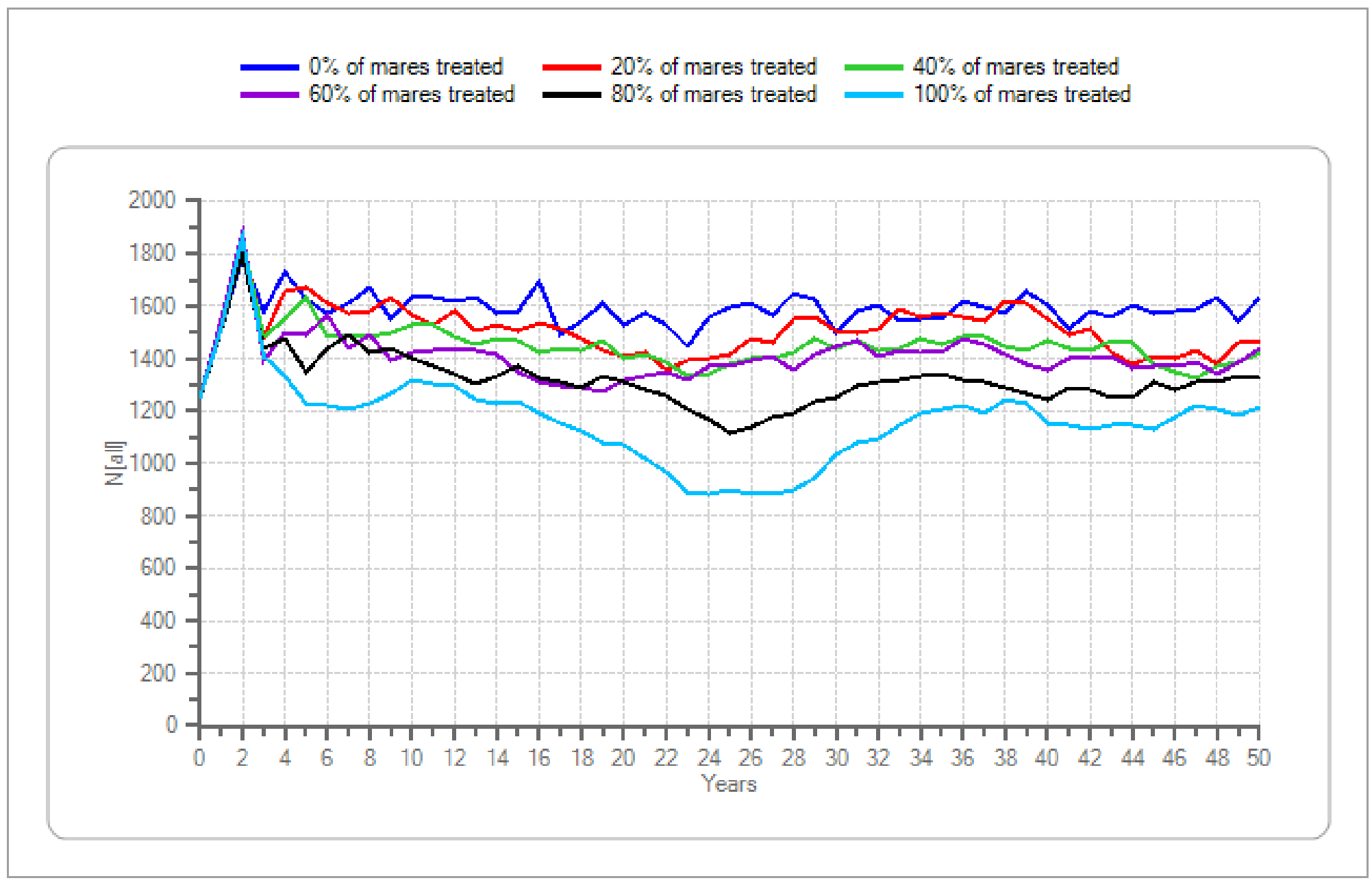

Figure 30. Initial population size $=1250$, lambda $=1.25$, genetic diversity $=$ low - Continued 
Mean population size after 20 years

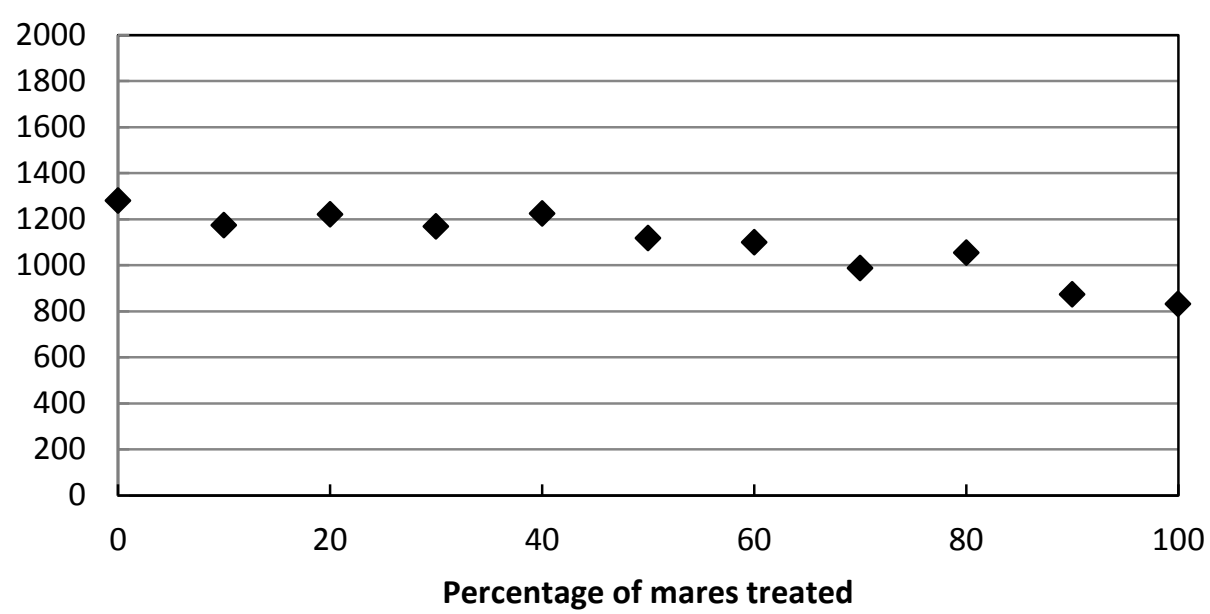

Mean probability of extirpation after 50 years

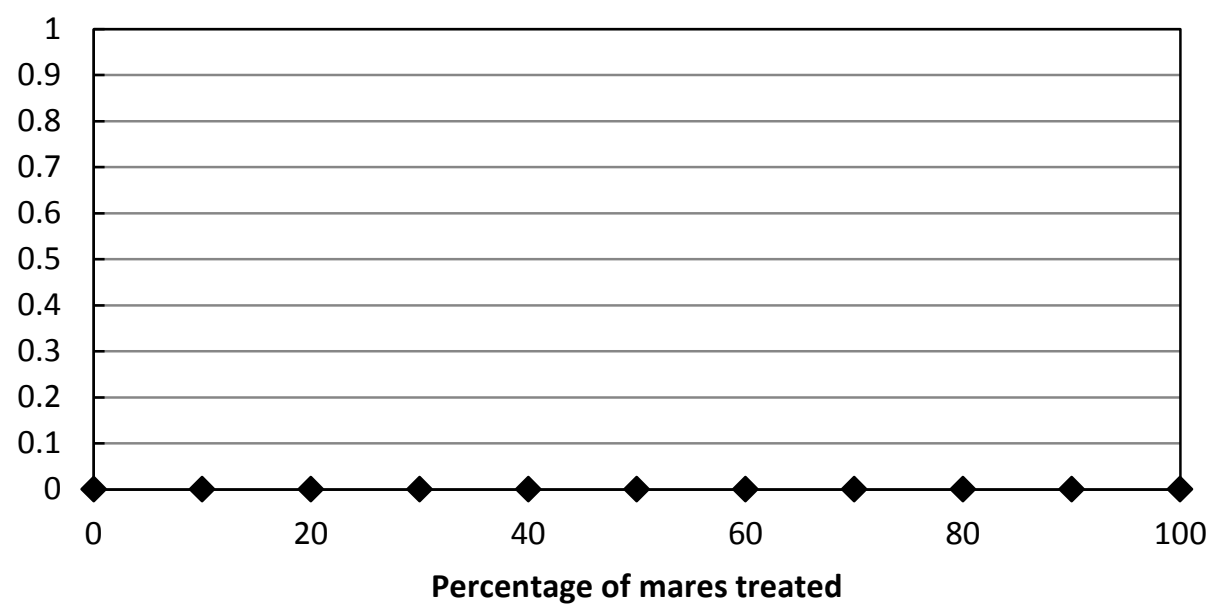

Figure 31. Initial population size $=1500$, lambda $=1.05$, genetic diversity $=$ low .
Mean number of removals after 20 years

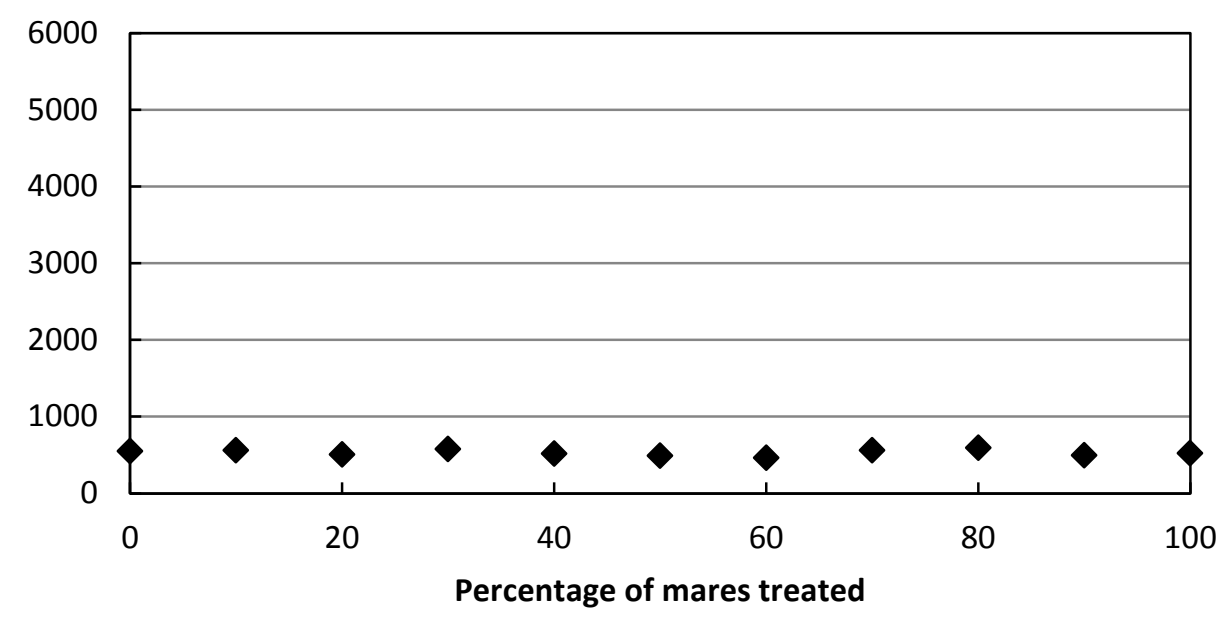

Mean heterozygosity after $\mathbf{5 0}$ years

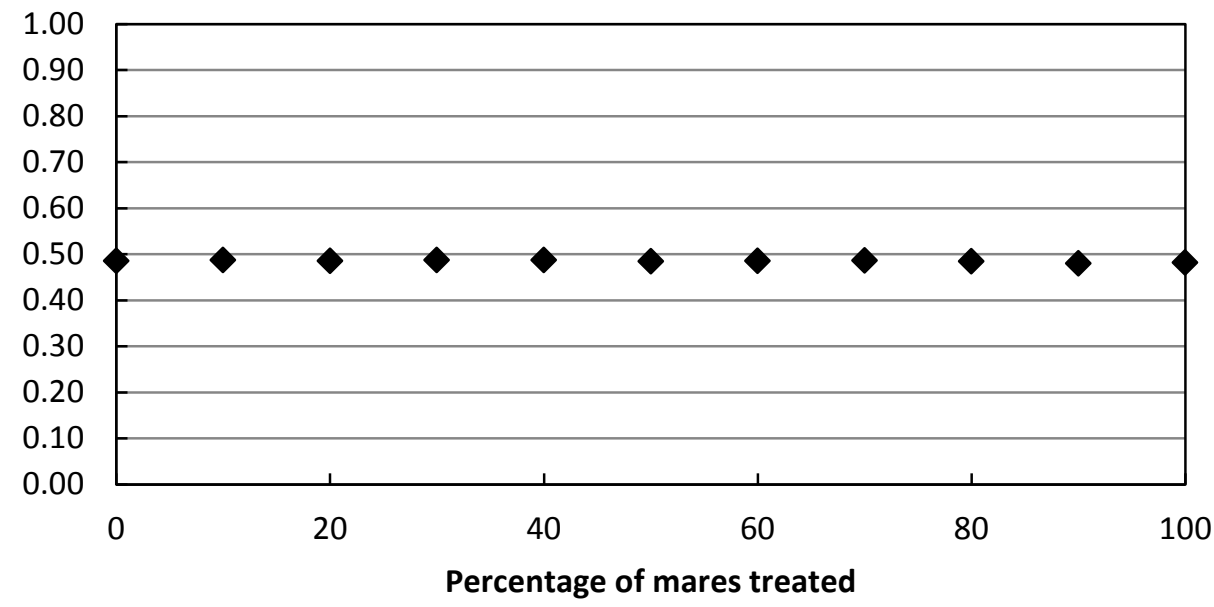




\begin{tabular}{lcccccccccccc}
\hline \multicolumn{1}{c}{ Percentage of mares treated } & $\mathbf{0}$ & $\mathbf{1 0}$ & $\mathbf{2 0}$ & $\mathbf{3 0}$ & $\mathbf{4 0}$ & $\mathbf{5 0}$ & $\mathbf{6 0}$ & $\mathbf{7 0}$ & $\mathbf{8 0}$ & $\mathbf{9 0}$ & $\mathbf{1 0 0}$ \\
\hline Mean population size after 20 years & 1279.4 & 1173.9 & 1220.8 & 1167.1 & 1224.9 & 1117.3 & 1098.7 & 987.4 & 1053.9 & 873.3 & 830.5 \\
Mean number of removals after 20 years & 547.92 & 557.55 & 504.35 & 573.75 & 514.62 & 487.4 & 459.94 & 558.95 & 591.28 & 496.54 & 524.2 \\
Mean probability of extirpation after 50 years & 0 & 0 & 0 & 0 & 0 & 0 & 0 & 0 & 0 & 0 & 0 \\
Mean heterozygosity after 50 years & 0.49 & 0.49 & 0.49 & 0.49 & 0.49 & 0.48 & 0.49 & 0.49 & 0.48 & 0.48 & 0.48 \\
\hline
\end{tabular}

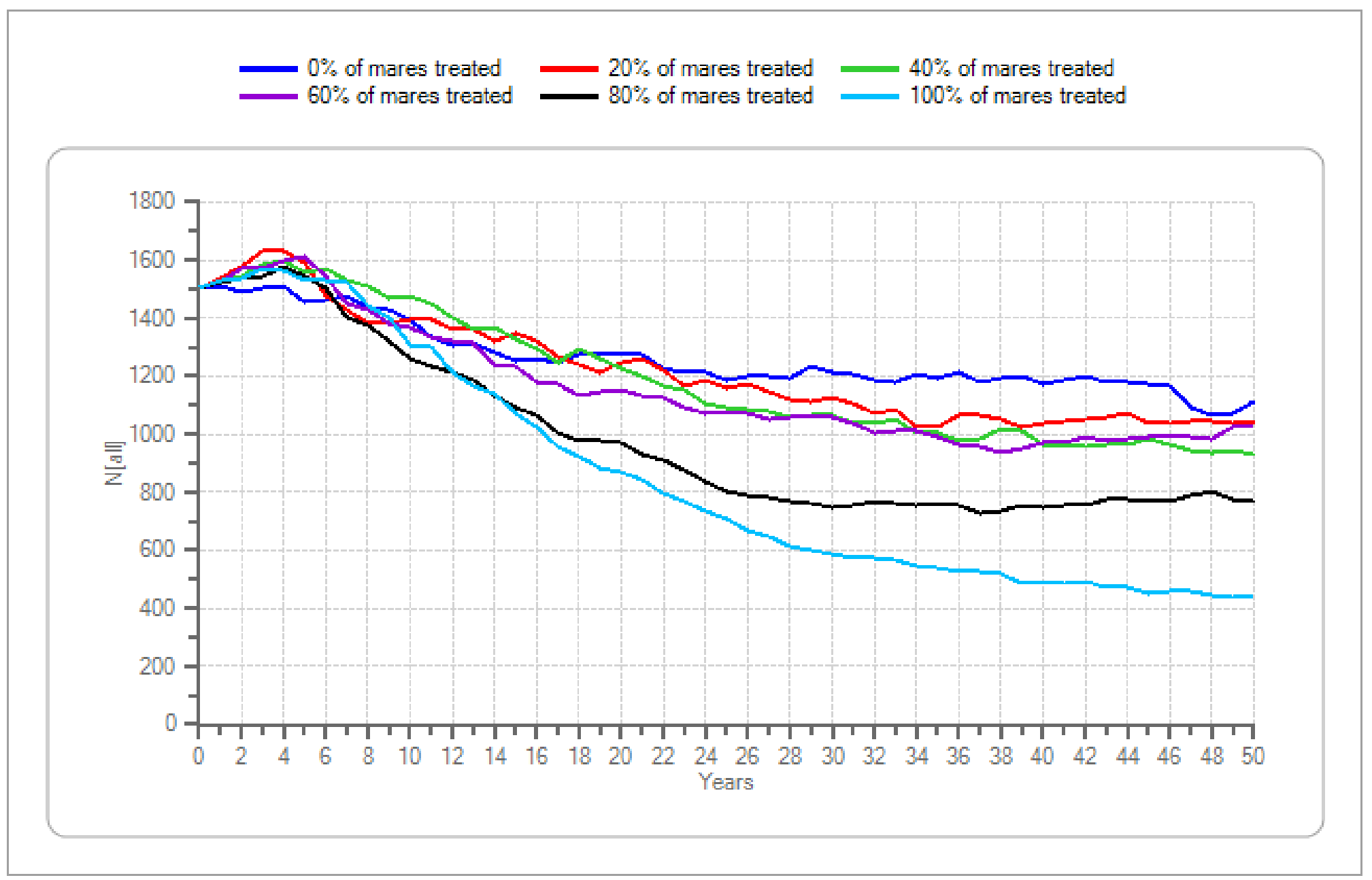

Figure 31. Initial population size $=1500$, lambda $=1.05$, genetic diversity $=$ low - Continued 
Mean population size after 20 years

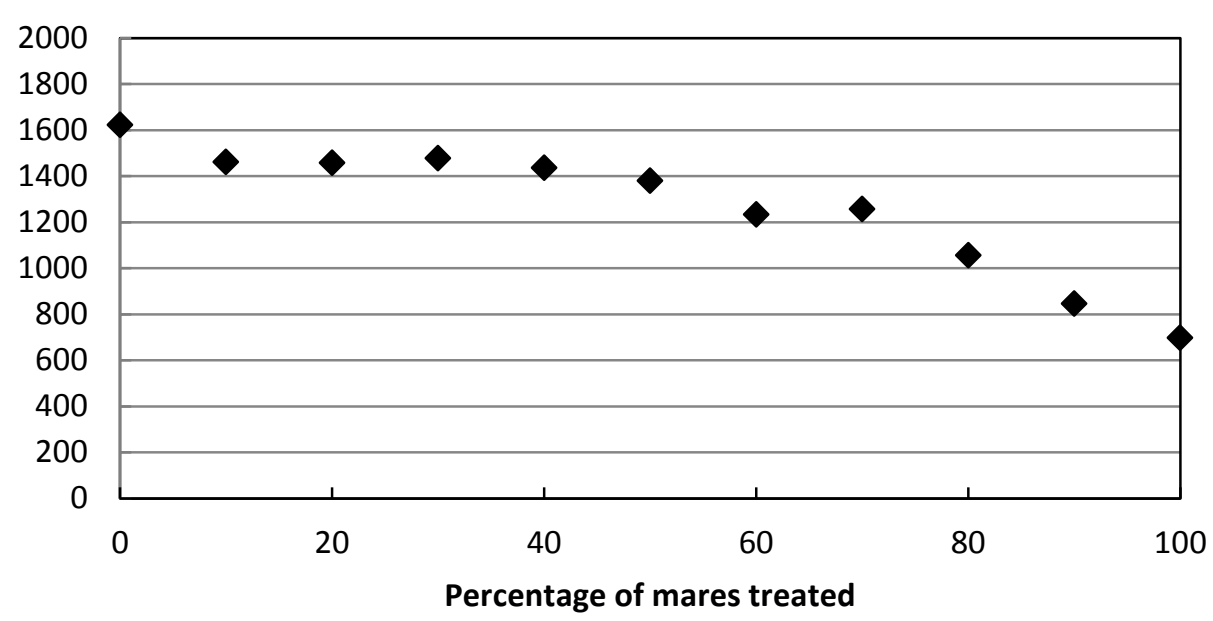

Mean probability of extirpation after $\mathbf{5 0}$ years

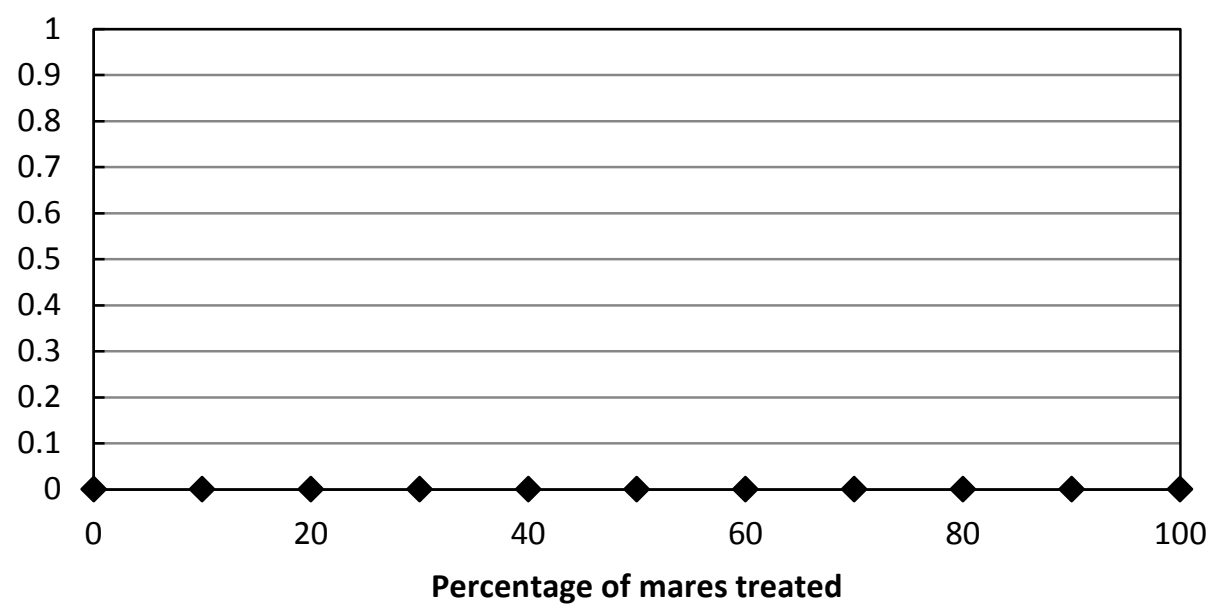

Figure 32. Initial population size $=1500$, lambda $=1.10$, genetic diversity $=$ low .
Mean number of removals after 20 years

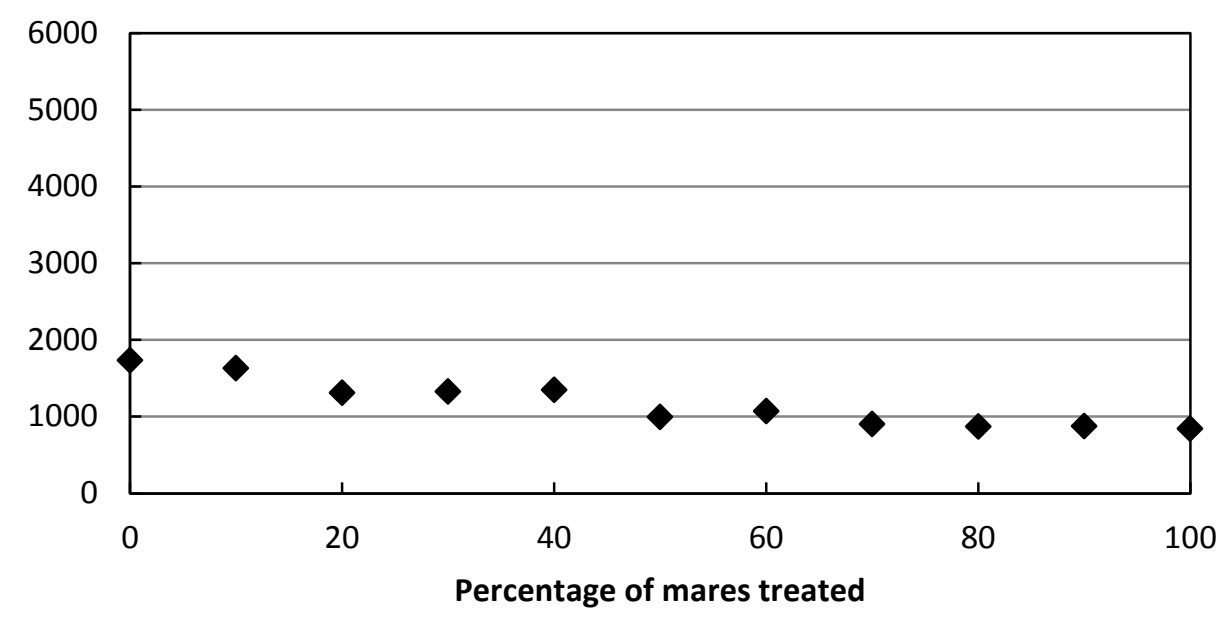

Mean heterozygosity after $\mathbf{5 0}$ years

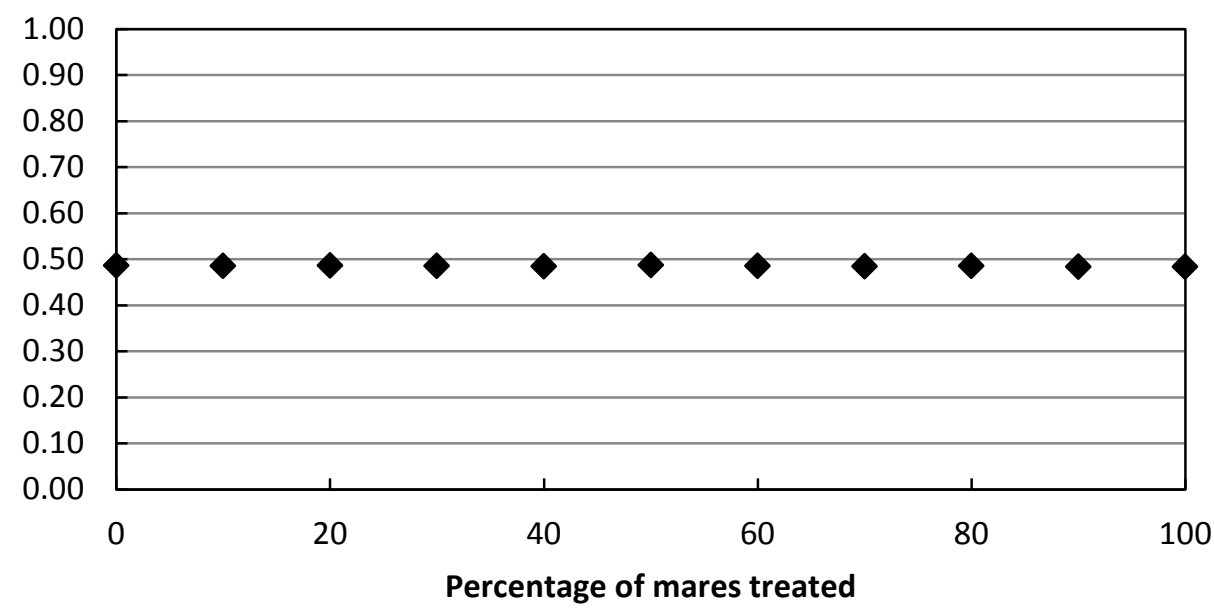




\begin{tabular}{lccccccccccc}
\hline \multicolumn{1}{c}{ Percentage of mares treated } & $\mathbf{0}$ & $\mathbf{1 0}$ & $\mathbf{2 0}$ & $\mathbf{3 0}$ & $\mathbf{4 0}$ & $\mathbf{5 0}$ & $\mathbf{6 0}$ & $\mathbf{7 0}$ & $\mathbf{8 0}$ & $\mathbf{9 0}$ & $\mathbf{1 0 0}$ \\
\hline Mean population size after 20 years & 1622.8 & 1461.2 & 1457.5 & 1477.4 & 1435.9 & 1380.1 & 1232.4 & 1255.8 & 1054.9 & 846.0 & 697.3 \\
Mean number of removals after 20 years & 1735.0 & 1628.0 & 1308.3 & 1327.2 & 1348.6 & 995.9 & 1072.9 & 903.0 & 868.5 & 872.8 & 843.8 \\
Mean probability of extirpation after 50 years & 0 & 0 & 0 & 0 & 0 & 0 & 0 & 0 & 0 & 0 & 0 \\
Mean heterozygosity after 50 years & 0.49 & 0.49 & 0.49 & 0.49 & 0.48 & 0.49 & 0.48 & 0.48 & 0.49 & 0.48 & 0.48 \\
\hline
\end{tabular}

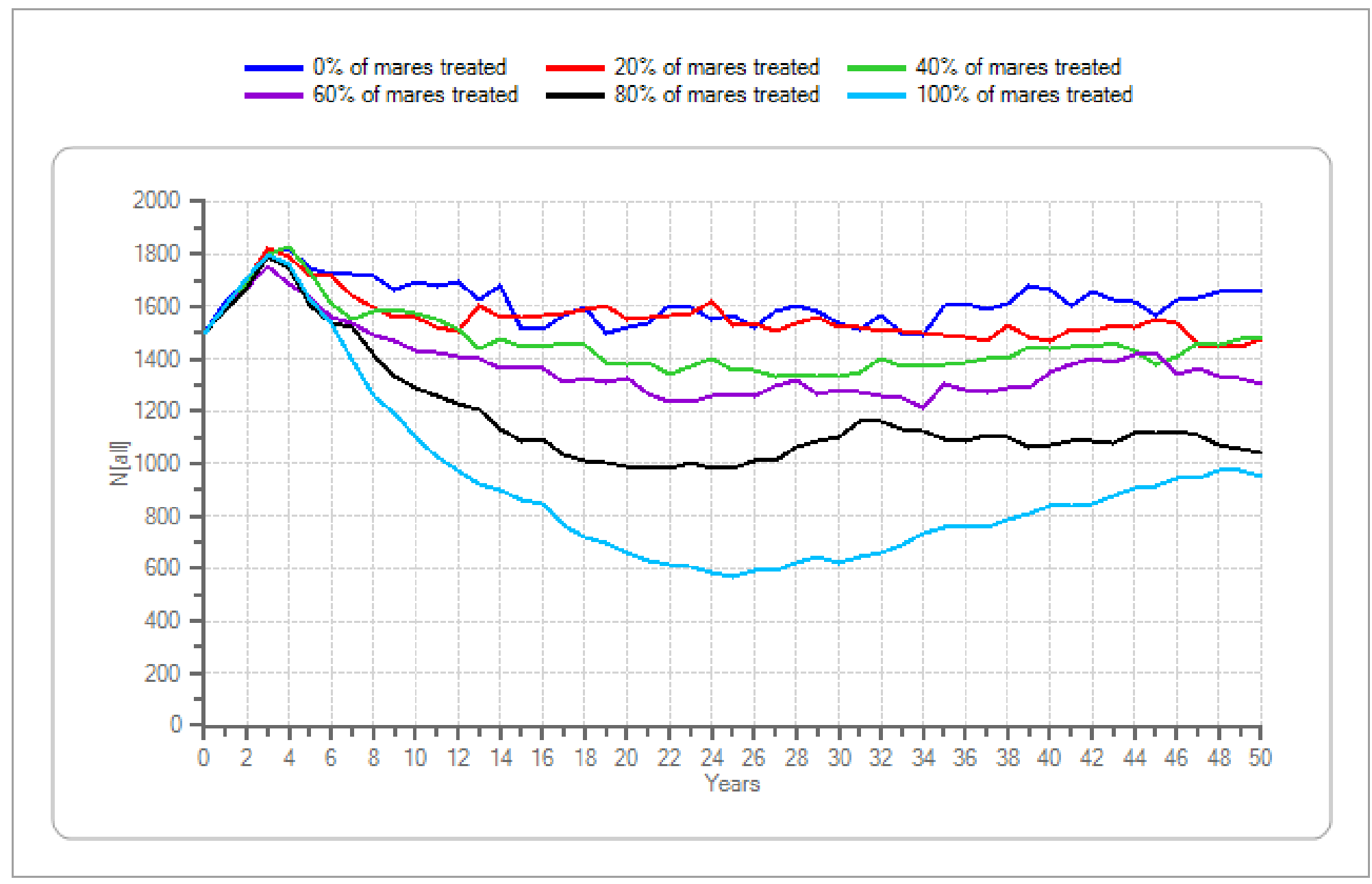

Figure 32. Initial population size $=1500$, lambda $=1.10$, genetic diversity $=$ low - Continued 
Mean population size after 20 years

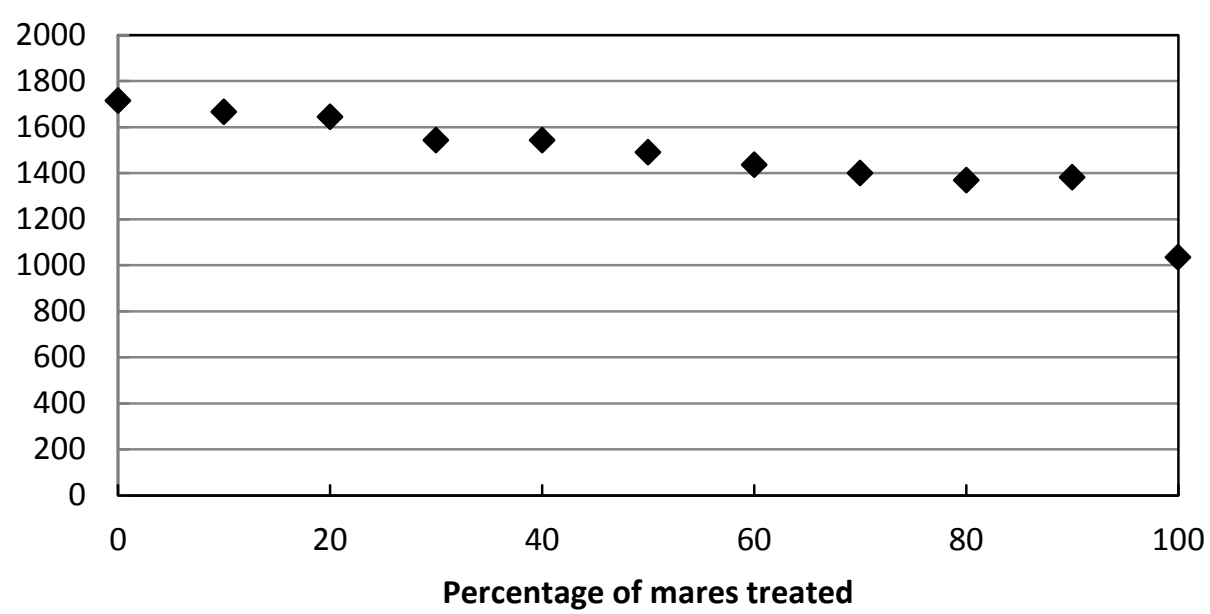

Mean probability of extirpation after 50 years

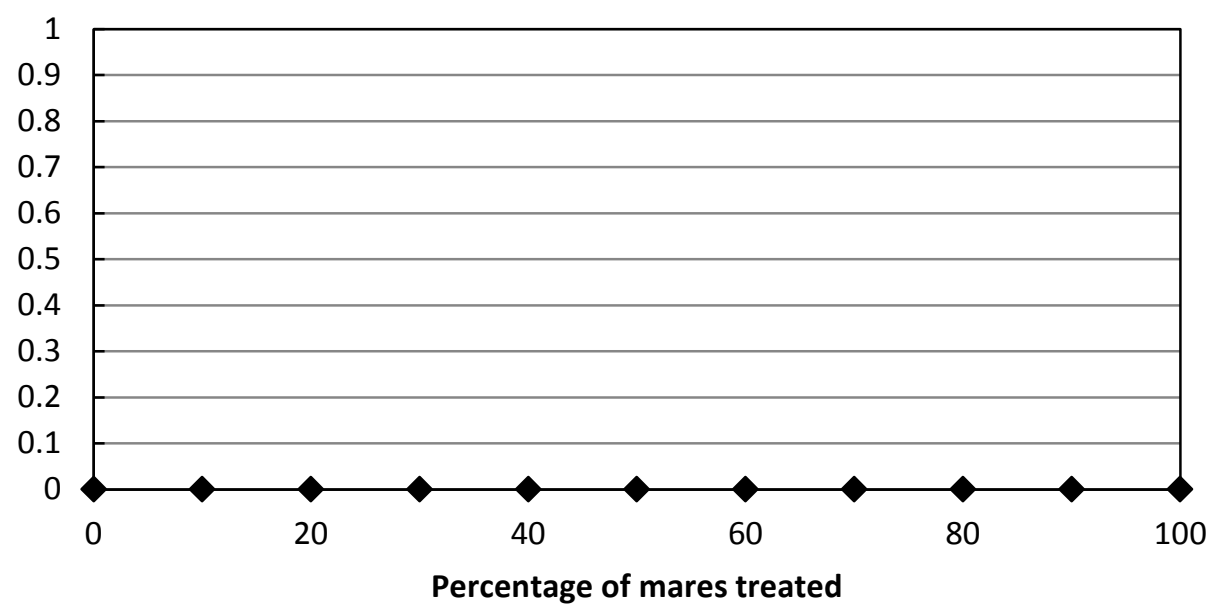

Figure 33. Initial population size $=1500$, lambda $=1.15$, genetic diversity $=$ low .
Mean number of removals after 20 years

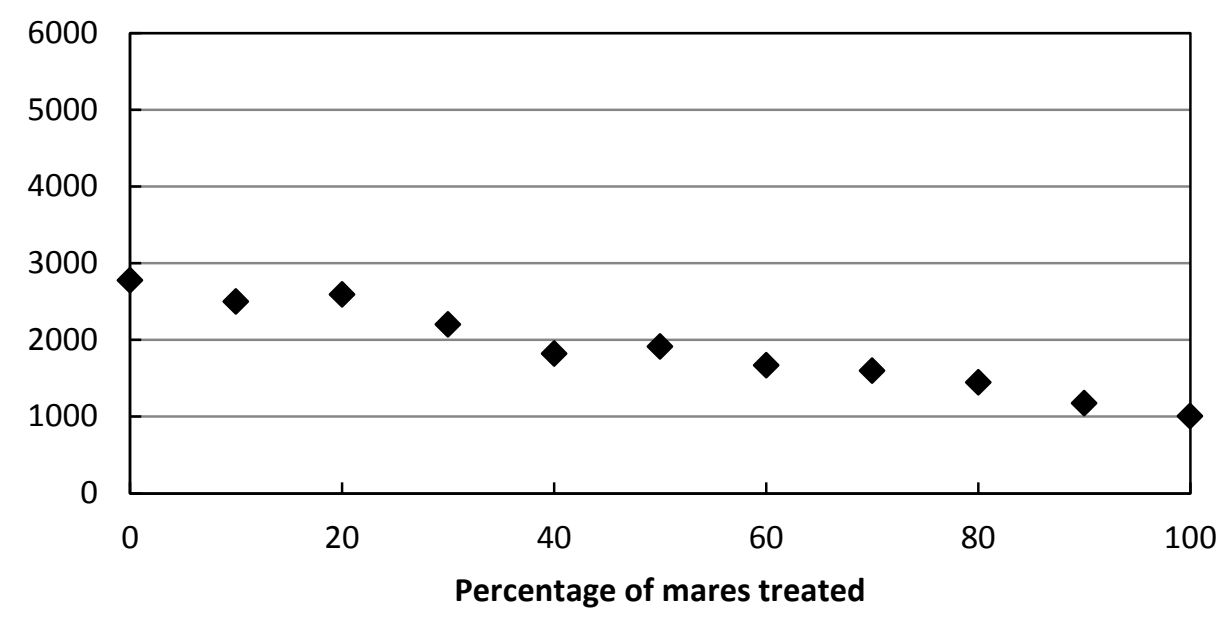

Mean heterozygosity after $\mathbf{5 0}$ years

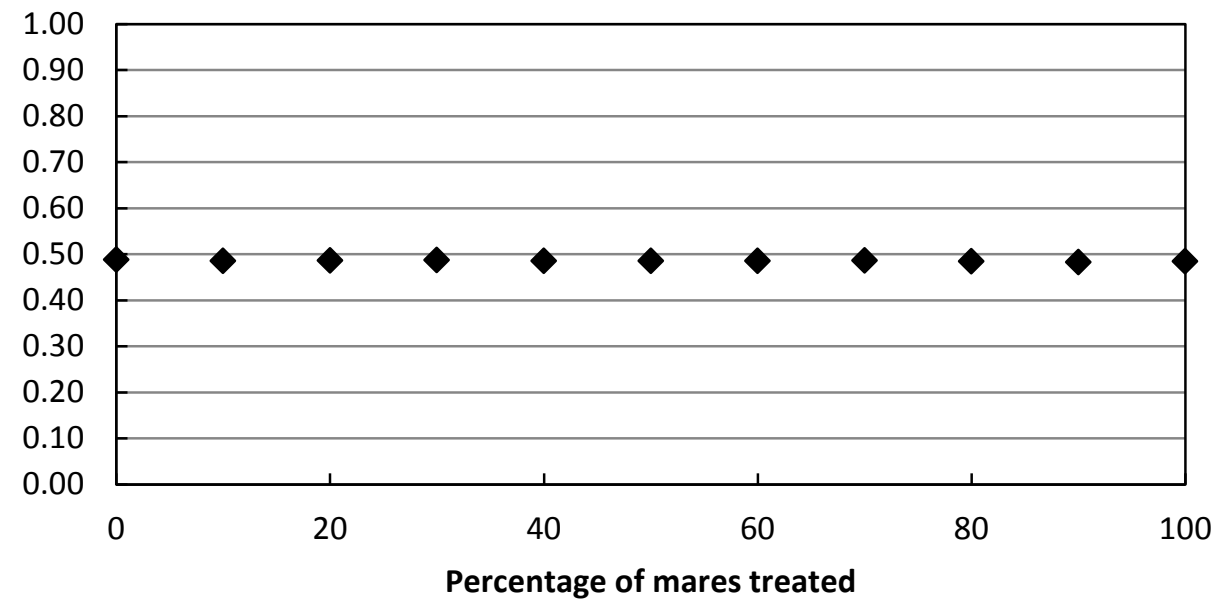




\begin{tabular}{lcccccccccccc}
\hline \multicolumn{1}{c}{ Percentage of mares treated } & $\mathbf{0}$ & $\mathbf{1 0}$ & $\mathbf{2 0}$ & $\mathbf{3 0}$ & $\mathbf{4 0}$ & $\mathbf{5 0}$ & $\mathbf{6 0}$ & $\mathbf{7 0}$ & $\mathbf{8 0}$ & $\mathbf{9 0}$ & $\mathbf{1 0 0}$ \\
\hline Mean population size after 20 years & 1714.8 & 1666.7 & 1643.6 & 1542.5 & 1542.7 & 1489.8 & 1436.2 & 1399.0 & 1368.2 & 1382.5 & 1033.5 \\
Mean number of removals after 20 years & 2777.7 & 2501.4 & 2591.7 & 2200.3 & 1822.5 & 1910.5 & 1669.9 & 1596.4 & 1444.0 & 1172.7 & 1004.9 \\
Mean probability of extirpation after 50 years & 0 & 0 & 0 & 0 & 0 & 0 & 0 & 0 & 0 & 0 & 0 \\
Mean heterozygosity after 50 years & 0.49 & 0.49 & 0.49 & 0.49 & 0.49 & 0.49 & 0.49 & 0.49 & 0.48 & 0.48 & 0.48 \\
\hline
\end{tabular}

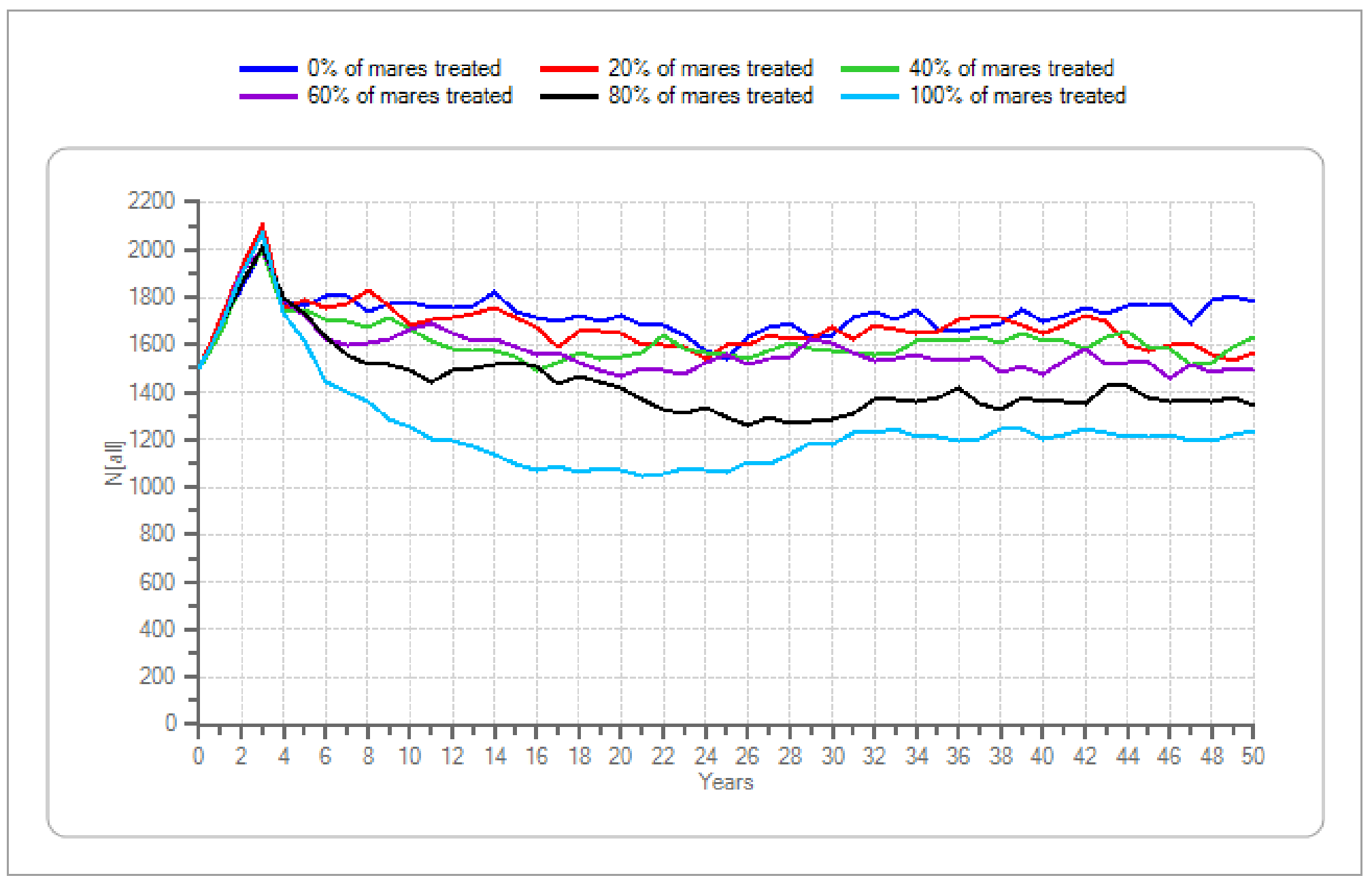

Figure 33. Initial population size $=1500$, lambda $=1.15$, genetic diversity $=$ low - Continued 
Mean population size after 20 years

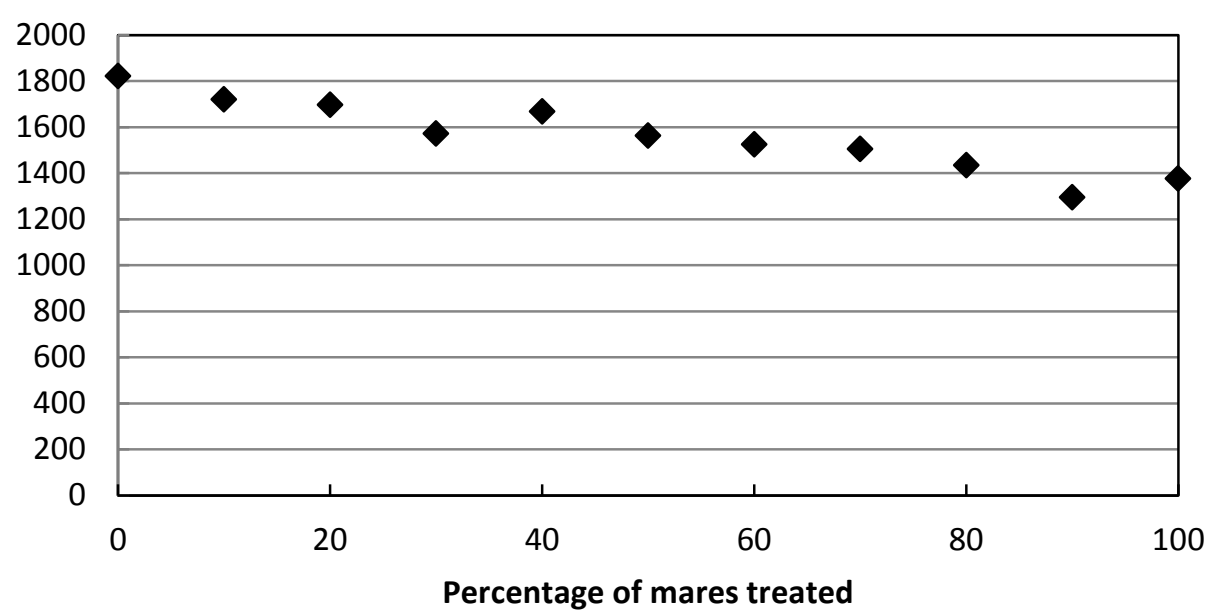

Mean probability of extirpation after 50 years

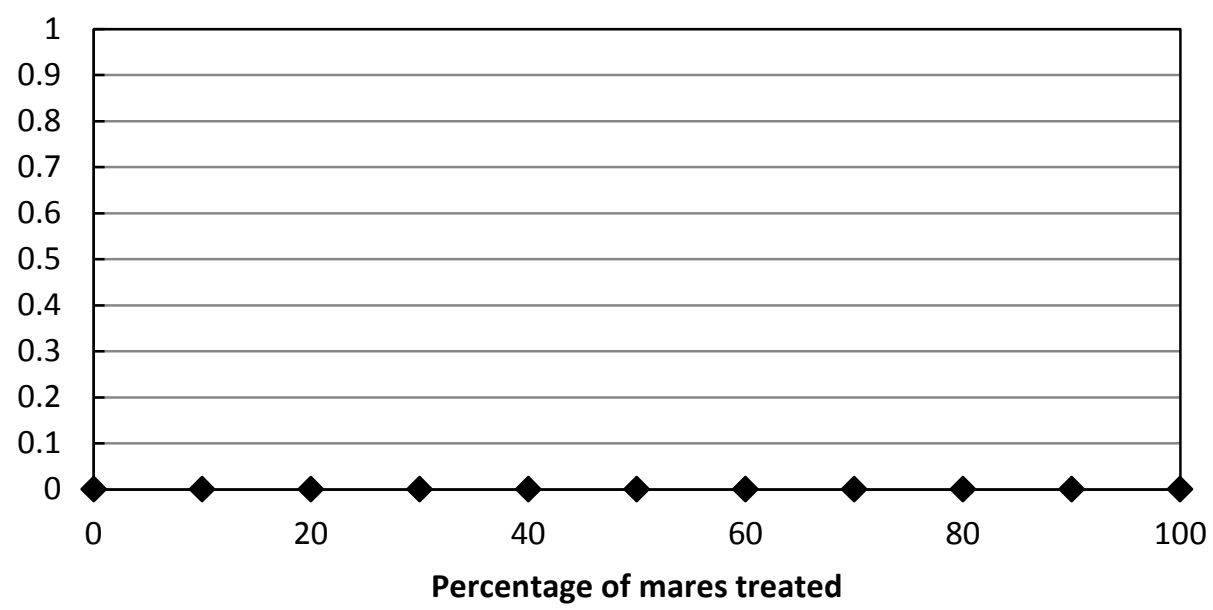

Figure 34. Initial population size $=1500$, lambda $=1.20$, genetic diversity $=$ low .
Mean number of removals after 20 years

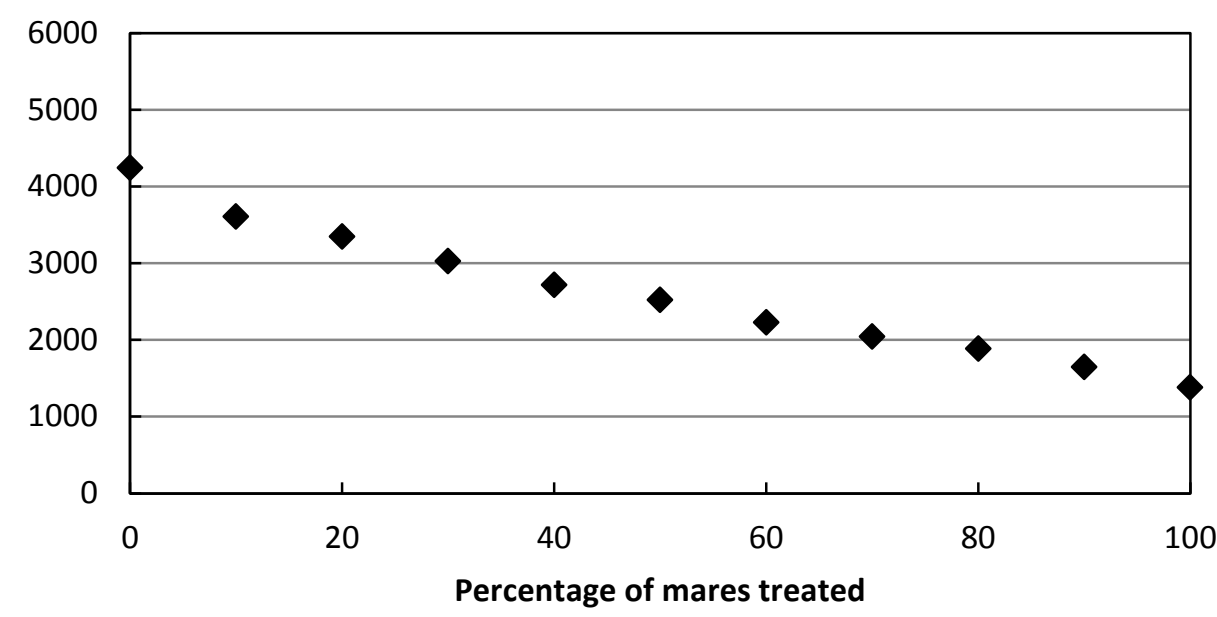

Mean heterozygosity after $\mathbf{5 0}$ years

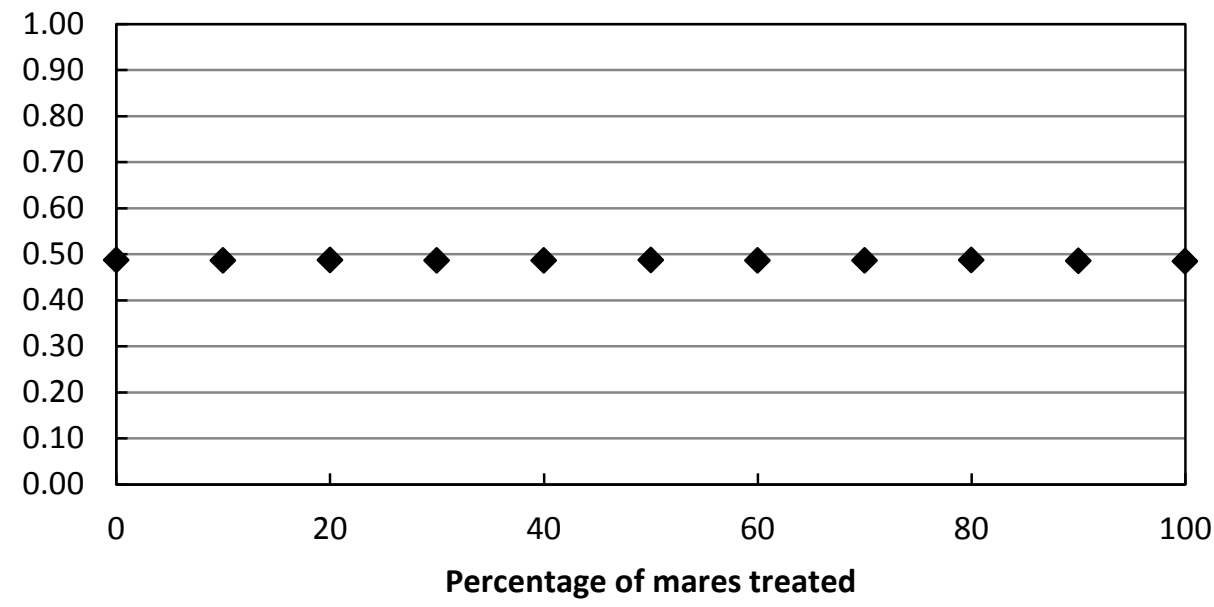




\begin{tabular}{lrccccccccccc}
\hline \multicolumn{1}{c}{ Percentage of mares treated } & $\mathbf{0}$ & $\mathbf{1 0}$ & $\mathbf{2 0}$ & $\mathbf{3 0}$ & $\mathbf{4 0}$ & $\mathbf{5 0}$ & $\mathbf{6 0}$ & $\mathbf{7 0}$ & $\mathbf{8 0}$ & $\mathbf{9 0}$ & $\mathbf{1 0 0}$ \\
\hline Mean population size after 20 years & 1821.8 & 1719.5 & 1697.0 & 1572.3 & 1667.0 & 1563.1 & 1523.7 & 1505.0 & 1433.3 & 1294.1 & 1376.3 \\
Mean number of removals after 20 years & 4241.3 & 3605.67 & 3348.38 & 3026.93 & 2716.34 & 2518.97 & 2225.75 & 2043.6 & 1886.32 & 1648.08 & 1381.6 \\
Mean probability of extirpation after 50 years & 0 & 0 & 0 & 0 & 0 & 0 & 0 & 0 & 0 & 0 & 0 \\
Mean heterozygosity after 50 years & 0.49 & 0.49 & 0.49 & 0.49 & 0.49 & 0.49 & 0.49 & 0.49 & 0.49 & 0.48 & 0.48 \\
\hline
\end{tabular}

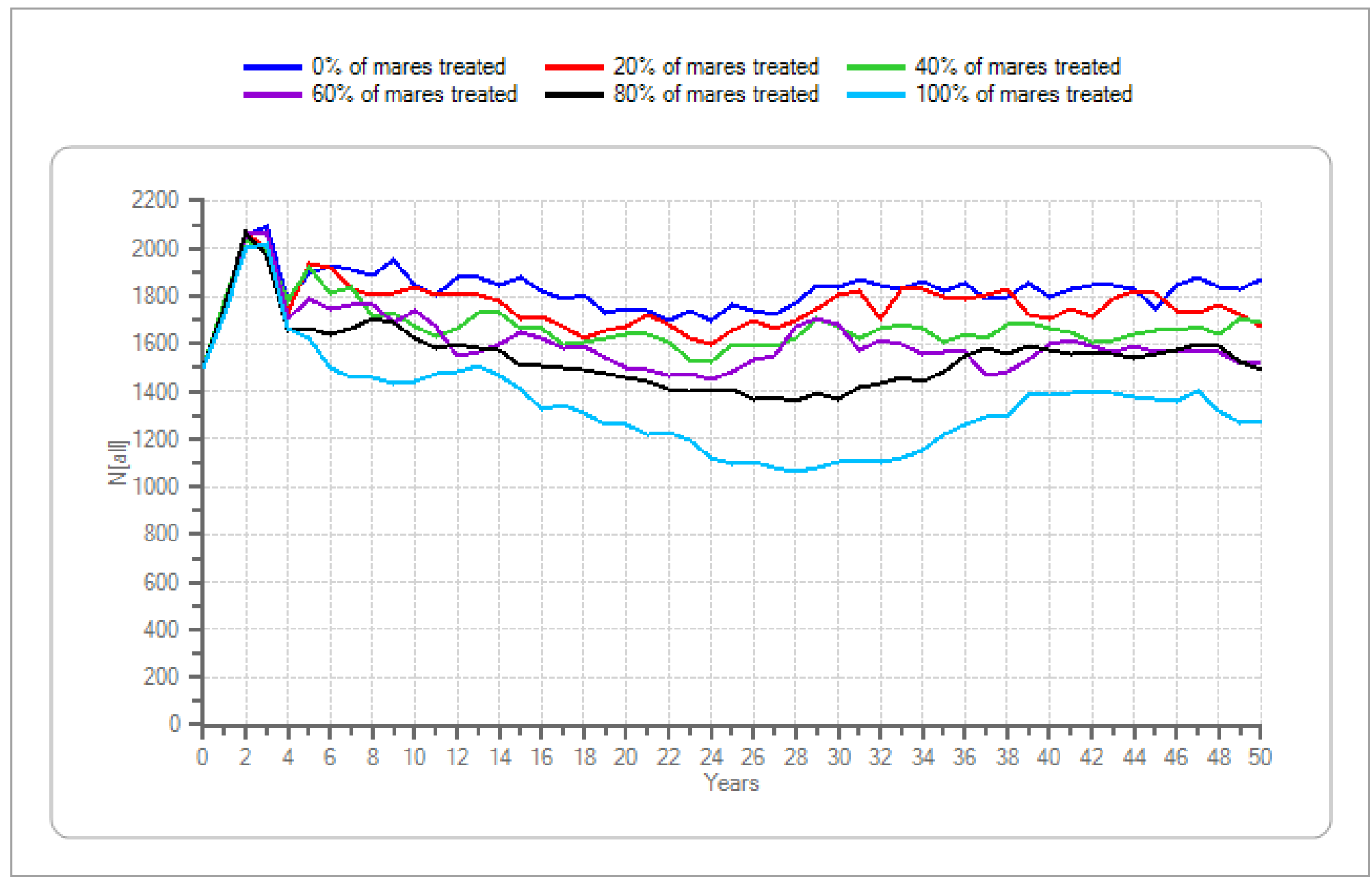

Figure 34. Initial population size $=1500$, lambda $=1.20$, genetic diversity $=$ low - Continued 
Mean population size after 20 years

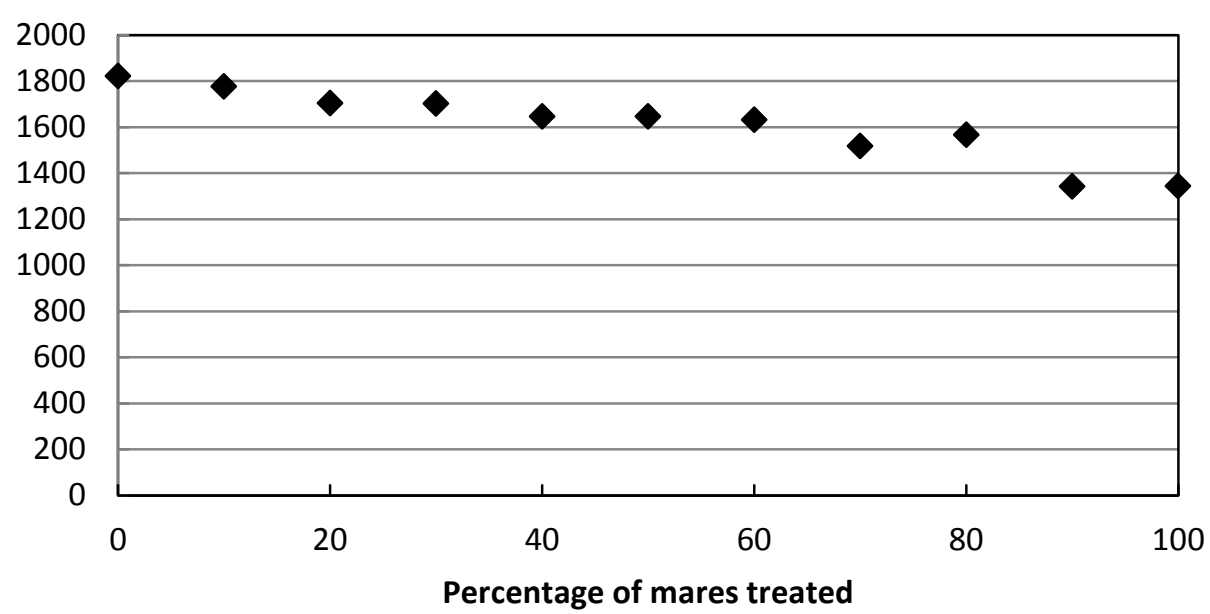

Mean probability of extirpation after 50 years

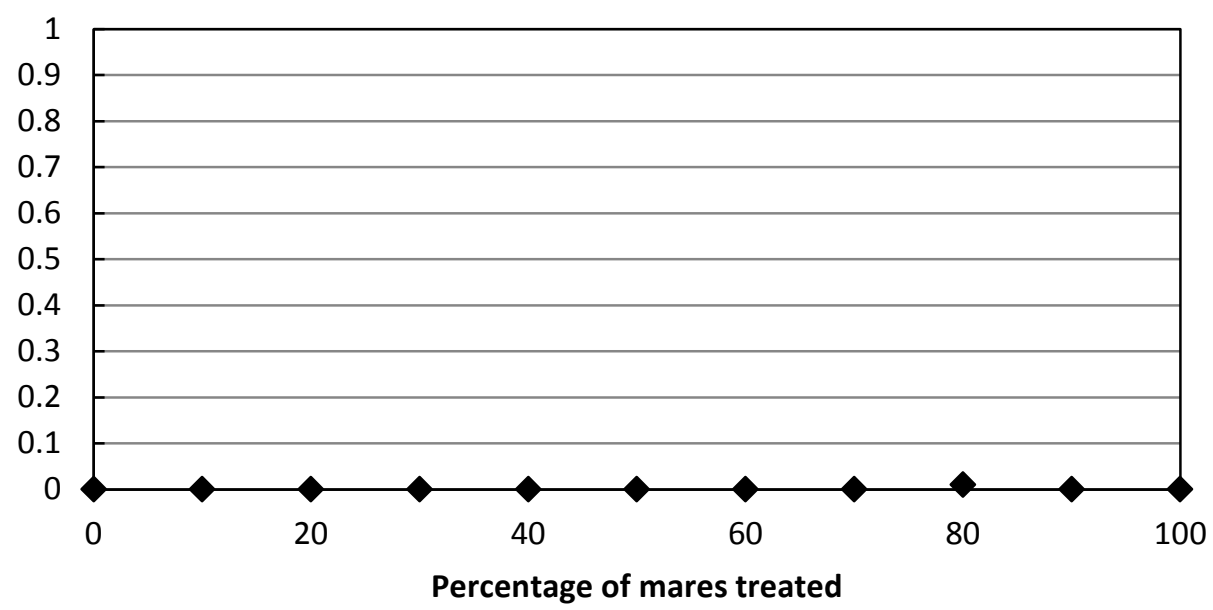

Figure 35. Initial population size $=1500$, lambda $=1.25$, genetic diversity $=$ low .
Mean number of removals after 20 years

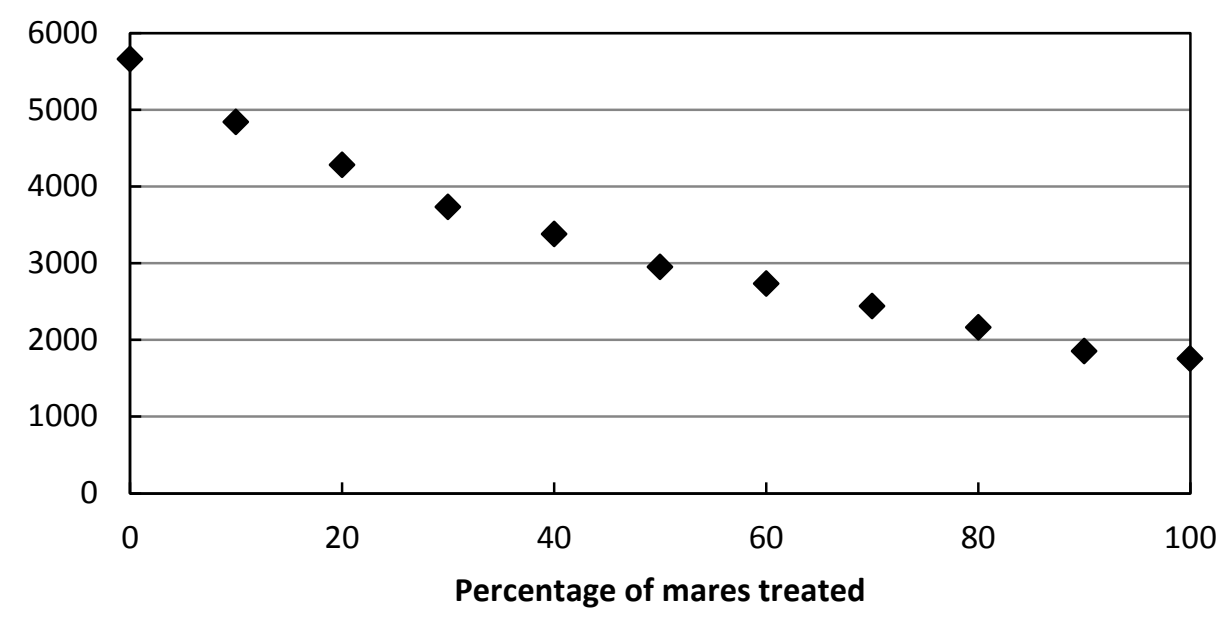

Mean heterozygosity after $\mathbf{5 0}$ years

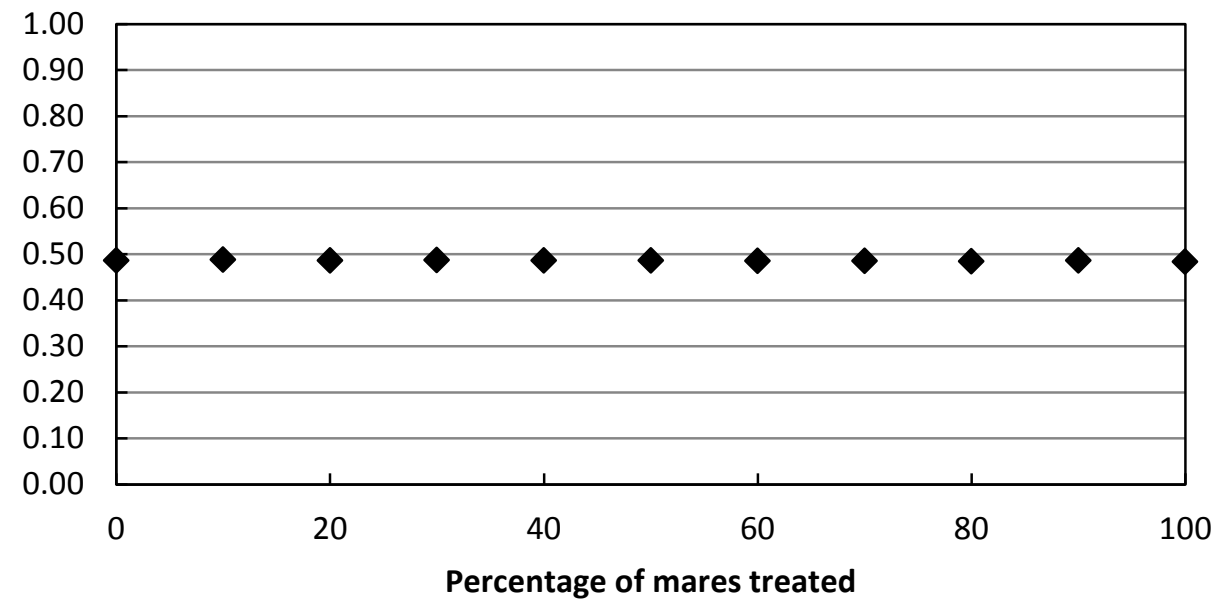




\begin{tabular}{lcccccccccccc}
\hline \multicolumn{1}{c}{ Percentage of mares treated } & $\mathbf{0}$ & $\mathbf{1 0}$ & $\mathbf{2 0}$ & $\mathbf{3 0}$ & $\mathbf{4 0}$ & $\mathbf{5 0}$ & $\mathbf{6 0}$ & $\mathbf{7 0}$ & $\mathbf{8 0}$ & $\mathbf{9 0}$ & $\mathbf{1 0 0}$ \\
\hline Mean population size after 20 years & 1821.3 & 1775.6 & 1704.2 & 1702.5 & 1645.8 & 1645.7 & 1631.5 & 1516.9 & 1566.6 & 1342.5 & 1343.4 \\
Mean number of removals after 20 years & 5662.1 & 4837.4 & 4282.2 & 3732.1 & 3380.2 & 2949.7 & 2731.2 & 2438.5 & 2164.2 & 1852.6 & 1756.3 \\
Mean probability of extirpation after 50 years & 0 & 0 & 0 & 0 & 0 & 0 & 0 & 0 & 0.01 & 0 & 0 \\
Mean heterozygosity after 50 years & 0.49 & 0.49 & 0.49 & 0.49 & 0.49 & 0.49 & 0.49 & 0.49 & 0.48 & 0.49 & 0.48 \\
\hline
\end{tabular}

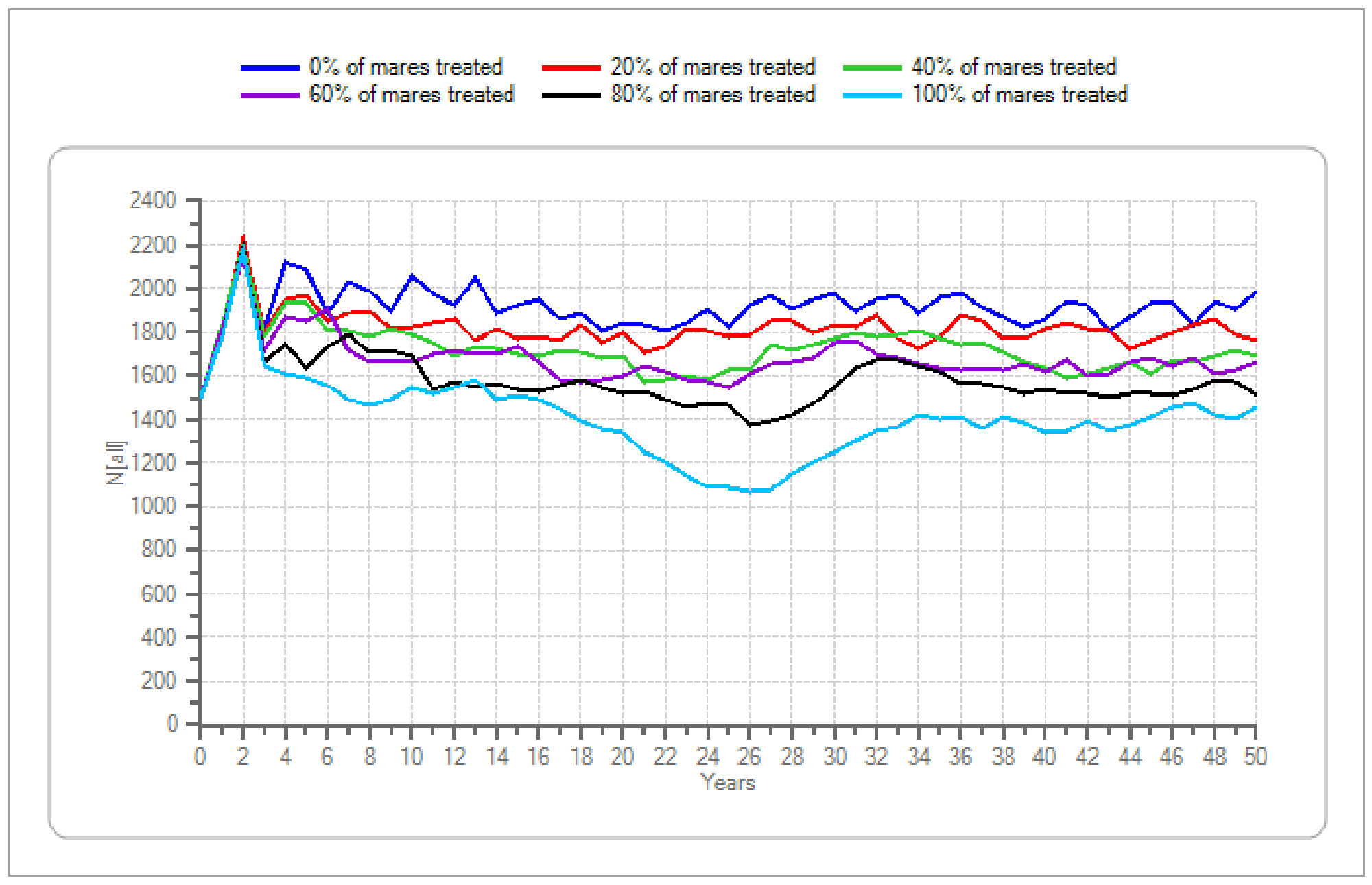

Figure 35. Initial population size $=1500$, lambda $=1.25$, genetic diversity $=$ low - Continued 
Mean population size after 20 years

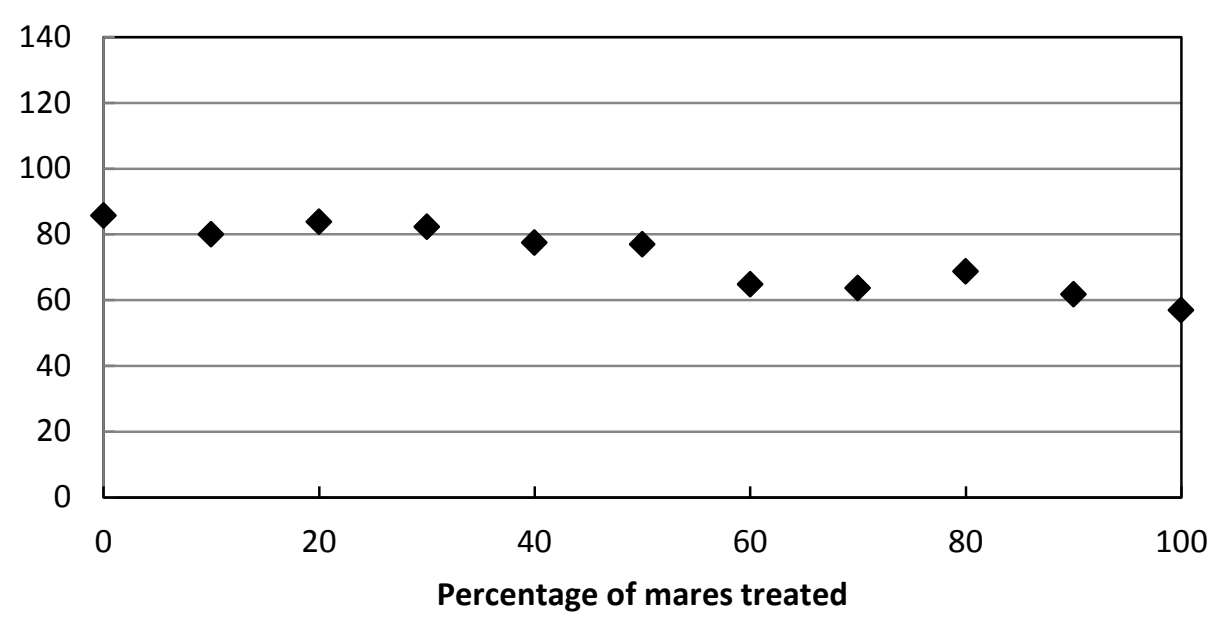

Mean probability of extirpation after $\mathbf{5 0}$ years

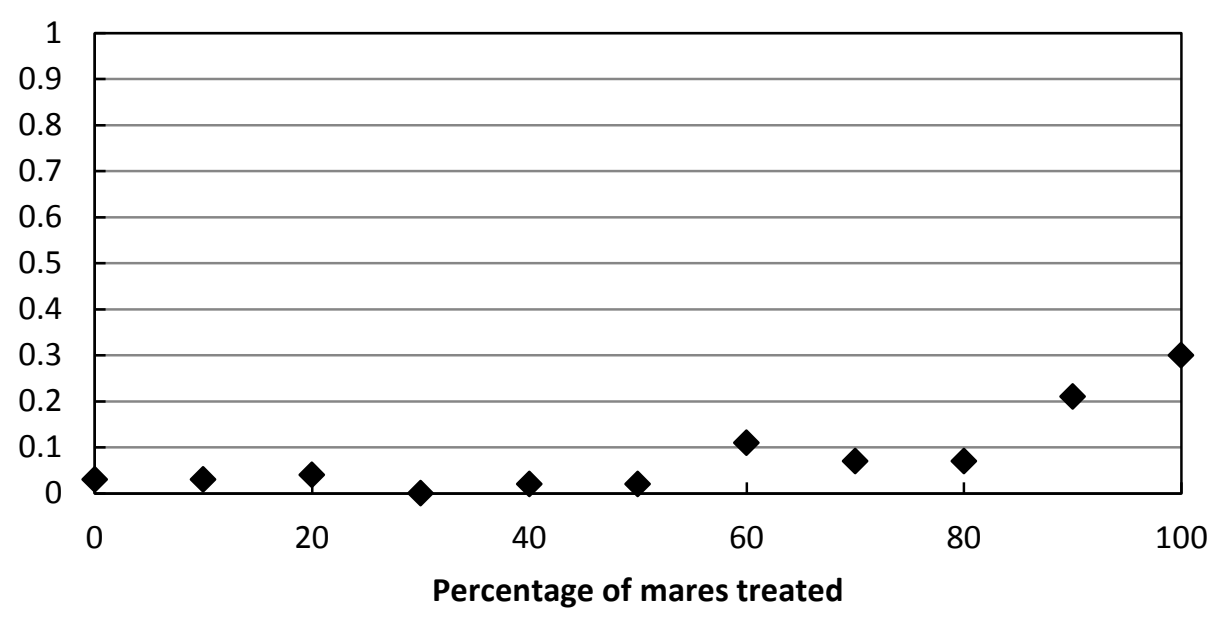

Figure 36. Initial population size $=100$, lambda $=1.05$, genetic diversity $=$ high .
Mean number of removals after 20 years

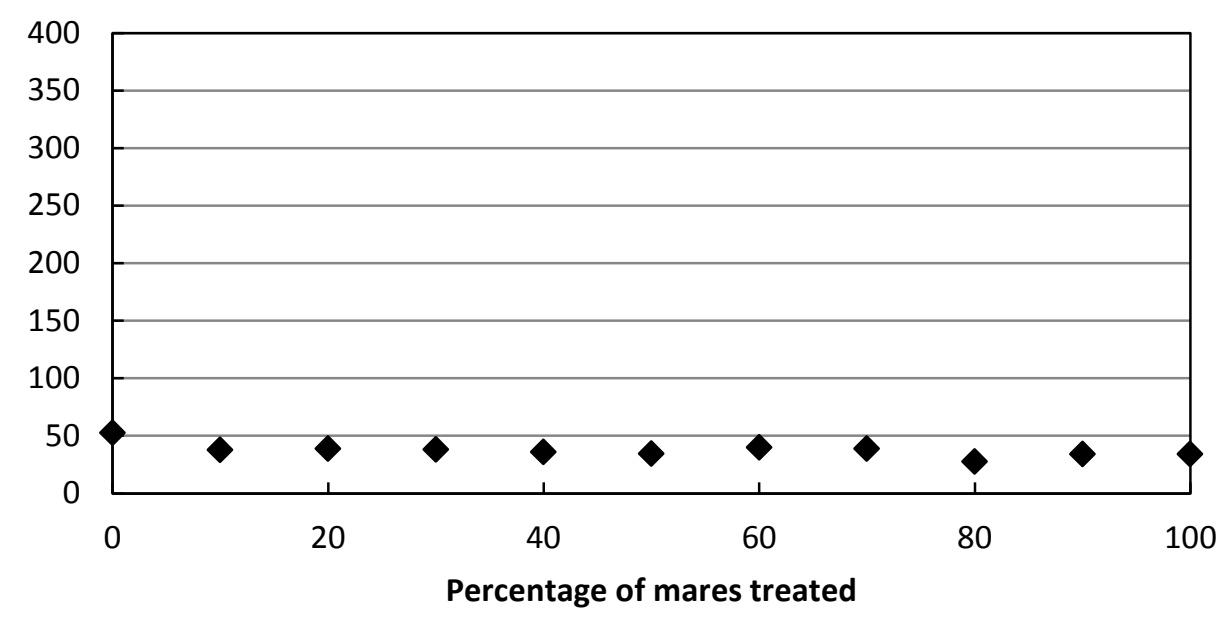

Mean heterozygosity after $\mathbf{5 0}$ years

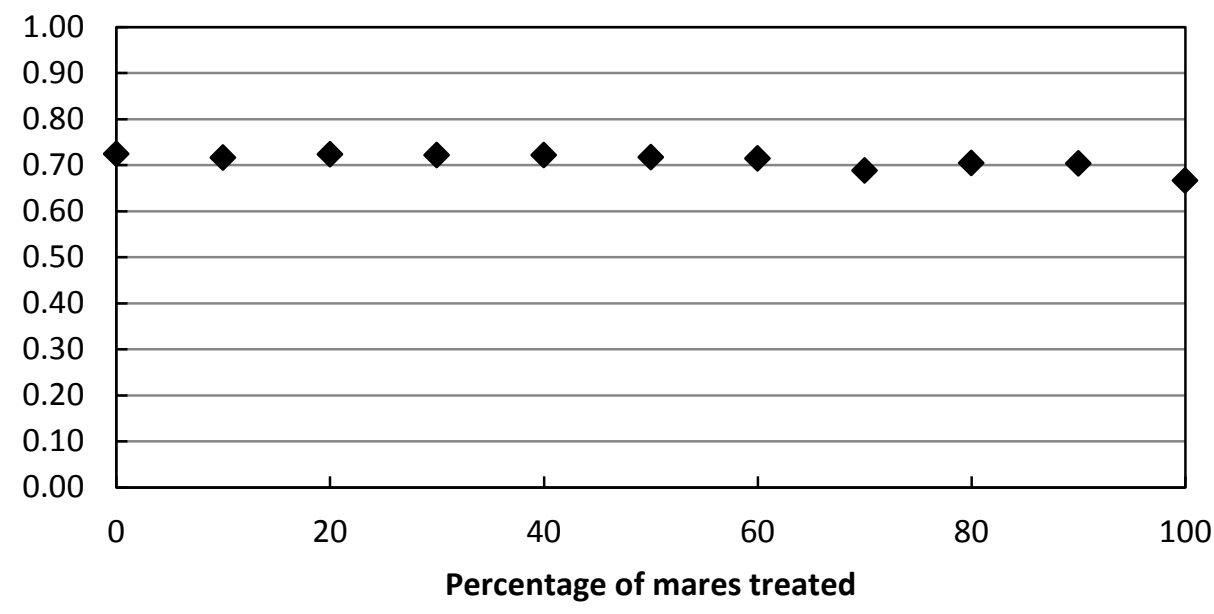




\begin{tabular}{|c|c|c|c|c|c|c|c|c|c|c|c|}
\hline Percentage of mares treated & 0 & 10 & 20 & 30 & 40 & 50 & 60 & 70 & 80 & 90 & 100 \\
\hline Mean population size after 20 years & 85.65 & 80.04 & 83.81 & 82.32 & 77.46 & 76.91 & 64.82 & 63.57 & 68.7 & 61.78 & 56.86 \\
\hline Mean number of removals after 20 years & 52.51 & 37.59 & 38.83 & 38.02 & 36.03 & 34.27 & 39.99 & 38.8 & 27.57 & 34.2 & 34.23 \\
\hline Mean probability of extirpation after 50 years & 0.03 & 0.03 & 0.04 & 0 & 0.02 & 0.02 & 0.11 & 0.07 & 0.07 & 0.21 & 0.3 \\
\hline Mean heterozygosity after 50 years & 0.72 & 0.72 & 0.72 & 0.72 & 0.72 & 0.72 & 0.71 & 0.69 & 0.70 & 0.70 & 0.67 \\
\hline
\end{tabular}

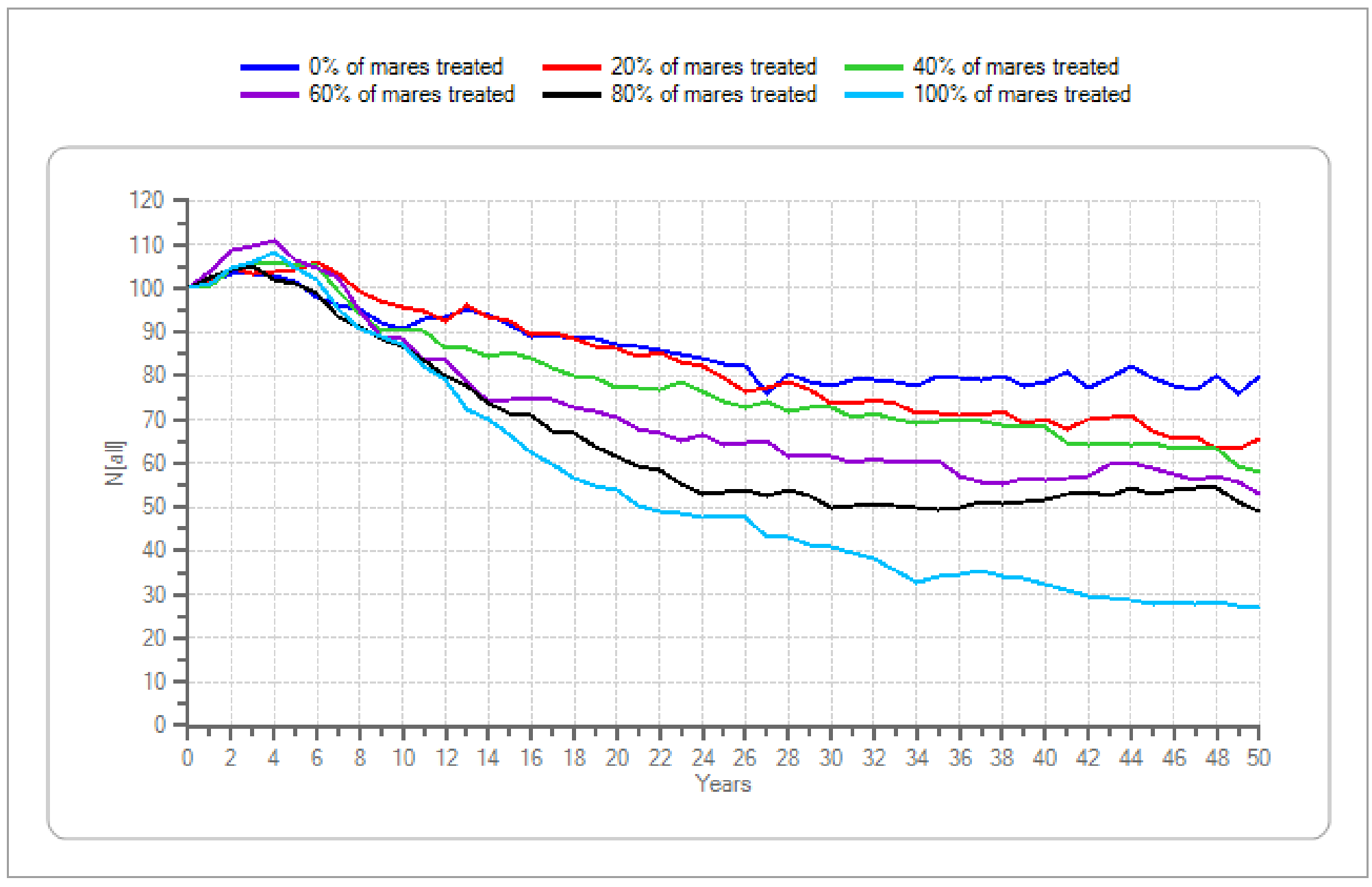

Figure 36. Initial population size $=100$, lambda $=1.05$, genetic diversity $=$ high - Continued 
Mean population size after 20 years

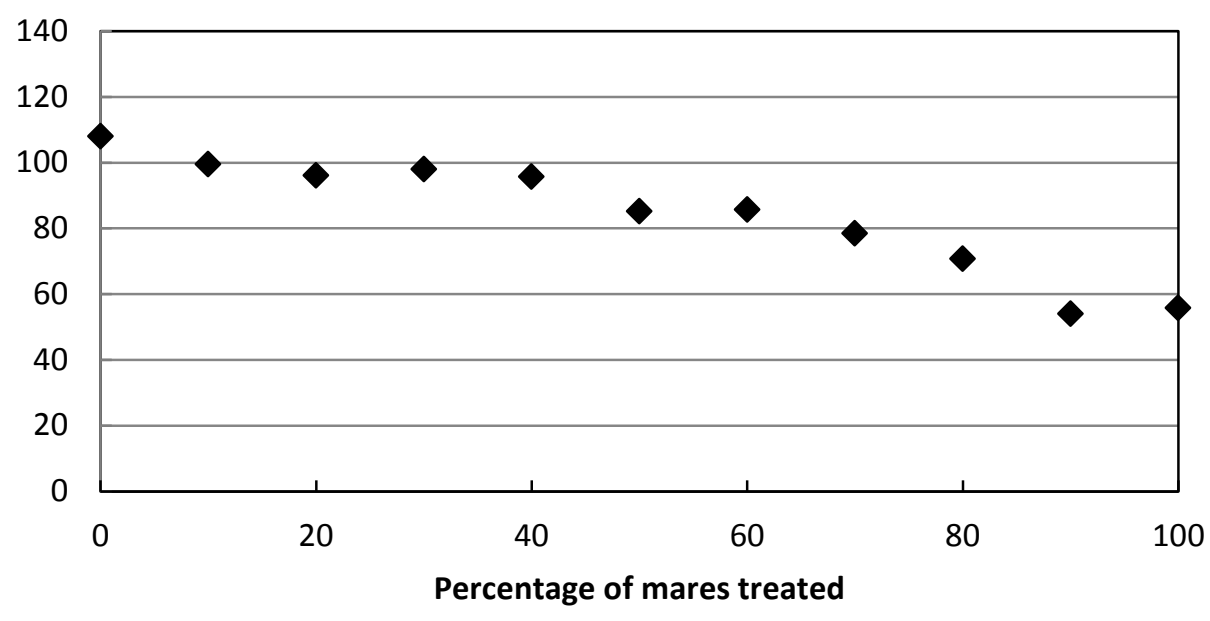

Mean probability of extirpation after $\mathbf{5 0}$ years

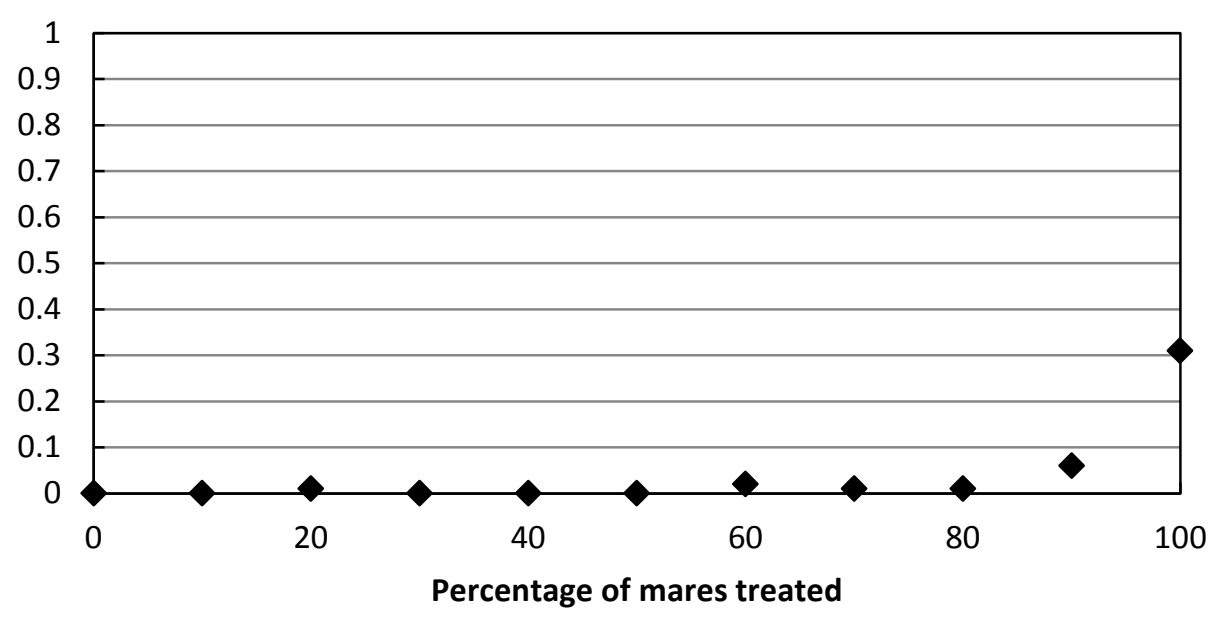

Figure 37. Initial population size $=100$, lambda $=1.10$, genetic diversity $=$ high .
Mean number of removals after 20 years

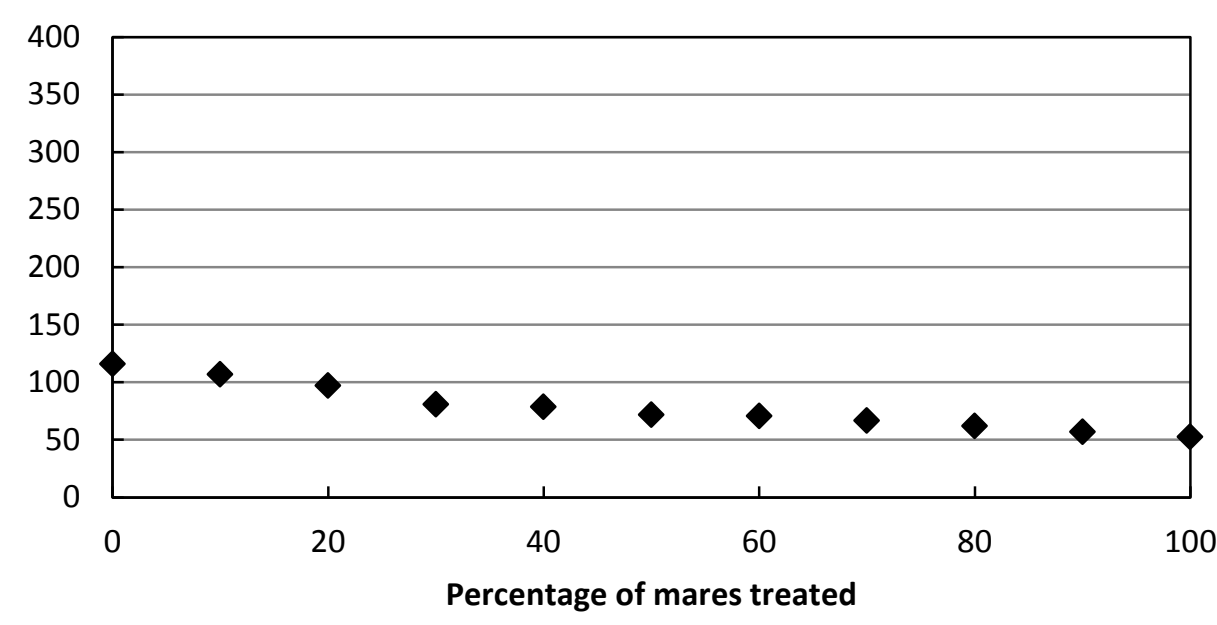

Mean heterozygosity after $\mathbf{5 0}$ years

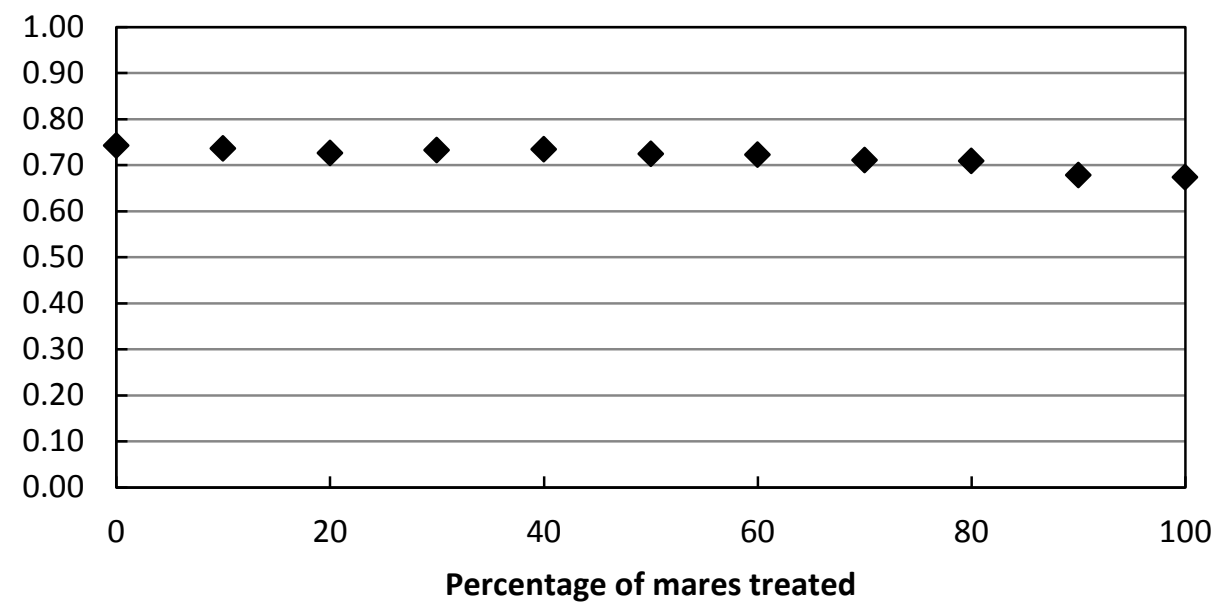




\begin{tabular}{|c|c|c|c|c|c|c|c|c|c|c|c|}
\hline Percentage of mares treated & 0 & 10 & 20 & 30 & 40 & 50 & 60 & 70 & 80 & 90 & 100 \\
\hline Mean population size after 20 years & 107.97 & 99.44 & 96.12 & 98.03 & 95.64 & 85.17 & 85.71 & 78.45 & 70.71 & 53.94 & 55.76 \\
\hline Mean number of removals after 20 years & 115.94 & 106.96 & 96.94 & 80.92 & 78.45 & 71.85 & 70.62 & 66.8 & 62.01 & 56.75 & 52.5 \\
\hline Mean probability of extirpation after 50 years & 0 & 0 & 0.01 & 0 & 0 & 0 & 0.02 & 0.01 & 0.01 & 0.06 & 0.31 \\
\hline Mean heterozygosity after 50 years & 0.74 & 0.74 & 0.73 & 0.73 & 0.73 & 0.72 & 0.72 & 0.71 & 0.71 & 0.68 & 0.67 \\
\hline
\end{tabular}

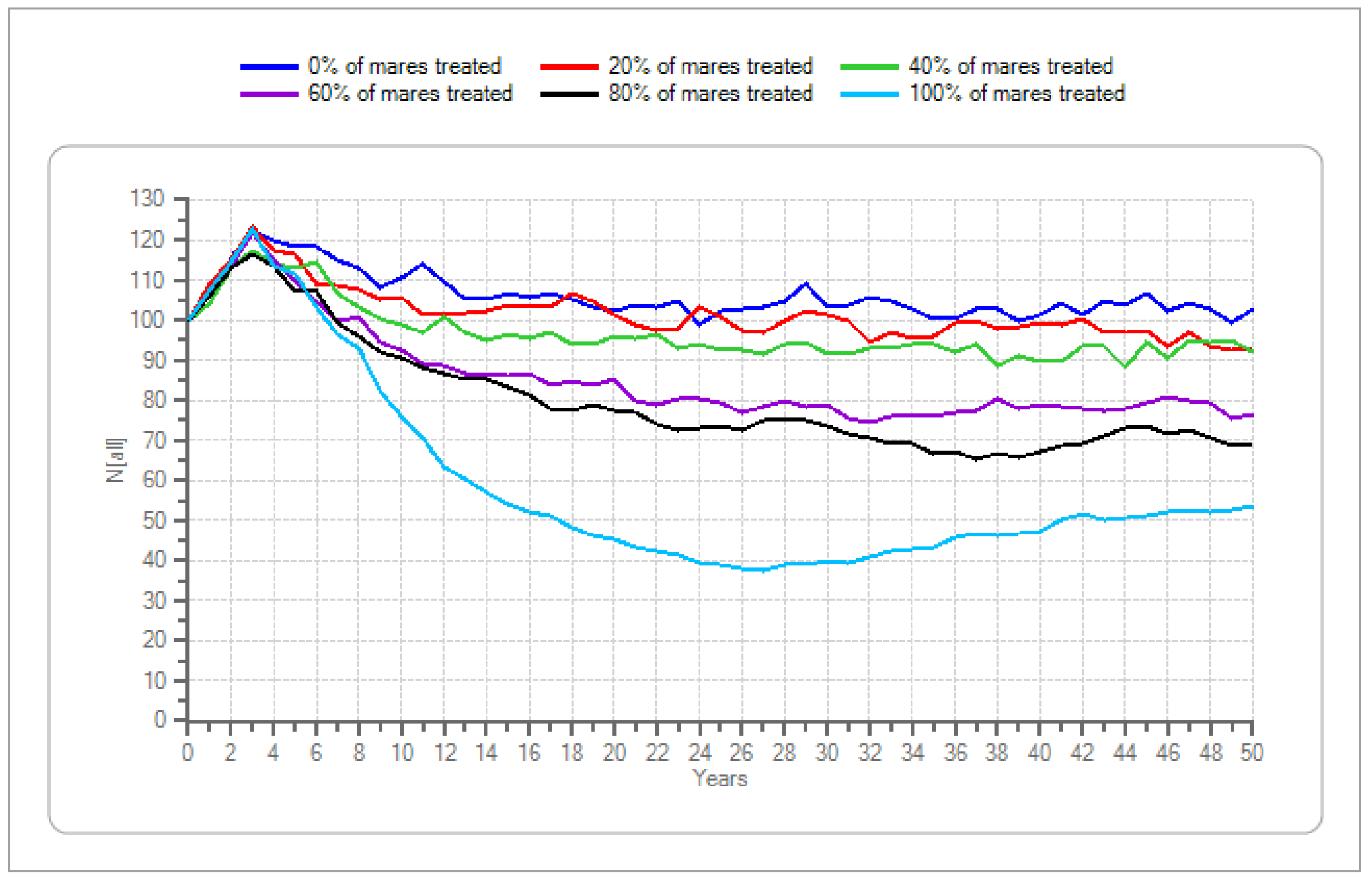

Figure 37. Initial population size $=100$, lambda $=1.10$, genetic diversity $=$ high - Continued 
Mean population size after 20 years

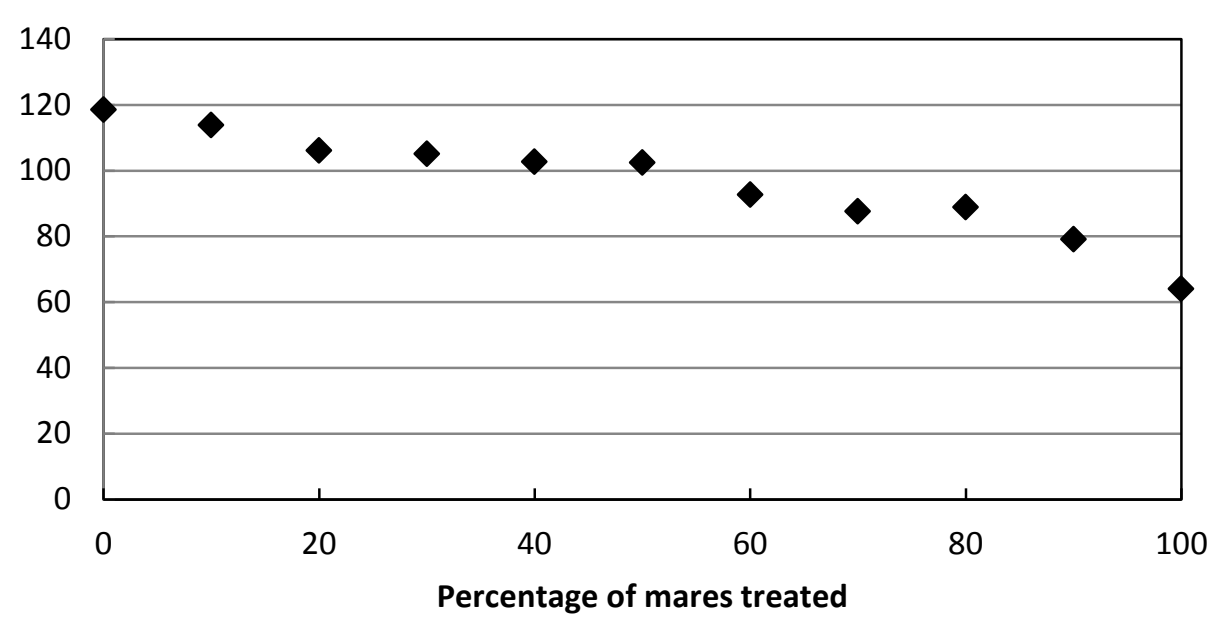

Mean probability of extirpation after $\mathbf{5 0}$ years

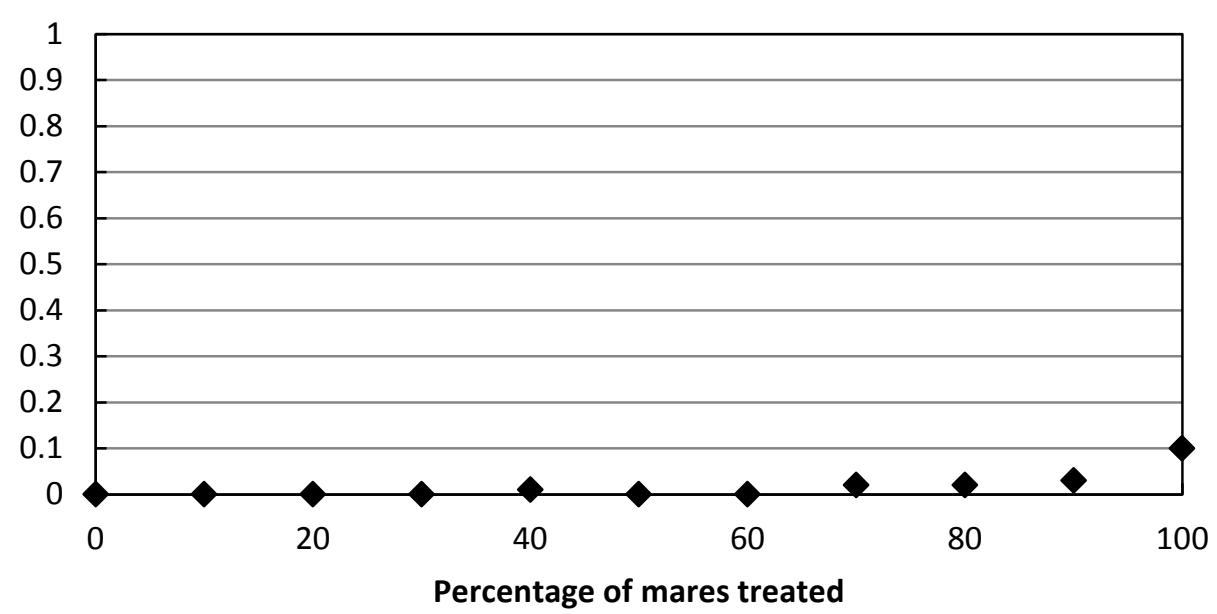

Figure 38. Initial population size $=100$, lambda $=1.15$, genetic diversity $=$ high .
Mean number of removals after 20 years

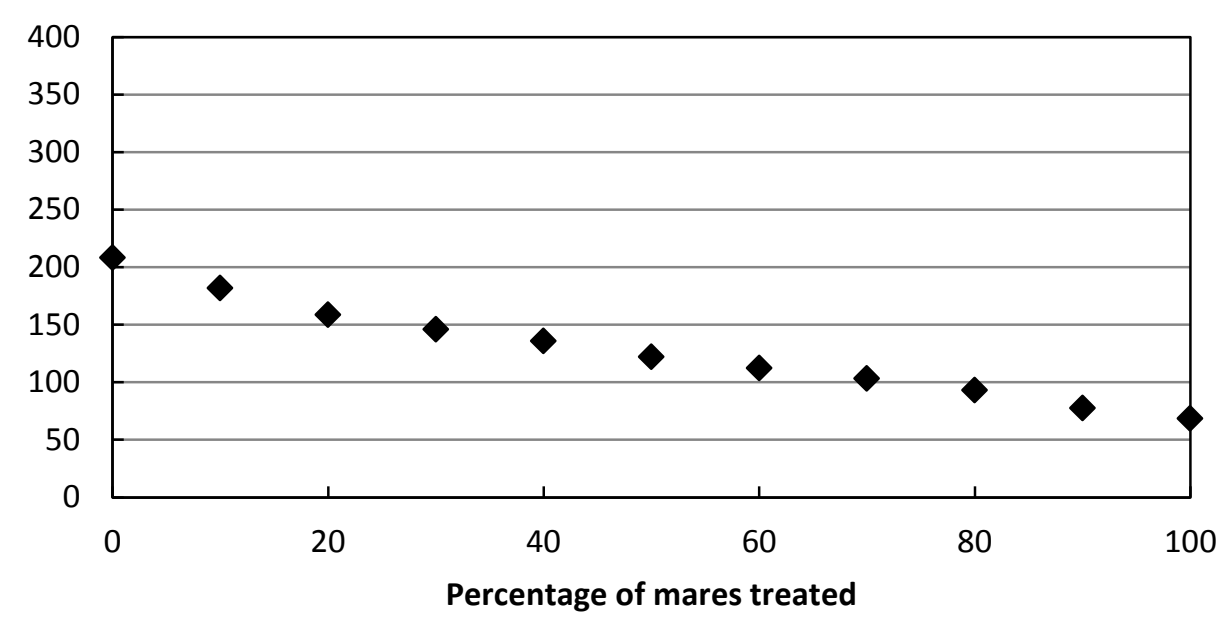

Mean heterozygosity after $\mathbf{5 0}$ years

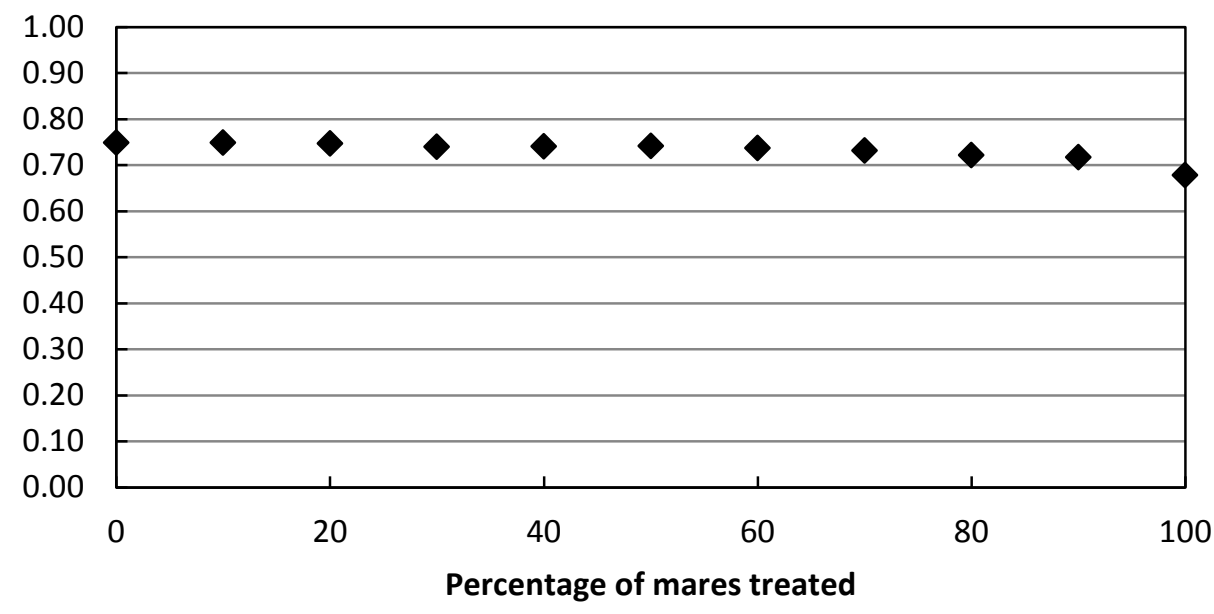




\begin{tabular}{lccccccccccc}
\hline \multicolumn{1}{c}{ Percentage of mares treated } & $\mathbf{0}$ & $\mathbf{1 0}$ & $\mathbf{2 0}$ & $\mathbf{3 0}$ & $\mathbf{4 0}$ & $\mathbf{5 0}$ & $\mathbf{6 0}$ & $\mathbf{7 0}$ & $\mathbf{8 0}$ & $\mathbf{9 0}$ & $\mathbf{1 0 0}$ \\
\hline Mean population size after 20 years & 118.53 & 113.82 & 106.1 & 105.09 & 102.72 & 102.37 & 92.64 & 87.6 & 88.79 & 79.15 & 63.95 \\
Mean number of removals after 20 years & 208.4 & 181.74 & 158.65 & 145.93 & 135.73 & 121.88 & 112.21 & 103.11 & 93.06 & 77.44 & 68.55 \\
Mean probability of extirpation after 50 years & 0 & 0 & 0 & 0 & 0.01 & 0 & 0 & 0.02 & 0.02 & 0.03 & 0.1 \\
Mean heterozygosity after 50 years & 0.75 & 0.75 & 0.75 & 0.74 & 0.74 & 0.74 & 0.74 & 0.73 & 0.72 & 0.72 & 0.68 \\
\hline
\end{tabular}

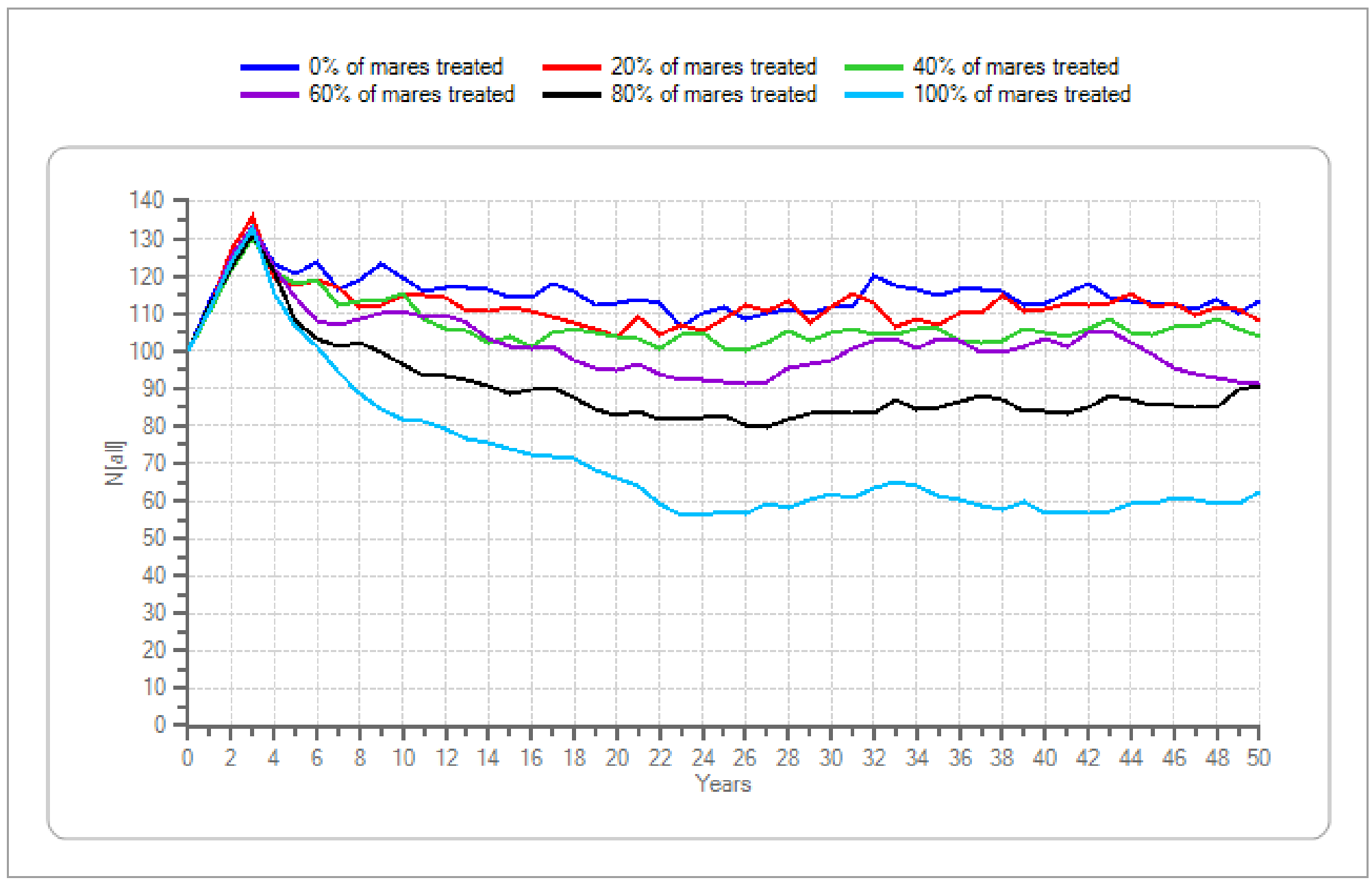

Figure 38. Initial population size $=100$, lambda $=1.15$, genetic diversity $=$ high - Continued 
Mean population size after 20 years

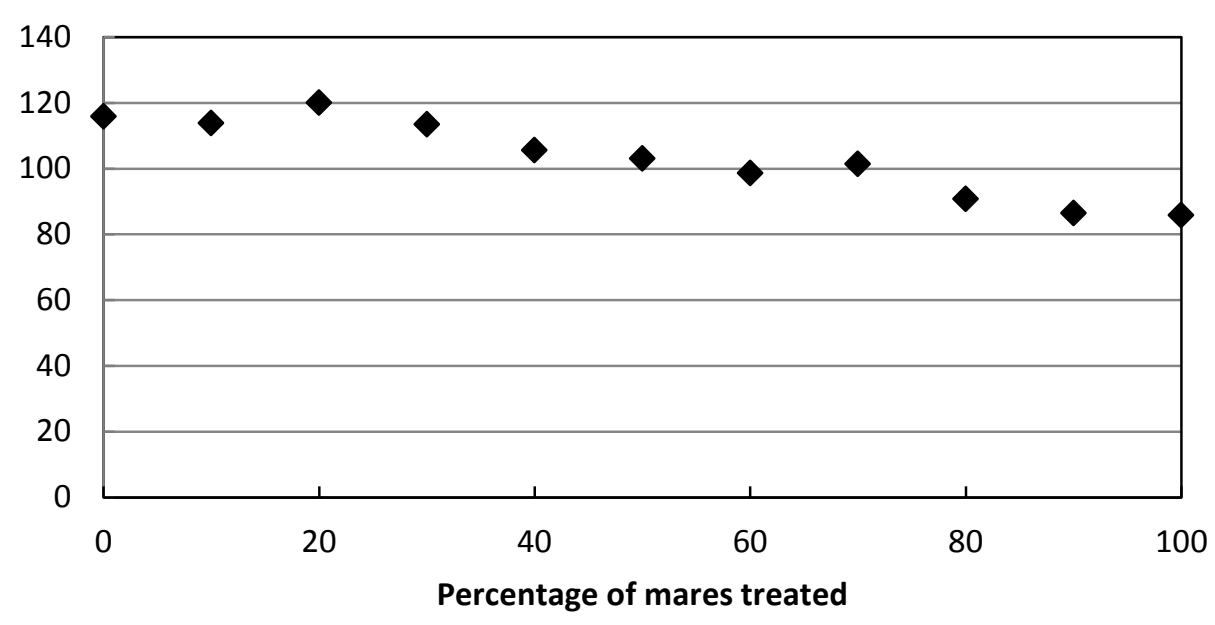

Mean probability of extirpation after $\mathbf{5 0}$ years

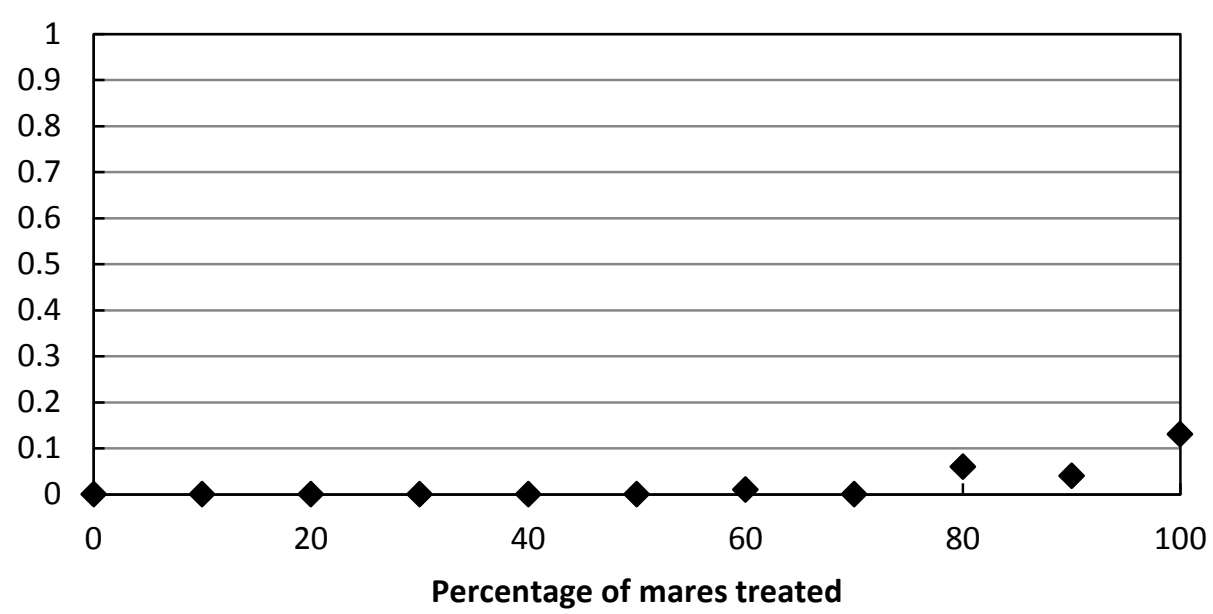

Figure 39. Initial population size $=100$, lambda $=1.20$, genetic diversity $=$ high .
Mean number of removals after 20 years

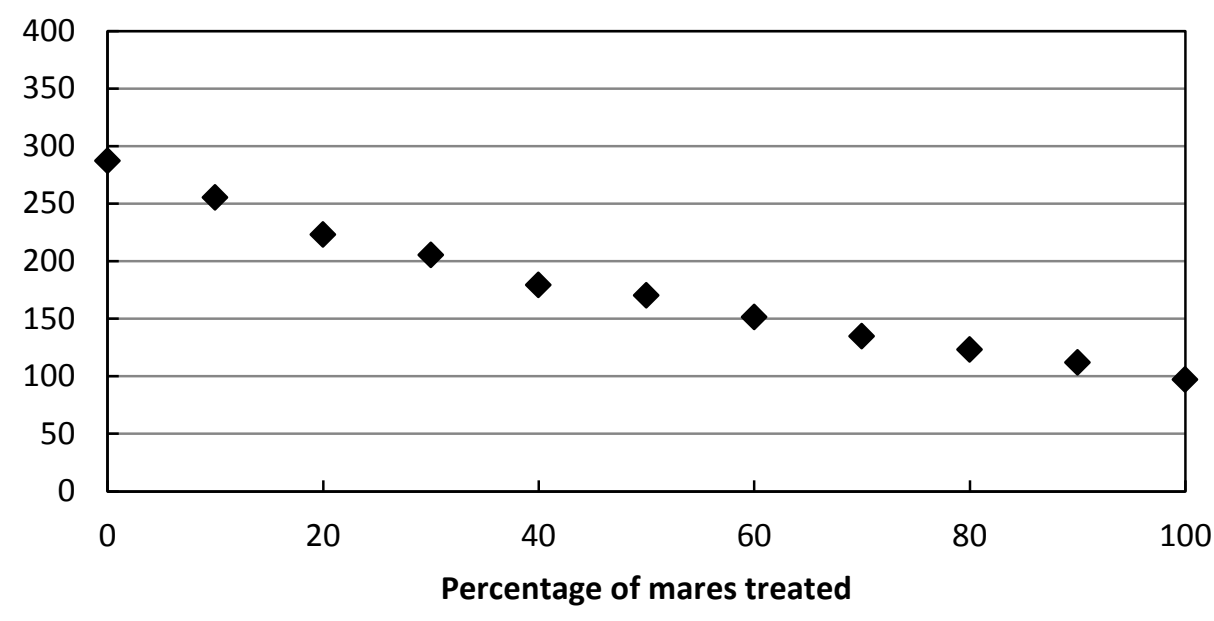

Mean heterozygosity after $\mathbf{5 0}$ years

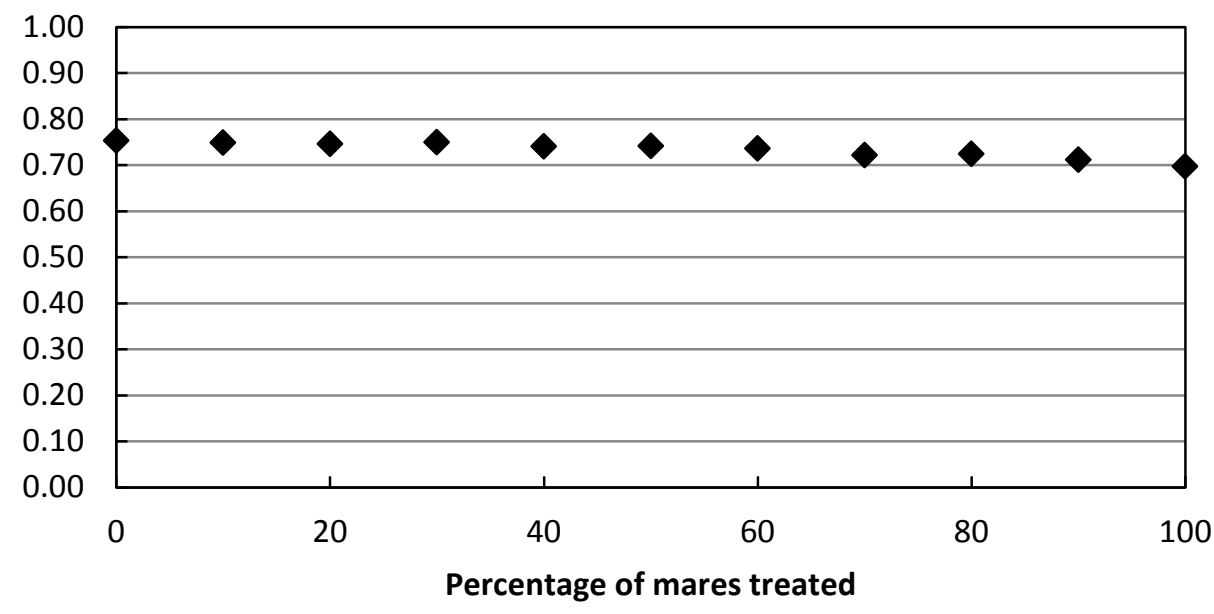




\begin{tabular}{|c|c|c|c|c|c|c|c|c|c|c|c|}
\hline Percentage of mares treated & 0 & 10 & 20 & 30 & 40 & 50 & 60 & 70 & 80 & 90 & 100 \\
\hline Mean population size after 20 years & 115.87 & 113.82 & 120.06 & 113.47 & 105.64 & 102.99 & 98.66 & 101.46 & 90.77 & 86.41 & 85.86 \\
\hline Mean number of removals after 20 years & 287.24 & 255.15 & 222.93 & 205.42 & 179.11 & 170.34 & 151.32 & 134.9 & 123.26 & 112.03 & 97.01 \\
\hline Mean probability of extirpation after 50 years & 0 & 0 & 0 & 0 & 0 & 0 & 0.01 & 0 & 0.06 & 0.04 & 0.13 \\
\hline Mean heterozygosity after 50 years & 0.75 & 0.75 & 0.75 & 0.75 & 0.74 & 0.74 & 0.74 & 0.72 & 0.72 & 0.71 & 0.70 \\
\hline
\end{tabular}

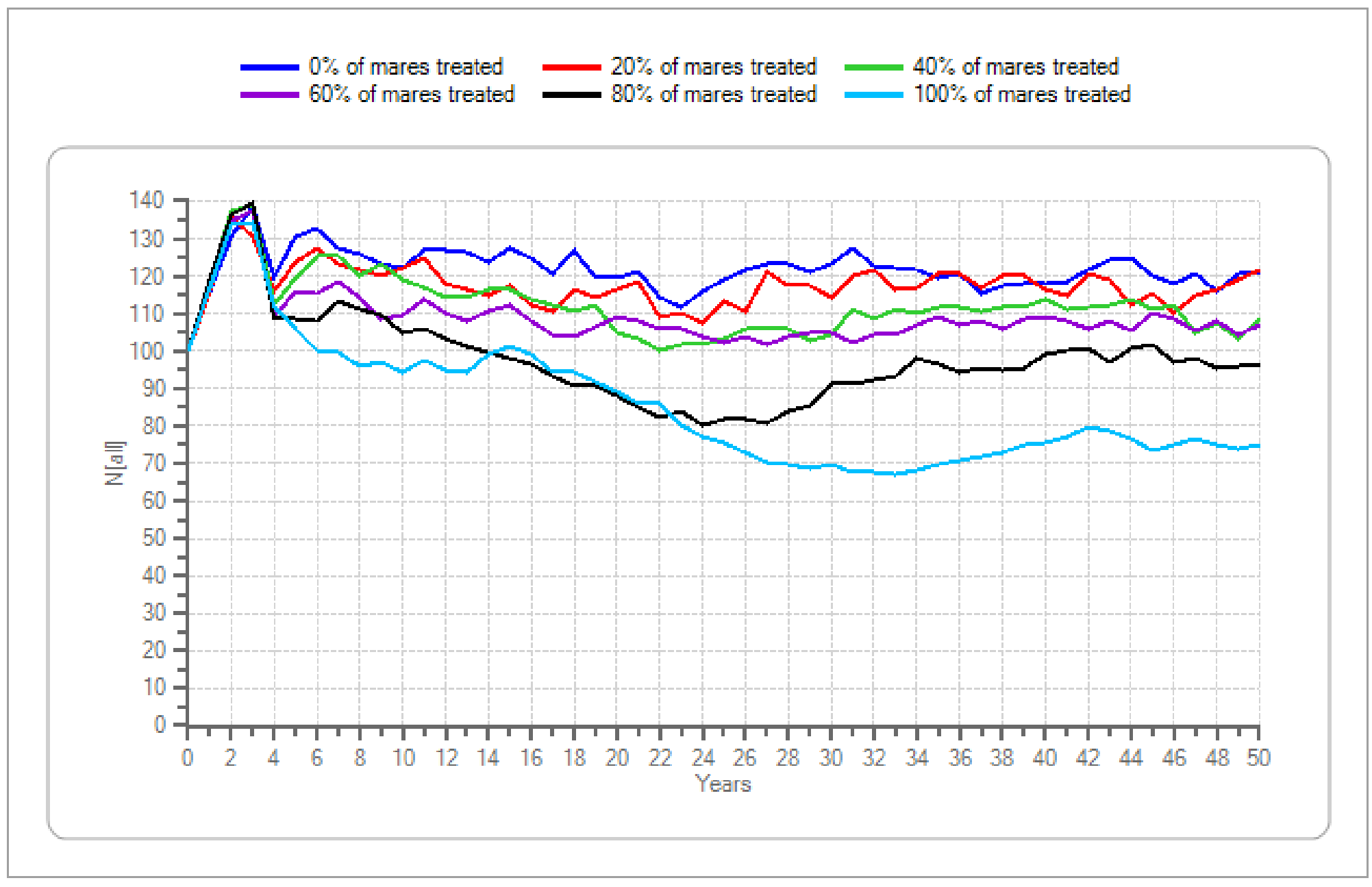

Figure 39. Initial population size $=100$, lambda $=1.20$, genetic diversity $=$ high - Continued 
Mean population size after 20 years

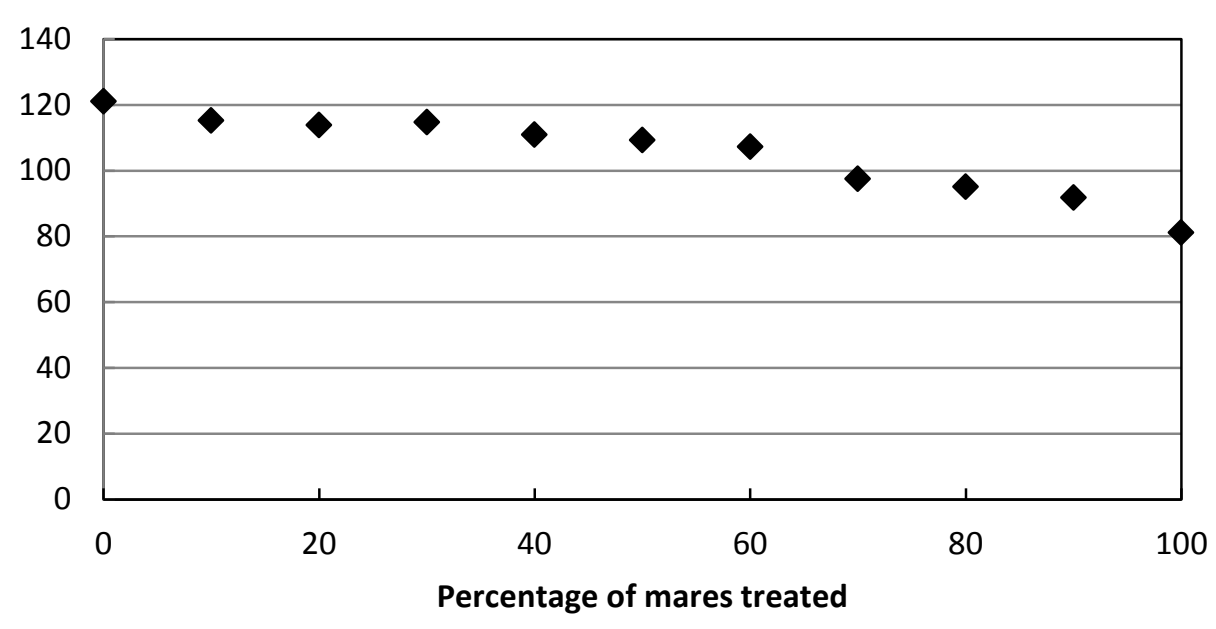

Mean probability of extirpation after $\mathbf{5 0}$ years

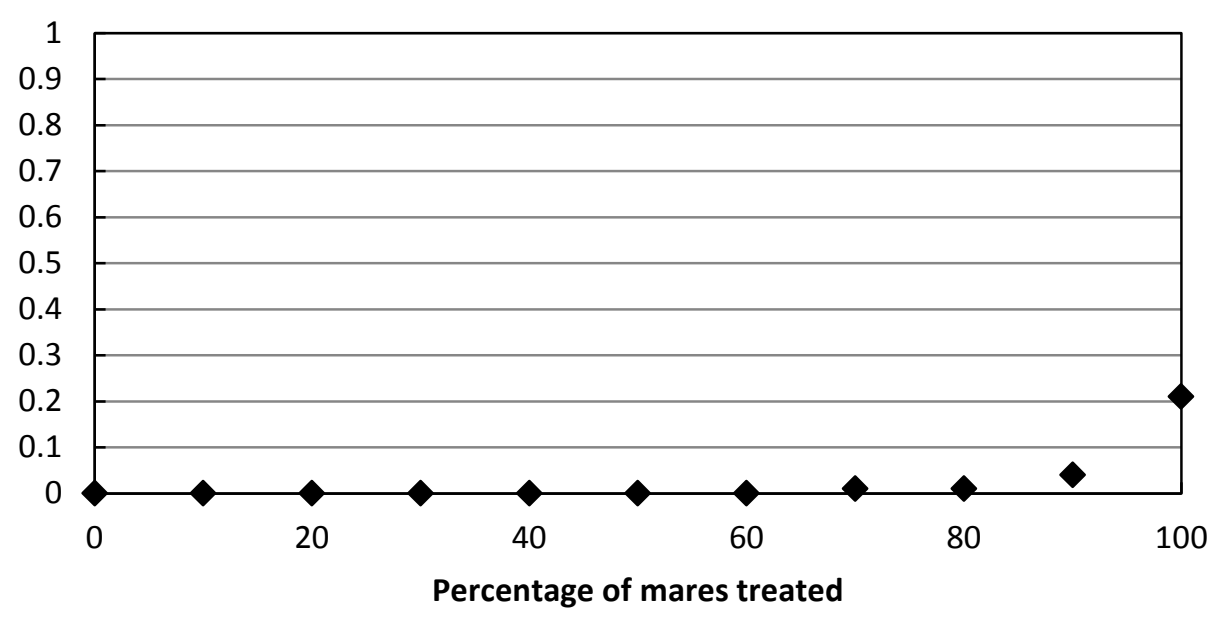

Figure 40. Initial population size $=100$, lambda $=1.25$, genetic diversity $=$ high .
Mean number of removals after 20 years

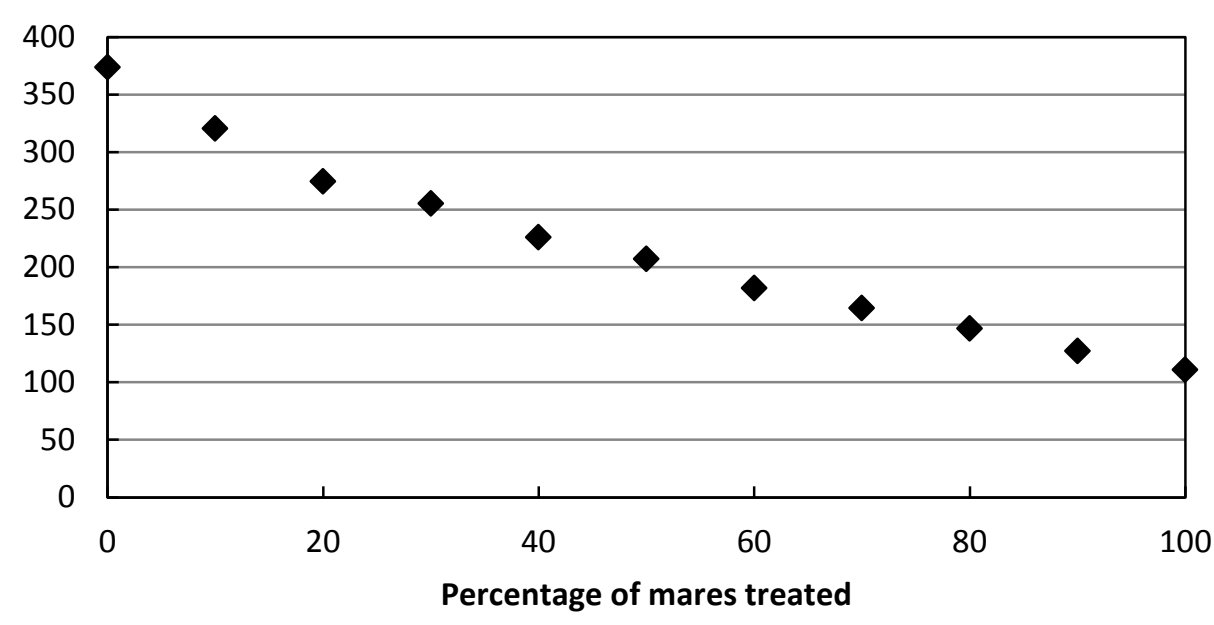

Mean heterozygosity after 50 years

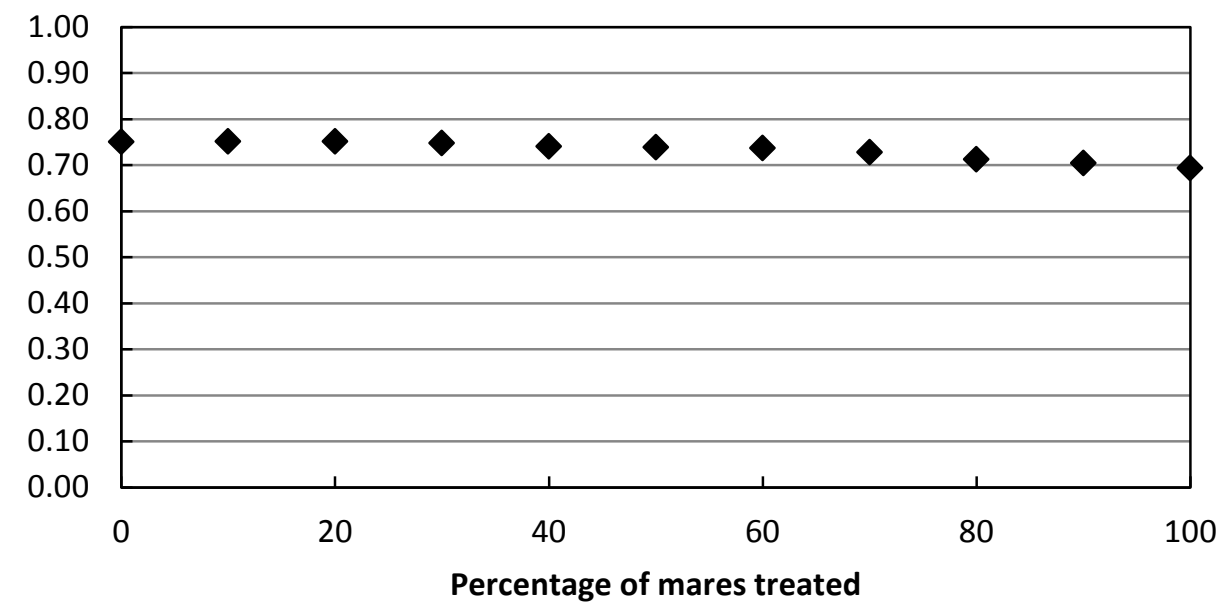




\begin{tabular}{lccccccccccc}
\hline \multicolumn{1}{c}{ Percentage of mares treated } & $\mathbf{0}$ & $\mathbf{1 0}$ & $\mathbf{2 0}$ & $\mathbf{3 0}$ & $\mathbf{4 0}$ & $\mathbf{5 0}$ & $\mathbf{6 0}$ & $\mathbf{7 0}$ & $\mathbf{8 0}$ & $\mathbf{9 0}$ & $\mathbf{1 0 0}$ \\
\hline Mean population size after 20 years & 121.02 & 115.17 & 113.84 & 114.66 & 110.88 & 109.29 & 107.18 & 97.48 & 95.07 & 91.82 & 81.06 \\
Mean number of removals after 20 years & 373.76 & 320.53 & 274.59 & 255.43 & 225.87 & 207.24 & 181.97 & 164.56 & 146.74 & 127.02 & 110.7 \\
Mean probability of extirpation after 50 years & 0 & 0 & 0 & 0 & 0 & 0 & 0 & 0.01 & 0.01 & 0.04 & 0.21 \\
Mean heterozygosity after 50 years & 0.75 & 0.75 & 0.75 & 0.75 & 0.74 & 0.74 & 0.74 & 0.73 & 0.71 & 0.70 & 0.69 \\
\hline
\end{tabular}

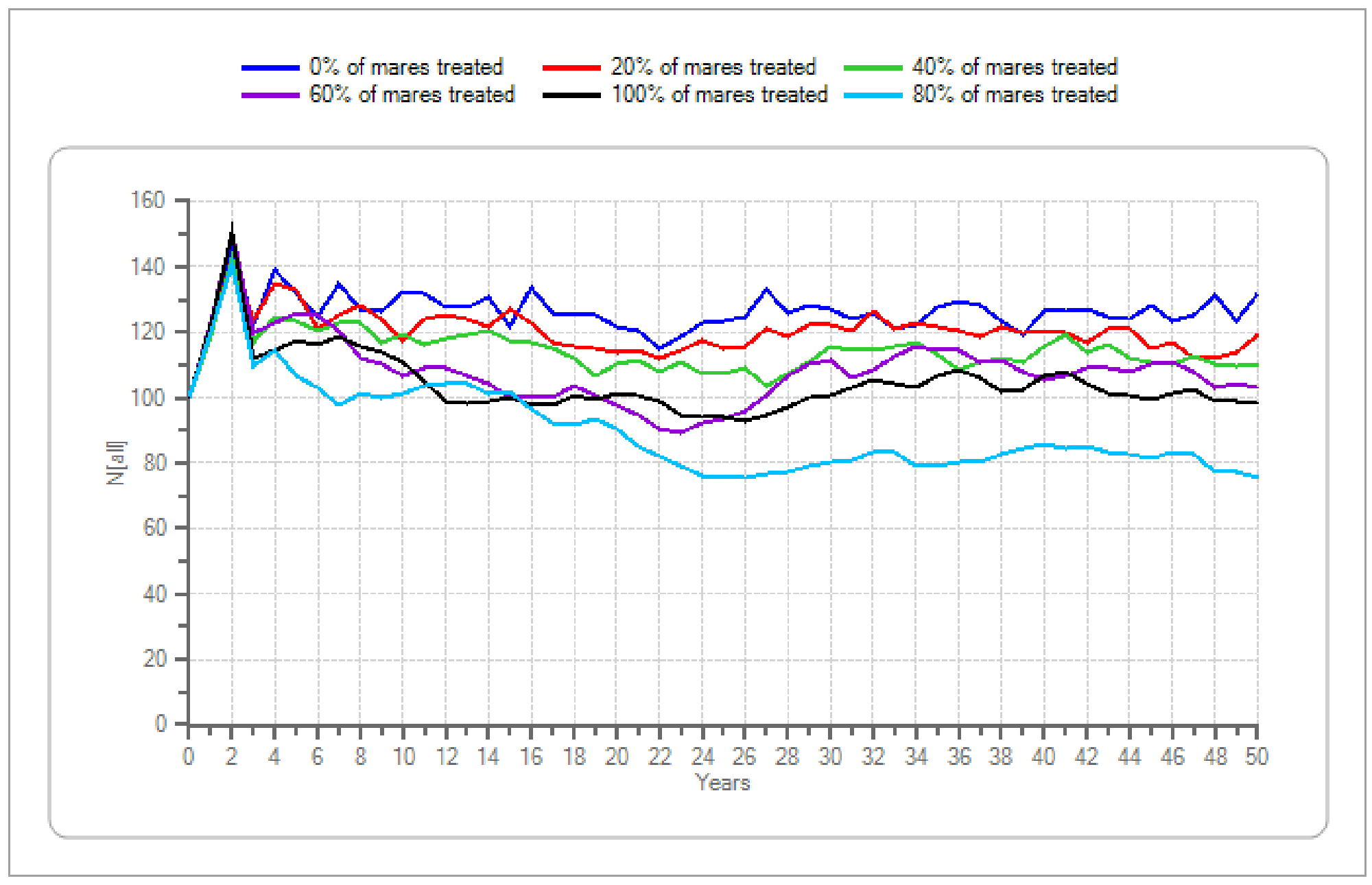

Figure 40. Initial population size $=100$, lambda $=1.25$, genetic diversity $=$ high. Continued 
Mean population size after 20 years

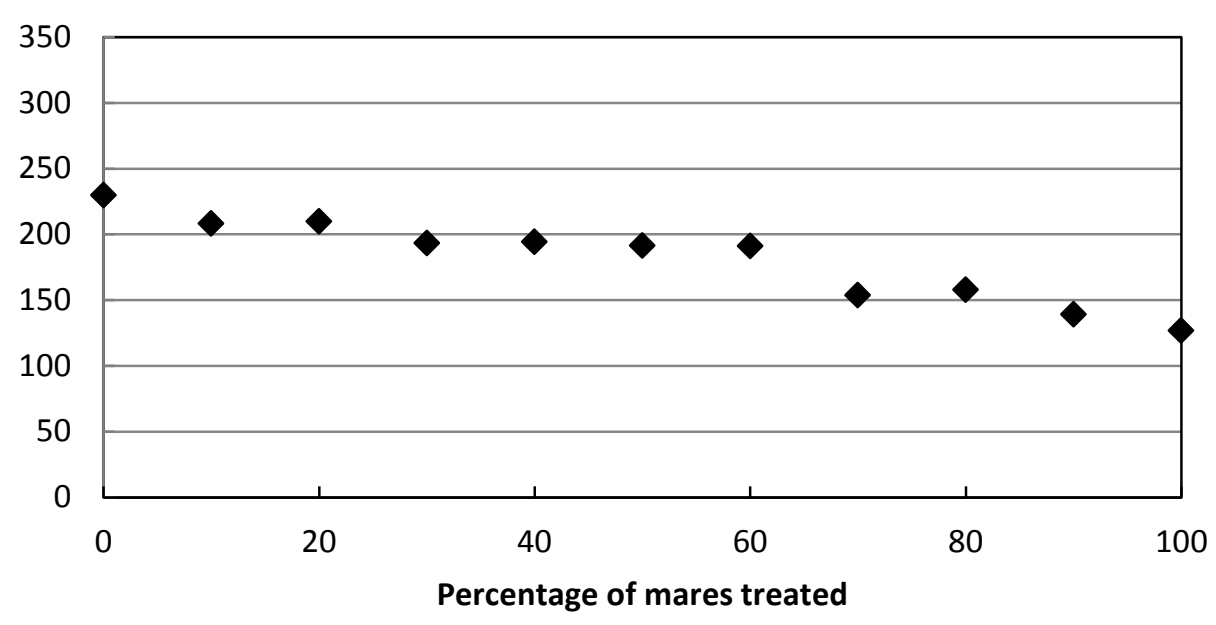

Mean probability of extirpation after $\mathbf{5 0}$ years

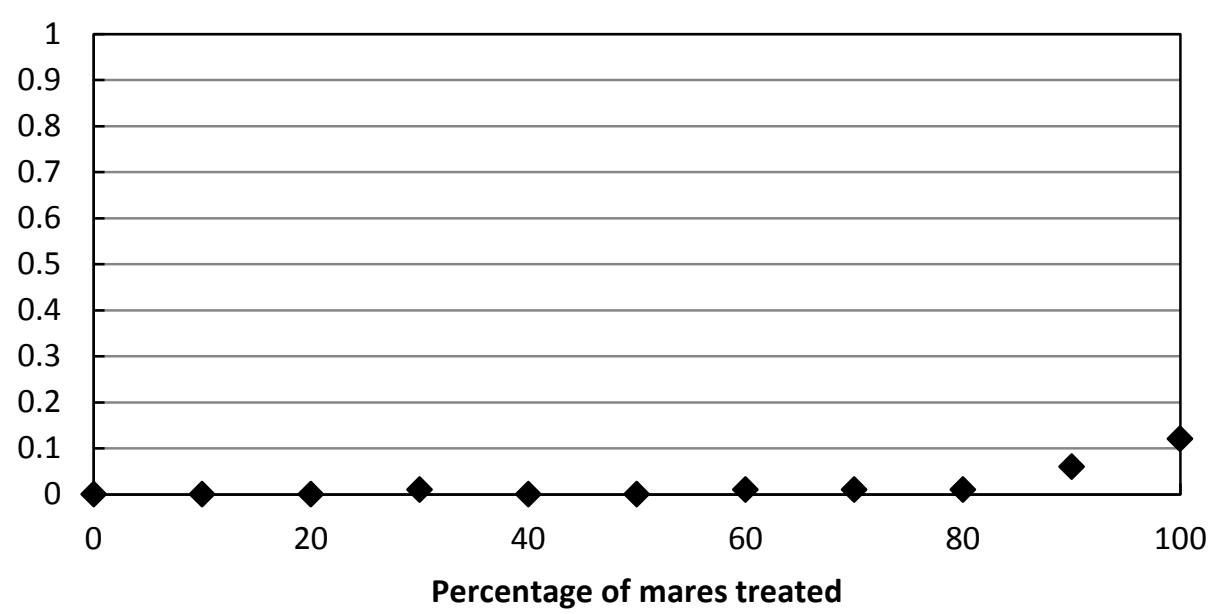

Figure 41. Initial population size $=250$, lambda $=1.05$, genetic diversity $=$ high .
Mean number of removals after 20 years

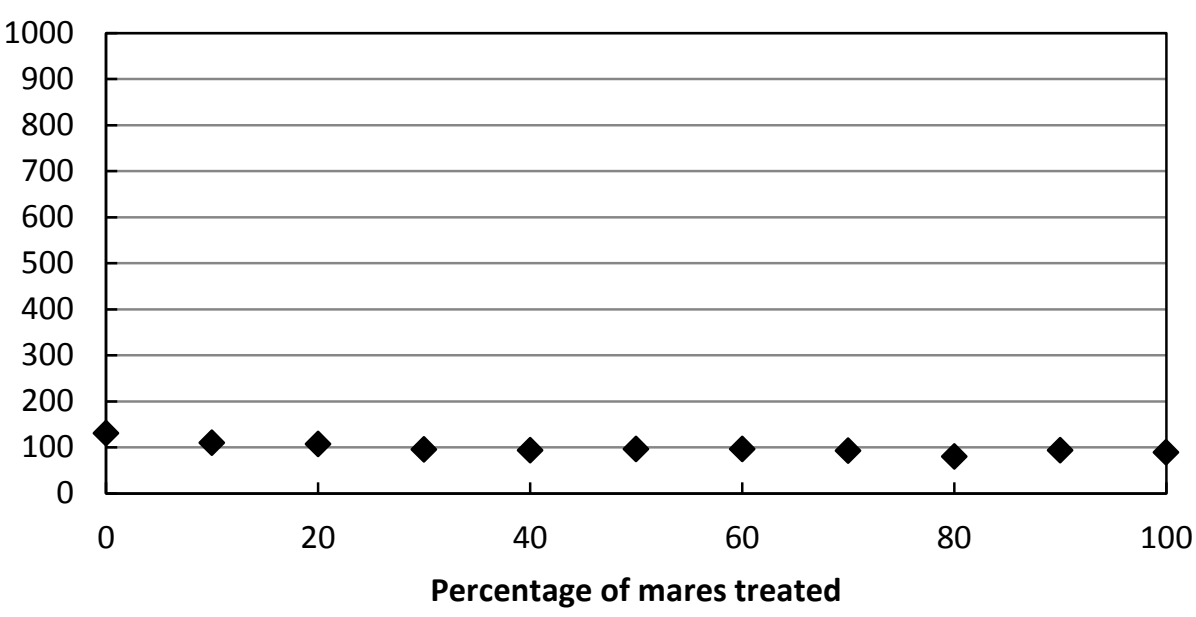

Mean heterozygosity after $\mathbf{5 0}$ years

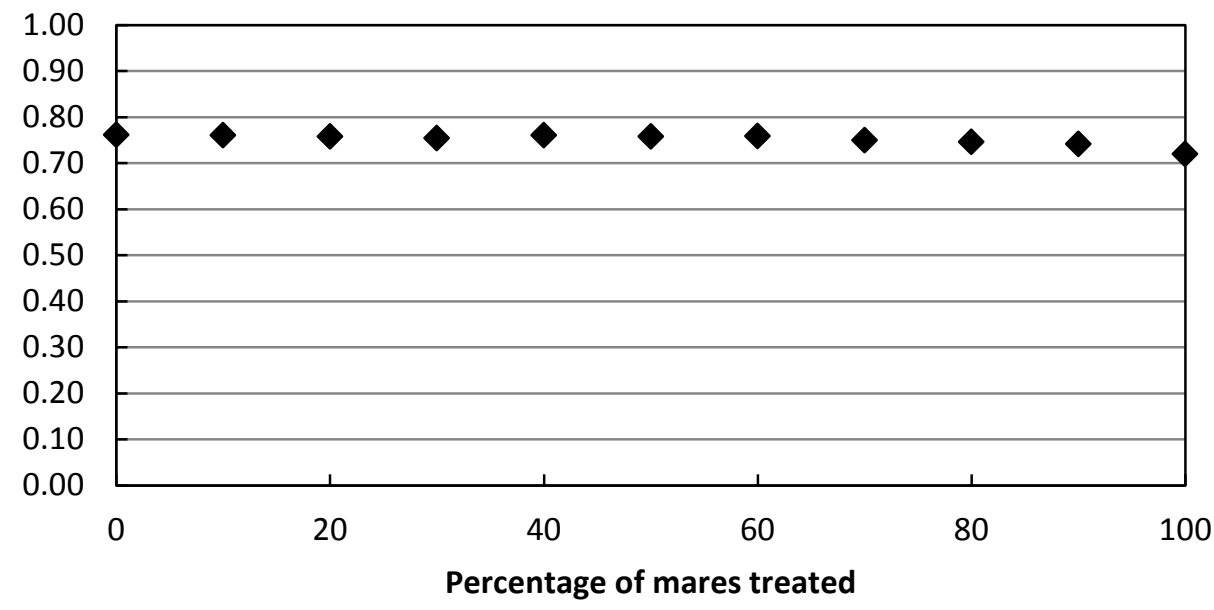




\begin{tabular}{lccccccccccc}
\hline \multicolumn{1}{c}{ Percentage of mares treated } & $\mathbf{0}$ & $\mathbf{1 0}$ & $\mathbf{2 0}$ & $\mathbf{3 0}$ & $\mathbf{4 0}$ & $\mathbf{5 0}$ & $\mathbf{6 0}$ & $\mathbf{7 0}$ & $\mathbf{8 0}$ & $\mathbf{9 0}$ & $\mathbf{1 0 0}$ \\
\hline Mean population size after 20 years & 229.82 & 208.28 & 209.81 & 193.14 & 194.37 & 191.52 & 191.17 & 153.65 & 157.95 & 139.22 & 126.74 \\
Mean number of removals after 20 years & 130.33 & 109.21 & 106.87 & 95.15 & 93.21 & 95.93 & 96.1 & 91.98 & 79.41 & 93.08 & 89.13 \\
Mean probability of extirpation after 50 years & 0 & 0 & 0 & 0.01 & 0 & 0 & 0.01 & 0.01 & 0.01 & 0.06 & 0.12 \\
Mean heterozygosity after 50 years & 0.76 & 0.76 & 0.76 & 0.75 & 0.76 & 0.76 & 0.76 & 0.75 & 0.75 & 0.74 & 0.72 \\
\hline
\end{tabular}

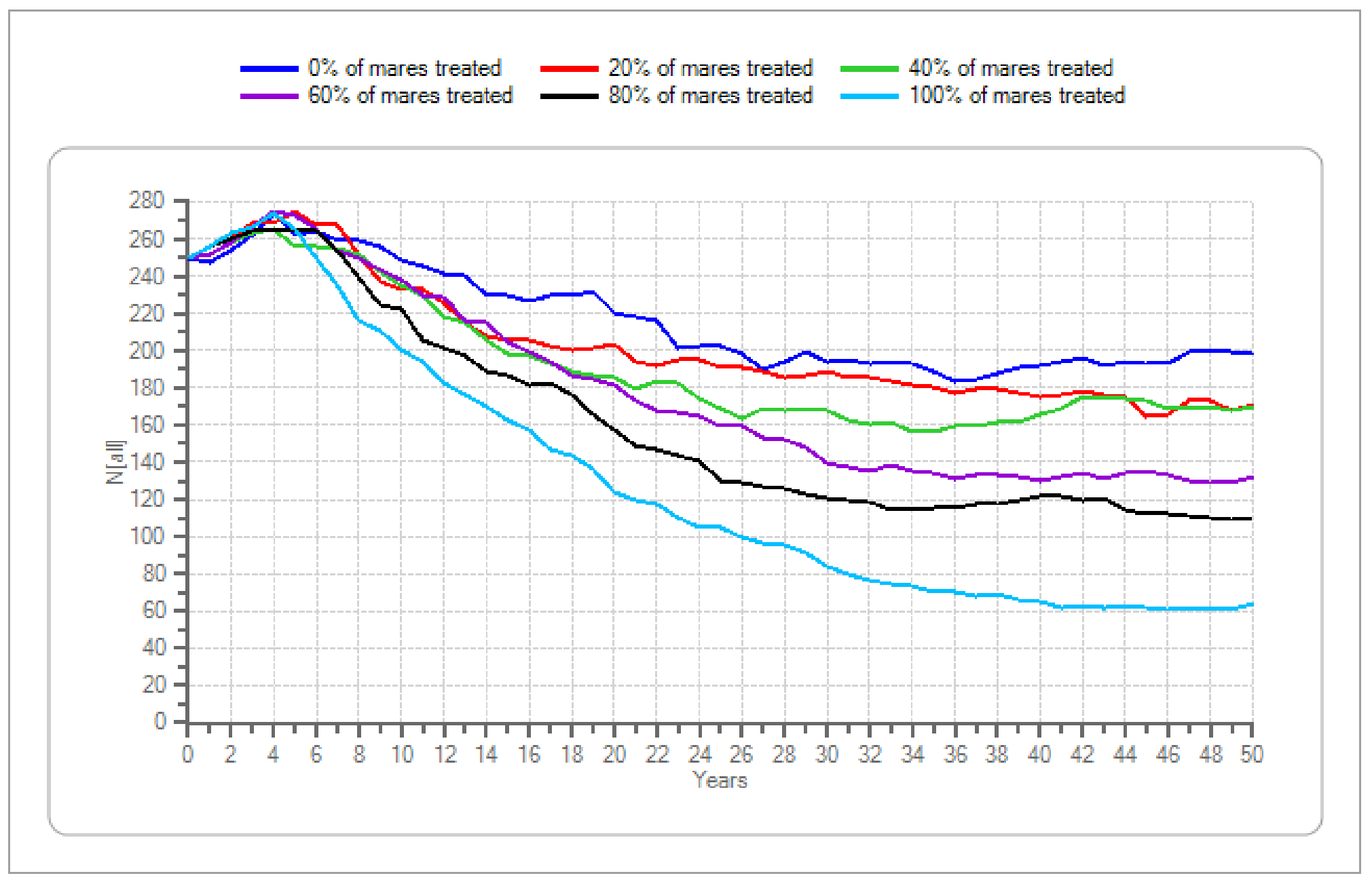

Figure 41. Initial population size $=250$, lambda $=1.05$, genetic diversity $=$ high. - Continued 
Mean population size after 20 years

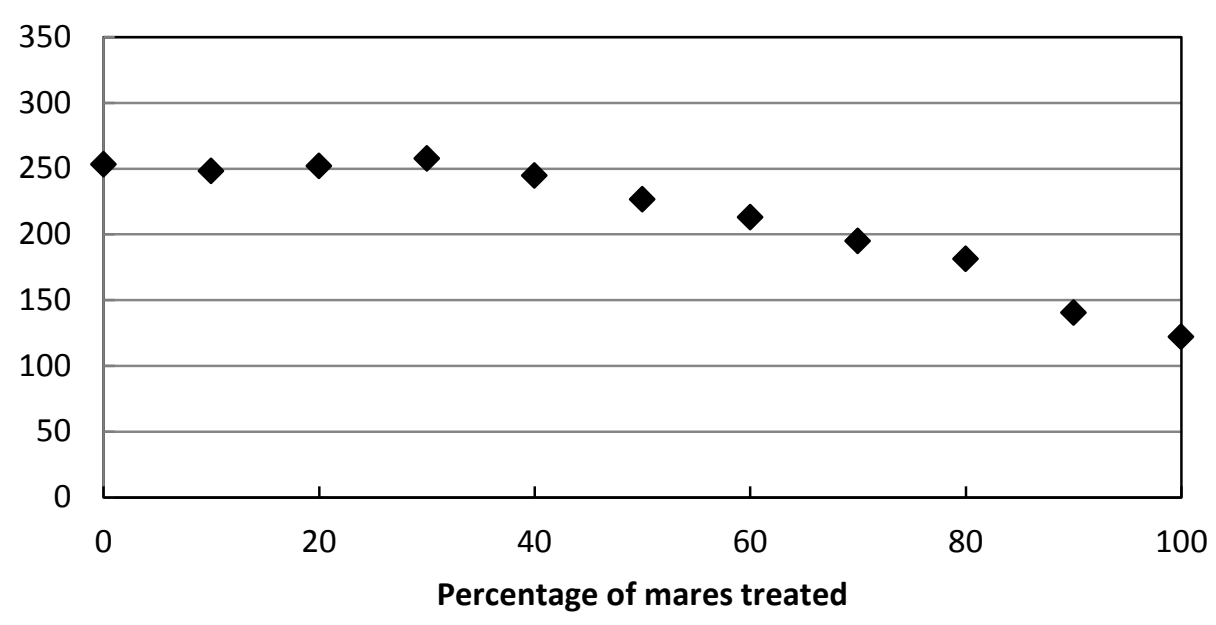

Mean probability of extirpation after $\mathbf{5 0}$ years

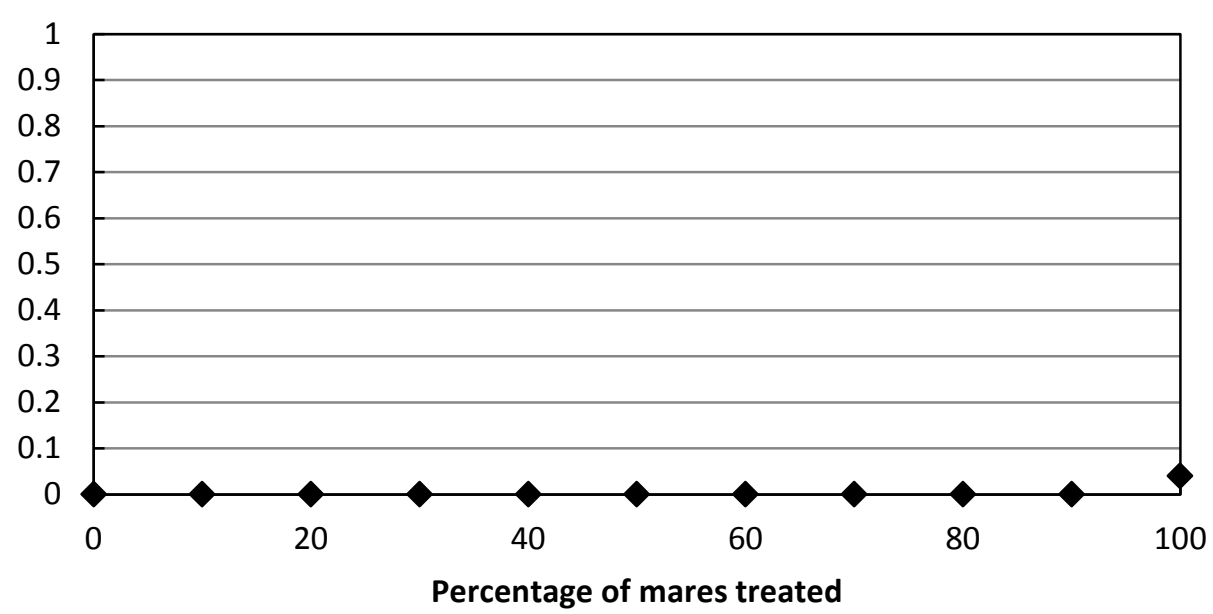

Figure 42. Initial population size $=250$, lambda $=1.10$, genetic diversity $=$ high .
Mean number of removals after 20 years

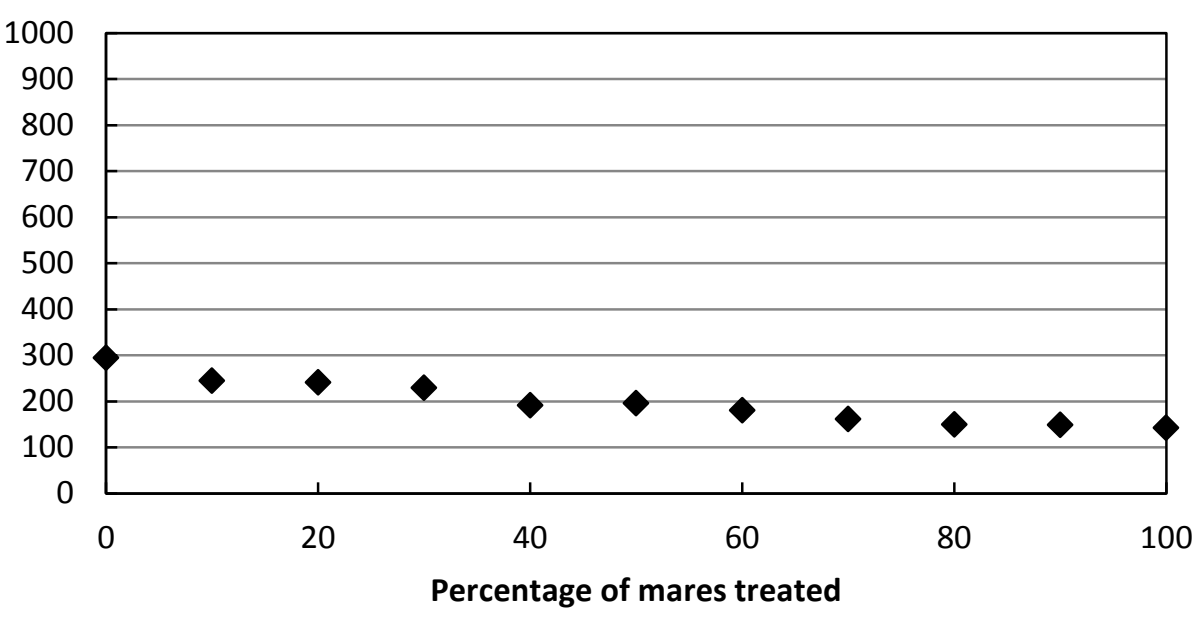

Mean heterozygosity after $\mathbf{5 0}$ years

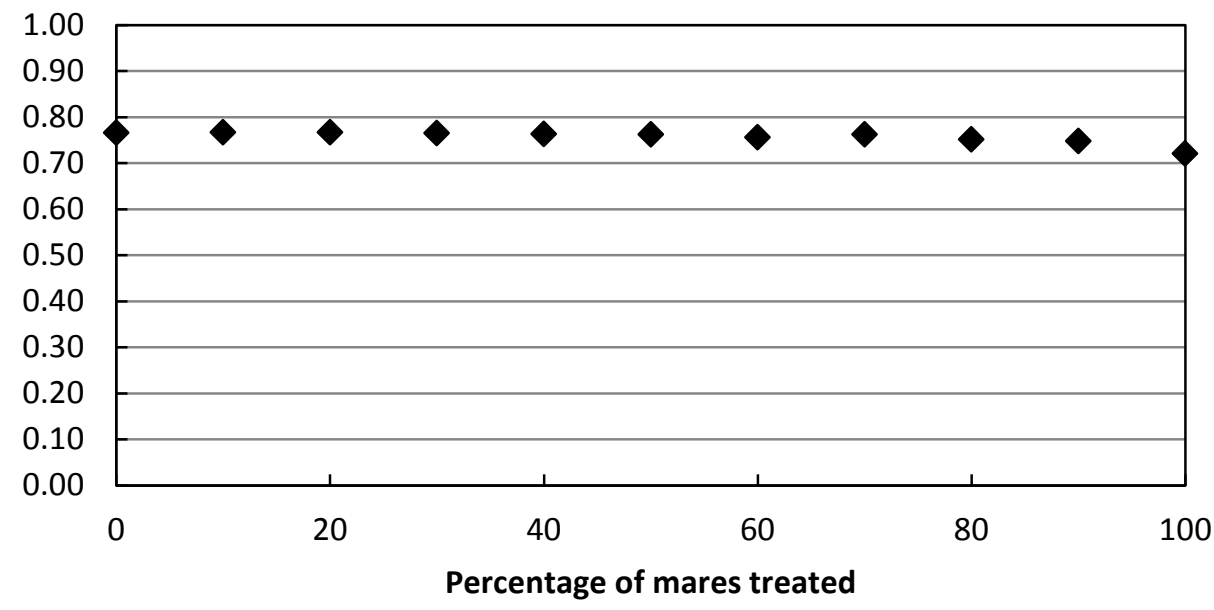




\begin{tabular}{lccccccccccc}
\hline \multicolumn{1}{c}{ Percentage of mares treated } & $\mathbf{0}$ & $\mathbf{1 0}$ & $\mathbf{2 0}$ & $\mathbf{3 0}$ & $\mathbf{4 0}$ & $\mathbf{5 0}$ & $\mathbf{6 0}$ & $\mathbf{7 0}$ & $\mathbf{8 0}$ & $\mathbf{9 0}$ & $\mathbf{1 0 0}$ \\
\hline Mean population size after 20 years & 253.29 & 248.13 & 251.86 & 257.66 & 244.53 & 226.55 & 212.8 & 194.92 & 181.23 & 140.26 & 121.89 \\
Mean number of removals after 20 years & 294.42 & 244.72 & 240.7 & 229.15 & 190.87 & 195.41 & 180.34 & 161.04 & 149.36 & 148.76 & 141.8 \\
Mean probability of extirpation after 50 years & 0 & 0 & 0 & 0 & 0 & 0 & 0 & 0 & 0 & 0 & 0.04 \\
Mean heterozygosity after 50 years & 0.77 & 0.77 & 0.77 & 0.77 & 0.76 & 0.76 & 0.76 & 0.76 & 0.75 & 0.75 & 0.72 \\
\hline
\end{tabular}

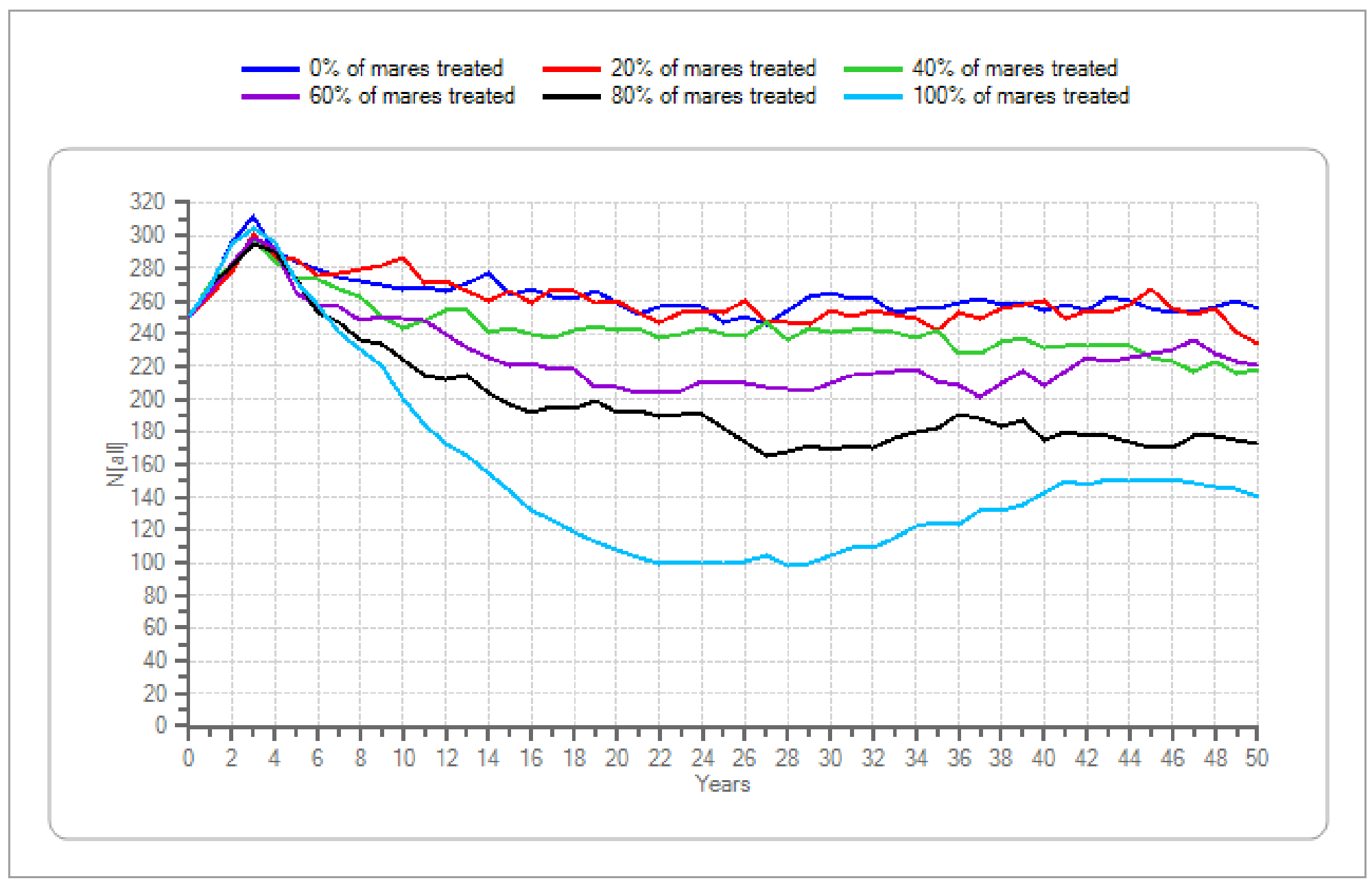

Figure 42. Initial population size $=250$, lambda $=1.10$, genetic diversity $=$ high. Continued 
Mean population size after 20 years

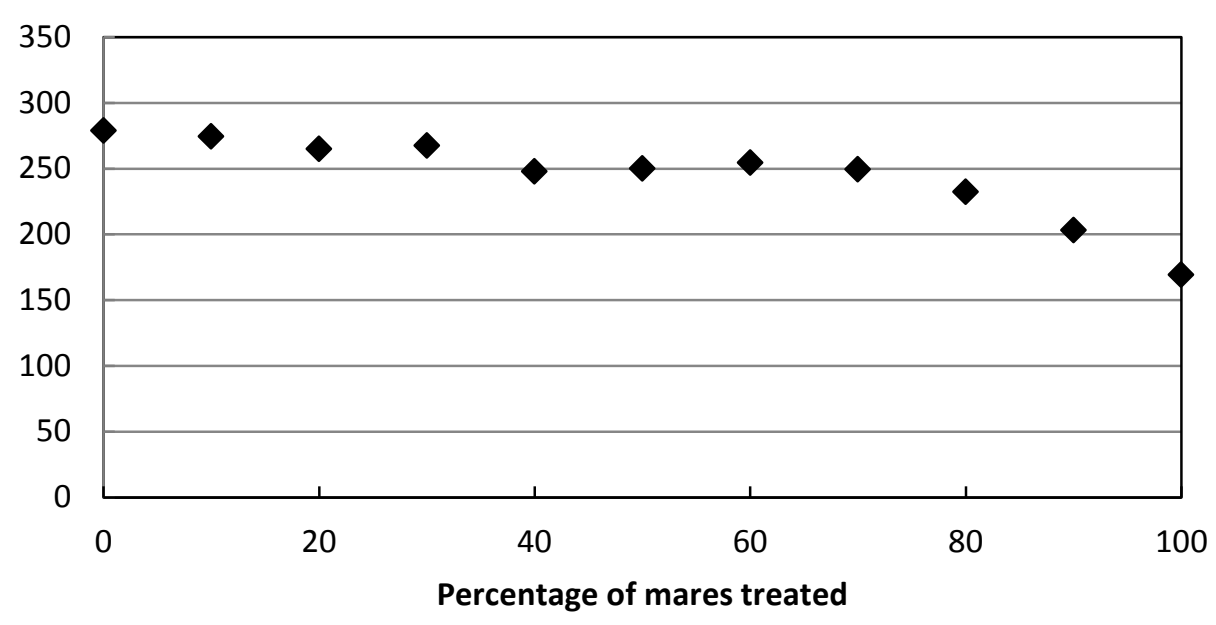

Mean probability of extirpation after $\mathbf{5 0}$ years

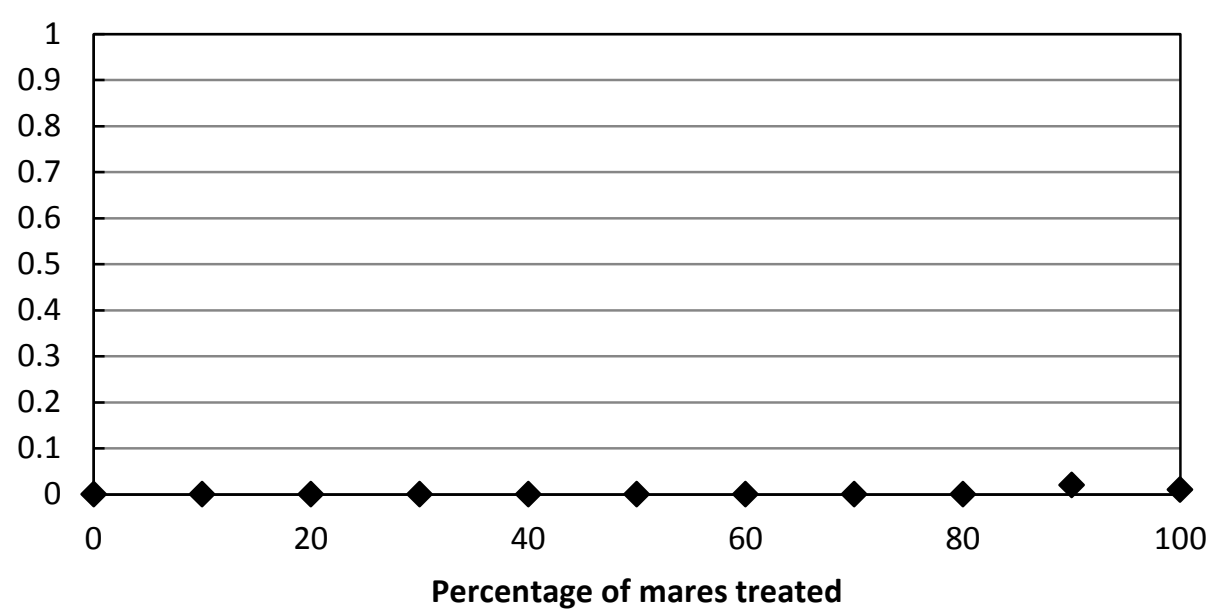

Figure 43. Initial population size $=250$, lambda $=1.15$, genetic diversity $=$ high .
Mean number of removals after 20 years

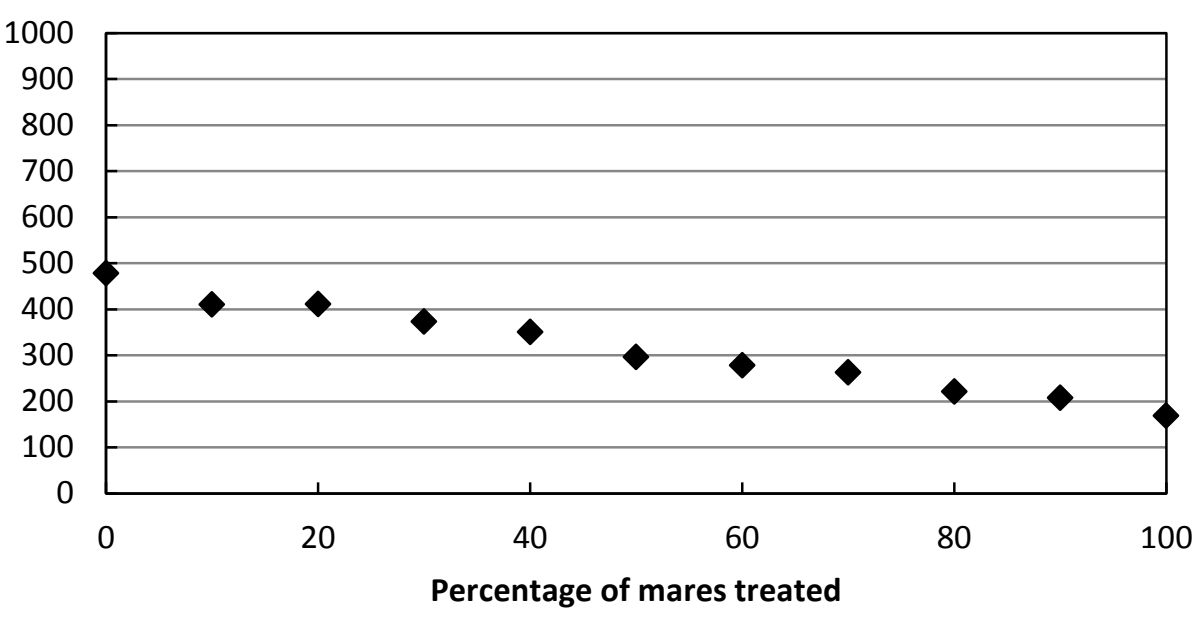

Mean heterozygosity after $\mathbf{5 0}$ years

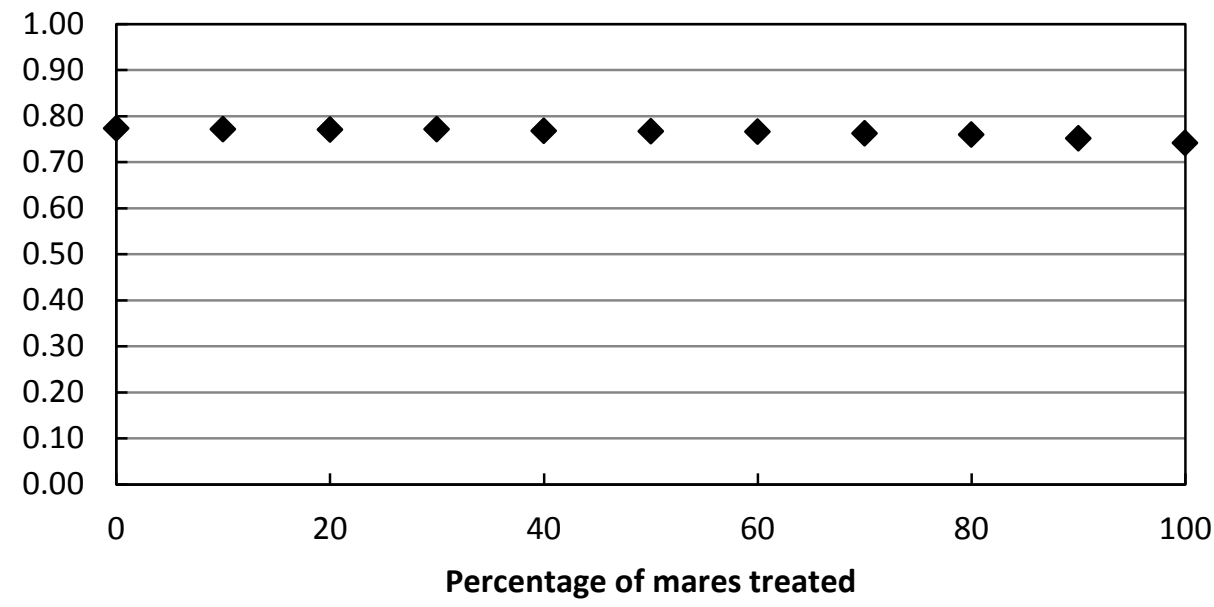




\begin{tabular}{lccccccccccc}
\hline \multicolumn{1}{c}{ Percentage of mares treated } & $\mathbf{0}$ & $\mathbf{1 0}$ & $\mathbf{2 0}$ & $\mathbf{3 0}$ & $\mathbf{4 0}$ & $\mathbf{5 0}$ & $\mathbf{6 0}$ & $\mathbf{7 0}$ & $\mathbf{8 0}$ & $\mathbf{9 0}$ & $\mathbf{1 0 0}$ \\
\hline Mean population size after 20 years & 278.95 & 274.39 & 264.77 & 267.48 & 247.95 & 249.86 & 254.3 & 249.36 & 232.19 & 203.01 & 169.11 \\
Mean number of removals after 20 years & 478.13 & 410.55 & 411.23 & 373.12 & 350.21 & 295.77 & 278.33 & 262.32 & 220.73 & 207.34 & 168.69 \\
Mean probability of extirpation after 50 years & 0 & 0 & 0 & 0 & 0 & 0 & 0 & 0 & 0 & 0.02 & 0.01 \\
Mean heterozygosity after 50 years & 0.77 & 0.77 & 0.77 & 0.77 & 0.77 & 0.77 & 0.77 & 0.76 & 0.76 & 0.75 & 0.74 \\
\hline
\end{tabular}

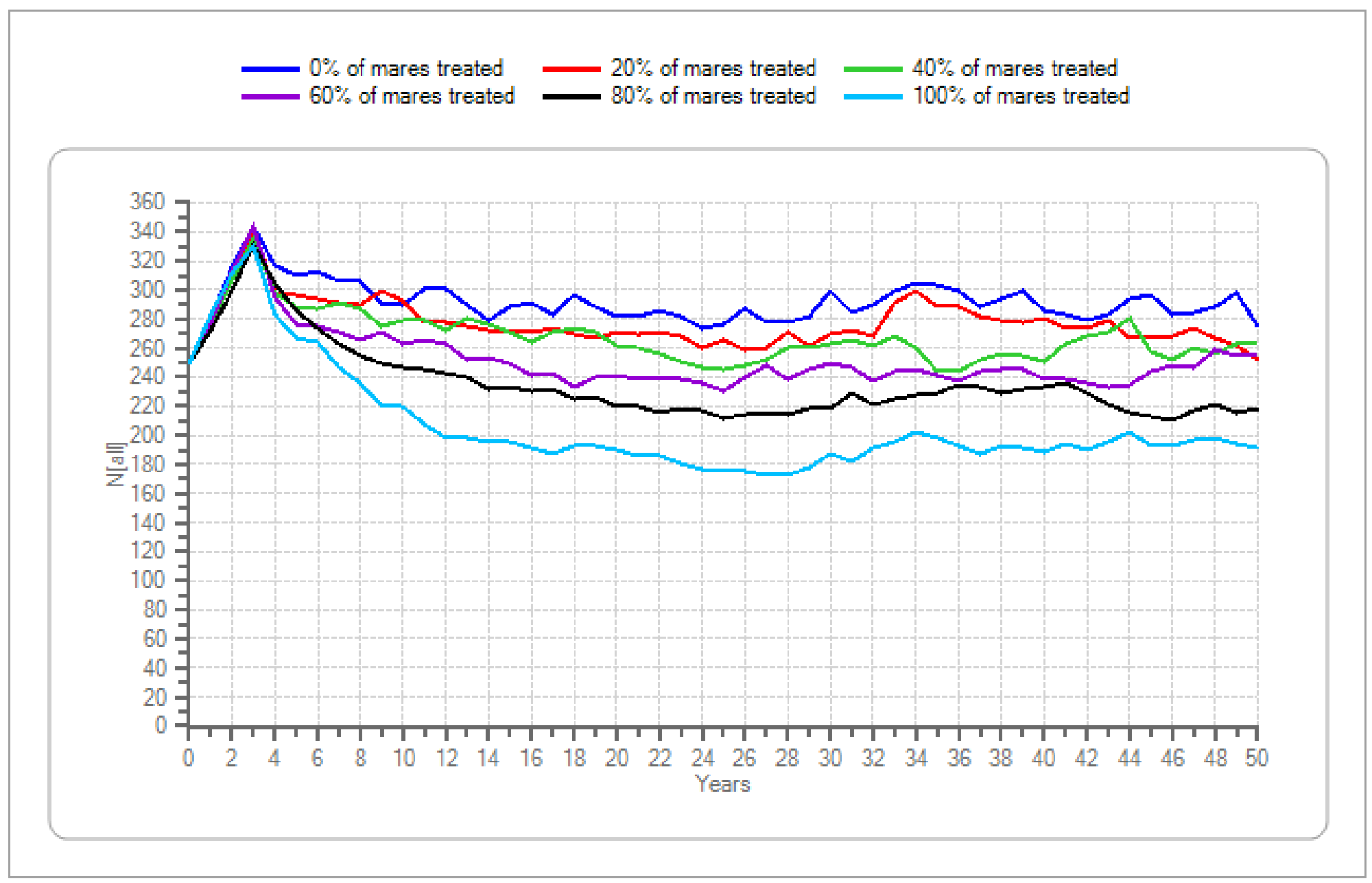

Figure 43. Initial population size $=250$, lambda $=1.15$, genetic diversity $=$ high. Continued 
Mean population size after 20 years

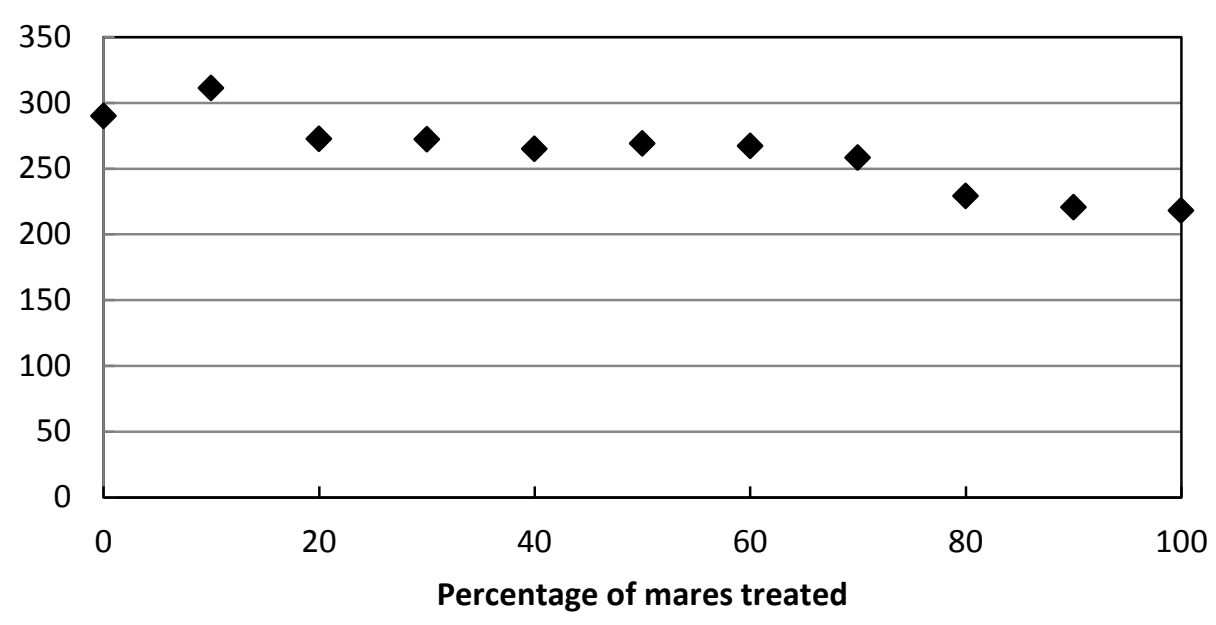

Mean probability of extirpation after $\mathbf{5 0}$ years

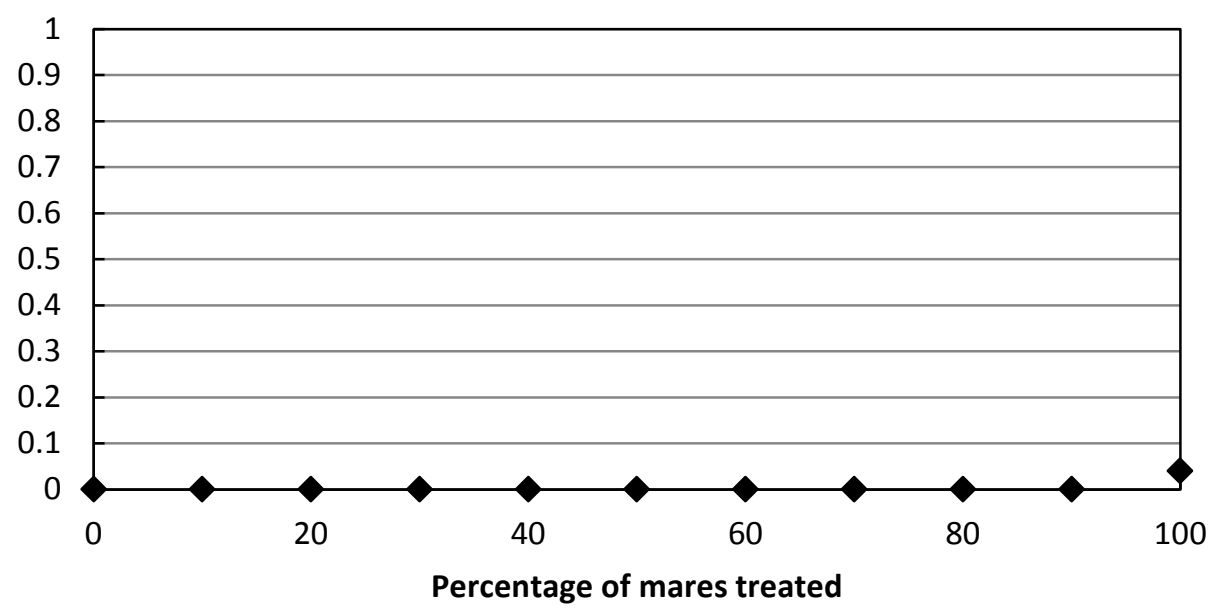

Figure 44. Initial population size $=250$, lambda $=1.20$, genetic diversity $=$ high .
Mean number of removals after 20 years

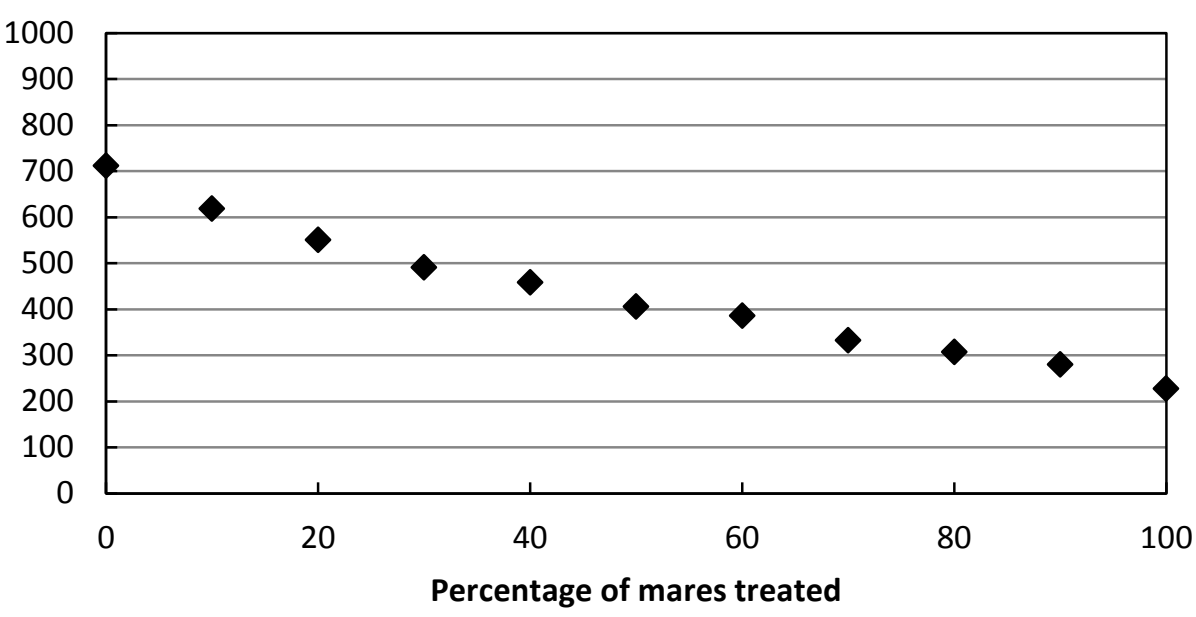

Mean heterozygosity after $\mathbf{5 0}$ years

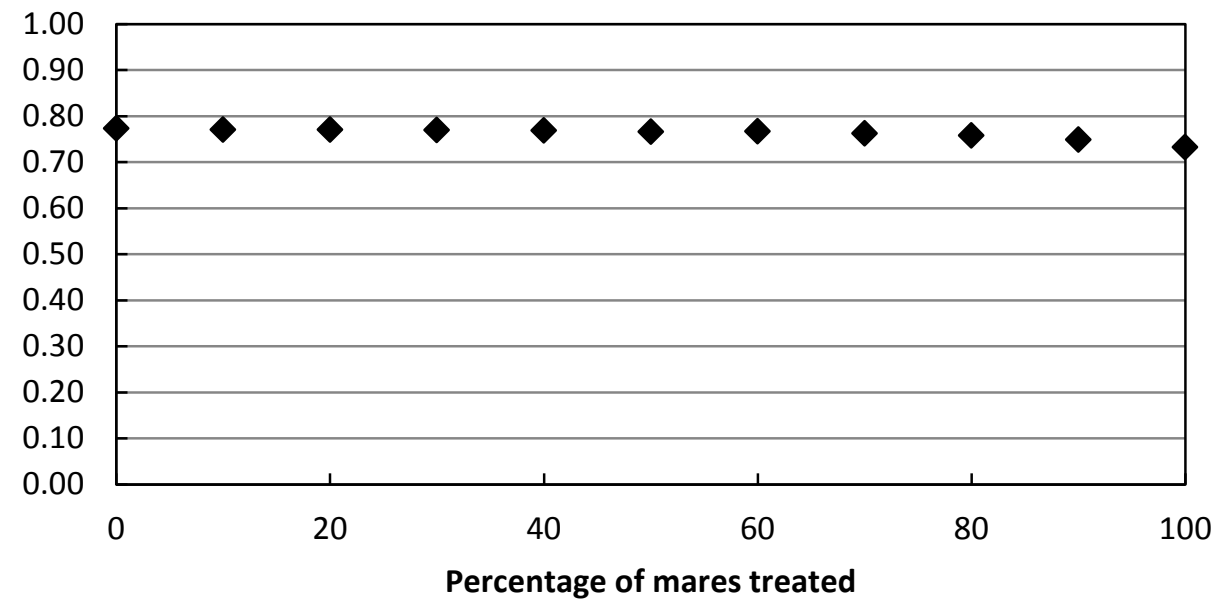




\begin{tabular}{lccccccccccc}
\hline \multicolumn{1}{c}{ Percentage of mares treated } & $\mathbf{0}$ & $\mathbf{1 0}$ & $\mathbf{2 0}$ & $\mathbf{3 0}$ & $\mathbf{4 0}$ & $\mathbf{5 0}$ & $\mathbf{6 0}$ & $\mathbf{7 0}$ & $\mathbf{8 0}$ & $\mathbf{9 0}$ & $\mathbf{1 0 0}$ \\
\hline Mean population size after 20 years & 290.06 & 311.21 & 272.6 & 272.05 & 264.99 & 269.08 & 267.14 & 258.33 & 229.2 & 220.46 & 218.12 \\
Mean number of removals after 20 years & 711.9 & 618.06 & 550.39 & 491.13 & 458.39 & 405.16 & 385.49 & 331.92 & 307.21 & 279.49 & 227.5 \\
Mean probability of extirpation after 50 years & 0 & 0 & 0 & 0 & 0 & 0 & 0 & 0 & 0 & 0 & 0.04 \\
Mean heterozygosity after 50 years & 0.77 & 0.77 & 0.77 & 0.77 & 0.77 & 0.77 & 0.77 & 0.76 & 0.76 & 0.75 & 0.73 \\
\hline
\end{tabular}

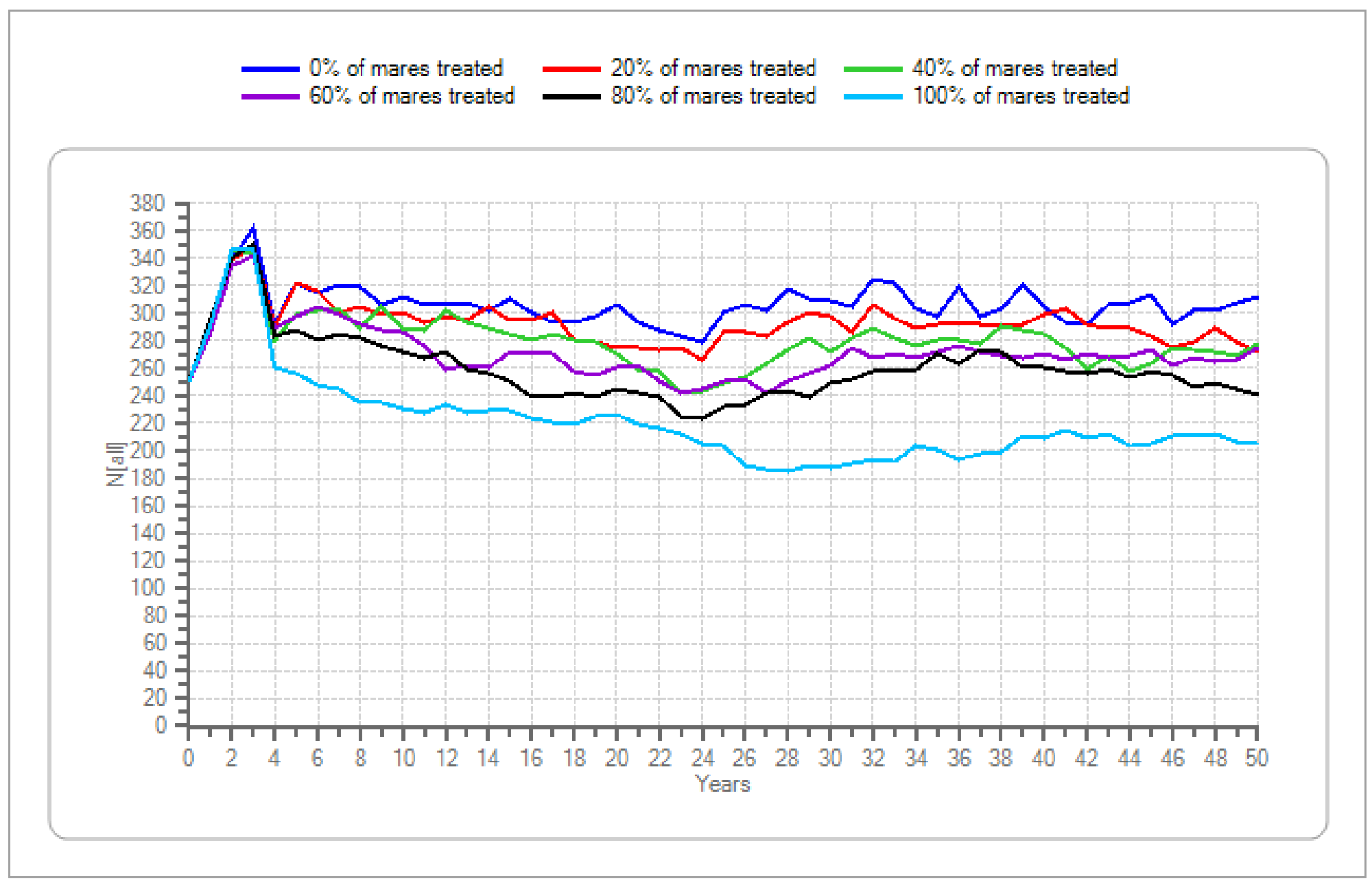

Figure 44. Initial population size $=250$, lambda $=1.20$, genetic diversity $=$ high. Continued 
Mean population size after 20 years

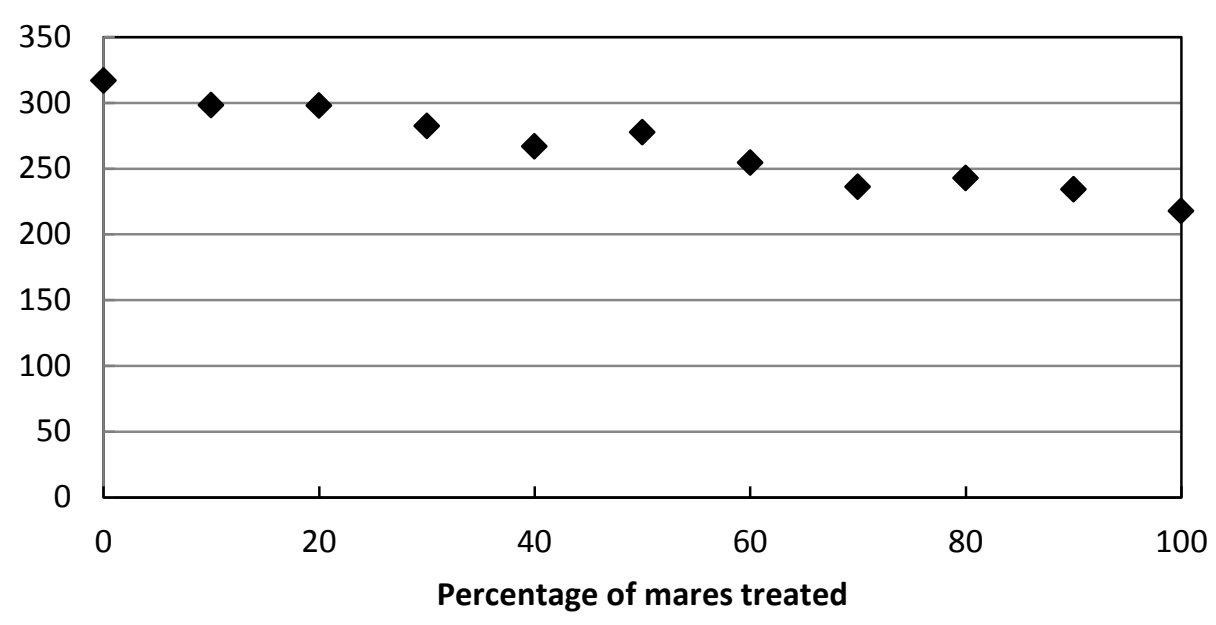

Mean probability of extirpation after $\mathbf{5 0}$ years

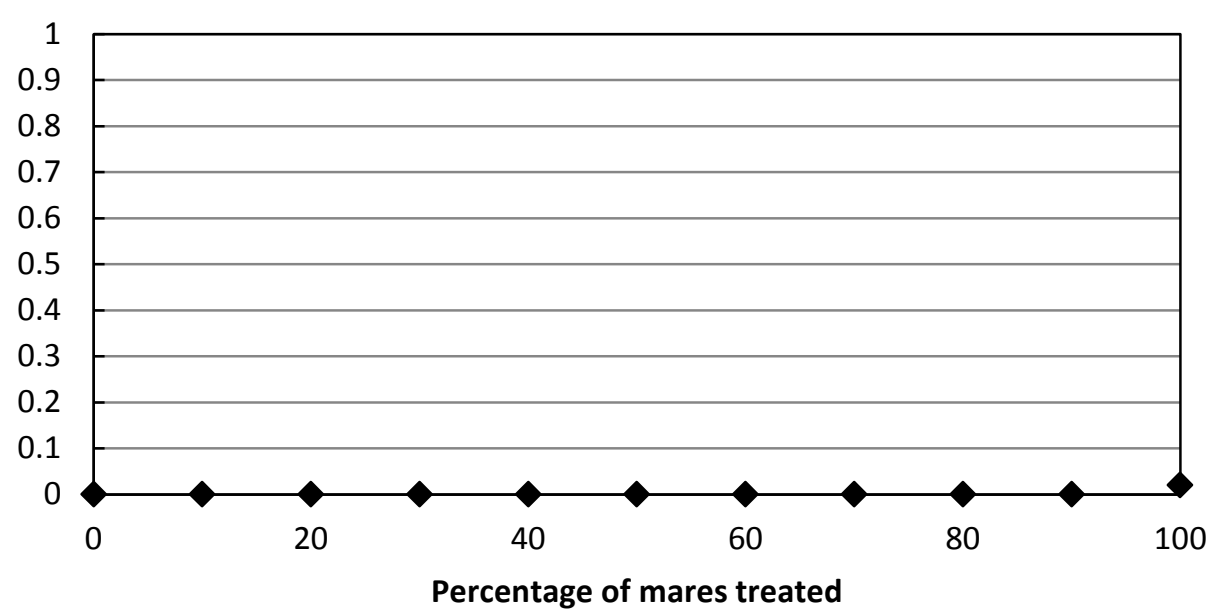

Figure 45. Initial population size $=250$, lambda $=1.25$, genetic diversity $=$ high .
Mean number of removals after 20 years

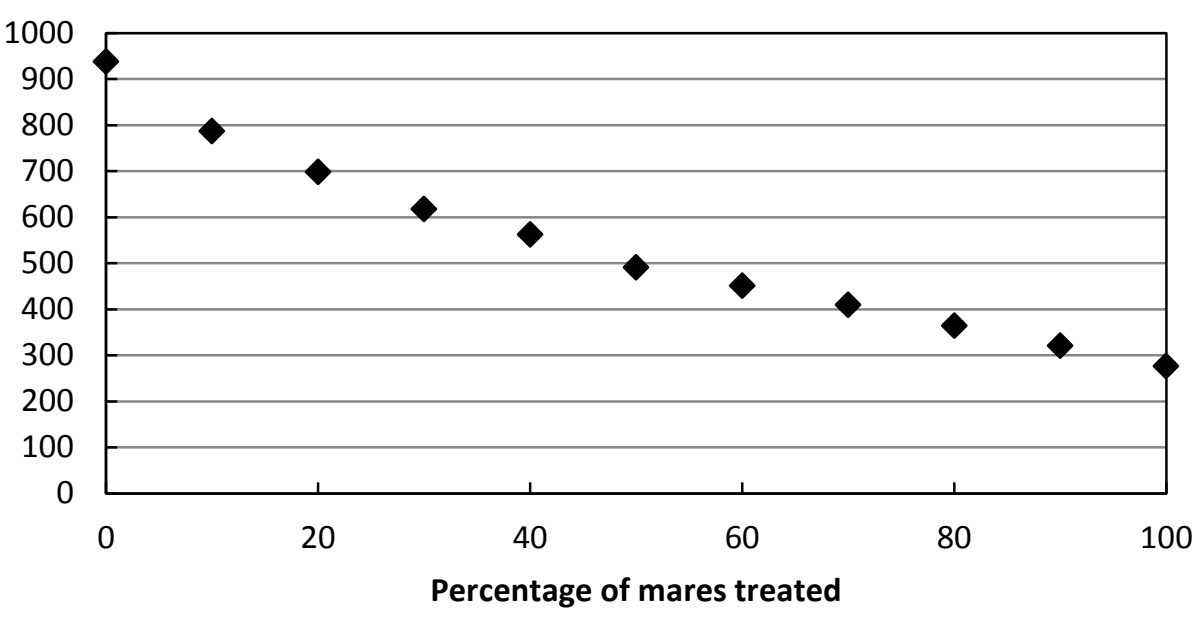

Mean heterozygosity after $\mathbf{5 0}$ years

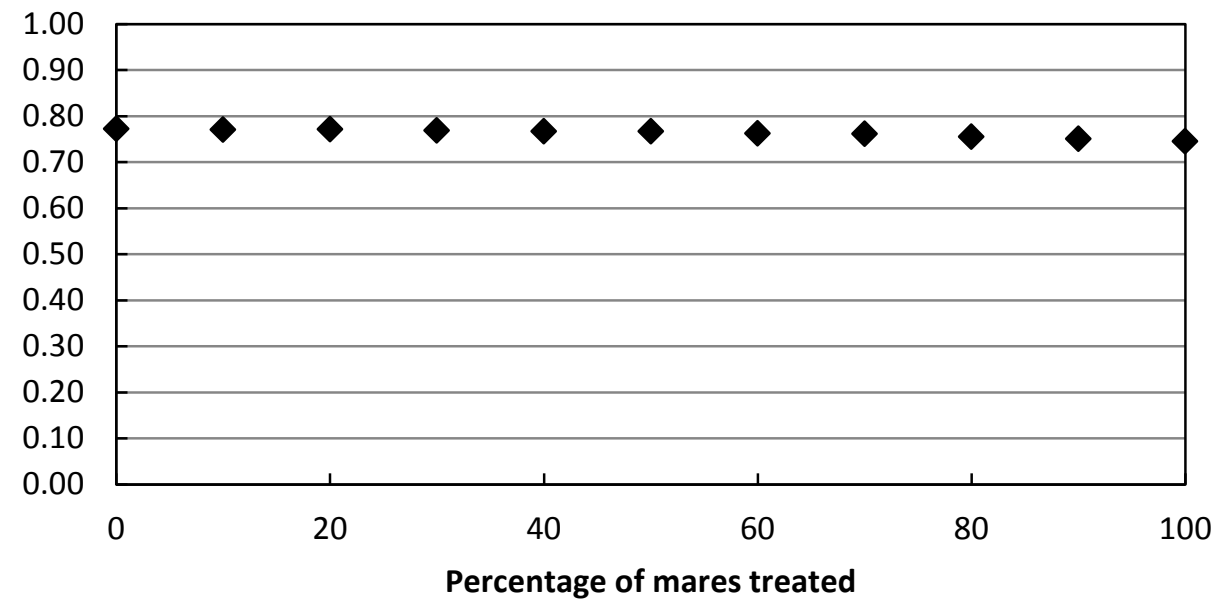




\begin{tabular}{lccccccccccc}
\hline \multicolumn{1}{c}{ Percentage of mares treated } & $\mathbf{0}$ & $\mathbf{1 0}$ & $\mathbf{2 0}$ & $\mathbf{3 0}$ & $\mathbf{4 0}$ & $\mathbf{5 0}$ & $\mathbf{6 0}$ & $\mathbf{7 0}$ & $\mathbf{8 0}$ & $\mathbf{9 0}$ & $\mathbf{1 0 0}$ \\
\hline Mean population size after 20 years & 316.89 & 298.09 & 297.94 & 282.24 & 266.75 & 277.65 & 254.56 & 236.14 & 242.76 & 234.27 & 217.59 \\
Mean number of removals after 20 years & 937.55 & 786.93 & 697.69 & 617.57 & 562.54 & 490.47 & 450.66 & 409.16 & 363.89 & 320.86 & 276.24 \\
Mean probability of extirpation after 50 years & 0 & 0 & 0 & 0 & 0 & 0 & 0 & 0 & 0 & 0 & 0.02 \\
Mean heterozygosity after 50 years & 0.77 & 0.77 & 0.77 & 0.77 & 0.77 & 0.77 & 0.76 & 0.76 & 0.76 & 0.75 & 0.75 \\
\hline
\end{tabular}

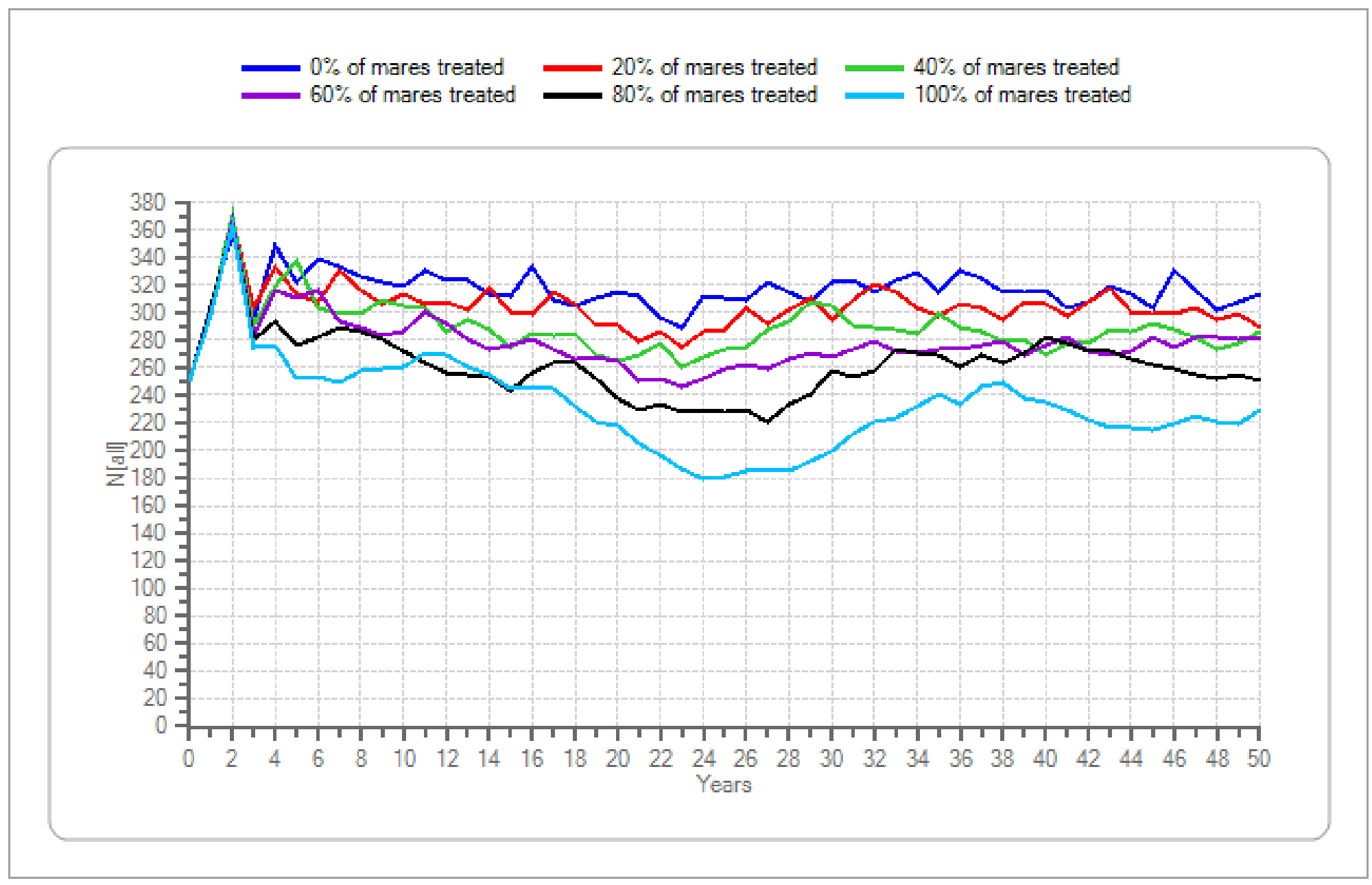

Figure 45. Initial population size $=250$, lambda $=1.25$, genetic diversity $=$ high - Continued 
Mean population size after 20 years

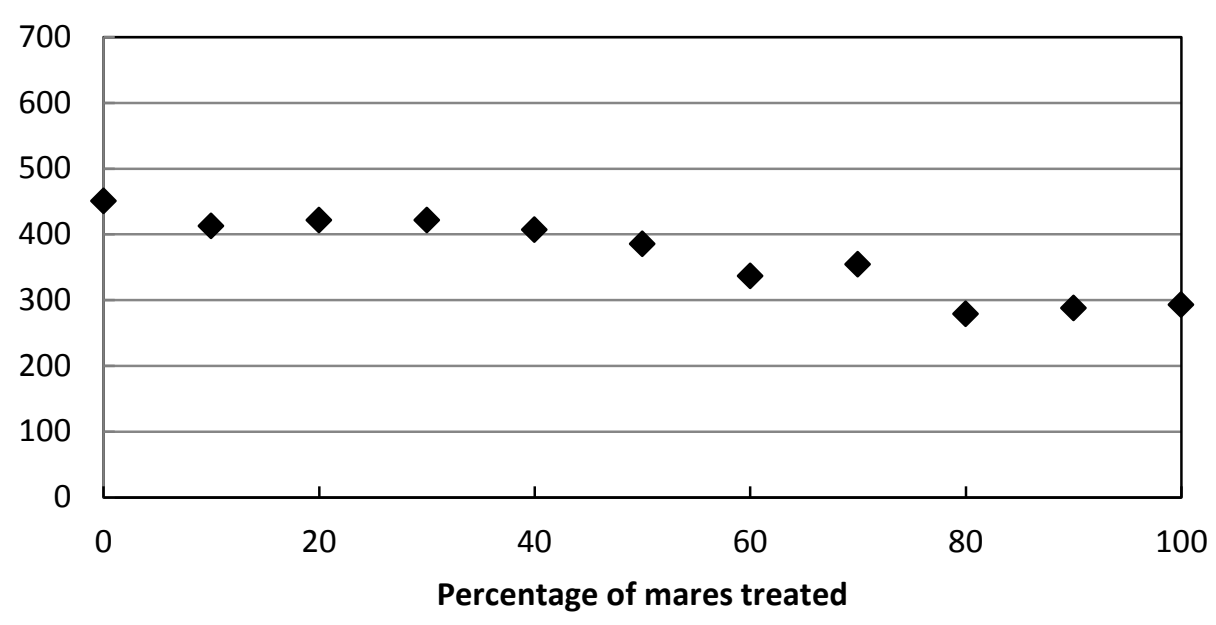

Mean probability of extirpation after $\mathbf{5 0}$ years

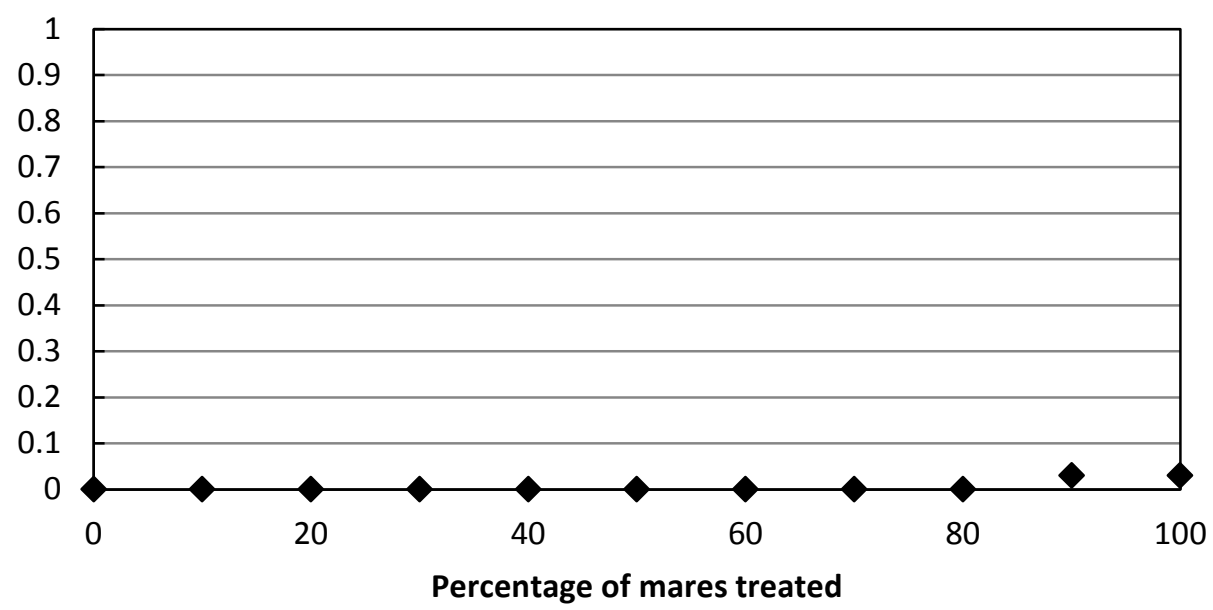

Figure 46. Initial population size $=500$, lambda $=1.05$, genetic diversity $=$ high .
Mean number of removals after 20 years

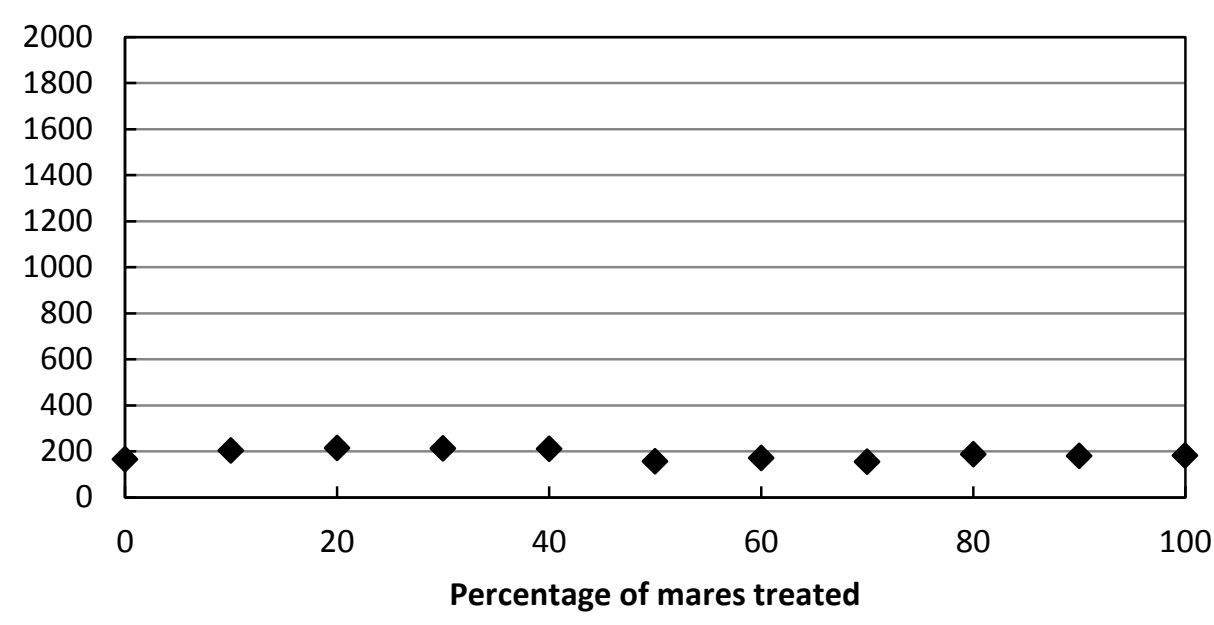

Mean heterozygosity after $\mathbf{5 0}$ years

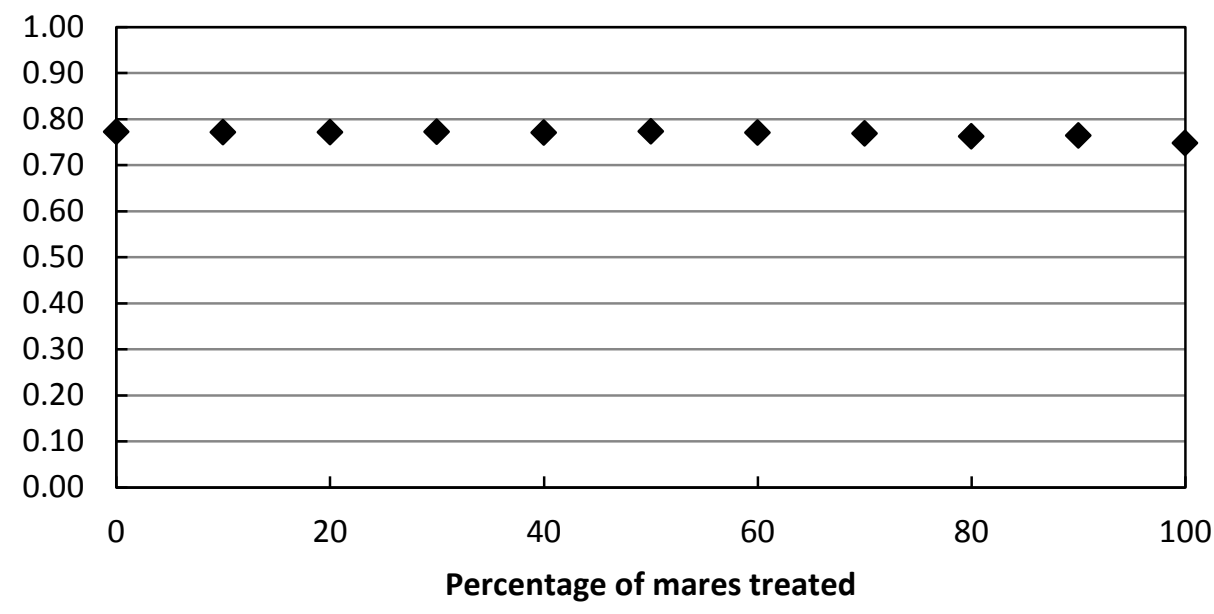




\begin{tabular}{lccccccccccc}
\hline \multicolumn{1}{c}{ Percentage of mares treated } & $\mathbf{0}$ & $\mathbf{1 0}$ & $\mathbf{2 0}$ & $\mathbf{3 0}$ & $\mathbf{4 0}$ & $\mathbf{5 0}$ & $\mathbf{6 0}$ & $\mathbf{7 0}$ & $\mathbf{8 0}$ & $\mathbf{9 0}$ & $\mathbf{1 0 0}$ \\
\hline Mean population size after 20 years & 450.57 & 412.67 & 421.58 & 421.47 & 407.01 & 385.44 & 336.29 & 354.49 & 278.69 & 287.98 & 292.7 \\
Mean number of removals after 20 years & 165.39 & 203.66 & 214.47 & 212.76 & 209.18 & 155.55 & 170.07 & 154.49 & 186.23 & 178.59 & 180.8 \\
Mean probability of extirpation after 50 years & 0 & 0 & 0 & 0 & 0 & 0 & 0 & 0 & 0 & 0.03 & 0.03 \\
Mean heterozygosity after 50 years & 0.77 & 0.77 & 0.77 & 0.77 & 0.77 & 0.77 & 0.77 & 0.77 & 0.76 & 0.76 & 0.75 \\
\hline
\end{tabular}

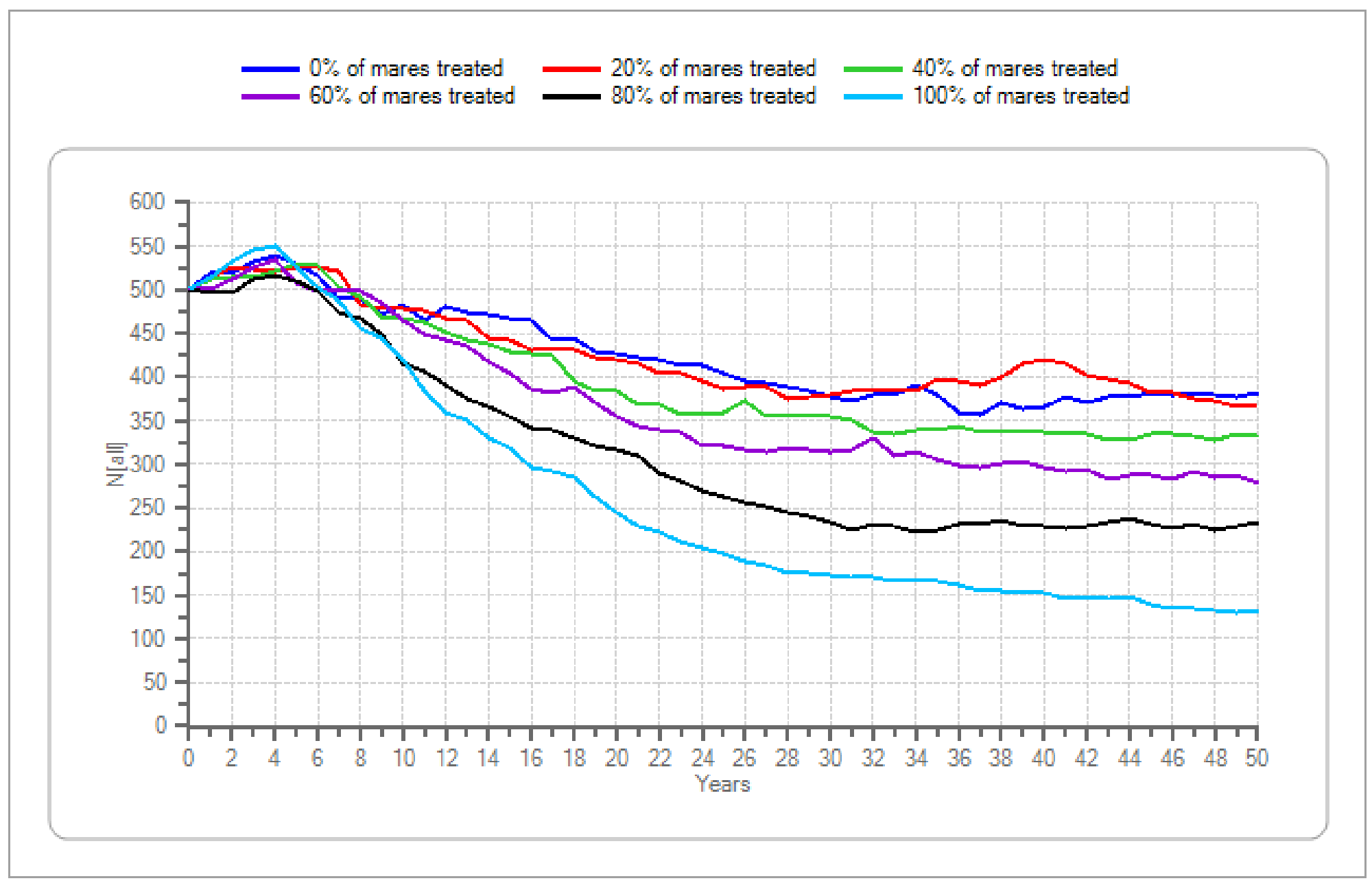

Figure 46. Initial population size $=500$, lambda $=1.05$, genetic diversity $=$ high. Continued 
Mean population size after 20 years

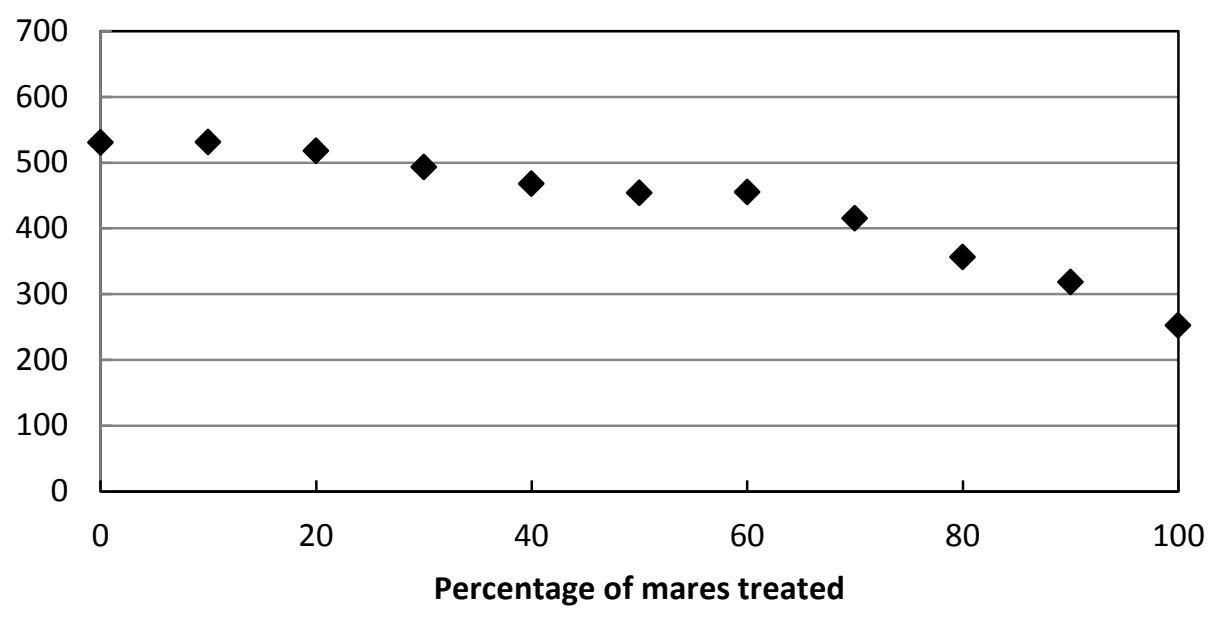

Mean probability of extirpation after $\mathbf{5 0}$ years

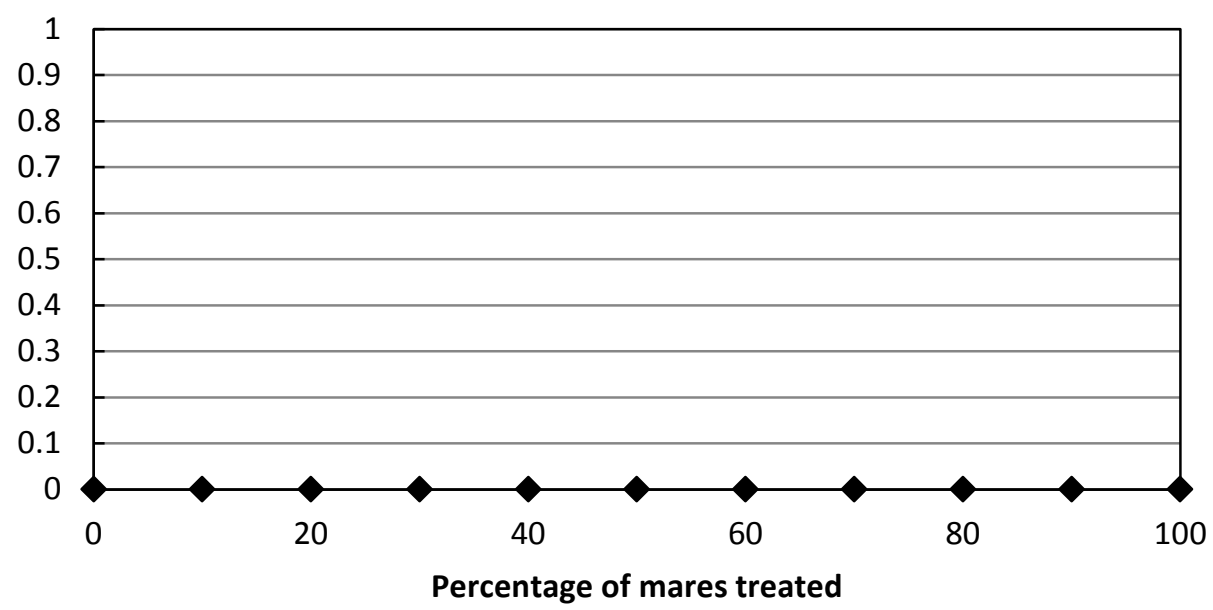

Figure 47. Initial population size $=500$, lambda $=1.10$, genetic diversity $=$ high .
Mean number of removals after 20 years

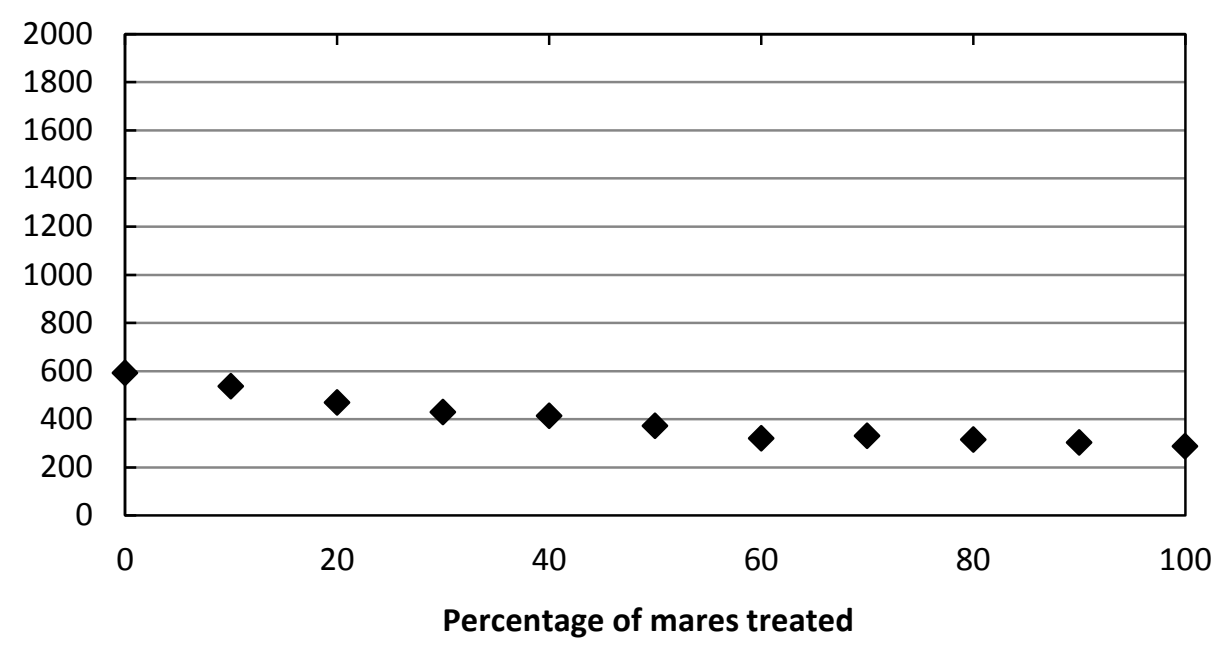

Mean heterozygosity after $\mathbf{5 0}$ years

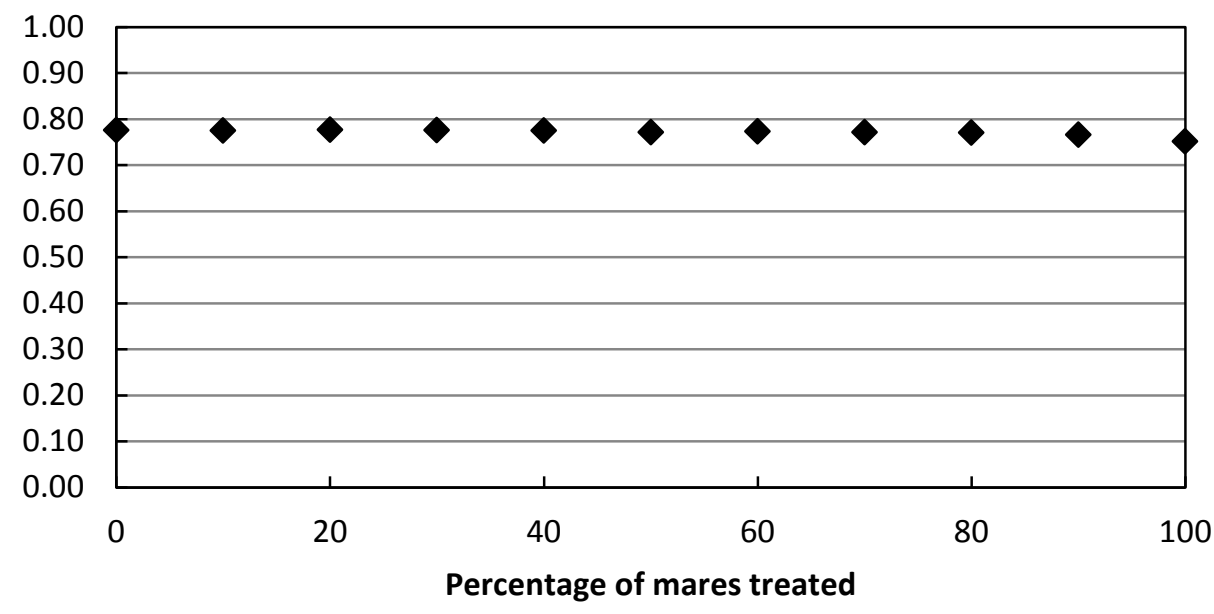




\begin{tabular}{lccccccccccc}
\hline \multicolumn{1}{c}{ Percentage of mares treated } & $\mathbf{0}$ & $\mathbf{1 0}$ & $\mathbf{2 0}$ & $\mathbf{3 0}$ & $\mathbf{4 0}$ & $\mathbf{5 0}$ & $\mathbf{6 0}$ & $\mathbf{7 0}$ & $\mathbf{8 0}$ & $\mathbf{9 0}$ & $\mathbf{1 0 0}$ \\
\hline Mean population size after 20 years & 530.61 & 530.85 & 517.52 & 493.18 & 467.76 & 453.59 & 454.75 & 415.17 & 356.16 & 318.2 & 252.43 \\
Mean number of removals after 20 years & 591.77 & 536.39 & 469.19 & 428.35 & 413.44 & 371.39 & 319.96 & 329.77 & 315.36 & 302.16 & 286.23 \\
Mean probability of extirpation after 50 years & 0 & 0 & 0 & 0 & 0 & 0 & 0 & 0 & 0 & 0 & 0 \\
Mean heterozygosity after 50 years & 0.78 & 0.78 & 0.78 & 0.78 & 0.77 & 0.77 & 0.77 & 0.77 & 0.77 & 0.77 & 0.75 \\
\hline
\end{tabular}

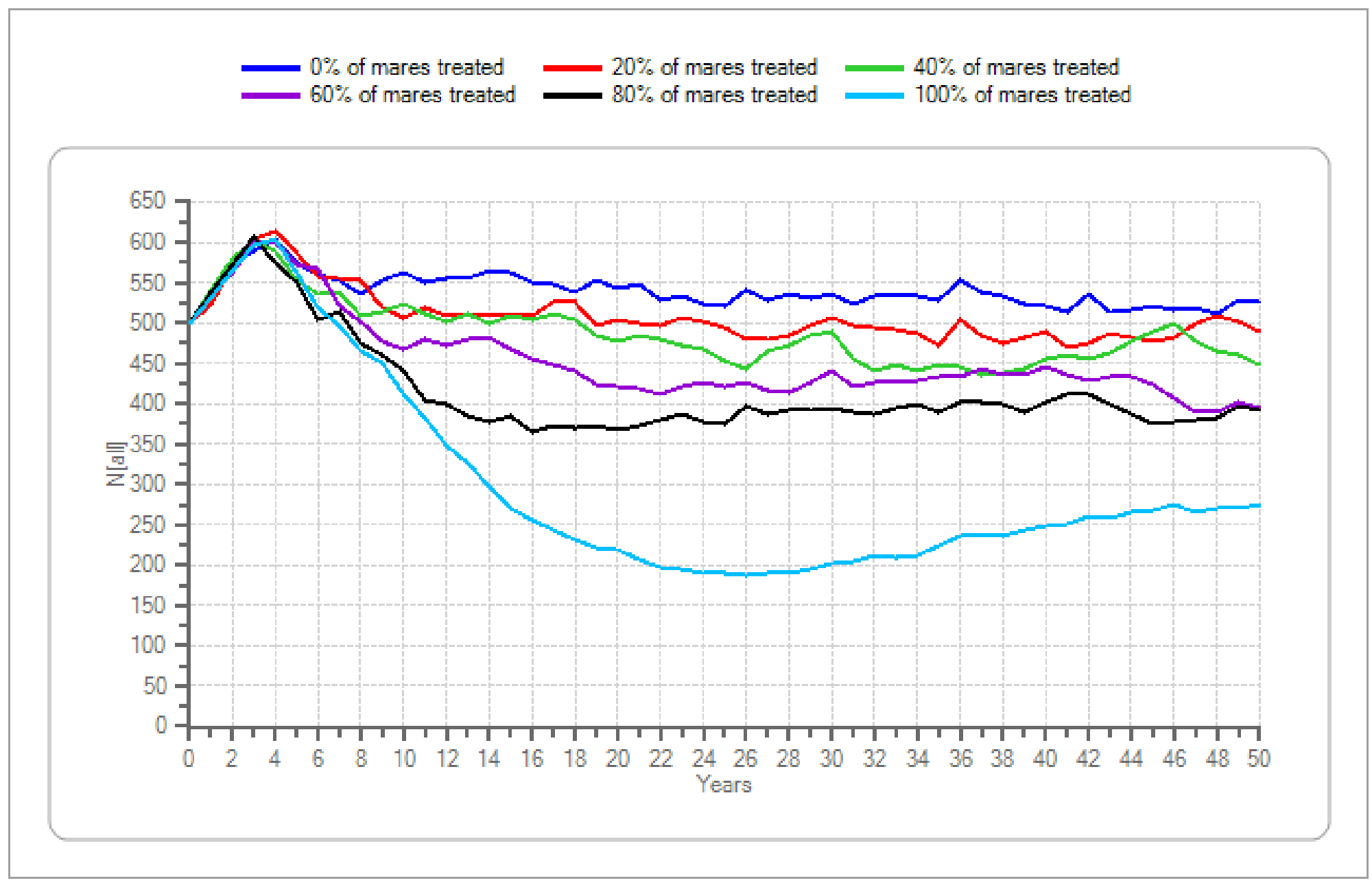

Figure 47. Initial population size $=500$, lambda $=1.10$, genetic diversity $=$ high. Continued 
Mean population size after 20 years

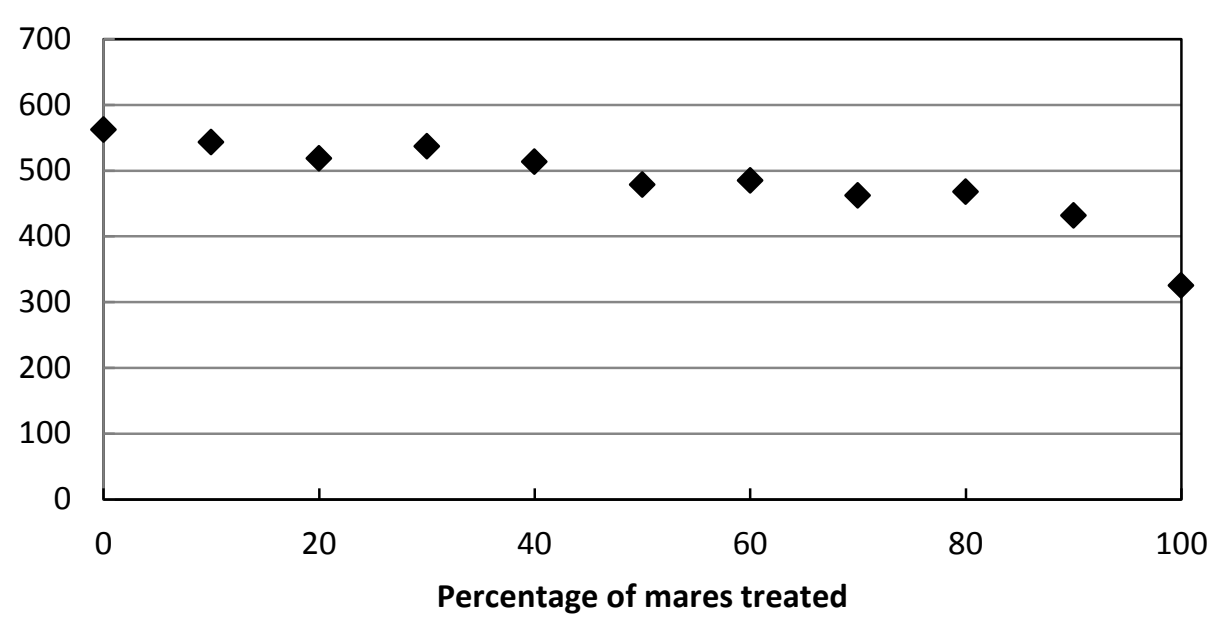

Mean probability of extirpation after $\mathbf{5 0}$ years

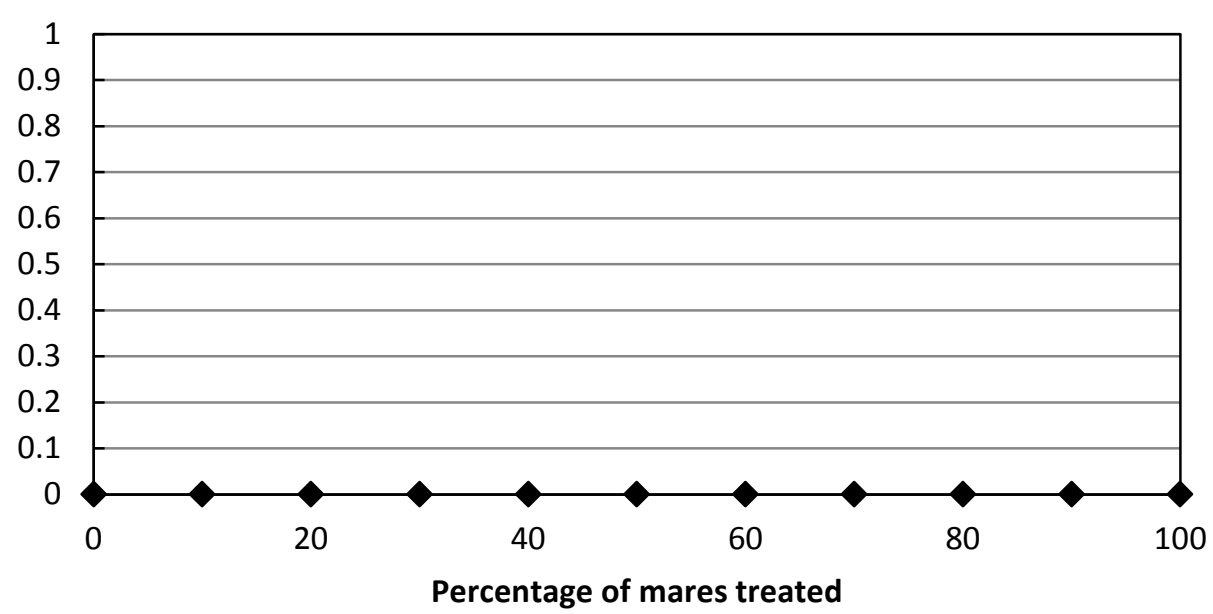

Figure 48. Initial population size $=500$, lambda $=1.15$, genetic diversity $=$ high .
Mean number of removals after 20 years

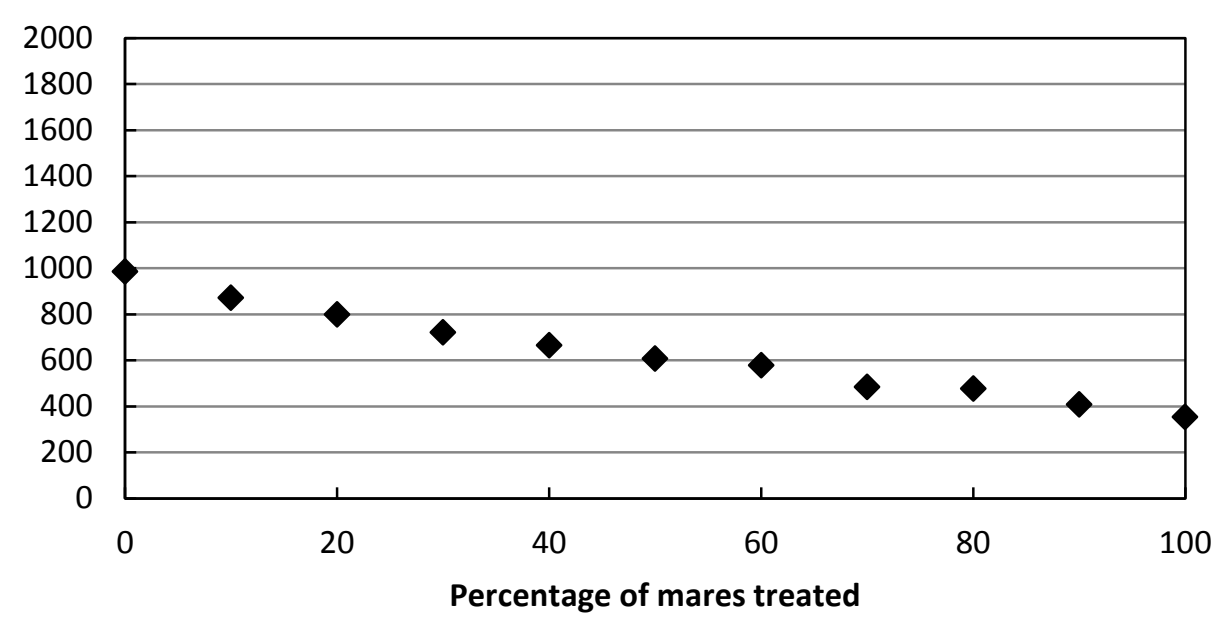

Mean heterozygosity after $\mathbf{5 0}$ years

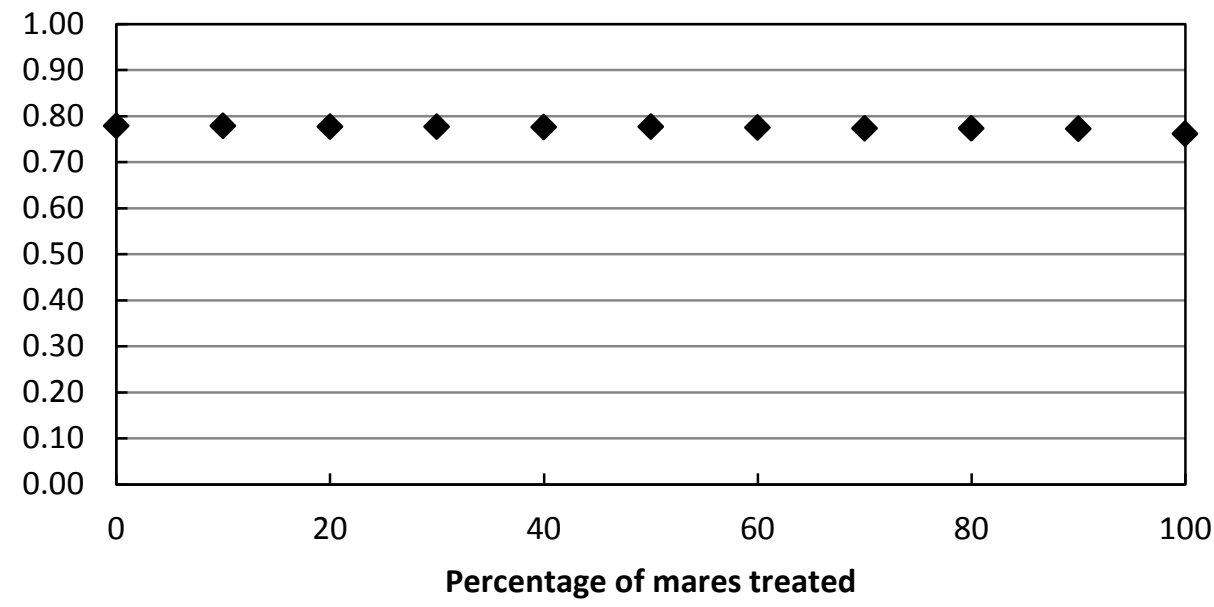




\begin{tabular}{lccccccccccc}
\hline \multicolumn{1}{c}{ Percentage of mares treated } & $\mathbf{0}$ & $\mathbf{1 0}$ & $\mathbf{2 0}$ & $\mathbf{3 0}$ & $\mathbf{4 0}$ & $\mathbf{5 0}$ & $\mathbf{6 0}$ & $\mathbf{7 0}$ & $\mathbf{8 0}$ & $\mathbf{9 0}$ & $\mathbf{1 0 0}$ \\
\hline Mean population size after 20 years & 562.32 & 542.84 & 518.23 & 536.63 & 513.12 & 478.55 & 484.51 & 462.25 & 467.67 & 431.71 & 325.38 \\
Mean number of removals after 20 years & 985.18 & 870.87 & 799.25 & 721.09 & 665.02 & 606.24 & 577.73 & 482.76 & 475.37 & 406.87 & 352.26 \\
Mean probability of extirpation after 50 years & 0 & 0 & 0 & 0 & 0 & 0 & 0 & 0 & 0 & 0 & 0 \\
Mean heterozygosity after 50 years & 0.78 & 0.78 & 0.78 & 0.78 & 0.78 & 0.78 & 0.77 & 0.77 & 0.77 & 0.77 & 0.76 \\
\hline
\end{tabular}

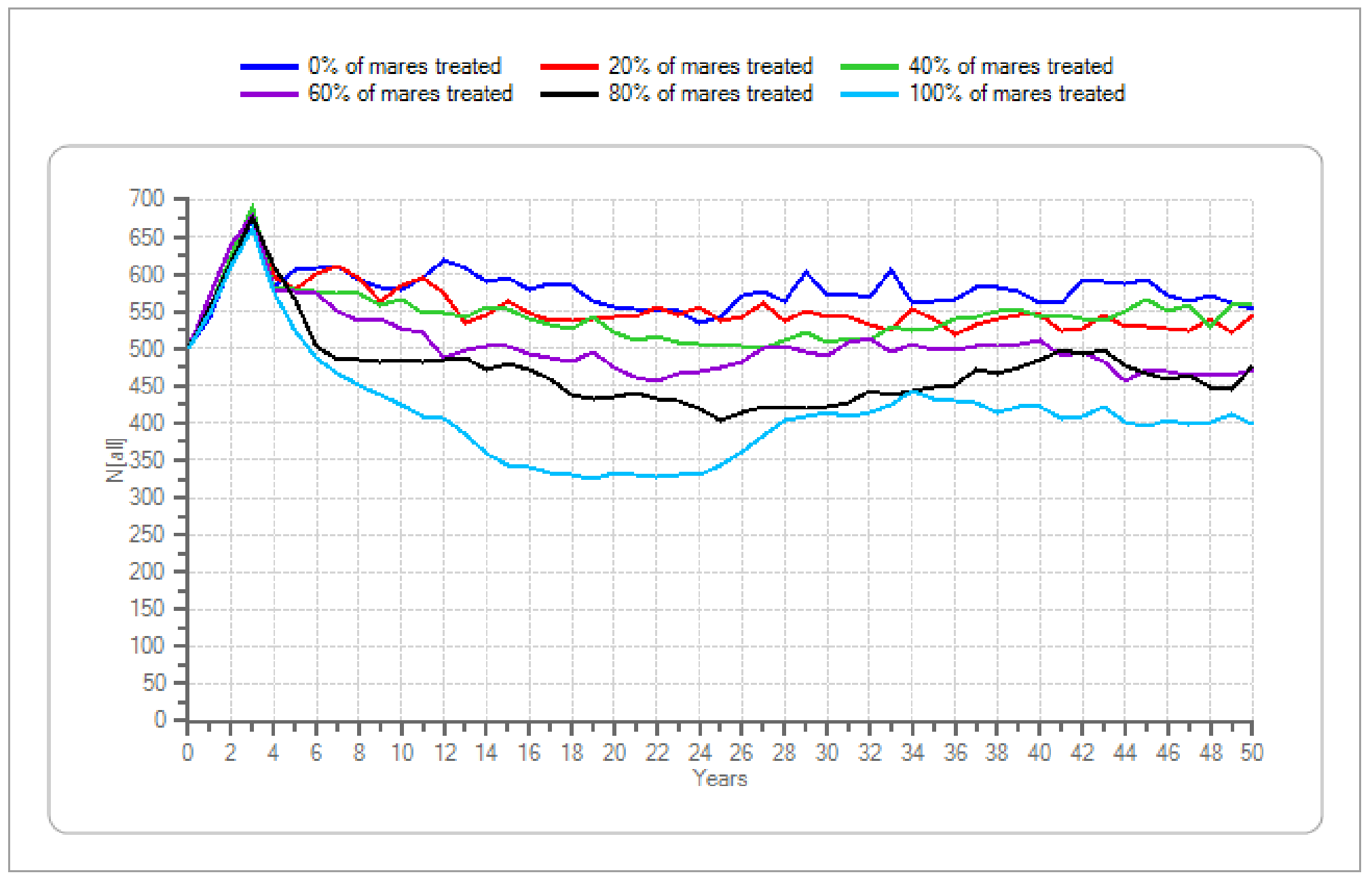

Figure 48. Initial population size $=500$, lambda $=1.15$, genetic diversity $=$ high - Continued 
Mean population size after 20 years

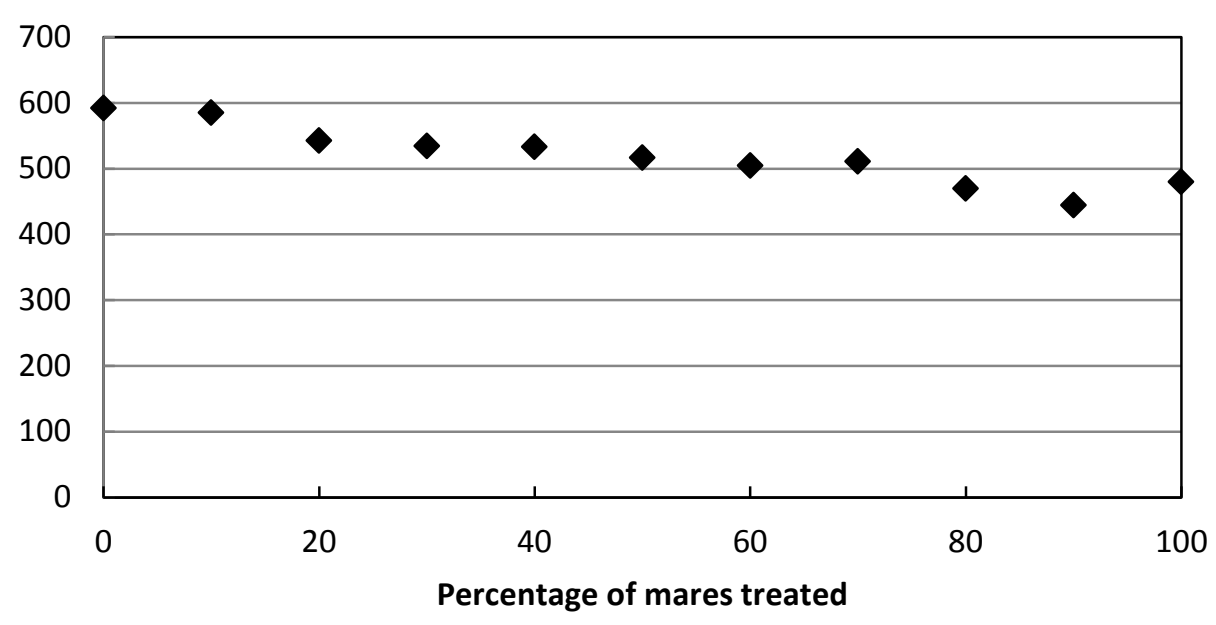

Mean probability of extirpation after $\mathbf{5 0}$ years

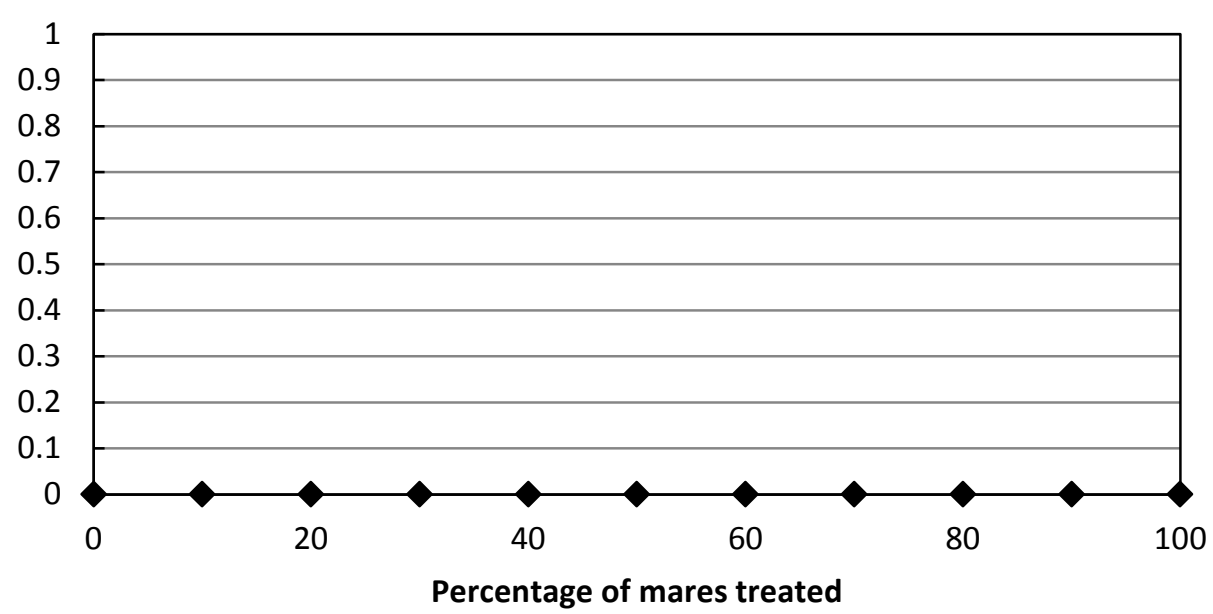

Figure 49. Initial population size $=500$, lambda $=1.20$, genetic diversity $=$ high .
Mean number of removals after 20 years

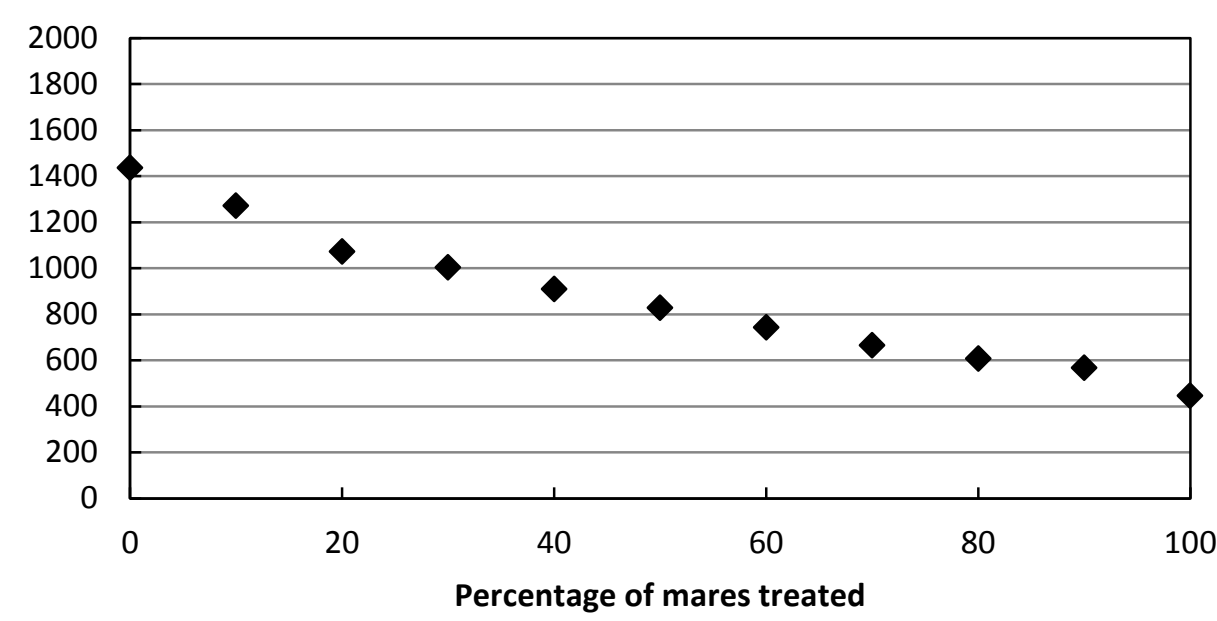

Mean heterozygosity after $\mathbf{5 0}$ years

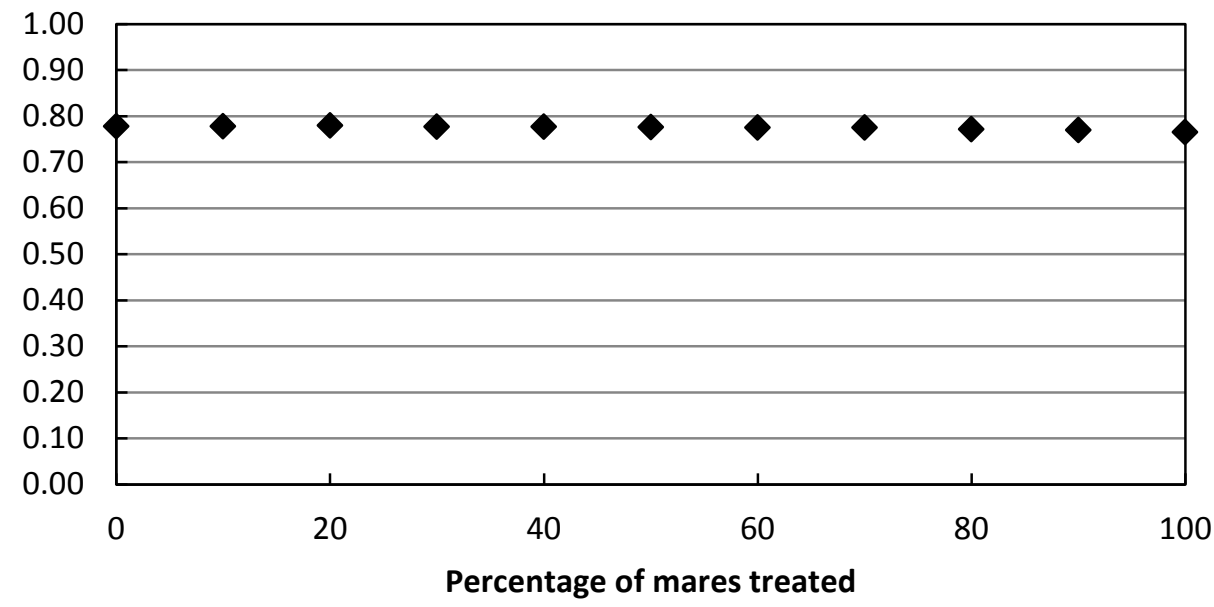




\begin{tabular}{lccccccccccc}
\hline \multicolumn{1}{c}{ Percentage of mares treated } & $\mathbf{0}$ & $\mathbf{1 0}$ & $\mathbf{2 0}$ & $\mathbf{3 0}$ & $\mathbf{4 0}$ & $\mathbf{5 0}$ & $\mathbf{6 0}$ & $\mathbf{7 0}$ & $\mathbf{8 0}$ & $\mathbf{9 0}$ & $\mathbf{1 0 0}$ \\
\hline Mean population size after 20 years & 591.83 & 584.93 & 542.73 & 534.42 & 532.9 & 516.29 & 504.75 & 510.67 & 469.76 & 444.23 & 479.71 \\
Mean number of removals after 20 years & 1435.1 & 1271.0 & 1072.6 & 1003.7 & 908.9 & 826.8 & 741.6 & 663.7 & 606.5 & 567.2 & 445.4 \\
Mean probability of extirpation after 50 years & 0 & 0 & 0 & 0 & 0 & 0 & 0 & 0 & 0 & 0 & 0 \\
Mean heterozygosity after 50 years & 0.78 & 0.78 & 0.78 & 0.78 & 0.78 & 0.78 & 0.78 & 0.78 & 0.77 & 0.77 & 0.76 \\
\hline
\end{tabular}

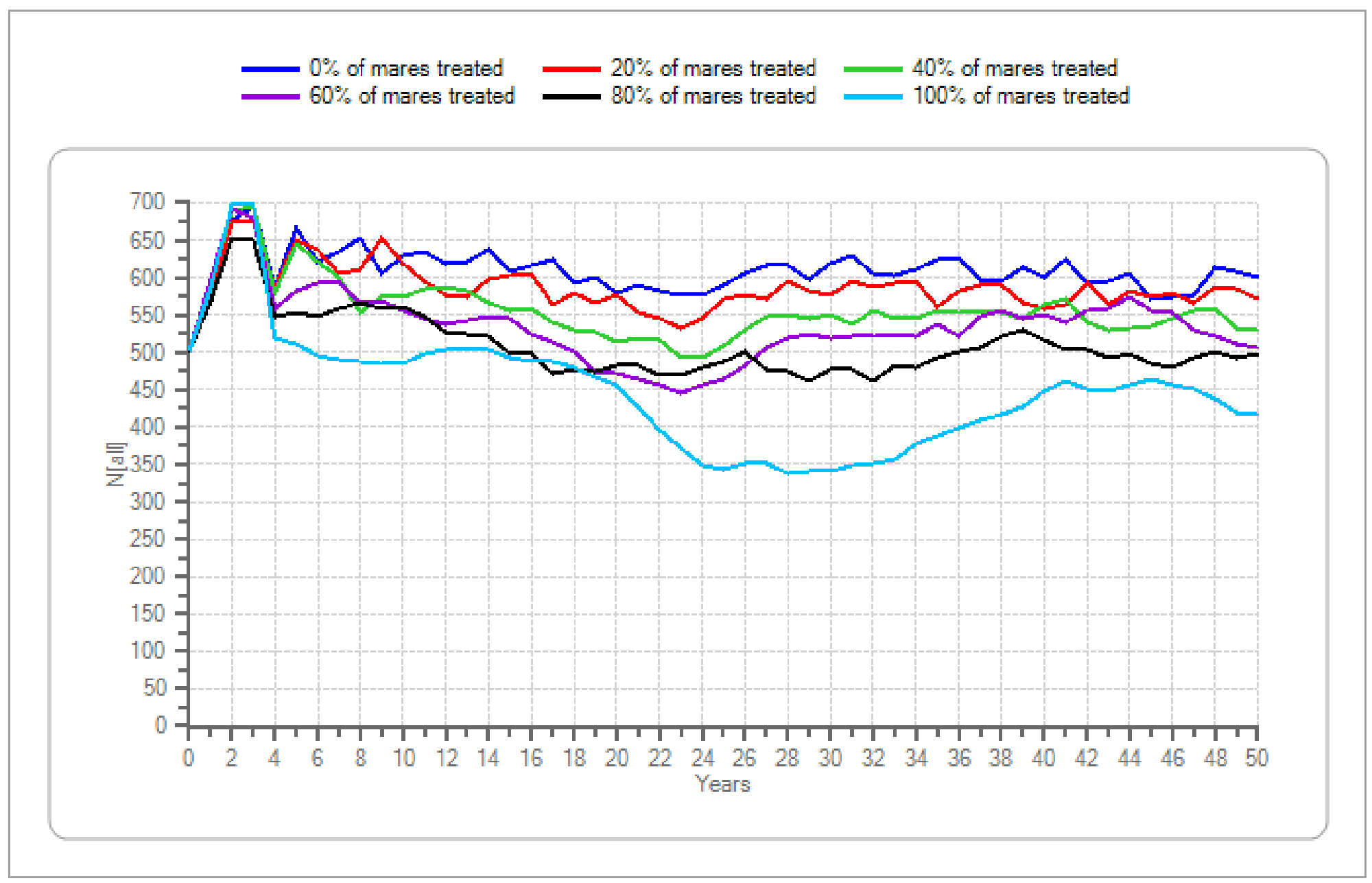

Figure 49. Initial population size $=500$, lambda $=1.20$, genetic diversity $=$ high. Continued 
Mean population size after 20 years

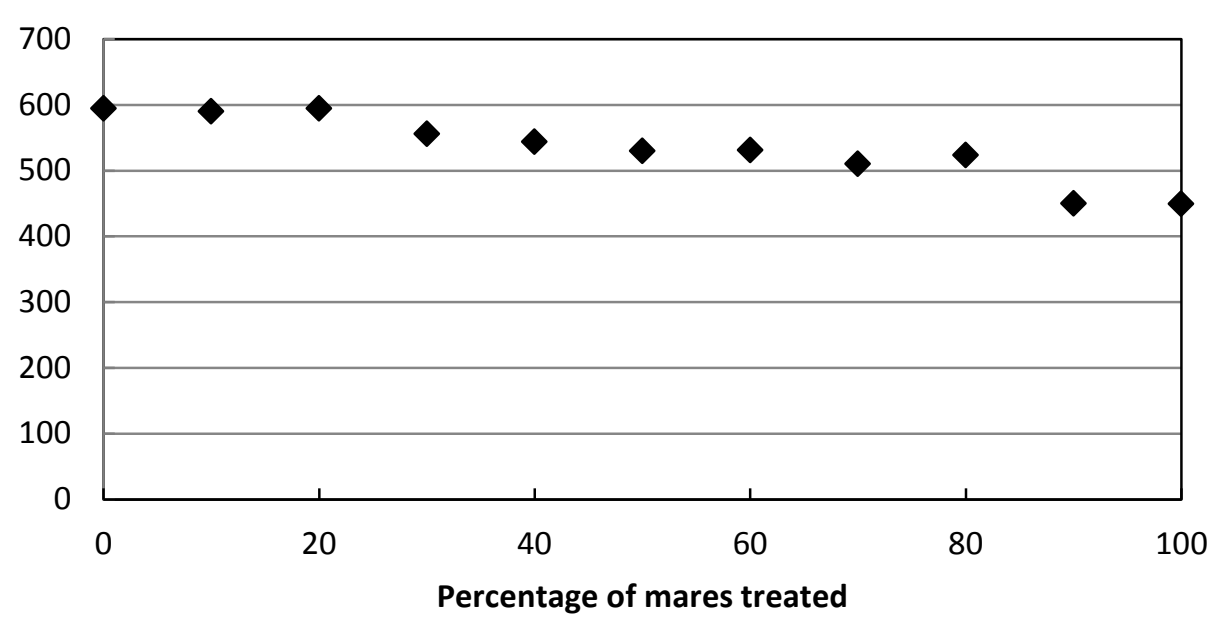

Mean probability of extirpation after $\mathbf{5 0}$ years

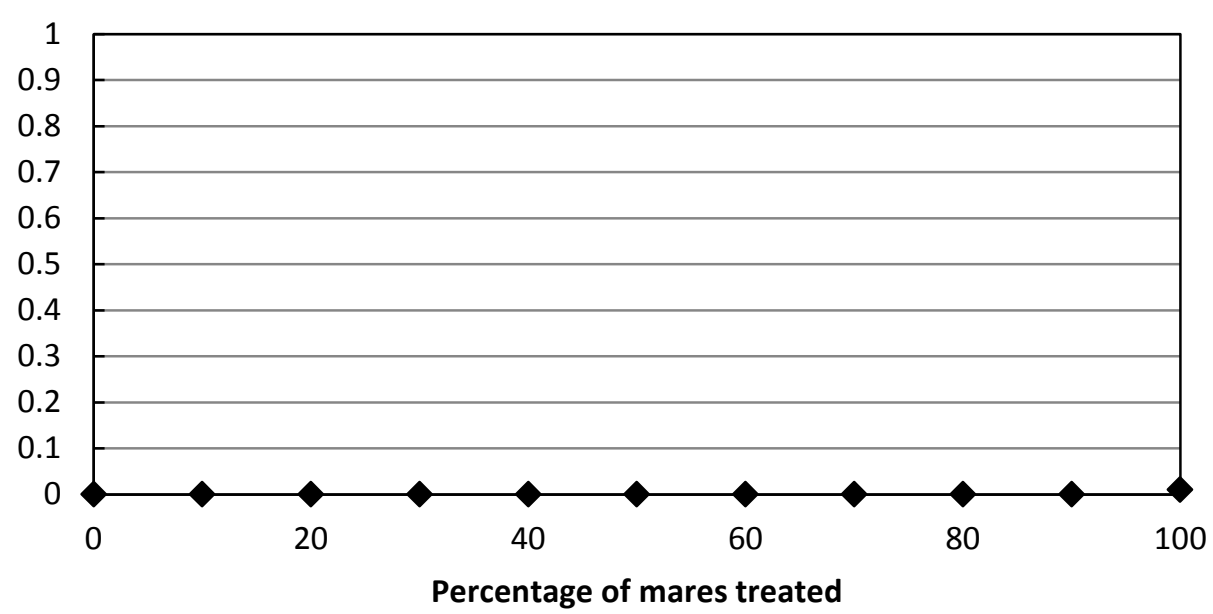

Figure 50. Initial population size $=500$, lambda $=1.25$, genetic diversity $=$ high .
Mean number of removals after 20 years

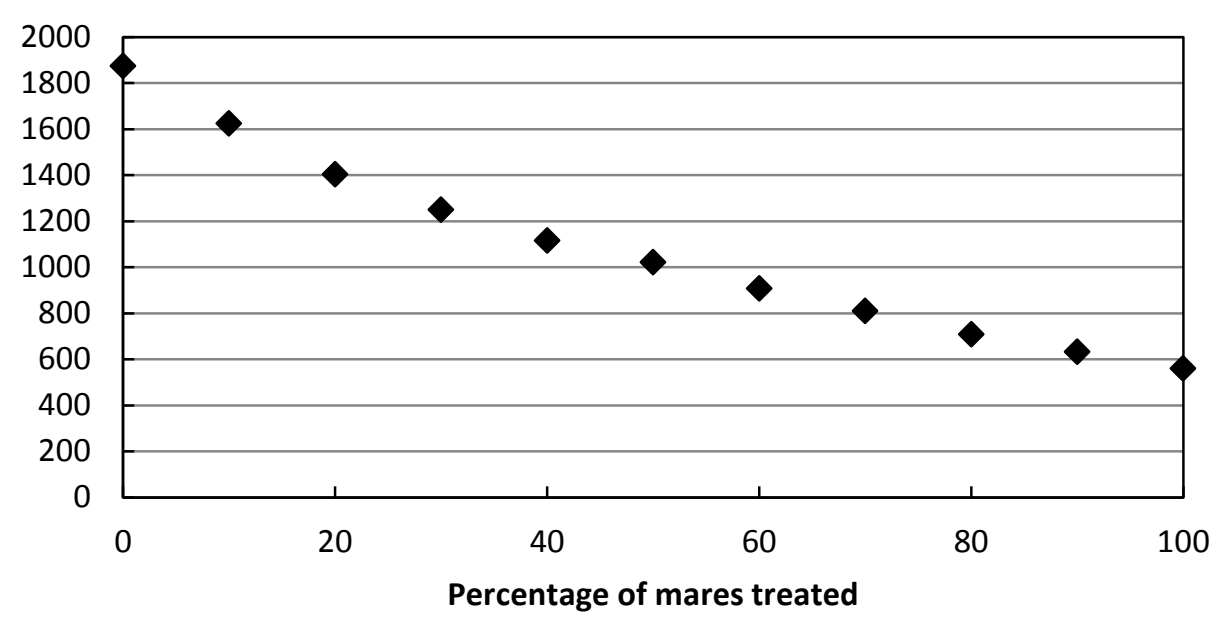

Mean heterozygosity after $\mathbf{5 0}$ years

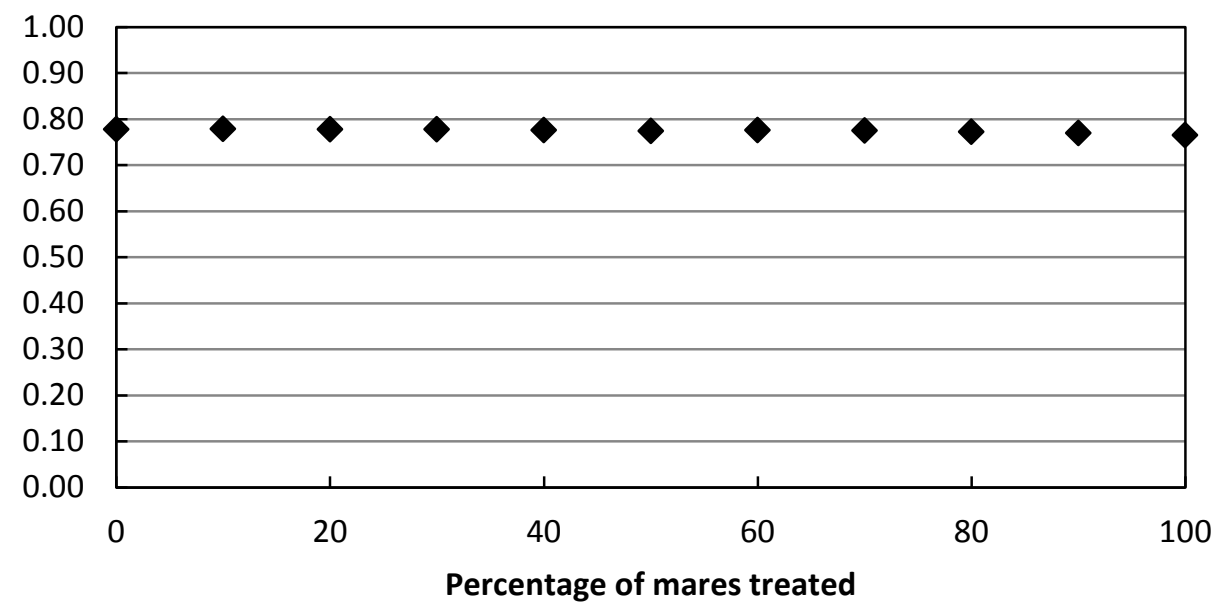




\begin{tabular}{lccccccccccc}
\hline \multicolumn{1}{c}{ Percentage of mares treated } & $\mathbf{0}$ & $\mathbf{1 0}$ & $\mathbf{2 0}$ & $\mathbf{3 0}$ & $\mathbf{4 0}$ & $\mathbf{5 0}$ & $\mathbf{6 0}$ & $\mathbf{7 0}$ & $\mathbf{8 0}$ & $\mathbf{9 0}$ & $\mathbf{1 0 0}$ \\
\hline Mean population size after 20 years & 594.45 & 589.9 & 594.5 & 555.72 & 543.85 & 529.95 & 531.28 & 510.02 & 523.65 & 449.97 & 449.55 \\
Mean number of removals after 20 years & 1874.5 & 1624.6 & 1402.6 & 1249.2 & 1116.2 & 1021.7 & 907.0 & 809.4 & 707.9 & 631.4 & 559.9 \\
Mean probability of extirpation after 50 years & 0 & 0 & 0 & 0 & 0 & 0 & 0 & 0 & 0 & 0 & 0.01 \\
Mean heterozygosity after 50 years & 0.78 & 0.78 & 0.78 & 0.78 & 0.78 & 0.77 & 0.78 & 0.78 & 0.77 & 0.77 & 0.76 \\
\hline
\end{tabular}

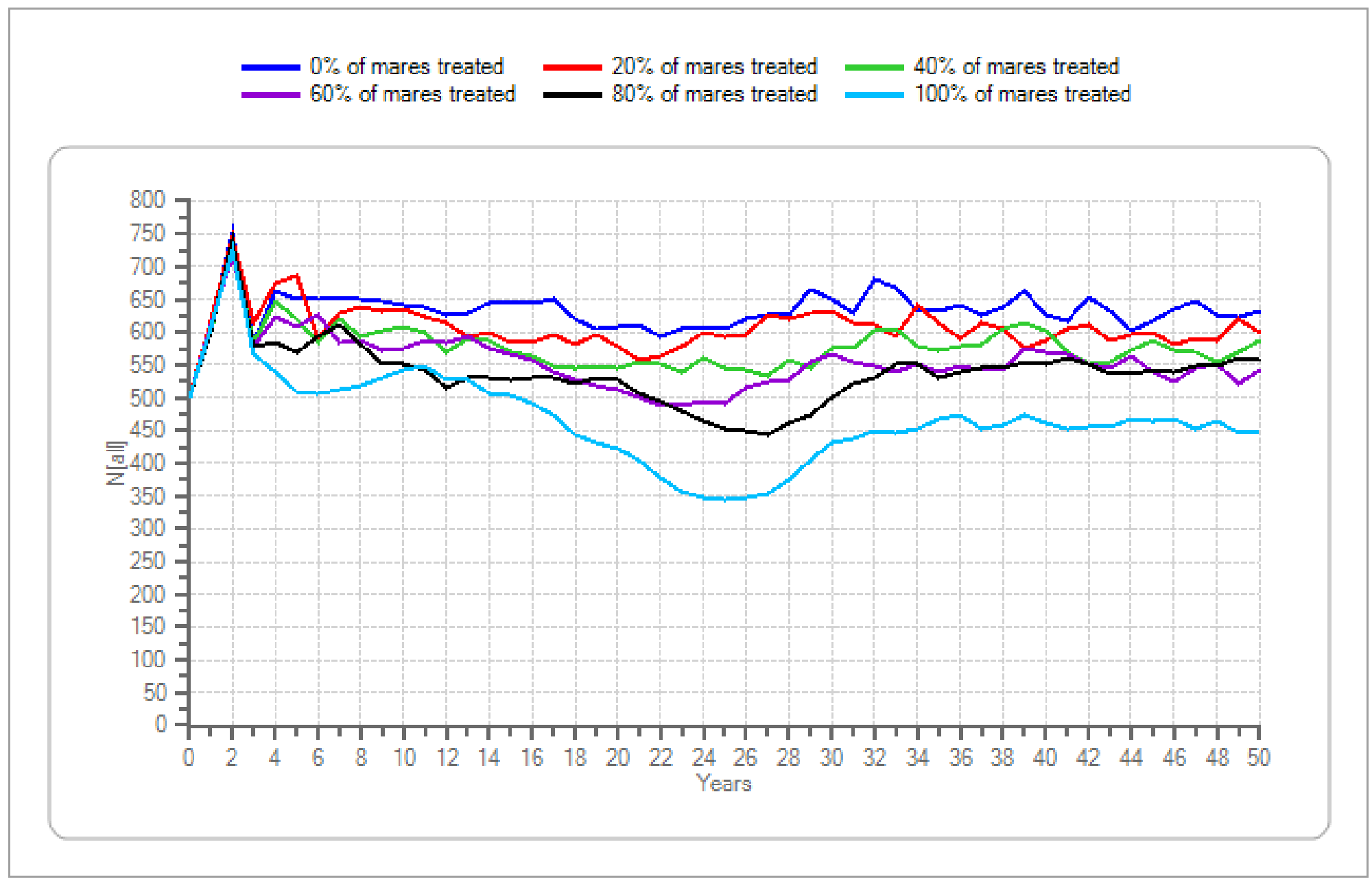

Figure 50. Initial population size $=500$, lambda $=1.25$, genetic diversity $=$ high - Continued 
Mean population size after 20 years

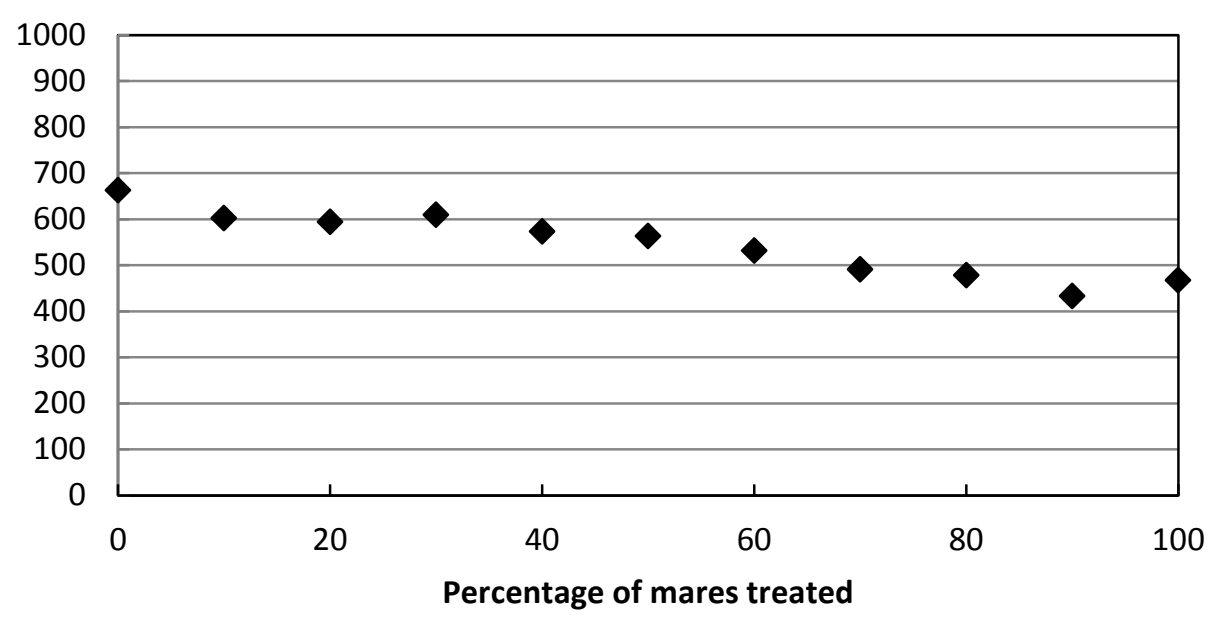

Mean probability of extirpation after $\mathbf{5 0}$ years

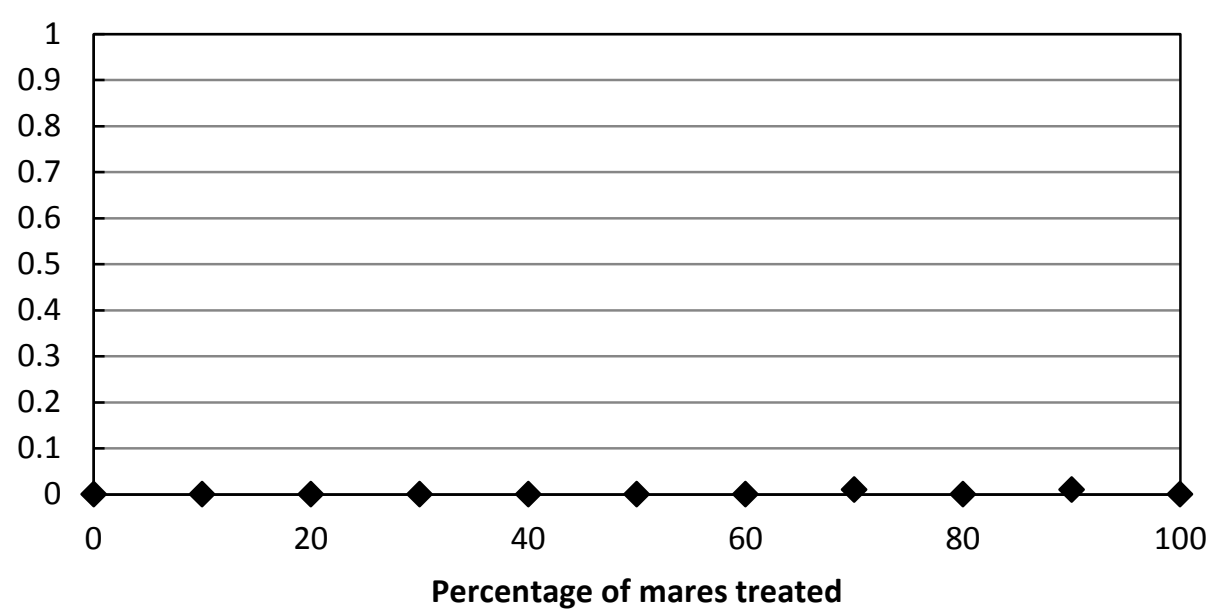

Figure 51. Initial population size $=750$, lambda $=1.05$, genetic diversity $=$ high .

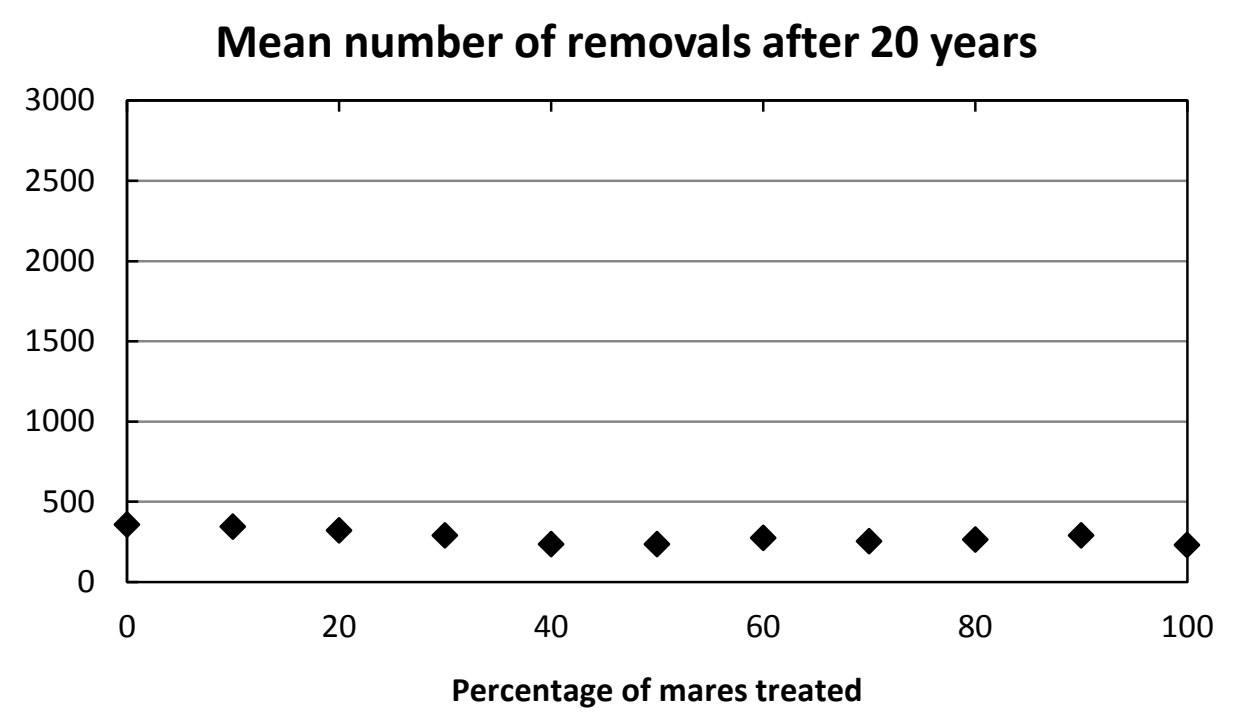

Mean heterozygosity after $\mathbf{5 0}$ years

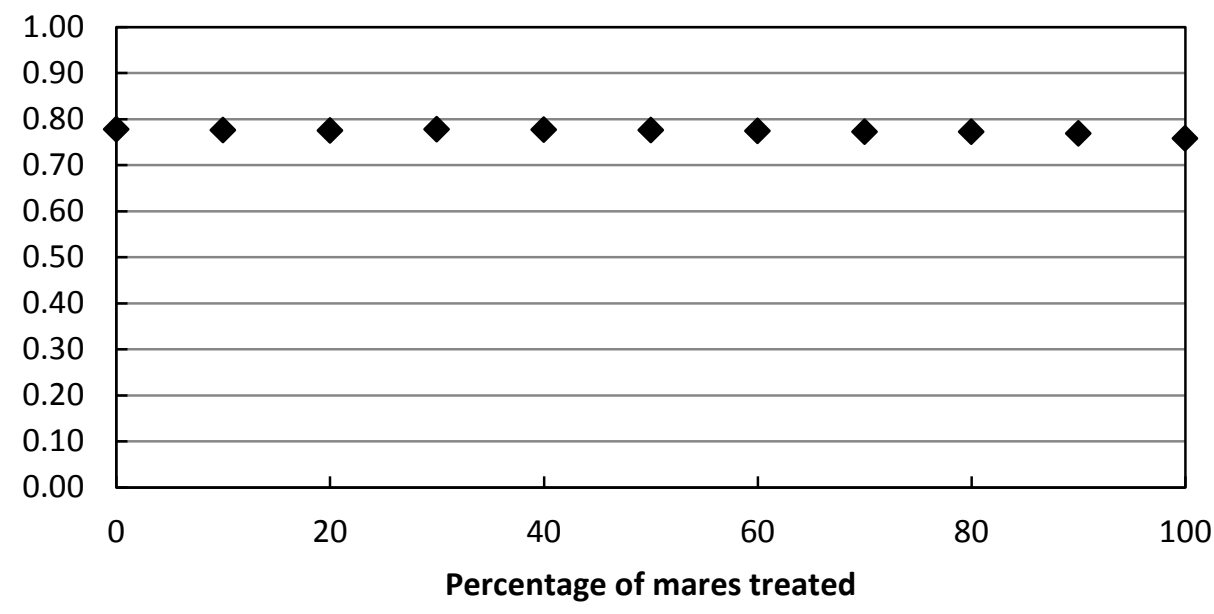




\begin{tabular}{lccccccccccc}
\hline \multicolumn{1}{c}{ Percentage of mares treated } & $\mathbf{0}$ & $\mathbf{1 0}$ & $\mathbf{2 0}$ & $\mathbf{3 0}$ & $\mathbf{4 0}$ & $\mathbf{5 0}$ & $\mathbf{6 0}$ & $\mathbf{7 0}$ & $\mathbf{8 0}$ & $\mathbf{9 0}$ & $\mathbf{1 0 0}$ \\
\hline Mean population size after 20 years & 662.85 & 602.11 & 594.21 & 609.04 & 573.37 & 563.41 & 531.5 & 490.61 & 478.09 & 432.79 & 467.39 \\
Mean number of removals after 20 years & 357.45 & 343.82 & 320.45 & 290.07 & 234.09 & 234 & 273.83 & 253.99 & 263.92 & 289.45 & 228.35 \\
Mean probability of extirpation after 50 years & 0 & 0 & 0 & 0 & 0 & 0 & 0 & 0.01 & 0 & 0.01 & 0 \\
Mean heterozygosity after 50 years & 0.78 & 0.78 & 0.77 & 0.78 & 0.78 & 0.78 & 0.77 & 0.77 & 0.77 & 0.77 & 0.76 \\
\hline
\end{tabular}

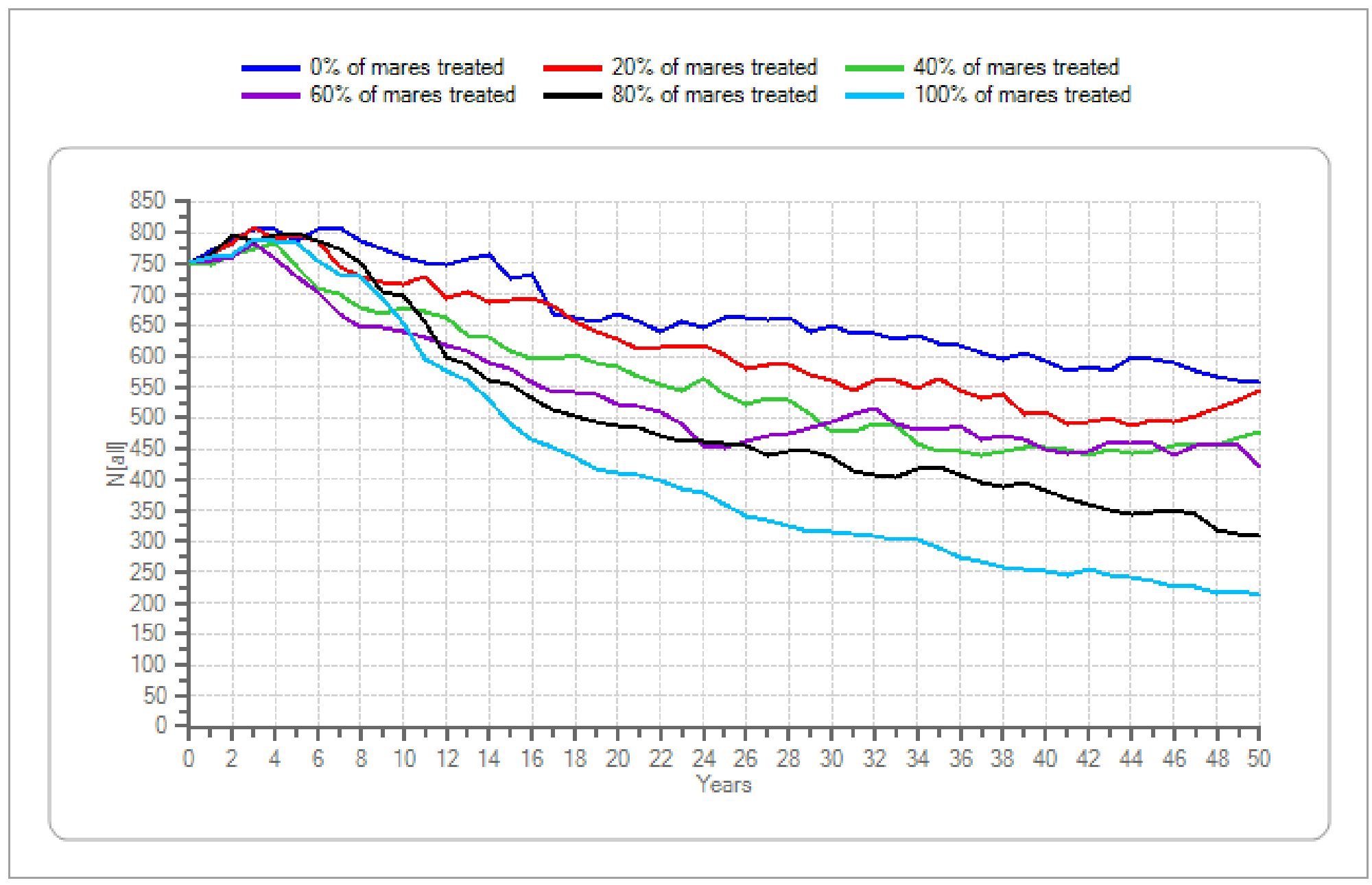

Figure 51. Initial population size $=750$, lambda $=1.05$, genetic diversity $=$ high - Continued 
Mean population size after 20 years

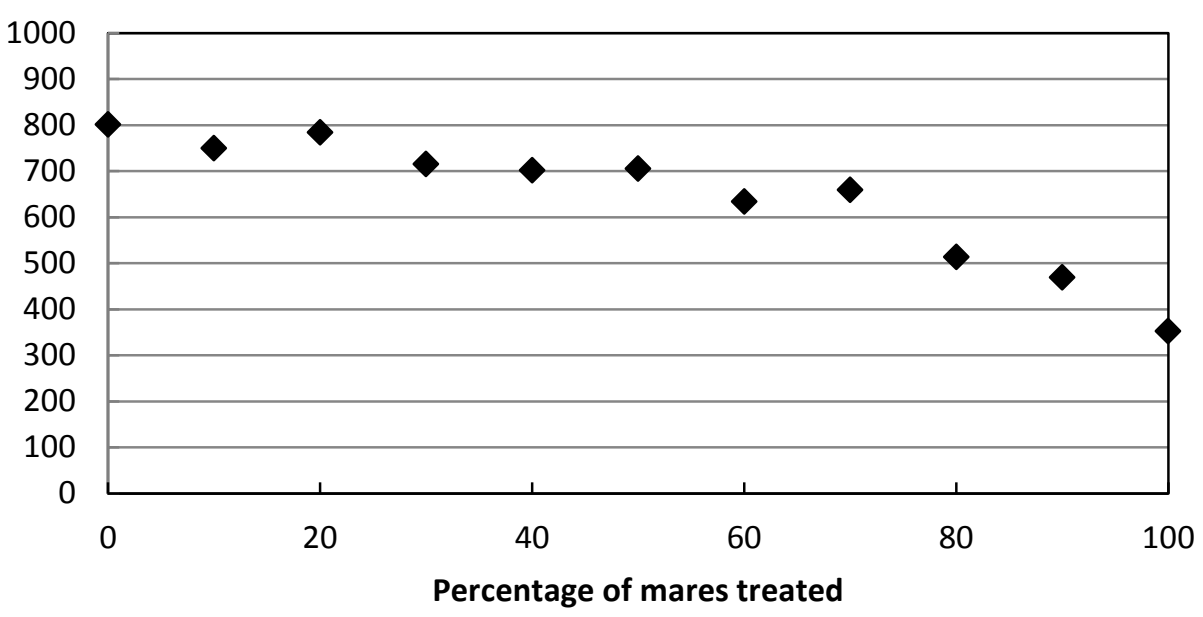

Mean probability of extirpation after 50 years

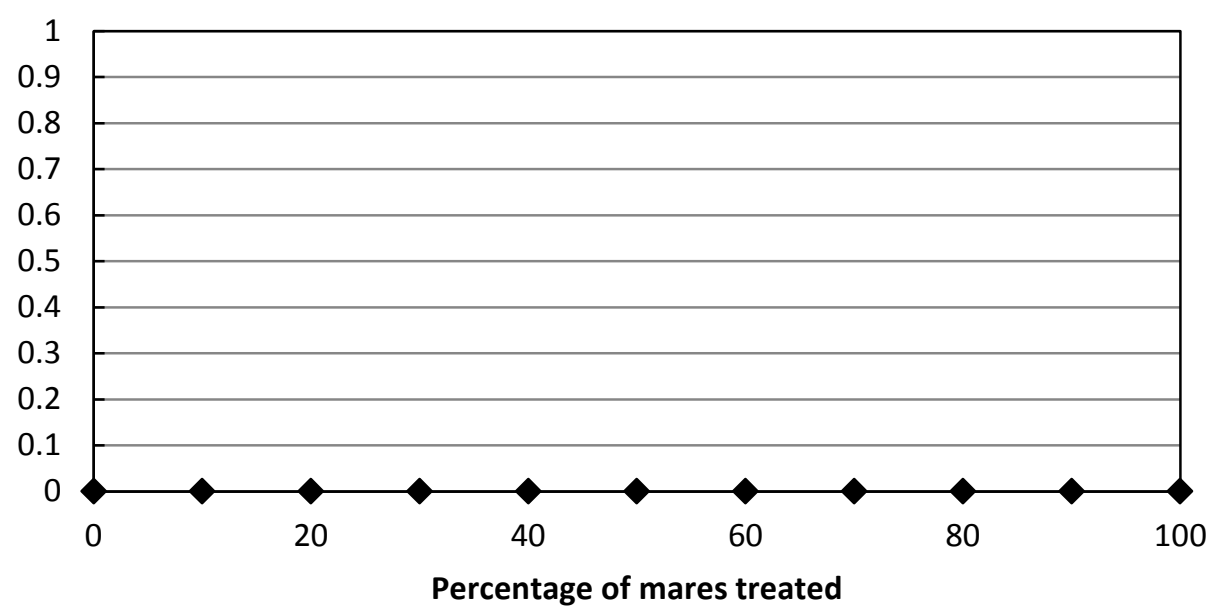

Figure 52. Initial population size $=750$, lambda $=1.10$, genetic diversity $=$ high .
Mean number of removals after 20 years

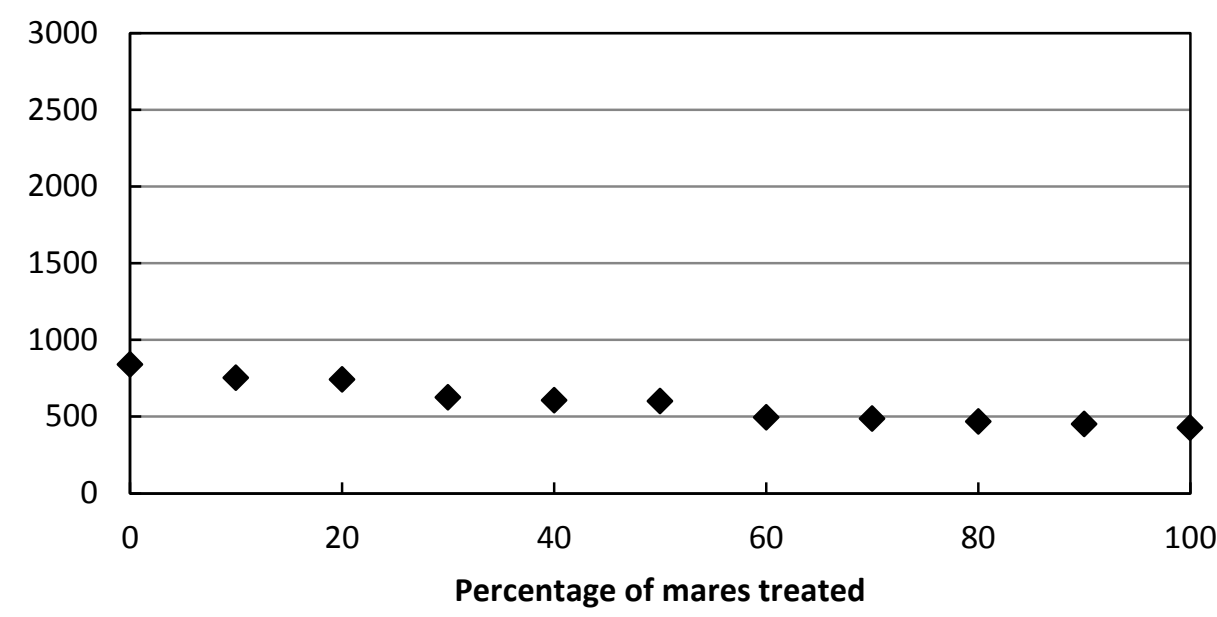

Mean heterozygosity after $\mathbf{5 0}$ years

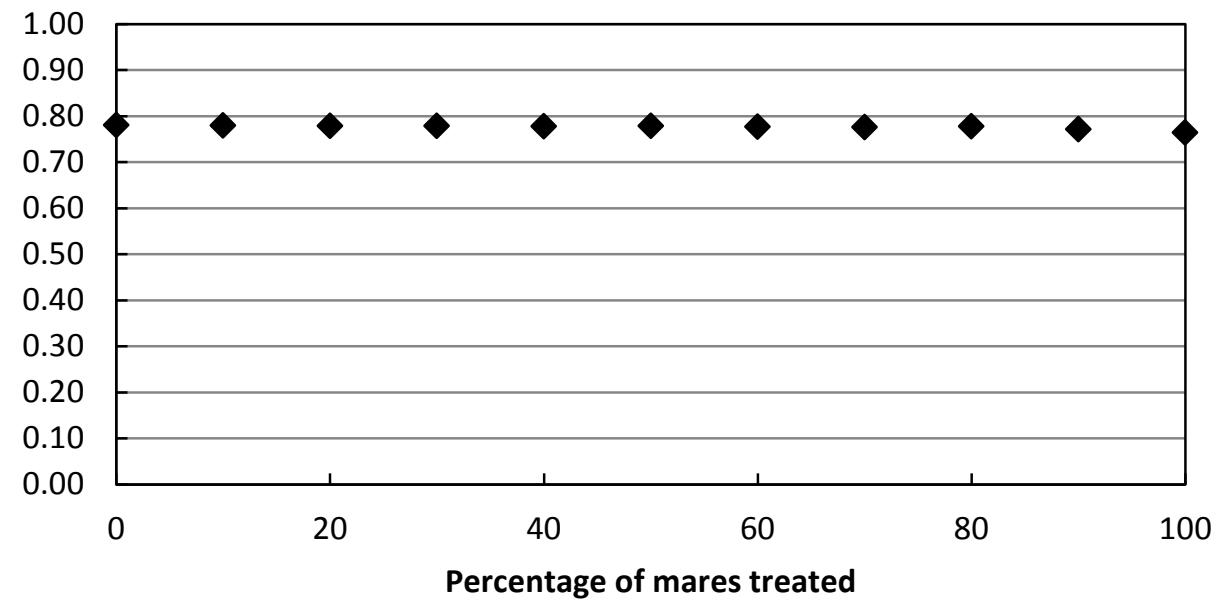




\begin{tabular}{lccccccccccc}
\hline \multicolumn{1}{c}{ Percentage of mares treated } & $\mathbf{0}$ & $\mathbf{1 0}$ & $\mathbf{2 0}$ & $\mathbf{3 0}$ & $\mathbf{4 0}$ & $\mathbf{5 0}$ & $\mathbf{6 0}$ & $\mathbf{7 0}$ & $\mathbf{8 0}$ & $\mathbf{9 0}$ & $\mathbf{1 0 0}$ \\
\hline Mean population size after 20 years & 800.78 & 749.94 & 784.34 & 714.78 & 701.29 & 705.25 & 633.52 & 658.99 & 513.24 & 468.63 & 351.92 \\
Mean number of removals after 20 years & 838.9 & 753.64 & 742.14 & 625.1 & 605.75 & 599.52 & 493.98 & 486.5 & 466.16 & 451.41 & 427.27 \\
Mean probability of extirpation after 50 years & 0 & 0 & 0 & 0 & 0 & 0 & 0 & 0 & 0 & 0 & 0 \\
Mean heterozygosity after 50 years & 0.78 & 0.78 & 0.78 & 0.78 & 0.78 & 0.78 & 0.78 & 0.78 & 0.78 & 0.77 & 0.76 \\
\hline
\end{tabular}

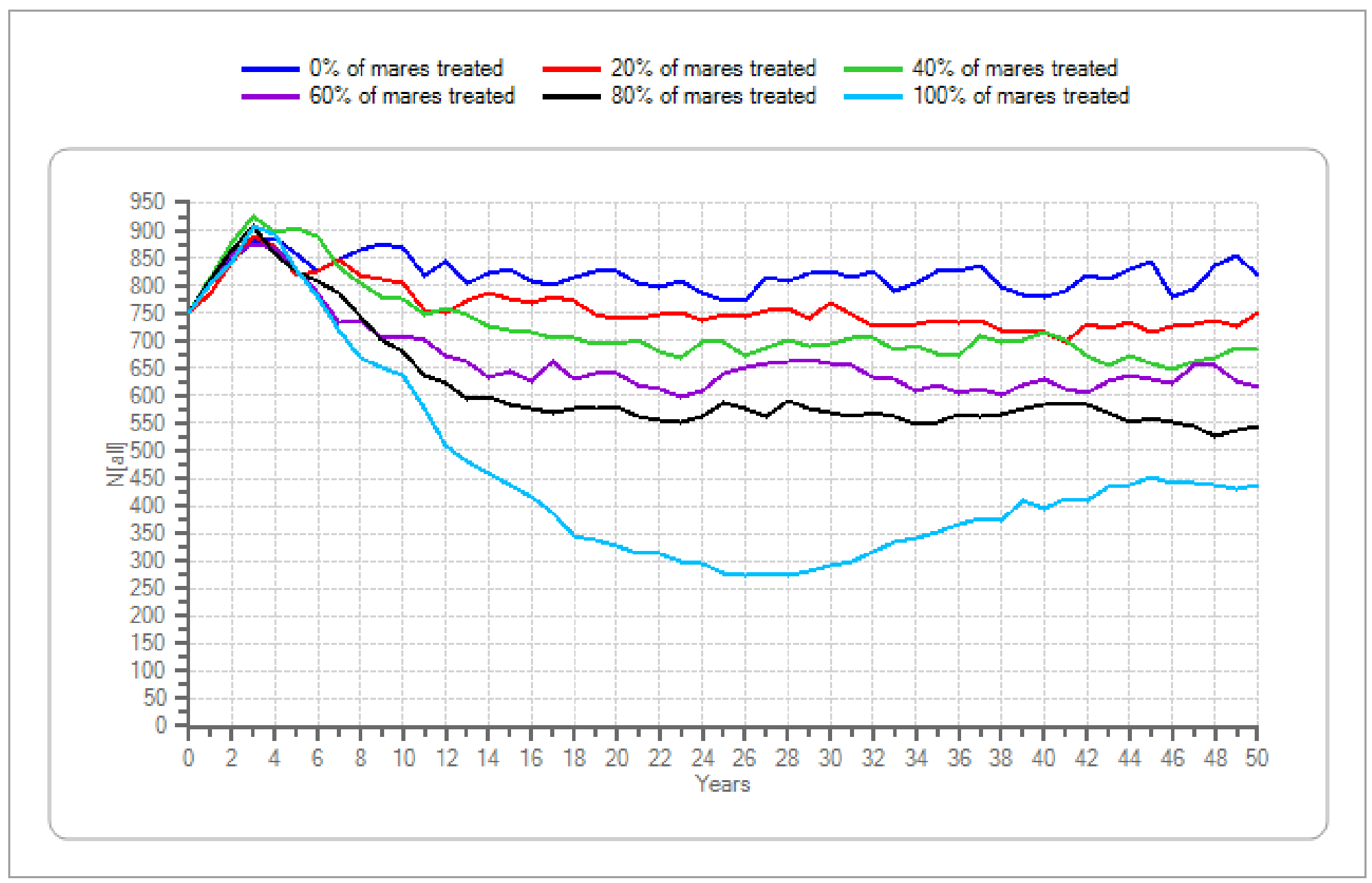

Figure 52. Initial population size $=750$, lambda $=1.10$, genetic diversity $=$ high. Continued 
Mean population size after 20 years

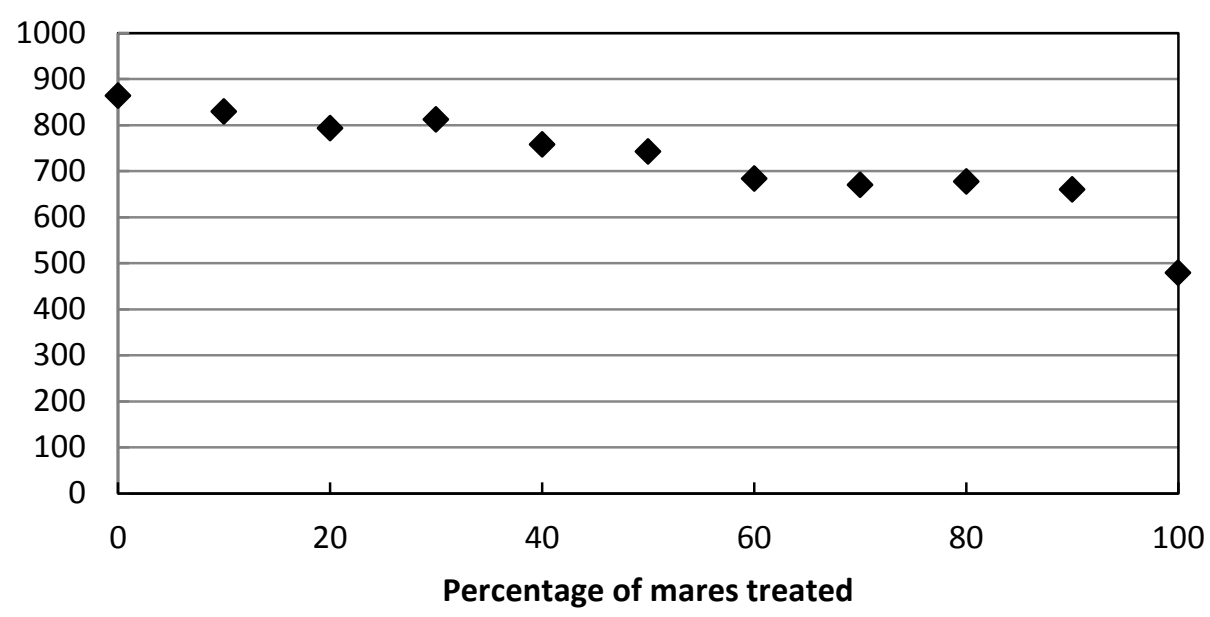

Mean probability of extirpation after $\mathbf{5 0}$ years

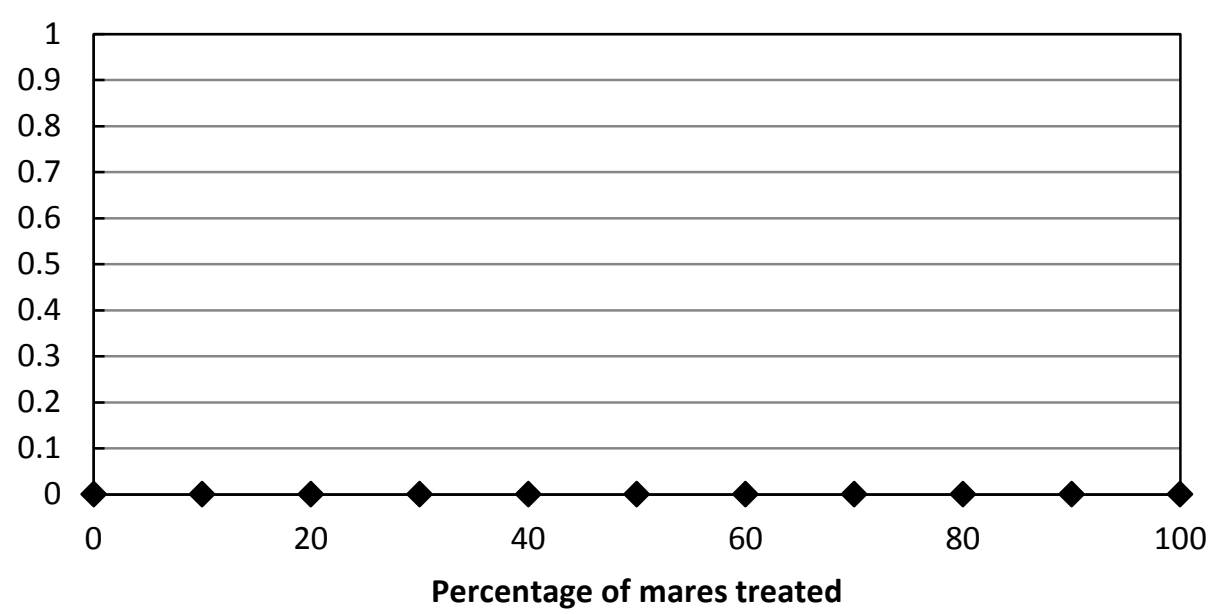

Figure 53. Initial population size $=750$, lambda $=1.15$, genetic diversity $=$ high .
Mean number of removals after 20 years

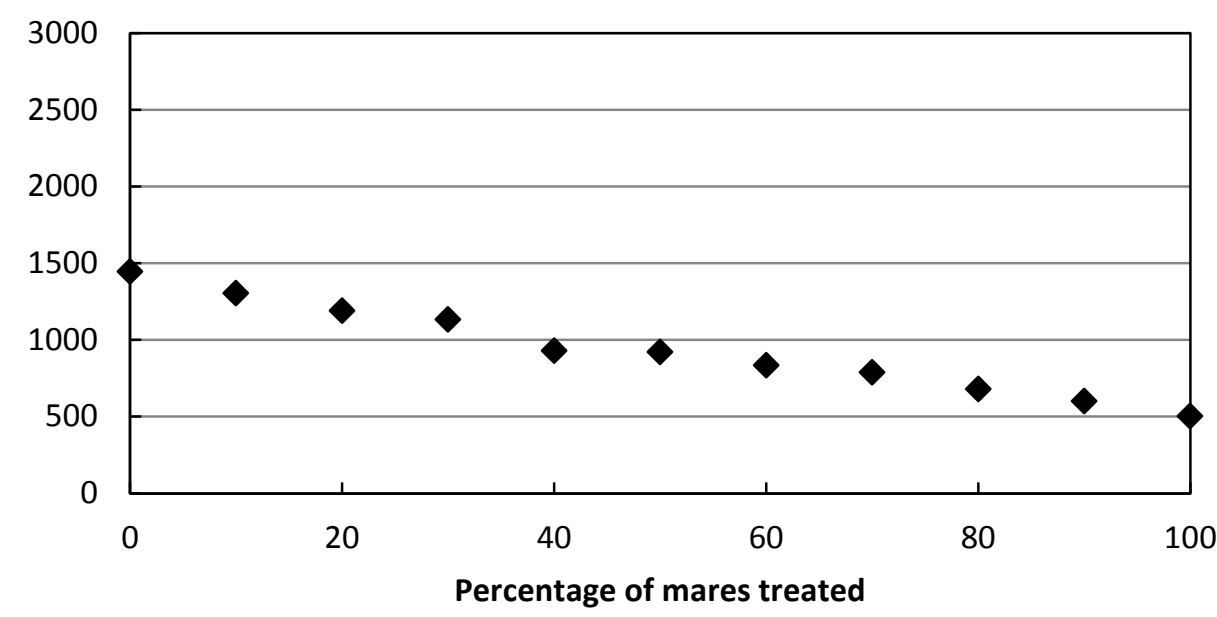

Mean heterozygosity after $\mathbf{5 0}$ years

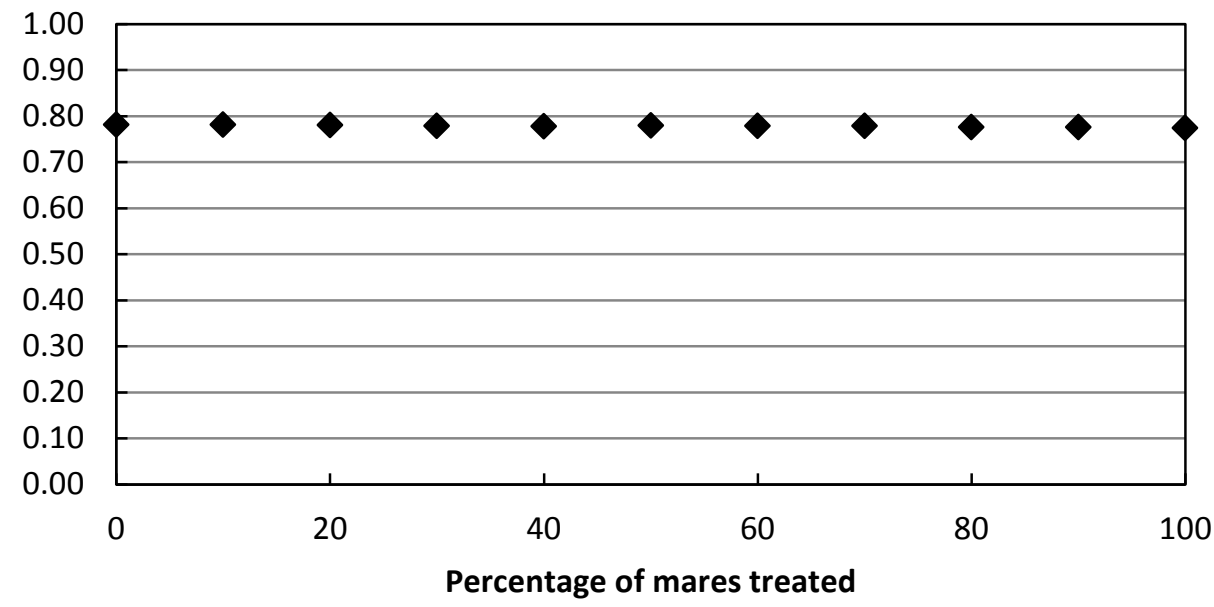




\begin{tabular}{lccccccccccc}
\hline \multicolumn{1}{c}{ Percentage of mares treated } & $\mathbf{0}$ & $\mathbf{1 0}$ & $\mathbf{2 0}$ & $\mathbf{3 0}$ & $\mathbf{4 0}$ & $\mathbf{5 0}$ & $\mathbf{6 0}$ & $\mathbf{7 0}$ & $\mathbf{8 0}$ & $\mathbf{9 0}$ & $\mathbf{1 0 0}$ \\
\hline Mean population size after 20 years & 863.26 & 828.87 & 793.09 & 812.32 & 758.17 & 742.73 & 683.78 & 670.07 & 677.22 & 660.32 & 478.72 \\
Mean number of removals after 20 years & 1444.8 & 1304.1 & 1189.2 & 1132.7 & 929.9 & 920.5 & 834.1 & 788.1 & 679.1 & 599.5 & 501.4 \\
Mean probability of extirpation after 50 years & 0 & 0 & 0 & 0 & 0 & 0 & 0 & 0 & 0 & 0 & 0 \\
Mean heterozygosity after 50 years & 0.78 & 0.78 & 0.78 & 0.78 & 0.78 & 0.78 & 0.78 & 0.78 & 0.78 & 0.78 & 0.77 \\
\hline
\end{tabular}

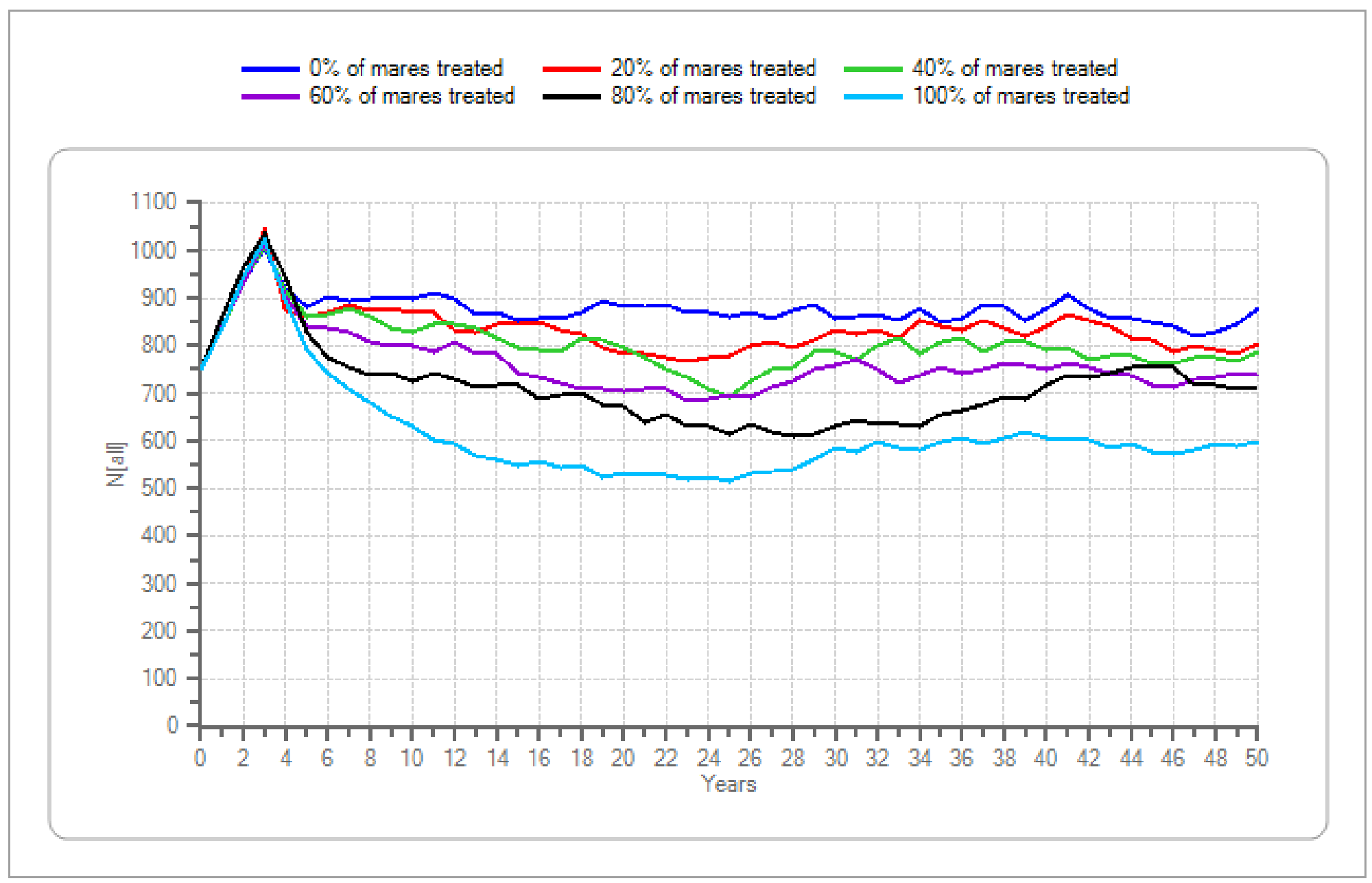

Figure 53. Initial population size $=750$, lambda $=1.15$, genetic diversity $=$ high. Continued 
Mean population size after 20 years

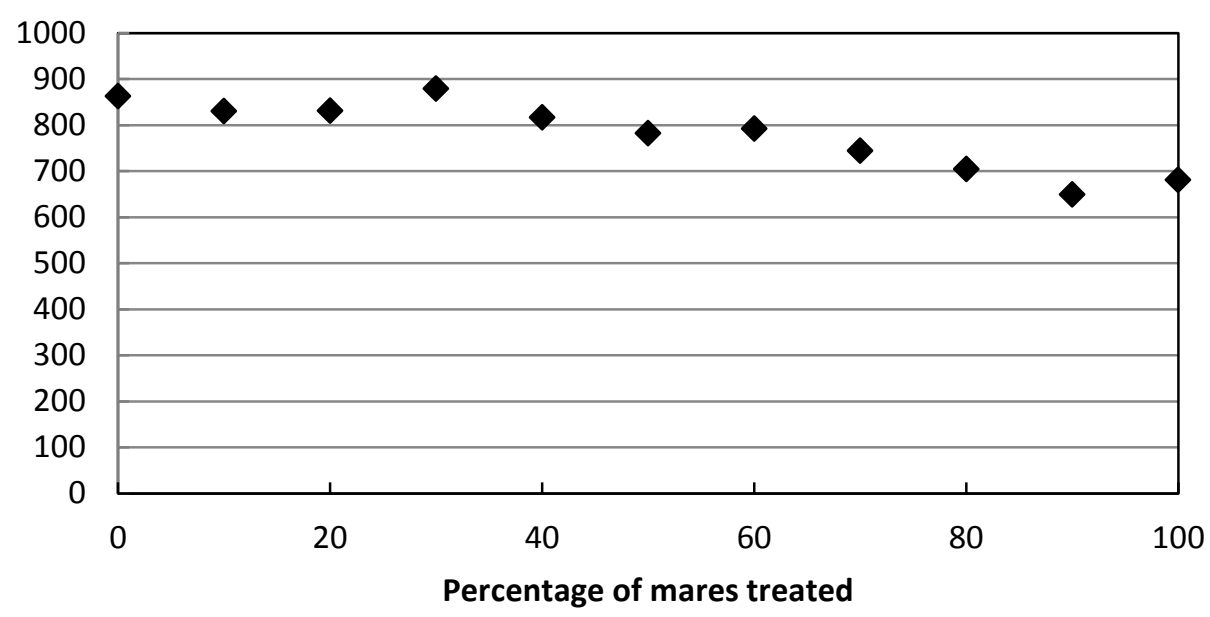

Mean probability of extirpation after $\mathbf{5 0}$ years

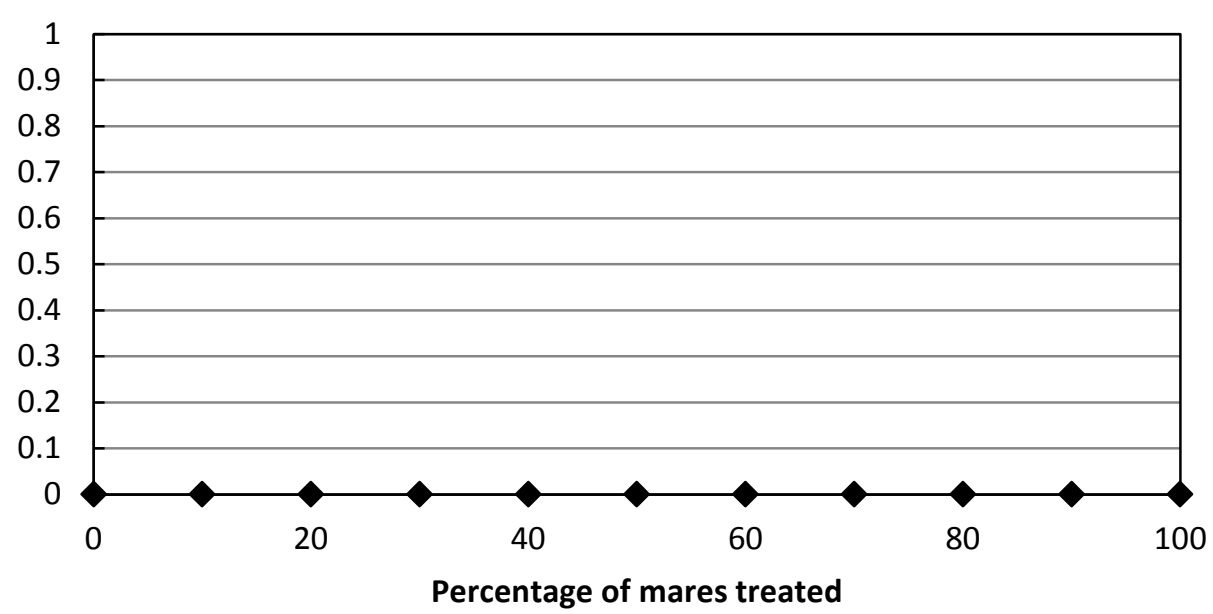

Figure 54. Initial population size $=750$, lambda $=1.20$, genetic diversity $=$ high .
Mean number of removals after 20 years

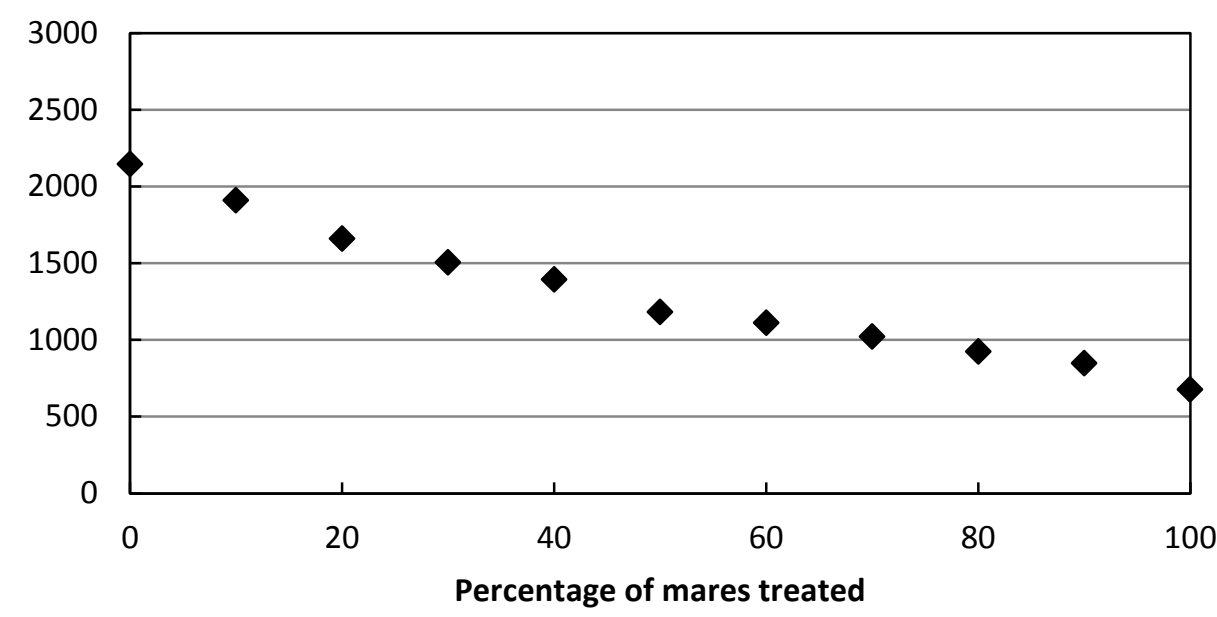

Mean heterozygosity after $\mathbf{5 0}$ years

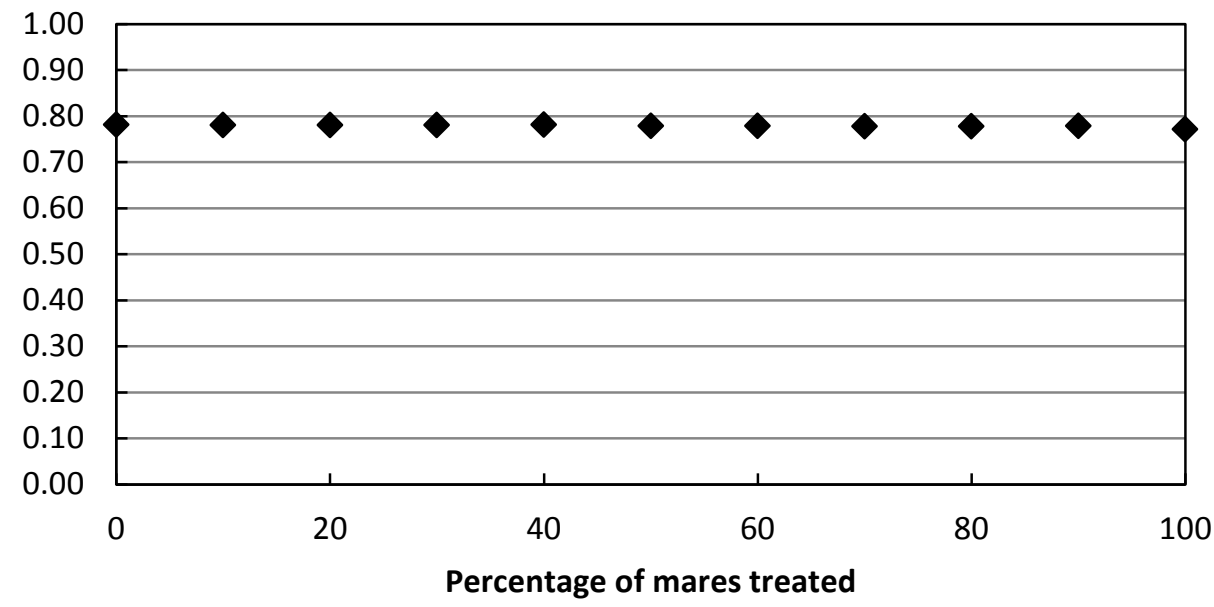




\begin{tabular}{lcccccccccccc}
\hline \multicolumn{1}{c}{ Percentage of mares treated } & $\mathbf{0}$ & $\mathbf{1 0}$ & $\mathbf{2 0}$ & $\mathbf{3 0}$ & $\mathbf{4 0}$ & $\mathbf{5 0}$ & $\mathbf{6 0}$ & $\mathbf{7 0}$ & $\mathbf{8 0}$ & $\mathbf{9 0}$ & $\mathbf{1 0 0}$ \\
\hline Mean population size after 20 years & 862.48 & 830.01 & 830.72 & 878.81 & 816.6 & 782.19 & 791.89 & 743.94 & 704.05 & 649.05 & 680.91 \\
Mean number of removals after 20 years & 2146.1 & 1909.2 & 1659.2 & 1506.0 & 1394.6 & 1181.1 & 1109.7 & 1021.6 & 923.1 & 847.1 & 675.6 \\
Mean probability of extirpation after 50 years & 0 & 0 & 0 & 0 & 0 & 0 & 0 & 0 & 0 & 0 & 0 \\
Mean heterozygosity after 50 years & 0.78 & 0.78 & 0.78 & 0.78 & 0.78 & 0.78 & 0.78 & 0.78 & 0.78 & 0.78 & 0.77 \\
\hline
\end{tabular}

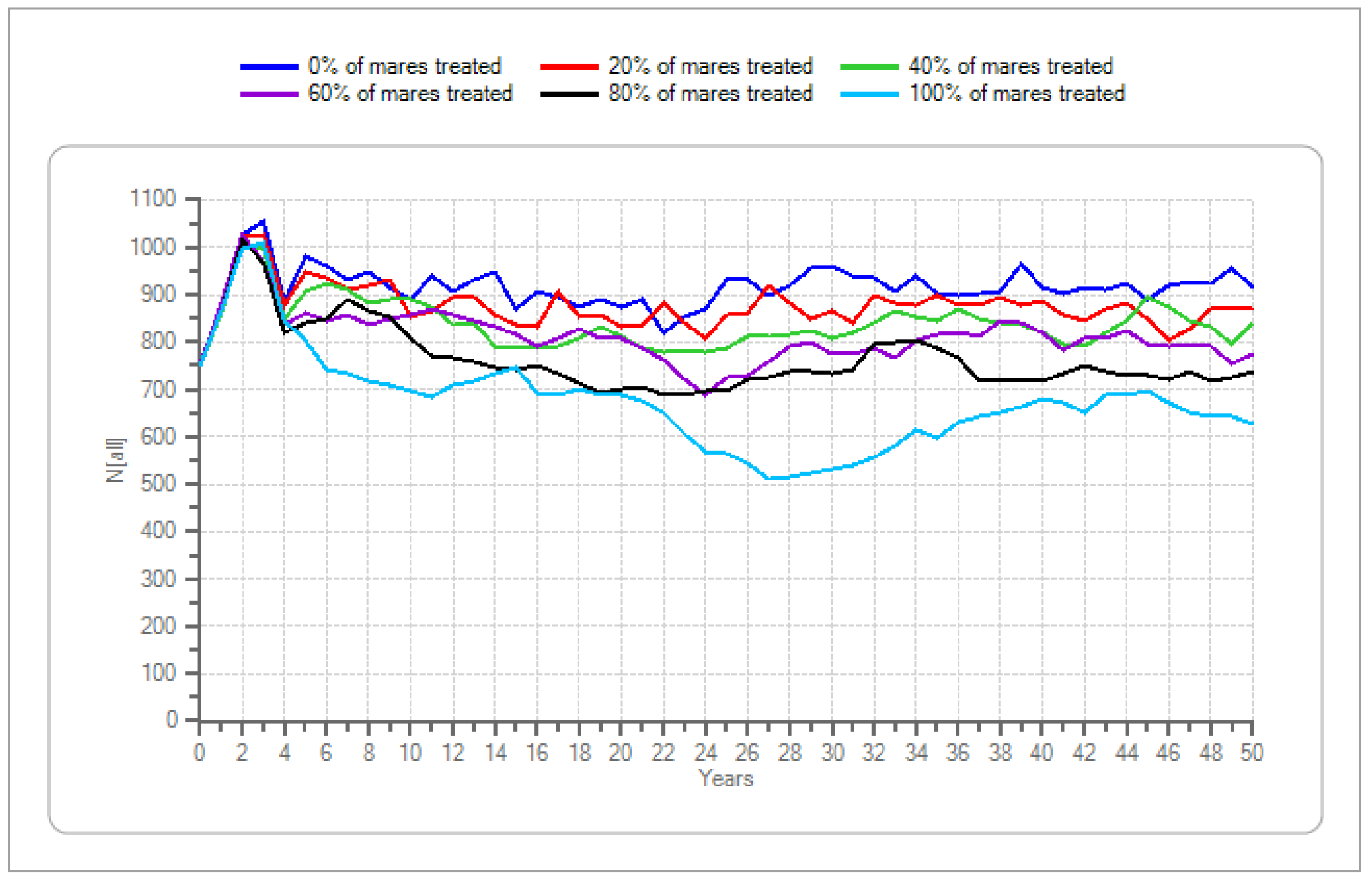

Figure 54. Initial population size $=750$, lambda $=1.20$, genetic diversity $=$ high - Continued 
Mean population size after 20 years

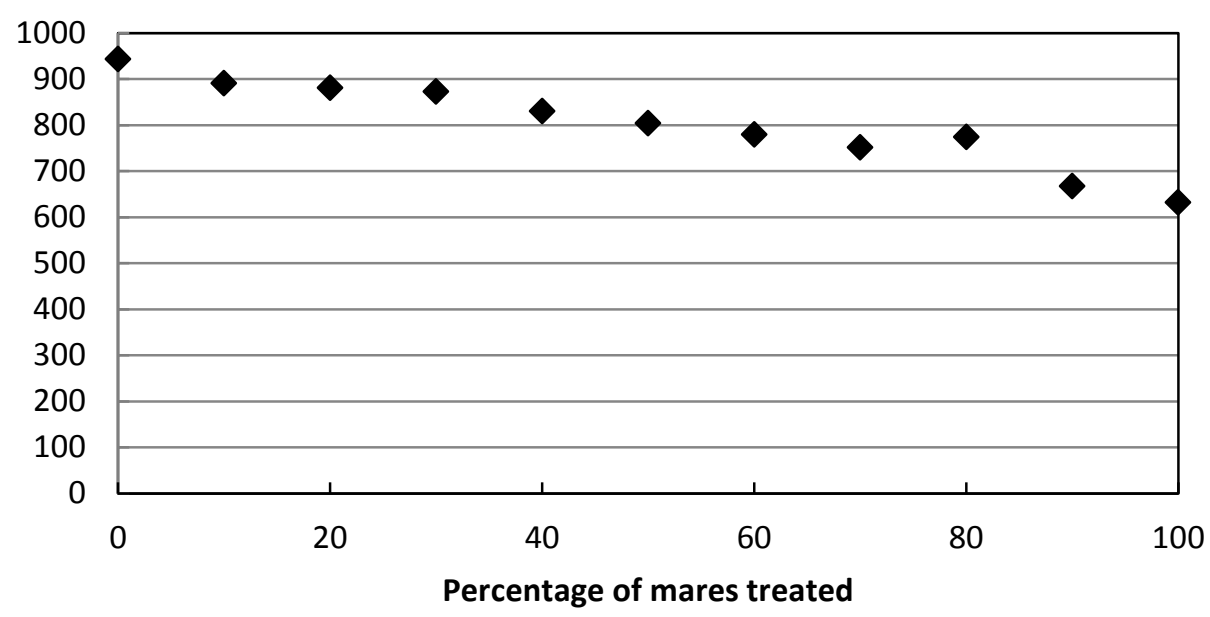

Mean probability of extirpation after $\mathbf{5 0}$ years

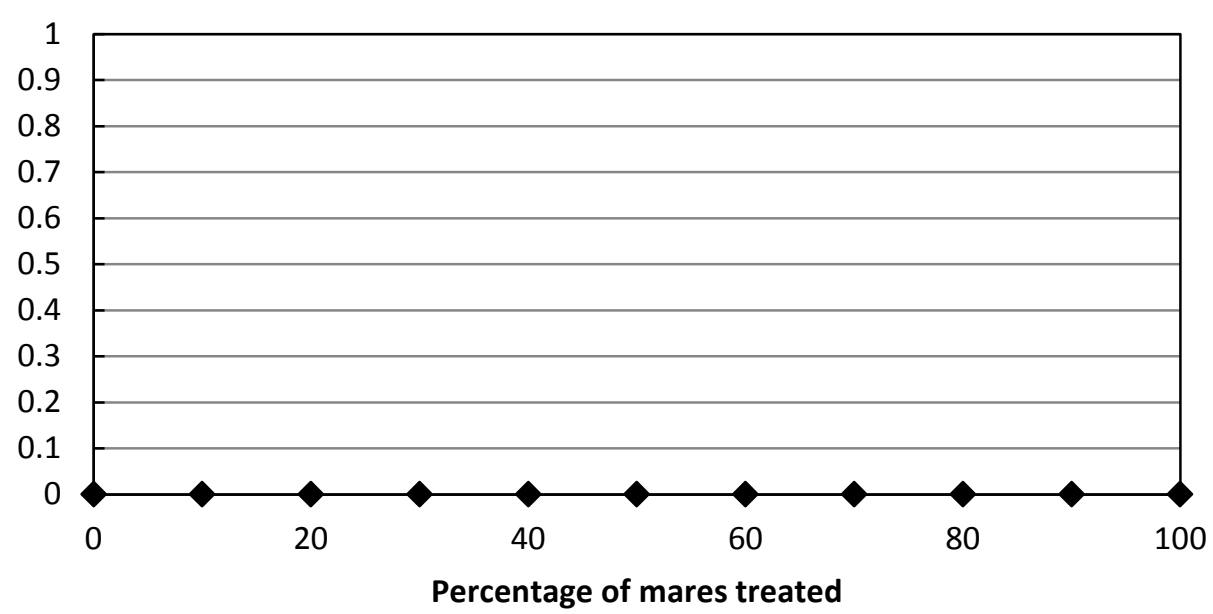

Figure 55. Initial population size $=750$, lambda $=1.25$, genetic diversity $=$ high .
Mean number of removals after 20 years

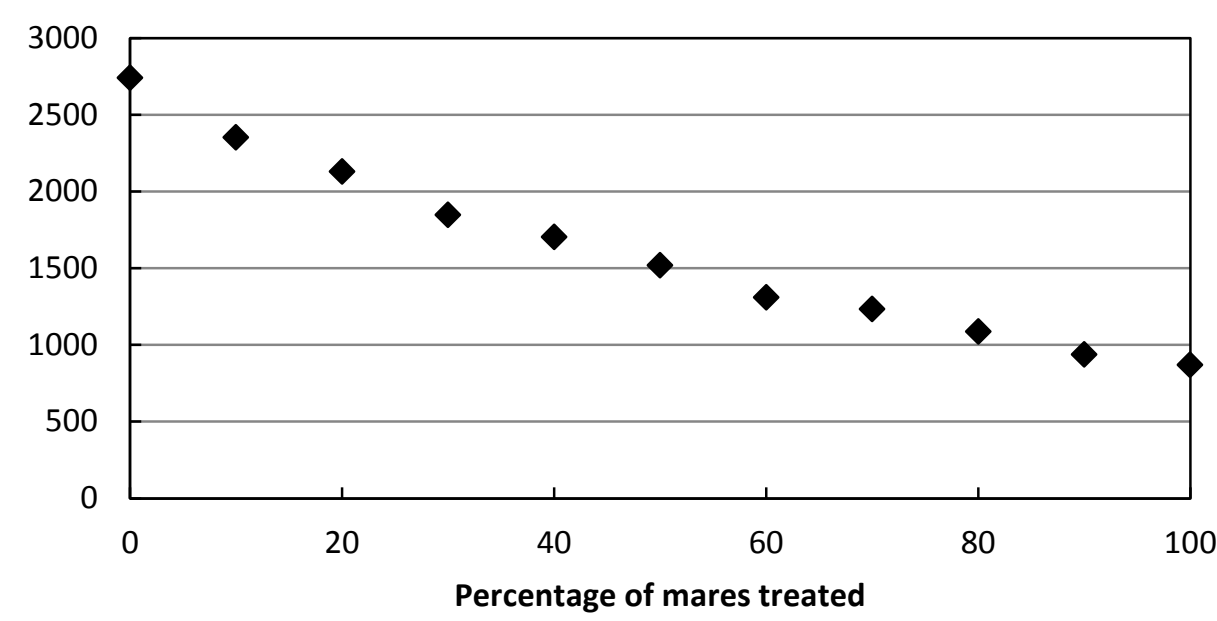

Mean heterozygosity after $\mathbf{5 0}$ years

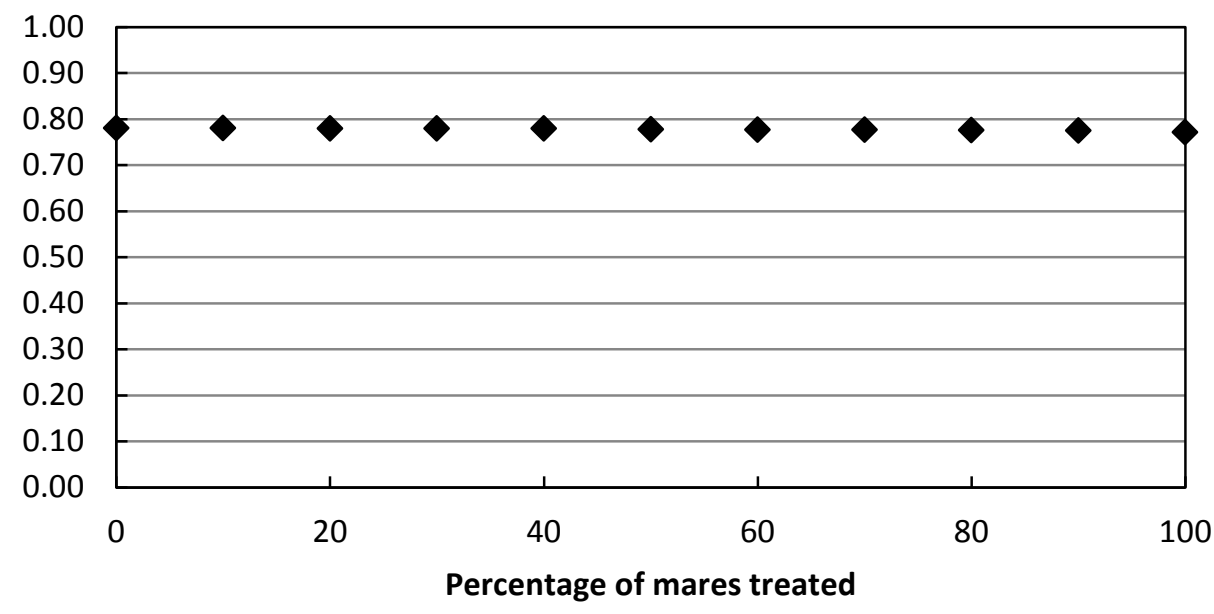




\begin{tabular}{lccccccccccc}
\hline \multicolumn{1}{c}{ Percentage of mares treated } & $\mathbf{0}$ & $\mathbf{1 0}$ & $\mathbf{2 0}$ & $\mathbf{3 0}$ & $\mathbf{4 0}$ & $\mathbf{5 0}$ & $\mathbf{6 0}$ & $\mathbf{7 0}$ & $\mathbf{8 0}$ & $\mathbf{9 0}$ & $\mathbf{1 0 0}$ \\
\hline Mean population size after 20 years & 943.03 & 890.54 & 880.47 & 872.85 & 830.01 & 804.21 & 779.9 & 751.3 & 773.8 & 666.84 & 631.64 \\
Mean number of removals after 20 years & 2741.1 & 2352.9 & 2128.9 & 1845.6 & 1703.7 & 1518.4 & 1309.1 & 1234.3 & 1086.5 & 937.0 & 868.1 \\
Mean probability of extirpation after 50 years & 0 & 0 & 0 & 0 & 0 & 0 & 0 & 0 & 0 & 0 & 0 \\
Mean heterozygosity after 50 years & 0.78 & 0.78 & 0.78 & 0.78 & 0.78 & 0.78 & 0.78 & 0.78 & 0.78 & 0.77 & 0.77 \\
\hline
\end{tabular}

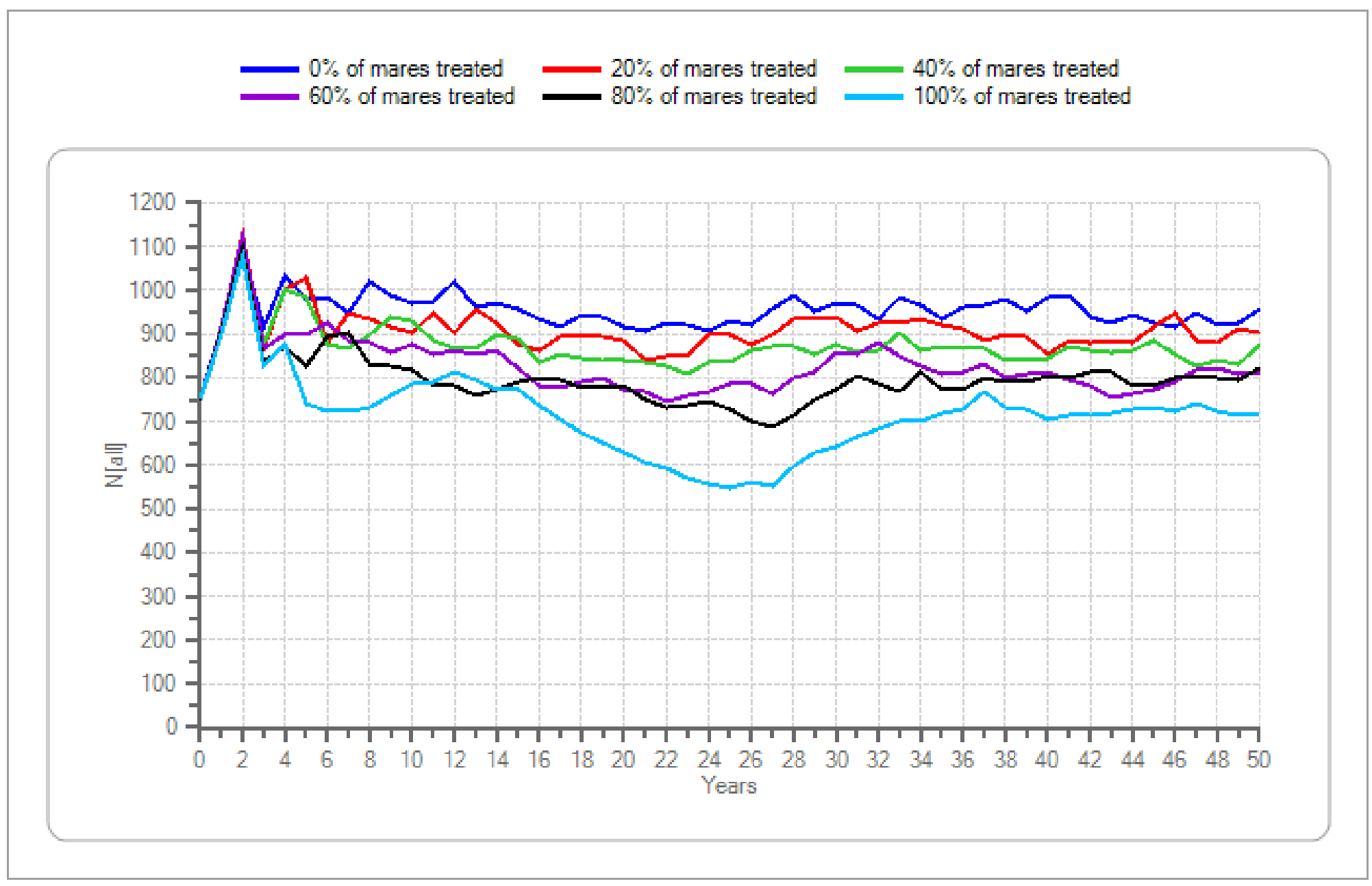

Figure 55. Initial population size $=750$, lambda $=1.25$, genetic diversity $=$ high - Continued 
Mean population size after 20 years

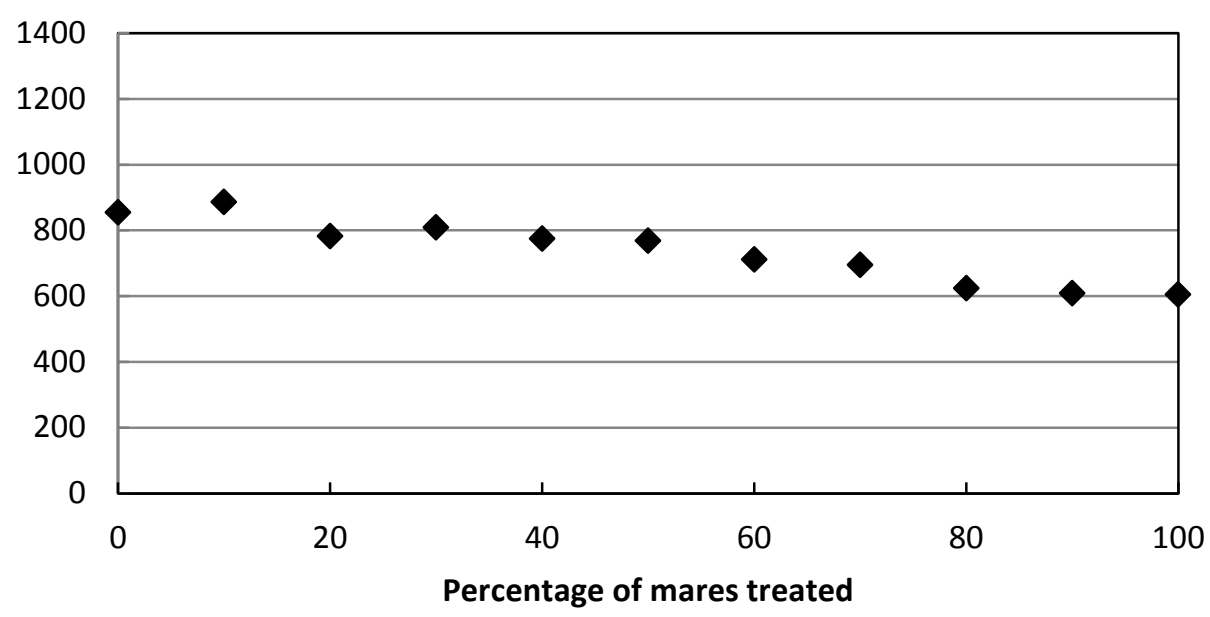

Mean probability of extirpation after 50 years

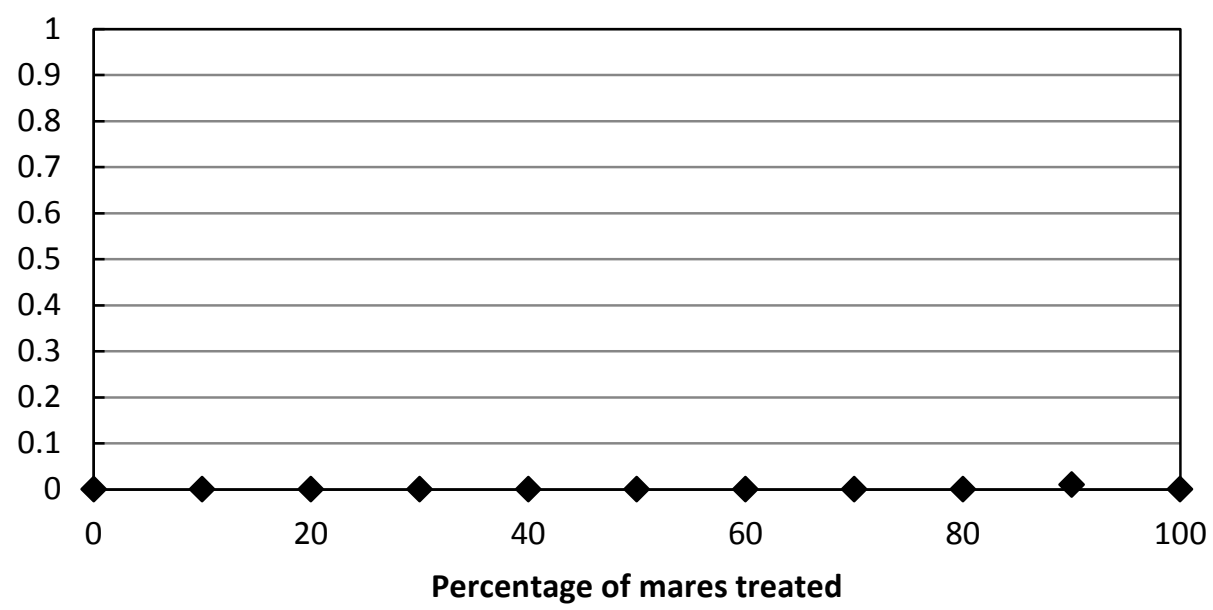

Figure 56. Initial population size $=1000$, lambda $=1.05$, genetic diversity $=$ high .
Mean number of removals after 20 years

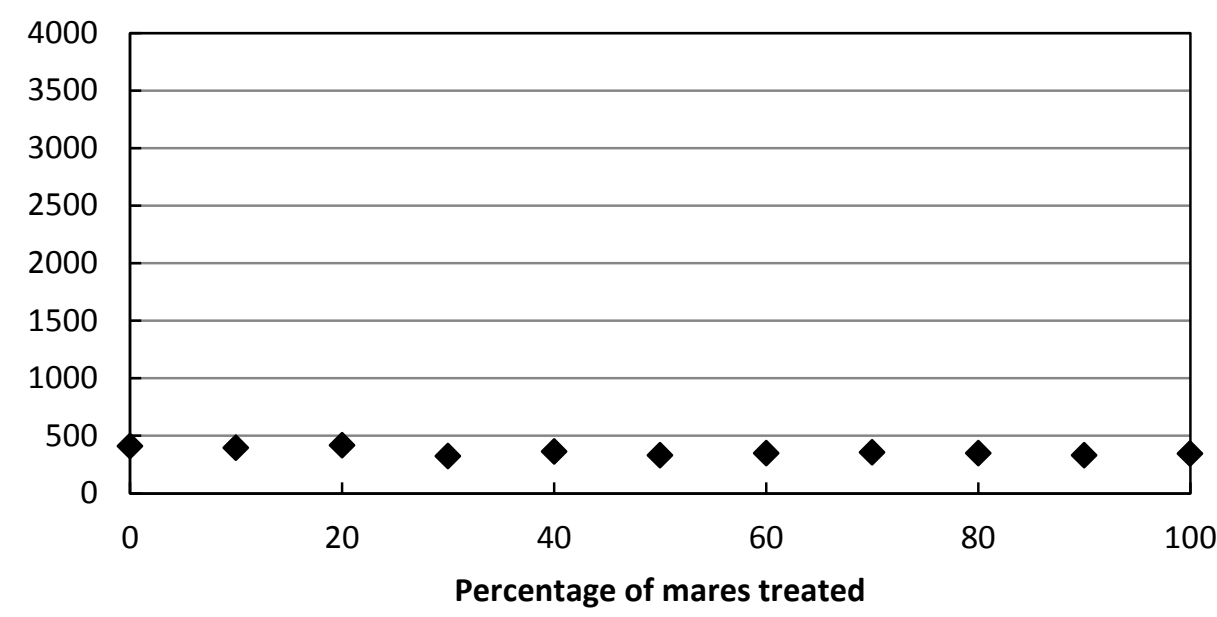

Mean heterozygosity after $\mathbf{5 0}$ years

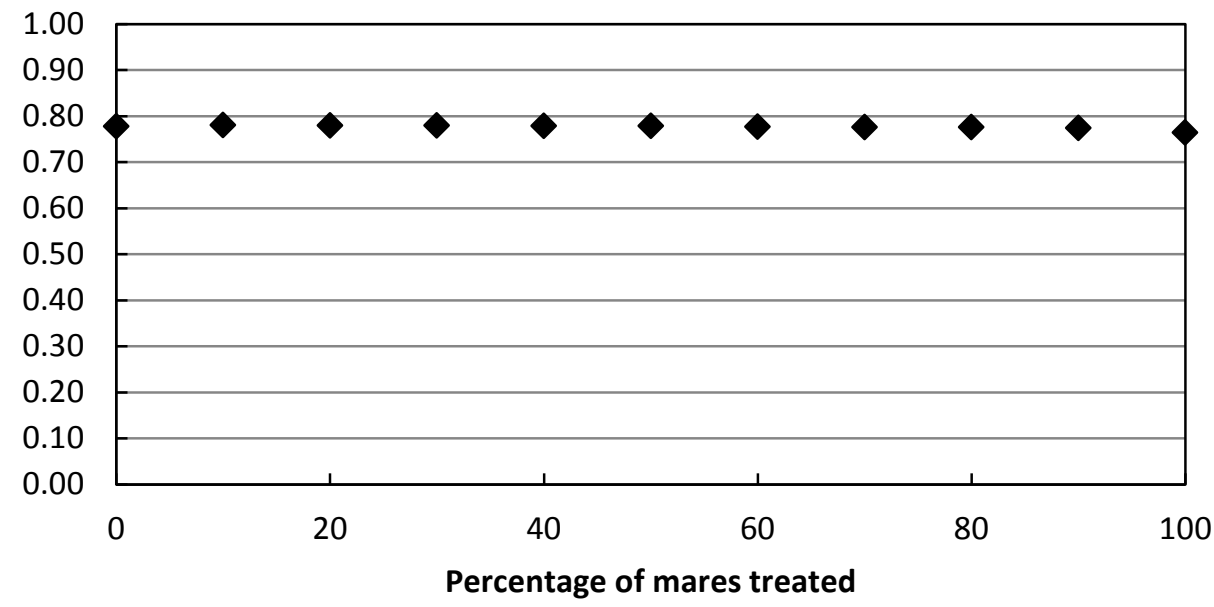




\begin{tabular}{lccccccccccc}
\hline \multicolumn{1}{c}{ Percentage of mares treated } & $\mathbf{0}$ & $\mathbf{1 0}$ & $\mathbf{2 0}$ & $\mathbf{3 0}$ & $\mathbf{4 0}$ & $\mathbf{5 0}$ & $\mathbf{6 0}$ & $\mathbf{7 0}$ & $\mathbf{8 0}$ & $\mathbf{9 0}$ & $\mathbf{1 0 0}$ \\
\hline Mean population size after 20 years & 854.42 & 886.33 & 782.46 & 808.7 & 774.68 & 767.58 & 711.38 & 694.91 & 623.41 & 608.7 & 604 \\
Mean number of removals after 20 years & 408.46 & 393.28 & 416.19 & 321.57 & 362.43 & 331.22 & 346.92 & 353.96 & 346.09 & 328.18 & 344.26 \\
Mean probability of extirpation after 50 years & 0 & 0 & 0 & 0 & 0 & 0 & 0 & 0 & 0 & 0.01 & 0 \\
Mean heterozygosity after 50 years & 0.78 & 0.78 & 0.78 & 0.78 & 0.78 & 0.78 & 0.78 & 0.78 & 0.78 & 0.77 & 0.76 \\
\hline
\end{tabular}

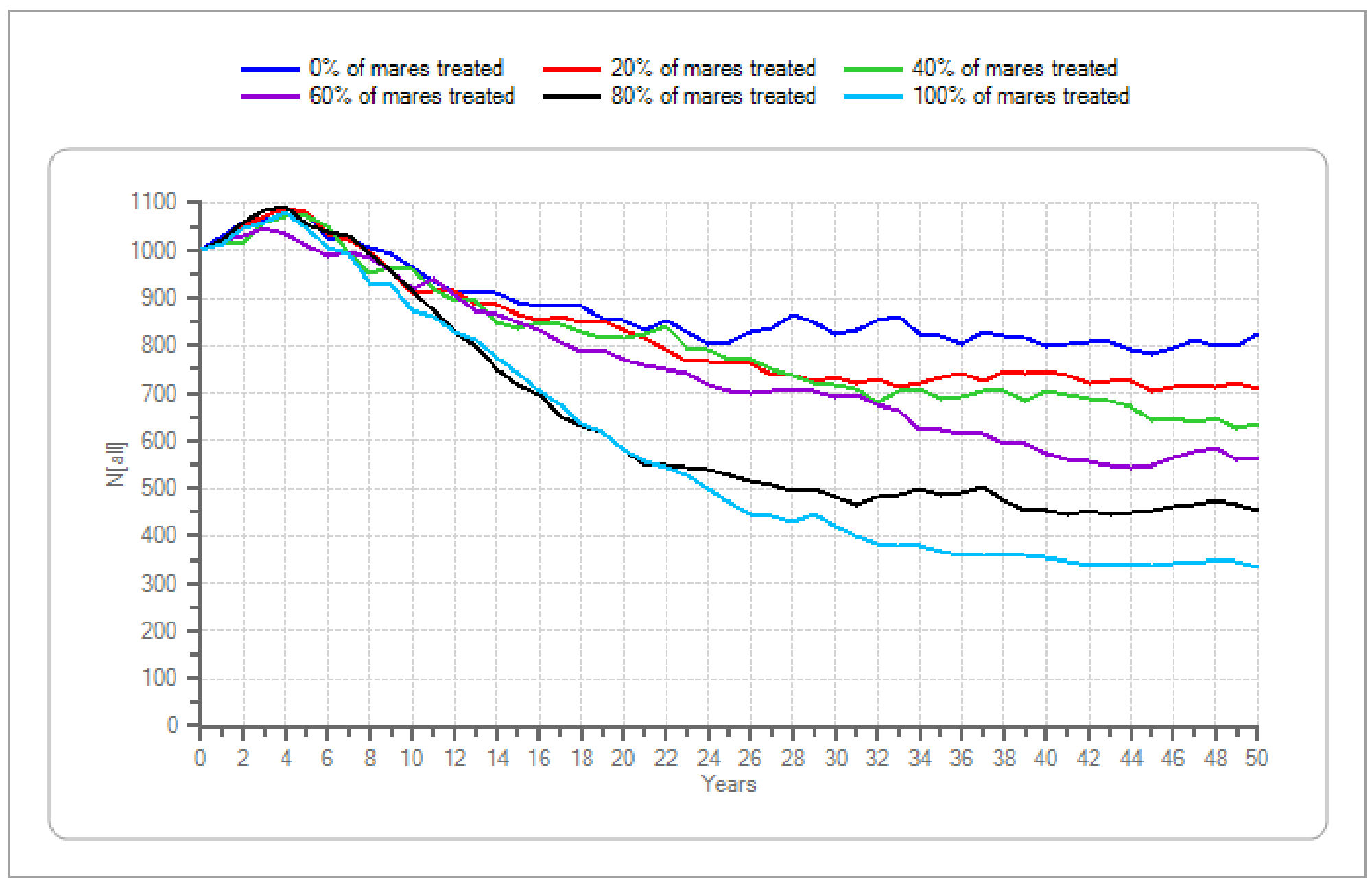

Figure 56. Initial population size $=1000$, lambda $=1.05$, genetic diversity $=$ high - Continued 
Mean population size after 20 years

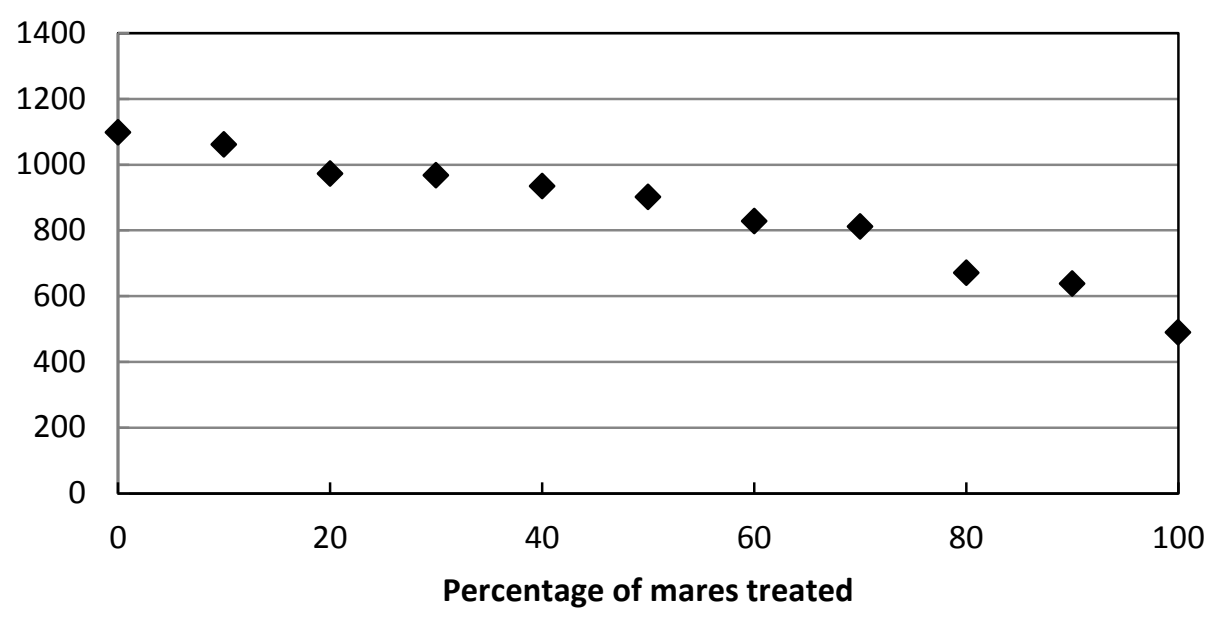

Mean probability of extirpation after 50 years

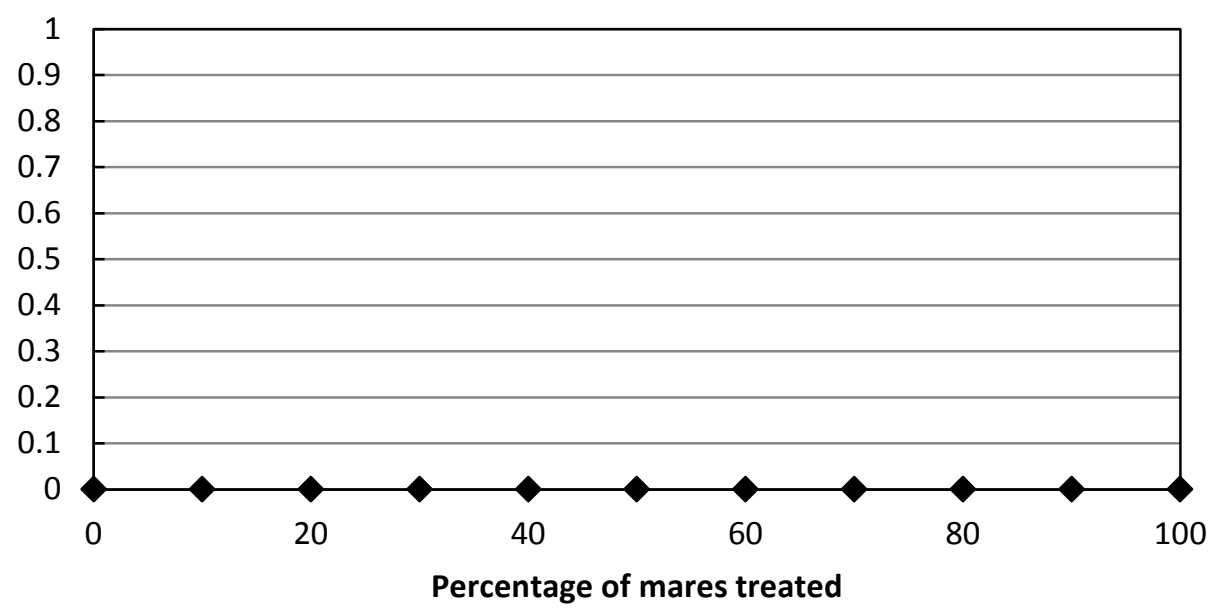

Figure 57. Initial population size $=1000$, lambda $=1.10$, genetic diversity $=$ high .
Mean number of removals after 20 years

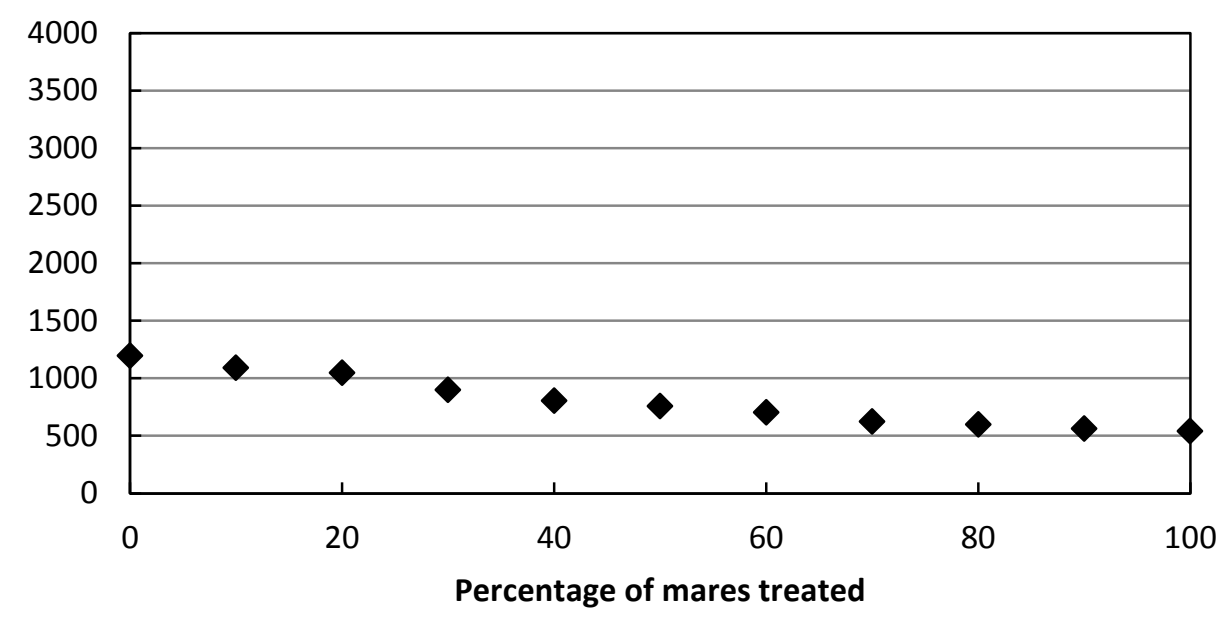

Mean heterozygosity after $\mathbf{5 0}$ years

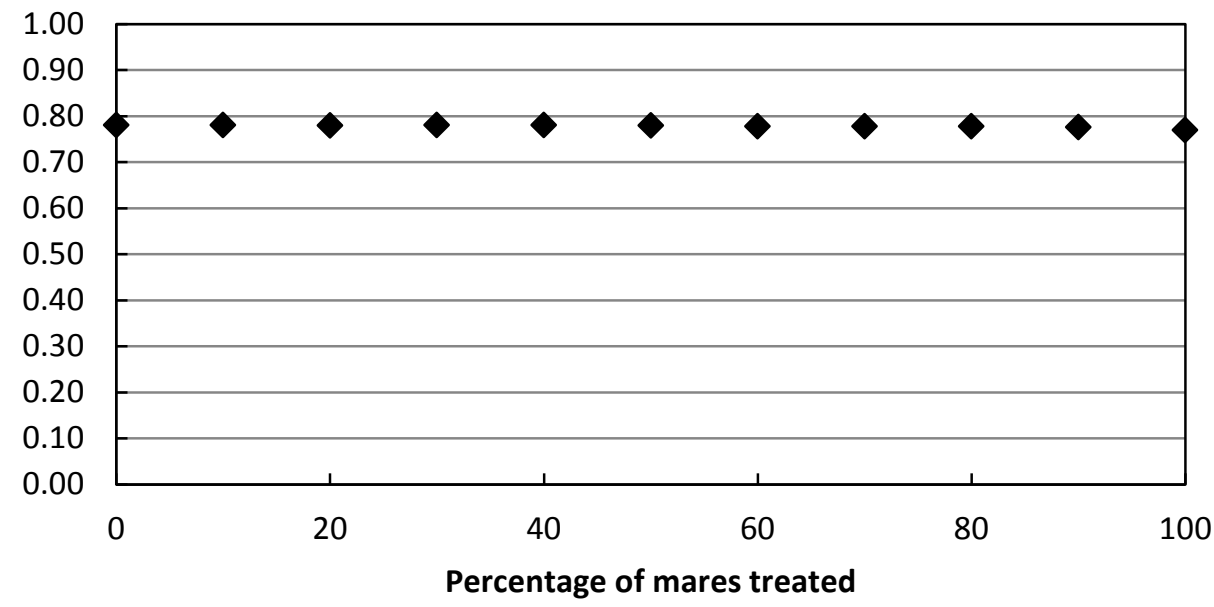




\begin{tabular}{lcccccccccccc}
\hline \multicolumn{1}{c}{ Percentage of mares treated } & $\mathbf{0}$ & $\mathbf{1 0}$ & $\mathbf{2 0}$ & $\mathbf{3 0}$ & $\mathbf{4 0}$ & $\mathbf{5 0}$ & $\mathbf{6 0}$ & $\mathbf{7 0}$ & $\mathbf{8 0}$ & $\mathbf{9 0}$ & $\mathbf{1 0 0}$ \\
\hline Mean population size after 20 years & 1098.09 & 1061.14 & 972.15 & 967.57 & 933.76 & 901.35 & 828.11 & 810.67 & 669.9 & 638.13 & 489.1 \\
Mean number of removals after 20 years & 1196.9 & 1091.1 & 1048.1 & 899.6 & 804.5 & 755.5 & 702.3 & 621.7 & 597.8 & 560.3 & 541.2 \\
Mean probability of extirpation after 50 years & 0 & 0 & 0 & 0 & 0 & 0 & 0 & 0 & 0 & 0 & 0 \\
Mean heterozygosity after 50 years & 0.78 & 0.78 & 0.78 & 0.78 & 0.78 & 0.78 & 0.78 & 0.78 & 0.78 & 0.78 & 0.77 \\
\hline
\end{tabular}

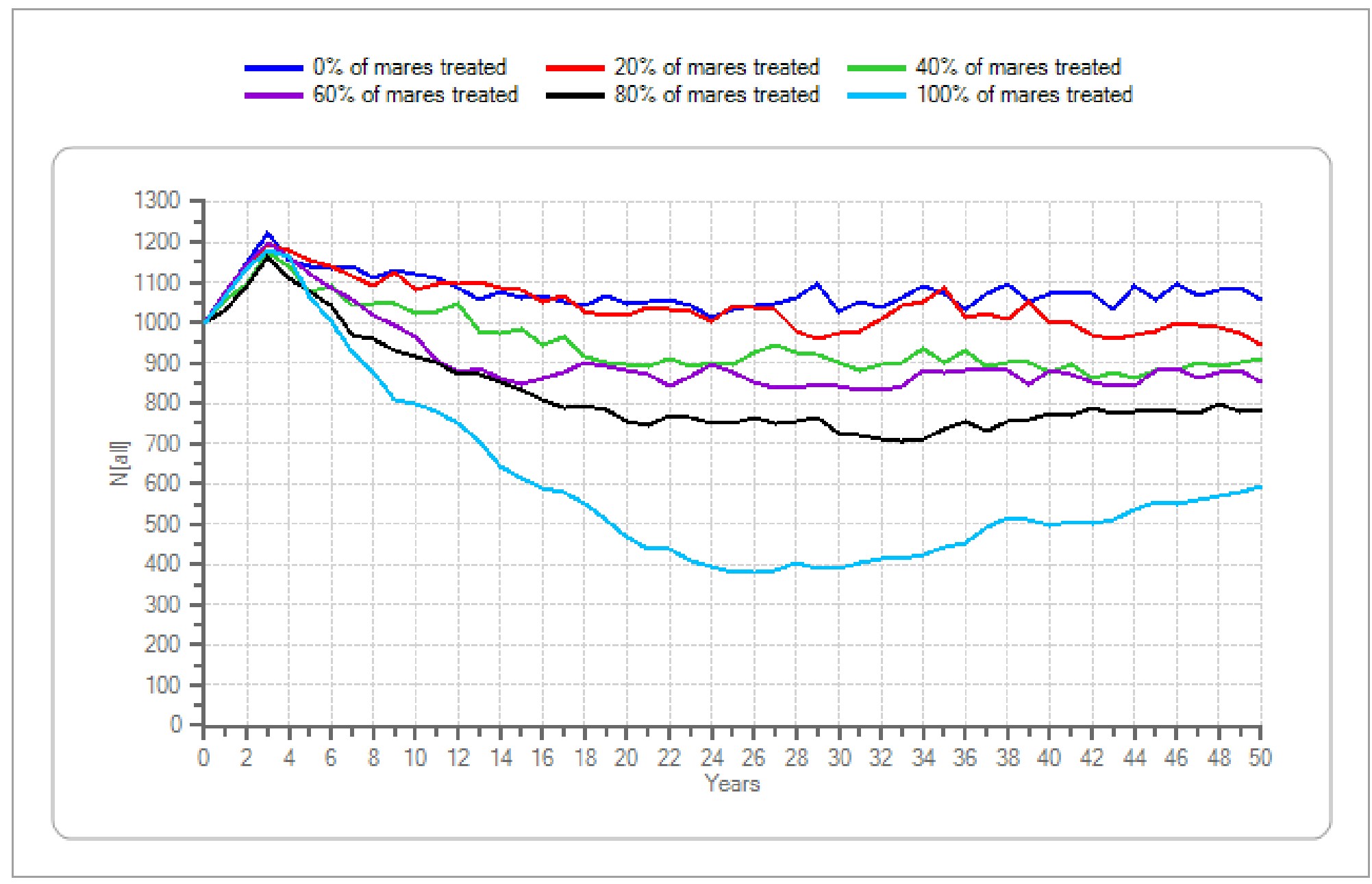

Figure 57. Initial population size $=1000$, lambda $=1.10$, genetic diversity $=$ high - Continued 
Mean population size after 20 years

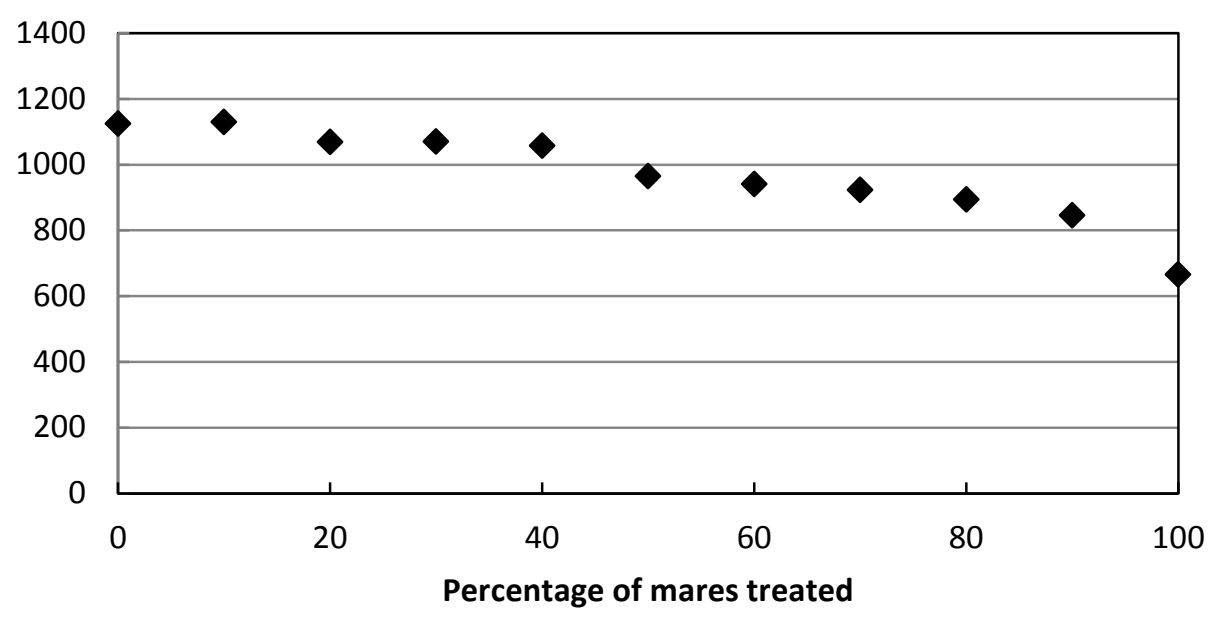

Mean probability of extirpation after 50 years

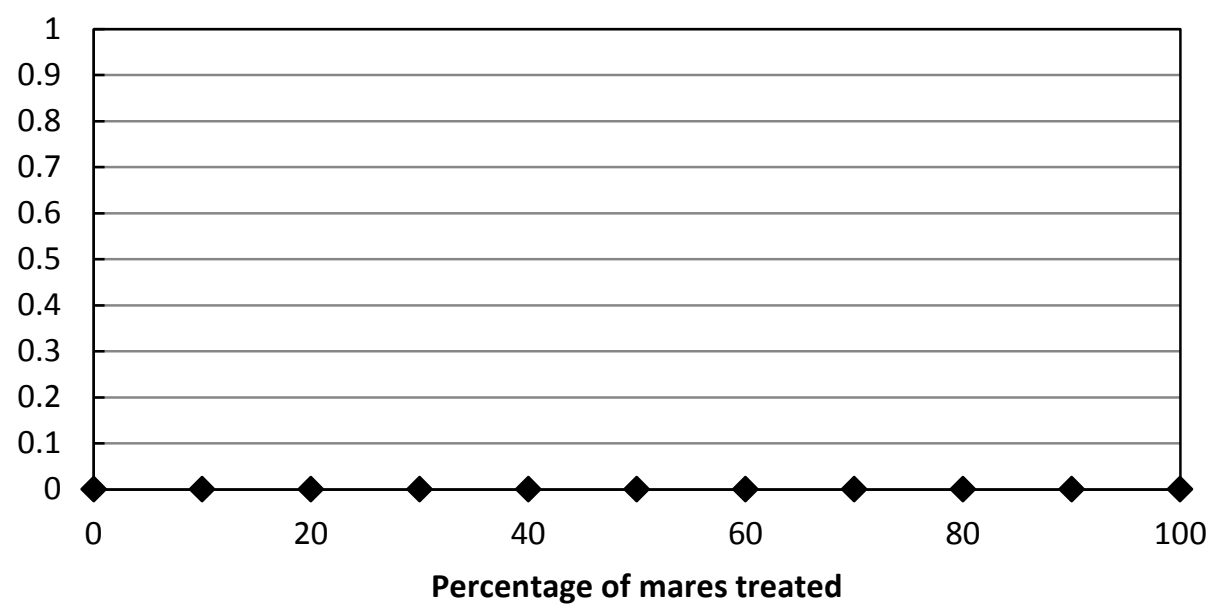

Figure 58. Initial population size $=1000$, lambda $=1.15$, genetic diversity $=$ high .
Mean number of removals after 20 years

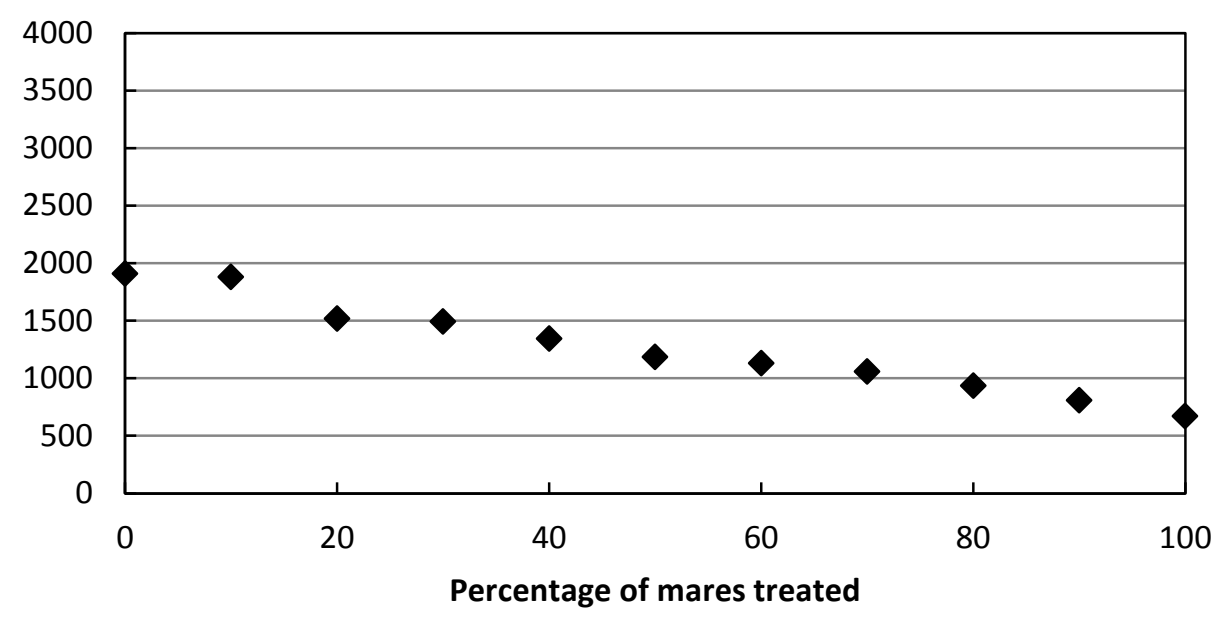

Mean heterozygosity after $\mathbf{5 0}$ years

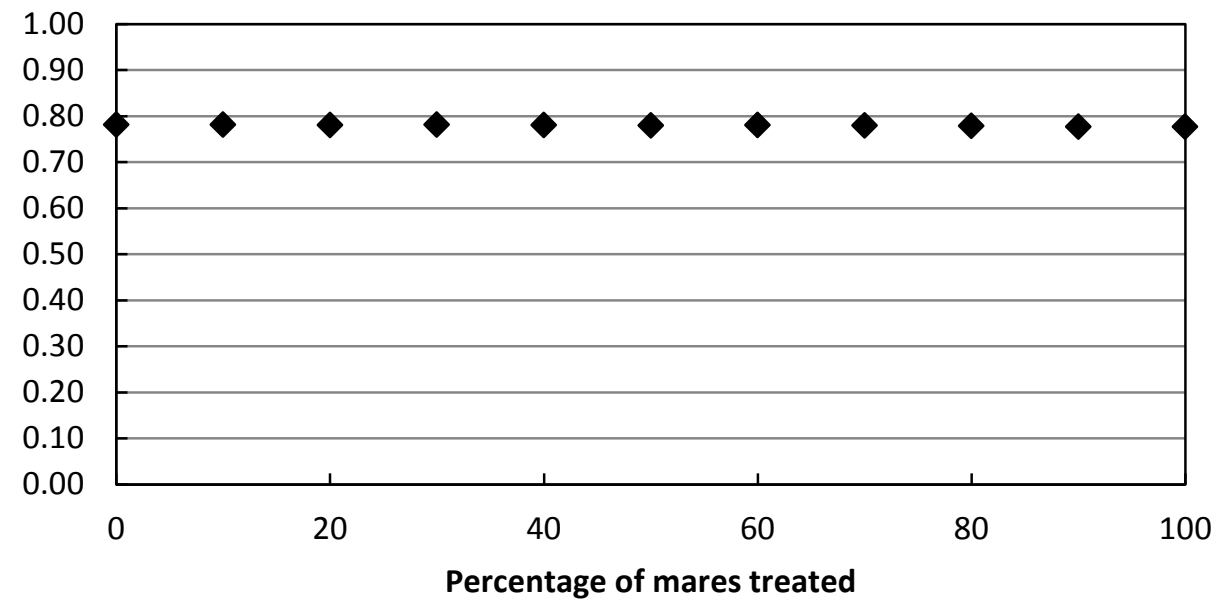




\begin{tabular}{lcccccccccccc}
\hline \multicolumn{1}{c}{ Percentage of mares treated } & $\mathbf{0}$ & $\mathbf{1 0}$ & $\mathbf{2 0}$ & $\mathbf{3 0}$ & $\mathbf{4 0}$ & $\mathbf{5 0}$ & $\mathbf{6 0}$ & $\mathbf{7 0}$ & $\mathbf{8 0}$ & $\mathbf{9 0}$ & $\mathbf{1 0 0}$ \\
\hline Mean population size after 20 years & 1124.15 & 1128.74 & 1069.1 & 1069.36 & 1056.52 & 964.86 & 940 & 922.21 & 893.25 & 845.4 & 665.68 \\
Mean number of removals after 20 years & 1907.4 & 1878.5 & 1517.7 & 1492.3 & 1343.0 & 1182.7 & 1130.2 & 1058.3 & 934.0 & 808.2 & 669.0 \\
Mean probability of extirpation after 50 years & 0 & 0 & 0 & 0 & 0 & 0 & 0 & 0 & 0 & 0 & 0 & 0 \\
Mean heterozygosity after 50 years & 0.78 & 0.78 & 0.78 & 0.78 & 0.78 & 0.78 & 0.78 & 0.78 & 0.78 & 0.78 & 0.78 \\
\hline
\end{tabular}

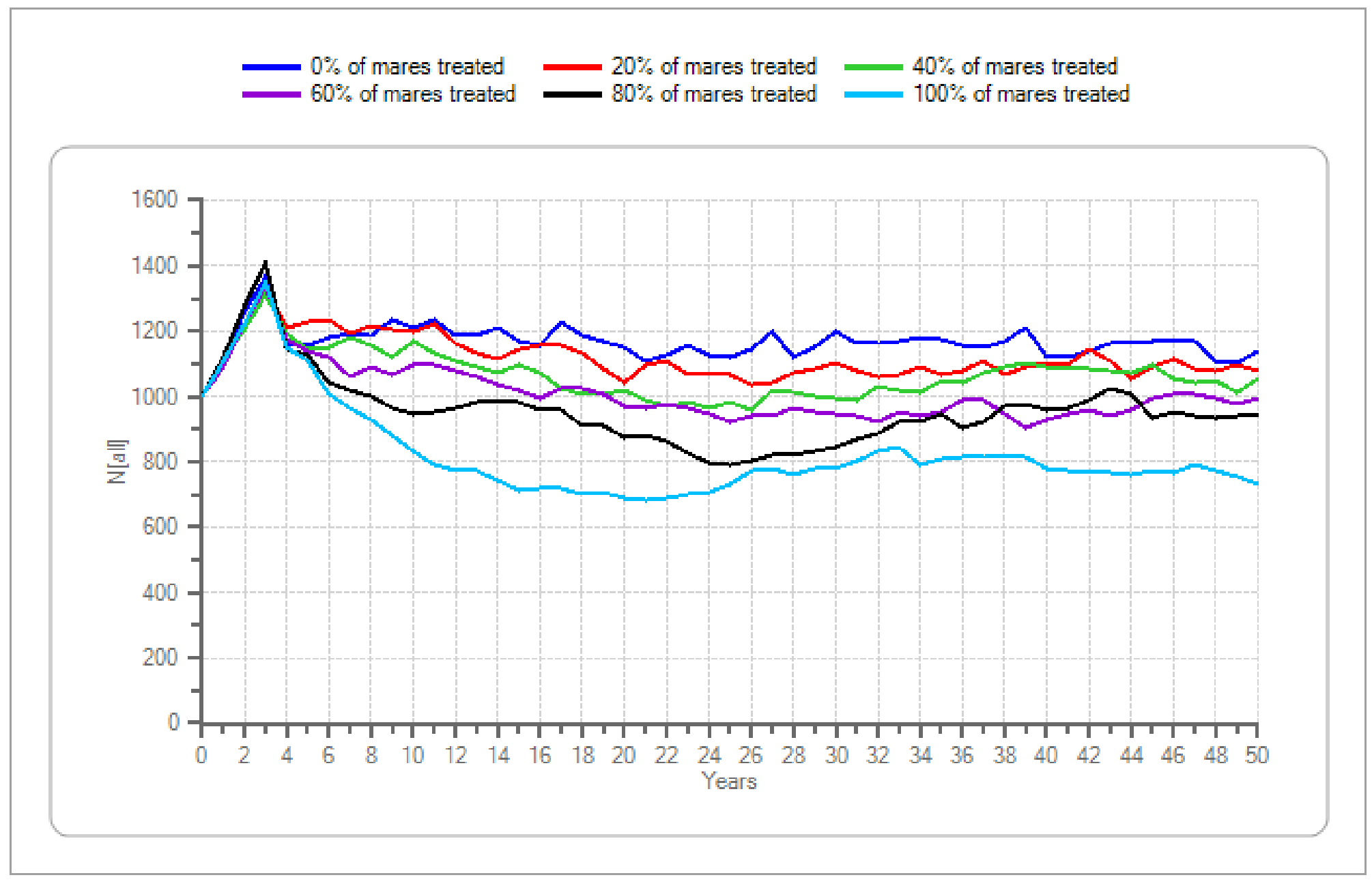

Figure 58. Initial population size $=1000$, lambda $=1.15$, genetic diversity $=$ high - Continued 
Mean population size after 20 years

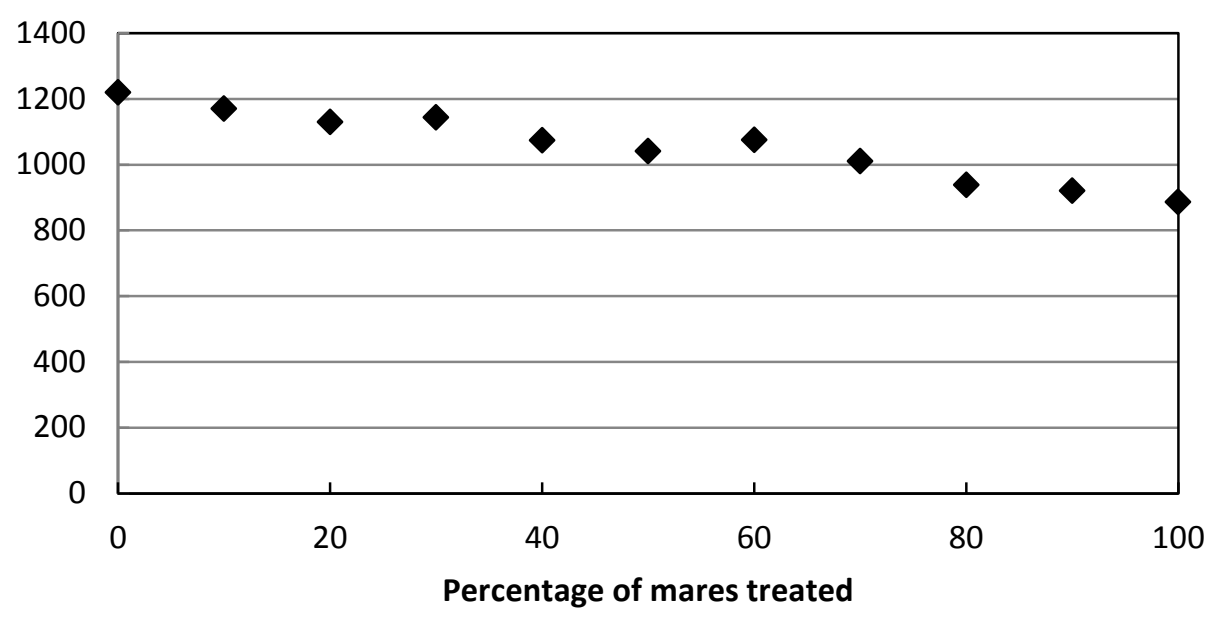

Mean probability of extirpation after 50 years

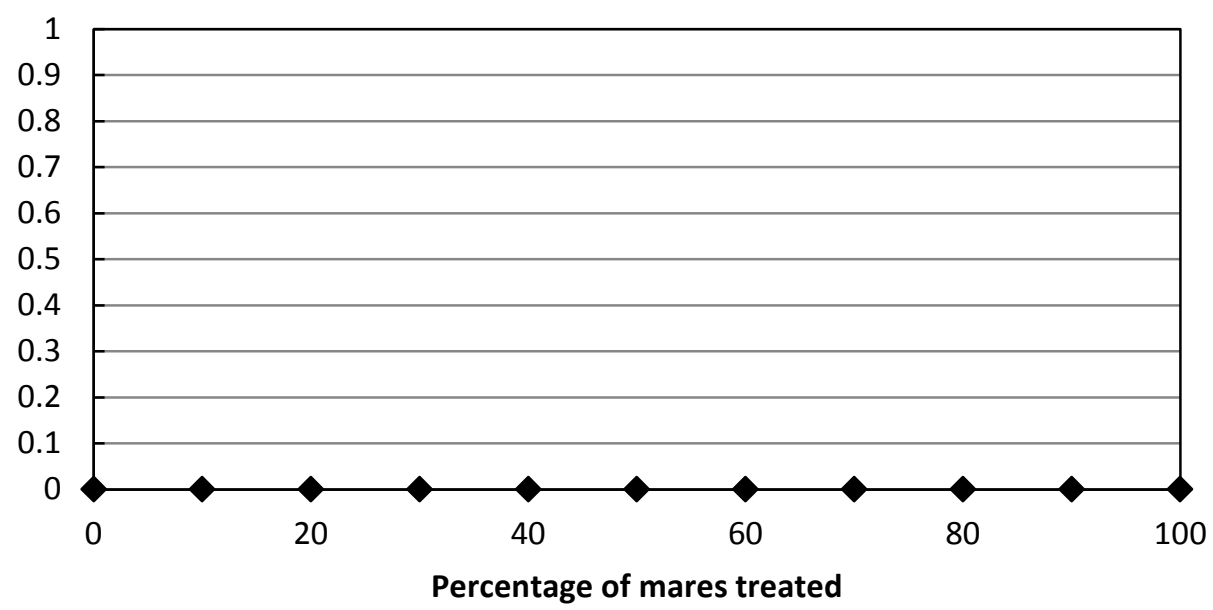

Figure 59. Initial population size $=1000$, lambda $=1.20$, genetic diversity $=$ high .
Mean number of removals after 20 years

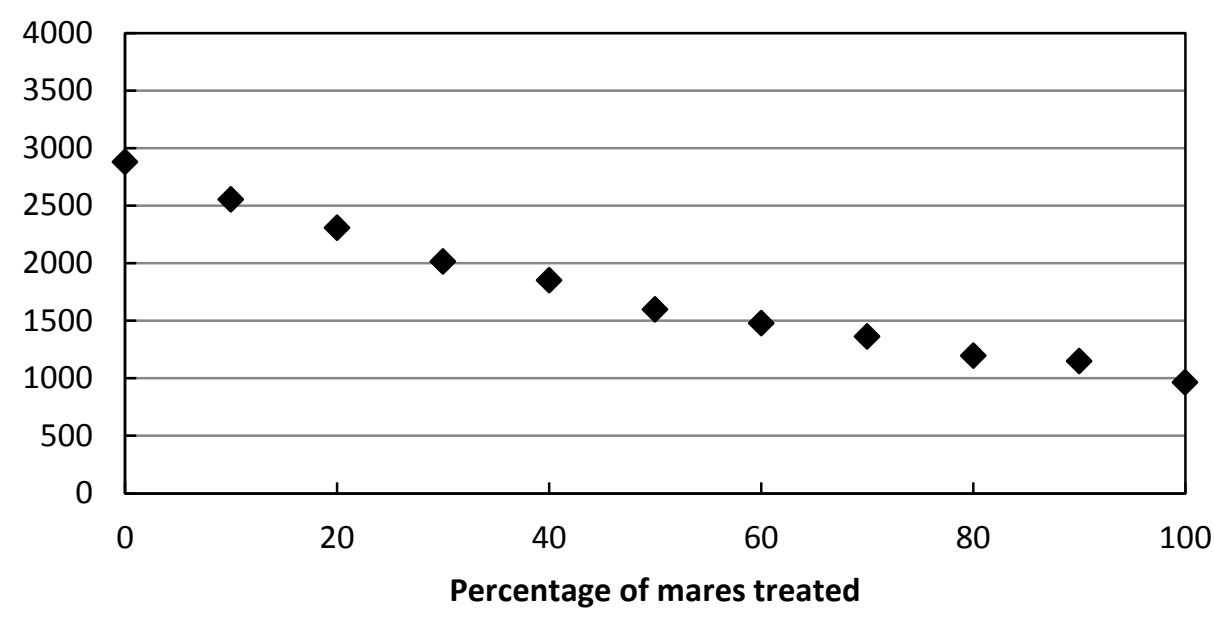

Mean heterozygosity after $\mathbf{5 0}$ years

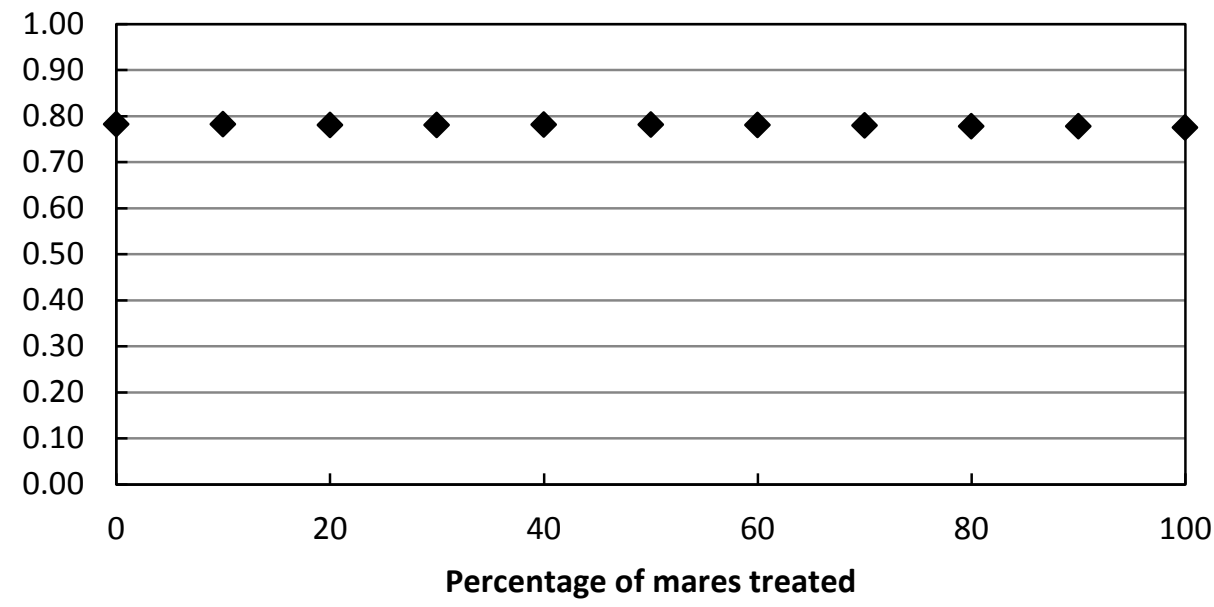




\begin{tabular}{lccccccccccc}
\hline \multicolumn{1}{c}{ Percentage of mares treated } & $\mathbf{0}$ & $\mathbf{1 0}$ & $\mathbf{2 0}$ & $\mathbf{3 0}$ & $\mathbf{4 0}$ & $\mathbf{5 0}$ & $\mathbf{6 0}$ & $\mathbf{7 0}$ & $\mathbf{8 0}$ & $\mathbf{9 0}$ & $\mathbf{1 0 0}$ \\
\hline Mean population size after 20 years & 1219.7 & 1169.6 & 1129.0 & 1143.8 & 1073.6 & 1041.0 & 1075.2 & 1009.6 & 938.2 & 920.0 & 886.5 \\
Mean number of removals after 20 years & 2877.8 & 2551.9 & 2307.0 & 2013.1 & 1851.7 & 1597.8 & 1478.2 & 1362.8 & 1196.4 & 1149.4 & 963.0 \\
Mean probability of extirpation after 50 years & 0 & 0 & 0 & 0 & 0 & 0 & 0 & 0 & 0 & 0 & 0 \\
Mean heterozygosity after 50 years & 0.78 & 0.78 & 0.78 & 0.78 & 0.78 & 0.78 & 0.78 & 0.78 & 0.78 & 0.78 & 0.78 \\
\hline
\end{tabular}

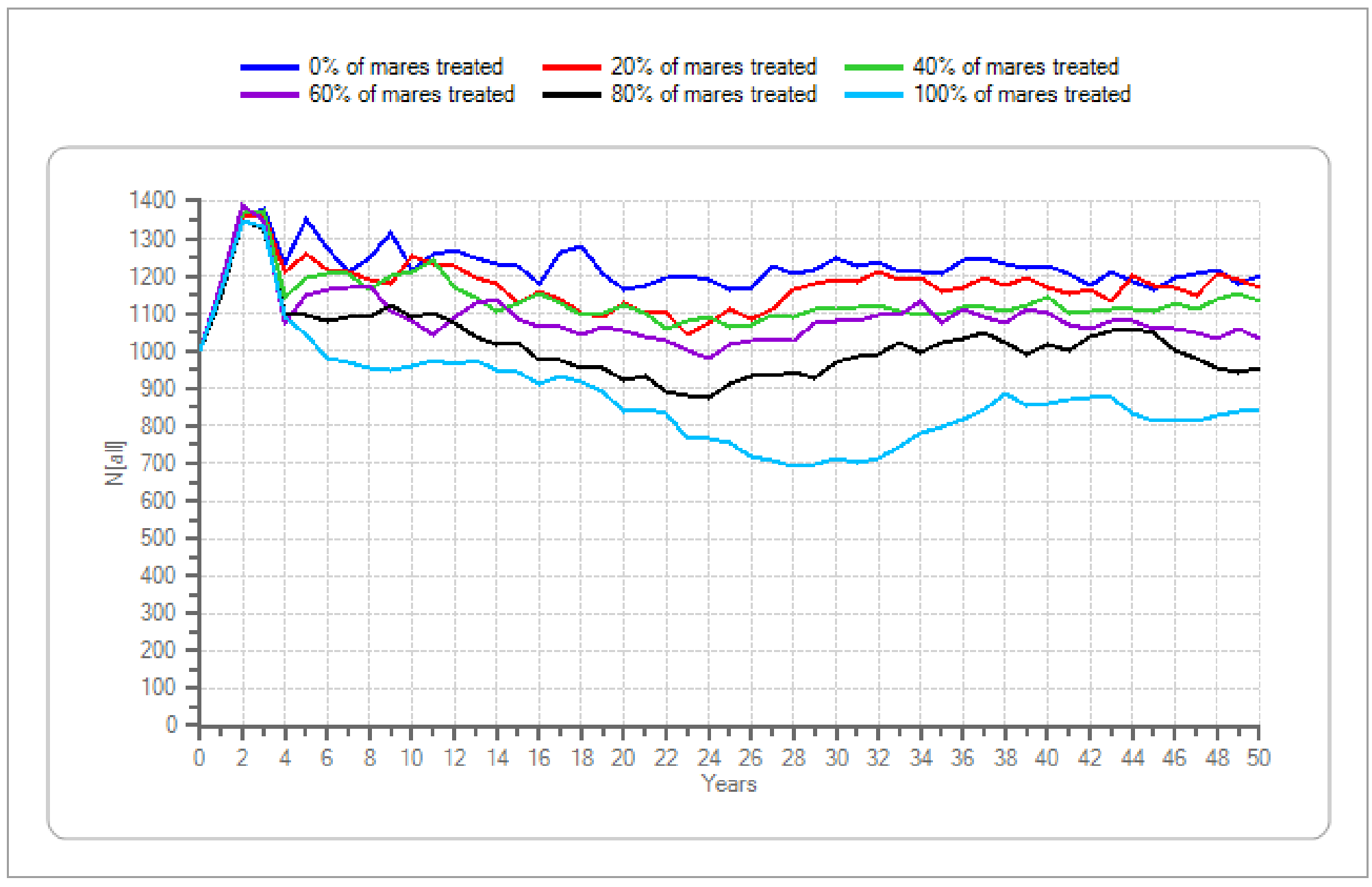

Figure 59. Initial population size $=1000$, lambda $=1.20$, genetic diversity $=$ high - Continued 
Mean population size after 20 years

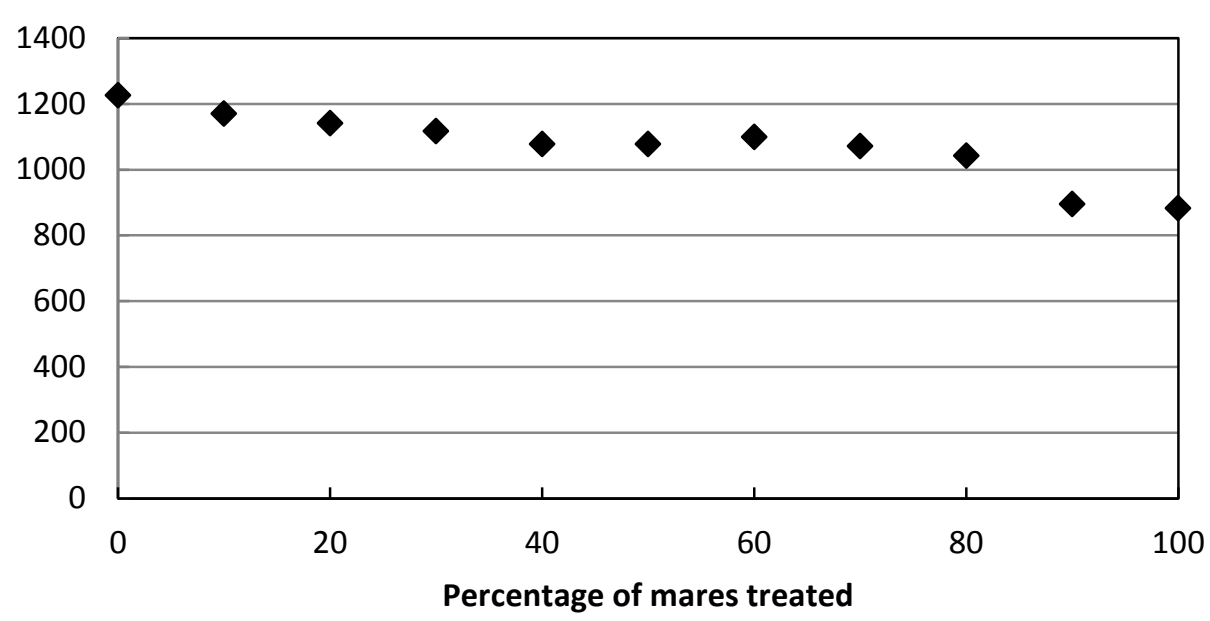

Mean probability of extirpation after 50 years

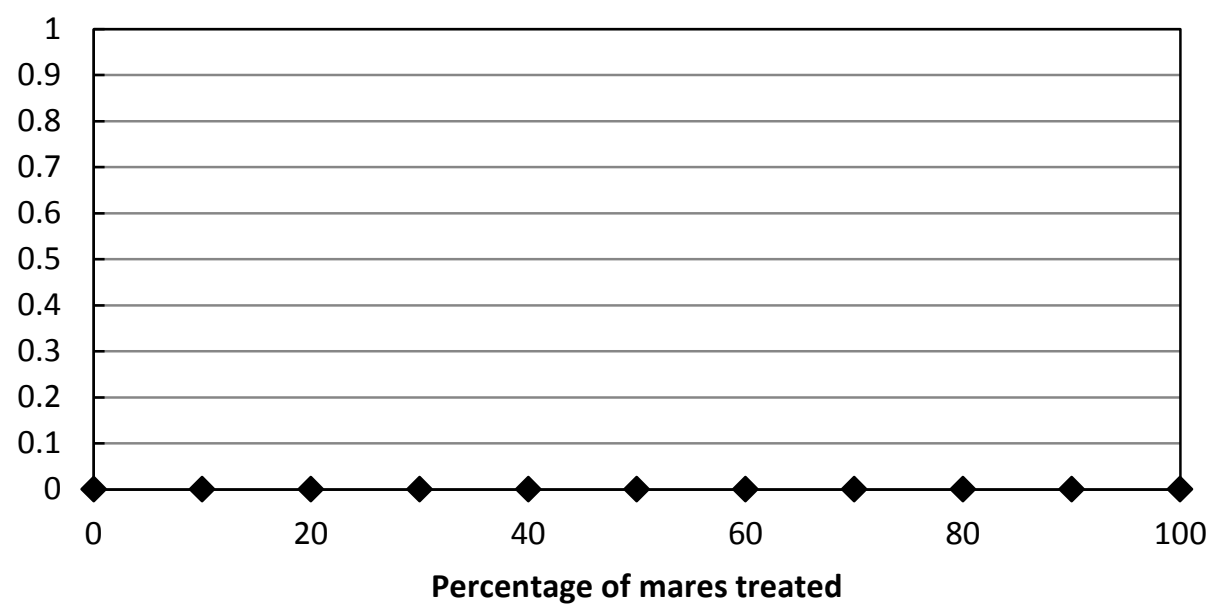

Figure 60. Initial population size $=1000$, lambda $=1.25$, genetic diversity $=$ high .
Mean number of removals after 20 years

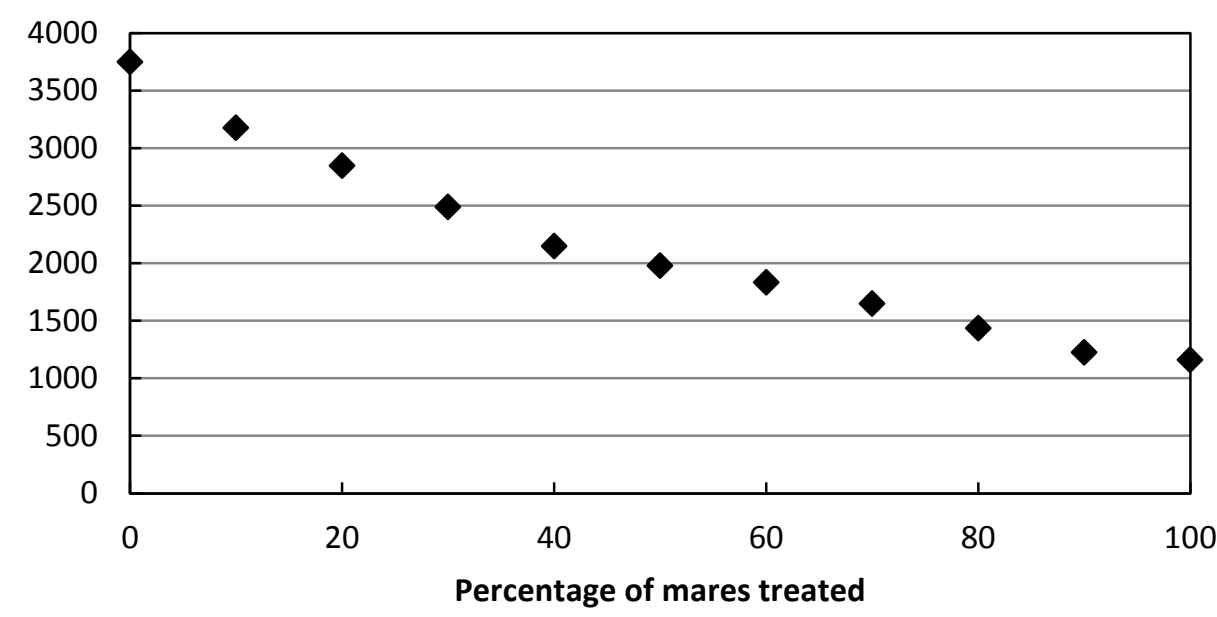

Mean heterozygosity after $\mathbf{5 0}$ years

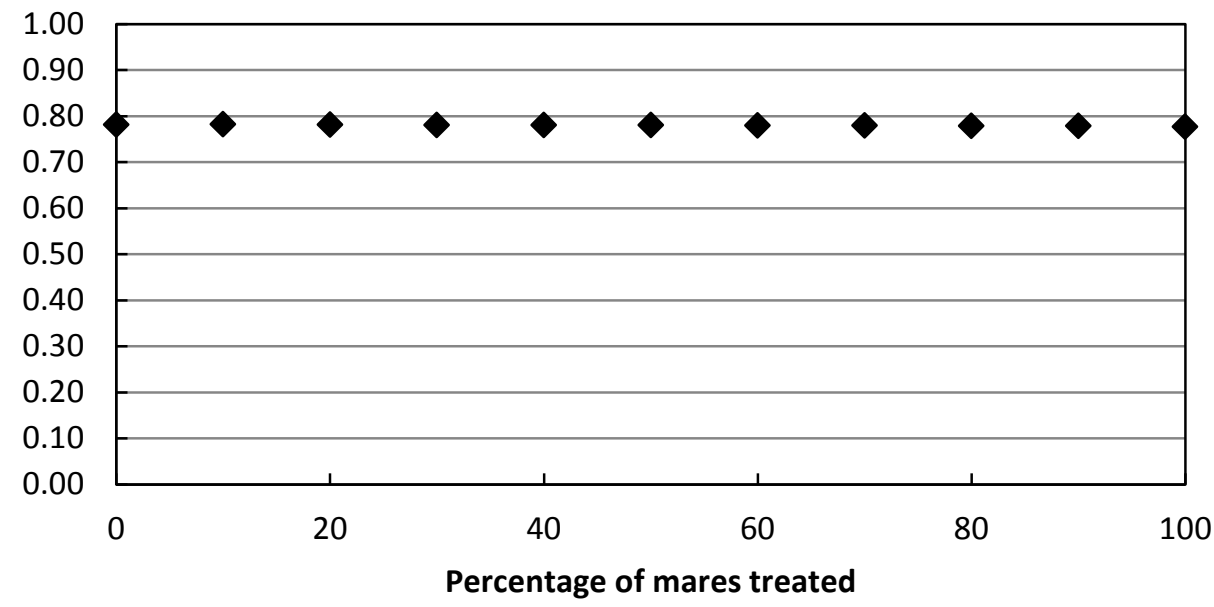




\begin{tabular}{lcccccccccccc}
\hline \multicolumn{1}{c}{ Percentage of mares treated } & $\mathbf{0}$ & $\mathbf{1 0}$ & $\mathbf{2 0}$ & $\mathbf{3 0}$ & $\mathbf{4 0}$ & $\mathbf{5 0}$ & $\mathbf{6 0}$ & $\mathbf{7 0}$ & $\mathbf{8 0}$ & $\mathbf{9 0}$ & $\mathbf{1 0 0}$ \\
\hline Mean population size after 20 years & 1225.4 & 1169.7 & 1140.7 & 1117.1 & 1077.6 & 1076.7 & 1098.6 & 1071.1 & 1041.7 & 894.4 & 882.7 \\
Mean number of removals after 20 years & 3749.6 & 3175.7 & 2844.7 & 2489.0 & 2147.9 & 1978.4 & 1831.9 & 1649.2 & 1432.6 & 1223.8 & 1159.1 \\
Mean probability of extirpation after 50 years & 0 & 0 & 0 & 0 & 0 & 0 & 0 & 0 & 0 & 0 & 0 \\
Mean heterozygosity after 50 years & 0.78 & 0.78 & 0.78 & 0.78 & 0.78 & 0.78 & 0.78 & 0.78 & 0.78 & 0.78 & 0.78 \\
\hline
\end{tabular}

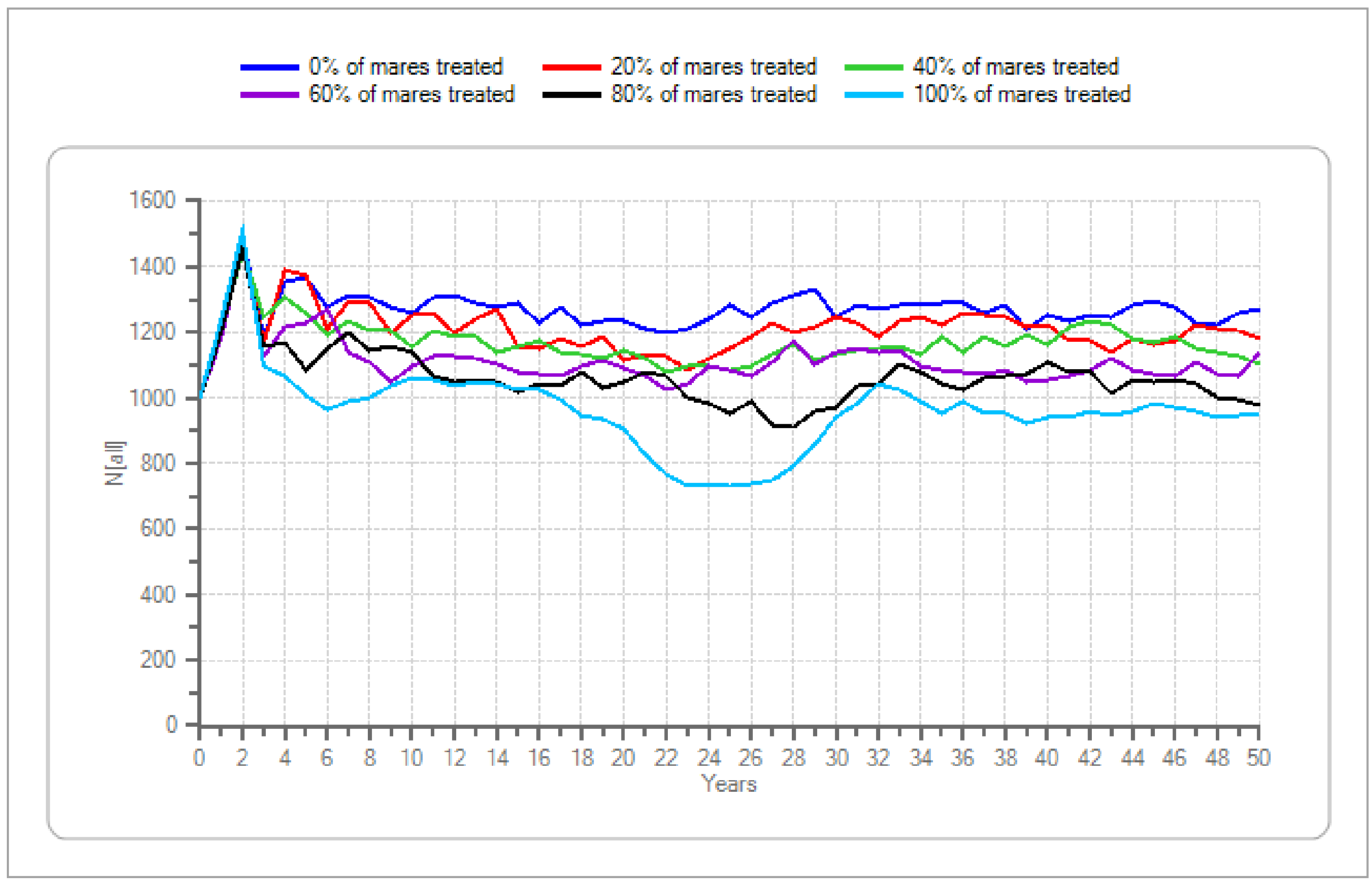

Figure 60. Initial population size $=1000$, lambda $=1.25$, genetic diversity $=$ high. Continued 
Mean population size after 20 years

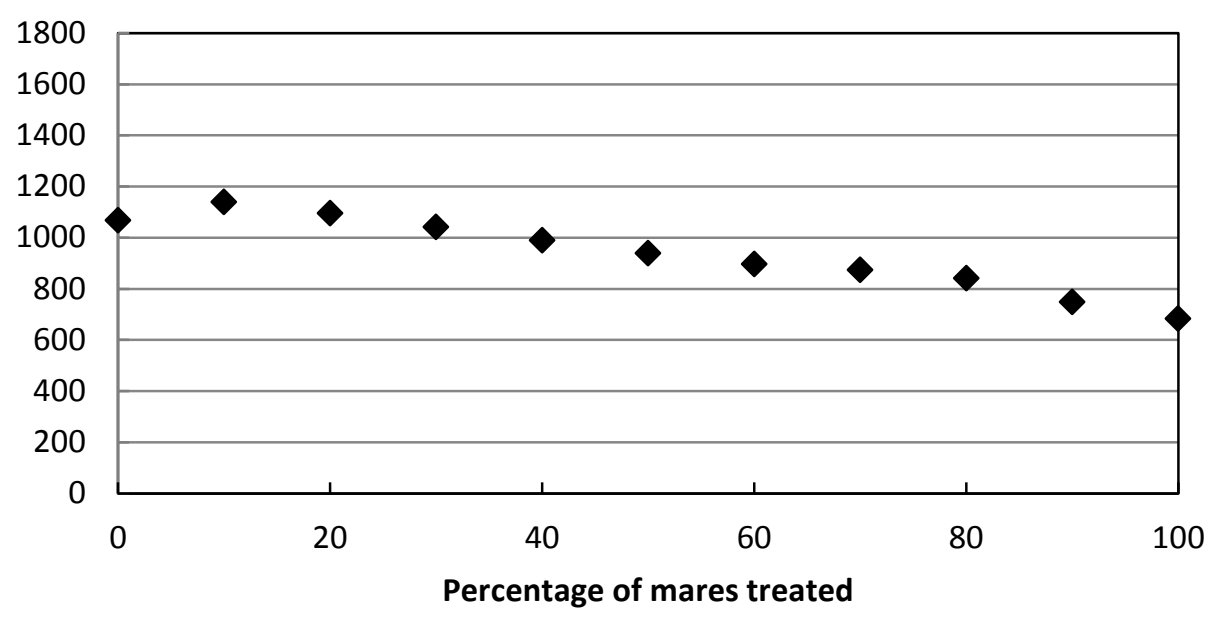

Mean probability of extirpation after $\mathbf{5 0}$ years

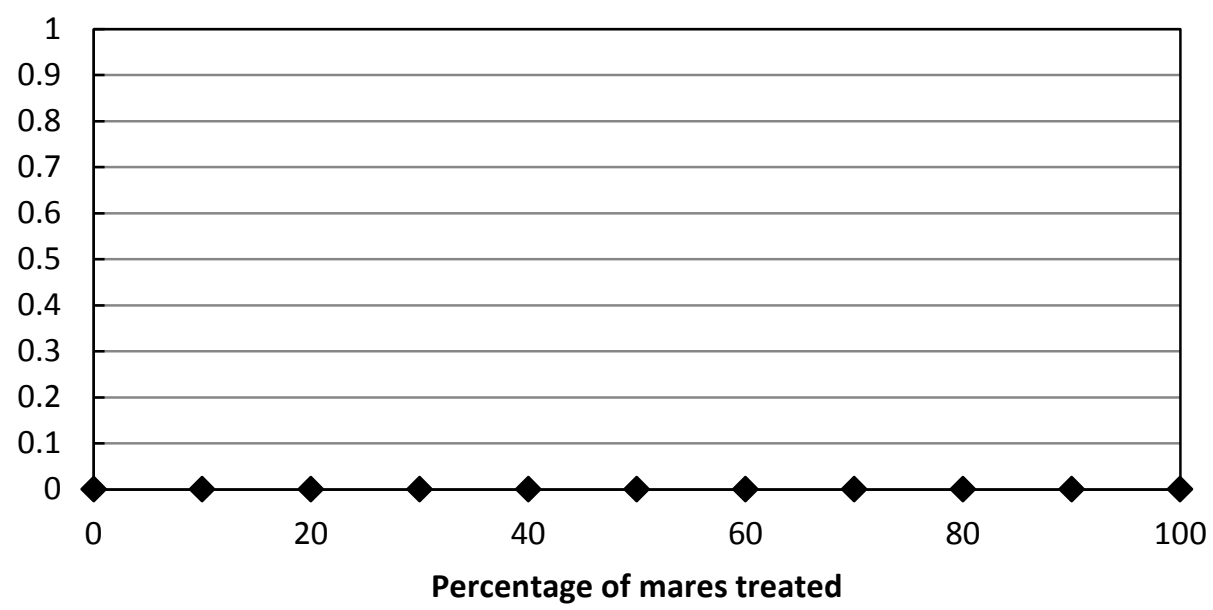

Figure 61. Initial population size $=1250$, lambda $=1.05$, genetic diversity $=$ high .
Mean number of removals after 20 years

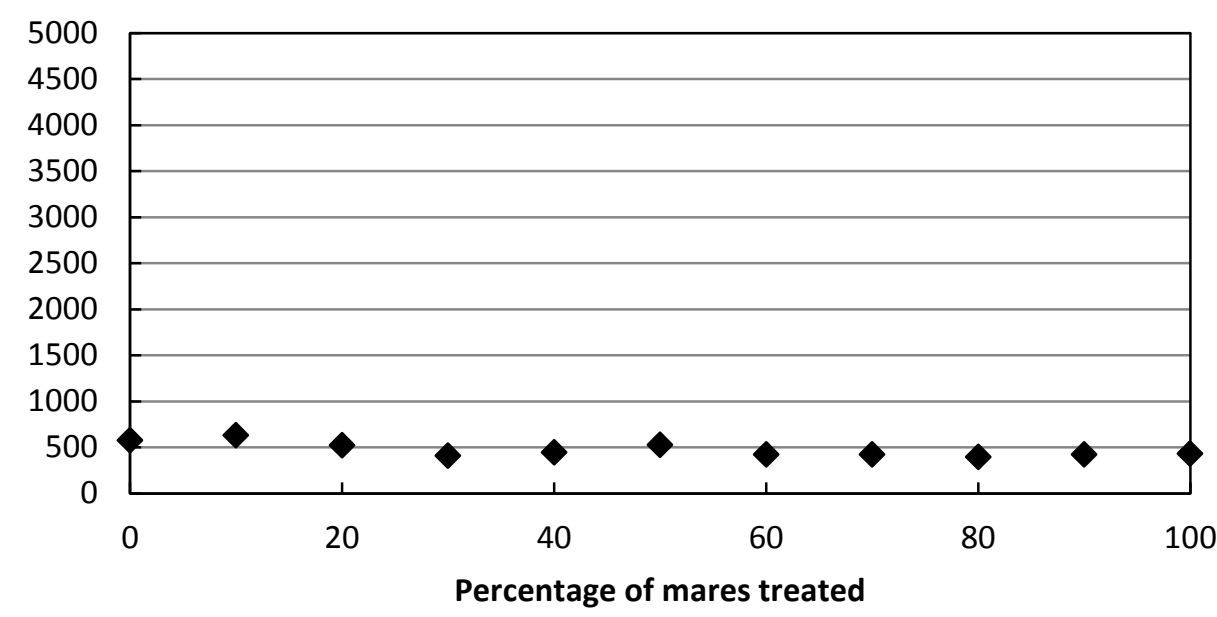

Mean heterozygosity after $\mathbf{5 0}$ years

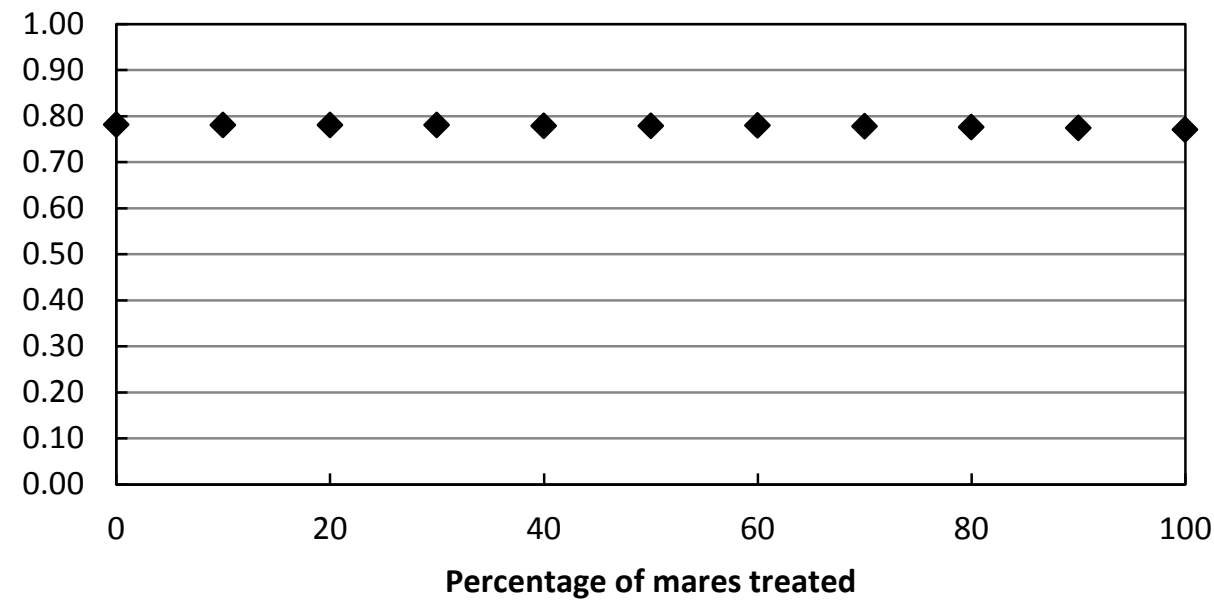




\begin{tabular}{lccccccccccc}
\hline \multicolumn{1}{c}{ Percentage of mares treated } & $\mathbf{0}$ & $\mathbf{1 0}$ & $\mathbf{2 0}$ & $\mathbf{3 0}$ & $\mathbf{4 0}$ & $\mathbf{5 0}$ & $\mathbf{6 0}$ & $\mathbf{7 0}$ & $\mathbf{8 0}$ & $\mathbf{9 0}$ & $\mathbf{1 0 0}$ \\
\hline Mean population size after 20 years & 1066.7 & 1139.5 & 1095.1 & 1041.0 & 989.2 & 938.4 & 896.1 & 874.0 & 841.0 & 747.5 & 683.3 \\
Mean number of removals after 20 years & 575.5 & 629.3 & 519.5 & 406.3 & 442.6 & 524.2 & 422.0 & 420.3 & 393.0 & 422.2 & 428.9 \\
Mean probability of extirpation after 50 years & 0 & 0 & 0 & 0 & 0 & 0 & 0 & 0 & 0 & 0 & 0 \\
Mean heterozygosity after 50 years & 0.78 & 0.78 & 0.78 & 0.78 & 0.78 & 0.78 & 0.78 & 0.78 & 0.78 & 0.77 & 0.77 \\
\hline
\end{tabular}

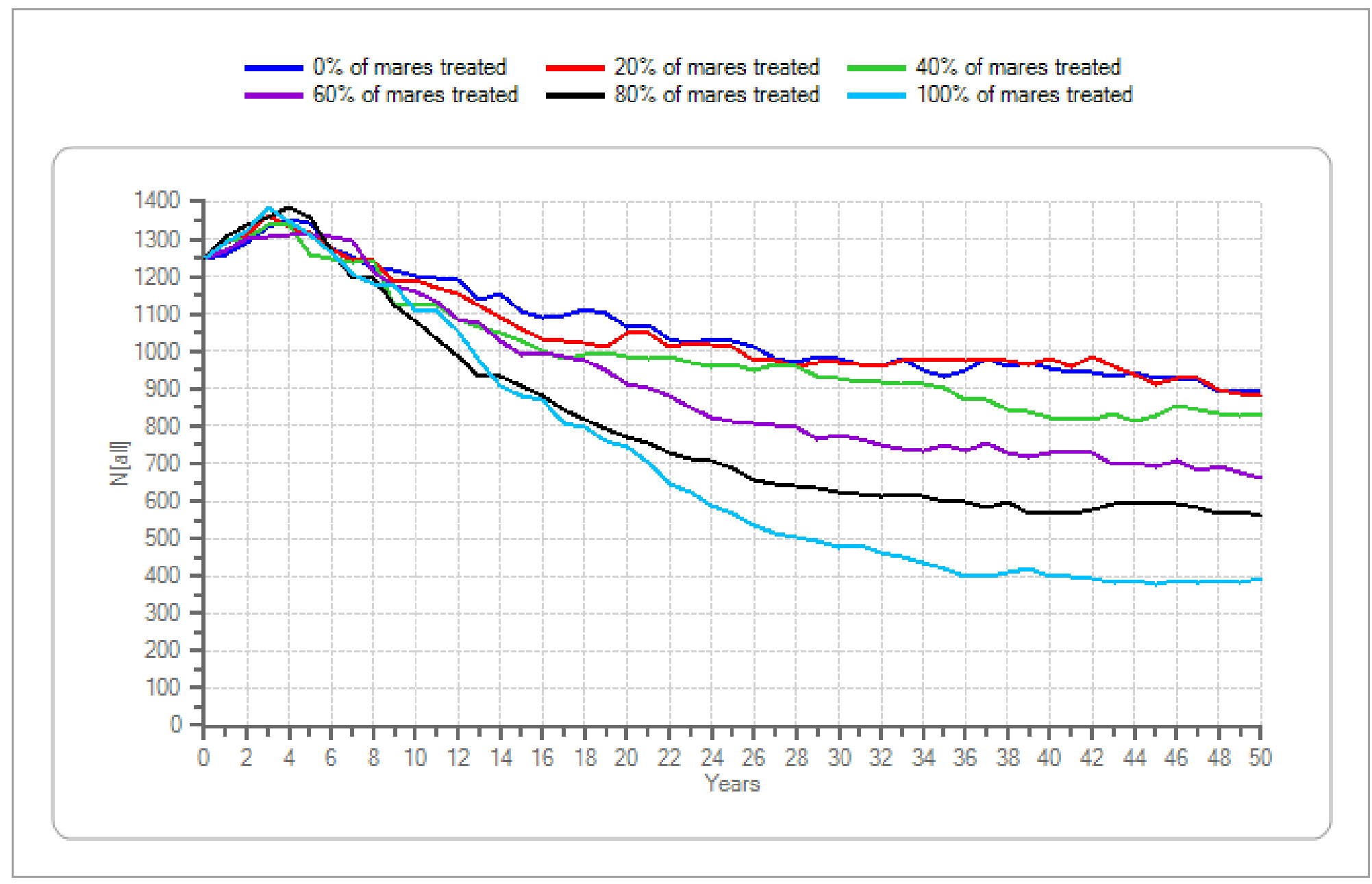

Figure 61. Initial population size $=1250$, lambda $=1.05$, genetic diversity $=$ high - Continued 
Mean population size after 20 years

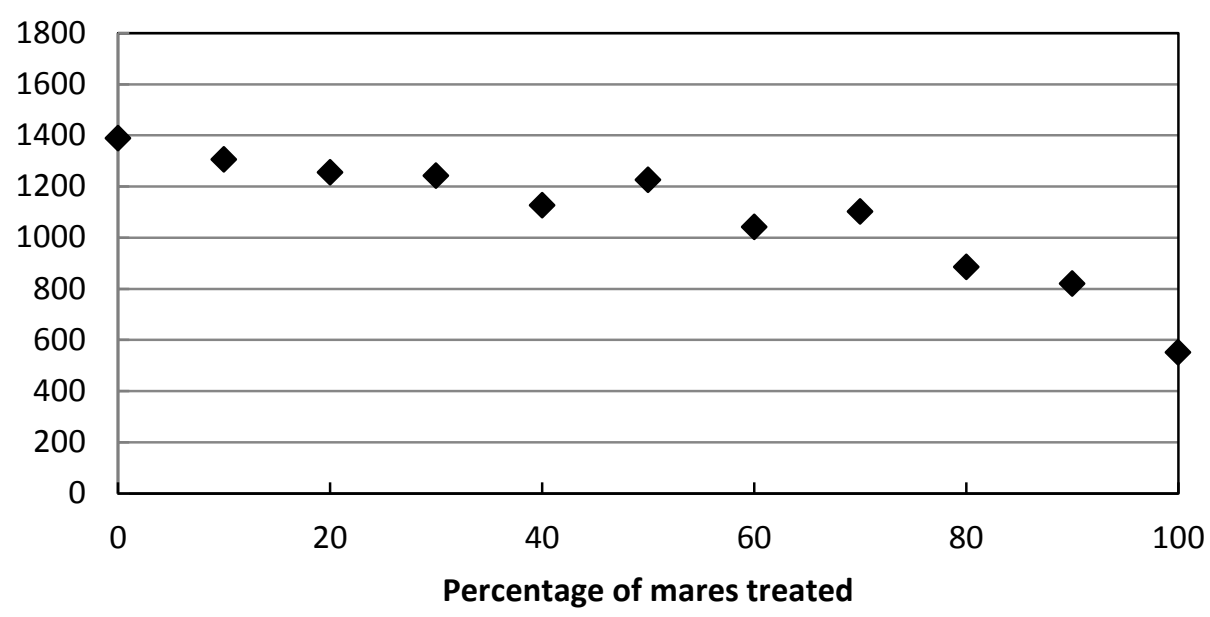

Mean probability of extirpation after $\mathbf{5 0}$ years

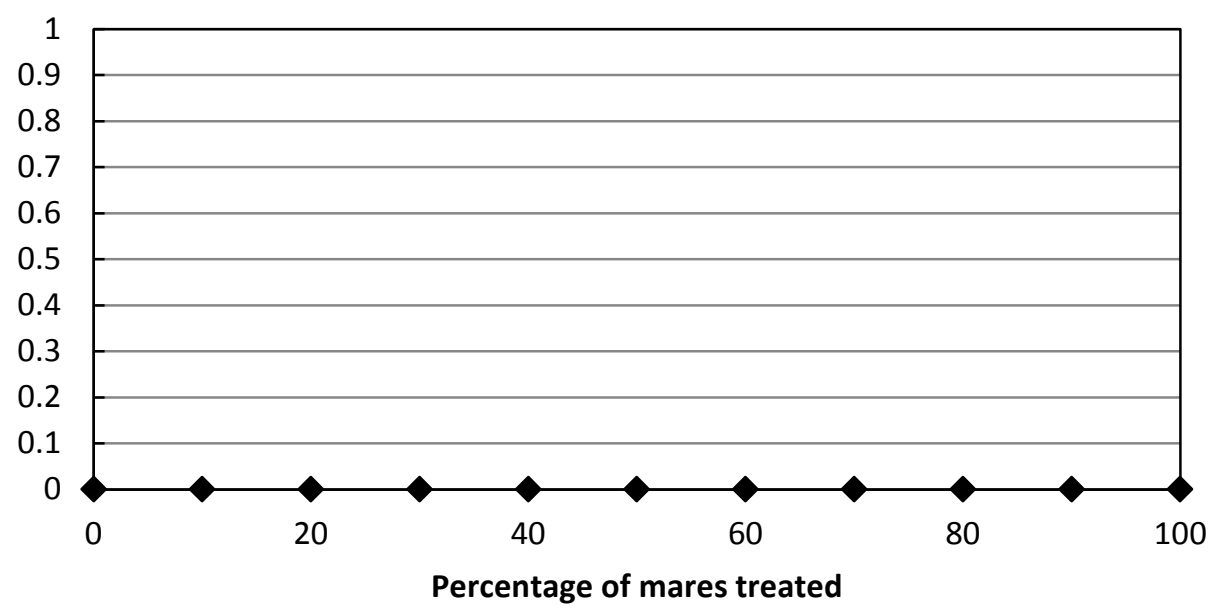

Figure 62. Initial population size $=1250$, lambda $=1.10$, genetic diversity $=$ high .
Mean number of removals after 20 years

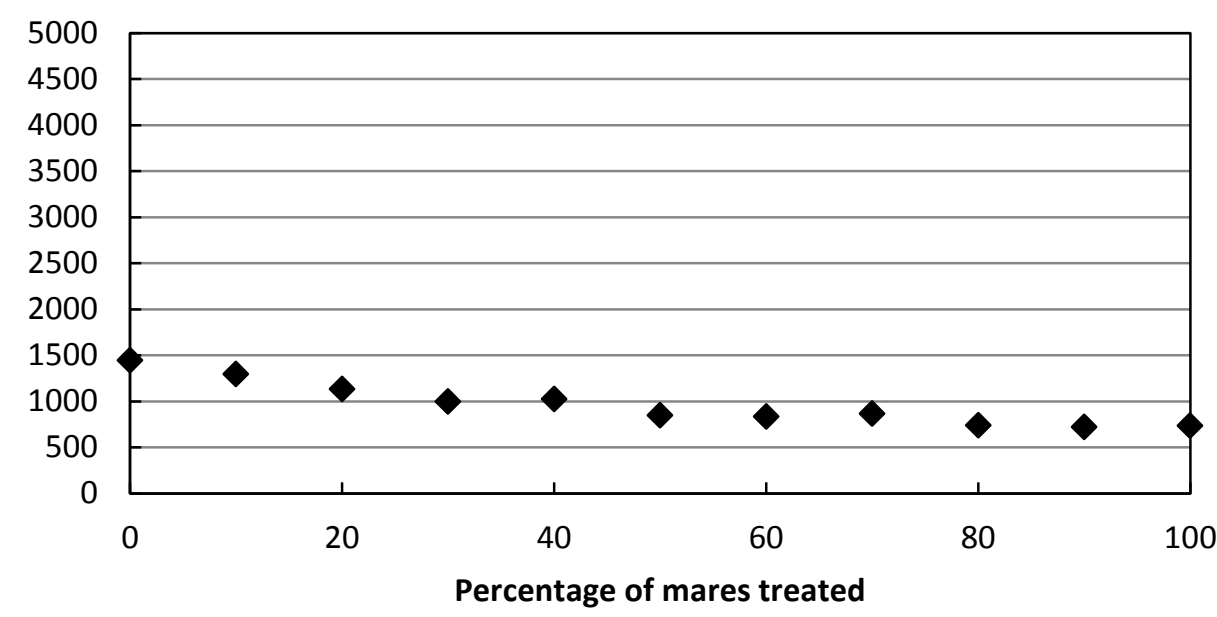

Mean heterozygosity after $\mathbf{5 0}$ years

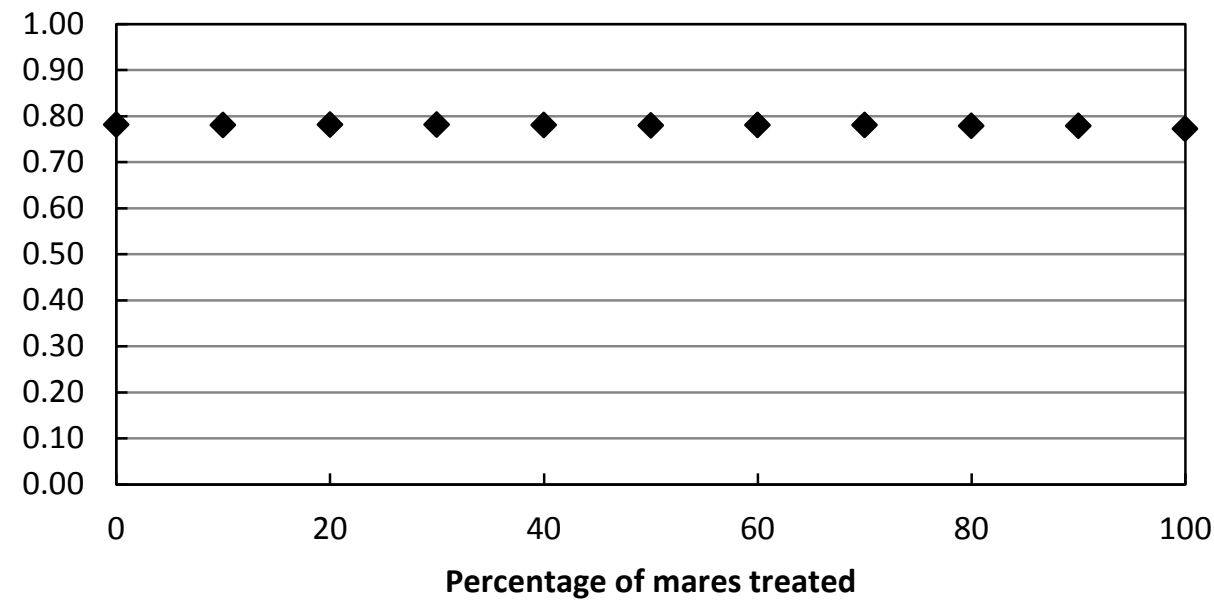




\begin{tabular}{lccccccccccc}
\hline \multicolumn{1}{c}{ Percentage of mares treated } & $\mathbf{0}$ & $\mathbf{1 0}$ & $\mathbf{2 0}$ & $\mathbf{3 0}$ & $\mathbf{4 0}$ & $\mathbf{5 0}$ & $\mathbf{6 0}$ & $\mathbf{7 0}$ & $\mathbf{8 0}$ & $\mathbf{9 0}$ & $\mathbf{1 0 0}$ \\
\hline Mean population size after 20 years & 1388.7 & 1304.7 & 1254.2 & 1242.3 & 1126.8 & 1225.7 & 1041.3 & 1101.1 & 885.0 & 820.1 & 551.3 \\
Mean number of removals after 20 years & 1442.2 & 1296.3 & 1129.6 & 995.3 & 1024.7 & 845.1 & 834.5 & 866.0 & 738.2 & 721.3 & 735.6 \\
Mean probability of extirpation after 50 years & 0 & 0 & 0 & 0 & 0 & 0 & 0 & 0 & 0 & 0 & 0 \\
Mean heterozygosity after 50 years & 0.78 & 0.78 & 0.78 & 0.78 & 0.78 & 0.78 & 0.78 & 0.78 & 0.78 & 0.78 & 0.77 \\
\hline
\end{tabular}

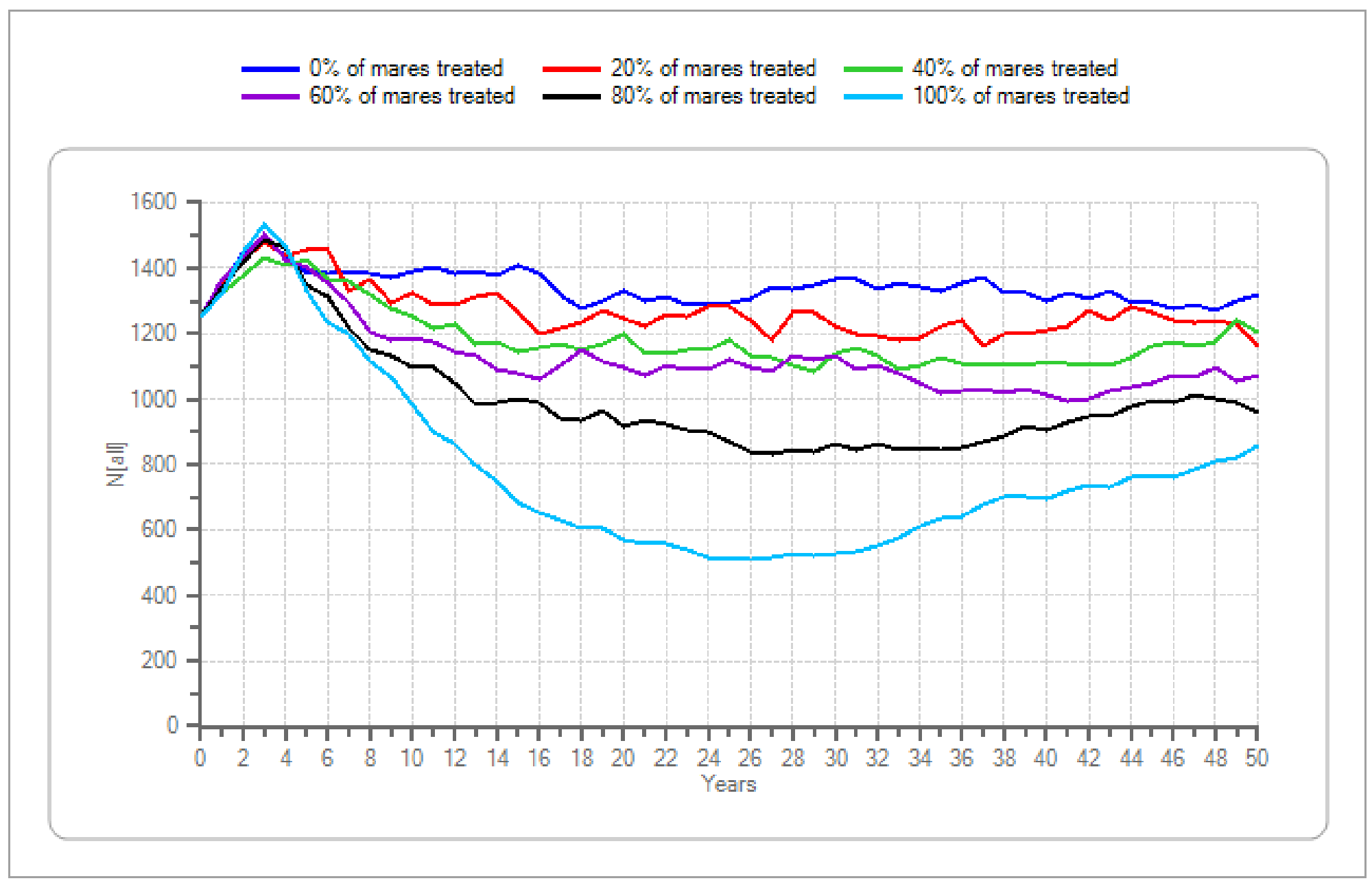

Figure 62. Initial population size $=1250$, lambda $=1.10$, genetic diversity $=$ high - Continued 
Mean population size after 20 years

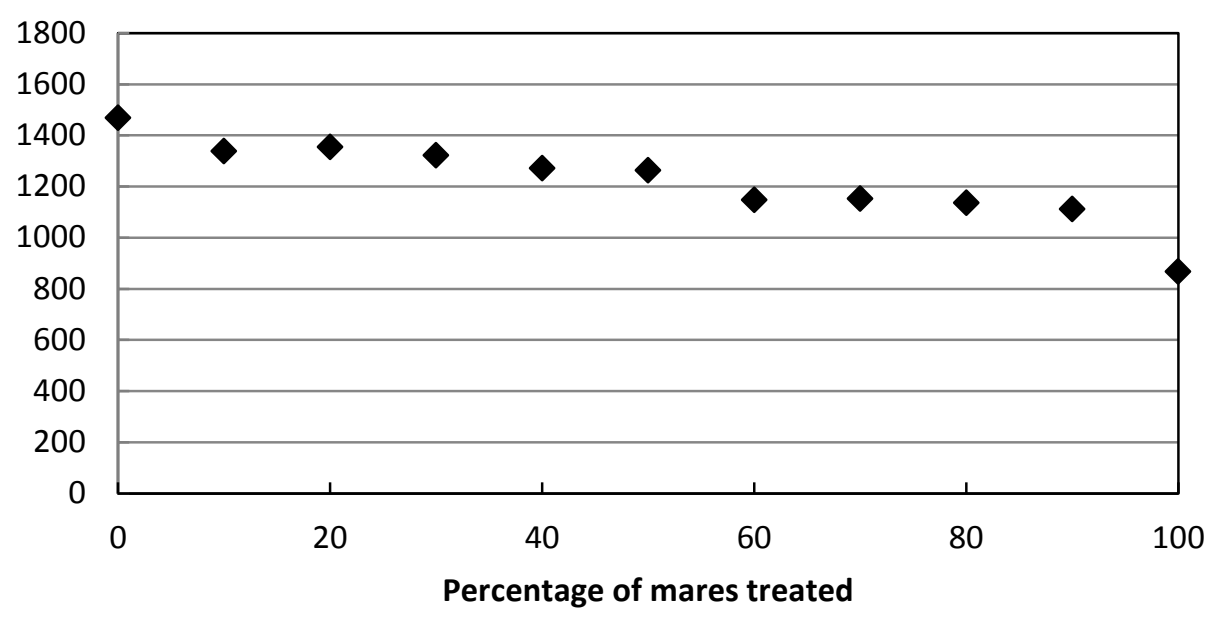

Mean probability of extirpation after $\mathbf{5 0}$ years

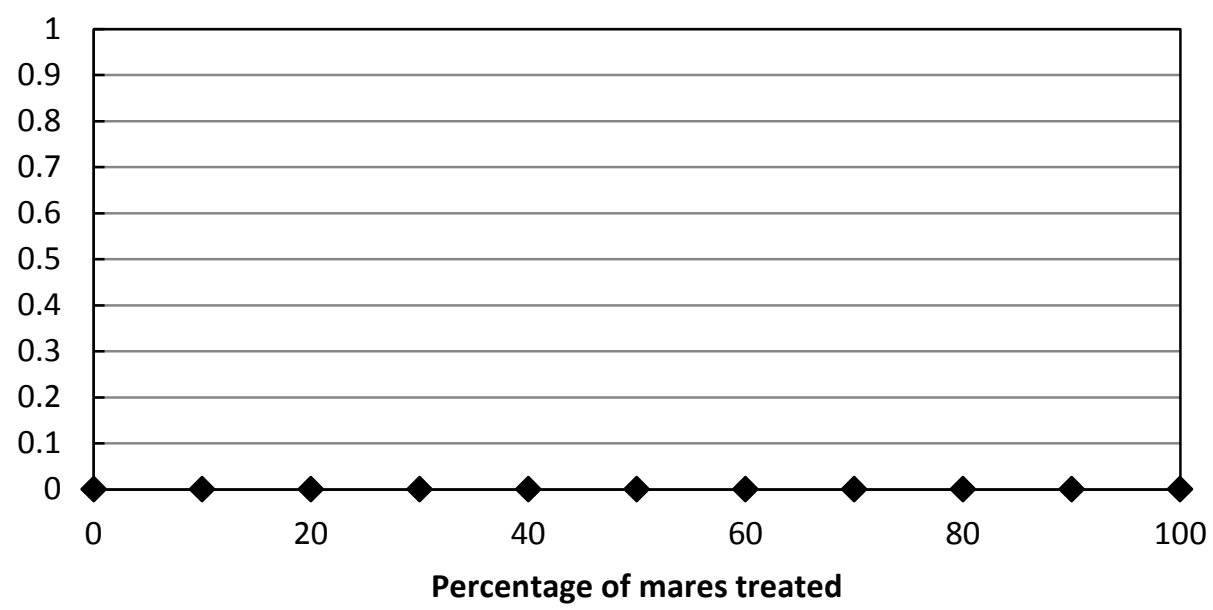

Figure 63. Initial population size $=1250$, lambda $=1.15$, genetic diversity $=$ high .
Mean number of removals after 20 years

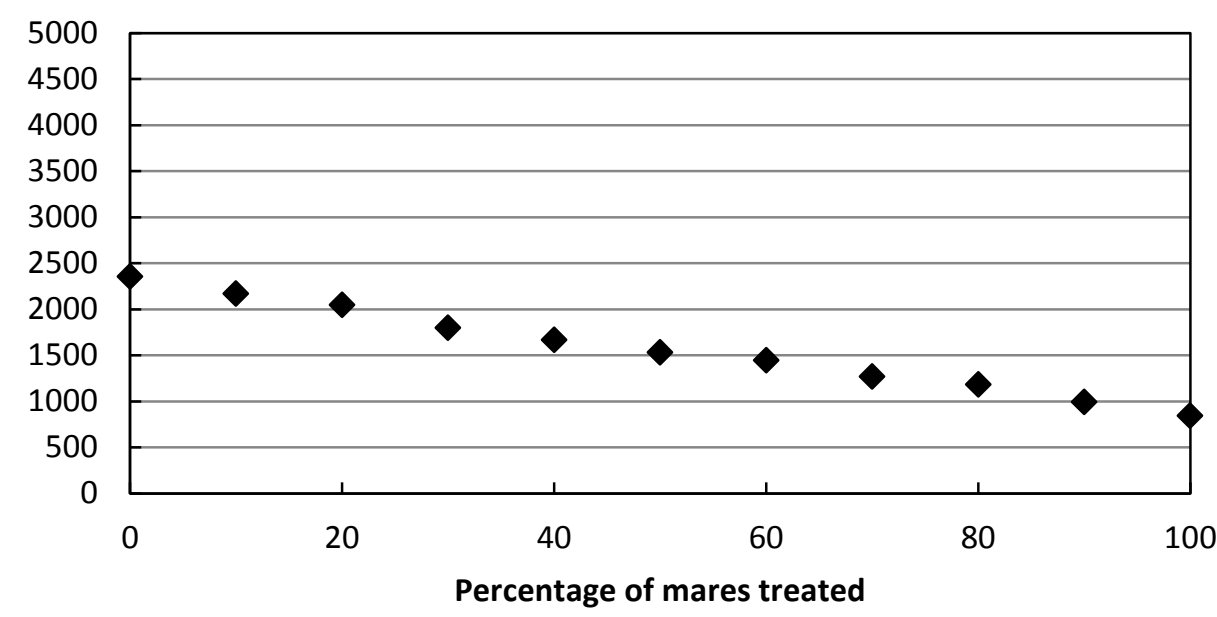

Mean heterozygosity after $\mathbf{5 0}$ years

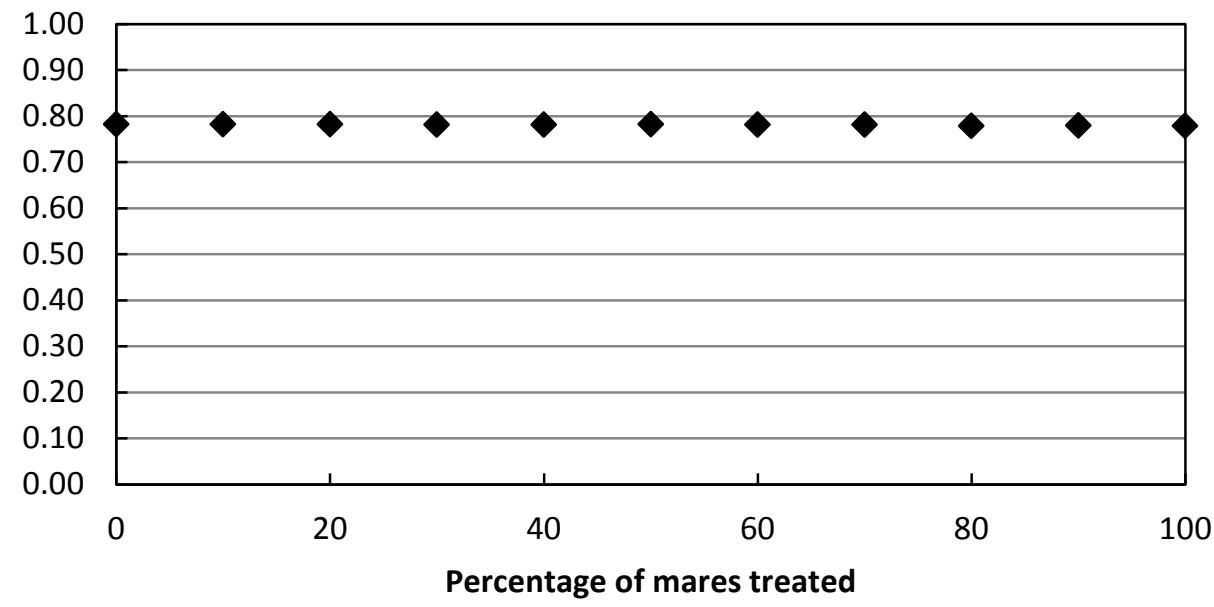




\begin{tabular}{lcccccccccccc}
\hline \multicolumn{1}{c}{ Percentage of mares treated } & $\mathbf{0}$ & $\mathbf{1 0}$ & $\mathbf{2 0}$ & $\mathbf{3 0}$ & $\mathbf{4 0}$ & $\mathbf{5 0}$ & $\mathbf{6 0}$ & $\mathbf{7 0}$ & $\mathbf{8 0}$ & $\mathbf{9 0}$ & $\mathbf{1 0 0}$ \\
\hline Mean population size after 20 years & 1468.9 & 1338.2 & 1354.4 & 1321.2 & 1271.8 & 1263.7 & 1147.8 & 1151.5 & 1135.1 & 1112.2 & 866.6 \\
Mean number of removals after 20 years & 2353.0 & 2166.1 & 2044.2 & 1797.3 & 1667.0 & 1529.1 & 1445.7 & 1267.1 & 1179.7 & 991.6 & 840.9 \\
Mean probability of extirpation after 50 years & 0 & 0 & 0 & 0 & 0 & 0 & 0 & 0 & 0 & 0 & 0 & 0 \\
Mean heterozygosity after 50 years & 0.78 & 0.78 & 0.78 & 0.78 & 0.78 & 0.78 & 0.78 & 0.78 & 0.78 & 0.78 & 0.78 \\
\hline
\end{tabular}

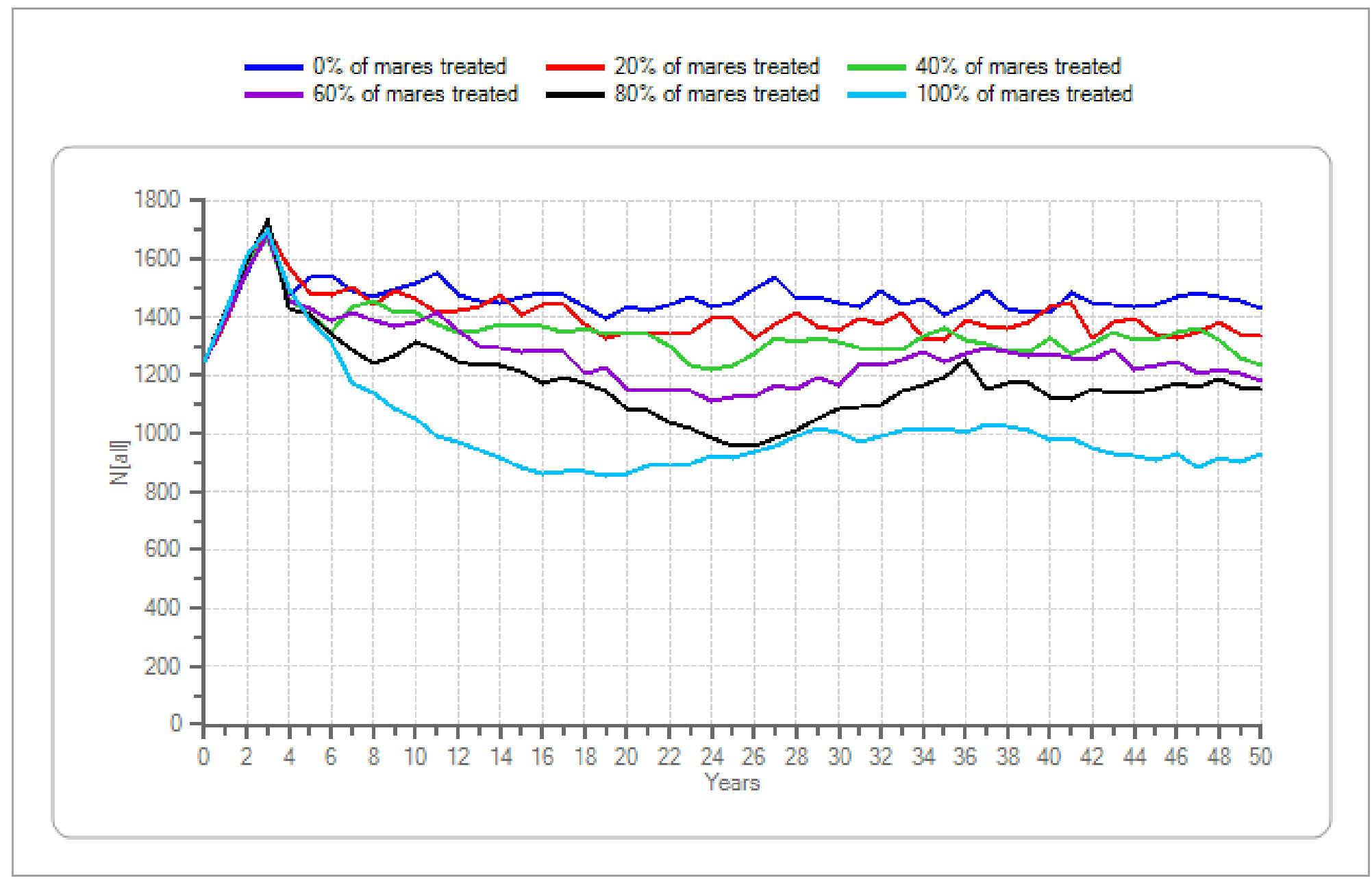

Figure 63. Initial population size $=1250$, lambda $=1.15$, genetic diversity $=$ high - Continued 
Mean population size after 20 years

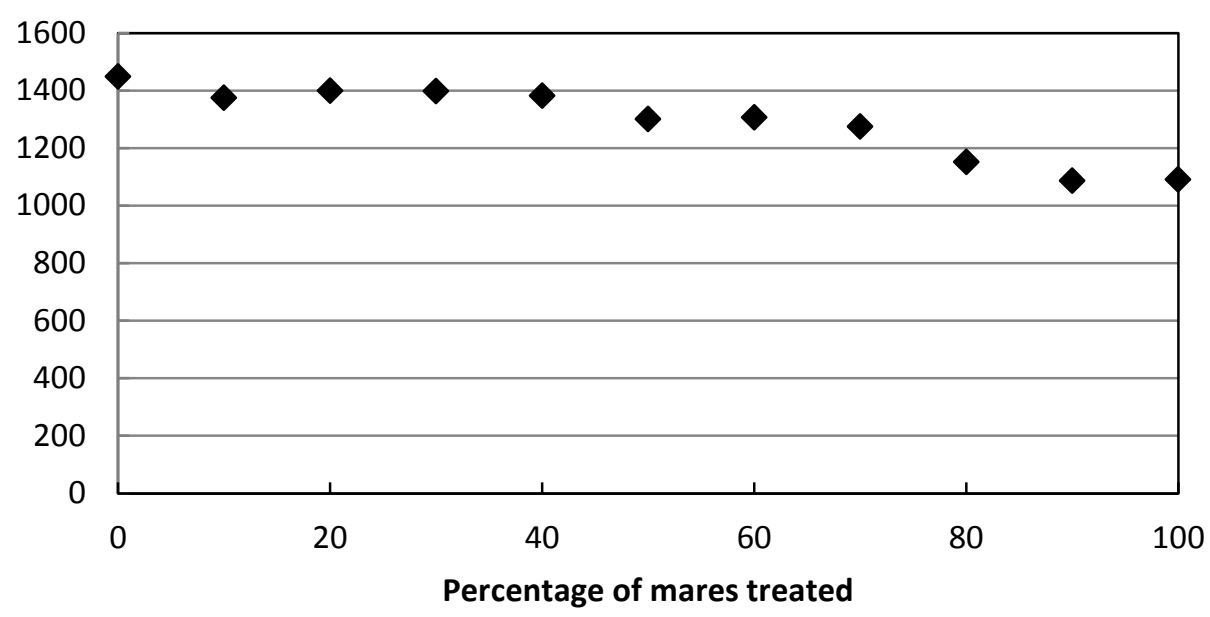

Mean probability of extirpation after $\mathbf{5 0}$ years

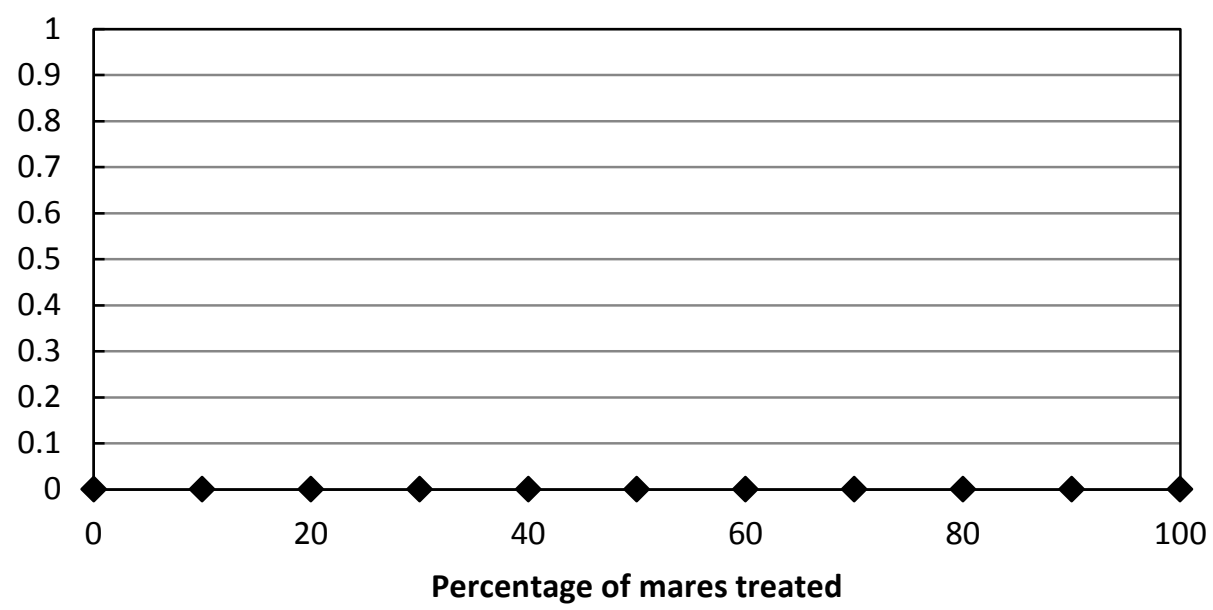

Figure 64. Initial population size $=1250$, lambda $=1.20$, genetic diversity $=$ low .
Mean number of removals after 20 years

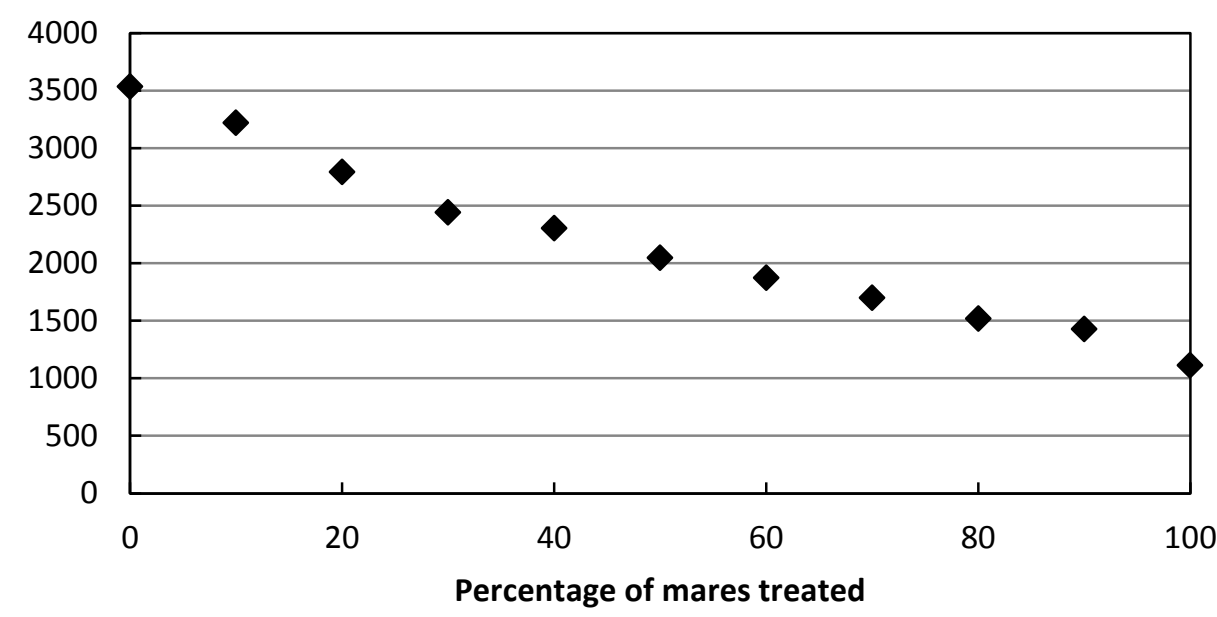

Mean heterozygosity after $\mathbf{5 0}$ years

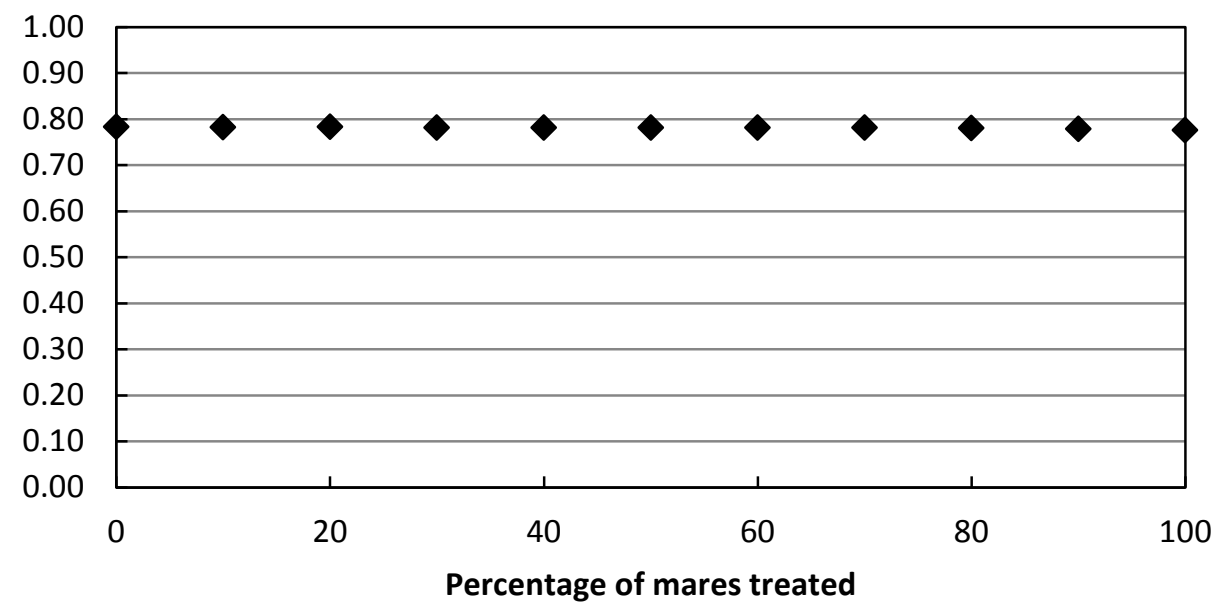




\begin{tabular}{lcccccccccccc}
\hline \multicolumn{1}{c}{ Percentage of mares treated } & $\mathbf{0}$ & $\mathbf{1 0}$ & $\mathbf{2 0}$ & $\mathbf{3 0}$ & $\mathbf{4 0}$ & $\mathbf{5 0}$ & $\mathbf{6 0}$ & $\mathbf{7 0}$ & $\mathbf{8 0}$ & $\mathbf{9 0}$ & $\mathbf{1 0 0}$ \\
\hline Mean population size after 20 years & 1449.1 & 1375.0 & 1398.9 & 1397.6 & 1381.6 & 1301.0 & 1306.8 & 1274.2 & 1152.3 & 1086.1 & 1090.1 \\
Mean number of removals after 20 years & 3536.0 & 3218.8 & 2791.6 & 2439.7 & 2302.0 & 2047.4 & 1874.1 & 1697.0 & 1517.3 & 1426.7 & 1110.3 \\
Mean probability of extirpation after 50 years & 0 & 0 & 0 & 0 & 0 & 0 & 0 & 0 & 0 & 0 & 0 \\
Mean heterozygosity after 50 years & 0.78 & 0.78 & 0.78 & 0.78 & 0.78 & 0.78 & 0.78 & 0.78 & 0.78 & 0.78 & 0.78 \\
\hline
\end{tabular}

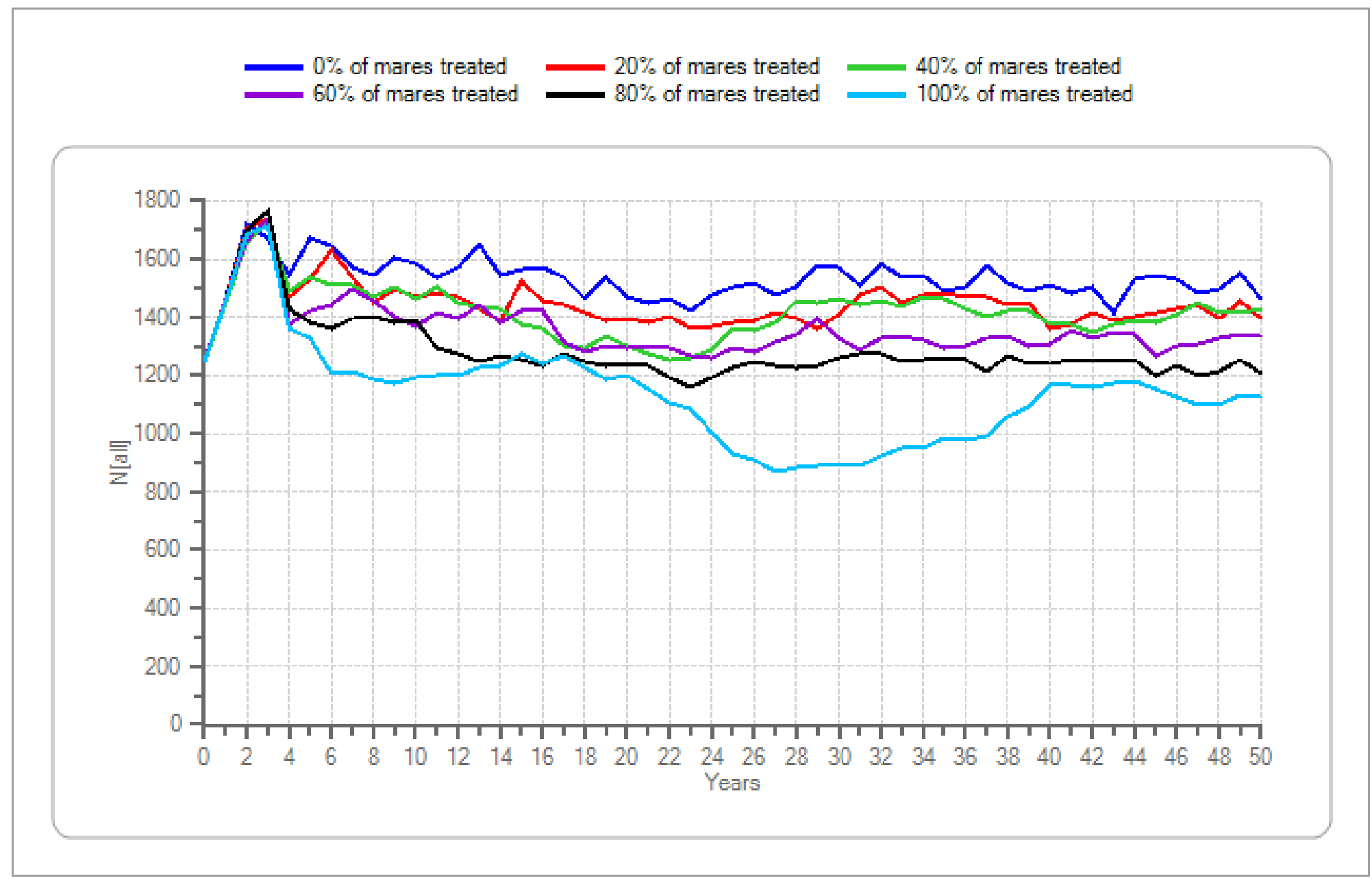

Figure 64. Initial population size $=1250$, lambda $=1.20$, genetic diversity $=$ low. - Continued 
Mean population size after 20 years

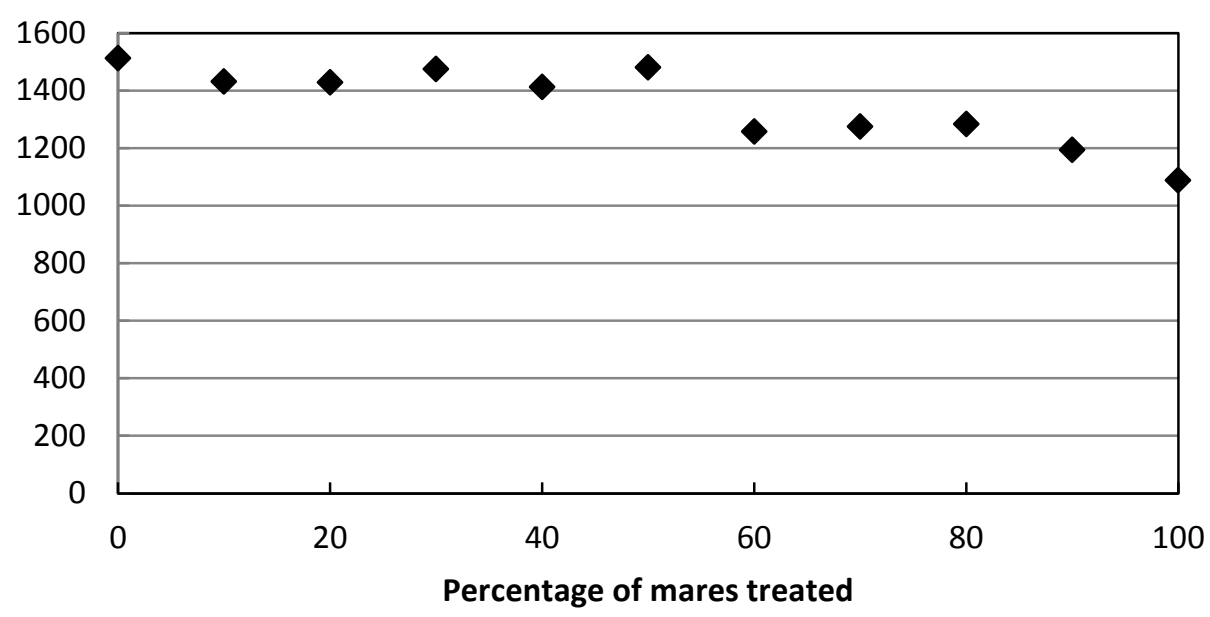

Mean probability of extirpation after $\mathbf{5 0}$ years

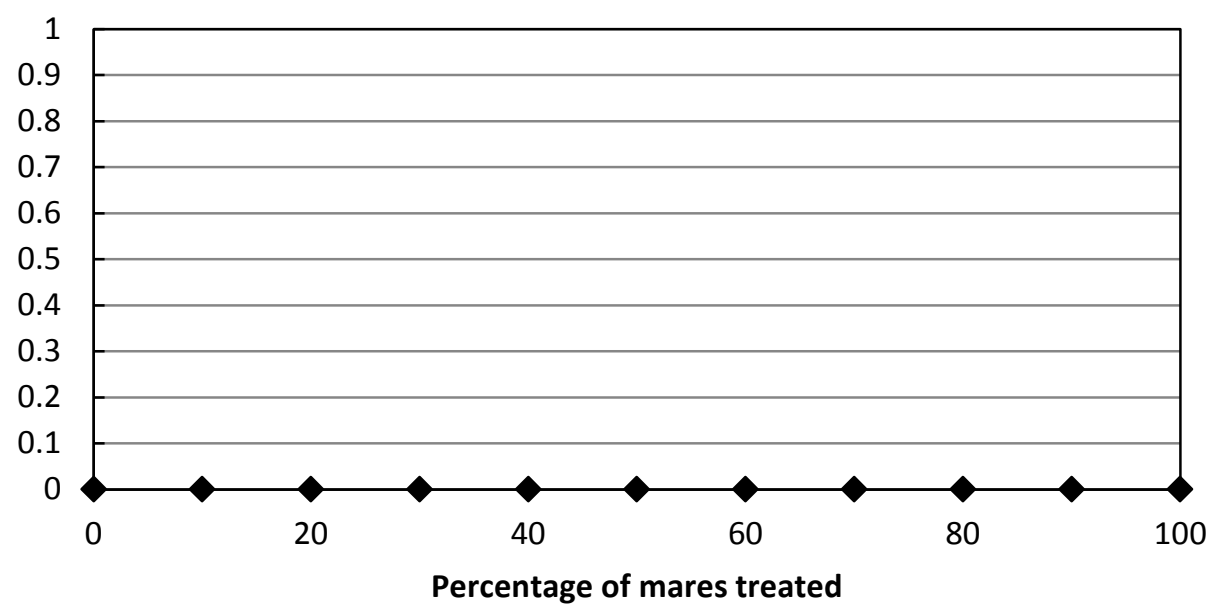

Figure 65. Initial population size $=1250$, lambda $=1.25$, genetic diversity $=$ low .
Mean number of removals after 20 years

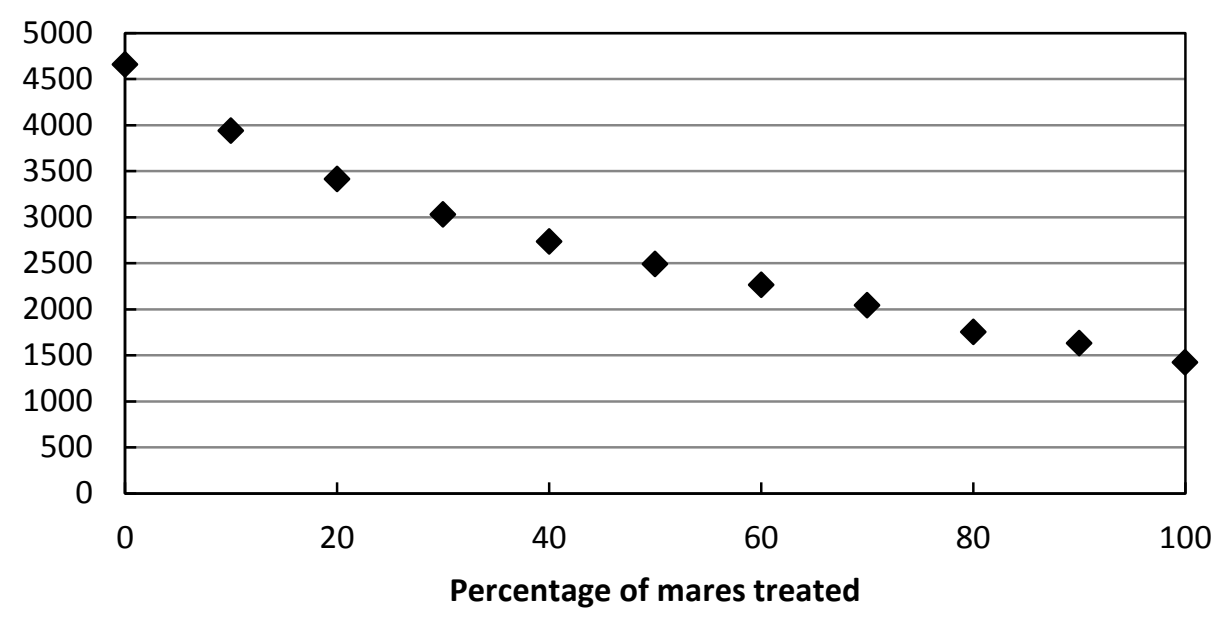

Mean heterozygosity after $\mathbf{5 0}$ years

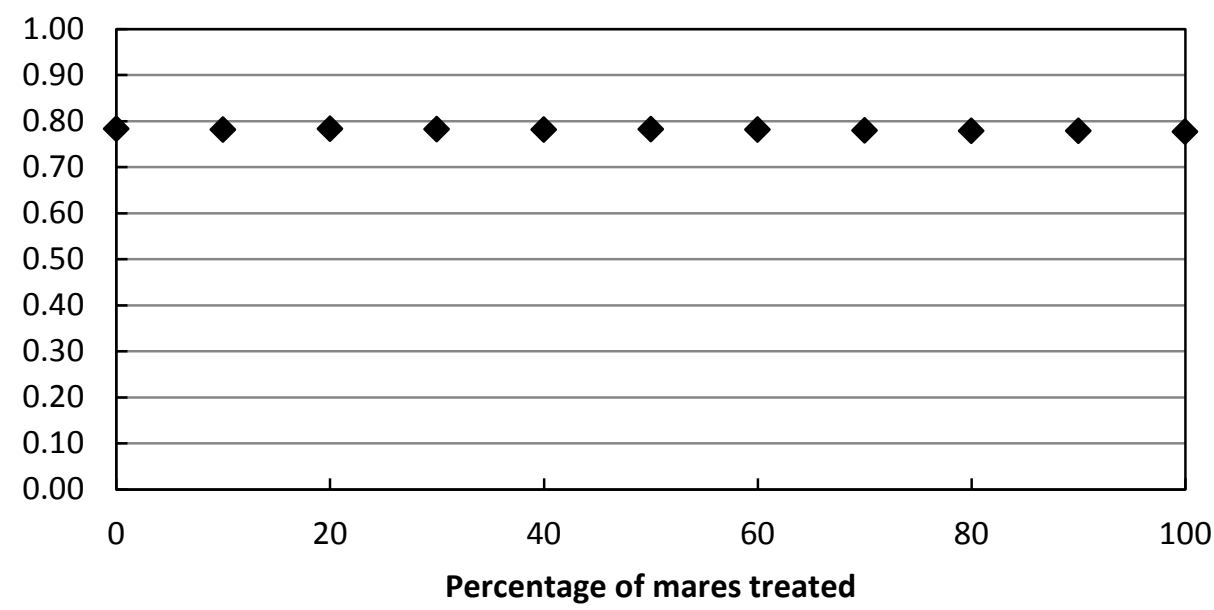




\begin{tabular}{lcccccccccccc}
\hline \multicolumn{1}{c}{ Percentage of mares treated } & $\mathbf{0}$ & $\mathbf{1 0}$ & $\mathbf{2 0}$ & $\mathbf{3 0}$ & $\mathbf{4 0}$ & $\mathbf{5 0}$ & $\mathbf{6 0}$ & $\mathbf{7 0}$ & $\mathbf{8 0}$ & $\mathbf{9 0}$ & $\mathbf{1 0 0}$ \\
\hline Mean population size after 20 years & 1512.7 & 1430.9 & 1428.7 & 1474.7 & 1412.0 & 1480.7 & 1257.7 & 1274.9 & 1284.0 & 1193.1 & 1087.6 \\
Mean number of removals after 20 years & 4658.4 & 3937.6 & 3415.0 & 3030.1 & 2732.3 & 2491.5 & 2261.5 & 2041.8 & 1751.8 & 1629.4 & 1422.0 \\
Mean probability of extirpation after 50 years & 0 & 0 & 0 & 0 & 0 & 0 & 0 & 0 & 0 & 0 & 0 \\
Mean heterozygosity after 50 years & 0.78 & 0.78 & 0.78 & 0.78 & 0.78 & 0.78 & 0.78 & 0.78 & 0.78 & 0.78 & 0.78 \\
\hline
\end{tabular}

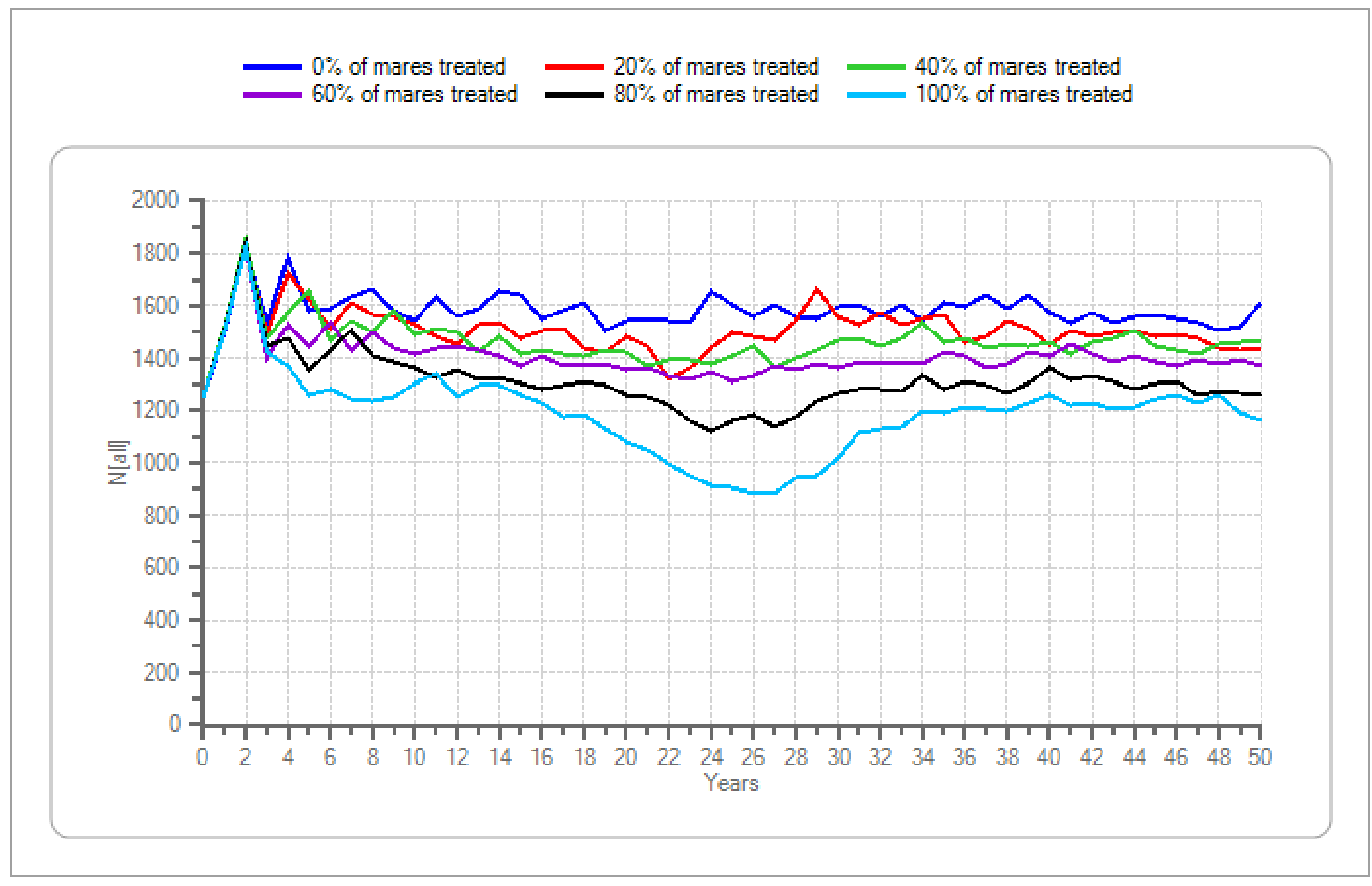

Figure 65 . Initial population size $=1250$, lambda $=1.25$, genetic diversity $=$ low - Continued 
Mean population size after 20 years

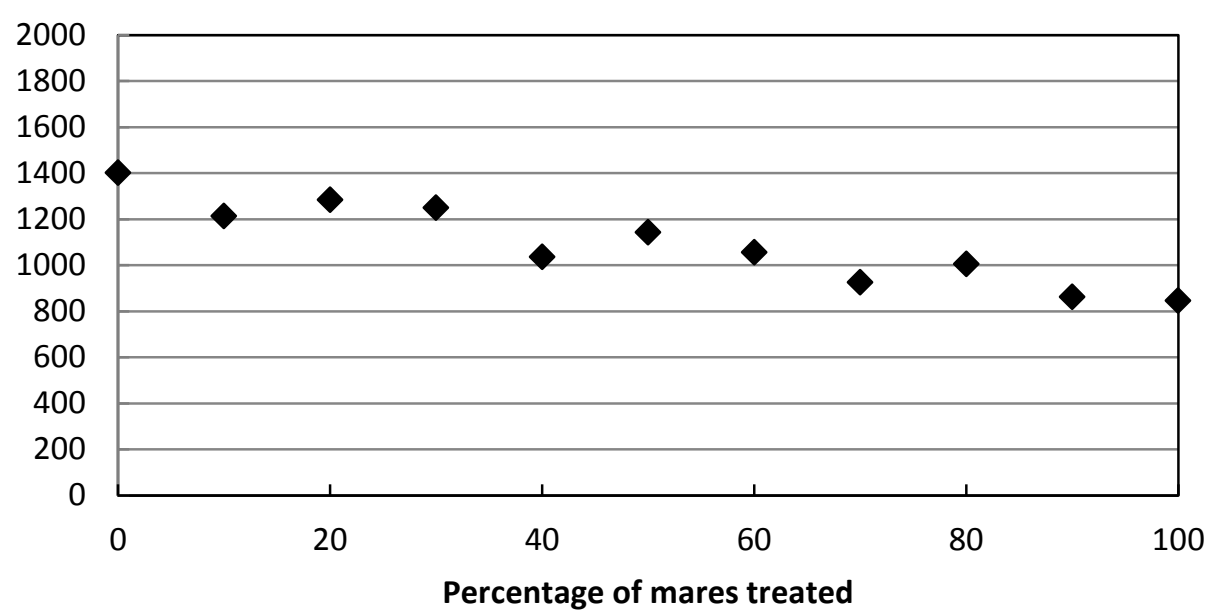

Mean probability of extirpation after 50 years

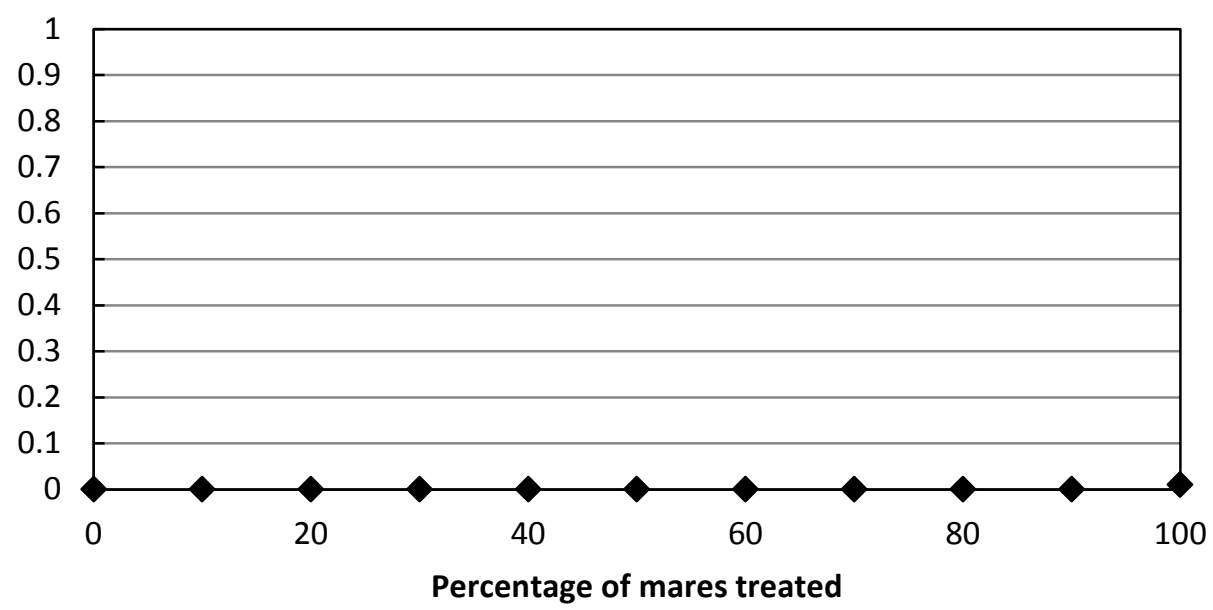

Figure 66. Initial population size $=1500$, lambda $=1.05$, genetic diversity $=$ high .
Mean number of removals after 20 years

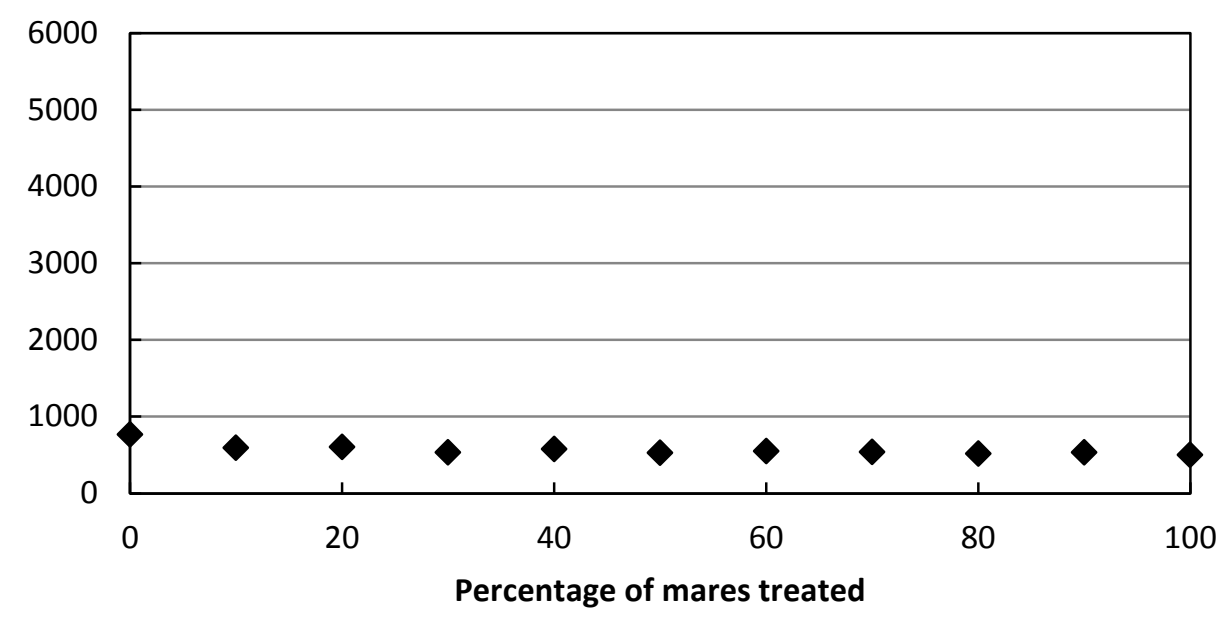

Mean heterozygosity after $\mathbf{5 0}$ years

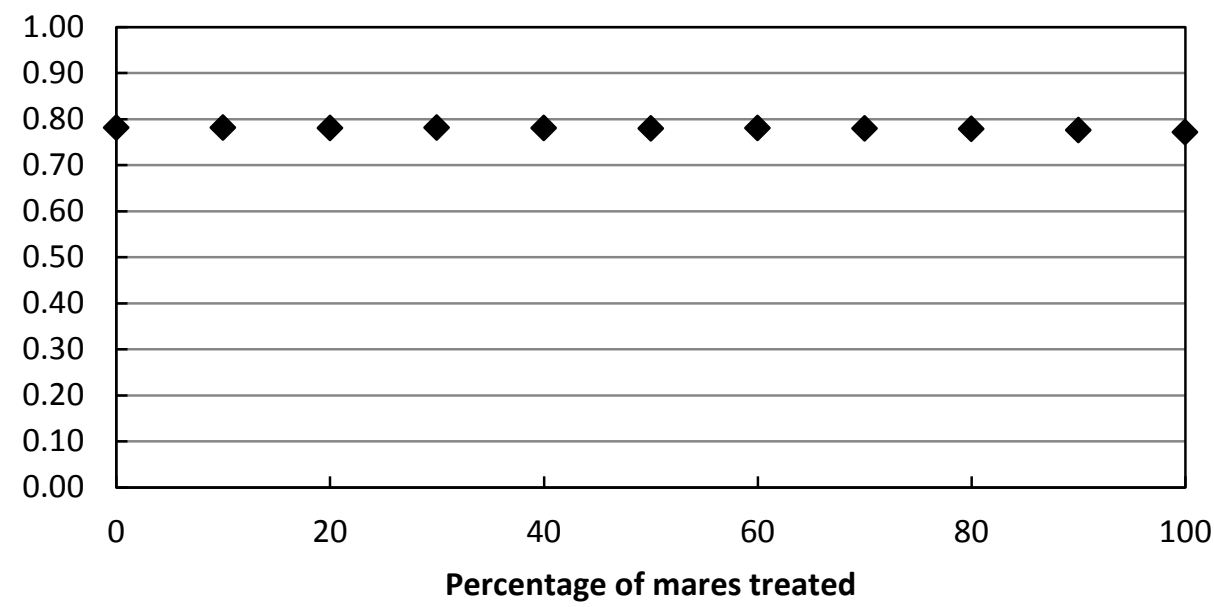




\begin{tabular}{lccccccccccc}
\hline \multicolumn{1}{c}{ Percentage of mares treated } & $\mathbf{0}$ & $\mathbf{1 0}$ & $\mathbf{2 0}$ & $\mathbf{3 0}$ & $\mathbf{4 0}$ & $\mathbf{5 0}$ & $\mathbf{6 0}$ & $\mathbf{7 0}$ & $\mathbf{8 0}$ & $\mathbf{9 0}$ & $\mathbf{1 0 0}$ \\
\hline Mean population size after 20 years & 1401.3 & 1213.9 & 1283.2 & 1249.7 & 1035.4 & 1142.4 & 1056.3 & 925.6 & 1005.1 & 862.6 & 845.7 \\
Mean number of removals after 20 years & 764.8 & 589.6 & 604.0 & 530.4 & 578.3 & 527.0 & 547.6 & 539.9 & 516.7 & 534.7 & 497.8 \\
Mean probability of extirpation after 50 years & 0 & 0 & 0 & 0 & 0 & 0 & 0 & 0 & 0 & 0 & 0.01 \\
Mean heterozygosity after 50 years & 0.78 & 0.78 & 0.78 & 0.78 & 0.78 & 0.78 & 0.78 & 0.78 & 0.78 & 0.78 & 0.77 \\
\hline
\end{tabular}

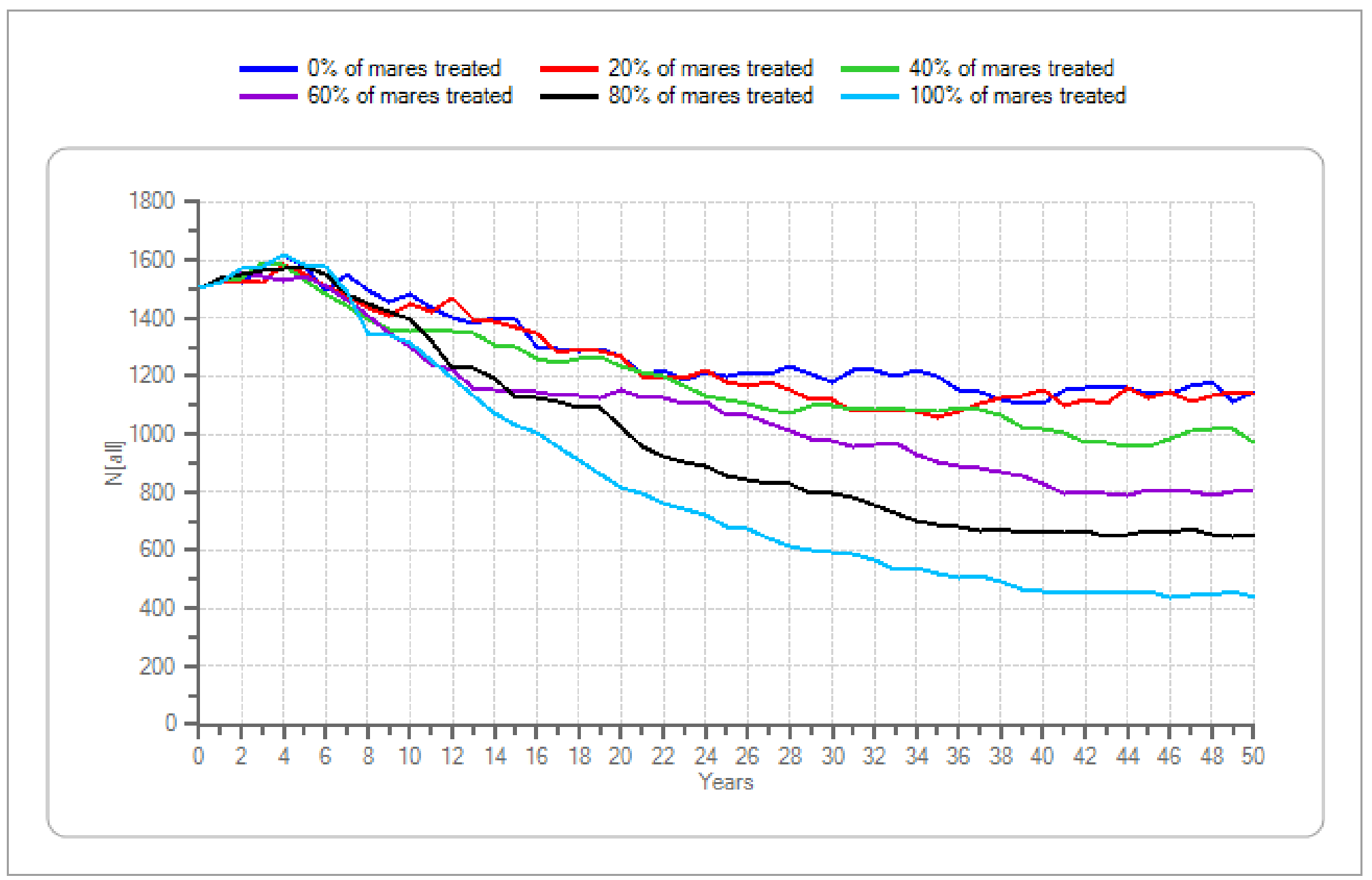

Figure 66. Initial population size $=1500$, lambda $=1.05$, genetic diversity $=$ high. Continued 
Mean population size after 20 years

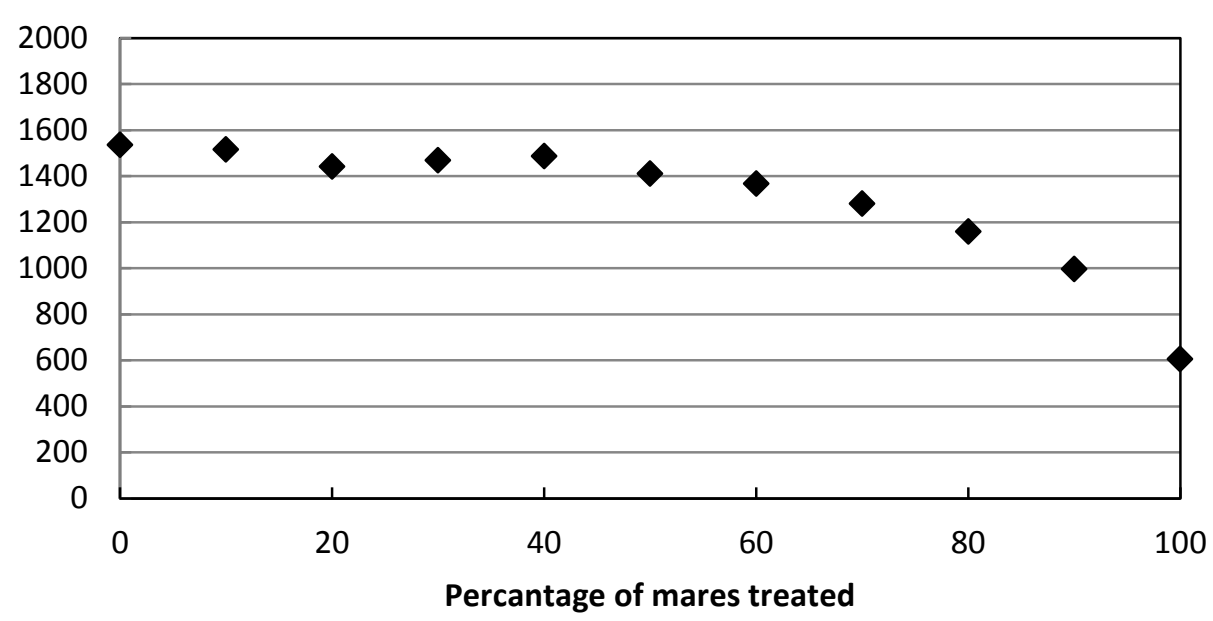

Mean probability of extirpation after 50 years

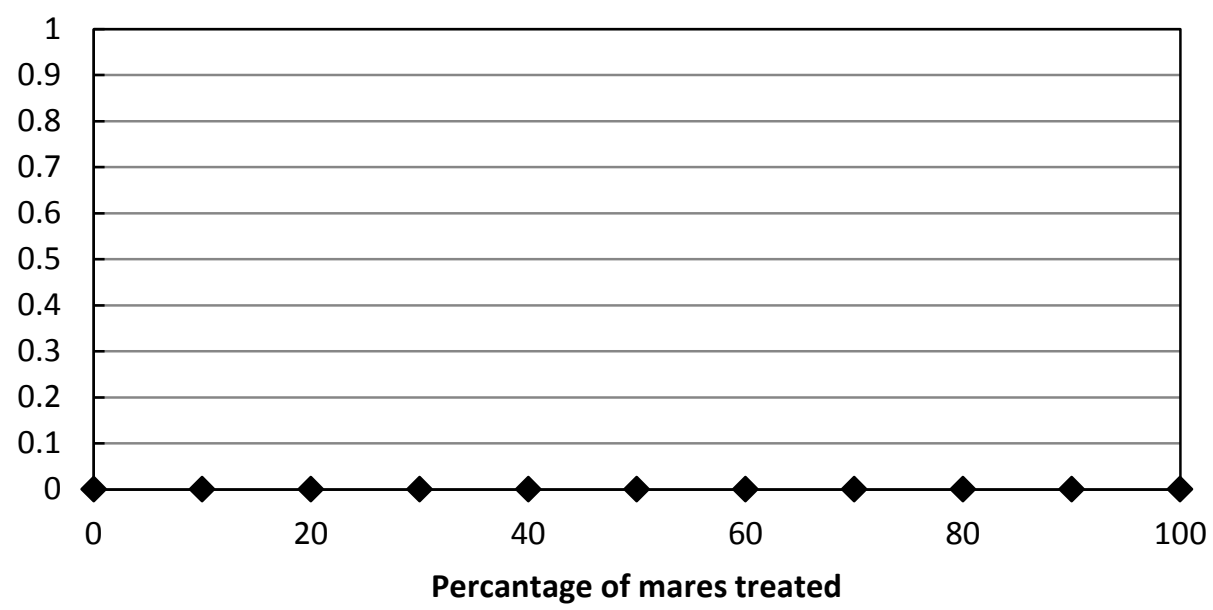

Figure 67. Initial population size $=1500$, lambda $=1.10$, genetic diversity $=$ high .
Mean number of removals after 20 years

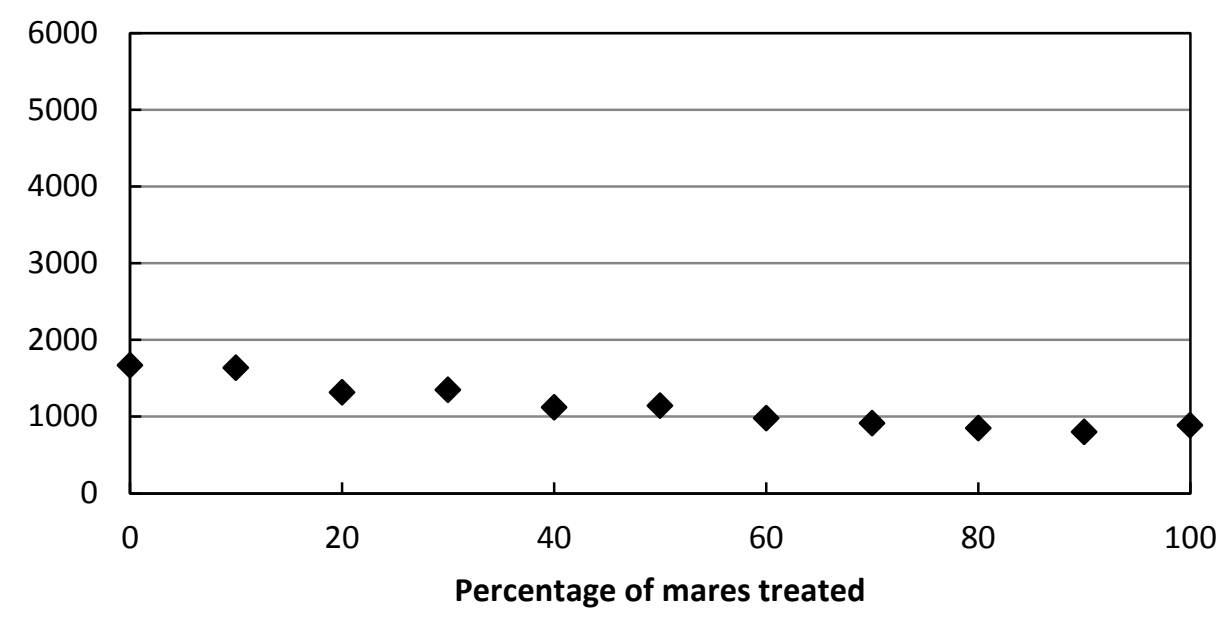

Mean heterozygosity after $\mathbf{5 0}$ years

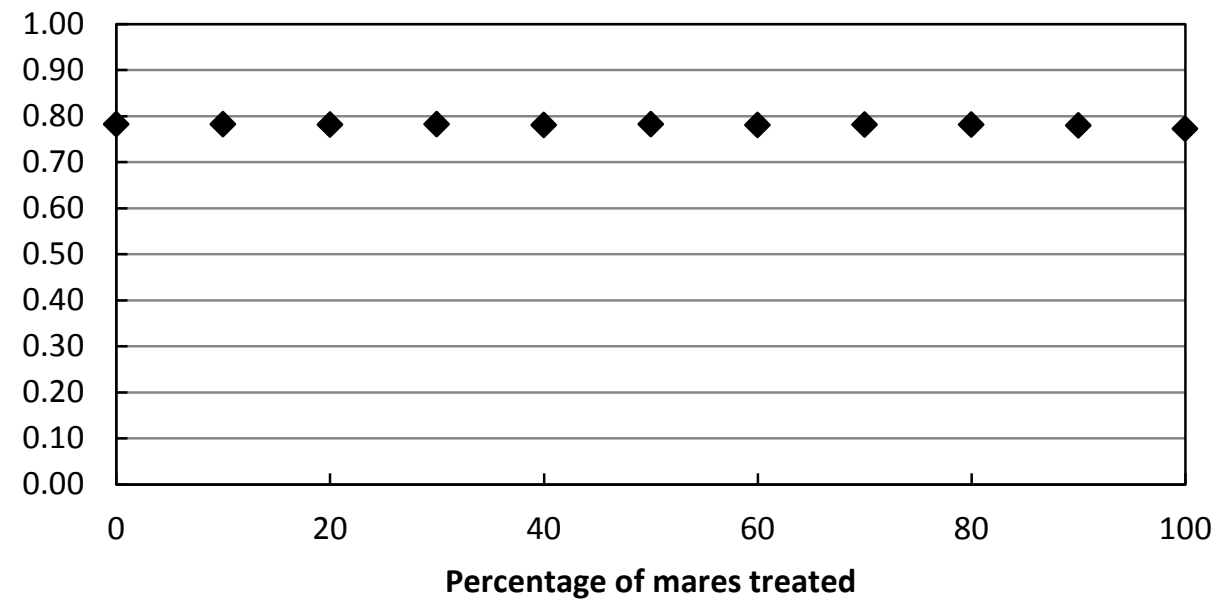




\begin{tabular}{lccccccccccc}
\hline \multicolumn{1}{c}{ Percentage of mares treated } & $\mathbf{0}$ & $\mathbf{1 0}$ & $\mathbf{2 0}$ & $\mathbf{3 0}$ & $\mathbf{4 0}$ & $\mathbf{5 0}$ & $\mathbf{6 0}$ & $\mathbf{7 0}$ & $\mathbf{8 0}$ & $\mathbf{9 0}$ & $\mathbf{1 0 0}$ \\
\hline Mean population size after 20 years & 1534.7 & 1515.0 & 1441.5 & 1468.4 & 1487.3 & 1411.4 & 1367.4 & 1280.2 & 1158.3 & 996.3 & 604.3 \\
Mean number of removals after 20 years & 1669.4 & 1636.8 & 1316.9 & 1346.6 & 1118.7 & 1139.7 & 979.0 & 911.8 & 846.6 & 799.3 & 886.0 \\
Mean probability of extirpation after 50 years & 0 & 0 & 0 & 0 & 0 & 0 & 0 & 0 & 0 & 0 & 0 \\
Mean heterozygosity after 50 years & 0.78 & 0.78 & 0.78 & 0.78 & 0.78 & 0.78 & 0.78 & 0.78 & 0.78 & 0.78 & 0.77 \\
\hline
\end{tabular}

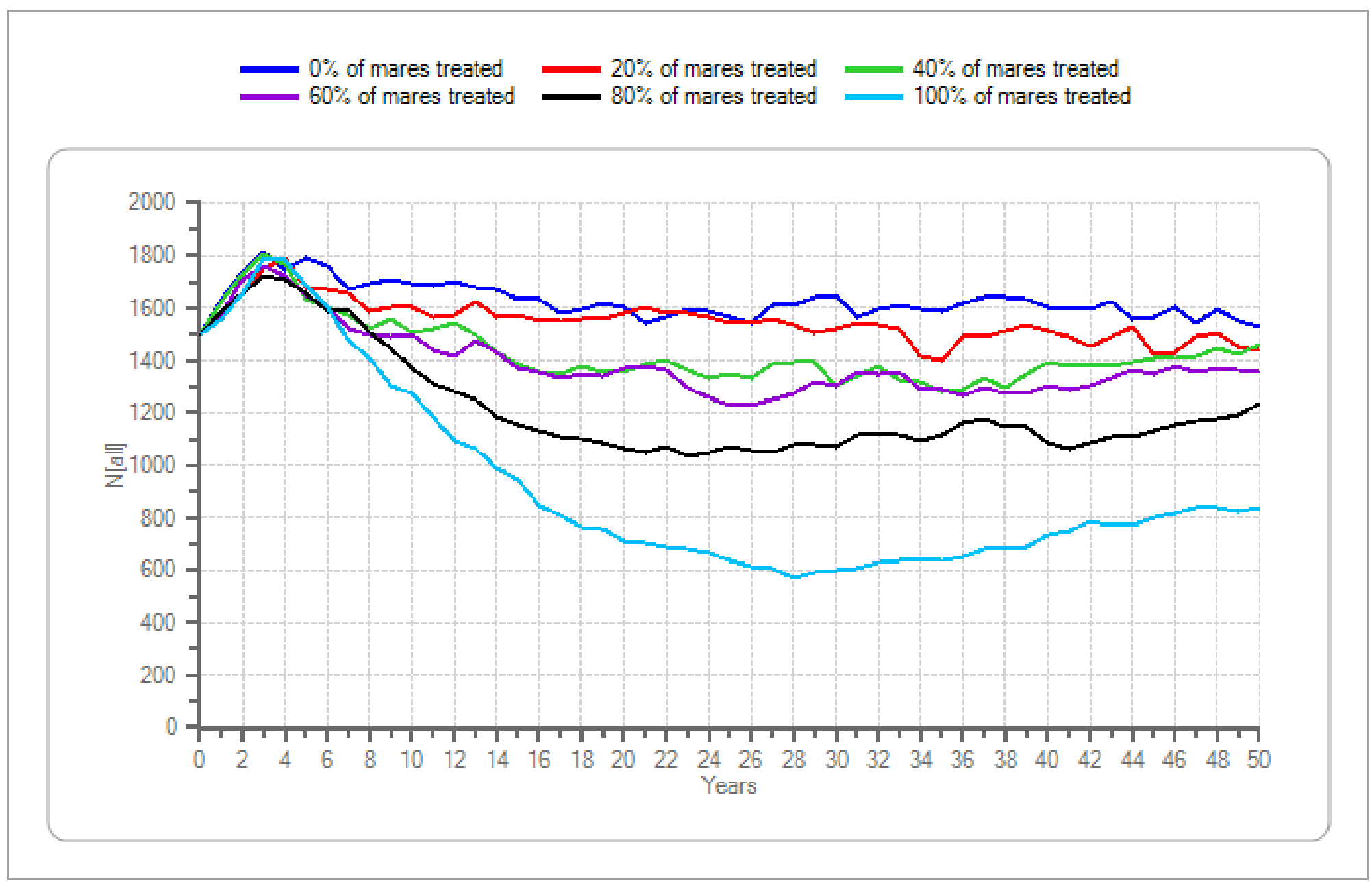

Figure 67. Initial population size $=1500$, lambda $=1.10$, genetic diversity $=$ high -Continued 
Mean population size after 20 years

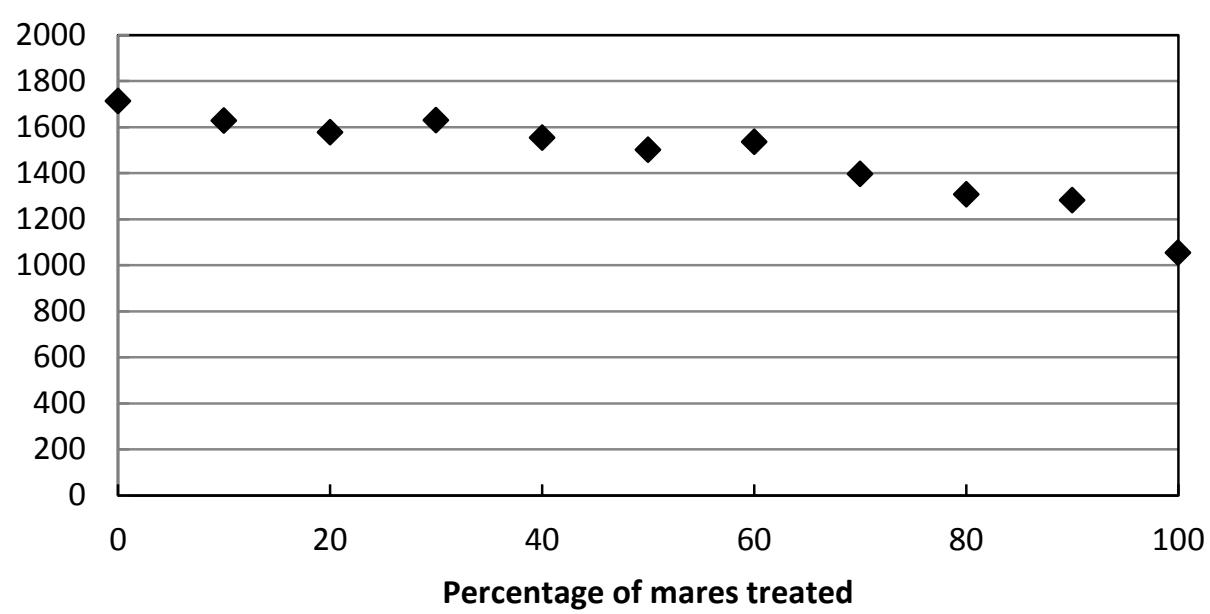

Mean probability of extirpation after $\mathbf{5 0}$ years

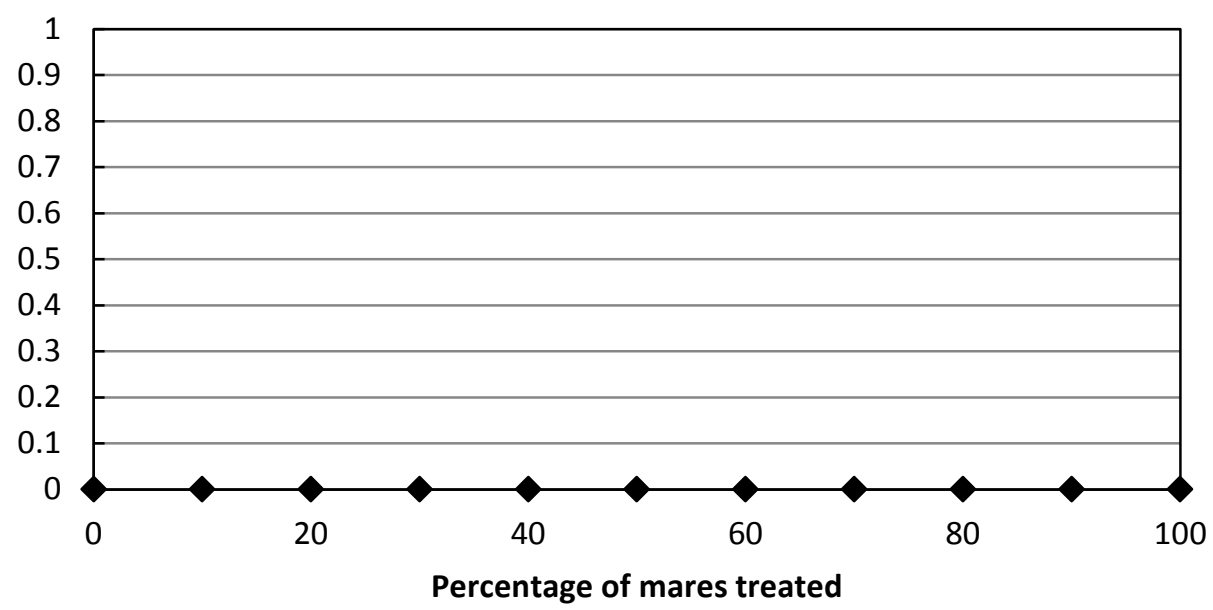

Figure 68. Initial population size $=1500$, lambda $=1.15$, genetic diversity $=$ high .
Mean number of removals after 20 years

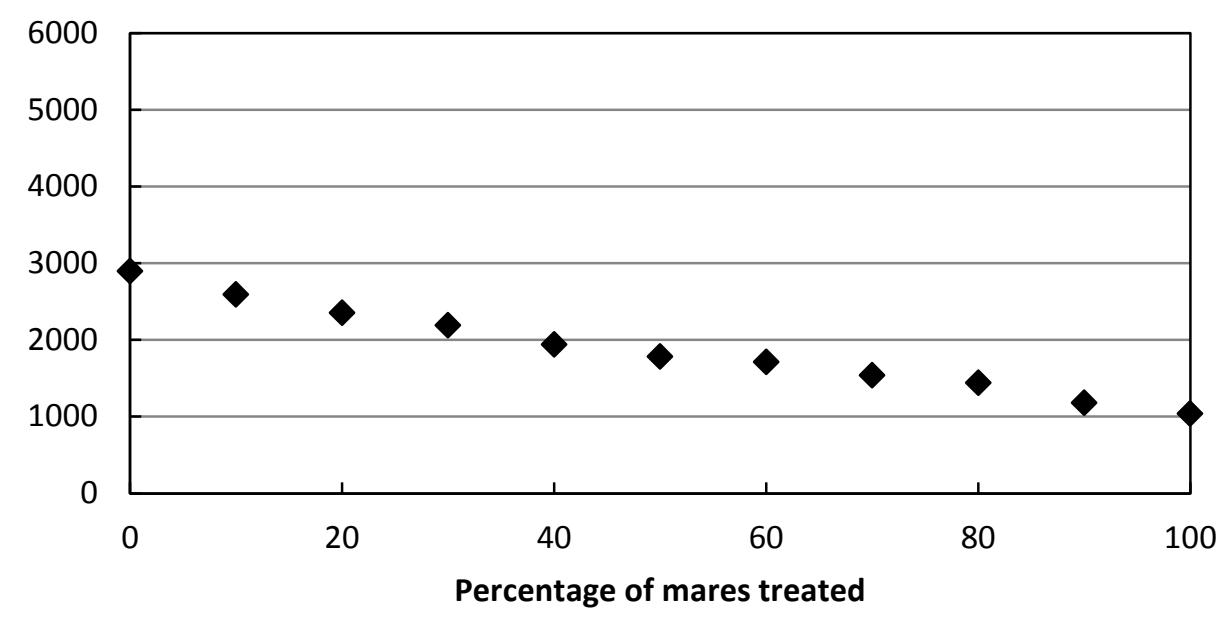

Mean heterozygosity after $\mathbf{5 0}$ years

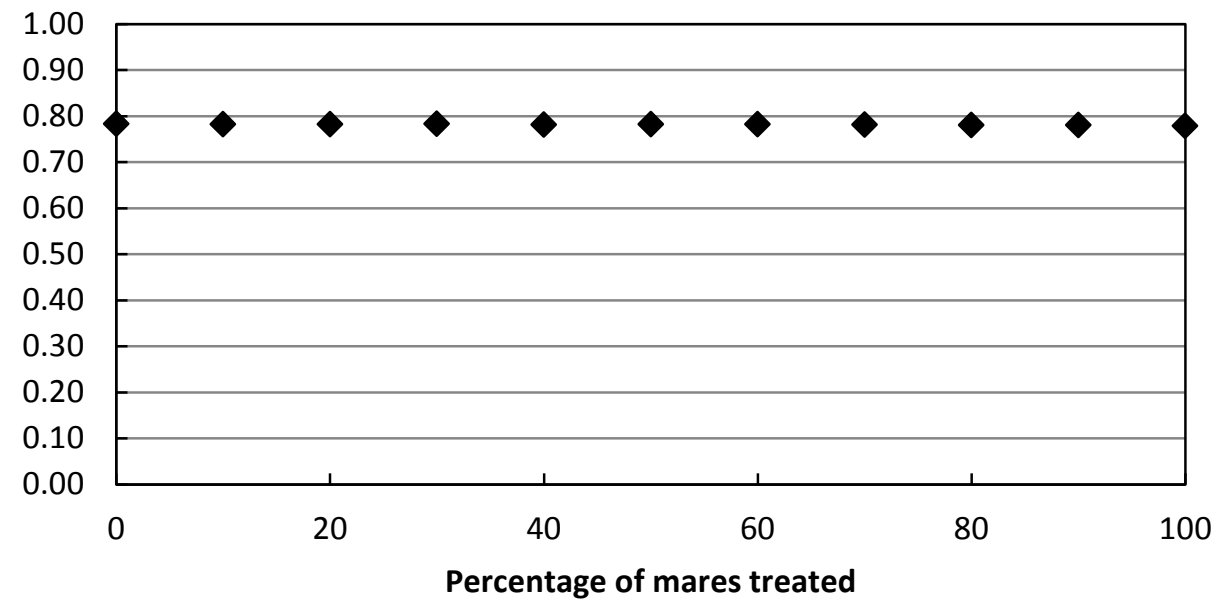




\begin{tabular}{lccccccccccc}
\hline \multicolumn{1}{c}{ Percentage of mares treated } & $\mathbf{0}$ & $\mathbf{1 0}$ & $\mathbf{2 0}$ & $\mathbf{3 0}$ & $\mathbf{4 0}$ & $\mathbf{5 0}$ & $\mathbf{6 0}$ & $\mathbf{7 0}$ & $\mathbf{8 0}$ & $\mathbf{9 0}$ & $\mathbf{1 0 0}$ \\
\hline Mean population size after 20 years & 1712.0 & 1627.6 & 1576.6 & 1629.3 & 1553.6 & 1500.3 & 1535.1 & 1396.9 & 1306.5 & 1281.2 & 1053.2 \\
Mean number of removals after 20 years & 2896.0 & 2590.5 & 2349.9 & 2187.2 & 1938.7 & 1780.1 & 1713.2 & 1538.8 & 1441.8 & 1180.9 & 1038.4 \\
Mean probability of extirpation after 50 years & 0 & 0 & 0 & 0 & 0 & 0 & 0 & 0 & 0 & 0 & 0 \\
Mean heterozygosity after 50 years & 0.78 & 0.78 & 0.78 & 0.78 & 0.78 & 0.78 & 0.78 & 0.78 & 0.78 & 0.78 & 0.78 \\
\hline
\end{tabular}

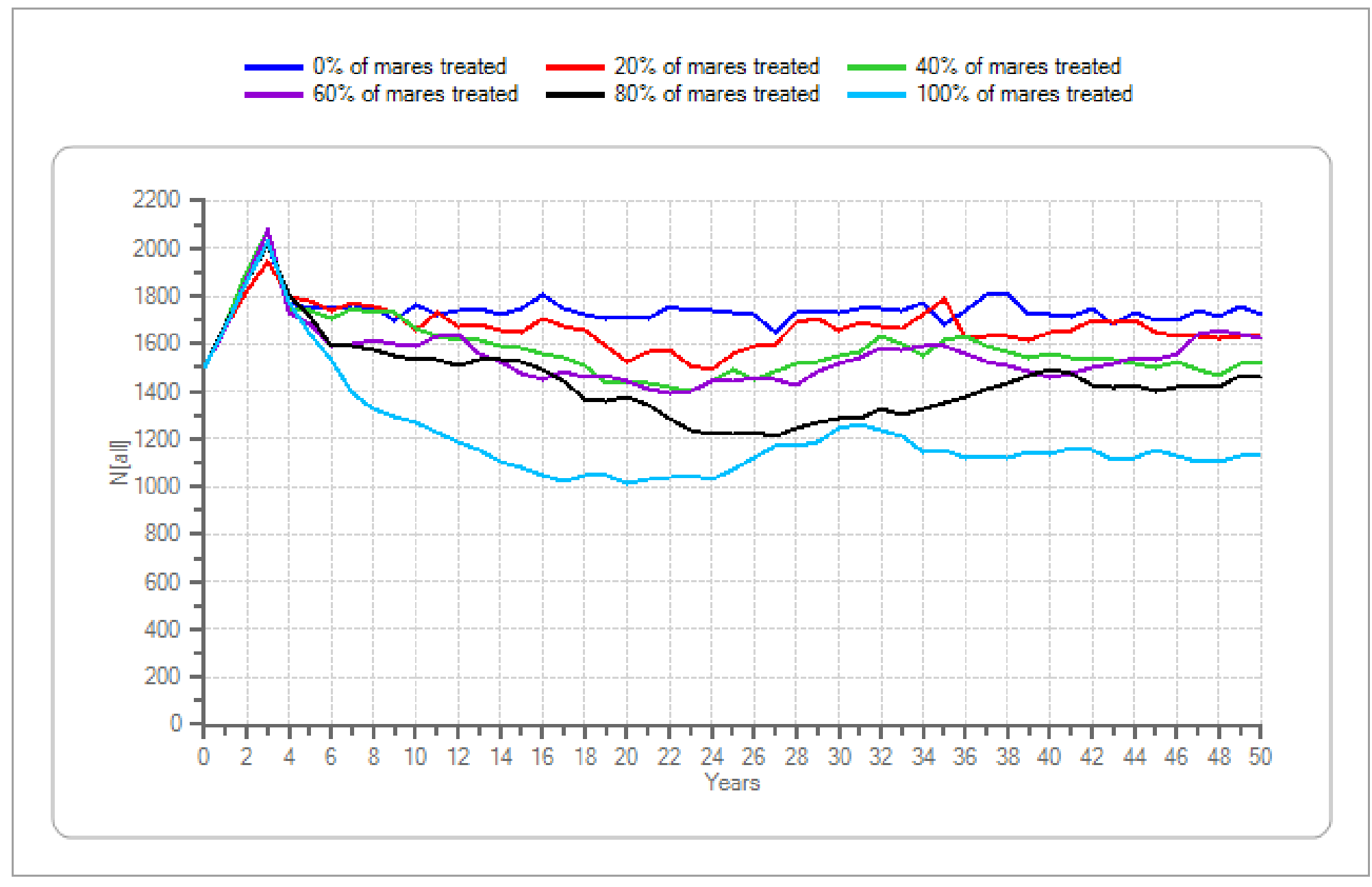

Figure 68. Initial population size $=1500$, lambda $=1.15$, genetic diversity $=$ high - Continued 
Mean population size after 20 years

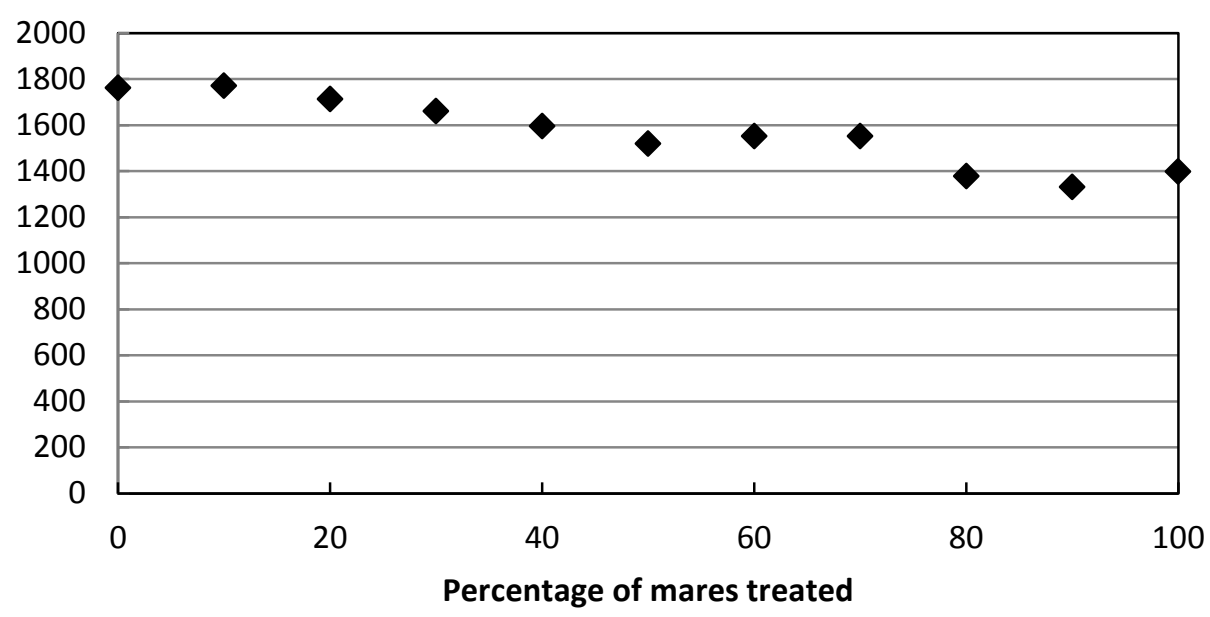

Mean probability of extirpation after 50 years

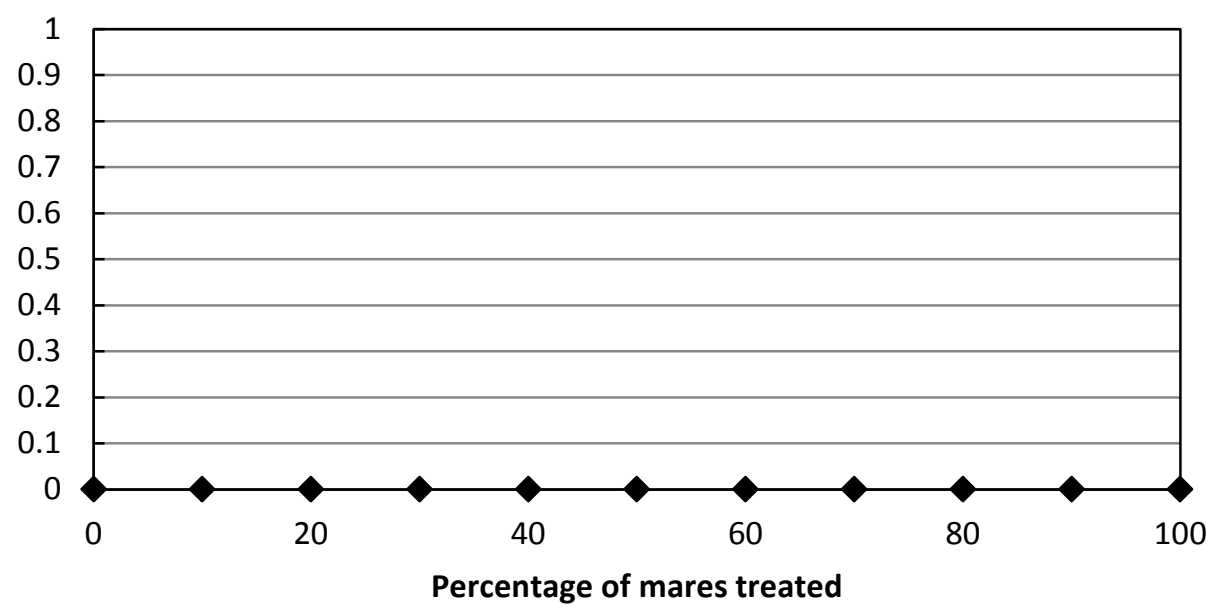

Figure 69. Initial population size $=1500$, lambda $=1.20$, genetic diversity $=$ high .
Mean number of removals after 20 years

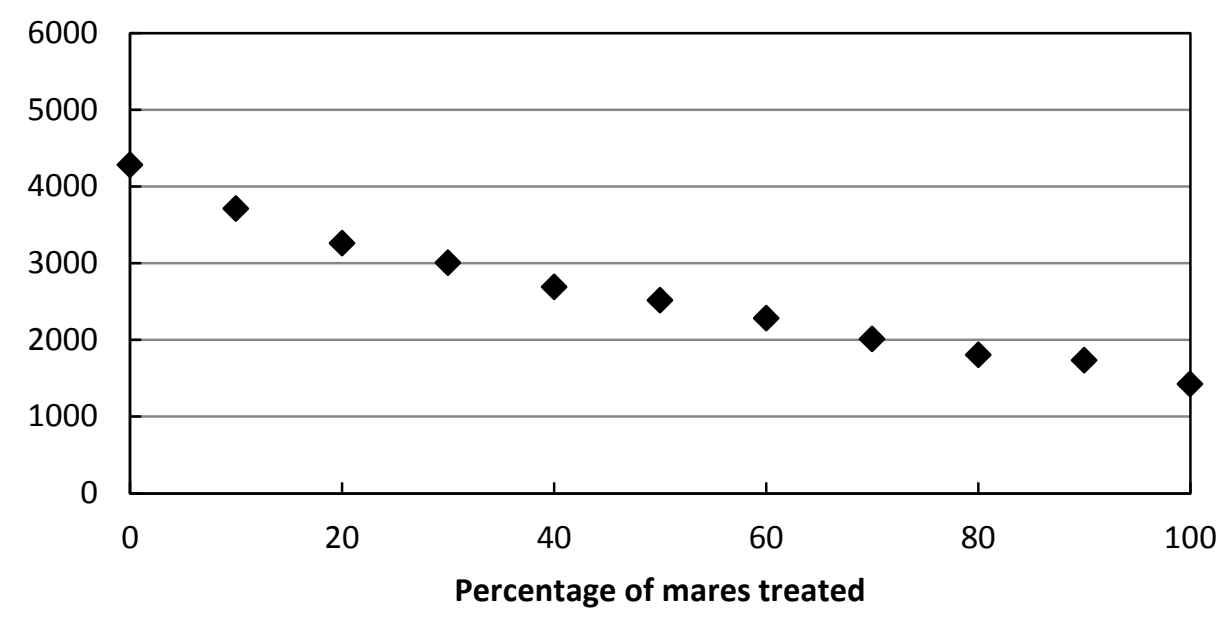

Mean heterozygosity after $\mathbf{5 0}$ years

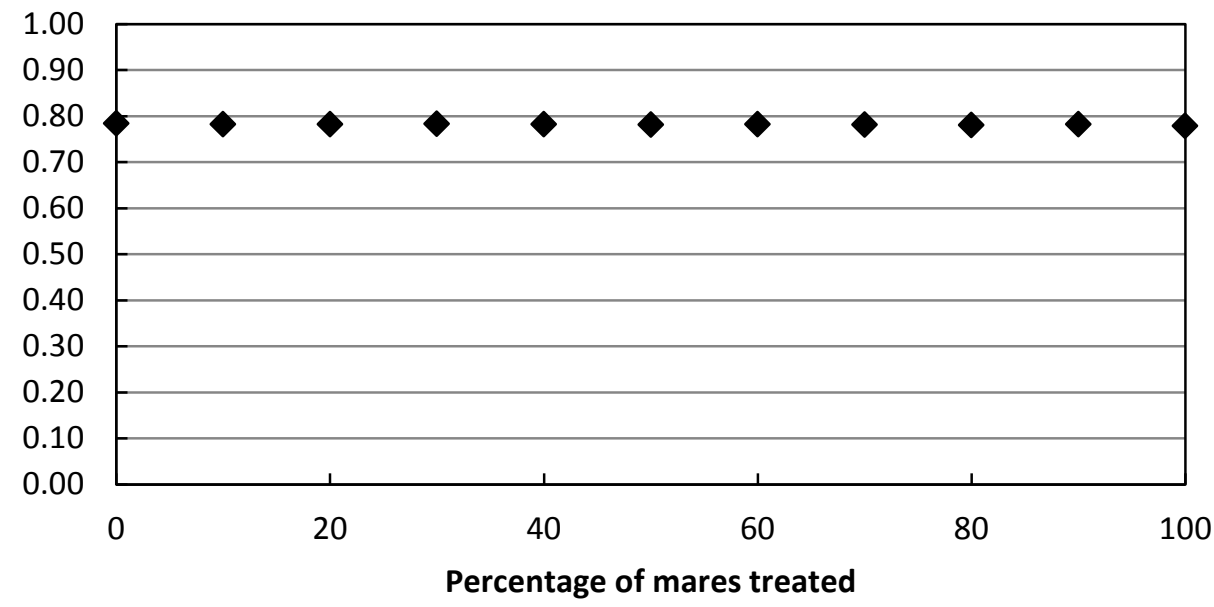




\begin{tabular}{lccccccccccc}
\hline \multicolumn{1}{c}{ Percentage of mares treated } & $\mathbf{0}$ & $\mathbf{1 0}$ & $\mathbf{2 0}$ & $\mathbf{3 0}$ & $\mathbf{4 0}$ & $\mathbf{5 0}$ & $\mathbf{6 0}$ & $\mathbf{7 0}$ & $\mathbf{8 0}$ & $\mathbf{9 0}$ & $\mathbf{1 0 0}$ \\
\hline Mean population size after 20 years & 1761.4 & 1771.4 & 1712.8 & 1660.2 & 1595.1 & 1518.3 & 1551.7 & 1551.3 & 1378.4 & 1331.5 & 1397.5 \\
Mean number of removals after 20 years & 4280.8 & 3712.2 & 3260.2 & 3005.3 & 2689.8 & 2516.5 & 2281.8 & 2007.8 & 1802.1 & 1735.3 & 1423.0 \\
Mean probability of extirpation after 50 years & 0 & 0 & 0 & 0 & 0 & 0 & 0 & 0 & 0 & 0 & 0 \\
Mean heterozygosity after 50 years & 0.78 & 0.78 & 0.78 & 0.78 & 0.78 & 0.78 & 0.78 & 0.78 & 0.78 & 0.78 & 0.78 \\
\hline
\end{tabular}

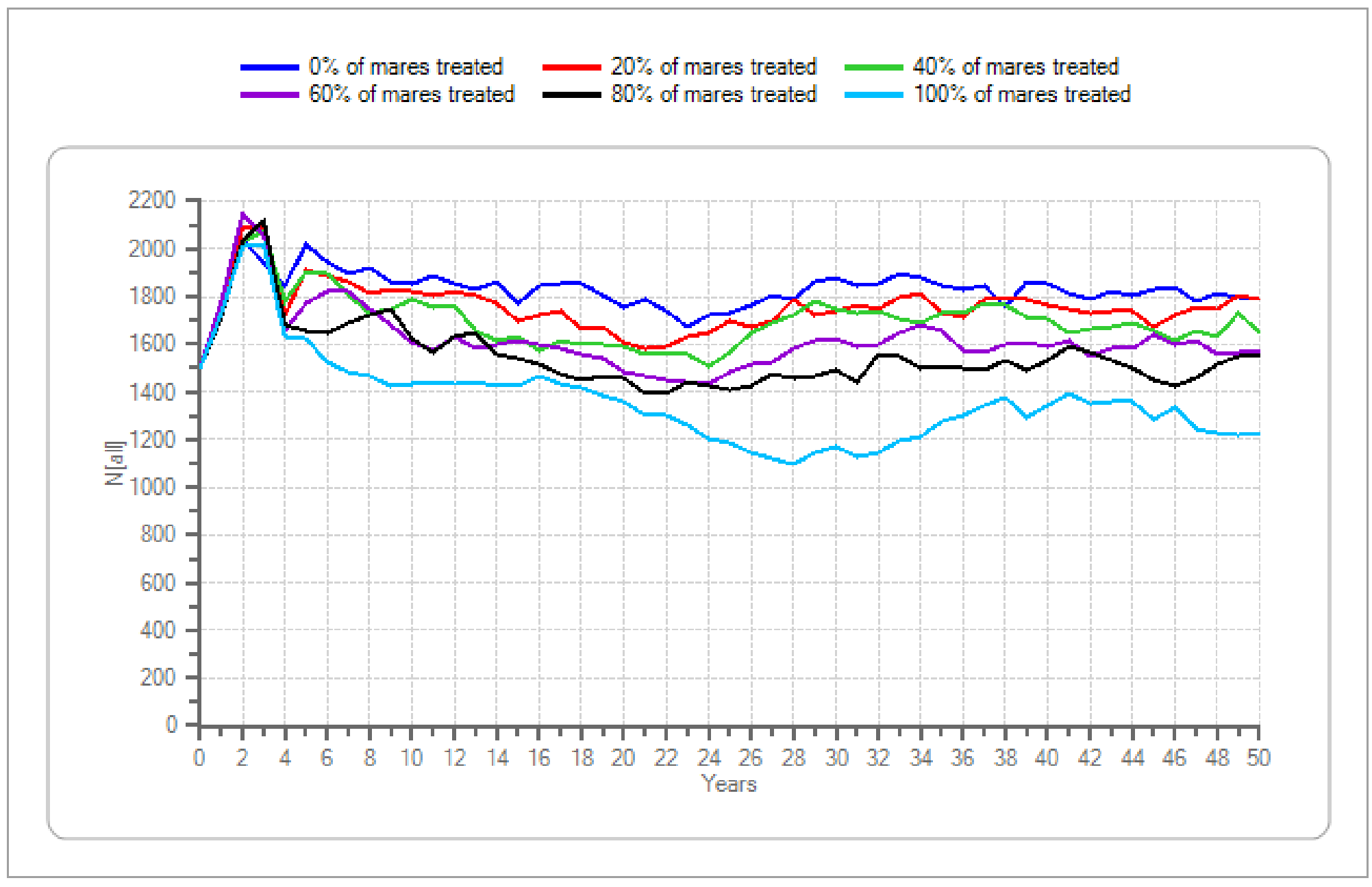

Figure 69. Initial population size $=1500$, lambda $=1.20$, genetic diversity $=$ high - Continued 
Mean population size after 20 years

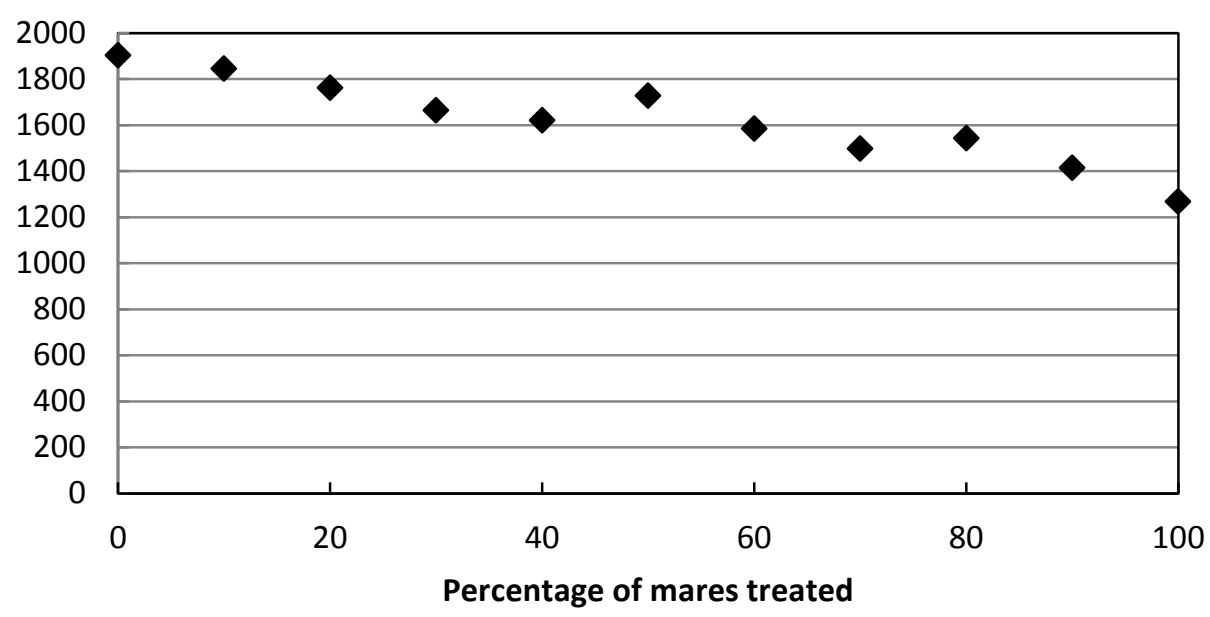

Mean probability of extirpation after 50 years

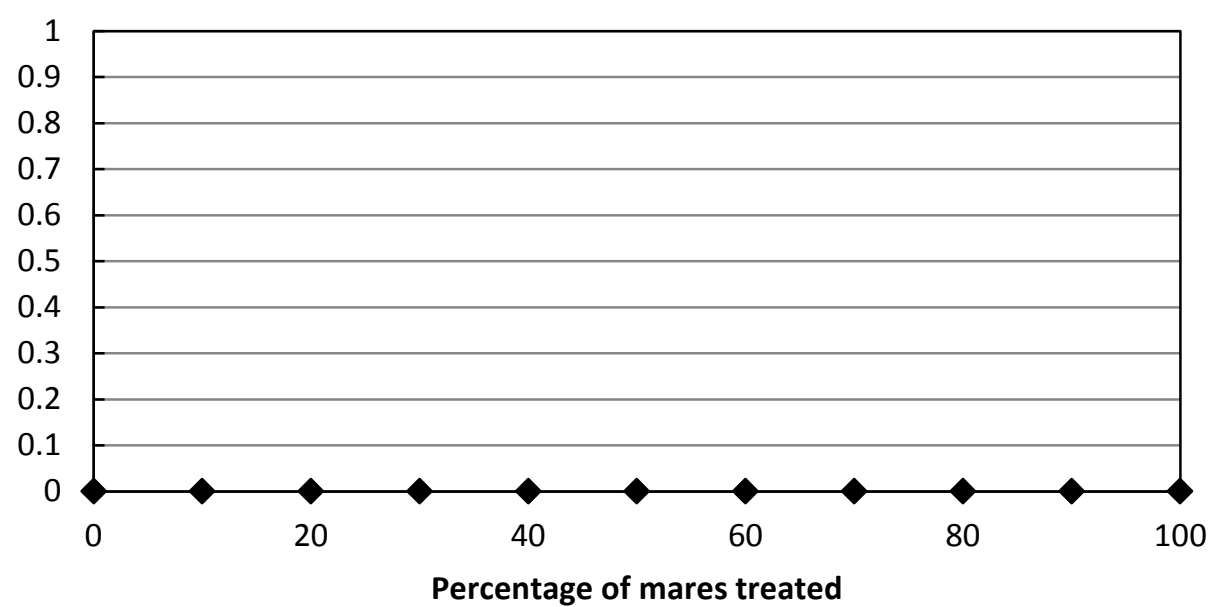

Figure 70. Initial population size $=1500$, lambda $=1.25$, genetic diversity $=$ high .
Mean number of removals after 20 years

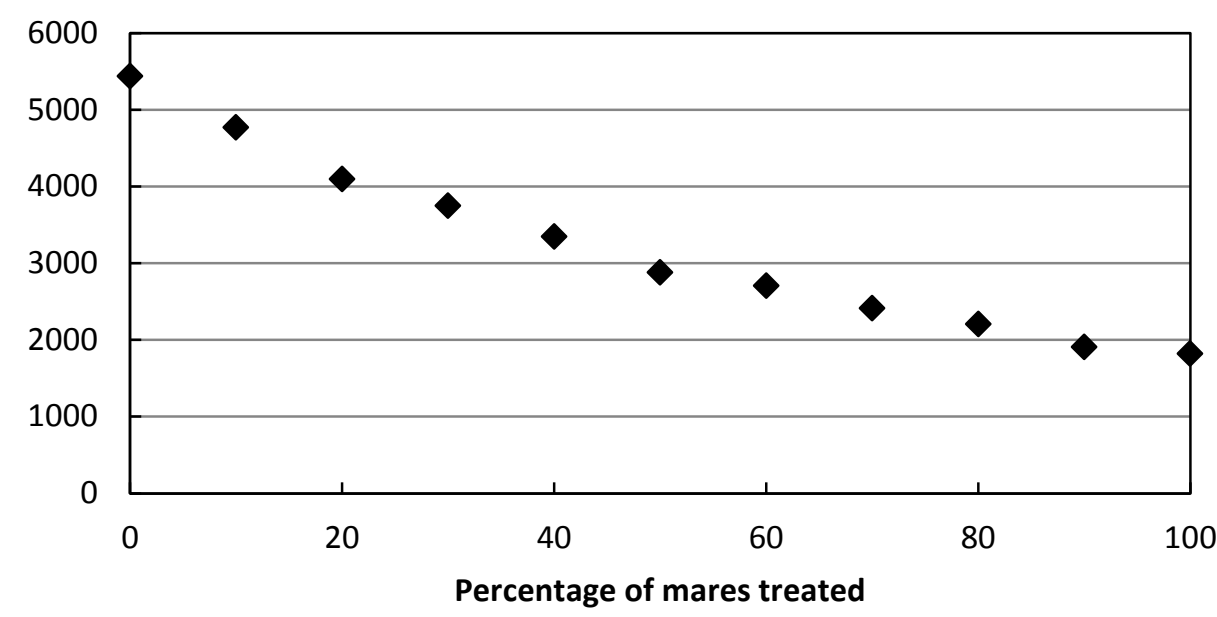

Mean heterozygosity after $\mathbf{5 0}$ years

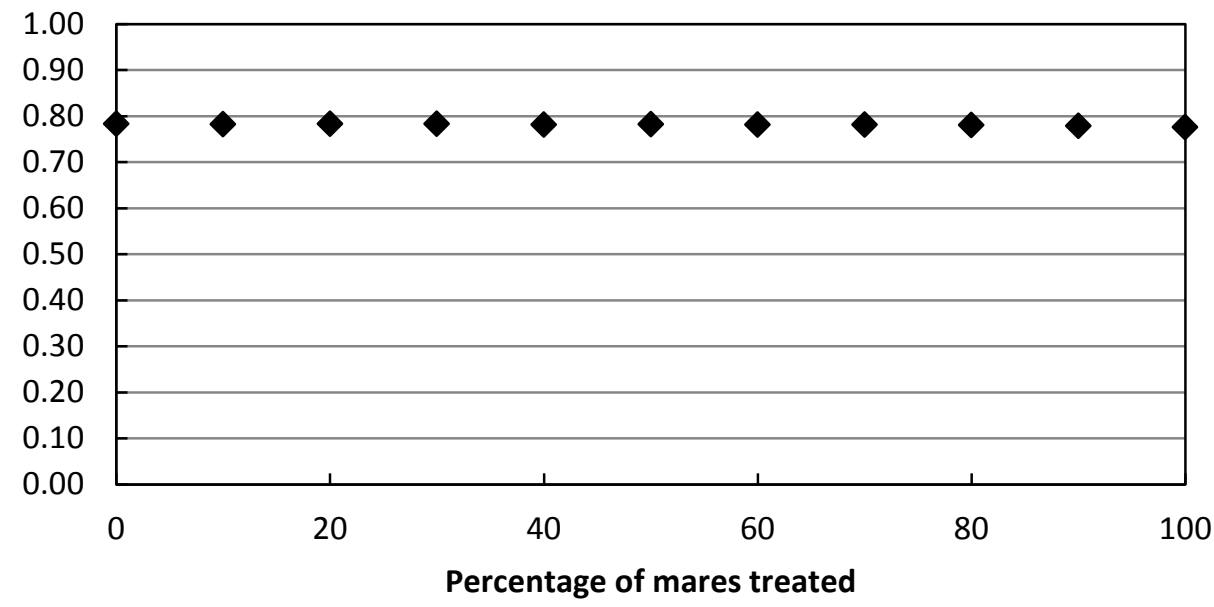




\begin{tabular}{lccccccccccc}
\hline \multicolumn{1}{c}{ Percentage of mares treated } & $\mathbf{0}$ & $\mathbf{1 0}$ & $\mathbf{2 0}$ & $\mathbf{3 0}$ & $\mathbf{4 0}$ & $\mathbf{5 0}$ & $\mathbf{6 0}$ & $\mathbf{7 0}$ & $\mathbf{8 0}$ & $\mathbf{9 0}$ & $\mathbf{1 0 0}$ \\
\hline Mean population size after 20 years & 1902.9 & 1845.4 & 1761.8 & 1664.4 & 1620.0 & 1726.7 & 1585.1 & 1496.6 & 1543.0 & 1414.6 & 1267.5 \\
Mean number of removals after 20 years & 5436.7 & 4770.0 & 4094.4 & 3748.1 & 3344.8 & 2876.4 & 2706.6 & 2411.4 & 2203.6 & 1906.3 & 1821.2 \\
Mean probability of extirpation after 50 years & 0 & 0 & 0 & 0 & 0 & 0 & 0 & 0 & 0 & 0 & 0 \\
Mean heterozygosity after 50 years & 0.78 & 0.78 & 0.78 & 0.78 & 0.78 & 0.78 & 0.78 & 0.78 & 0.78 & 0.78 & 0.78 \\
\hline
\end{tabular}

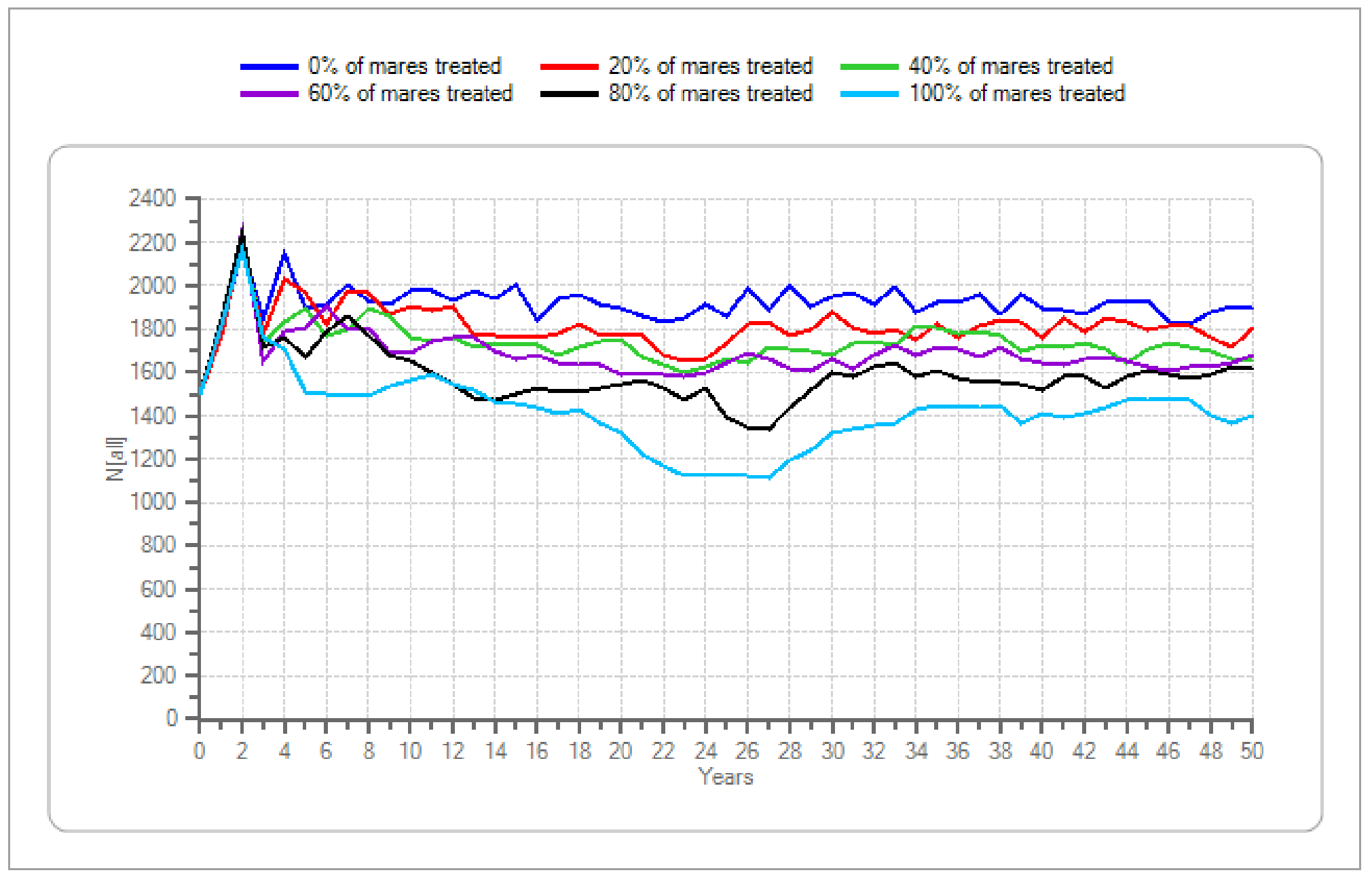

Figure 70. Initial population size $=1500$, lambda $=1.25$, genetic diversity $=$ high - Continued 